\title{
On the operation of visual cortical gamma in the light of frequency variation
}

Citation for published version (APA):

Lowet, E. P. (2016). On the operation of visual cortical gamma in the light of frequency variation. [Doctoral Thesis, Maastricht University]. Maastricht University. https://doi.org/10.26481/dis.20160323el

Document status and date:

Published: 01/01/2016

DOI:

10.26481/dis.20160323el

Document Version:

Publisher's PDF, also known as Version of record

Document license:

Unspecified

\section{Please check the document version of this publication:}

- A submitted manuscript is the version of the article upon submission and before peer-review. There can be important differences between the submitted version and the official published version of record.

People interested in the research are advised to contact the author for the final version of the publication, or visit the DOI to the publisher's website.

- The final author version and the galley proof are versions of the publication after peer review.

- The final published version features the final layout of the paper including the volume, issue and page numbers.

Link to publication

\footnotetext{
General rights rights.

- You may freely distribute the URL identifying the publication in the public portal. please follow below link for the End User Agreement:

www.umlib.nl/taverne-license

Take down policy

If you believe that this document breaches copyright please contact us at:

repository@maastrichtuniversity.nl

providing details and we will investigate your claim.
}

Copyright and moral rights for the publications made accessible in the public portal are retained by the authors and/or other copyright owners and it is a condition of accessing publications that users recognise and abide by the legal requirements associated with these

- Users may download and print one copy of any publication from the public portal for the purpose of private study or research.

- You may not further distribute the material or use it for any profit-making activity or commercial gain

If the publication is distributed under the terms of Article $25 \mathrm{fa}$ of the Dutch Copyright Act, indicated by the "Taverne" license above, 


\section{On the operation of visual cortical gamma in the light of frequency variation}

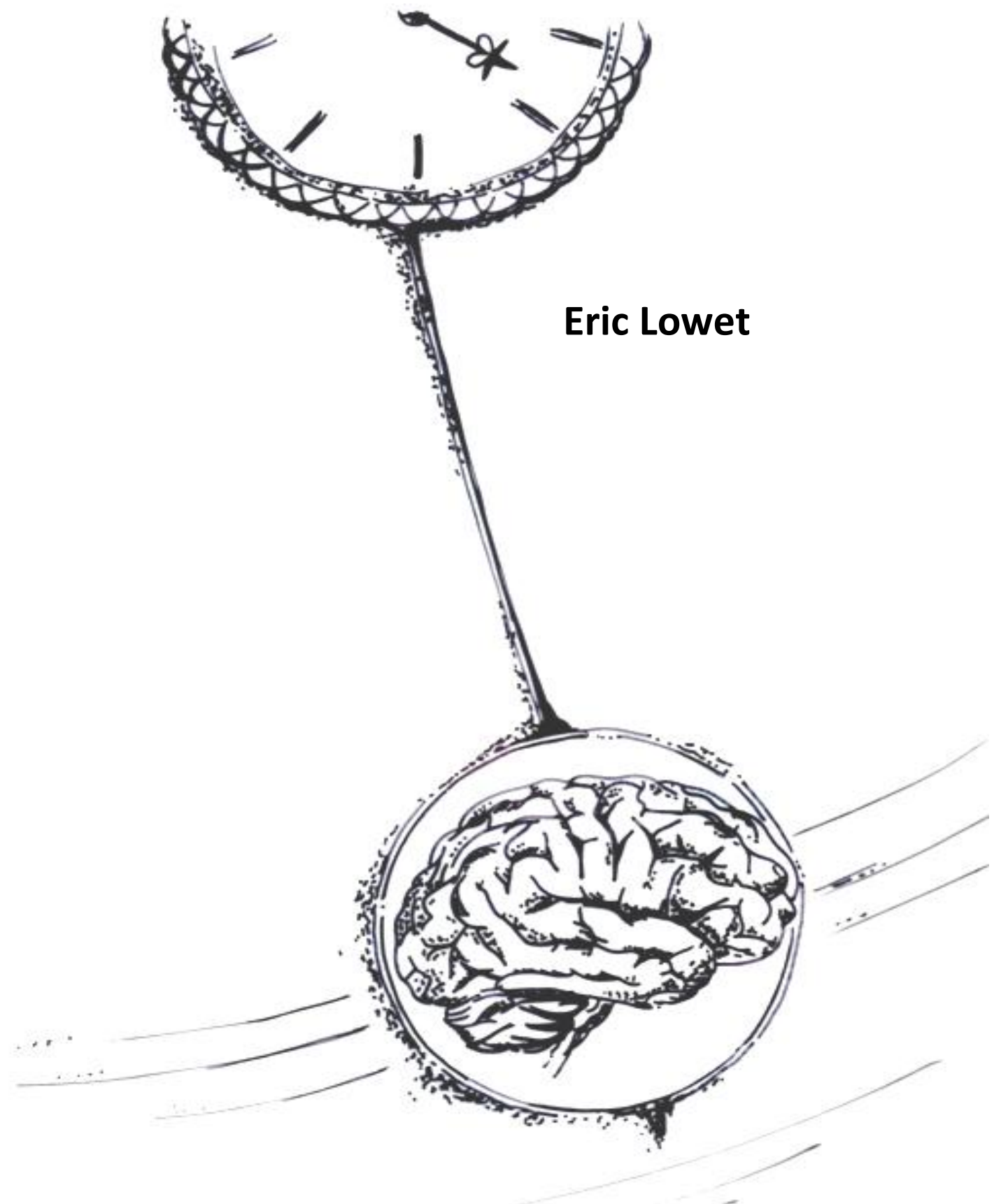


No part of this publication may be reproduced, stored in an automated data system or transmitted in any form or by any means, electronic, mechanical or photocopying, recording or otherwise, without prior permission of the author.

(C) Eric Lowet, Maastricht, 2016

Cover Pixel Art by

ISBN 978-94-6203-612-3 


\title{
On the operation of visual cortical gamma in the light of frequency variation
}

\author{
Dissertation \\ to obtain the degree of Doctor at \\ Maastricht University, \\ on the authority of the Rector Magnificus, Prof.dr. L.L.G Soete \\ in accordance with the decision of the Board of Deans, \\ to be defended in public \\ on Wednesday 23 March 2016, at 16.00 hours \\ by
}

Eric Lowet 


\section{Supervisor:}

Prof. dr. Peter De Weerd

\section{Co-supervisor:}

Dr. Mark Roberts

\section{Assessment committee:}

Prof. dr. Bernadette Jansma (chair)

Prof. dr. Rainer Goebel

Dr. Judith Peters

Prof dr. Wolf Singer (Ernst Strüngmann Institute, Frankfurt, Germany)

Dr. Eric Maris (Radboud University, Nijmegen, Netherlands) 


\section{Contents}

$\begin{array}{llr}\text { Chapter } 1 \text { Introduction } & \text { 4- } 20\end{array}$

Chapter 2 Robust gamma coherence between macaque V1 21-61 and $\mathrm{V} 2$ by dynamic frequency matching

Chapter 3 Parametric variation of gamma frequency and 62-90 power with luminance contrast: A comparative study of human MEG and monkey LFP and spike responses

Chapter 4 Areas V1 and V2 show microsaccade-related 3$4 \mathrm{~Hz}$ covariation in gamma power and frequency

Chapter 5 Input-dependent frequency modulation of 105-161 cortical gamma oscillations shapes spatial synchronization and enables phase coding

Chapter 6 Problems with spectral coherence as a measure 162-199 for quantifying oscillatory synchronization

Chapter 7 Gamma-band coordination of synchrony and 200-231 spike timing in visual cortex predicted by the theory of weakly coupled oscillators

Chapter 8 Summary and Conclusion 


\section{Chapter 1: Introduction}

The emergence of intelligent organisms, being able to move, sense and interact with their environment, is one of the most fascinating phenomena occurring in our universe. Neuroscience is the scientific endeavor to understand the biophysical principles underlying the intelligence of neural systems. Neuroscience is a relatively new and interdisciplinary research field, which has become established during the course of the 20th century, integrating investigators from computer science, physics, biology, psychology, philosophy and many more disciplines. Today, neuroscience investigates neural systems using fascinating theoretical and experimental methods in various organisms including insects, amphibians, reptiles, birds and mammals with the most important being rodents and primates. Amazing progress in the understanding of neural systems has been achieved. Examples of the amazing progress afforded by modern neuroscience include insights into the mechanism of action potential generation or 'spiking', (Catterall et al., 2012), into synaptic plasticity underlying learning (Kandel, 2009), and into cortical architecture (Hubel and Wiesel, 1969, 1959; Mountcastle, 1957). Yet many fundamental questions remain largely open, the principles of information coding during sensory processing and cognition being a prime example (Ainsworth et al., 2012; Ferster and Spruston, 1995). To address this question, both experimental as well as theoretical neurosciences have contributed their part to the progress. While a close and mutual interaction of theory and empirical testing is critical, as in many other science fields, the gap between researchers with a theoretical focus and those with an empirical focus has remained difficult to bridge. The present doctoral thesis focuses on the role of oscillatory neural activity in information coding and neural communication. In this work, we use both theoretical and empirical techniques to improve the understanding of oscillatory activity in neural networks.

\section{General introduction into cortical dynamics}

The information processing capacity of organisms is largely based on a class of highly specialized cells, the neurons. Neurons communicate with each other. They send information in the form of small electrical impulses over their axons, which can be compared to electrical wires. These electrical impulses are referred to as action potentials (spikes). At the end of the axon, neurons make connections with other neurons through synapses, where spikes are converted into a chemical response affecting the membrane of the receiving neuron. This puts in motion a cascade of cellular events that under specific conditions can generate an action potential in the receiving neuron, which can in turn send that action potential forward.

Synapses are highly complex structures that determine how neurons will 'listen' to each other. Many neurons constitute neural networks (neural circuits) that, through the collective coordinated behavior of many neurons, have amazing computational power (Hinton, 2000). Over the long time scale of evolution, the complexity of animal behavior has increased and so has the number and complexity of neurons and their networks. The mammalian brain, in particular the cerebral cortex, is constituted of many billions of neurons and the complexity and function of brain regions until today is poorly understood. 
Neurons coordinate their activity among each other (spatially) in a temporally precise manner on a millisecond scale. Neurons likely do not process information independent from their neighbors, but because each neuron is influenced by thousands of other neurons, information processing is largely a collective endeavor. A spike of single neurons can be understood only in the context of the spiking patterns of other neurons. Analogously, from letters alone the message cannot be understood. One needs to understand the combination of letters ('words', 'sentences') to appreciate the message. Hence, the investigation of the spatio-temporal patterns of neural spiking is key to get an understanding of how our nervous system implements computations and how it generates meaningful 'messages' for 'internal communication'. The technological evolution in neurophysiological recordings, which evolved from single cell neurophysiology to a variety of techniques permitting the recording of population activity, has contributed tremendously to our ability to start decoding 'messages' in patterns of activity in neuronal networks. On the one hand, there is constant improvement in our ability to record spikes from significant numbers of neurons (on the order of 10 to several hundreds) at the same time. On the other hand, modern recording equipment and storage capabilities allow one to measure local electrical field potentials, the so-called local field potential (LFP). The LFP constitutes a local neural population signal arising from changes in the electrical potential of the extracellular space due to neural activity in large numbers of neurons. A change in the LFP signifies that many neurons (on the order of hundreds of thousands) surrounding the measuring electrode are collectively changing activity. The LFP has spatial resolution of less than $1 \mathrm{~mm}$ and permits the investigation of coordinated activity across cortical columns and cortical lamina. Hence, LFPs are a crucial tool in studying network activity with relatively high spatial resolution, and when LFPs are combined with simultaneously recorded spiking activity in a comparatively small set neurons, one has excellent tools in hand to study network activity and coding. Note that Electroencephalography (EEG) and the magneto-encephalography (MEG) in essence measure the electro- or magnetic fields, respectively, around the brain, which ultimately are derived from the combined electrical changes in the brain. These field potentials allow investigation of collective neuronal activity and their dynamics non-invasively in humans. However, the spatial resolution of these signals unfortunately is poor, and sources of the electromagnetic fields can be localized only at a centimeter scale. Hence, there is so far no alternative for invasive recording methods if one wants to understand the neural signature of information coding and communication.

\section{What are oscillations?}

An oscillation refers to a repetitive re-occurrence of a change in state in a system. Oscillations have been described in many physical systems (Pikovsky, 2003), from molecular to astronomical level. Examples of oscillations are oscillations in the earth climate (Scafetta, 2010), in animal population size (Nicholson, 1954), or our heart (Shaffer et al., 2014). Oscillatory patterns in neural activity have also been observed in various structures of the nervous system (neocortex, thalamus, basal ganglia, retina, spinal cord and many more) and in essentially all animal species (insects, amphibians, reptiles, birds, mammals). This indicates that the capacity of generating oscillations is a general property of neural networks in the animal kingdom that likely arose early during evolution. 
In a neural network, at any moment in time, many neurons in the network will spike and influence each other. Hence, the probability of a neuron to spike will strongly depend on the input from other neurons it receives from the network it is embedded in. When a neuron receives many spikes from surrounding neurons, the neuron will likely also spike. Hence, the connectivity of a network induces correlations of spike timing among neurons. This means that the likelihood of a spike of neuron $X$ depends on whether a connected neuron $Y$ has fired or not. Correlation peaks in cross-correlograms between the spiking of two neurons has been well documented over the last decades (e.g., (Hirabayashi and Miyashita, 2005; Ts'o et al., 1986). When correlation peaks in the cross-correlation histogram are not localized on zero, but instead are shifted from zero, then this is suggestive of a time lag, meaning that neuron $X$ will fire with a time-lag $t$ after or before neuron $Y$. If neurons spike precisely at the same time (time lag 0 ), neurons are often termed as to spiking in synchrony. These observed correlations have fundamental implications for the nature of coding and computations used by our nervous system (Salinas and Sejnowski, 2001). Moreover, these correlations are indicative of timing relationships between spiking in different neurons, which are exploited by mechanisms of learning and memory according to principles of Hebbian plasticity (Caporale and Dan, 2008; Gallistel and Matzel, 2011).

In networks, correlations often go hand in hand with the emergence of waves of activity that can occur periodically over time (frequency), which are termed oscillations. Oscillations in neural networks have been already been described in the early 20th century by Berger (Tudor et al., 2005) using EEG. He found that electrical fields generated by the brain had distinct oscillatory components. Classically, periodic component in neural signals can be demonstrated using Fourier analysis. The Fourier analysis (developed by Joseph Fourier in 1822) decomposes signals into sinusoids of different frequencies, amplitudes and phases. By applying Fourier analysis one can transform signals from the time-domain to the frequency-domain (Bracewell, 1989). The power spectrum (periodogram) shows which frequencies contribute most power (squared amplitude) to the signal. If a signal is evolving purely randomly, the power spectrum will be smooth and flat (white noise). If the signal has a periodic component, particular frequencies will contain most of the power of the signal. An oscillatory process will therefore induce a clear peak in Fourier power spectrum. However, not every peak in the power spectrum necessarily signifies an oscillatory process. Further steps (more detailed analysis of the temporal characteristics) need to be undertaken to demonstrate that an oscillation is really present. The power spectrum of neural signals is often characterized by two key features: The power-law scaling and the presence of several distinct peaks. The former means that the power decays with increasing frequency (power law exponent of -1). Power law behavior is often observed in natural phenomena and often indicates hierarchical and scale invariant processes (Miller et al., 2009; Pritchard, 1992). The latter (presence of several peaks) means that there are several particular 'frequency bands' which contain a major part of the power, indicating that the recorded neural signal contains several periodic components or oscillations.

The documentation and investigation of particular frequency bands has attracted scientific interest already early in the 20th century. These periodic components can be observed both in population signals like LFP/EEG and in single-cell recordings (in spike trains, and in variations of intracellular voltage). Peaks in the power spectrum are classified based on historical/pragmatic reasons according to 
frequency bands including delta $(2-4 \mathrm{~Hz})$, theta $(4-8 \mathrm{~Hz})$, alpha $(8-12 \mathrm{~Hz})$, beta $(12-25 \mathrm{~Hz})$, gamma $(25-80 \mathrm{~Hz})$ and high gamma/epsilon $(80-200 \mathrm{~Hz})$. For example, prominent oscillations in the alpha range $(8-12 \mathrm{~Hz})$ had been already found by Hans Berger in the 1930s in humans EEG recordings of the visual cortex (Berger, 1935). Throughout history, more or less successful attempts have been done to associate certain frequency bands with specific functions. For example, the power of alpha increases strongly if eyes are closed (Redlich et al., 1946), which has led to suggestions that alpha is related to awareness. Another prominent example of the functional significance of neural oscillations is the classification of sleep stages (Loomis et al., 1937). Sleep is characterized by a succession of different stages (e.g. rapid eye-movement stage, REM), which can be distinguished based on the oscillatory content of neural signals. This has been shown to be very useful for the monitoring of human patients. Neural signals often consist of several distinct oscillatory components occurring in different frequency bands. It has moreover been shown that the different oscillations interact with each other. The trajectory of a higher frequency oscillation can depend on the trajectory of a slower oscillation. This interaction has been termed cross-frequency coupling (CFC) and has reached broad scientific interest only in the last decades (Canolty and Knight, 2010; Jensen and Colgin, 2007).

The complex dynamics of these oscillations as well as the exact biophysical principles underlying them, is untill today a tremendous challenge. How these different oscillations and their interactions are produced in the highly complex neural networks, as for example in the mammalian neocortex, is challenging to approach with theoretical modeling techniques due to many unknown variables. Experimentally they are difficult to approach for two major reasons. First, one needs to measure the dynamics with high spatial-temporal resolution and be able to measure all essential processes in the network. Unfortunately it is difficult to know which elements of the network activity are essential to improve understanding. Secondly, methodologically, many data analysis techniques have difficulty to deal with data complexity (e.g., non-stationarity and non-linearity). These challenges are particularly exemplified in the investigation of cortical gamma oscillations; the topic of this thesis. In this work, it will be shown that by using a combined approach of precise theoretical modeling, experimental testing and use of state-of-the art data analysis techniques an improved understanding about the underlying principles governing the complex dynamics of gamma oscillations can be achieved.

\section{What are gamma oscillations?}

Gamma oscillations, sometimes also called beta-gamma oscillations (Feng et al., 2010b), are periodic components in neural signals in the frequency range between $25 \mathrm{~Hz}$ up to $80 \mathrm{~Hz}$ (there is no agreed exact definition of the range among researchers). In general, empirical and theoretical research has indicated that increased gamma oscillation power is a signature of activated neuronal networks. For example, electrical stimulation of the cholinergic fibers in the nucleus of Meynert, which is critical for arousal regulation, induces widespread gamma oscillations in the neocortex (Goard and Dan, 2009). Gamma can also be induced by external stimuli (Gray and Singer, 1989) and top-down attention (Fries et al., 2001) and is related with the metabolism of the cortical tissue (Niessing et al., 2005). Direct application of glutamate (Traub et al., 1996) increases gamma oscillation power and frequency. In sedated and sleep brain states, gamma oscillation emerge in 'spontaneous up-states' (Haider and McCormick, 2009). The 
underlying generation mechanisms of gamma oscillations are still not completely understood. Yet, the critical role of inhibitory neurons is well established (X.-J. Wang \& Buzsaki, 1996; Buzsáki \& Wang, 2012). Inhibitory neurons exhibit resonance properties in the gamma frequency range (Cardin et al., 2009; Fellous et al., 2001). The inhibitory neurons get input from surrounding excitatory neurons (Tiesinga and Sejnowski, 2009) which get rhythmically inhibited and entrained by the inhibitory neurons. If certain inhibitory neurons are depolarized enough they will silence the whole network, both excitatory and other inhibitory neurons, for a certain time. The inhibitory effect of the most depolarized neurons will entrain the other into an oscillation rhythm, because they will tend to fire spikes between the time when inhibition has decayed and the time at which a new volley of inhibition is arising. The oscillation period is mainly defined by the decay time constant of inhibition and the ability of neurons to recover from inhibition (Traub et al., 1996). Gamma oscillations networks need a sufficient amount of excitatory drive to start oscillating (Tiesinga and Sejnowski, 2009; Traub et al., 1996), and hence neurons need to interact sufficiently (through spikes) to entrain or synchronize each other.

The detailed description of gamma oscillations has occurred later in neuroscience history than for their lower frequency counterparts (particular alpha rhythm). This is because it is only with the emergence of digitalization of EEG that the resolution of recordings became sufficient to study faster rhythms in detail. However, early reports of 'fast rhythms' can already be found around the 1930s (Adrian and Matthews, 1934). In the primate visual cortex, Hughes (1964) published one of the first reports of gamma oscillations recorded using microelectrodes. Since then, a vast number of studies have shown that gamma oscillations emerge in various brain structures and in various animals (see Fries, 2009). Since the late eighties the studies on gamma oscillations has strongly increased with a peak in popularity in the nineties (Crick and Koch, 1990; Engel et al., 1999; Gold, 1999). Yet, the understanding of gamma oscillations has remained until today a field of great interest shared by many neuroscientists (Buzsaki \& Wang, 2012; Fries, 2009). It was especially in the eighties and early nineties that due to a series of experimental findings in cat and primate visual system, scientific attention towards gamma oscillations dramatically increased. It was found that gamma oscillation pattern were robustly present in stimulated visual cortical neurons (Gray and Singer, 1989). Moreover, gamma oscillation power was reported to increase strongly especially when the neurons were stimulated with their preferred visual stimulus. The emergence of gamma was hence stimulus-input specific. Further, nearby neurons exhibited synchronous or coherent oscillations. That means that they showed a cross-correlation peak in the gamma oscillation range. It was further observed that the neurons were gamma coherent if they shared the same (visual) stimulus input coding preference and moreover, it was discovered that gamma coherence was more likely coherent if the neurons were stimulated by the same visual object (Eckhorn et al., 2001; Gray and Singer, 1989; König and Schillen, 1991). To summarize, the accumulated experimental evidence indicated that gamma oscillations emerge in a stimulus-input specific manner and that neighboring neurons synchronize to a shared oscillation when coding for similar input properties (Engel et al., 1999; König et al., 1996; Singer, 1999a, 1999b). 


\section{What is the function of gamma oscillations?}

The idea that neurons would engage in the same gamma rhythm if they are engaged in the encoding of the same object or object feature has led to several profound theoretical proposals for the function of gamma oscillations. One such proposal is that gamma oscillation may be critical for solving the 'binding problem' (Malsburg, 1995; Treisman, 1996). We perceive in our visual consciousness objects as coherent entities characterized by form, motion, color, textures and other properties. Yet, in our brain, the information on difference stimulus dimensions (e.g., color and motion) are processed in distinct brain networks. How is the separate information put together such that we can perceive objects as a coherent whole? It was suggested by several leading researchers that gamma oscillatory synchronization could be the underlying mechanism to integrate information over the different brain regions. Neurons coding for different parameters of the same object would be coherent to a common gamma rhythm (Crick and Koch, 1990; Engel et al., 1999; Gold, 1999; Meador et al., 2002), permitting communication and linking of disparate information. In this context, during the 90's and early 2000s, the gamma rhythm was a 'hot' topic in neuroscience and was considered as an elementary process for 'perception' and 'consciousness'.

However, a number of studies have reported data that was interpreted as contradictory with the view that gamma oscillation synchrony could solve the binding problem (Palanca and DeAngelis, 2005; Shadlen and Movshon, 1999). It was found that gamma synchronization is a local and spatially specific phenomenon (Eckhorn et al., 2001; Ray and Maunsell, 2010; Shadlen and Movshon, 1999). In addition, neurons could be de-coherent in the gamma range even if coding for the same object (Gail et al., 2000; Ray and Maunsell, 2010) and gamma oscillations seemed not always present or weak during visual processing (Berens et al., 2008; Brunet et al., 2013; Hermes et al., 2014; Kayser, 2003). These findings did not support the 'binding-by-synchrony' hypothesis. According to a number of the cited investigators, the local, variable and complex nature of gamma oscillations make it a poor candidate to solve the (global) binding problem as posed by (Malsburg, 1995). Importantly, it is possible to distinguish a global and a more local component in the binding-by-gamma hypothesis. The global hypothesis emphasizes the role of gamma for integrating information among disparate populations of neurons, spread over the whole brain. However, it is also possible that the primary function of gamma is more local, and that its primary role is to integrate information in smaller spatially restricted networks. There are several studies supporting a role of gamma in more local 'binding' or 'feature integration' processes (Eckhorn, 2000, 1999; Singer and Gray, 1995). Perhaps this view of the role of gamma was more defensible, but this idea has not been followed up. This is likely due to the fact that gamma oscillations lost much of their attraction to many neuroscientists as the limitations of the hypothesis became clear, and, as another extreme, a viewpoint gained influence in which gamma was seen as unimportant and uninteresting.

Nevertheless, in the last decade, a number of investigators have continued to work on gamma, and considerable advances in the understanding of gamma oscillation have been achieved. It has been found that neural populations for distant cortical locations can be coherent in the gamma range, yet only if they are directly connected with each other (Bosman et al., 2012; Gregoriou et al., 2009; Roberts et al., 2013). This contrasts with previous suggestions (Crick and Koch, 1990; Engel et al., 1999; Malsburg, 1995 ) that gamma would engage into widespread synchronization in the brain (spatially unspecific). The 
strength of the coherence was found to be sensitive to the behavioral state of the animal, particularly with attention (Bosman et al., 2012; Fries et al., 2001; Womelsdorf et al., 2007). In these studies, when an animal focused its attention to a particular part of the visual space, it was found that neurons with RFs in the regions selected by attention started to exhibit stronger coherent gamma oscillations to higher-order cortical regions. This is of high relevance as cortical areas are hierarchically organized (Van Essen and Maunsell, 1983) so that lower-order areas (e.g. V1) have strong convergent connectivity to higher-order areas (e.g. V4 or TEO). From the perspective of high-order neurons, it means that they receive various inputs from lower-order neurons from different cortical locations, creating the so-called 'bottleneck problem' (Moran and Desimone, 1985). It has been argued that a solution to this mass of bottom-up input is an effective and flexible selection mechanism such that a higher-order neuron reduces its sensitivity only to a fraction of the input for a given time moment (Desimone, 1998; Kastner et al., 1998; Moran and Desimone, 1985). It was proposed that this could be the underlying mechanism for attention selection. For any given time moment, we selectively process in depth only a fraction of all information present in our environment, due to limitation of information processing capacities. A series of papers have suggested that gamma synchronization between cortical regions may be critical for the enhanced and selective information routing in the brain during attention. The hypothesis has been termed 'communication through coherence' (CTC, (Fries, 2005)). The idea is that enhanced coherence of gamma oscillating neurons enhances communication among them and blocks other neurons from communicating if they are not coherent (Tiesinga and Sejnowski, 2010). Recent experimental findings in the macaque visual cortex have given support to the hypothesis (Bosman et al., 2012). However, a weakness of the hypothesis is that the implied causation of increased communication through coherence is not well established yet (Rolls et al., 2012). Theoretically, both causal directions are possible and an experimental clarification has not yet been achieved.

There are strong similarities between the 'binding hypothesis' and the 'communication by coherence' hypotheses of gamma oscillations. In both hypotheses, the ability of gamma oscillating neurons to functionally engage or disengage into synchronous 'ensembles' is critical. In the binding hypothesis, this functional grouping is used for grouping neurons coding for the same object (Engel et al., 1999; Meador et al., 2002) or to group local neurons according to their coding similarity (Eckhorn et al., 2001; Singer, 1999b). The 'grouping by gamma coherence' is used here as an organization tool for representing and decomposing the bottom-up input into meaningful clusters/segments (Eckhorn, 1999; König and Schillen, 1991; Kuntimad and Ranganath, 1999). The focus of experimental testing for this hypothesis has been gamma oscillation synchronization within a cortical area (particularly V1). In the communication by coherence hypothesis, the functional grouping is used to select some neurons over others for selective information transmission to the next cortical processing level. The 'grouping by gamma coherence' is used here to group neurons of 'interest' (selected by attention) from noninteresting neurons and to share the information they are processing across cortical areas (Börgers et al., 2005; Zeitler et al., 2008). The focus of experimental testing involves the measurement of gamma oscillation synchronization among cortical areas. From the summary of the two hypotheses, it becomes clear that they are neither contradictory nor fundamentally different in their key description of gamma oscillatory mechanism. They differ in their functional association ('visual segmentation/binding' vs. 'attentional selection'), yet these functions are related to each other or even complementary. Indeed, 
the 'pre-attentive' segmentation of incoming input is a good basis for further attentional selection (Treisman, 1985).

Given the fact that the two models share their most important assumptions, it is not surprising that they also share the same weaknesses. Both of the proposed models define insufficiently how the grouping/selection by gamma oscillations is done. On one possible model, neurons are either synchronized or not to determine their relatedness (Börgers et al., 2005), with synchrony/coherence defined as sharing of a common frequency (frequency matching). In another possible model, neurons are synchronized to a common frequency, yet they spike at different phases within the oscillation cycle (McLelland and Paulsen, 2009; Montemurro et al., 2008). This can be considered as being a phase code (whereas the former is rather a frequency code). For example, theoretical models of the 'gamma binding hypothesis' define neural grouping either in terms of modulations in coherence (König and Schillen, 1991) or in terms of different phases (Wang and Terman, 1997). The same can be seen to some extent for the 'communication by coherence' hypothesis (Fries, 2005; Hoppensteadt and Izhikevich, 1998; Tiesinga and Sejnowski, 2010; Zeitler et al., 2008). It is not clear whether gamma oscillating networks use frequency matching versus phase-locking and phase differences as organization codes, and it is not specified how these different mechanisms interact with each other. Interestingly, there is experimental evidence for both types of organization. There is substantial evidence that phase-locking (defined as the co-occurrence of spikes at the same phase in a shared gamma rhythm) between nearby neurons can be dependent on stimulus-input properties and hence that it can change adaptively depending on input conditions (Gail et al., 2000; Gray and Singer, 1989). On the other hand, there is also evidence for gamma phase coding in visual cortex (defined as the coding by differences in phase among different neurons contributing to the same gamma rhythm at a shared frequency) (Maris et al., 2013; Vinck et al., 2010). Thus, these two types of order appear to coexist in cortical networks. Future research will need to address how the two coordination-by-gamma mechanisms relate to each other.

As can be suspected from the existence of many frequency bands in the brain other than gamma, it is clear that the coordination of neural activity is not organized only by gamma. Gamma oscillations depend on many other processes occurring in neural networks, among them other oscillatory processes. For example, it has been shown that gamma oscillation fluctuate strongly in power with delta/theta oscillations (3-8Hz, (Lakatos et al., 2005)), as is particularly clear in the rodent hippocampus (Belluscio et al., 2012; Lisman and Jensen, 2013). Alpha (8-12Hz)-gamma coupling has also been shown (Osipova et al., 2008; Spaak et al., 2012). Furthermore, saccadic and micro-saccadic eye movements, that occur 1-4 times a second, have strong impact on gamma oscillation dynamics (Bosman et al., 2009; Brunet et al., 2013). As gamma oscillations display complex interactions with other rhythmic process in the brain, they should not be studied and modeled in isolation.

The brief overview in the present section of the function of gamma was limited to its potential contribution to grouping/binding as well as neural communication. In this overview, it was implicitly assumed that the connections in the studied neural network are fixed. However, the brain continually adapts to experience, and it has been proposed that gamma oscillation might be well-suited to promote and support the underlying synaptic plasticity processes. This is in line with the observation that electrical stimulation protocols using pulses in the gamma frequency range successfully induce long- 
term potentiating of synapses (Lisman and Spruston, 2005). This is also in line with the idea that precise synchrony and time-delays are essential in inducing plasticity (spike-timing dependent plasticity, STDP, (Caporale and Dan, 2008; Knoblauch et al., 2012; Lee et al., 2009; Masquelier et al., 2009)). Neurons synchronized in the gamma range do exhibit precise spike synchrony in a millisecond range which may facilitate synaptic plasticity. The relationship between gamma and plasticity falls outside the scope of the present doctoral thesis, but increased insight into the function of gamma and its coding mechanisms will facilitate subsequent research on the role of gamma in neural plasticity and learning.

\section{Current challenges in understanding the role of gamma oscillations}

Neural communication in the gamma frequency range requires that there is a sufficient match in frequency among communicating neural populations, as well as a systematic phase relationship that permits the exchange of information (spikes). However, it is currently not understood if and how gamma frequency and phase are used to code information and to transmit that information (previous section). Recently, several investigators have formulated strong challenges against the role of gamma oscillations in information processing (e.g., Merker, 2013; Ray \& Maunsell, 2014). These challenges were based on findings that the precise frequency of gamma oscillations varies strongly in short time intervals (Burns et al., 2011, 2010; Xing et al., 2012) and that frequency changes as a function of stimulus-input properties like stimulus contrast (Jia et al., 2013; Ray and Maunsell, 2010; Roberts et al., 2013), orientation (Feng, Havenith, Wang, Singer, \& Nikolic, 2010; Jia et al., 2013) and size (Gieselmann and Thiele, 2008; Jia et al., 2013). These important findings challenged previous conceptions of gamma oscillations as being a rather stable ('clock-like') timing framework (Buzsáki and Draguhn, 2004; Gold, 1999; Steriade et al., 1996; Traub et al., 1996). In the 90's the conception of gamma oscillations as a stable timing reference was popular and the term of ' $40 \mathrm{~Hz}^{\prime}$ oscillations suggested that gamma oscillation have a stable frequency over time (e.g. Desmedt \& Tomberg, 1994; Fisahn, Pike, Buhl, \& Paulsen, 1998; K.-H. Lee, Williams, Haig, Goldberg, \& Gordon, 2001). Nevertheless, already during that time several researchers had observed that the frequency of gamma oscillations is flexible (Gray and Singer, 1989; Steriade et al., 1996). For example, it was shown by Steriade et al. (1996) that gamma oscillations shift frequency on a relatively fast time scale during slower fluctuations of neural excitability (Steriade et al., 1996). However, at that time the significance of variations in oscillation frequency was neither further explored experimentally nor studied theoretically. It was not until the recent studies by Burns who investigated the moment-by-moment gamma frequency variation in awake monkey cortex that the flexible nature of gamma oscillation frequency was described and discussed. In fact, the variation is so complex that it was described as being 'random' or 'chaotic' (Burns et al., 2011). The apparently random variability of gamma oscillation frequency seemed to be in contradiction with a possible role of gamma in information processing, although this view was directly questioned by other researchers (Nikolić et al., 2013).

Among the studies that showed a relationship between gamma frequency and stimulus properties, studies using contrast manipulations had a strong impact (Jia et al., 2013; Ray and Maunsell, 2010). This is because the relationship between gamma frequency and stimulus contrast is particularly clear, and also because conditions were described in which the precise frequency of gamma oscillations could be 
different among nearby cortical locations all coding for the same stimulus (Ray and Maunsell, 2010). These findings were backed up by studies investigating the gamma response to natural stimuli (consisting of rapidly changing lower-level visual features, such as spatio-temporal contrast changes). These studies showed that gamma oscillations exhibit spatially and temporally differentiated responses to natural stimuli (Brunet et al., 2013; Hermes et al., 2014) that are quite different from the responses elicited by gratings. These findings questioned the role of gamma in meaningful functional grouping of neurons by synchronization, because oscillations can be in synchrony only if they share the same frequency (Ray and Maunsell, 2010). In other words, any form of phase-locking or any form of a systematic phase relation that would permit the exchange of spikes between populations of neurons would require a sufficient match in frequency. Hence, taking these findings together, significant doubt is bestowed upon the idea that neurons can be grouped into meaningful synchronous ensembles according to a fixed (' $\left.40 \mathrm{~Hz}^{\prime}\right)$ gamma frequency channel operating in a widespread manner in the brain.

To summarize, not much is known about the role and significance of flexible changes in the precise frequency of gamma oscillation, as particularly demonstrated by recent experimental observations. So far the variability in oscillation frequency has been seen as problematic and contradictory with the theoretical models of gamma oscillations and their contribution to information processing. This is understandable, because the theoretical models proposed so far have not explicitly described and included the role of oscillation frequency in their framework.

\section{Aim and outline of the thesis}

The primary aim of this thesis is to understand the mechanisms and the significance of the frequency variations of gamma oscillation for its synchronization behavior and for information processing, using theoretical as well as experimental techniques. The theoretical techniques included numerical simulations of biophysical plausible neural networks as well as more abstract mathematical models. In essence, neurons as well their synaptic interactions can be modeled by a set of differential equations, which over the last decades have been continuously refined. The neural networks composed of units interacting according to those differential equations can be studied with respect to their oscillation and synchronization properties and are an essential tool in helping to understand the experimental observations (Catterall et al., 2012) and in generating meaningful predictions. The empirical methods used to address the aims of the thesis consisted of experimental techniques for the measurement of spikes and LFPs through microelectrodes and depth probes in early visual cortical areas (V1 and V2) in awake macaque monkeys. The thesis is built around a productive interaction in which empirical data uncover the need for more quantitative models, and in which the models in turn generate predictions that are experimentally tested in empirical experiments. In the thesis, we used advanced data analysis techniques, and contributed to the development of new techniques, in particular to new techniques that can correctly estimate synchronization (phase relationships) in the context of the non-stationary properties of gamma oscillation dynamics. These new methods were necessary to be able to test model predictions in our data. 
In Chapter 2, we study the robustness of gamma oscillation coherence between macaque visual cortical areas V1 and V2 as a function of different stimulus contrasts, which are known to modulate the frequency of gamma. We also studied spontaneous frequency modulations at fixed contrasts. Moreover, we studied whether gamma coherence displayed cortical laminar specificities. To that aim we inserted two microelectrodes ('depth probes') simultaneously in V1 and V2 and recorded LFP as well spikes at different cortical depths. With this experiment we could explicitly test whether frequency variations, were spontaneous or induced by stimulus contrast. Indeed limits robust neural communication across areas. In Chapter 3, we aimed to replicate in the human visual cortex the finding of stimulus contrast dependency of the frequency of gamma oscillation, as reported in macaque monkeys. We measured gamma oscillatory signals in 9 humans using MEG and beamforming techniques (Vrba and Robinson, 2001). In this study, we systematically quantified spectral gamma properties, particular the dominant frequency, as a function of visual stimulus contrast. This study verified to what extent mechanisms underlying synchronization behavior in macaque visual cortex would be generalizable to humans. In Chapter 4, we used recording data from macaque V1 and V2 to test to what extent rapid fluctuations of gamma frequency, which had been described in the literature as random, could be related to lower rhythms. Furthermore, we aimed to test whether any structure hidden in the apparent randomness might be linked with could be linked with particular brain processed, such as eye movements (Bosman et al., 2009). In Chapter 5 the main theoretical work of the thesis is described. Using biophysically realistic neural networks (Hodgkin-Huxley type) as well more abstract mathematical models (phaseoscillator models) we systematically investigated the role of oscillation frequency in determining phaselocking and phase-relation among neighboring units, and the role of this frequency/phase interaction in information processing. This led to a specific theoretical model yielding precise predictions on the frequency and phase relations among neighboring, interacting neuronal populations exposed to differential input. To test these predictions in empirical data, we realized that common Fourier-based coherence methods are ill-suited to estimate phase-locking in empirical oscillatory synchronization data, which are by nature non-stationary. In Chapter 6 , we describe the nature of oscillatory synchronization in more detail with an emphasis on the partially synchronized state. We then show that synchronization occurring in the partially non-synchronized state cannot be captured by traditional Fourier-based methods. An alternative method will be outlined that is based on singular-spectrum decomposition of the signal (SSD) and the Hilbert Transform. This alternative method gave us a more robust and valid method to estimate phase-locking and phase-relation in fast frequency fluctuating oscillations. This method was applied in Chapter 7, where we experimentally test the predictions made from our theoretical work presented in Chapter 5. To that goal, we inserted three depth probes 2 to $3 \mathrm{~mm}$ apart in macaque visual cortex $\mathrm{V} 1$. We systematically modulated the frequency of gamma at the three probe locations using local stimulus contrast variation in order to test how the precise frequency influenced the phase-locking and phase-relation among nearby neural populations generating gamma oscillations. In Chapter 8 we will shortly summarize the work that has been done, indicate the contributions to current literature, and outline future directions of research. 


\section{References}

Adrian, E.D., Matthews, B.H.C., 1934. The interpretation of potential waves in the cortex. J. Physiol. 81, 440-471. doi:10.1113/jphysiol.1934.sp003147

Ainsworth, M., Lee, S., Cunningham, M.O., Traub, R.D., Kopell, N.J., Whittington, M.A., 2012. Rates and rhythms: a synergistic view of frequency and temporal coding in neuronal networks. Neuron 75, 572-583. doi:10.1016/j.neuron.2012.08.004

Belluscio, M.A., Mizuseki, K., Schmidt, R., Kempter, R., Buzsaki, G., 2012. Cross-Frequency Phase-Phase Coupling between Theta and Gamma Oscillations in the Hippocampus. J. Neurosci. doi:10.1523/JNEUROSCI.4122-11.2012

Berens, P., Keliris, G.A., Ecker, A.S., Logothetis, N.K., Tolias, A.S., 2008. Feature selectivity of the gammaband of the local field potential in primate primary visual cortex. Front Neurosci 2, 199-207. doi:10.3389/neuro.01.037.2008

Berger, H., 1935. Über das Elektrenkephalogramm des Menschen. Arch. Psychiatr. Nervenkr. 103, 444454. doi:10.1007/BF02024891

Börgers, C., Epstein, S., Kopell, N.J., 2005. Background gamma rhythmicity and attention in cortical local circuits: a computational study. Proc. Natl. Acad. Sci. U. S. A. 102, 7002-7. doi:10.1073/pnas.0502366102

Bosman, C.A., Schoffelen, J.-M., Brunet, N., Oostenveld, R., Bastos, A.M., Womelsdorf, T., Rubehn, B., Stieglitz, T., De Weerd, P., Fries, P., 2012. Attentional stimulus selection through selective synchronization between monkey visual areas. Neuron $75,875-888$. doi:10.1016/j.neuron.2012.06.037

Bosman, C.A., Womelsdorf, T., Desimone, R., Fries, P., 2009. A microsaccadic rhythm modulates gammaband synchronization and behavior. J Neurosci 29, 9471-9480. doi:10.1523/JNEUROSCI.119309.2009

Bracewell, R.N., 1989. The Fourier transform. Sci. Am. 260, 86-89, 92-95.

Brunet, N., Bosman, C. a, Roberts, M., Oostenveld, R., Womelsdorf, T., De Weerd, P., Fries, P., 2013. Visual Cortical Gamma-Band Activity During Free Viewing of Natural Images. Cereb. Cortex 1-9. doi:10.1093/cercor/bht280

Burns, S.P., Xing, D., Shapley, R.M., 2011. Is gamma-band activity in the local field potential of V1 cortex a "clock" or filtered noise? J Neurosci 31, 9658-9664. doi:10.1523/JNEUROSCI.0660-11.2011

Burns, S.P., Xing, D., Shelley, M.J., Shapley, R.M., 2010. Searching for autocoherence in the cortical network with a time-frequency analysis of the local field potential. J Neurosci 30, 4033-4047. doi:10.1523/JNEUROSCI.5319-09.2010

Buzsáki, G., Draguhn, A., 2004. Neuronal oscillations in cortical networks. Science 304, 1926-9. doi:10.1126/science.1099745

Buzsáki, G., Wang, X.-J., 2012a. Mechanisms of gamma oscillations. Annu. Rev. Neurosci. 35, 203-25. doi:10.1146/annurev-neuro-062111-150444

Buzsáki, G., Wang, X.-J., 2012b. Mechanisms of gamma oscillations. Annu Rev Neurosci 35, 203-225. doi:10.1146/annurev-neuro-062111-150444

Canolty, R.T., Knight, R.T., 2010. The functional role of cross-frequency coupling. Trends Cogn. Sci. 14, 506-15. doi:10.1016/j.tics.2010.09.001

Caporale, N., Dan, Y., 2008. Spike timing-dependent plasticity: a Hebbian learning rule. Annu. Rev. Neurosci. 31, 25-46. doi:10.1146/annurev.neuro.31.060407.125639

Cardin, J.A., Carlén, M., Meletis, K., Knoblich, U., Zhang, F., Deisseroth, K., Tsai, L.-H., Moore, C.I., 2009. Driving fast-spiking cells induces gamma rhythm and controls sensory responses. Nature 459, 663667. doi:10.1038/nature08002 
Catterall, W.A., Raman, I.M., Robinson, H.P.C., Sejnowski, T.J., Paulsen, O., 2012. The Hodgkin-Huxley Heritage: From Channels to Circuits. J. Neurosci. doi:10.1523/JNEUROSCI.3403-12.2012

Crick, F., Koch, C., 1990. Some Reflections on Visual Awareness. Cold Spring Harb. Symp. Quant. Biol. 55, 953-962. doi:10.1101/SQB.1990.055.01.089

Desimone, R., 1998. Visual attention mediated by biased competition in extrastriate visual cortex. Philos. Trans. R. Soc. Lond. B. Biol. Sci. 353, 1245-55. doi:10.1098/rstb.1998.0280

Desmedt, J.E., Tomberg, C., 1994. Transient phase-locking of $40 \mathrm{~Hz}$ electrical oscillations in prefrontal and parietal human cortex reflects the process of conscious somatic perception. Neurosci. Lett. 168, 126-129. doi:10.1016/0304-3940(94)90432-4

Eckhorn, R., 2000. Cortical synchronization suggests neural principles of visual feature grouping. Acta Neurobiol Exp 60, 261-269.

Eckhorn, R., 1999. Neural mechanisms of scene segmentation: recordings from the visual cortex suggest basic circuits for linking field models. IEEE Trans Neural Netw 10, 464-479. doi:10.1109/72.761705

Eckhorn, R., Bruns, A., Saam, M., Gail, A., Gabriel, A., Brinksmeyer, H.J., 2001. Flexible cortical gammaband correlations suggest neural principles of visual processing. Vis. cogn. 8, 519-530. doi:10.1080/13506280143000098

Engel, A.K., Fries, P., König, P., Brecht, M., Singer, W., 1999. Temporal binding, binocular rivalry, and consciousness. Conscious. Cogn. 8, 128-51. doi:10.1006/ccog.1999.0389

Fellous, J.M., Houweling, A.R., Modi, R.H., Rao, R.P., Tiesinga, P.H., Sejnowski, T.J., 2001. Frequency dependence of spike timing reliability in cortical pyramidal cells and interneurons. J Neurophysiol 85, 1782-1787.

Feng, W., Havenith, M.N., Wang, P., Singer, W., Nikoli?, D., 2010a. Frequencies of gamma/beta oscillations are stably tuned to stimulus properties. Neuroreport 21, 680-684. doi:10.1097/WNR.0b013e32833ae9d1

Feng, W., Havenith, M.N., Wang, P., Singer, W., Nikolić, D., 2010b. Frequencies of gamma/beta oscillations are stably tuned to stimulus properties. Neuroreport 21, 680-4. doi:10.1097/WNR.0b013e32833ae9d1

Ferster, D., Spruston, N., 1995. Cracking the neural code. Science (80-. ). 270, 756-757.

Fisahn, A., Pike, F.G., Buhl, E.H., Paulsen, O., 1998. Cholinergic induction of network oscillations at $40 \mathrm{~Hz}$ in the hippocampus in vitro. Nature 394, 186-9. doi:10.1038/28179

Fries, P., 2009. Neuronal gamma-band synchronization as a fundamental process in cortical computation. Annu Rev Neurosci 32, 209-224. doi:10.1146/annurev.neuro.051508.135603

Fries, P., 2005. A mechanism for cognitive dynamics: neuronal communication through neuronal coherence. Trends Cogn. Sci. 9, 474-80. doi:10.1016/j.tics.2005.08.011

Fries, P., Reynolds, J.H., Rorie, A.E., Desimone, R., 2001. Modulation of oscillatory neuronal synchronization by selective visual attention. Science 291, 1560-1563. doi:10.1126/science.1055465

Gail, A., Brinksmeyer, H.J., Eckhorn, R., 2000. Contour decouples gamma activity across texture representation in monkey striate cortex. Cereb Cortex 10, 840-850.

Gallistel, C.R., Matzel, L.D., 2011. The Neuroscience of Learning: Beyond the Hebbian Synapse. Annu. Rev. Psychol. doi:10.1146/annurev-psych-113011-143807

Gieselmann, M. a, Thiele, a, 2008. Comparison of spatial integration and surround suppression characteristics in spiking activity and the local field potential in macaque V1. Eur. J. Neurosci. 28, 447-59. doi:10.1111/j.1460-9568.2008.06358.x

Goard, M., Dan, Y., 2009. Basal forebrain activation enhances cortical coding of natural scenes. Nat. Neurosci. 12, 1444-9. doi:10.1038/nn.2402

Gold, I., 1999. Does 40-Hz oscillation play a role in visual consciousness? Conscious. Cogn. 8, 186-95. doi:10.1006/ccog.1999.0399 
Gray, C.M., Singer, W., 1989. Stimulus-specific neuronal oscillations in orientation columns of cat visual cortex. Proc. Natl. Acad. Sci. U. S. A. 86, 1698-702.

Gregoriou, G.G., Gotts, S.J., Zhou, H., Desimone, R., 2009. High-frequency, long-range coupling between prefrontal and visual cortex during attention. Science 324, 1207-1210. doi:10.1126/science.1171402

Haider, B., McCormick, D.A., 2009. Rapid neocortical dynamics: cellular and network mechanisms. Neuron 62, 171-89. doi:10.1016/j.neuron.2009.04.008

Hermes, D., Miller, K.J., Wandell, B. a, Winawer, J., 2014. Stimulus Dependence of Gamma Oscillations in Human Visual Cortex. Cereb. Cortex 1-9. doi:10.1093/cercor/bhu091

Hinton, G.E., 2000. Computation by neural networks. Nat. Neurosci. 3 Suppl, 1170. doi:10.1038/81442

Hirabayashi, T., Miyashita, Y., 2005. Dynamically modulated spike correlation in monkey inferior temporal cortex depending on the feature configuration within a whole object. J. Neurosci. 25, 10299-10307. doi:10.1523/JNEUROSCI.3036-05.2005

Hoppensteadt, F.C., Izhikevich, E.M., 1998. Thalamo-cortical interactions modeled by weakly connected oscillators: could the brain use FM radio principles? Biosystems 48, 85-94.

Hubel, D.H., Wiesel, T.N., 1969. Anatomical demonstration of columns in the monkey striate cortex. Nature 221, 747-750. doi:10.1038/221747a0

Hubel, D.H., Wiesel, T.N., 1959. Receptive fields of single neurones in the cat's striate cortex. J. Physiol. 148, 574-591. doi:10.1113/jphysiol.2009.174151

Hughes, J.R., 1964. International Review of Neurobiology Volume 7, International Review of Neurobiology, International Review of Neurobiology. Elsevier. doi:10.1016/S0074-7742(08)60266-4

Jensen, O., Colgin, L.L., 2007. Cross-frequency coupling between neuronal oscillations. Trends Cogn. Sci. 11, 267-9. doi:10.1016/j.tics.2007.05.003

Jia, X., Xing, D., Kohn, A., 2013. No consistent relationship between gamma power and peak frequency in macaque primary visual cortex. J Neurosci 33, 17-25. doi:10.1523/JNEUROSCI.1687-12.2013

Kandel, E.R., 2009. The biology of memory: a forty-year perspective. J. Neurosci. 29, 12748-12756. doi:10.1523/JNEUROSCI.3958-09.2009

Kastner, S., De Weerd, P., Desimone, R., Ungerleider, L.G., 1998. Mechanisms of directed attention in the human extrastriate cortex as revealed by functional MRI. Science 282, 108-111. doi:10.1126/science.282.5386.108

Kayser, C., 2003. Responses to Natural Scenes in Cat V1. J. Neurophysiol. 90, 1910-1920. doi:10.1152/jn.00195.2003

Knoblauch, A., Hauser, F., Gewaltig, M.-O., Körner, E., Palm, G., 2012. Does spike-timing-dependent synaptic plasticity couple or decouple neurons firing in synchrony? Front. Comput. Neurosci. 6, 55. doi:10.3389/fncom.2012.00055

König, P., Engel, A.K., Singer, W., 1996. Integrator or coincidence detector? The role of the cortical neuron revisited. Trends Neurosci. doi:10.1016/S0166-2236(96)80019-1

König, P., Schillen, T.B., 1991. Stimulus-Dependent Assembly Formation of Oscillatory Responses: I. Synchronization. Neural Comput. 3, 155-166. doi:10.1162/neco.1991.3.2.155

Kuntimad, G., Ranganath, H.S., 1999. Perfect image segmentation using pulse coupled neural networks. IEEE Trans. Neural Netw. 10, 591-8. doi:10.1109/72.761716

Lakatos, P., Shah, A.S., Knuth, K.H., Ulbert, I., Karmos, G., Schroeder, C.E., 2005. An oscillatory hierarchy controlling neuronal excitability and stimulus processing in the auditory cortex. J Neurophysiol 94, 1904-1911. doi:10.1152/jn.00263.2005

Lee, K.-H., Williams, L.M., Haig, A., Goldberg, E., Gordon, E., 2001. An integration of $40 \mathrm{~Hz}$ Gamma and phasic arousal: novelty and routinization processing in schizophrenia. Clin. Neurophysiol. 112, 1499-1507. doi:10.1016/S1388-2457(01)00584-3 
Lee, S., Sen, K., Kopell, N., 2009. Cortical gamma rhythms modulate NMDAR-mediated spike timing dependent plasticity in a biophysical model. PLoS Comput Biol 5, e1000602. doi:10.1371/journal.pcbi.1000602

Lisman, J., Spruston, N., 2005. Postsynaptic depolarization requirements for LTP and LTD: a critique of spike timing-dependent plasticity. Nat. Neurosci. 8, 839-841. doi:10.1038/nn0705-839

Lisman, J.E., Jensen, O., 2013. The ?-? neural code. Neuron 77, 1002-1016. doi:10.1016/j.neuron.2013.03.007

Loomis, A.L., Harvey, E.N., Hobart, G.A., 1937. Cerebral states during sleep, as studied by human brain potentials. J. Exp. Psychol. doi:10.1037/h0057431

Malsburg, C. von der, 1995. Binding in models of perception and brain function. Curr. Opin. Neurobiol. 5, 520-526. doi:10.1016/0959-4388(95)80014-X

Maris, E., Womelsdorf, T., Desimone, R., Fries, P., 2013. Rhythmic neuronal synchronization in visual cortex entails spatial phase relation diversity that is modulated by stimulation and attention. Neuroimage 74, 99-116. doi:10.1016/j.neuroimage.2013.02.007

Masquelier, T., Hugues, E., Deco, G., Thorpe, S.J., 2009. Oscillations, phase-of-firing coding, and spike timing-dependent plasticity: an efficient learning scheme. J. Neurosci. 29, 13484-93. doi:10.1523/JNEUROSCI.2207-09.2009

McLelland, D., Paulsen, O., 2009. Neuronal oscillations and the rate-to-phase transform: mechanism, model and mutual information. J. Physiol. 587, 769-785. doi:10.1113/jphysiol.2008.164111

Meador, K.J., Ray, P.G., Echauz, J.R., Loring, D.W., Vachtsevanos, G.J., 2002. Gamma coherence and conscious perception. Neurology 59, 847-854. doi:10.1212/WNL.59.6.847

Merker, B., 2013. Cortical gamma oscillations: the functional key is activation, not cognition. Neurosci. Biobehav. Rev. 37, 401-17. doi:10.1016/j.neubiorev.2013.01.013

Miller, K.J., Sorensen, L.B., Ojemann, J.G., Den Nijs, M., 2009. Power-law scaling in the brain surface electric potential. PLoS Comput. Biol. 5. doi:10.1371/journal.pcbi.1000609

Montemurro, M.A., Rasch, M.J., Murayama, Y., Logothetis, N.K., Panzeri, S., 2008. Phase-of-firing coding of natural visual stimuli in primary visual cortex. Curr. Biol. 18, 375-80. doi:10.1016/j.cub.2008.02.023

Moran, J., Desimone, R., 1985. Selective attention gates visual processing in the extrastriate cortex. Science (80-. ). 229, 782-784. doi:10.1126/science.4023713

Mountcastle, V.B., 1957. Modality and topographic properties of single neurons of cat's somatic sensory cortex. J. Neurophysiol. 20, 408-434.

Nicholson, A., 1954. An outline of the dynamics of animal populations. Aust. J. Zool. 2, 9. doi:10.1071/ZO9540009

Niessing, J., Ebisch, B., Schmidt, K.E., Niessing, M., Singer, W., Galuske, R.A.W., 2005. Hemodynamic signals correlate tightly with synchronized gamma oscillations. Science 309, 948-951. doi:10.1126/science.1110948

Nikolić, D., Fries, P., Singer, W., 2013. Gamma oscillations: precise temporal coordination without a metronome. Trends Cogn. Sci. 17, 54-5. doi:10.1016/j.tics.2012.12.003

Osipova, D., Hermes, D., Jensen, O., 2008. Gamma power is phase-locked to posterior alpha activity. PLoS One 3, e3990. doi:10.1371/journal.pone.0003990

Palanca, B.J.A., DeAngelis, G.C., 2005. Does neuronal synchrony underlie visual feature grouping? Neuron 46, 333-46. doi:10.1016/j.neuron.2005.03.002

Pikovsky, A., 2003. Synchronization: A Universal Concept in Nonlinear Sciences. Cambridge University Press.

Pritchard, W.S., 1992. The brain in fractal time: $1 /$ f-like power spectrum scaling of the human electroencephalogram. Int. J. Neurosci. 66, 119-129. doi:10.3109/00207459208999796 
Ray, S., Maunsell, J.H.R., 2014. Do gamma oscillations play a role in cerebral cortex? Trends Cogn. Sci. 19, 78-85. doi:10.1016/j.tics.2014.12.002

Ray, S., Maunsell, J.H.R., 2010. Differences in gamma frequencies across visual cortex restrict their possible use in computation. Neuron 67, 885-896. doi:10.1016/j.neuron.2010.08.004

Redlich, F.C., Callahan, A., Mendelson, R.H., 1946. Electroencephalographic changes after eye opening and visual stimulation. Yale J. Biol. Med. 18, 367-76.

Roberts, M.J., Lowet, E., Brunet, N.M., Ter Wal, M., Tiesinga, P., Fries, P., De Weerd, P., 2013. Robust gamma coherence between macaque V1 and V2 by dynamic frequency matching. Neuron 78, 52336. doi:10.1016/j.neuron.2013.03.003

Rolls, E.T., Webb, T.J., Deco, G., 2012. Communication before coherence. Eur. J. Neurosci. 36, 2689-709. doi:10.1111/j.1460-9568.2012.08188.x

Salinas, E., Sejnowski, T.J., 2001. Correlated neuronal activity and the flow of neural information. Nat Rev Neurosci 2, 539-550. doi:10.1038/35086012

Scafetta, N., 2010. Empirical evidence for a celestial origin of the climate oscillations and its implications. J. Atmos. Solar-Terrestrial Phys. 72, 951-970. doi:10.1016/j.jastp.2010.04.015

Shadlen, M.N., Movshon, J.A., 1999. Synchrony Unbound. Neuron 24, 67-77. doi:10.1016/S08966273(00)80822-3

Shaffer, F., McCraty, R., Zerr, C.L., 2014. A healthy heart is not a metronome: an integrative review of the heart's anatomy and heart rate variability. Front. Psychol. 5, 1040. doi:10.3389/fpsyg.2014.01040

Singer, W., 1999a. Time as coding space? Curr. Opin. Neurobiol. 9, 189-194. doi:10.1016/S09594388(99)80026-9

Singer, W., 1999b. Neuronal Synchrony: A Versatile Code for the Definition of Relations? Neuron 24, 4965. doi:10.1016/S0896-6273(00)80821-1

Singer, W., Gray, C.M., 1995. Visual Feature Integration and the Temporal Correlation Hypothesis. Annu Rev Neurosci. doi:10.1146/annurev.ne.18.030195.003011

Spaak, E., Bonnefond, M., Maier, A., Leopold, D. a, Jensen, O., 2012. Layer-specific entrainment of gamma-band neural activity by the alpha rhythm in monkey visual cortex. Curr. Biol. 22, 2313-8. doi:10.1016/j.cub.2012.10.020

Steriade, M., Amzica, F., Contreras, D., 1996. Synchronization of fast (30-40 Hz) spontaneous cortical rhythms during brain activation. J. Neurosci. 16, 392-417.

Tiesinga, P., Sejnowski, T.J., 2009. Cortical enlightenment: are attentional gamma oscillations driven by ING or PING? Neuron 63, 727-32. doi:10.1016/j.neuron.2009.09.009

Tiesinga, P.H., Sejnowski, T.J., 2010. Mechanisms for Phase Shifting in Cortical Networks and their Role in Communication through Coherence. Front Hum Neurosci 4, 196. doi:10.3389/fnhum.2010.00196

Traub, R.D., Whittington, M.A., Colling, S.B., Buzsaki, G., Jefferys, J.G., 1996. Analysis of gamma rhythms in the rat hippocampus in vitro and in vivo. J. Physiol. 493, 471-484.

Treisman, A., 1996. The binding problem. Curr. Opin. Neurobiol. 6, 171-178. doi:10.1016/S09594388(96)80070-5

Treisman, A., 1985. Preattentive processing in vision. Comput. Vision, Graph. Image Process. 31, 156177. doi:10.1016/S0734-189X(85)80004-9

Ts'o, D.Y., Gilbert, C.D., Wiesel, T.N., 1986. Relationships between horizontal interactions and functionalarchitecture in cat striate cortex as revealed by cross-correlationanalysis. J. Neurosci. 6 , 1160-1170.

Tudor, M., Tudor, L., Tudor, K.I., 2005. Hans Berger (1873-1941)--the history of electroencephalography. Acta Med. Croatica 59, 307-313. 
Van Essen, D.C., Maunsell, J.H.R., 1983. Hierarchical organization and functional streams in the visual cortex. Trends Neurosci. doi:10.1016/0166-2236(83)90167-4

Vinck, M., Lima, B., Womelsdorf, T., Oostenveld, R., Singer, W., Neuenschwander, S., Fries, P., 2010. Gamma-phase shifting in awake monkey visual cortex. J Neurosci 30, 1250-1257. doi:10.1523/JNEUROSCI.1623-09.2010

Vrba, J., Robinson, S.E., 2001. Signal processing in magnetoencephalography. Methods 25, 249-71. doi:10.1006/meth.2001.1238

Wang, D., Terman, D., 1997. Image Segmentation Based on Oscillatory Correlation. Neural Comput. 9, 805-836. doi:10.1162/neco.1997.9.4.805

Wang, X.-J., Buzsaki, G., 1996. Gamma Oscillation by Synaptic Inhibition in a Hippocampal Interneuronal Network Model. J. Neurosci. 16, 6402-6413.

Womelsdorf, T., Schoffelen, J.-M., Oostenveld, R., Singer, W., Desimone, R., Engel, A.K., Fries, P., 2007. Modulation of neuronal interactions through neuronal synchronization. Science 316, 1609-1612. doi:10.1126/science.1139597

Xing, D., Shen, Y., Burns, S., Yeh, C.-I., Shapley, R., Li, W., 2012. Stochastic generation of gamma-band activity in primary visual cortex of awake and anesthetized monkeys. J. Neurosci. 32, 13873-80a. doi:10.1523/JNEUROSCI.5644-11.2012

Zeitler, M., Fries, P., Gielen, S., 2008. Biased competition through variations in amplitude of ??oscillations. J. Comput. Neurosci. 25, 89-107. doi:10.1007/s10827-007-0066-2 


\section{Chapter 2}

\section{Robust gamma coherence between macaque V1 and V2 by dynamic frequency matching}

Based on:

M. J. Roberts, E. Lowet, N. M. Brunet, M. Ter Wal, P. Tiesinga, P. Fries, and P. De Weerd, "Robust gamma coherence between macaque V1 and V2 by dynamic frequency matching.," Neuron, vol. 78, no. 3, pp. 523-36, 2013. 


\section{ABSTRACT}

Current theories propose that coherence of oscillatory brain activity in the gamma band $(30-80 \mathrm{~Hz})$ constitutes an avenue for communication among remote neural populations. However, reports documenting stimulus dependency and time variability of gamma frequency suggest that distant neuronal populations may, at any one time, operate at different frequencies precluding synchronization. To test this idea, we recorded from macaque V1 and V2 simultaneously while presenting gratings of varying contrast. Although gamma frequency increased with stimulus contrast in V1 and V2 (by $25 \mathrm{~Hz}$ ), V1-V2 gamma coherence was maintained for all contrasts. Moreover, while gamma frequency fluctuated by $\sim 15 \mathrm{~Hz}$ during constant contrast stimulation, this fluctuation was highly correlated between V1 and V2. The strongest coherence connections showed a layer-specific pattern, matching feedforward anatomical connectivity. Hence, gamma coherence among remote populations can occur despite large stimulus-induced and time-dependent changes in gamma frequency, allowing communication through coherence to operate without a stimulus independent, fixed-frequency gamma channel. 


\section{Introduction}

Cortical activity is characterized by oscillatory processes segregated into distinct frequency bands. According to the 'Communication through Coherence' (CTC) hypothesis (Fries, 2005), coherent oscillations in the so-called 'gamma' band $(30-80 \mathrm{~Hz})$ contribute importantly to long-range information transmission among different hierarchical processing levels of the brain during sensory and cognitive processing (Fries, 2005; Salinas and Sejnowski, 2001; Tiesinga et al., 2002; Wildie and Shanahan, 2012). Coherence is facilitated when oscillations in distant areas, or distant regions of the same areas (Gray et al., 1989), occur at the same frequency on a moment-to-moment basis (Buzsaki and Draguhn, 2004; Rosenblum et al., 2001). Until recently it was thought that gamma frequency was highly stable over time and across brain areas in a given individual (Hoogenboom et al., 2006; Muthukumaraswamy et al., 2010), which would ensure efficient communication among remote neuronal populations.

However, the idea that gamma frequencies in different areas are by default matched across the brain, runs counter the fact that gamma depends on local network properties (Buia and Tiesinga, 2006; Fries, 2005), which likely will differ among areas. Therefore, the gamma frequencies exhibiting maximum power ('gamma peak frequencies') in a given stimulus condition could differ substantially between areas. Moreover, a number of reports have demonstrated strong dependencies of gamma band frequencies on visual stimulus parameters (Feng et al., 2010; Gieselmann and Thiele, 2008; Jia et al., 2013; Ray and Maunsell, 2010, 2011; Swettenham et al., 2009). In view of likely differences among visual areas, variations in low-level stimulus parameters may therefore affect gamma frequency in a way that differs substantially between areas. As a result, gamma frequency differences, coherence, and thus the efficiency of information transfer between remote populations in different visual areas would be stimulus dependent, thereby rendering CTC implausible (Jia et al., 2013). Additionally, gamma power and frequency in V1 have been shown to change rapidly in an apparently random manner during constant stimulation (Burns et al., 2011; Xing et al., 2012a), or in response to fluctuations in the internal state of the animal (Gray and McCormick, 1996). If these rapid variations would occur in an uncoordinated fashion in different visual areas, a possibility that so far has not been tested, then this would constitute another challenge to CTC. Hence, whenever the frequency in a given area shifts, CTC can only operate efficiently if the gamma frequency in communicating areas is dynamically matched.

Here, we aimed to address the fundamental question of whether gamma frequency modulations induced either by stimulus variations or occurring spontaneously during constant stimulation, do or do not prevent coherence of oscillatory brain activity between different visual cortical areas. To that goal, we performed simultaneous recordings in awake macaque areas V1 and V2 and determined within-area gamma peak frequencies and across-area coherence during the presentation of visual stimuli of varying luminance contrast. Furthermore, we tested the extent to which coherence was consistent with a role in neuronal communication by examining the directionality and laminar distribution of gamma band coherence. 


\section{Results}

\section{In V1 and V2 gamma peak frequency is stimulus dependent, but coherence is maintained}

To test the robustness of gamma coherence across varying stimulus conditions, we simultaneously recorded neuronal activity of V1 and V2 neurons with overlapping or near-overlapping receptive fields (RFs) in two awake macaque monkeys (Fig S1). Spikes and local field potentials (LFPs) were recorded from V1 and V2 using linear arrays of 8 recording contacts with a $200 \mu \mathrm{m}$ inter-contact spacing in each area. The monkeys fixated the centre of a computer screen while static square-wave gratings of varying luminance contrast were presented in the RFs. In 17 recording sessions from monkey $S$ and 17 from monkey K, we obtained a total of 202 recording sites in V1, and 220 in V2 (monkey S V1=107, V2=109; monkey K V1=95, V2=111). To investigate cross-area coherence, we transformed our data into current source density (CSD) by taking the second spatial derivative of the LFP along the linear array electrodes (Mitzdorf, 1985). Using CSD rather than LFP enhances the spatial specificity of coherence measurements and removes the common reference, thereby eliminating major sources of spurious coherence (Mitzdorf, 1985). In total, 780 cross-area coherence measurements were made (monkey S=408, monkey K=372). For details, see Supplemental Experimental Procedures.

In a first test of the stimulus dependency of gamma, we computed time-resolved, induced LFP powerspectra in areas V1 and V2 for a single session from monkey $S$ for gratings of two different contrasts. Fig. $1 \mathrm{~A}$ (top left) shows a distinct gamma band in V1 with peak power at approximately $40 \mathrm{~Hz}$ during $50.3 \%$ contrast stimulation. A reduction of grating contrast to $16.3 \%$ shifted the gamma band in $\mathrm{V} 1$ down to about $30 \mathrm{~Hz}$ (Fig. 1A, bottom left). Remarkably, exactly the same shift in the gamma frequency was also observed in V2 (center column), as well as in V1-V2 coherence between CSD channels (right column).

Fig. 1B shows the population average induced power spectra for each contrast condition in both monkeys, and reveals a large contrast-induced shift of the gamma band in V1 (top row), with frequencies at peak power shifting from $\sim 20 \mathrm{~Hz}$ for low contrast stimuli to $\sim 45 \mathrm{~Hz}$ for high contrast stimuli. Very similar effects were observed in V2 (Fig. 1B, middle row) and in the cross-area coherence (Fig. 1B, bottom row). We used a Gaussian fitting approach to determine peaks of V1 and V2 power spectra and cross-area coherence spectra (see Fig. S2). Note that we use the term 'peak frequency' as shorthand for the frequency with the highest power/coherence in the gamma range. We found a strong dependence of peak frequency on contrast which was similar for LFP power and for cross-area coherence (Fig. 1C). A two-way ANOVA with factors contrast and data-type (V1, V2 or coherence) for each monkey separately confirmed that gamma peak frequency was significantly affected by stimulus contrast (monkey S F(7,279)=128.1, $p<0.01$; Monkey K $F(7,269)=196, p<0.001$ ) but not data-type (monkey S $F(2,279)=1.8, p=0.17$; Monkey $\mathrm{K} \mathrm{F}(2,269)=5.4, \mathrm{p}=0.29)$. Interactions were not significant (monkey $S(F(14,279)=1.2, p=0.25$; monkey $K F(14,269)=1.29 p=0.21)$. Interestingly, we found that in a minority of sessions at the lower two contrasts in monkey $S$ (Fig. 1C, left), gamma peak frequency was unexpectedly high, reaching to up to $30 \mathrm{~Hz}$ during $2.5 \%$ contrast stimulation (note the wider standard error bars at low contrasts, also see figures 2 and 3 ). 
A Monkey S Session Example
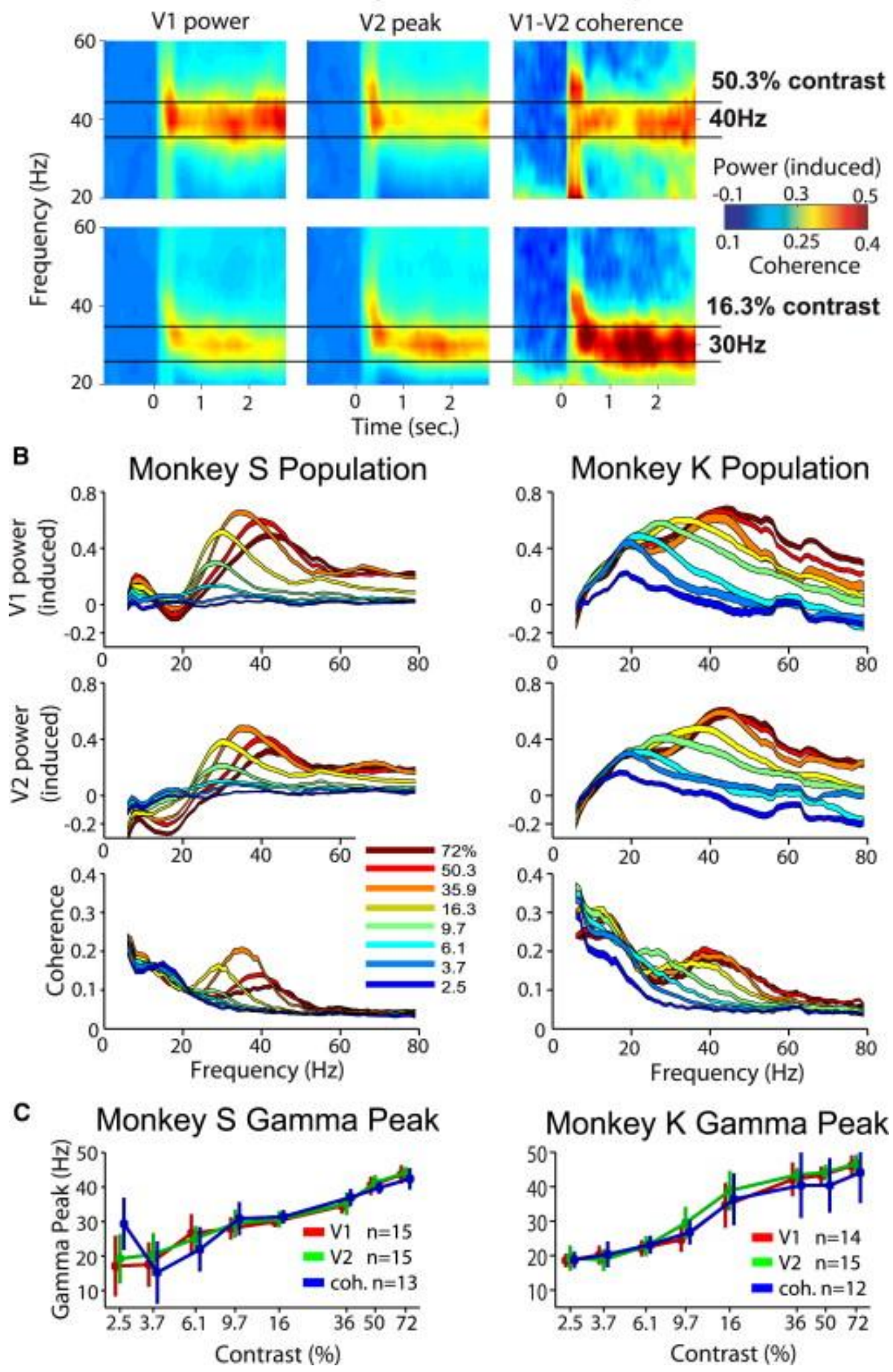

Figure 1: Contrast-dependent shift in gamma frequency band. A) Time-frequency representation of stimulus-induced LFP power in area V1 (left column) and V2 (middle), and V1-to-V2 coherence (right). Black lines contain the gamma band, which is at a different frequency for different rows (top 50.3\% contrast; bottom 16.3\%). B) Stimulus-induced LFP power-spectra in V1 (top 
row), V2 (middle) and of V1-V2 coherence (bottom) during the sustained period (from 350ms post stimulus onset) of the response. Line color indicates contrast condition (legend); line thickness indicates standard error. Data from two monkeys are shown separately (columns). C) Gamma frequency at peak power (derived from data in B, see Fig S2 for details) increases as a function of grating contrast for LFP power in V1 (red line) and V2 (green line), and for V1-V2 coherence (blue line) in monkey S and $\mathrm{K}$, error bars show standard deviation.
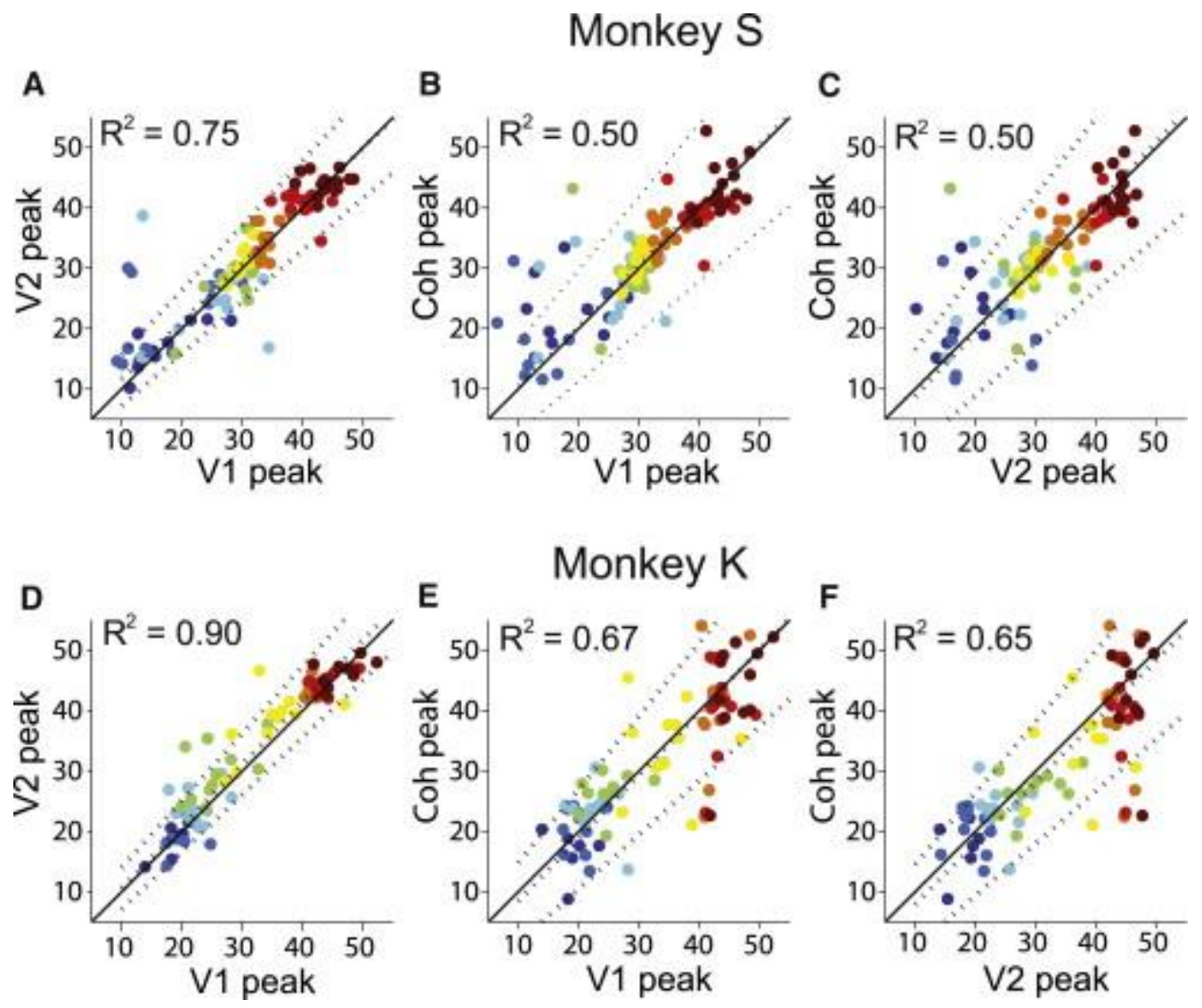

Figure 2: Correlations among gamma frequencies showing peak power in V1 (V1 peak), in V2 (V2 peak), and V1-V2 coherence (Coh peak) in the gamma range. Points show peak frequency per session for each contrast condition (dot color codes contrast as in Fig.1 B) calculated as the median of the peak frequency recorded at each recording contact, or the median the peak frequency of all coherence measurements. Solid black line gives the diagonal. Dashed lines show regression line (largely overlapping with the diagonal) and upper and lower $95 \%$ confidence intervals.

Fig. 2 further illustrates the close relationships among gamma band peak frequencies in areas V1 and V2 and cross-area coherence (see squared correlation values in figure). Fig. 2 shows for all contrasts and sessions a linear relationship between peak frequency in V1 and in V2 (Fig. 2A,D), between peak frequency in $\mathrm{V} 1$ and $\mathrm{V} 1-\mathrm{V} 2$ coherence (Fig. 2B,E), and between peak frequency in V2 and V1-V2 coherence (Fig. 2C,F), with data in each case fit by a regression line with a slope close to 1 . These data indicate that $\mathrm{V} 1-\mathrm{V} 2$ coherence was robust against large shifts in the gamma frequency band and argues against the view that coherence can only be efficient in a restricted and fixed band of gamma frequencies. Instead, the data indicate that coherence can sustain long-range communication according to the CTC mechanism across a broad spectrum of stimulus dependent frequencies. 


\section{Laminar distribution of gamma coherence agrees with anatomical feedforward connectivity}

Spike-field coherence spectra and band-limited power spectra show that gamma-band activity is stronger in superficial layers than in deep cortical layers (Buffalo et al., 2011; Maier et al., 2011; Smith et al., 1213; Xing et al., 2012b). We hypothesized that also gamma cross-area coherence may show layerspecificity. Specifically, if band-limited coherence is related to neuronal communication, the coherence may be most prominent between sites known to be strongly connected anatomically. V1 origins of anatomical feedforward connections are situated in layers 2, 3, 4A, and 4B (Callaway, 1998; Lund et al., 1975). V1 layers 2 and 3A (i.e., top two thirds layer 3), which receive LGN input indirectly via 4C, provide relatively weak output to V2. However, V1 layer 4B provides a strong magnocellular output directly to V2. In addition, V1 layer 3B, which receives input from sub-layers $4 C \alpha$ and $4 C \beta$ (and also $4 A$ ), is also a major source of output to V2 (for reviews see Bastos et al., 2013; Callaway, 1998; Nassi and Callaway, 2009). Thus, the strongest anatomical connection between V1 and V2 is the feedforward projection from superficial V1 layers $3 \mathrm{~B}$ and $4 \mathrm{~B}$, which projects to the full extent of layer 4 in V2 (Douglas and Martin, 2004; Felleman and Van Essen, 1991). Since the stimuli were irrelevant to the task and hence were likely to be ignored, we expected functional connectivity to be dominated by a feedforward flow of information. Therefore, as a minimal hypothesis we expected the pattern of functional connections, revealed by the strongest coherence measurements, to link relatively shallow sites in V1 with relatively deeper sites in V2. To test this prediction, an alignment of the recording sites from the different depth probe placements over sessions was necessary. This was accomplished by making use of the characteristic reversal in layer 4 from positive visually evoked potentials (VEPs) in superficial layers to negative VEPs in deeper layers (Supplemental Material, Fig. S3). In the V1 data aligned to the VEP reversal point, we also found a source-sink reversal in the Current Source Density (CSD) map, which, in V1, has been documented to indicate the top of input layer 4C (Maier et al., 2011; Schroeder et al., 1991). The pattern of VEPs across depth in V2 was highly similar to that observed in V1 (note that we were recording on the upper surface of the pre-lunate gyrus) and we therefore used the same criteria for aligning the V2 data. The point of VEP reversal in V2 was also found to match the point of the early sink-source reversal in the CSD map in that area, which we therefore assume to correspond to the top of layer 4 in V2. In Figure 3, we have set the top of layer 4C as depth zero in V1, and the top of layer 4 in V2 as depth zero in V2. Not only CSD maps that were obtained after alignment, but also the layer-specific distribution of gamma, and of spiking response latencies supported the validity of our alignment procedure (see Supplemental Experimental Procedures). 


\section{Monkey S}


Monkey K


Figure 3: Depth-specific pattern of V1-V2 gamma coherence is preserved across contrast conditions. For each of two monkeys (monkey $\mathrm{S}$ on the left and monkey $\mathrm{K}$ on the right), there are 8 panels. The color surfaces show LFP induced power as a function of frequency ( $\mathrm{X}$-axis) and depth ( $\mathrm{Y}$ axis) at each contrast for V1 (leftward) and for V2 (rightward). Horizontal dashed lines in each color surface highlight the 0-depth (top layer 4c in V1, top layer 4 in V2). Thin grey lines linking V1 and V2 data indicate all pairs of V1/V2 depth positions for which CSD-CSD coherence was recorded. Thick black lines highlight those pairs which showed the strongest coherence (top 5\%). Note that there are more depth positions than there were contact points on a single 8-contact probe. This reflects the depth-alignment of data coming from different sessions, in which depth probes showed some variability in their physical depth relative to cortex (for details, see Supplementary Materials, Fig. S2).

After alignment, our minimal hypothesis of a shallower-to-deeper pattern of coherence connections could be reformulated as an expectation of preferential functional connectivity between above-zero V1 depths and below-zero V2 depths. As an initial test, we divided all CSD coherence pairs into four groups, with pairs in group 1 linking all V1-V2 sites above zero-depth, pairs in group 2 linking all V1-V2 sites below zero-depth, pairs in group 3 linking above-zero V1 and below-zero V2 sites, and pairs in group 4 linking below-zero V1 and above-zero V2 sites. A two-way ANOVA, with factors group (4 data groupings) 
and contrast ( 8 contrasts) conducted for each monkey separately confirmed that gamma coherence significantly depended on data grouping (monkey S: $F(3,3232)=107.2, \quad p<0.001$; monkey $\mathrm{K}$ : $F(3,2800)=55.9, p<0.001$ ). Post-hoc testing (Tukey-Kramer method) showed that in both monkeys the above-zero V1 depth to below-zero V2 depth data grouping had significantly higher coherence than other data groupings $(p<0.05)$, in line with the minimal hypothesis outlined above. In agreement with other analyses, the effect of contrast was significant in both monkeys (monkey S: $F(7,3232)=67.7$; $p<0.001$, monkey $K: F(7,2800)=13.4 ; p<0.001)$. Interactions were significant in monkey $S$ $(F(21,3232)=3.3 ; p<0.001)$ but not in monkey $K(F(21,2800)=0.7, p=0.84)$.

To investigate the pattern of functional connectivity in the depth-aligned data in a more fine-grained matter, we calculated coherence between all available V1-V2 pairs of CSD contacts. The computation of coherence for V1-V2 pairs of CSD contacts was done for all contrasts in the two monkeys. Grey lines in the 16 panels in Fig. 3 indicate all position pairs which were available in our data. Black lines highlight the connections with the strongest coherence (top $5 \%$ of the population). In line with the previous analysis, the black lines indicate that the bulk of strong connectivity pairs linked superficial V1 (zerodepth and above) with relatively deeper V2 layers (zero-depth and below). In monkey S, 63.7\% of all strongest coherence pairs linked superficial sites in V1 with deep sites in V2, and in monkey $\mathrm{K}$ this was $64.4 \%$.

We then calculated the V1 and V2 center locations of the strong coherence connections as the mean of the depth positions in V1 and V2 of each of the strongest connections. In Monkey $\mathrm{S}$, the sites contributing to the strongest connectivity were centered at $0.42 \mathrm{~mm}$ in V1 and at $-0.24 \mathrm{~mm}$ in V2. In monkey $\mathrm{K}$ this was $0.58 \mathrm{~mm}$ in $\mathrm{V} 1$ and $-0.25 \mathrm{~mm}$ in $\mathrm{V} 2$ (data combined over contrasts). These values were largely unaffected by stimulus contrast (see Fig. S4) and were remarkably similar between monkeys. We tested the significance of the observed center locations of V1 and V2 against distributions of center locations obtained from a bootstrapping procedure. This procedure confirmed in V2 of both monkeys, and in V1 of monkey $\mathrm{S}$, that these center locations were significantly different from those expected by chance (this procedure did not yield significant results in V1 of monkey $\mathrm{K}$ due to lack of data in deep layers, for details see Fig S4).

We also tested whether the depth range of V1 sites and the depth range of V2 sites forming the pairs of strongest coherence were anatomically plausible. Anatomical studies have shown that the V1 layers most strongly involved in feedforward connectivity comprise layers $4 B$ to $3 B$, extending about $1 \mathrm{~mm}$ above the top of layer 4 C, i.e., about $1 \mathrm{~mm}$ above the zero-alignment depth in Fig. 3 (see also Fig. S3). V2 layer 4, is about $0.4 \mathrm{~mm}$ thick (de Sousa et al., 2010; Lund, 1988), and should therefore extend below the zero-alignment depth shown in Fig. 3 by about $0.4 \mathrm{~mm}$ (see also Fig. S3). Hence, the strongest anatomical connections originate in a 0 to $1 \mathrm{~mm}$ depth range in $\mathrm{V} 1$, and terminate in a 0 to $-0.4 \mathrm{~mm}$ depth range in V2. These depth ranges capture the large bulk of V1 and V2 depths $(79.4 \%$ in monkey $\mathrm{S}$ and $78.5 \%$ in monkey $\mathrm{K}$, pooled over contrasts) contributing to functional connectivity as described in Fig. 3. Moreover, the $\mathrm{V} 1$ center points $(0.42$ in monkey $\mathrm{S}, 0.58 \mathrm{~mm}$ in monkey $\mathrm{K}$ ) fall well within the expected 0 to $1 \mathrm{~mm}$ range, and likewise, the $\mathrm{V} 2$ center points $(-0.24$ in monkey $\mathrm{S},-0.25 \mathrm{~mm}$ in monkey $\mathrm{K})$ fall well within the expected 0 to $-0.4 \mathrm{~mm}$ range (averaged over contrasts). Hence, we found a remarkable match 
between the layer-specific pattern of strong functional connectivity (coherence) and the layered pattern of feedforward anatomical connectivity.

\section{Directionality analysis shows that Gamma coherence is predominantly feedforward}

The coherence-based connectivity displayed in Figure 3 is in line with the known feedforward anatomical connectivity. This suggests that functional directionality analysis for the top $5 \%$ strongest coherence pairs in Figure 3 should reveal a predominant feedforward term. To test this idea, we determined Granger causal influences using non-parametric spectral matrix factorization (Dhamala et al., 2008) for the top $5 \%$ coherence connections (Fig. 4). To quantify the effective directionality, we subtracted the feedback term from the feedforward term. Significance was tested with a bootstrap technique (see Experimental Procedures).
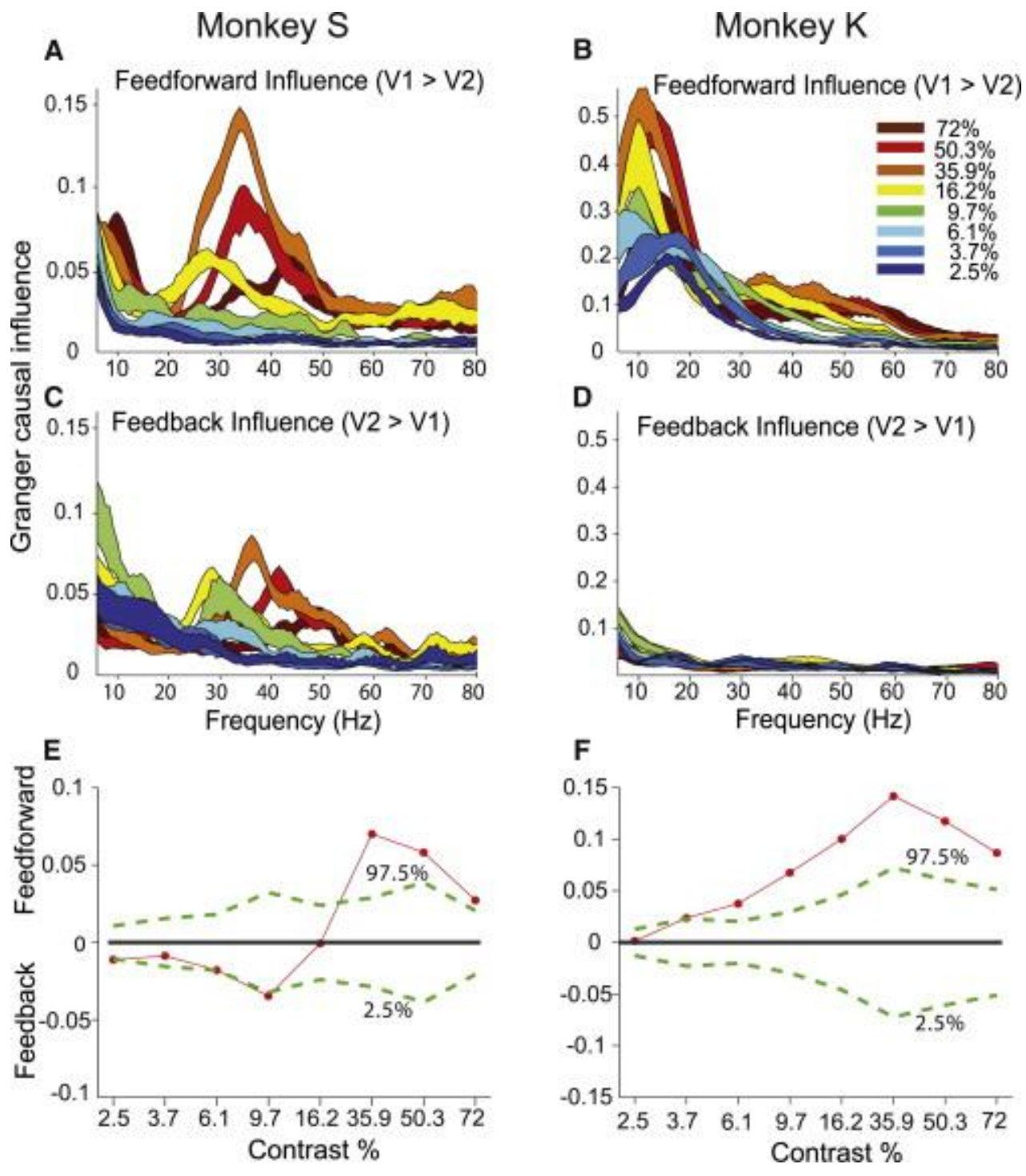
Figure 4: V1-V2 non-parametric granger causality for different stimulus contrasts (line color). Data are shown separately for the two monkeys (columns). Panels A and B show feedforward (V1 to V2) term, panels C and D show feed-back term (V2 to V1). Panels $E$ and $F$ show the subtraction of the feedback term from the feedforward term, and the results of the subtractions are shown by red dots and line. Thin green dashed lines show confidence intervals derived from bootstrap testing (described in main text). The significant bias towards feedforward connectivity at higher contrasts in monkey S (contrasts of $35.9 \%$ and above) and in monkey $\mathrm{K}$ (contrasts of $6.1 \%$ and above) remained significant after correction for multiple comparisons (correction for 8 tests; resulting in confidence interval with lower bound $0.31 \%$ and upper bound $99.69 \%$ ).

We found that the feedforward term (Fig. 4A,B) was significantly higher than the feedback term (Fig. 4C,D) at the 7 highest contrasts in monkey $\mathrm{K}$ (Fig. 4F) and at the three highest contrasts in monkey $\mathrm{S}$ (Fig. $4 \mathrm{E}$ ), in line with the previously proposed feedforward function of gamma processes (Buffalo et al., 2011; Bosman et al., 2012). At lower contrasts in monkey $S$ the feedback term tended to be stronger than the feedforward term and this difference was significant at the $0.05 \%$ level for the $9.7 \%$ contrast condition. Note that it was in the same monkey that we observed in a subset of sessions an increase in gamma frequency at the lowest few contrasts compared with higher contrasts (see Figure 1c, 2 and 3). The current Granger analysis suggests that feedback from higher areas could underlie this frequency increase. Granger causal influence spectra showed a shift in peak frequency with stimulus contrast, matching the findings of gamma power and gamma coherence. Hence, despite some differences between monkeys at lower contrasts, Granger causality analysis support a feedforward flow of information in both monkeys at higher contrasts.

\section{The similarity of V1-V2 gamma coherence networks is maintained over contrast}

The coherence networks presented in Fig. 3 appear highly similar for different contrast conditions. In Fig. 5, we tested this similarity, and moreover we tested whether the layer-specific similarity of functional connections for different contrasts was limited to the gamma band. To quantify similarity we first defined the pattern observed at the maximum power (peak) gamma frequency at a mid-level contrast of $35.9 \%$ as a 'reference pattern' of layer-specific coherence. In Fig. 5, the reference is labeled ' $R$ ' in the similarity matrices of monkey $S$ and $K$. We compared this reference to 'test patterns' observed with the other contrasts, with 4 example test patterns labeled A-D in the similarity matrices. To do the comparison in a robust way, we rank-ordered the coherence values and subsequently calculated a measure of similarity as the percentage of variance in the test pattern explained by the refence pattern. The small panels in Fig. 5 show visual illustrations of comparison tests between the refence pattern (red lines) and test patterns (black lines). For example, for test $\mathrm{A}$ in monkey $\mathrm{S}$ test pattern $\mathrm{A}$ shown in black lines showed poor overlap with the reference pattern shown in red lines, resulting in a low similarity index (yellow color) in the similarity matrix. Test B in monkey $\mathrm{S}$ revealed better referencetest similarity, resulting in a higher similarity index (orange color). The similarity was determined between the reference and test patterns for all stimulus contrasts in 25 non-overlapping $3 \mathrm{~Hz}$ frequency windows (i.e. frequency ranges of a $3 \mathrm{~Hz}$ width, with their center frequencies increasing from 3 to $78 \mathrm{~Hz}$ in steps of $3 \mathrm{~Hz}$; e.g. the first bin containing frequencies from 2 to $4 \mathrm{~Hz}$ ). In both monkeys, the highest similarity values were confined to the gamma range (which was broader in monkey $\mathrm{K}$ than monkey $\mathrm{S}$, see Fig. 1). The difference between similarity values for all coherence patterns within $10 \mathrm{~Hz}$ of the peak gamma frequency and values obtained outside that frequency range was highly significant for both 
monkeys (two sample t-test: monkey $\mathrm{S}: \mathrm{t}(174)=11.9, \mathrm{p}<0.001$; monkey $\mathrm{K}: \mathrm{t}(155)=6.5, \mathrm{p}<0.001$ ). These findings show that the preservation of layer-specific patterns of V1-V2 functional connectivity is specific to the gamma range. This further supports gamma as a means of stable neural communication despite large stimulus-induced gamma frequency changes. In addition, the tight link between the stable V1-V2 coherence connections and gamma gives further support to the notion that these connections are predominantly feedforward, as in other studies gamma has indeed been linked with feedforward information transmission (Buffalo et al., 2011).
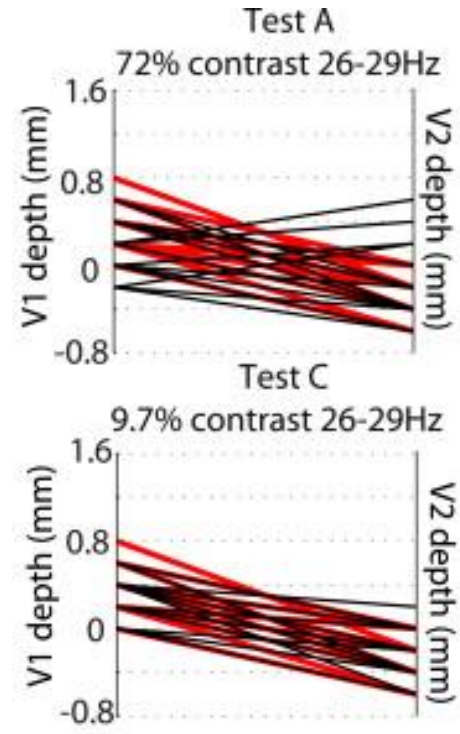

Test A
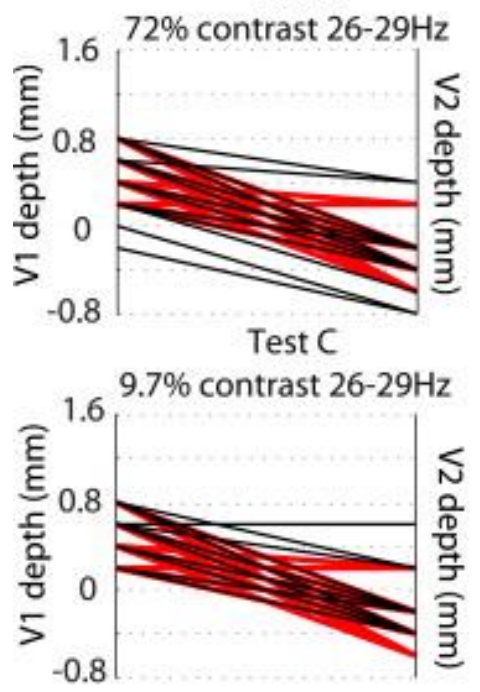

Monkey S

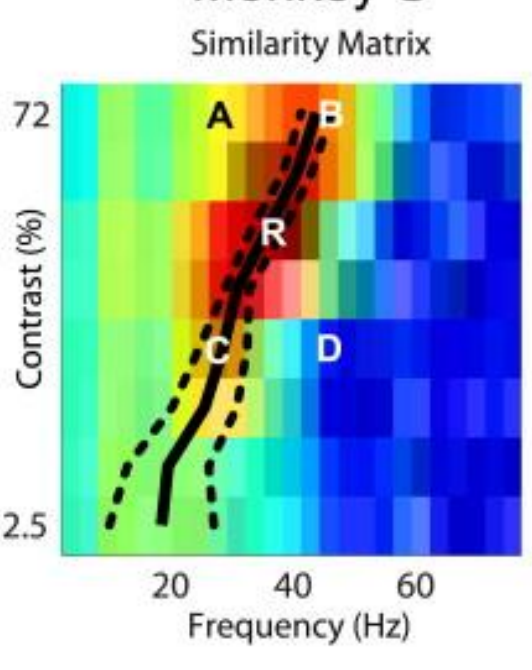

Monkey K

Similarity Matrix

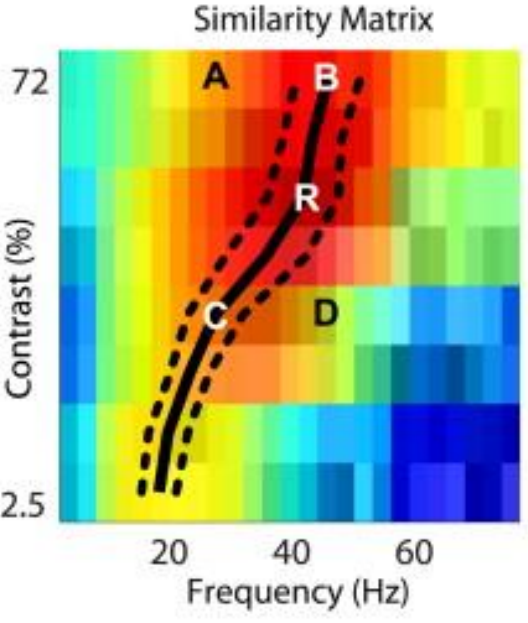

Test B

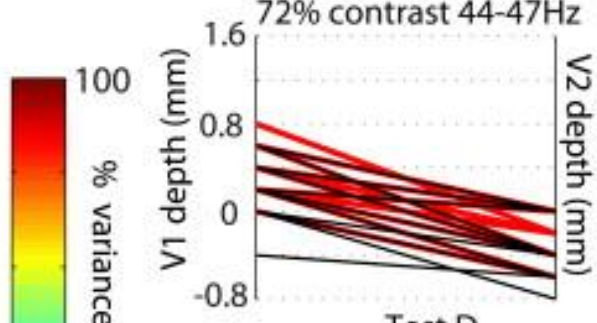

Test D

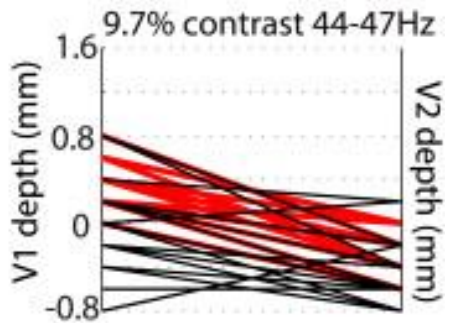

Figure 5: Quantitative comparison of the cross area coherence pattern across frequencies and contrast conditions in monkeys $\mathrm{S}$ and $\mathrm{K}$. Surface color shows the similarity (percentage variance explained) between the test coherence patterns for each contrast at each $3 \mathrm{~Hz}$ frequency window and the reference pattern (marked by an R on the color surface). For each contrast, V1V2 coherence was computed in each $1 \mathrm{~Hz}$ bin (Hanning tapered), and bins were grouped in $3 \mathrm{~Hz}$ windows within which coherence values were averaged. Similarity values were computed by comparing reference to test patterns of connectivity at a 
frequency resolution of $3 \mathrm{~Hz}$. For each contrast, reference-test similarity was shown for 25 non-overlapping $3 \mathrm{~Hz}$ frequency windows (with center frequencies from 3 to $78 \mathrm{~Hz}$ increasing with a step size of 3). Thus, for example, the first window was centered on $3 \mathrm{~Hz}$ containing frequencies from 2 to $4 \mathrm{~Hz}$; the second window was centered on $6 \mathrm{~Hz}$ containing 5 to $7 \mathrm{~Hz}$, etc. Overlaid black line in the color surfaces for each monkey shows mean peak gamma frequency per contrast condition (mean calculated from data from V1 and V2 combined, flanking dashed lines indicate \pm 1 standard deviation, data show separately in Fig1C). This line closely tracks the peak coherence in the color surfaces. Surrounding plots (Test A-D) show examples comparisons of connectivity patterns for selected test patterns (A-D as marked in color surfaces) and the reference pattern R. Red lines show the top $5 \%$ coherence pairs in the reference pattern, black lines show the top $5 \%$ coherence pairs for the test patterns. Each comparison of reference and test patterns yields a similarity index shown on a color scale in the color surface representing the similarity matrix. Letters marking test and reference patterns are colored black or white visibility.

\section{Rapid shifts in gamma band frequency under constant stimulus conditions}

Rapid, apparently random shifts in frequency as observed in striate cortex (Burns et al., 2011; Xing et al., 2012a) could present an important barrier for effective communication though coherence, in addition to stimulus dependent shifts. These shifts could represent physiological fluctuations in stimulus drive as well as noise within cortical networks. Since we observed robust coherence between V1 and V2, this suggests the existence of a mechanism that limits frequency differences between V1 and V2 on brief time scales. To test whether our data support such mechanism, we characterized moment-by-moment variation in gamma peak frequency in V1 and V2. Following Burns et al. (2011), one might expect higher areas (V2) to be unlikely to keep up with changing frequency in lower areas (V1). Fig. 6A shows V1 frequency versus $V 2$ frequency joint probability scatter diagrams for 3 contrasts from a single session of monkey $\mathrm{S}$, with marginal distributions of the probability of frequency in V1 and V2 along $\mathrm{X}$ and $\mathrm{Y}$ axes, respectively. Fig. $6 B$ shows population data from the two monkeys for 4 contrasts using joint probability surfaces. Figures $6 \mathrm{~A}$ and $\mathrm{B}$ show that the frequency estimates within $\mathrm{V} 1$ and $\mathrm{V} 2$ varied over up to $15 \mathrm{~Hz}$ confirming Burn's (2011) findings in V1. Given this large frequency range in each area, a high correlation is not expected unless there is a mechanism that helps to constrain frequency differences between the two areas. In our data, we find a strong probability for frequency-frequency matches (bottom-left to top-right diagonal in joint probability surfaces) and a significant moment-to-moment frequencyfrequency correlation. Pearson V1-V2 correlations in moment-to-moment gamma frequency are illustrated in Fig. 6C for 4 contrasts. These correlations were in the order of 0.1 when based on all possible V1-V2 contact pairs, pooled over both monkeys and all sessions (dark grey symbols in Fig. 6C). For the 5\% strongest connections shown in Fig. 3, correlation coefficients were in the order of 0.3 pooled over monkeys and contrasts (red symbols in Fig. 6C). To test the significance of the correlations we randomly shuffled trial labels between V1 and V2 and re-calculated correlation coefficients over 1000 iterations. The distribution of correlation coefficients were found to be not significantly different from 0 in shuffled data, and correlation coefficients in the unshuffled data were significantly above the shuffled distribution (all $p<0.005$ for both monkeys). These data support the idea of a functional architecture that helps constrain frequency-frequency differences on short time scales during communication (coherence) between two recorded cortical areas. The high correlation of rapid frequency shifts in the two cortical areas could reflect rapid shifts in the internal state of the animal (Gray and McCormick, 1996) which simultaneously and equally affects both cortical areas. However, granger causality analysis (Fig. 5) indicates a predominantly feedforward influence of V1 onto V2, 
especially at high stimulus contrasts, thus it is also possible that the gamma peak frequency in V2 is dynamically matched to the incoming V1 gamma signal.

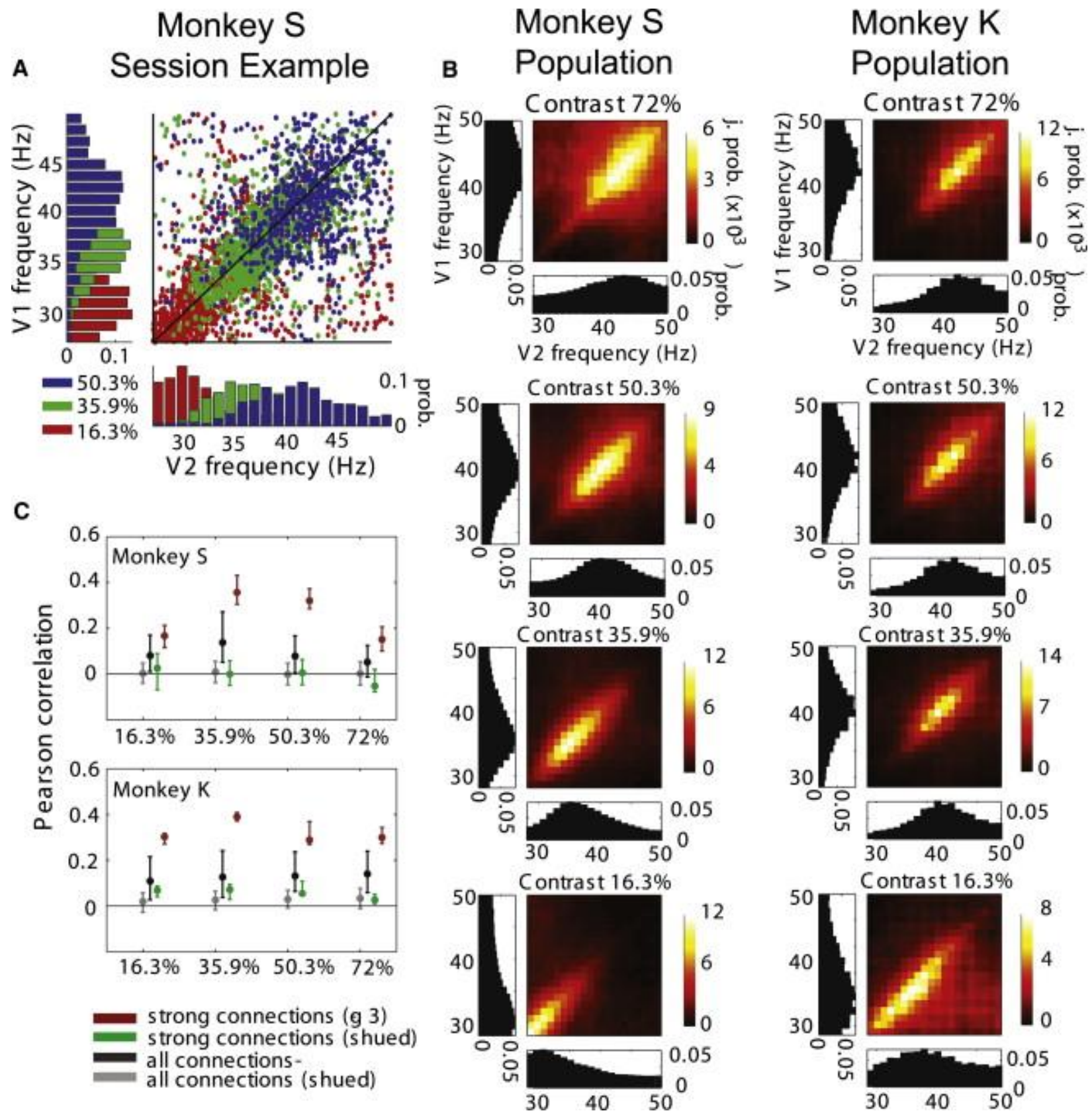

Figure 6: Evidence for frequency-frequency matching A) Moment-by-moment frequency-frequency correlation from a single V1-V2 pair of sites, from a single example session in monkey $S$ at three stimulus contrasts. Each dot represents the frequency of the ongoing LFP of V1 ( $Y$ ordinate) and V2 (X ordinate) at a specific 1ms time bin. Black line gives the diagonal. Histograms along the axes show the probability distribution per frequency. B) Population joint probability (j. prob.) of V1/V2 frequency-frequency coupling for four contrasts (row titles) for monkey S (left) and monkey K (right). Histograms along the axes show the probability distribution per frequency. C) Median of frequency-frequency correlation coefficients for different contrasts in the population of V1-V2 connections. Correlations are shown without shuffling based on all connections (black symbols) or based on the top $5 \%$ strongest coherence connections (red symbols). In addition, correlations are shown after trial shuffling, again for all connections (grey symbols) and for the $5 \%$ strongest connections (green symbols). Error bars show upper and lower quartiles. 


\section{Dynamic frequency matching is obtained in coupled PING models}

To elucidate the mechanism by which gamma frequency in a downstream area dynamically shifts in order to match the frequency of an upstream area we have constructed a computational model in which local V1 and V2 networks were modeled explicitly by spiking neurons with Hodgkin-Huxley voltage-gated channels. The V1 network consisted of reciprocally connected excitatory (E) and inhibitory (I) neurons. V2 was modeled in the same way, with the $E$ and I cells in addition receiving direct excitatory inputs from V1 E cells. We have illustrated these networks as respectively representing superficial V1 and layer 4 of V2 (Fig 7A). Within each network, all cells received noise currents (details in Supplementary Material). Each network synchronized through the Pyramidal Inter-Neuron Gamma (PING) mechanism, in which a volley of E cells recruits a volley of I cells. This shuts the network down for a gamma period, and when the E cells recover, the cycle starts anew. For the oscillations to be present, the E cells need to be depolarized (for example by presentation of a stimulus), and the frequency of oscillations then depends on the level of depolarization in the $E$ and I cells. The level of depolarizing current in the model was set at 6 levels, simulating a variation in contrast (Sanchez-Vives et al., 2000) from low to high (dark blue to red colors in Fig. 7B-F). This accounts for the stimulus-induced oscillations in V1 at different simulated contrasts (Fig. 7B). Stimulus-induced oscillations in V2 can arise by the same mechanism, but in the simulation the stimulus-related inputs to $\mathrm{V} 2$ come from V1, and therefore are already oscillating at gamma. Hence, the V2 network activity locks to the oscillating input, which is indicated by power peaks in corresponding frequencies in V1 and V2 (Fig. 7B, C) and coherence between V1 and V2 in the same frequency band (Fig. 7D). Simulated contrast increases led to enhanced power reaching a maximum at intermediate frequencies, with diminishing power for further simulated contrast increases (Fig. 7B \& C). During the time-interval that the simulated stimulus was present, the V1 gamma frequency fluctuated in a $5-10 \mathrm{~Hz}$ frequency band, reflecting simulated variations in driving current (noise, details in Supplementary Material). Despite these rapid frequency fluctuations, there were significant correlations of moment-to-moment peak frequencies in V1 and V2 (Fig. 7E), indicating a form of dynamic frequency matching between V1 and V2. Correlation coefficients for different contrasts ranged between 0.24 and 0.48 and were all significant (based on the same approach and criteria as used for empirical data). The correlations were non-significant when the peak-frequency time series was shuffled (Fig. 7F). There were however limitations to the frequency range within which, and the rate of frequency change for which, frequency matching between PING networks could be obtained. For example, when V1 frequency changed by more than $20 \mathrm{~Hz}$ in $200 \mathrm{~ms}$, V2 frequencies no longer matched V1 frequencies. Taken together, our modeling study shows that two PING networks in which one provides forward drive to the other produce an output that closely resembles our empirical observations (compare Fig. 7B-D with $1 \mathrm{~B}$ and $7 \mathrm{E}$ to $6 \mathrm{~A}$ ). Hence, the frequency of gamma oscillations in different populations exchanging information may not only be determined by local architecture in each area, but appears to be influenced by interactions among those populations. The interconnection of PING networks thus provides a mechanism that may be highly relevant for our empirical observations. This mechanism may underlie the entrainment or mutual interactions between communicating neural populations that are necessary to offset initial differences in oscillation frequencies, to thereby initiate and maintain communication between distant neural populations. 


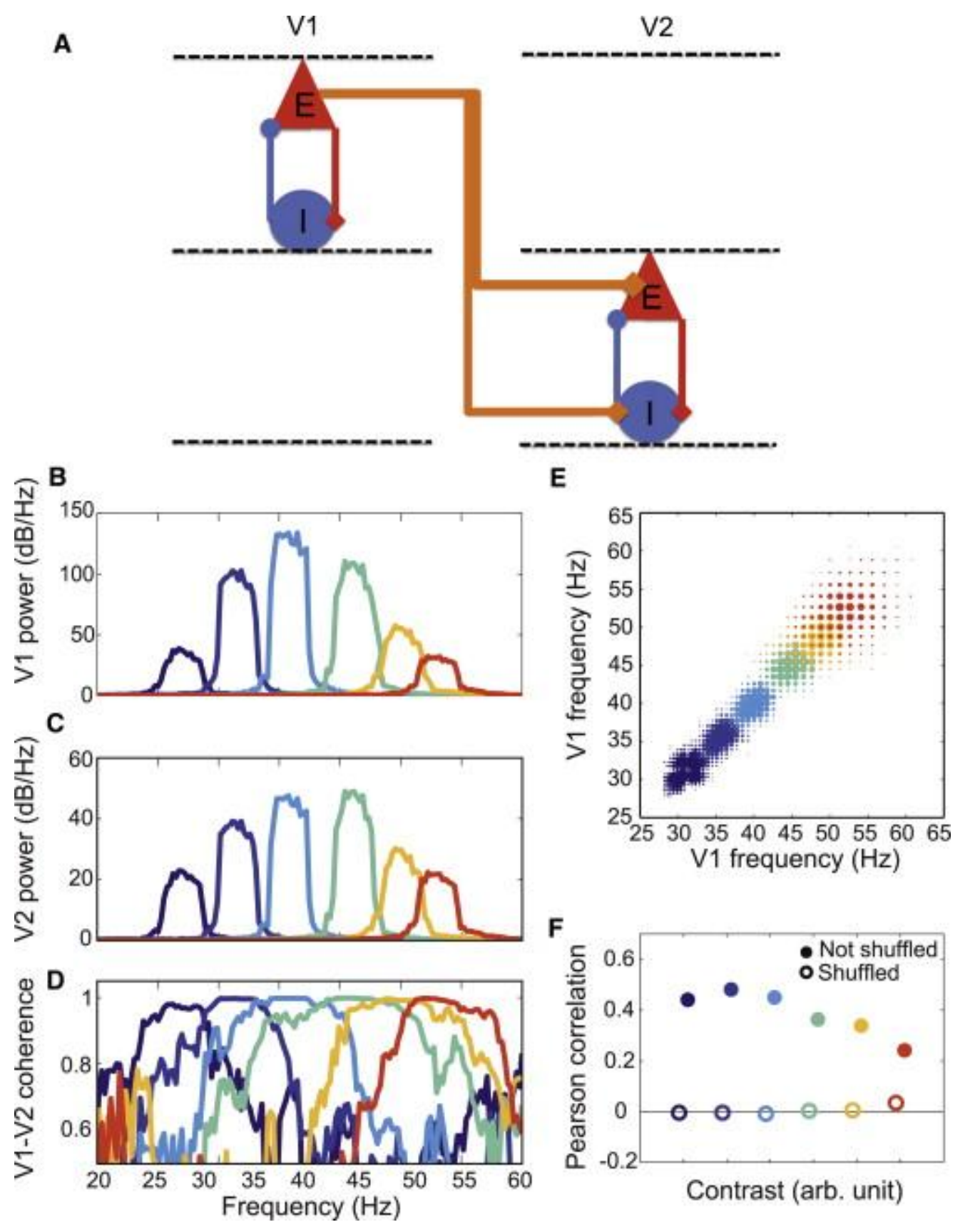

Figure 7: Simulation of frequency-frequency matching between V1 and V2 (A) Schematic representation of reciprocally coupled network of excitatory ( $\mathrm{E}, \mathrm{red}$ ) and inhibitory cells (I, blue) within V1 and V2. In each area, the network contained $400 \mathrm{E}$ cells and 100 I cells. Forward projection was modeled by E cells in superficial V1 projecting to both E as well as I cells in layer 4 of V2 (orange lines); the projections between areas were stronger than within area projections of the same type. $E$ to $E$ projections were a factor 4 stronger and $E$ to I projections were 5/3 times stronger (see Table S2). The visual stimulation was modeled by a constant depolarizing current to the $\mathrm{E}$ and I cells in V1, together with a slowly fluctuating, zero-mean drive. Stochastic fluctuations represented stimulus-induced stochastic fluctuations in the layer 4 activity which provides the input to V1 superficial layer. Stimulus contrast was modeled as the level of depolarization, and the time-varying firing rate of the $E$ cells was taken as a proxy for the LFP. Additional model description and parameter settings are presented in the Supplementary Materials. (B-F) Frequency analysis of simulated oscillations in V1 and V2 for different contrast values, varying from the lowest contrast in blue to the highest contrast in red. The gamma band shifted to higher frequencies with increased 'contrast' in V1 (B) 
and V2 (C), which is also reflected in the near-unity value for coherence between V1 and V2 at those common peak frequencies (D). The power at the peak frequency varied non-monotonously with a maximum at intermediate frequencies, which reflects an optimal frequency for within-area synchronization set by the synaptic time scale of fast inhibition. The analysis in panels B-D was based on average power over a long time range (3s). Despite modeled fluctuations in driving current, there was frequency matching between $V 1$ and $V 2$ on a short time scale ( $E$, dot size represents number of observations, ranging between 1 and 352 per dot). This led to significant correlations in the time-resolved peak frequencies (for all contrasts $p<0.0001$ ), which were lost when $\mathrm{V} 2$ time series were shuffled (F).

\section{Discussion}

We have shown that communication through coherence (CTC) between V1 and V2 remains possible despite significant stimulus and time-dependent changes in gamma frequency, because these changes occur in a coordinated fashion between areas. Other authors have proposed that coherence (synchrony) may be an important mechanism for 'binding' the representation of single stimuli among neurons within the same area - the so called 'binding by synchrony hypothesis' (Gray et al., 1989). This hypothesis is conceptually distinct from the communication-through-coherence hypothesis, which emphasizes the contribution of coherence to long-range, inter-areal communication. Note however that there is also some overlap between the two hypotheses, as coherent activity from distributed neurons at a lower stage of the cortical hierarchy may be more effectively integrated by later-stage neurons, indicating that binding implies also coherent inter-areal communication. Therefore, critical ideas on the relevance of synchrony/coherence for binding have immediate implications for CTC, and vice versa. Hence, although our data only addressed CTC directly, we here discuss our findings in the context of both theories.

\section{Binding by synchrony}

The theoretical proposal of binding by synchrony has attracted both support (Gray, 1999; Singer and Gray, 1995) and criticism (Palanca and DeAngelis, 2005; Thiele and Stoner, 2003). Of particular relevance for the idea of binding through within-area synchronous activity to distributed object parts, Ray and Maunsell (2010) showed that pairs of V1 neurons responding to different parts of a single contrastvarying grating (Gabor stimulus), responded with differing peak gamma frequencies, in line with our own data. Moreover, they showed reduced gamma coherence compared to conditions where contrast did not vary over the object surface, suggesting that binding-by-synchrony may not operate effectively for parts within an object characterized by different contrast and different gamma frequency. Hence the Ray and Maunsell (2010) and ours both point to the importance of a sufficient frequency match between different neuronal populations for maximizing neuronal communication by synchrony or coherence. From their interesting data, Ray and Maunsell (2010) concluded that binding by synchrony may be problematic for contrast-varying objects in V1. This prediction however, should be followed up by further experiments before we can fully exclude a synchrony based mechanism for binding. For example, Ray and Maunsell (2010) did not assess the monkey's perceptual experience of the object. It is possible that for large contrast differences, bright parts of the stimulus are seen as the foreground and darker as the background, or vice versa (Manjunath and Chellappa, 1993). Furthermore, we have recently shown attentional modulation of gamma frequency (Bosman et al., 2012) and we therefore 
speculate that attentional mechanisms could potentially reduce gamma frequency differences between neurons representing an attended object, which may be especially relevant in stimuli, or stimulus parts, showing smaller variations in contrast. Hence, we suggest that not only during CTC, but also during binding the presence or absence of frequency differences is perceptually relevant, and may within constraints be exploited by executive mechanisms (such as attention).

\section{Communication through coherence}

The peak-power frequency of gamma oscillations has been shown to vary considerably between individuals but has been thought to be largely stable within individuals (Hoogenboom et al., 2006; Muthukumaraswamy et al., 2010). A stable frequency might be considered to be an attractive attribute for theories that propose neuronal synchrony to underlie long-range communication (Fries, 2005; Salinas and Sejnowski, 2001; Tiesinga et al., 2002; Wildie and Shanahan, 2012), as stability would help to ensure that distant cortical areas maintain matching oscillation frequencies. Under conditions of nonstable frequency, an additional assumption must be made, namely that frequency shifts in separate areas occur in unison.

Until recently only minor shifts in peak frequency with changes in stimulus characteristics (Gray et al., 1990; Swettenham et al., 2009) and state of the animal (Feng et al., 2010) were demonstrated. These effects were generally relatively small (on the order of $5 \mathrm{~Hz}$ or less, but see Gieselmann and Thiele, 2008), and were not widely considered to represent a major challenge to the assumption of a stable intra-individual gamma band. However, we found a much stronger stimulus dependency of gamma frequency, varying from $18 \mathrm{~Hz}$ at the lowest contrast $(2.8 \%)$ to $45 \mathrm{~Hz}$ at the highest (78\%), in line with Ray and Maunsell (2010). Interestingly, recordings in anesthetized macaque, reported no change in frequency with varied contrast (Henrie and Shapley, 2005), which may be due to anesthesia: Xing et al (2012a) demonstrated a substantial reduction in gamma frequency during anesthesia which may limit the dynamic range for further modulation of frequency by stimulus contrast. Large stimulus-dependent shifts in frequency agree with computational network models (Buia and Tiesinga, 2006; Traub et al., 1996, but see also Vida et al., 2006), as well as in vitro (Llinas et al., 1991; Traub et al., 1996) and in vivo experiments (Atallah and Scanziani, 2009) linking oscillation frequency with excitatory drive (see also Fig. 7), which in those studies may be equated with stimulus contrast (Sanchez-Vives et al., 2000).

As local architecture of visual areas may lead to differences in their stimulus-dependency of gamma (Buzsaki and Chrobak, 1995), gamma power may be contained in different frequency bands for the same stimulus in different areas. As a sufficient frequency match of oscillatory activity is important to achieve phase coupling and coherence, CTC (Fries, 2005) can be expected to be limited if gamma frequencies in different areas are too disparate. In the latter case, the relationship between excitable periods in different populations of neurons would vary over time, thereby limiting neuronal communication. This limitation in principle could be circumvented without any need for an active frequency matching mechanism if the gamma band were sufficiently broad, shifted only slightly with stimulus variations, and maintained sufficient overlap in V1 and V2 for any stimulus condition. In that case, neural communication between areas could be maintained through coherence between common frequencies in the power spectra for each area, even if the power at these overlapping frequencies would be limited. 
However, our data demonstrate that the impact of stimulus contrast on V1 gamma frequency is powerful enough that, were V2 gamma frequency unaffected by contrast, changes in local gamma due to stimulus contrast could potentially move the frequency band of gamma synchrony in V1 so far away from the frequency band of gamma synchrony in V2 that gamma as a channel for communication would become unlikely. Furthermore, gamma frequency was found to be unstable in both areas, varying over a range of $\sim 15 \mathrm{~Hz}$ from moment to moment. Were this variation to occur independently, gamma frequencies in the two areas would be incompatible for a large part of the time, even if time-averaged peak frequencies matched, thus, rapid and non-stimulus dependent shifts in frequency would present an additional obstacle to effective communication.

However, we found (I) that the power spectra of the LFP in V1 and V2 showed similar shifts in peak power as a function of stimulus contrast; (II) that the same peak shift occurred in the coherence between V1 and V2; (III) that the pattern of coherence between V1 and V2 was layer-specific and matched the pattern expected according to feedforward anatomical connectivity; (IV) that the pattern was robust against large variations in stimulus contrast; and (V) that rapid and non-stimulus dependent shifts in gamma frequency were highly correlated in V1 and V2 consistent with a frequency matching mechanism that was modeled successfully by a computational neuronal network comprising two interconnected PING networks. Thus, cross-area coherence was maintained in an anatomically consistent pattern over conditions of widely and rapidly varying gamma frequency, in line with a role of coherence in neural communication. The evidence emerging in this research field that neural communication through coherence does not require a fixed gamma frequency, and that gamma frequency differences can make or break communication links, suggests that frequency differences could be exploited as a mechanism to route information in the brain during perceptual and cognitive operations.

\section{Experimental Procedures}

Species used and surgical procedures: Two male Macaca mulatta were used in this study. All procedures were in accordance with the European Communities Council Directive 1986 (86/609/EEC), and approved by the local ethics committee. Following initial training, monkeys were implanted with a titanium head holder (Crist instruments), and a recording chamber ( $\mathrm{NaN}$ instruments) above V1/V2 under general anesthesia and sterile conditions. In a second surgery, after further training, a craniotomy was made above V1/V2.

Stimuli: Stimuli were presented on a Samsung TFT screen (SyncMaster $940 \mathrm{bf}, 38^{\circ} \times 30 \circ 60 \mathrm{~Hz}$ ). Stimuli were circular patches (typical diameter of $5^{\circ}$, but varied in some sessions between $1^{\circ}$ and $9^{\circ}$ ) of static (not drifting) square-wave gratings (2 cycles/degree), at luminance contrasts of $2.5 \%, 3.7 \% 6.1 \% 9.7 \%$ $16.3 \% 35.9 \% 50.3 \%$ or $72 \%$. Stimuli were presented at two orthogonal orientations. Average luminance matched the background $\left(125 \mathrm{~cd} / \mathrm{m}^{2}\right)$. Stimulus presentation time was randomized between 750 and $4000 \mathrm{~ms}$ and preceded by $1000 \mathrm{~ms}$ pre-stimulus time. During stimulation and pre-stimulus time the monkey maintained eye position (measured by infra-red camera, Arrington $60 \mathrm{~Hz}$ sampling rate) within a 
square window of $2 \times 2^{\circ}$. This window was relatively large to allow for noise associated with the camera. Eye position was considerably more stable than the window allowed. The median difference in eye position from one trial to the next was 0.23 o in Monkey $\mathrm{S}$ and 0.5 o degrees in Monkey $\mathrm{K}$. This excludes the possibility that the large grating stimuli centered on the RFs of the recorded neurons would have left the RFs on any trials. The median total range in eye position within trials, as measured with the Arrington system, was 0.8 in monkey $S$ and 0.45 o in monkey $\mathrm{K}$. These values likely underestimate the true accuracy of fixation. In later experiments in monkey $\mathrm{S}$, we have used a high speed infra-red camera system (Thomas Recording $245 \mathrm{~Hz}$ system), in conjunction with the Arrington system. Using the Thomas recording system we recorded a more stable eye position, with within trial median total range in eye position of 0.3 . Since RFs were small relative to the size and spatial frequency of the grating, eye movements in the order of a few tenths of a degree would be too small to radically change the nature of the stimulation.

Recording methods: Spikes and LFP were recorded using 2 linear arrays of 8 recording contacts (Plexon inc.) with $200 \mu \mathrm{m}$ inter-contact spacing. Recording arrays were manipulated using NaN instruments micro-drives. LFPs were filtered $(0.7$ to $300 \mathrm{~Hz})$ and recorded at $1 \mathrm{KHz}$ (Plexon MAP system). The probes were placed $4 \mathrm{~mm}$ to $6 \mathrm{~mm}$ apart such that RFs from the two areas were overlapping or near-overlapping (mean overlap of simultaneously recorded V1 and V2 RF areas=14.3\%; standard deviation 22.3\%). Regions with close RFs are likely to have stronger anatomical connections (Lund et al., 2003), and stronger coherence (Bosman et al., 2012; Nowak et al., 1999), than regions with distant RFs. In our data cross area CSD coherence was not found to be correlated with V1-V2 RF distance in monkey $S$ $\left(R^{2}=0.0002, p=0.5\right.$ robust linear regression) and was only weakly (negatively) correlated in monkey $K\left(R^{2}\right.$ $=0.003, p=0.039$ ). This indicates that the daily variation in the amount of RF overlap was insufficient to affect coherence.

Data are included from 17 recording sessions from monkey $S$ and 17 from monkey $\mathrm{K}$. In total, data are included from 202 recording sites in V1, and 220 in V2 (monkey S V1=107, V2=109, monkey K V1=95, $\mathrm{V} 2=111$ ). Deeper sites, of which we could document, based on receptive field mapping, that they did not belong to the area of interest and were excluded from these counts (see Supplemental Online Material for details). We collected an average of 62 trials per condition per session in monkey $\mathrm{S}$, and 53 trials per condition per session in monkey $\mathrm{K}$. In analyses of responses in single areas (V1 or V2 separately), all available data were used. In analysis of V1-V2 coherence, functional connectivity and frequencyfrequency correlation, less data could be included because data were missing in either V1 or V2 of some sessions, for example, because of a probe not sufficiently entering cortex. Coherence and functional connectivity analyses in monkey $\mathrm{S}$ were based on 13 sessions with data from both areas (2 session rejected because of missing V1 data; 2 sessions rejected because of missing V2 data). In monkey K, these analyses were based on 12 sessions with data from both areas ( 3 sessions rejected because of missing V1 data, and 2 sessions rejected because of missing V2 data).

Receptive fields (RFs) were mapped using high contrast black and white squares presented individually at a fast rate, on a $10 \times 10^{\circ}$ grid, with square sizes varying from $0.1^{\circ}$ to $1^{\circ}$. Averaged over monkeys (who showed similar values) RF sizes were $.75^{\circ}$ in V1 (5.4 eccentricity) and $0.91^{\circ}$ in V2 (5.9 eccentricity). V2 
recordings were done on the pre-lunate gyrus. Data were classified as belonging to V1 or V2 based on conventional criteria (Gattass et al., 1981; see Supplemental Materials).

Data selection: The first $500 \mathrm{~ms}$ following fixation onset, and the first $350 \mathrm{~ms}$ following stimulus onset were discarded from data analysis to avoid effects of fixation onset and stimulus onset transients. In addition to the large shift $(\sim 25 \mathrm{~Hz})$ of peak gamma frequency with increases in grating contrast, we also observed much smaller shifts $(\sim 1-2 \mathrm{~Hz})$ as a function of stimulus size and orientation. In the analyses presented in the Main Text, data were pooled across all size and orientation conditions per contrast.

Power and coherence computations: Stimulus-induced power (Psi) in the LFP signal was computed from non-overlapping $500 \mathrm{~ms}$ (or $1000 \mathrm{~ms}$ for analysis shown in Fig. 5) time windows starting $350 \mathrm{~ms}$ after stimulus onset (S). LFP spectra were computed using a multi-taper method with discrete prolate spheroid sequences (DPSS) for frequencies 6 to $80 \mathrm{~Hz}$ (smoothing $\pm 3 \mathrm{~Hz}$ ), or for analysis of $1000 \mathrm{~ms}$ snippets (Fig. 4), using Hanning tapers for frequencies between 2 and $80 \mathrm{~Hz}$ and $2 \mathrm{~Hz}$ frequency resolution (Oostenveld et al., 2011). Power generally decreases as a function of frequency (f) in a $1 / f$ manner. Hence power in higher-frequency bands can be difficult to discern, and power spectra recorded in the stimulation period $(S)$ was indexed against power spectra recording during the pre-stimulus baseline (B) computed over the $500 \mathrm{~ms}$ prior to stimulus onset $[\mathrm{Psi}=(\mathrm{S}-\mathrm{B}) /(\mathrm{S}+\mathrm{B})]$. The $1 /$ frequency dropoff of power spectra was thus removed from the data. Coherence was calculated as the magnitude of the summed cross-spectral density between two CSD time series, normalized by respective power spectra (Oostenveld et al., 2011).

Granger calculation: We used a non-parametric spectral matrix factorization of the CSD cross-spectral density (calculated from the period of $350 \mathrm{~ms}$ after stimulus onset, to the trial end) to estimate feedforward and feedback influences for a given V1-V2 contact pair (Dhamala et al., 2008), using the Fieldtrip toolbox (Oostenveld et al., 2011). To test for significance for an unidirectional dominance in granger influence over different contrasts the difference between feedforward and feedback terms were recomputed 10000 times with randomized feedback and feedforward labels of V1-V2 pairs to estimate the null distribution of the effective directionality. For significance testing the frequency range of $20-55 \mathrm{~Hz}$ was used. At each randomization iteration, we subtracted the maximum value of the feedback term from the maximum value of the feedforward term. Correspondingly, a unidirectional dominance was considered to be significant if it reached the top or bottom 2.5 percentile of the nulldistribution. In Fig. 5 data were pooled over all V1-V2 contact pairs with 5\% strongest coherence values.

Frequency-Frequency locking estimation: To assess the moment-to-moment evolution of oscillation frequency in the gamma range we estimated the frequency based on complex Morlet wavelet timefrequency CSD representations for each trial (wavelet width $\sigma=5 \mathrm{~ms}$ ). For a given $1 \mathrm{~ms}$ time bin the peak power in the frequency range $25-55 \mathrm{~Hz}$ was recorded and only peak power that differed by $>2 \sigma$ from baseline power was included. Four different contrasts $(16.3 \%, 35.9 \%, 50.3 \%, 72 \%)$ were included. At lower contrasts gamma oscillations were partly intermixed with other processes operating at alpha/beta frequency regime. In Fig. 6a an example of a V1-V2 contact pair (from Monkey S) is shown with frequency-frequency scatter-plot and marginal distribution for three different contrasts $(16 \%, 35.9 \%$, $50.3 \%)$. Fig. $6 \mathrm{~b}$ shows combined joint probability surfaces and marginal distributions from all contact 
pairs in the population. To quantify the strength of the correlation we then computed the Pearson correlation for each contact pair. To test for significance a randomization procedure was implemented by shuffling trial labels. Significance testing was done as for Granger estimates. In Fig. $6 \mathrm{c}$ the median and inter-quartile range of the distribution is shown for randomized and non-randomized overall distribution as well as the distribution of $5 \%$ strongest coherence connections highlighted in Fig. 3 . We found significant correlation coefficients (that is outside of $97.5 \%$ of the shuffled data distribution) over the population and especially for the strongest coherence connections.

\section{Acknowledgments}

Research supported by NWO VICl grant to P.D.W. (\#453-04-002), NWO VENI grant to M.R. (\#451-09025), and Maastricht University Graduate funding in cooperation with Donders Institute for Brain Behavior and Cognition to P.D.W. Experiments were designed by P.D.W. and M.R. Measurements were carried out by M.R. and E.L. with assistance from N.B. Analysis was carried out by M.R. and E.L. and modeling by M.T.W. with guidance from P.T., P.F., and P.D.W. The paper was written by M.R., E.L and P.D.W in collaboration with the other authors. 


\section{References}

Atallah, B. V., \& Scanziani, M. (2009). Instantaneous modulation of gamma oscillation frequency by balancing excitation with inhibition. Neuron, 62, 566-577. doi: 10.1016/j.neuron.2009.04.027

Bastos, A. M., Usrey, W. M., Adams, R. A., Mangun, G. R., Fries, P., \& Friston, K. J. (2013). Canonical microcircuits for predictive coding . Neuron, 76(4), 695-711. doi:

10.1016/j.neuron.2012.10.03810.1016/j.neuron.2012.10.038

Bosman, C. A., Schoffelen, J., Brunet, N., Oostenveld, R., Bastos, A. M., Womelsdorf, T., ... Fries, P. (2012). Attentional Stimulus Selection through Selective Synchronization between Monkey Visual Areas. Neuron, 75, 875-888.

Buffalo, E. A., Fries, P., Landman, R., Buschman, T. J., \& Desimone, R. (2011). Laminar differences in gamma and alpha coherence in the ventral stream. Proc Natl Acad Sci U S A, 108(27), 1126211267. doi: 10.1073/pnas.1011284108

Buia, C., \& Tiesinga, P. (2006). Attentional modulation of firing rate and synchrony in a model cortical network. [Research Support, Non-U.S. Gov't]. J Comput Neurosci, 20(3), 247-264. doi: 10.1007/s10827-006-6358-0

Burns, S. P., Xing, D., \& Shapley, R. M. (2011). Is gamma-band activity in the local field potential of v1 cortex a "clock" or filtered noise? J Neurosci, 31, 9658-9664. doi: 10.1523/JNEUROSCI.066011.2011

Buzsaki, G., \& Chrobak, J. J. (1995). Temporal structure in spatially organized neuronal ensembles: a role for interneuronal networks. Curr Opin Neurobiol, 5(4), 504-510.

Buzsaki, G., \& Draguhn, A. (2004). Neuronal oscillations in cortical networks. Science, 304, 1926-1929. doi: $10.1126 /$ science.1099745

Callaway, E. M. (1998). Local circuits in primary visual cortex of the macaque monkey. Annu Rev Neurosci, 21, 47-74. doi: 10.1146/annurev.neuro.21.1.47

de Sousa, A. A., Sherwood, C. C., Schleicher, A., Amunts, K., MacLeod, C. E., Hof, P. R., \& Zilles, K. (2010). Comparative cytoarchitectural analyses of striate and extrastriate areas in hominoids. Cereb Cortex, 20, 966-981. doi: 10.1093/cercor/bhp158

Dhamala, M., Rangarajan, G., \& Ding, M. (2008). Analyzing information flow in brain networks with nonparametric Granger causality. Neuroimage, 41, 354-362. doi:

10.1016/j.neuroimage.2008.02.020

Douglas, R. J., \& Martin, K. A. (2004). Neuronal circuits of the neocortex. Annu Rev Neurosci, 27, 419451. doi: 10.1146/annurev.neuro.27.070203.144152

Felleman, D. J., \& Van Essen, D. C. (1991). Distributed hierarchical processing in the primate cerebral cortex.Cereb Cortex, 1(1), 1-47.

Feng, W., Havenith, M. N., Wang, P., Singer, W., \& Nikolic, D. (2010). Frequencies of gamma/beta oscillations are stably tuned to stimulus properties. [Research Support, Non-U.S. Gov't]. Neuroreport, 21(10), 680-684. doi: 10.1097/WNR.0b013e32833ae9d1

Fries, P. (2005). A mechanism for cognitive dynamics: neuronal communication through neuronal coherence. Trends Cogn Sci, 9(10), 474-480. 
Gieselmann, M. A., \& Thiele, A. (2008). Comparison of spatial integration and surround suppression characteristics in spiking activity and the local field potential in macaque V1. Eur J Neurosci, 28(3), 447-459.

Gray, C. M. (1999). The temporal correlation hypothesis of visual feature integration: still alive and well. Neuron, 24, 31-47, 111-125.

Gray, C. M., Engel, A. K., Konig, P., \& Singer, W. (1990). Stimulus-Dependent Neuronal Oscillations in Cat Visual Cortex: Receptive Field Properties and Feature Dependence. Eur J Neurosci, 2(7), 607-619.

Gray, C. M., Konig, P., Engel, A. K., \& Singer, W. (1989). Oscillatory responses in cat visual cortex exhibit inter-columnar synchronization which reflects global stimulus properties. Nature, 338(6213), 334-337. doi: 10.1038/338334a0

Gray, C. M., \& McCormick, D. A. (1996). Chattering cells: superficial pyramidal neurons contributing to the generation of synchronous oscillations in the visual cortex.Science, 274(5284), 109-113.

Henrie, J. A., \& Shapley, R. (2005). LFP power spectra in V1 cortex: the graded effect of stimulus contrast. J Neurophysiol, 94(1), 479-490.

Hoogenboom, N., Schoffelen, J. M., Oostenveld, R., Parkes, L. M., \& Fries, P. (2006). Localizing human visual gamma-band activity in frequency, time and space. Neuroimage, 29(3), 764-773.

Jia, X., Xing, D., \& Kohn, A. (2013). No Consistent Relationship between Gamma Power and Peak Frequency in Macaque Primary Visual Cortex. J Neurosci, 33, 17-25. doi: 10.1523/JNEUROSCI.1687-12.2013

Llinas, R. R., Grace, A. A., \& Yarom, Y. (1991). In vitro neurons in mammalian cortical layer 4 exhibit intrinsic oscillatory activity in the 10- to 50-Hz frequency range. Proc Natl Acad Sci U S A, 88(3), 897-901.

Lund, J. S. (1988). Anatomical organization of macaque monkey striate visual cortex. Annu Rev Neurosci, 11, 253-288. doi: 10.1146/annurev.ne.11.030188.001345

Lund, J. S., Angelucci, A., \& Bressloff, P. C. (2003). Anatomical substrates for functional columns in macaque monkey primary visual cortex. Cereb Cortex, 13(1), 15-24.

Lund, J. S., Lund, R. D., Hendrickson, A. E., Bunt, A. H., \& Fuchs, A. F. (1975). The origin of efferent pathways from the primary visual cortex, area 17 , of the macaque monkey as shown by retrograde transport of horseradish peroxidase. [Research Support, U.S. Gov't, P.H.S.]. J Comp Neurol, 164(3), 287-303. doi: 10.1002/cne.901640303

Maier, A., Aura, C. J., \& Leopold, D. A. (2011). Infragranular sources of sustained local field potential responses in macaque primary visual cortex. J Neurosci, 31(6), 1971-1980.

Manjunath, B. S., \& Chellappa, R. (1993). A unified approach to boundary perception: edges, textures, and illusory contours. IEEE Trans Neural Netw, 4(1), 96-108. doi: 10.1109/72.182699

Mitzdorf, U. (1985). Current source-density method and application in cat cerebral cortex: investigation of evoked potentials and EEG phenomena. Physiol Rev, 65(1), 37-100.

Muthukumaraswamy, S. D., Singh, K. D., Swettenham, J. B., \& Jones, D. K. (2010). Visual gamma oscillations and evoked responses: variability, repeatability and structural MRI correlates. Neuroimage, 49(4), 3349-3357.

Nassi, J. J., \& Callaway, E. M. (2009). Parallel processing strategies of the primate visual system. Nat Rev Neurosci, 10, 360-372. doi: 10.1038/nrn2619 
Nowak, L. G., Munk, M. H., James, A. C., Girard, P., \& Bullier, J. (1999). Cross-correlation study of the temporal interactions between areas V1 and V2 of the macaque monkey. J Neurophysiol, 81(3), 1057-1074.

Oostenveld, R., Fries, P., Maris, E., \& Schoffelen, J. M. (2011). FieldTrip: Open source software for advanced analysis of MEG, EEG, and invasive electrophysiological data. Comput Intell Neurosci, Article ID 156869. doi: 10.1155/2011/156869

Palanca, B. J., \& DeAngelis, G. C. (2005). Does neuronal synchrony underlie visual feature grouping? Neuron, 46, 333-346. doi: 10.1016/j.neuron.2005.03.002

Ray, S., \& Maunsell, J. H. (2010). Differences in gamma frequencies across visual cortex restrict their possible use in computation. Neuron, 67(5), 885-896.

Ray, S., \& Maunsell, J. H. (2011). Different origins of gamma rhythm and high-gamma activity in macaque visual cortex. PLoS Biol, 9(4), e1000610. doi: 10.1371/journal.pbio.1000610

Rosenblum, M., Pikovsky, A., \& Kurths, J. (2001). Synchronization: A Universal Concept in Nonlinear Sciences (Cambridge Nonlinear Science Series). Cambridge, United Kingdom: Cambridge University Press.

Salinas, E., \& Sejnowski, T. J. (2001). Correlated neuronal activity and the flow of neural information. Nat Rev Neurosci, 2, 539-550. doi: 10.1038/35086012

Sanchez-Vives, M. V., Nowak, L. G., \& McCormick, D. A. (2000). Membrane mechanisms underlying contrast adaptation in cat area 17 in vivo. J Neurosci, 20, 4267-4285.

Schroeder, C. E., Tenke, C. E., Givre, S. J., Arezzo, J. C., \& Vaughan, H. G., Jr. (1991). Striate cortical contribution to the surface-recorded pattern-reversal VEP in the alert monkey. Vision Res, 31, 1143-1157.

Singer, W., \& Gray, C. M. (1995). Visual feature integration and the temporal correlation hypothesis. [Comparative Study Review]. Annu Rev Neurosci, 18, 555-586. doi:

10.1146/annurev.ne.18.030195.003011

Smith, M. A., Jia, X., Zandvakili, A., \& Kohn, A. (1213). Laminar dependence of neuronal correlations in visual cortex. J Neurophysiol. doi: 10.1152/jn.00846.2012

Swettenham, J. B., Muthukumaraswamy, S. D., \& Singh, K. D. (2009). Spectral properties of induced and evoked gamma oscillations in human early visual cortex to moving and stationary stimuli. $J$ Neurophysiol, 102(2), 1241-1253.

Thiele, A., \& Stoner, G. (2003). Neuronal synchrony does not correlate with motion coherence in cortical area MT. Nature, 421, 366-370. doi: 10.1038/nature01285

Tiesinga, P. H., Fellous, J. M., Jose, J. V., \& Sejnowski, T. J. (2002). Information transfer in entrained cortical neurons.Network, 13(1), 41-66.

Traub, R. D., Whittington, M. A., Colling, S. B., Buzsaki, G., \& Jefferys, J. G. (1996). Analysis of gamma rhythms in the rat hippocampus in vitro and in vivo. J Physiol, 493(Pt 2), 471-484.

Vida, I., Bartos, M., \& Jonas, P. (2006). Shunting inhibition improves robustness of gamma oscillations in hippocampal interneuron networks by homogenizing firing rates. Neuron, 49(1), 107-117.

Wildie, M., \& Shanahan, M. (2012). Establishing communication between neuronal populations through competitive entrainment. Frontiers in computational neuroscience, 5(62), 1-16. 
Xing, D., Shen, Y., Burns, S., Yeh, C. I., Shapley, R., \& Li, W. (2012). Stochastic generation of gamma-band activity in primary visual cortex of awake and anesthetized monkeys. $J$ Neurosci, 32, 1387313880. doi: 10.1523/JNEUROSCI.5644-11.2012

Xing, D., Yeh, C. I., Burns, S., \& Shapley, R. M. (2012). Laminar analysis of visually evoked activity in the primary visual cortex. Proc Natl Acad Sci U S A, 109, 13871-13876. doi:

10.1073/pnas.1201478109

\section{Supplemental Information}

Robust Gamma Coherence between Macaque V1 and V2 by Dynamic Frequency Matching

Mark J. Roberts, Eric Lowet, Nicolas M. Brunet, Marije Ter Wal, Paul Tiesinga, Pascal Fries, and Peter De Weerd

Inventory

Supplemental Data

Figures S1-S4

Tables S1, S2

Supplemental Experimental Procedures

Supplemental References

\section{Links between figures in main text and items in Supplemental Data}

Figure 1 (Contrast-dependent shift in gamma frequency band)

Figure S1 (stimulation setup and paradigm)

Figure 2 (Correlations among gamma frequencies)

Figure S2 (spectral peak determination)

Figure 3 (Depth-specific pattern of V1-V2 gamma coherence is preserved across contrast conditions)

Figure S3 (depth alignment procedure),

Figure 5 (Quantitative comparison of the cross area coherence pattern across frequencies and contrast conditions)

Figure S4 (statistical evaluation of connectivity pattern)

Figure 7 (Simulation of frequency-frequency matching between V1 and V2)

Tables S1 S2 


\section{Supplemental Data}

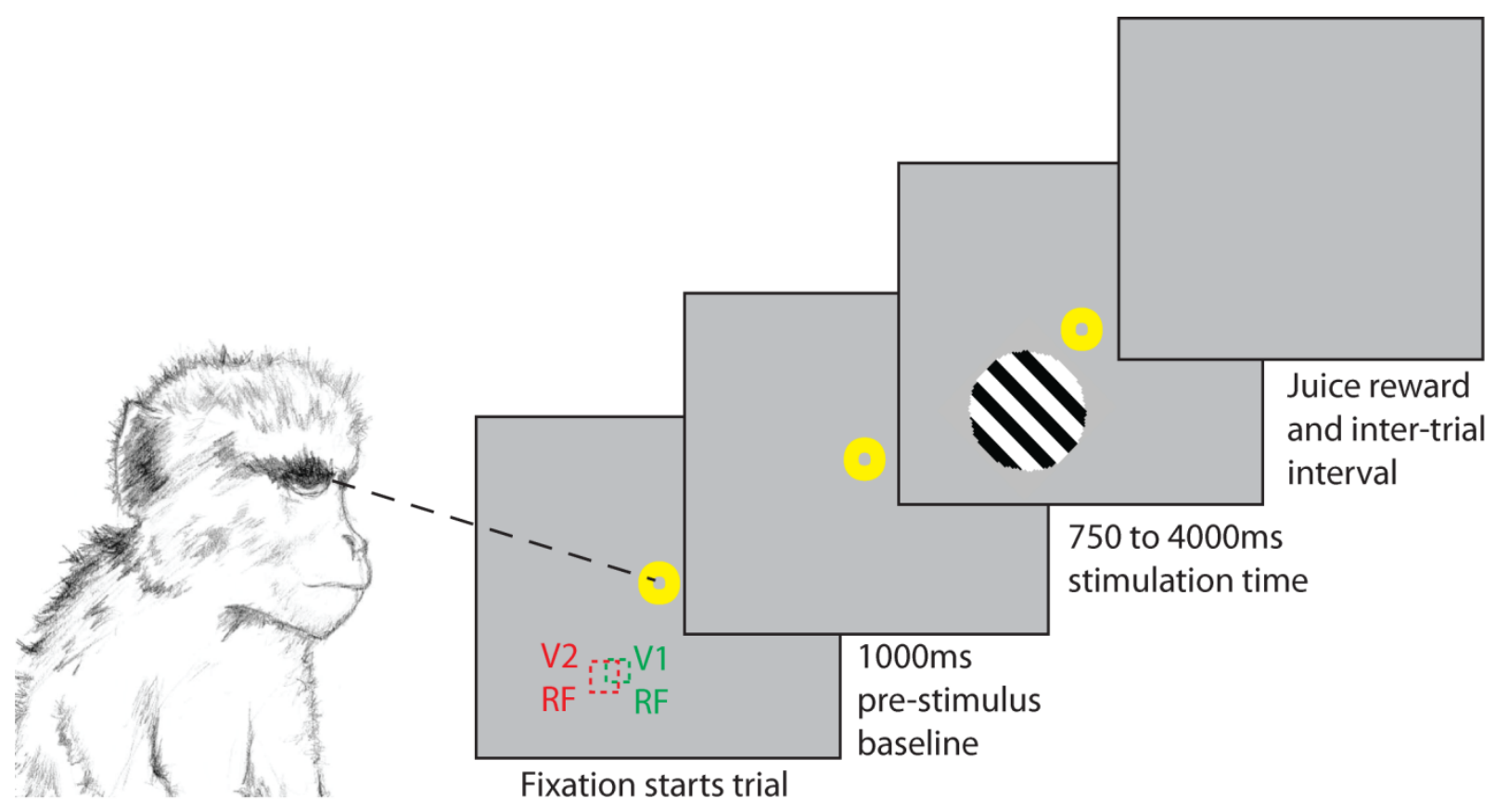

Figure S1: Stimulation setup and paradigm (underlying data shown in main text Fig. 1). Epochs in the trial are shown as grey screen shots as seen by the monkey. The monkey fixated its gaze on central fixation spot (yellow annulus of which the center had to be fixated, enlarged for illustrative purposes) which started the trial. The red and green dashed square outlines represent $\mathrm{V} 1$ and $\mathrm{V} 2$ receptive fields (RFs) mapped prior to the beginning of the experiment. The trials started with a 1000ms baseline preceding stimulus presentation. Stimuli were circular patches of square-wave gratings ( 2 cycles/degree) presented at the location of the V1 and V2 RFs. Stimulus presentation time was randomized between 750 and $4000 \mathrm{~ms}$. If the monkey's eye position did not move from the fixation point for the duration of the trial the monkey received a juice reward at the end of the trial. The monkey was free to start a new trial after a minimum period of $1000 \mathrm{~ms}$. The minimum wait period was included to prevent muscle artifacts associated with juice licking being included in the baseline period of the subsequent trial. For further details, see Supplemental Experimental Procedures. 

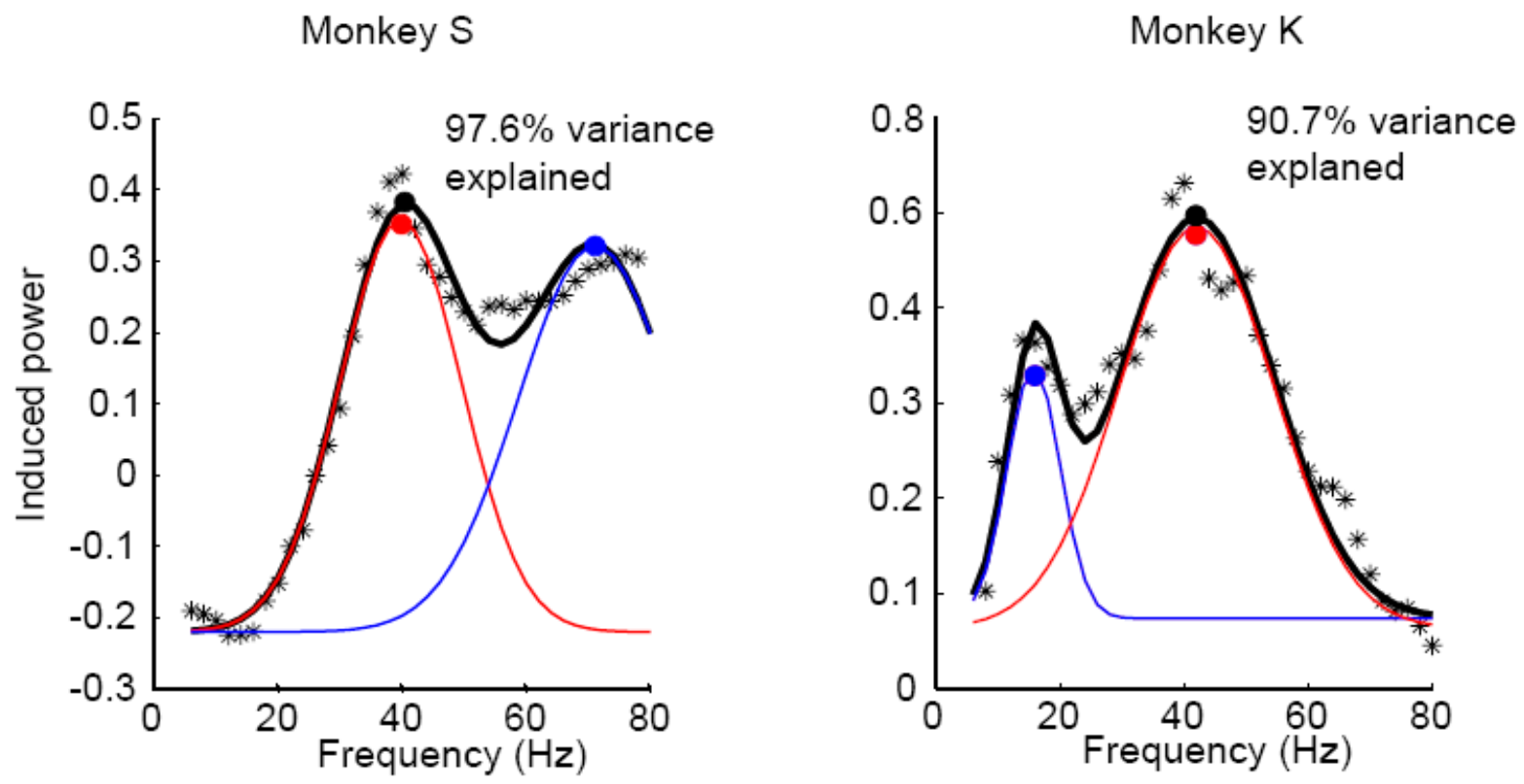

Figure S2: Spectral peak determination (underlying peak power gamma frequencies in main Figs. 1c and 2). Single session examples of induced power data (black stars) fitted with double Gaussian function (black line). The function is the combination of two Gaussians, one corresponding to the gamma band (red curve) and the second curve (blue curve) corresponding to a broad-spectrum high frequency elevation, typical in monkey S (left panel), or a Beta band, typical in monkey $\mathrm{K}$ (right panel). Black, blue and red dots show respectively, peak of double Gaussian function (used for analysis in main Figs $1 \mathrm{c}$ and 2 ) and peaks of both constituent Gaussian curves. Further details of fitting procedures can be found in Supplemental Experimental Procedures. 
A

LFP visually evoked potential
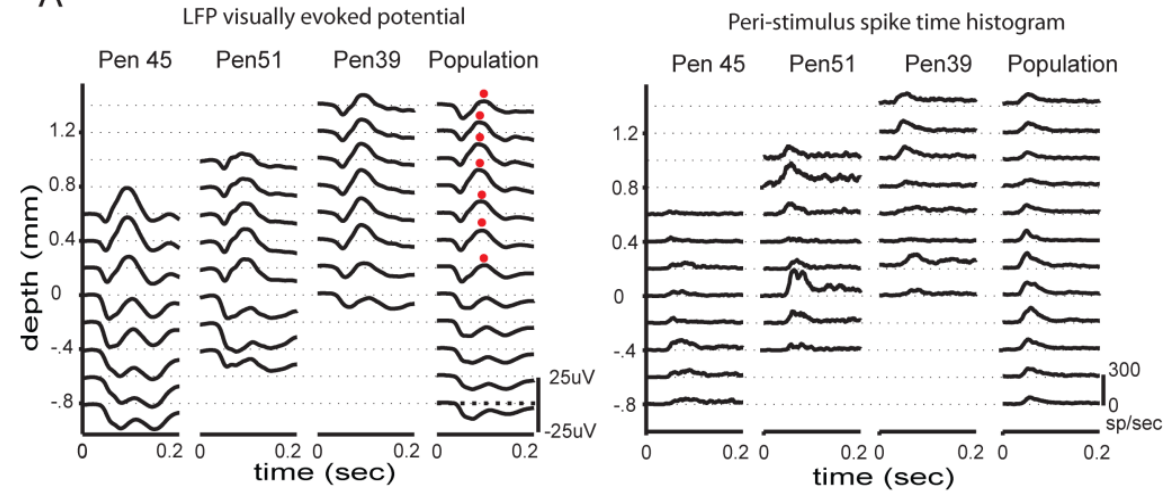

B



V1 $Y=.96 X+0 R=0.5 P<0.01$

V2 $Y=.82 X+0.1 R=0.3 P<0.05$

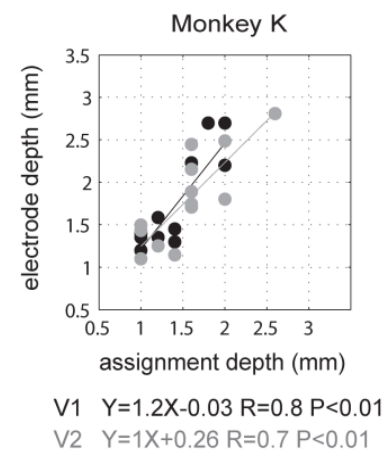

C
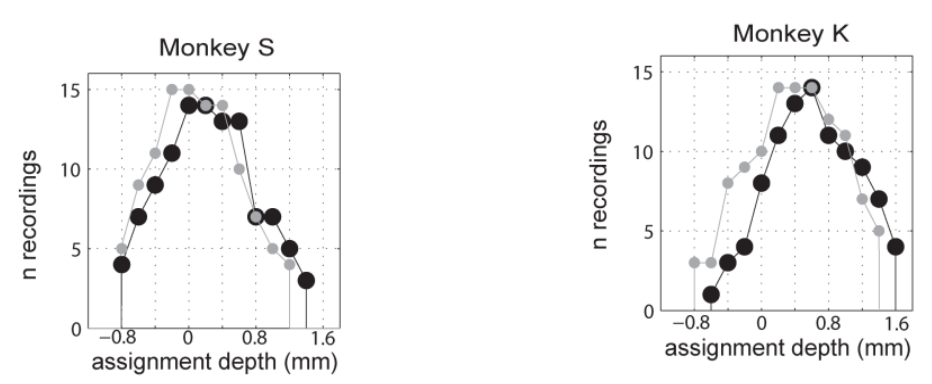

D

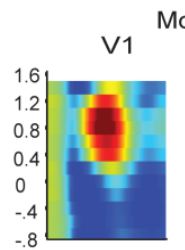

Monkey S
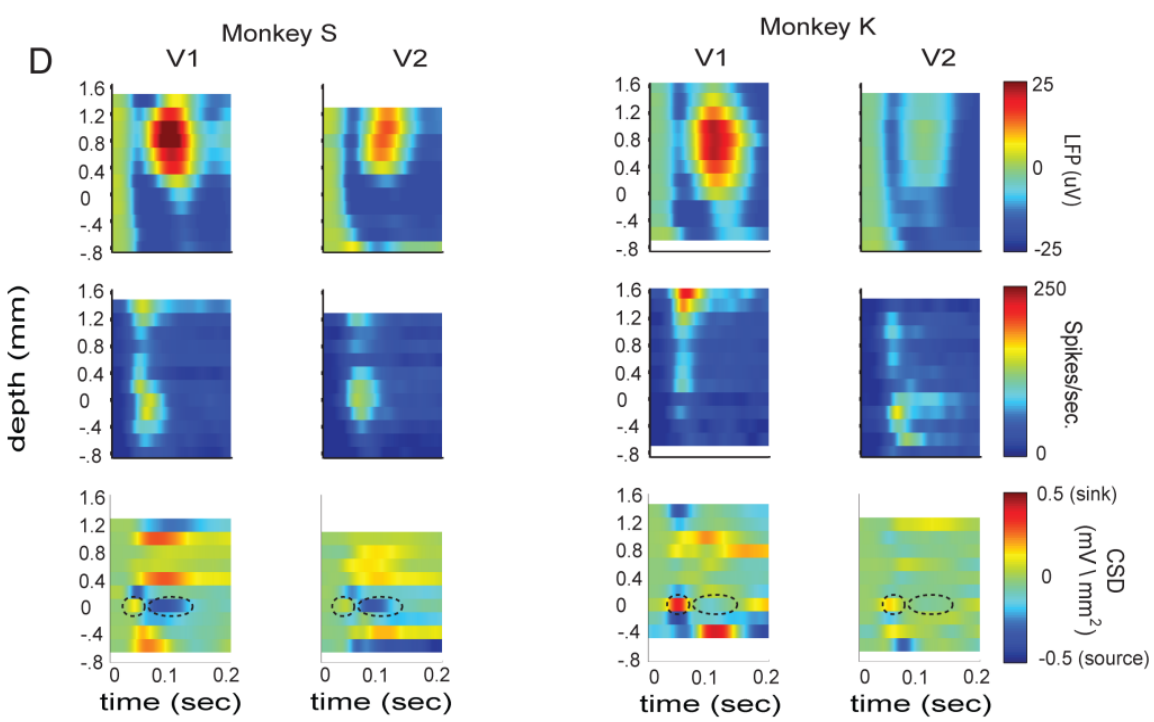
Figure S3: Depth alignment procedure, and resulting time and depth-resolved VEPS, CSD and spiking responses to the experimental grating onset after depth alignment. The depth alignment described here underlies main text Fig. 3 and derived main text Figs. 4-6. A) Demonstration of depth alignment according to visually-evoked potential (VEP) at different depth contacts in monkey S (left) with corresponding spiking data (right). The VEP data consist of 4 columns. The three leftmost columns show 3 examples of aligned VEP data sets from monkey $S$ area V1 (Pen 45, 51, 39). These data are flanked by the fourth column showing the average shape of VEPs at all recorded depths (200um spacing) after pooling of all available data (Population). Data from different sessions (penetrations) could be aligned to one another in depth by carefully comparing the shapes of VEPs. Final probe positions taken together yielded probe contacts covering the full thickness of cortex (see population average). Therefore, for any individual session, the difference between the depth of the deepest contact estimated from aligned data, and the shallowest depth showing a VEP response in population data provided an alignment-based estimate of depth for each final probe position ( $Y$ axis values). After alignment, a clear pattern emerged in which at shallow depths, there were strong positive deflections in the VEP about $100 \mathrm{~ms}$ post stimulus onset (marked by red dots in population average), and a disappearance of these deflections a greater depths. Spiking data (peri-stimulus time histograms) in response to the experimental grating were variable from session to session, but on average indicate a patterned response across layers with variation in response latency in accordance with the known literature (see below, Depth alignment procedure and validation). B) In addition to depth alignment based on VEP shape, we also tracked physical probe depth. For each penetration the physical probe depth during experimental recordings was measured relative to the shallowest depth of the probe at which a VEP could be elicited by the brief presentation $(250 \mathrm{~ms})$ of a full-screen checkerboard stimulus (indicating the first depth of entering the beginning of the first layer of cortex). Thus, the physically 'measured' depth of the probe could be compared with the alignment-based depth for each session. Note that these two depth estimates are not necessarily equal, as the movement of the probe through the tissue may deform the tissue to different extents in different sessions, however over sessions we found a high correspondence of physically measured depth and 'assigned depth', as demonstrated by scatter plots in monkeys $\mathrm{S}$ and $\mathrm{K}$ showing a close correlation between physically measured depth and alignment-based depth of the deepest contact of the probe in both monkeys for V1 (black regression line and symbols) and for V2 (grey regression line and symbols). Example sessions shown in panel A are circled. C) Histogram of the number of observations made at each depth level, which were more numerous around the center of the cortex. V1 and V2 data are shown in black and grey respectively. D) LFP (top row), spiking (middle) and CSD data (bottom) onset response during $72 \%$ contrast stimulation after VEP-based data alignment computed on all sessions for V1 and V2 from the two monkeys. 'Zero' depth corresponded to the first contact position below the strong positive deflection of VEPs at $\sim 100 \mathrm{~ms}$ after stimulus onset (see panel A red dots). Average visually evoked LFP, spiking and CSD over cortical depth for V1 and V2 are shown as colored surface plots, with color scaling identical along each row. In CSD surface plots, blue/green and yellow/red respectively represent sources and sinks. CSD surface plots show a strong source/sink reversal at depth zero, most clearly present in V1 and V2 of monkey S, and to a lesser extent in monkey $\mathrm{K}$ (see dashed outlines superimposed on color surfaces) at depth zero. 
Monkey S

V1

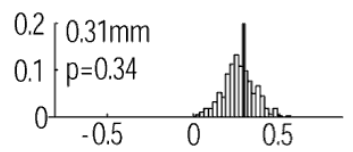

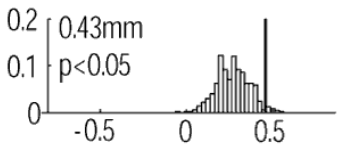

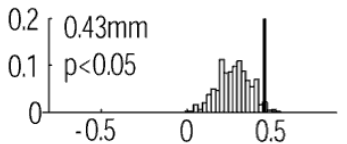

$0.2[0.35 \mathrm{~mm}$

0.1 . $p=0.1$
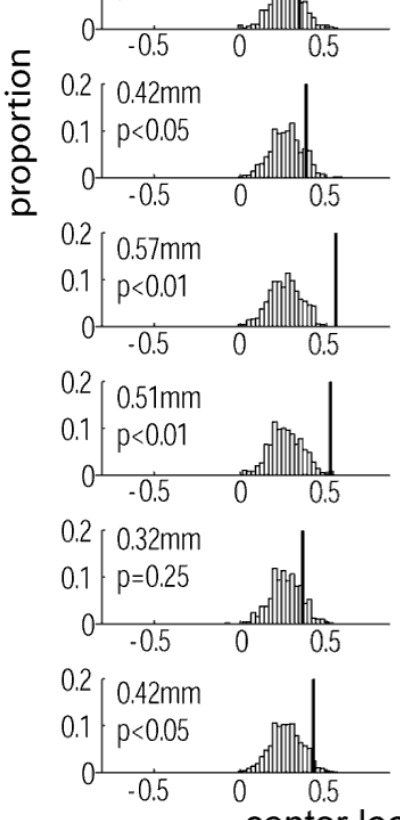

V2



$0.2-0.18 \mathrm{~mm}$



$0.2-0.20 \mathrm{~mm}$

$0.1 p<0.01$

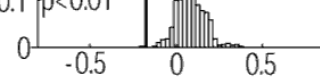

$0.2-0.25 \mathrm{~mm}$



$0.2-0.24 \mathrm{~mm}$

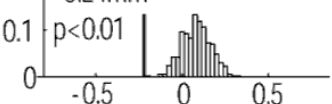

$0.2-0.23 \mathrm{~mm}$

$0.1 p<0.01$

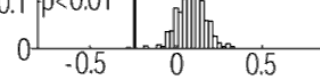

$0.2[-0.29 \mathrm{~mm}$

$0.1 p<0.01$

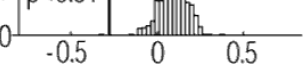

$0.2[-0.32 \mathrm{~mm}$

$0.1-p<0.01$
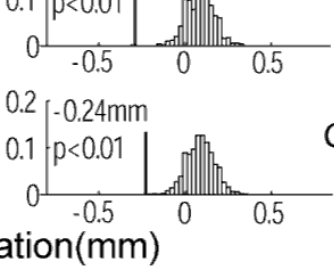

Monkey K

V1

V2



$3.7 \%$
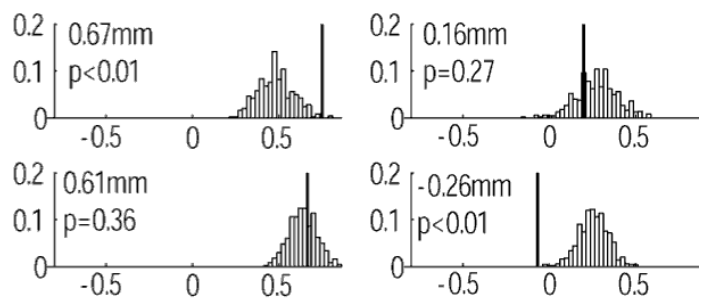

$0.2[-0.26 \mathrm{~mm}$

$0.1-p<0.01$

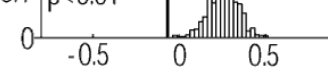

$6.1 \%$
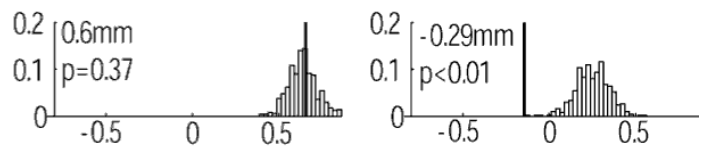

$9.7 \%$
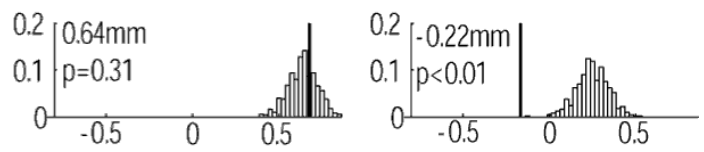

$16.3 \%$
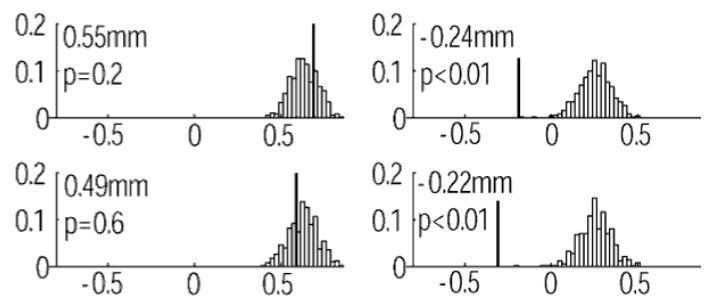

$0.2-0.22 \mathrm{~mm}$

$35.9 \%$

$0.1-p<0.01$
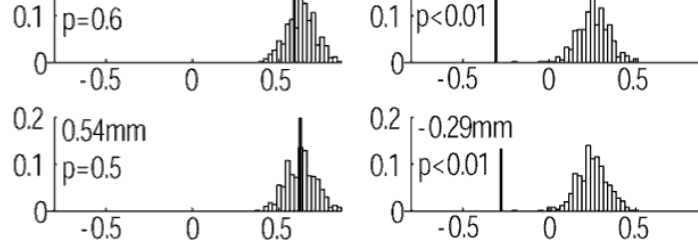

$0.2-0.29 \mathrm{~mm}$
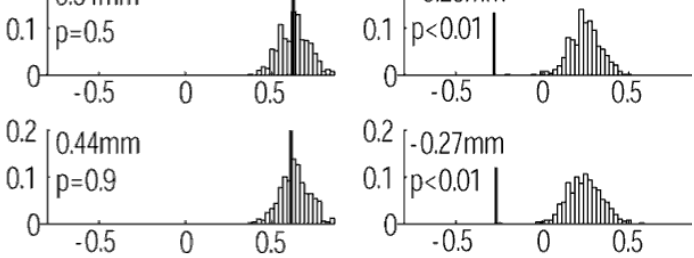

$0.2[-0.27 \mathrm{~mm}$

$0.1 p<0.01$

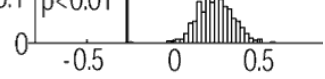

Combined
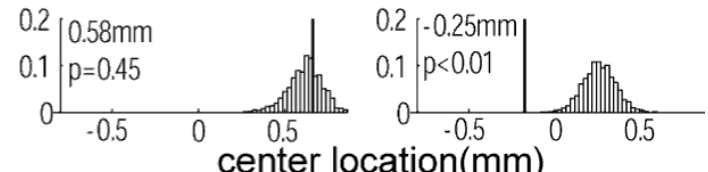

Figure S4: Statistical evaluation of V1-V2 coherence pattern presented in figure 5. The probability that empirically determined center locations of $\mathrm{V} 1$ and $\mathrm{V} 2$ sites linked by the strongest coherence connections (top 5\%) would have been found in observed locations by chance was tested by bootstrapping. The empirical center locations of V1 sites and V2 sites in the original data (vertical lines indicate empirical depth on X-axis) are compared with a distribution of center V1 and V2 sites obtained from randomly shuffling the connectivity pairs over all available sites in 1000 iterations (histograms, y axis values indicate proportions). Text in figures gives the center location of the strongest coherence connections in the original data and the percentage of values in the shuffled data that are equal to or greater than the empirical depth value (V1 columns) or equal to or less than the empirical depth value (V2 columns). Depth positions are considered significant if these percentages are less than 2.5\%. Rows 1 to 8 show data from individual contrasts. Row 9 shows data combined over contrasts. Data from monkeys $S$ and $\mathrm{K}$ are shown separately. Details of procedure are described in Supplemental Experimental Procedures. 


\section{Supplemental Experimental Procedures}

Receptive field mapping: Receptive fields (RFs) were mapped by presenting high contrast black and white squares at pseudo-random locations on a 10x10 grid while monkeys fixated centrally on the screen. Square size was adjusted between $1^{\circ}$ and $0.1^{\circ}$ to optimally map concurrently recorded RFs (150ms presentation time, $150 \mathrm{~ms}$ inter-stimulus interval). The mean response at each stimulus location was determined and the RF defined to include locations where the response was above the 75 percentile of the distribution. RFs reported here were generally larger than reported in other studies (Gattass, Gross, \& Sandell, 1981; Gattass, Sousa, \& Gross, 1988; Roberts, Delicato, Herrero, Gieselmann, $\&$ Thiele, 2007), and this difference may be explained by the relatively large mapping stimuli used here, which were adjusted to stimulate several simultaneously recorded cells. We carefully examined the RF maps of each contact point of each probe to check for shifts in receptive field location or size, which would indicate that deeper channels were located in a separate column, and likely a separate area (Gattass et al., 1981), to shallower channels. According to these constraints we removed 50 channels (monkey S V1=13, V2=11; monkey K V1=17, V2=9) from the population.

Definition of the V1/V2 border: Area V2 was accessed on the upper surface of the pre-lunate gyrus. We localized the transition between area V1 and area V2 by determining cortical locations where RFs were positioned along the vertical meridian, and additional locations on both sides of this boundary for which RFs shifted away from the vertical meridian in opposite directions. Area V2 was on the anterior side of this boundary while area V1 was on the posterior side (Gattass et al., 1981). V1 RFs were smaller than V2 RFs (monkey $\mathrm{K}$ median V1 diameter $=0.84^{\circ}, \mathrm{V} 2=0.94, \mathrm{t}=16.8, \mathrm{p}=0.019$; monkey $\mathrm{S} \mathrm{V} 1$ median diameter $=0.67, \mathrm{~V} 2=0.88, \mathrm{t}=65.3 \mathrm{p}<0.005$, two sample $\mathrm{t}$-test) in line with previous reports (Felleman \& Van Essen, 1991; Gattass et al., 1981). V1 spiking onset response was significantly earlier than the V2 onset (monkey S V1 onset median $=0.38 \mathrm{~ms}$, V2 $44 \mathrm{~ms}$ for high contrast stimuli $t=6.4, p<0.001$ two sample t-test; monkey K V1 onset $=39 \mathrm{~ms} V 2$ onset $=43 \mathrm{~ms}, \mathrm{t}=4.6 \mathrm{p}<0.001)$. RF eccentricity in monkey $\mathrm{S}$ was $5.5^{\circ}$ in area V1 (standard deviation $=0.6$ ), and 5.8 in V2 (standard deviation $=0.6$ ). In monkey K V1 RF eccentricity was 5.3으 (standard deviation $=0.5 \circ$ ) and 5.9을 $\mathrm{V} 2$ (standard deviation = 0.6ㅇ) .

Spectral peak determination: We tried several methods for reliably quantifying the peak, or centerfrequency, of the gamma band. Because of the complex shape of the induced-power-spectrum and coherence spectrum, and because of the wide shift in center frequency, simply choosing the frequency with the highest power proved to be unreliable. We therefore used a fitted-function, in which some parameters of the function accounted specifically for the gamma band, and other parameters accounted for other aspects of the data. Specifically, we quantified the center gamma frequency by fitting a double Gaussian model to the data according to the function:

$$
Y=G a *\left(\exp -\left(\frac{2 * \text { frequency }-P a}{S a}\right)^{2}\right)+G b *\left(\exp -\left(\frac{2 * \text { frequency }-P b}{S b}\right)^{2}\right)+\text { offset }
$$

where $\mathrm{Ga}$ and $\mathrm{Gb}$ correspond to the gain (strength), $\mathrm{Pa}$ and $\mathrm{Pb}$ correspond to the centre peak and Sa and $\mathrm{Sb}$ correspond to the width of the first and second Gaussian respectively, the final free parameter Offset 
corresponds to the baseline of the function. The seven free parameters were optimized by minimizing the summed squared difference between the model $Y$, and the data. The function provided good fits to the data, explaining mean $96.2 \%$ (2.9\% standard deviation) of the variance (See Fig. S2). In this function, one Gaussian reflected the gamma band while the second Gaussian reflected either a lower frequency band (e.g. Beta, which was present in only some recordings) or reflected general trends in the data (e.g. higher induced power at higher frequency especially present in monkey S). The peak gamma frequency was taken by the center of the Gaussian with the higher-frequency center where this did not exceed $70 \mathrm{~Hz}$ (near to the highest analyzed frequency, $80 \mathrm{~Hz}$ ).

Depth alignment procedure and validation: The probes we have used were too short to record the entire cortical thickness simultaneously, and hence data from different recording sessions had to be aligned based on a consistent functional criterion (Fig. S3). As a criterion, we used the systematic change in the shape of the LFP onset response (also called the visually evoked potential or VEP) as a function of cortical depth. Hence, we aligned the data in depth by combining data with similar patterns of onset response (Fig. S3A). In addition, we carefully measured the physical depth at which a strong VEP could first be induced by visual stimulation (i.e. the start of the cortex), and the depth at which experimental recordings were carried out (computer controlled electrode advancer (NAN instruments; electrode moving at $0.002 \mathrm{~mm} / \mathrm{sec}$ ). Our experimental data could thus be aligned in cortical lamina (within 13 cortical depth bins) using the shape of the onset response, cross-referenced by the physical depth of the electrode. Physical depth and assigned depth of electrode placements were significantly correlated (Fig. S3B) indicating our method for data alignment was robust. More data were obtained around the middle of cortex, and less data for the shallowest and deepest cortical layers (Fig. S3C). Data averaged over sessions (see population average LFP data Fig. S3A) revealed a region of positive deflection across the more shallow recording contacts. The bottom of this region was assigned a 0-depth bin for V1 and V2 data in both monkeys (Fig. S3A, D). In Figure S3D, for V1 and V2 of both monkeys, the aligned LFP stimulus onset data are shown in depth-time color-coded surface plots, with below them depth-resolved current-source-density maps and spiking responses shown in similar formats. Note that the depth distribution of CSD and spiking response data resulted from the VEP-alignment procedure. We found an early source-sink reversal in the CSD data, corresponding to the top of layer 4C in area V1 (Bollimunta, Chen, Schroeder, \& Ding, 2008; Maier, Aura, \& Leopold, 2011; Mitzdorf, 1985; Schroeder, Tenke, Givre, Arezzo, \& Vaughan, 1991).

As a validation of the depth alignment procedure, we tested spiking latency, which is known to vary over depth. Previous reports have shown that spiking response latencies in V1 superficial layers (2 and 3) and granular layer (4) exhibit earlier spiking onset than deeper layers (5 and 6) (Nowak, Munk, Girard, \& Bullier, 1995). Accordingly, we rearranged our data into 3 groups corresponding to supragranular (estimated to be above $0.2 \mathrm{~mm})$, granular $(0.2 \mathrm{~mm}$ to $-0.4 \mathrm{~mm})$, and infragranular (below $0.4 \mathrm{~mm}$ ). Differences in latency between layers were confirmed by a 3 way ANOVA with factors (layer, contrast separately for each monkey) in which both factors were significant for both monkeys $\mathrm{S}$ (layer: $\mathrm{F}(2,955)=3.2, \mathrm{p}<0.01$; area: $\mathrm{F}(1,955)=55.3, \mathrm{p}<0.001$; contrast: $\mathrm{F}(7,955)=70.7, \mathrm{p}<0.001)$ and monkey $\mathrm{K}$ layer: $\mathrm{F}(2,1244)=9.9, \mathrm{p}<0.001$; area: $\mathrm{F}(1,1244)=22.5, \mathrm{p}<0.001$; contrast: $\mathrm{F}(7,1244)=54.7, \mathrm{p}<0.001)$ Interactions were not significant. Post-hoc tests (Tukey-Kramer method) showed that response latencies 
in layers $5 / 6$ (population marginal mean monkey $S=49.4 \mathrm{~ms}$ standard error $=0.8 \mathrm{~ms}$; monkey $\mathrm{K}=50.1 \mathrm{~ms}$ standard error $=1.9 \mathrm{~ms}$ ) were significantly later than in shallower layers, while response latencies in layers $2 / 4$ (population marginal mean monkey $\mathrm{S}=47.5 \mathrm{~ms}$ standard error $=0.4 \mathrm{~ms}$; monkey $\mathrm{K}=45.2 \mathrm{~ms}$ standard error $=0.4 \mathrm{~ms}$ ) and layer 4 (population marginal mean monkey $\mathrm{S}=46.9 \mathrm{~ms}$ standard error $=$ $0.5 \mathrm{~ms}$; monkey $\mathrm{K}=44.1 \mathrm{~ms}$ standard error $=0.3 \mathrm{~ms}$ ) were not significantly different at the 0.05 level. Onset response latencies were defined by fitting a sigmoid function to the cumulative response (Falzett, Moore, Petry, \& Powers, 1985). Only well-defined spike responses were included in the analysis (i.e., response during stimulation above response during baseline with a receiver operating characteristic value above $\mathbf{0 . 7}$, corresponding to $72.1 \%$ of spike recordings in monkey $\mathrm{S}$ and $85.8 \%$ in monkey $\mathrm{K}$ ).

We also tested the effects of cortical depth, cortical area, and stimulus contrast on gamma power. In line with previous studies (Buffalo, Fries, Landman, Buschman, \& Desimone, 2011; Maier et al., 2011; Smith, Jia, Zandvakili, \& Kohn, 1213; Xing, Yeh, Burns, \& Shapley, 2012b), gamma power in the LFP was strongest in supra-granular layers of both V1 and V2, shown in Fig. 3 as the tendency for reddish colors (highest power gamma) to be above the dotted zero-depth reference lines in V1 and V2, for all contrasts, and for both monkeys. A 3-way ANOVA of gamma power confirmed that the factor depth (above or below zero-depth) was highly significant in both monkeys (monkey S $F(1,1696)=320.1$, $\mathrm{p}<0.001$; monkey $\mathrm{K}(1,1555)=166.6, \mathrm{p}<0.001)$. There was less gamma power in $\mathrm{V} 2$ than in $\mathrm{V} 1$, confirmed by a significant effect of the factor area $(\mathrm{V} 1, \mathrm{~V} 2)$ in both monkeys (monkey S: $F(1,1696)=54.6, p<0.001$; monkey K: $F(1,1555)=61.6, p<0.001)$. The factor contrast also was highly significant (monkey $S$ : $F(7,1696)=298.9, p<0.001)$; monkey $K: F(7,1555)=60.74, p<0.001)$. In monkey $S$, there was a significant interaction between contrast and depth $(F(7,1696)=13.5, p<0.001)$, and area $(F(7,1696)=9.4, p<0.001)$, but there was no interaction between area and depth $(F(1,1696)=0.3, p=0.58)$. Monkey $K$ showed the opposite pattern, with an, interaction between area and depth $(F(1,1555)=9.8, p<0.005)$ but no interactions of contrast with depth $F(7,1555)=1.54, p=0.15)$ or area $(F(7,1555)=1.1, p=0.37)$.

Statistical evaluation of V1-V2 coherence pattern: We tested the probability that the specific V1 and V2 center locations corresponding to the average sites linked by the top 5\% coherence connections (Fig. 3 ) would be found by chance. To that aim, we used a bootstrap technique (Fig. S4). In 1000 iterations we randomly (with replacement) shuffled all coherence values (for a given monkey at a given contrast) for all recorded depth pairings. For the randomized pattern of coherence connections resulting from each iteration, we estimated the mean depth in V1 and V2 for the top 5\% coherence pairs. Thus, the bootstrapping procedure produced a distribution comprising 1000 estimated V1 and V2 center sites for top $5 \%$ coherence pairs. This procedure was done for each contrast and both monkeys, and permitted in each case a comparison between the V1 and V2 center locations linked by the greatest coherence found empirically or determined by bootstrapping (Fig. S4). In Fig S4, data for different contrasts are shown in rows 1 to 8, with at the bottom (row 9) an estimation of center locations after averaging all data from different contrasts, which are the center locations reported in the main text. The grand average locations were compared with the combined distribution of bootstrapped center locations from each contrast. Locations found in the empirical data were considered significant if they fell above or below $97.5 \%$ of the distribution of bootstrapped locations. In monkey S the V1 center location observed in the unshuffled data was significantly above the bootstrapped distribution of center locations, while the V2 
location was significantly below. In monkey $\mathrm{K}$, the V1 observed center location in the unshuffled data was not significantly different from the distribution of center locations from shuffled data (due to the lack of recordings in deeper V1 layers in this monkey). However, the V2 location was significantly below the distribution (see Fig. S4 for details). Taken together, this analysis confirms that the observed connectivity pattern was not likely to be due to chance or sampling bias.

\section{Computational model}

\section{$1.1 \quad$ Model summary}

We used a generic model of two interconnected local cortical circuits to model V1 and V2 dynamics, based on the work by (Buia \& Tiesinga, 2006). Each area consisted of 400 excitatory pyramidal cells and 100 inhibitory interneurons. We used single compartment neurons with Hodgkin-Huxley type voltagegated channels for both the pyramidal cells (Golomb \& Amitai, 1997) and interneurons (Wang \& Buzsaki, 1996). The full model is described in section (1.4). Each synaptic connection was made randomly between presynaptic and postsynaptic cells according to the probabilities listed in Table S1. Inhibitory connections were mediated by GABAA type synapses and excitatory connection by AMPA synapses. Within an area there was low probability for $E$ to $E$ connections and high interneuron-pyramidal cell connectivity, based on experimental findings (Fino \& Yuste, 2011; Holmgren, Harkany, Svennenfors, \& Zilberter, 2003; Markram et al., 2004). Connections between areas were purely excitatory. Unitary synaptic strengths, both for connections within and between areas, are given in Table S1. Synaptic delays were homogeneous and were chosen to be $1 \mathrm{~ms}$ within and $5 \mathrm{~ms}$ between areas The model was implemented in Matlab (The MathWorks, 2012a). The differential equations were integrated using a $4^{\text {th }}$ order Runge-Kutta algorithm, with a time step of $0.05 \mathrm{~ms}$.

\begin{tabular}{l|l|l}
\hline Connection type & Connection probability $(\%)$ & Synaptic strength $\left(\mathrm{mS} / \mathrm{cm}^{2}\right)$ \\
\hline E to E within area & 10 & 0.0012 \\
E to I within area & 30 & 0.0010 \\
I to I within area & 20 & 0.0120 \\
I to E within area & 60 & 0.0050 \\
E to E between areas & 5 & 0.0100 \\
E to I between areas & 10 & 0.0050 \\
\hline
\end{tabular}

Table. S1: Connection probabilities and synaptic strengths for all connections in the network model.

\subsection{Input to the areas}

Neurons in area V1 were activated by injecting a depolarizing current: $I_{\operatorname{lnj}}=I_{0}+I_{\sigma}+I_{\text {noise }}$. The tonic $I_{0}$ current applied to interneurons was kept at $0.5 \mu \mathrm{A} / \mathrm{cm}^{2}$, while the current to the pyramidal cells was varied between runs, simulating different stimulus contrasts, between 0.8 and $2.6 \mu \mathrm{A} / \mathrm{cm}^{2}$. The $\mathrm{I}_{\sigma}$ current was constant in time, but differed between neurons. $I_{\sigma}$ was drawn from a Gaussian distribution with mean 0 and standard deviation $0.1 \mu \mathrm{A} / \mathrm{cm}^{2}$ for both interneurons and pyramidal cells. The noise current $I_{\text {noise }}$ fluctuated over time independently for each area, but was the same for all neurons in an 
area. It was constructed by filtering white noise. The resulting trace had a frequency spectrum with an approximate $1 / \mathrm{f}$ power fall off.

V2 neurons received tonic $I_{0}$ currents of $0.2 \mu \mathrm{A} / \mathrm{cm}^{2}$ for the interneurons and $0.5 \mu \mathrm{A} / \mathrm{cm}^{2}$ for the pyramidal cells. $I_{\sigma}$ currents had standard deviations of $0.1 \mu \mathrm{A} / \mathrm{cm}^{2}$ for both neuron types. $\mathrm{V} 2$ neurons received a noise current with the same spectral characteristics as the noise in V1.

\subsection{Analysis}

Spikes of all neurons were detected by determining when the membrane potential crossed a threshold, which was $-20 \mathrm{mV}$ for pyramidal cells and $0 \mathrm{mV}$ for interneurons. Spike times were saved and spike time histograms were produced with 0.5 ms bins, according to:

$$
\begin{aligned}
& \operatorname{STH}(t)=\frac{1000}{\Delta t N} \sum_{i} X_{i}(t) \\
& X_{i}(t)=\left\{\begin{array}{c}
1 \text { if } t_{j}^{i} \in[t, t+\Delta t) \\
0 \text { otherwise }
\end{array}\right.
\end{aligned}
$$

Power spectral densities (PSD) for both areas and the coherence between the areas were obtained from the spike time histograms of the pyramidal cell populations using Chronux (Bokil, Andrews, Kulkarni, Mehta, \& Mitra, 2010). For the multitaper analysis, a time-bandwidth product of 5, 9 tapers and 3 seconds of simulated data with a sampling rate of $2000 \mathrm{~Hz}$ was used. The instantaneous oscillation frequencies of the areas were obtained from a wavelet transform performed with the Wavelet Toolbox for Matlab. A complex Morlet wavelet was used, with a centre frequency $f_{\mathrm{c}}$ of $1 \mathrm{~Hz}$ and a frequency bandwidth $f_{\mathrm{b}}$ of $1 \mathrm{~Hz}$. For the mother wavelet:

(3)

$$
\Psi(t)=\frac{1}{\sqrt{\pi f b}} e^{2 i \pi f_{0} t} e^{-t^{2} / f b}
$$

\section{$1.4 \quad$ Neuron models}

Interneurons:

A single area contains 100 inhibitory, fast-spiking interneurons. The membrane potential $V_{i}(\mathrm{mV})$ of these interneurons obeys:

$$
C_{m} \frac{d V}{d t}=-I_{N a}-I_{K}-I_{L}-I_{G A B A}-I_{A M P A}-I_{i n j}+C_{m} \xi
$$


The RHS terms represent, respectively, the sodium and potassium currents, a leak current and synaptic inputs (Wang \& Buzsaki, 1996). $C_{m}$ is the membrane capacitance $\left(\mu \mathrm{F} / \mathrm{cm}^{2}\right)$ and $C_{m} \xi$ is a noise term. $I_{n j}$ represents all external input currents into the cell. The currents in (4) are modeled as:

$$
\begin{aligned}
& I_{L}=g_{L}\left(V-E_{L}\right) \\
& I_{N a}=g_{N a} m_{\infty}^{3} h\left(V-E_{N a}\right) \\
& I_{K}=g_{K} n^{4}\left(V-E_{K}\right) \\
& I_{G A B A}=g_{i i} s_{i i}^{\text {tot }}(t)\left(V-E_{G A B A}\right) \\
& I_{A M P A}=g_{e i} s_{e i}^{\text {tot }}(t)\left(V-E_{\text {AMPA }}\right)
\end{aligned}
$$

Where $m, h, n$ and $s$ are gating variables. Gating variable $m$ has fast dynamics and can be replaced by its asymptotic value. The dynamics of $h$ and $n$ are described by differential equations:

$$
\begin{aligned}
& m_{\infty}=\frac{\alpha_{m}}{\alpha_{m}+\beta_{m}} \\
& \frac{d h}{d t}=\varphi\left(\alpha_{h}(1-h)-\beta_{h} h\right. \\
& \frac{d n}{d t}=\varphi\left(\alpha_{n}(1-n)-\beta_{n} n\right.
\end{aligned}
$$

With rate constants:

$$
\begin{aligned}
& \alpha_{m}=\frac{-0.1(V+35)}{\exp (-0.1(V+35))-1} \\
& \beta_{m}=4 \exp (-(V+60) / 18) \\
& \alpha_{h}=0.07 \exp (-(V+58) / 20) \\
& \beta_{h}=[\exp (-0.1(V+28))+1]^{-1} \\
& \alpha_{n}=\frac{-0.01(V+34)}{\exp (-0.1(V+34))-1} \\
& \beta_{n}=0.125 \exp (-(V+44) / 80)
\end{aligned}
$$

The parameter values used in the model can be found in Table S2.

\section{Pyramidal cells}

The model for the 400 pyramidal cells in the local circuit is slightly different from that of the interneurons described above (Buia \& Tiesinga, 2006; Golomb \& Amitai, 1997). It incorporates a sodium current, persistent sodium current, delayed rectifier potassium current and the A-type potassium current, respectively: 


$$
C_{m} \frac{d V}{d t}=-I_{N a}-I_{N a P}-I_{K d r}-I_{K A}-I_{L}-I_{G A B A}-I_{A M P A}-I_{i n j}+C_{m} \xi
$$

The currents in (8) are described by:

(9)

$$
\begin{aligned}
& I_{L}=g_{L}\left(V-E_{L}\right) \\
& I_{N a}=g_{N a} m_{\infty}^{3} h\left(V-E_{N a}\right) \\
& I_{N a P}=g_{N a P} p_{\infty}\left(V-E_{N a}\right) \\
& I_{K d r}=g_{K d r} n^{4}\left(V-E_{K}\right) \\
& I_{K A}=g_{K A} a_{\infty}^{3} b\left(V-E_{K}\right) \\
& I_{G A B A}=g_{i e} s_{i e}^{t o t}(t)\left(V-E_{G A B A}\right) \\
& I_{A M P A}=g_{e \theta} s_{e \theta}^{t o t}(t)\left(V-E_{A M P A}\right)
\end{aligned}
$$

Here, the gating variables $m, p$ and $a$ are fast and are replaced by their asymptotic values $m 1, p 1$ and $a 1$, respectively. The dynamics of the other gating variables are given by:

$$
\begin{aligned}
& \frac{d h}{d t}=\left(h_{\infty}(V)-h\right) / \tau_{h} \\
& \frac{d n}{d t}=\left(n_{\infty}(V)-n\right) / \tau_{n} \\
& \frac{d b}{d t}=\left(b_{\infty}(V)-b\right) / \tau_{b}
\end{aligned}
$$

The rate constants in the above equations are:

$$
\begin{aligned}
& m_{\infty}=[\exp (-(V+30) / 9.5)+1]^{-1} \\
& h_{\infty}=[\exp ((V+53) / 7)+1]^{-1} \\
& \tau_{h}=0.37+2.78[\exp ((V+40.5) / 6)+1]^{-1} \\
& p_{\infty}=[\exp (-(V+40) / 5)+1]^{-1} \\
& n_{\infty}=[\exp (-(V+30) / 10)+1]^{-1} \\
& \tau_{n}=0.37+1.85[\exp ((V+27) / 15)+1]^{-1} \\
& a_{\infty}=[\exp (-(V+50) / 20)+1]^{-1} \\
& \tau_{b}=15 \mathrm{~ms}
\end{aligned}
$$

Noise 
At each time step and for each neuron independently, the noise term $C_{m} \xi$ was drawn from a uniform distribution between $-\sqrt{ } 6 \lambda / d t$ and $\sqrt{ } 6 \lambda / d t$ (see table $S 2$ ).

\section{Synapses}

The equations for the connection type specific synaptic input current were given above and have the general form:

$$
I_{s y n}=g_{k l} S_{k l}^{\text {tot }}(t)\left(V-E_{s y n}\right)
$$

Here, $g \mathrm{kl}$ is the unitary synaptic conductance $\left(\mathrm{mS} / \mathrm{cm}^{2}\right)$ for connections from the presynaptic neurons of type $\mathrm{k}$ to a postsynaptic neuron of type I. Variable $\mathrm{s}_{\mathrm{kl}}^{\text {tot }}$ represents the total input to the postsynaptic cell and depends on the network structure and the synaptic gating variables s of the presynaptic cells:

$$
\begin{gathered}
\frac{d s}{d t}=\alpha F\left(V_{p r e}\right)(1-s)-\beta s \\
F\left(V_{p r e}\right)=\frac{1}{1+\exp \left(-\left(V_{p r e}-\theta_{s}\right) / \sigma_{s}\right)}
\end{gathered}
$$

Parameters used in the equations can be found in Table S2.

\begin{tabular}{l:l:l}
\hline Parameter (unit) & Pyramidal cells & Interneurons \\
\hline $\mathrm{E}_{\mathrm{L}}(\mathrm{mV})$ & -70 & -65 \\
$\mathrm{E}_{\mathrm{Na}}(\mathrm{mV})$ & 55 & 55 \\
$\mathrm{E}_{\mathrm{K}}(\mathrm{mV})$ & -90 & -90 \\
$\mathrm{E}_{\mathrm{AMPA}}(\mathrm{mV})$ & 0 & 0 \\
$\mathrm{E}_{\mathrm{GABA}}(\mathrm{mV})$ & -75 & -75 \\
$\mathrm{~g}_{\mathrm{L}}\left(\mathrm{mS} / \mathrm{cm}^{2}\right)$ & 0.02 & 0.1 \\
$\mathrm{~g}_{\mathrm{Na}}\left(\mathrm{mS} / \mathrm{cm}^{2}\right)$ & 24 & 35 \\
$\mathrm{~g}_{\mathrm{NaP}}\left(\mathrm{mS} / \mathrm{cm}^{2}\right)$ & 0.07 & - \\
$\mathrm{g}_{\mathrm{Kdr}}\left(\mathrm{mS} / \mathrm{cm}^{2}\right)$ & 3 & - \\
$\mathrm{g}_{\mathrm{KA}}\left(\mathrm{mS} / \mathrm{cm}^{2}\right)$ & 1.4 & - \\
$\mathrm{g}_{\mathrm{K}}\left(\mathrm{mS} / \mathrm{cm}^{2}\right)$ & - & 9 \\
$\mathrm{C}_{\mathrm{M}}\left(\mu \mathrm{F} / \mathrm{cm}^{2}\right)$ & 1 & 1 \\
$\varphi$ & - & 5 \\
$\lambda\left(\mathrm{mV} / \mathrm{ms}^{2}\right)$ & 0.06 & 0.02 \\
Delay within area $(\mathrm{s})$ & 1 & 1 \\
Delay between areas $(\mathrm{ms})$ & 5 & 5 \\
\hline & AMPA & GABA \\
\hline$\theta(\mathrm{mV})$ & -20 & 0 \\
$\alpha\left(\mathrm{ms}{ }^{-1}\right)$ & 0.8 & 10 \\
$\beta\left(\mathrm{ms}{ }^{-1}\right)$ & 0.5 & 0.2 \\
$\sigma_{\mathrm{s}}(\mathrm{mV})$ & 2 & 2 \\
\hline
\end{tabular}

Table. S2: Parameters for pyramidal cells, interneurons and synapses. 


\section{Supplementary References}

Bokil, H., Andrews, P., Kulkarni, J. E., Mehta, S., \& Mitra, P. P. (2010). Chronux: a platform for analyzing neural signals. J Neurosci Methods, 192, 146-151. doi: 10.1016/j.jneumeth.2010.06.020

Bollimunta, A., Chen, Y., Schroeder, C. E., \& Ding, M. (2008). Neuronal mechanisms of cortical alpha oscillations in awake-behaving macaques. J Neurosci, 28, 9976-9988. doi: 10.1523/JNEUROSCI.2699-08.2008

Buffalo, E. A., Fries, P., Landman, R., Buschman, T. J., \& Desimone, R. (2011). Laminar differences in gamma and alpha coherence in the ventral stream. Proc Natl Acad Sci U S A, 108(27), 1126211267. doi: $10.1073 /$ pnas. 1011284108

Buia, C., \& Tiesinga, P. (2006). Attentional modulation of firing rate and synchrony in a model cortical network. J Comput Neurosci, 20(3), 247-264. doi: 10.1007/s10827-006-6358-0

Falzett, M., Moore, R. K., Petry, H. M., \& Powers, M. K. (1985). A method for determining threshold from single-unit neural activity. Brain Res, 347, 127-131.

Felleman, D. J., \& Van Essen, D. C. (1991). Distributed hierarchical processing in the primate cerebral cortex. Cereb Cortex, 1(1), 1-47.

Fino, E., \& Yuste, R. (2011). Dense inhibitory connectivity in neocortex. Neuron, 69, 1188-1203. doi: 10.1016/j.neuron.2011.02.025

Gattass, R., Gross, C. G., \& Sandell, J. H. (1981). Visual topography of V2 in the macaque. J Comp Neurol, 201(4), 519-539. doi: 10.1002/cne.902010405

Gattass, R., Sousa, A. P., \& Gross, C. G. (1988). Visuotopic organization and extent of V3 and V4 of the macaque. J Neurosci, 8(6), 1831-1845.

Golomb, D., \& Amitai, Y. (1997). Propagating neuronal discharges in neocortical slices: computational and experimental study. J Neurophysiol, 78(3), 1199-1211.

Holmgren, C., Harkany, T., Svennenfors, B., \& Zilberter, Y. (2003). Pyramidal cell communication within local networks in layer 2/3 of rat neocortex. J Physiol, 551, 139-153. doi: 10.1113/jphysiol.2003.044784

Maier, A., Aura, C. J., \& Leopold, D. A. (2011). Infragranular sources of sustained local field potential responses in macaque primary visual cortex. J Neurosci, 31(6), 1971-1980.

Markram, H., Toledo-Rodriguez, M., Wang, Y., Gupta, A., Silberberg, G., \& Wu, C. (2004). Interneurons of the neocortical inhibitory system. Nat Rev Neurosci, 5, 793-807. doi: 10.1038/nrn1519

Mitzdorf, U. (1985). Current source-density method and application in cat cerebral cortex: investigation of evoked potentials and EEG phenomena. Physiol Rev, 65(1), 37-100.

Nowak, L. G., Munk, M. H., Girard, P., \& Bullier, J. (1995). Visual latencies in areas V1 and V2 of the macaque monkey.Vis Neurosci, 12(2), 371-384.

Roberts, M., Delicato, L. S., Herrero, J., Gieselmann, M. A., \& Thiele, A. (2007). Attention alters spatial integration in macaque V1 in an eccentricity-dependent manner. Nat Neurosci, 10, 1483-1491. doi: $10.1038 / \mathrm{nn} 1967$ 
Schroeder, C. E., Tenke, C. E., Givre, S. J., Arezzo, J. C., \& Vaughan, H. G., Jr. (1991). Striate cortical contribution to the surface-recorded pattern-reversal VEP in the alert monkey. Vision Res, 31, 1143-1157.

Smith, M. A., Jia, X., Zandvakili, A., \& Kohn, A. (1213). Laminar dependence of neuronal correlations in visual cortex. J Neurophysiol. doi: 10.1152/jn.00846.2012

Wang, X. J., \& Buzsaki, G. (1996). Gamma oscillation by synaptic inhibition in a hippocampal interneuronal network model.J Neurosci, 16(20), 6402-6413.

Xing, D., Yeh, C. I., Burns, S., \& Shapley, R. M. (2012). Laminar analysis of visually evoked activity in the primary visual cortex. Proc Natl Acad Sci U S A, 109, 13871-13876. doi:

10.1073/pnas.1201478109 


\section{Chapter 3}

\section{Parametric variation of gamma frequency and power with luminance contrast: A comparative study of human MEG and monkey LFP and spike responses}

\section{Based on:}

A. Hadjipapas", E. Lowet", M. J. Roberts, A. Peter, and P. De Weerd, "Parametric variation of gamma frequency and power with luminance contrast: A comparative study of human MEG and monkey LFP and spike responses.," Neuroimage, Mar. 2015.

"First shared authorship 


\begin{abstract}
Gamma oscillations vary in their dominant frequency. This frequency is key in terms of structuring unit activity into functional assemblies, and has been shown to vary with several stimulus parameters. Variation of gamma frequency with luminance contrast is perhaps the most instructive paradigm for understanding the organization of the visual-cortical network because contrast maps well onto the level of excitatory input to the network from the retinogeniculate pathway. In network models, increasing input increases oscillation frequency. Consistently, increasing stimulus contrast increases gamma frequencies of LFPs in monkey V1. Here, we compare spectral responses to varying contrast in a monkey dataset acquired previously (Roberts et al., 2013) to a new human MEG dataset. In doing so, we match stimulus parameters to the monkey dataset as much as possible while maintaining sufficient SNR in MEG. For the first time we show parametric frequency shifts in human MEG analogous to the ones observed in the monkey both at the single- subject and single-trial level. Additionally, we report parametric modulations of spectral asymmetry, consistent across spikes, LFP and MEG, which suggest a role for resonance in gamma generation. However, we also observe a striking difference: while gamma power scales linearly with contrast in MEG, at high contrasts saturation is observed in the LFP and spike trains. Thus gamma frequency appears to be a more stable parameter across scales of measurements than gamma power. The comparative approach undertaken here delineates a fruitful path towards a better understanding of gamma oscillations in the human.
\end{abstract}




\section{Introduction}

In monkey visual cortex, gamma oscillations have been studied for many years, and are thought to mediate a number of computational functions [Buzsáki and Wang, 2012; Engel et al., 1999; Fries et al., 2007; Fries, 2009; Tiesinga and Sejnowski, 2010]. One of the key parameters of an oscillation is its dominant frequency. An influential framework of coordinated neural action views neurons as relaxation oscillators [Buzsáki and Draguhn, 2004], that is, oscillators exhibiting longer phases during which input (information) is integrated and short fire phases, during which output is produced. Excitatory neurons are thought to be under a constant inhibitory barrage [Buzsáki, 2006] such that their excitability is generally low. It has been proposed that (local) field oscillations serve to modulate neuronal excitability rhythmically and in this way define windows of opportunity for neurons to fire [Buzsáki and Draguhn, 2004; Csicsvari et al., 1999; Lakatos et al., 2005]. The length of information-integration phases, the spacing between them and the duration of the fire phases is governed by the frequency of the field oscillation. Influential theories of neural communication also implicate oscillation frequency, in that common frequency is a prerequisite for appropriate alignment of excitability phases between interacting populations[Fries, 2005]. Recently, it has been shown that gamma frequency in areas V1 and V2 are dynamically matched to facilitate such alignment[Roberts et al., 2013]. Consistently, the theory of weakly coupled oscillators predicts that similar frequencies are a prerequisite for synchronization of nearby neuronal populations (see Arnold tongues e.g. [Pikovsky, 2003]). Empirically, gamma peak frequency shows correlations with perceptual performance [Edden et al., 2009], age [Gaetz et al., 2012], cortical structure[Schwarzkopf et al., 2012] and GABA concentration [Muthukumaraswamy et al., 2009] among other parameters. Thus, oscillation frequency is a network parameter of utmost functional relevance and theoretical interest [Barardi et al., 2014; Cohen, 2014; Colgin et al., 2009].

In the last decade or so MEG beamformer source reconstruction has enabled the study of gamma oscillations localized to human visual cortex [Adjamian et al., 2004; Hall et al., 2005a; Hoogenboom et al., 2006]. Using these methods, several MEG studies reported human gamma oscillations to exhibit marked stimulus specificity [Adjamian et al., 2004; Duncan et al., 2010; Hadjipapas et al., 2007; Hall et al., 2005a; Koelewijn et al., 2010; Muthukumaraswamy and Singh, 2008; Swettenham et al., 2009], paralleling neurophysiological findings in the macaque [Berens et al., 2008; Friedman-Hill et al., 2000; Frien et al., 2000; Gray and Singer, 1989; Henrie and Shapley, 2005a]. Although these stimulusdependent effects in monkeys and humans were recorded in experiments that are difficult to compare, they suggest that gamma may be generated by neural mechanisms, which if not common, are at least partially shared across the two species and across recoding modalities. Yet, it is still not clear whether characteristics of gamma-band activity observed in macaque experiments can be linked in a straightforward manner to characteristics observed from human non-invasive recordings. More specifically, can observed modulations in gamma frequency or power in a human MEG experiment be thought of as equivalent to modulations in monkey electrophysiological recordings? This is a non-trivial question of crucial importance for non-invasive EEG/MEG studies on human brain functions that base their hypothesis on prior monkey electrophysiology studies. 
Here we set out to ask whether the functional behaviour of gamma oscillation frequency and power in the human is consistent with the behaviour observed in the macaque and further whether it is a function of the scales of measurement (spikes, LFP, MEG). To investigate this hypothesis, it is necessary to identify a stimulus parameter with robust and well-understood effects on gamma frequency and manipulate this parameter in both human and non-human primates. In computational studies, gamma is often modeled in terms of an interaction between excitation (E) and inhibition (I). In Pyramidal Inhibitory Neuronal Gamma PING network [Tiesinga and Sejnowski, 2009], spikes from E-neurons quickly lead to spiking in I-neurons, which in turn briefly shut down the network. The recurrence of this process leads to oscillations, which are in the gamma range due to the time constants of the neurons involved [Börgers and Kopell, 2005]. Increasing E-drive leads to a faster recovery from inhibition, resulting in a higher oscillation frequency [Brunel and Wang, 2003; Buia and Tiesinga, 2006; Tiesinga and Sejnowski, 2009; Traub et al., 1996]. The gamma frequency increase predicted to follow from increased E-drive has been confirmed in neurophysiological studies in the macaque, in which gamma oscillation frequency as measured in the LFP recorded from early visual cortex was shown to increase strongly with stimulus contrast [Jia et al., 2013; Ray and Maunsell, 2010a; Roberts et al., 2013]. Stimulus contrast can be thought of as an experimentally accessible proxy for E-drive to primary visual cortex [Contreras and Palmer, 2003; Sclar et al., 1990]. Hence, parametric variation of contrast is a paradigm that is theoretically well understood such that it may allow the identification of biophysical mechanisms and at the same time it produces robust modulations of gamma frequency in nonhuman primates.

We applied this rationale to a human MEG experiment, which was compared to a study in macaque V1 previously conducted in our group [Roberts et al., 2013]. The task of experimental design is not trivial: on one hand, one needs to match monkey and human experiments as closely as possible; at the same time due to the low gamma SNR in the MEG for a number of experimental conditions, some parameters in the MEG experiment need to be adapted such that sufficient SNR can be obtained. Beyond the use of a visual parameter whose effects on the visual system are well-understood, our study benefits from a parametric stimulus variation used in both macaque and human experiments in order to facilitate functional interpretations and also from identical spectral analyses.

Here, we provide the first evidence that MEG-measured gamma frequency in human visual cortex increases with increasing stimulus contrast. We also analyzed the gamma response in macaque singleunit spike trains as a function of visual contrast. We present the first evidence that those single units, which exhibited a strong gamma oscillatory component, also shifted their frequency of oscillation in a similar manner to the LFP and MEG. Further, we report spectral asymmetries, which were consistently modulated by contrast in all recording modalities (single unit spikes, LFP, MEG). This in turn may suggest a role for resonance in the generation of gamma rhythms. Alongside the many similarities between MEG, LFP and spikes, we also observed a major difference: while there was a linear increase in the gamma oscillation power with stimulus contrast in the MEG data consistently with previous observations [Hall et al., 2005a], in the LFP data, saturation and even reduction of power were observed at high contrasts.

Overall, the pronounced similarity of these findings across measurement scales and across human and macaque suggests a common generative model of gamma-band activity that can be measured using 
non-invasive techniques in humans. However, the pronounced difference in contrast-dependent power modulation indicates that MEG-measured gamma power cannot be easily equated to LFP gamma power. These results as well as theoretical considerations indicate that oscillation frequency might be a more robust parameter for comparing findings across species and spatial scales of measurement.

\section{Materials and Methods}

\section{HUMAN MEG RECORDING}

Participants: All 9 participants provided informed consent according to the guidelines of the local ethics committee (Commissie Mensgebonden Onderzoek / Committee on Research Involving Human Participants, Region Arnhem-Nijmegen, The Netherlands). The sample consisted of 4 male and 5 female participants. The age range was from 25 to 41 and all were healthy and had normal or corrected-tonormal vision.

Task: Participants were positioned comfortably in the MEG scanner, and instructed to sit still while looking at a fixation spot in the middle of the translucent back-projection screen (EIKI LCD projector that was outside of the shielded room, projection dimensions: $45 \times 34 \mathrm{~cm}, \mathrm{WxH}$, refresh rate: $60 \mathrm{~Hz}$ ), which was located 70 to $80 \mathrm{~cm}$ in front of them. The monitor was calibrated to linearize luminance as a function of RGB values. Each experimental trial consisted of a $2 \mathrm{~s}$ interstimulus interval and a $2 \mathrm{~s}$ stimulation period. The fixation spot was present at all times. Stimuli were square-wave gratings of $4^{\circ}$ size positioned in the lower right visual quadrant (the left upper corner of the square was offset $0.15^{\circ}$ from fixation spot vertically and horizontally) presented on a gray background with the same mean luminance as the stimulus. Stimulus presentation was in Matlab, using the Psychophysics Toolbox extensions [Brainard, 1997; Kleiner et al., 2007; Pelli, 1997]. Stimuli were static and had a spatial frequency of 3 cycles per degree. Stimulus luminance contrast was defined as Michelson contrast:

$C m=100 \frac{L_{\max }-L_{\min }}{L_{\max }+L_{\min }}$

where $L_{\max }$ and $L_{\min }$ denote the maximal and minimal luminance of the stimuli. Stimulus contrast was varied between five values (20\%, 36\%, 48\%, 66\%, 96\%) and selected pseudo-randomly for each trial (100 trials per contrast condition, per participant). After each block of 50 trials the participants had a small break of 10 s. To minimize long-term adaptation effects, orientation ( 0 ㅇ or $90^{\circ}$ ) and phase (8 phases) of the grating was randomized trial-by-trial.

Recording: Magnetic fields of the brain were sampled at $1200 \mathrm{~Hz}$ by a 275 axial gradiometer whole-head magnetoencephalography system (Omega 2000; CTF Systems, Canada) in a magnetically shielded room. A third-order synthetic gradiometer was applied on the sensor data. The MEG signals were high (cutoff $0.5 \mathrm{~Hz}$ ) and low pass filtered (cutoff $120 \mathrm{~Hz}$ ) based on data segments of $10 \mathrm{~s}$. The head position was localized with respect to the MEG sensors using fiducials (small coils) attached at anatomical landmarks (the nasion, the left and right ear canal). Head movements were continuously monitored during the 
MEG recording and kept below $0.5 \mathrm{~cm}$. For anatomical source reconstruction of the MEG signal anatomical MRIs (T1-weighted, MPRAGE) were recorded using a $1.5 \mathrm{~T}$ whole-body scanner (Siemens Avanto, Erlangen, Germany) with anatomical reference markers (left and right ear canal) at the same locations as the MEG fiducials.

Beamforming: Synthetic Aperture Magnetometry, a minimum variance beamformer [Robinson and Vrba, 1999; Vrba and Robinson, 2001], was used to generate maps of power change on a 1 cubic mm grid throughout the brain for each participant. We compared the power in the $20-80 \mathrm{~Hz}$ band between the baseline ( $-1.8 \mathrm{sec}$ to $0 \mathrm{~s})$ and stimulus $(0.2$ to $2 \mathrm{~s}$ ) periods. Data from all luminance contrast conditions were used to generate the volumetric maps. For each participant, we identified the peak voxel showing the largest stimulus-induced change in power compared to the baseline, which was quantified as the pseudo-t parameter in Synthetic Aperture Magnetometry [Robinson and Vrba, 1999; Vrba and Robinson, 2001]. We then reconstructed a time-varying estimate of the electrical activity for the peak location of the image for each participant (this was the estimated source signal). Briefly, this estimate was based on a spatial filter constructed from a covariance matrix comprising baseline and combined luminance contrast conditions. The covariance window for the source signal estimation was $-2 \mathrm{~s}$ to $2 \mathrm{~s}$, 20-80 Hz. All luminance contrast conditions were used in the construction of the spatial filter (the beamformer weights) and sensor data were then projected through this common spatial filter to yield a time-varying estimate at the image peak location (source signal) of each trial. All subsequent analyses were based on these estimated source signals.

\section{MONKEY ELECTROPHYSIOLOGICAL RECORDING}

Details of the experimental methods in the monkey experimental have been described in [Roberts et al., 2013].

Subjects and surgical procedure: Two adult male rhesus monkeys (Macaca mulatta) weighing 7 and $9 \mathrm{~kg}$ took part in this experiment. Two chambers were implanted above early visual cortex, one positioned over V1 and V2 and the second over V4. For the experiment reported here we used data from V1 only. A head post was implanted to head-fix the monkey during the experiment. All the procedures were in accordance with the European council directive 2010/63/EU, the Dutch 'experiments on animal acts' (1997) and approved by the Radboud University ethical committee on experiments with animals (Dier-Experimenten-Commissie, DEC).

Task: The monkeys were head-fixed and placed in a Faraday-isolated darkened box at a distance of $57 \mathrm{~cm}$ from a computer monitor (refresh rate $60 \mathrm{~Hz}$; resolution $1280 \times 1024$ pixels). The monitor was calibrated to linearize luminance as function of RGB values. The position of one eye was monitored with an infrared eye-tracking system (Arrington, $60 \mathrm{~Hz}$ temporal resolution). The stimulus presentation, reward delivery and behavioral control were managed by the CORTEX software package (http://www.cnbc.cmu.edu/ rickr/ctxman5.html). Square wave gratings (2 cycles per degree, 3-5 diameter) were shown on an isoluminant gray background. Stimulus position was based on the mean receptive field position of the recorded neurons in V1 and V2 (4-6 eccentricity). Stimuli were presented at 8 different luminance contrasts $(2 \%, 3.5 \%, 6 \%, 9.7 \%, 16.3 \%, 35.9 \%, 50.3 \%, 72 \%)$ and two orientations 
$\left(45^{\circ}\right.$ and $135^{\circ}$ in monkey $\mathrm{S}, 0^{\circ}$ and $90^{\circ}$ in monkey $\mathrm{K}$ ) in pseudo-random order. Data from the two orientation conditions were pooled. The behavioral task was to hold fixation on a fixation spot in the middle of the computer screen during stimulus presentation. The within-trial median total range eye position was $0.3^{\circ}$ in monkey $\mathrm{K}$ and $0.8^{\circ}$ in monkey $\mathrm{S}$. If the eye position exceeded a maximally $3 \times 3^{\circ}$ window centered on the fixation spot, the trial was aborted. Maintaining fixation was rewarded with a drop of fruit juice. A trial consisted of a $1 \mathrm{~s}$ pre-stimulus baseline, followed by a stimulus randomized in duration between 0.75 and $4 \mathrm{~s}$. Trials with $<1.5 \mathrm{sec}$ stimulus duration were excluded because of insufficient frequency resolution.

Electrophysiological recording: V1 recordings were made with Plexon U-probes (Plexon Inc.) consisting of 8 carbon nanotube coated contacts $(10 \mu \mathrm{m}$ diameter, $0.5-1 \mathrm{~m} \Omega$ impedance, and $200 \mu \mathrm{m}$ inter-contact spacing). The probe was inserted through a sharp guide tube, which was lowered through granulation tissue just above the level of the dura surface. The probe was then advanced by a separate microdrive (Nan Instruments LTD.). The probe was connected to a headstage of high input impedance, and data were acquired via the Plexon 'Multichannel Acquisition system' (MAP, Plexon Inc.). The measured extracellular signal was filtered online between $150 \mathrm{~Hz}$ and $8 \mathrm{kHz}$ to extract spiking activity and filtered between $0.7 \mathrm{~Hz}$ and $300 \mathrm{~Hz}$ to obtain the 'local field potential' (LFP). The signal was amplified and digitized with $1 \mathrm{kHz}$ for the LFP and $40 \mathrm{kHz}$ for the spike signal. For analysis on LFPs, the quantifications obtained from each contact of a laminar probe were averaged over the 8 contacts of each laminar probe to obtain an overall estimate irrespective of the layering of visual cortex. Spike sorting: Multi-unit spike channels were spike sorted using an adapted Waveclus approach [Quiroga et al., 2004]. A k-mean clustering was used, where the optimal cluster size was chosen by using the cluster size with the best ratio of between-cluster-distance to within-cluster-distance. The quality of the clustering was manually checked and in certain cases regrouping was performed.

Selection of neurons based on gamma-band component: For our spectral analysis we quantified parameters (peak power, peak frequency and skewness index) that required a sufficiently strong gamma power induction in the spectrum for reliable estimation (see selection criterion below). Moreover, we used analysis on spike trains only, as opposed to the more commonly used spike-field coherence (SFC) measures, because our objective was to have a local measure of gamma periodicity in spikes, that was independent of LFP properties. Due to these restrictions, we ended up with only a few cells that fulfilled these criteria. However, it is not surprising that we obtained such a low number of cells. Previous experimental studies [Buzsáki and Wang, 2012; Fries et al., 2007; Tiesinga and Sejnowski, 2009; Vinck et al., 2013] have shown that neurons, and in particular pyramidal cells, display only weak locking to network population oscillations and further, that spike rates are often lower than the population gamma frequency (in other words, that single units skip cycles). The selected neurons ( $n=5$ in monkey $S$ and $n=2$ in monkey K) however, fired spikes on every gamma cycle and thus exhibited a strong periodic component in their spike trains. We must emphasize that these neurons are not necessarily representative for the whole population of cortical cells but rather of that subclass of cells that exhibits strong gamma periodicity. Here we tested the hypothesis whether these cells exhibit the same spectral modulations as a function of contrast as those observed in the LFP and MEG. 


\section{DATA ANALYSIS COMMON FOR HUMAN MEG AND MONKEY MICROELECTRODE DATA}

Spectral analysis: The FIELDTRIP MATLAB toolbox (http://www.ru.nl/neuroimaging/fieldtrip [Oostenveld et al., 2011] was used for spectral analysis. For the human MEG power spectra time windows of [ $[-1.8 \mathrm{~s}$ to $0 \mathrm{~s}]$ as baseline and $[0.2 \mathrm{~s}$ to $2 \mathrm{~s}]$ as stimulus period were used. For monkey power spectra time windows [- $1 \mathrm{~s}$ to $0 \mathrm{~s}$ ] as baseline and [0.2s $1.5 \mathrm{~s}$ ] for stimulus period were used. The minimum trial duration of $1.5 \mathrm{~s}$ for the monkey data was chosen because, although stimuli were presented for up to $4 \mathrm{~s}$, selecting only trials with maintained fixation for longer durations would lead to a strong decline in the number of trials available for analysis. The FFT power spectra were computed using a multi-taper method using discrete prolate spheroid sequences (DPSS) with a spectral smoothing of $\pm 3 \mathrm{~Hz}$. For timefrequency analysis (TFR), we also used FFT DPSS computed on sliding windows of $0.25 \mathrm{sec}$ length with $10 \mathrm{~ms}$ spacing. Prior to computation of any spectral parameters we calculated the power ratio between the stimulus period compared to the baseline period. Thus subsequent calculations were based on such baseline-corrected spectra. The power ratio was defined as:

Power ratio $=\frac{(\text { stim-base) }}{\text { base }}$ where stim is the stimulation period (presentation of the visual stimulus) and base is the baseline period (absence of the visual stimulus).

Asymmetric Gaussian fitting for spectral parameter estimation: To estimate the peak frequency, power and asymmetry we used a fitting approach, where the two sides of the Gaussian were separately fitted.

$\lambda(x)=\lambda_{0} e^{-x^{2} / 2 \sigma^{2}}\left\{\begin{array}{l}\sigma=\sigma L, \text { if } x<0 \\ \sigma=\sigma R, \text { if } x \geq 0\end{array}\right.$

where $x$ represents the frequency of the spectrum, $\lambda_{0}$ is the amplitude of the Gaussian and $\sigma L$ and $\sigma R$ are the left and right standard deviations respectively. The fitting was implemented using an unconstrained nonlinear minimization algorithm (fminsearch, Matlab, MathWorks). Before Gaussian fitting the spectra of a subject where normalized to 1 (over all contrasts). The frequency range was from 25 to $70 \mathrm{~Hz}$ in human MEG and 20 to $60 \mathrm{~Hz}$ in monkey data.

Gamma power: was estimated as the area under the curve of the fitted Gaussian. This measure takes changes in the bandwidth into account in comparison to peak power measures. Certain contrast conditions might lead to stronger moment-by-moment frequency fluctuations leading to broader gamma power distributions. Taking the area under the curve ensured that power estimates were not confounded by changes in the gamma band bandwidth.

Gamma frequency: was estimated by two methods. We computed the peak power frequency (mode) or the center of weight (mean) frequency. They were estimated based on the fitted Gaussian function: For each power spectrum, we fitted the asymmetric Gaussian using the nonlinear minimization algorithm. We then derived the mode as well as the mean of the fitted asymmetric Gaussian. This gave use reliable estimates for human MEG as well as for monkey LFP/spike data. We could use the mode and mean estimates of the Gaussian to compute an asymmetry index. This is because distribution asymmetry affects the mode and the mean differently. Moreover, the mean frequency compared to the more commonly used peak power frequency is a more robust measure since it is less affected by noise in the 
power estimate, which can strongly affect peak power frequency (small variations on a broader gamma peak can lead to strong variations in peak power frequency estimates).

Distribution asymmetry: was quantified by an index of skewness [Doane and Seward, 2011]

$\rho=(\mu-\kappa) / \sigma$

Where $\rho$ is the nonparametric Pearson's first skewness coefficient, $\mu$ the mean, $\kappa$ the mode and $\sigma$ the standard deviation of the distribution. To approximate $\sigma$ we used the standard deviation of the fitted Gaussians.

Statistical Analysis: Statistical analysis was restricted to trials with a sufficiently long stimulus duration to permit sufficient frequency resolution ( $>1500 \mathrm{~ms}$ in monkey and $2000 \mathrm{~ms}$ in human data), and the first 200ms following stimulus onset were excluded from analysis in both species (see Spectral Analysis). Thus, analysis windows were $1300 \mathrm{~ms}$ and $1800 \mathrm{~ms}$ in monkey and human data, respectively. Analysis of spectral power in Figure 2 included data from a single session in 9 human participants (100 trials per contrast). Analysis of spectral power in Figure 3 included data from 15 sessions from Monkey S (59.0 \pm 2.7 trials per session and contrast) and 13 sessions from monkey $\mathrm{K}(42.8 \pm 1.2$ trials per session and contrast). When quantifying specific features of the spectral distribution in Figures 4 and 5 (peak frequency, asymmetry), analysis was restricted to data that showed a clear oscillatory gamma component. Thus, all monkey sessions that showed significant gamma power induction (if t-test p-value $(25-60 \mathrm{~Hz})>0.05$ ) in at least three contrasts were included in the analysis; sessions not satisfying this criterion were excluded as a whole. Experimental sessions in the monkey showing no or weak induced gamma response were due either to an insufficient number of trials or due to the positioning of the laminar probes largely in the deep cortical layers where gamma is weak [Roberts et al., 2013; Xing et al., 2012]. Data then included 11 sessions from monkey $S$ (average of 700.1 trials per contrast pooled over all sessions) and 11 sessions from monkey K (average of 470.5 trials per contrast pooled over all sessions). The same selection rule (at least 3 contrasts with significant gamma power) was applied to the human population at the participant level, leading to a selection of 7 of 9 subjects (who each contributed a single session with 100 trials per contrast). Within this data selection, we excluded the lowest contrast for MEG frequency and asymmetry estimations (20\%), and the three lowest contrasts for LFP/spike-based frequency and asymmetries estimations $(2 \%, 3.5 \%, 6 \%)$, because of a lack of gamma power ( $t$-test $p$ value $[25-60 \mathrm{~Hz}]>0.05)$. Hence, in these cases, monkey recording analysis was restricted to contrasts $9.7 \%, 16.3 \%, 35.9 \%, 50.3 \%, 72 \%$, and human MEG analysis to $36 \%, 48 \%, 66 \%, 96 \%$. Statistical regression analysis was done on spectral parameters of interest over contrast on a single trial level basis for each subject individually (human participant or monkey). To accomplish this, we used a robust fitting approach (reducing effects of outliers) to assess significance and effect size estimates on the level of individual subjects (monkeys or human participants) or sessions (in monkey data). We also applied regression fitting on the trial-averaged spectra of all subjects to estimate population effects. Note that the total pooled sample of trials available per individual contrast from the two monkeys (1170.6) was comparable to the total pooled sample of trials available per individual contrast from the 9 human participants (900). In the analysis of frequency and asymmetry, analysis was restricted to 7 participants (700 trials per contrast, see above). In regression analyses we fitted both linear and quadratic models in 
a stepwise fashion - quadratic fits were only reported if they substantially improved the explained variance $\left(R^{2}\right)$ compared to linear models.

\section{Results}

\section{Comparison of stimuli and experimental designs}

In a previous study from our group, Roberts et al. (2013) reported a $\sim 25 \mathrm{~Hz}$ increase in LFP frequency in $\mathrm{V} 1$ and $\mathrm{V} 2$ of 2 macaque monkeys as luminance contrast was increased in square-wave gratings from $2 \%$ to $72 \%$ (grating diameter $3-5^{\circ}$, eccentricity $4-6^{\circ}$, spatial frequency $2 c / d e g$, lower visual field quadrant, $1300 \mathrm{~ms}$ analysis time window). To test the generality of the relationship between grating contrast and gamma frequency, we compared this monkey data with MEG data collected from 9 human participants also viewing square wave gratings. Luminance contrast was increased from 20 to $96 \%$ (grating diameter $4^{\circ}$, eccentricity $3^{\circ}$, spatial frequency 3 cycles/deg, lower visual field quadrant, $1800 \mathrm{~ms}$ analysis time window). In both experiments (Figure 1, A-B), the subjects' task was to fixate a central fixation spot; the grating stimuli were irrelevant to the observer. MEG provides a less direct measurement of activity than invasive measurements of LFPs and spiking (Figure 1C). Accordingly, some specific stimulus adaptations in the MEG experiment were necessary to counteract the lower signal-to-noise ratio (SNR) of MEG gamma. The low SNR becomes exacerbated for small cortical stimulus representations, i.e. when eccentricity is high [van Pelt and Fries, 2013; Swettenham et al., 2009] or stimulus size small [Gieselmann and Thiele, 2008]. Hence, to increase SNR in the MEG experiment the stimuli were placed at a smaller eccentricity and presented in a square aperture to restrict their presentation to one hemifield. Additionally, the contrast manipulation was shifted to higher contrast values. The final stimulus parameters were a result of a series of pilot experiments where the smallest departure from the monkey experiment that still allowed for discernible gamma response in the MEG was sought. Despite the necessary differences stimulus parameters outlined above, the similarity of the experimental manipulation permitted a comparison of the spectral properties of the MEG signals (top panels in Figures 1D, 1E) with the LFP signals (middle panels Figures 1D, 1E), and the spiking signals (bottom panels figures 1D, 1E). 
A



B

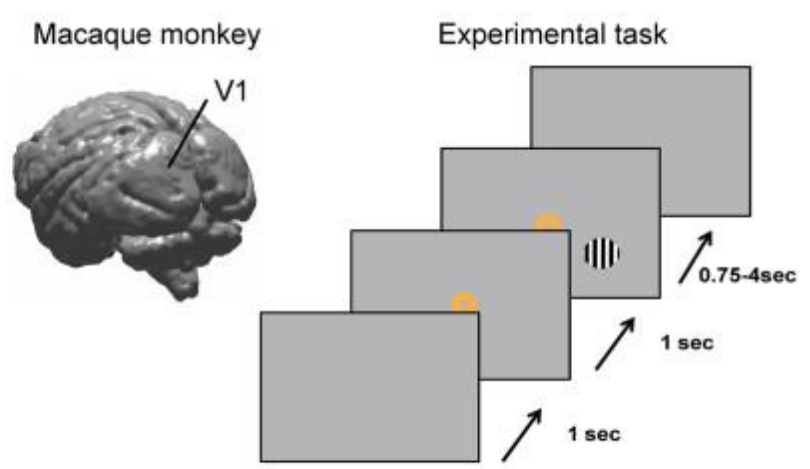

C

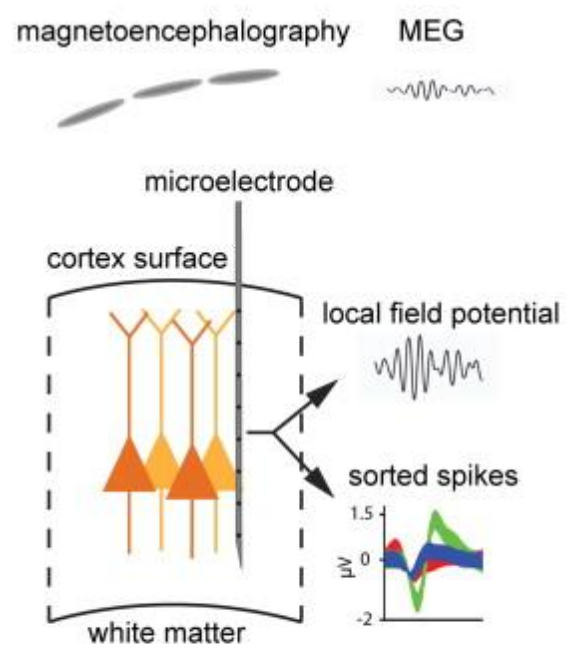

D


E
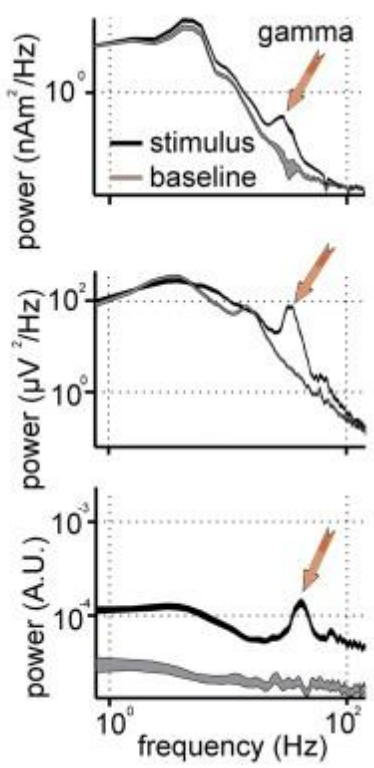

Figure 1. Overview of experimental tasks and recording signals A) Left: A beamformer-reconstructed gamma source projected on an MRI-reconstructed human brain. The peak voxel (located in the area of highest activation as indicated by the yellow color) for each subject was chosen for source signal reconstruction using the beamformer weights for that location. Right: human experimental paradigm B) Left: Illustration of a macaque brain. Right: monkey experimental paradigm C) Illustration of the three signal sources compared in this study: MEG, LFP and extracellular unit activity. MEG collects magnetic signals (fT-pT) over larger cortical space from outside the brain; it is sensitive to highly synchronized events representing mostly dendrosomatic currents $(>5 \mathrm{~mm})$. The LFP (microV) is predominantly sensitive to dendro-somatic currents locally $(<0.5 \mathrm{~mm}$ to the electrode). Extracellular spikes represent action potentials. After spike sorting a specific set of spikes can be attributed to one cell. D) Example single trial data are shown for three different signal types. The spike train was converted into spike density to facilitate the observation of the gamma rhythm. E) The log-log power spectra of a baseline compared to high contrast grating stimulation (single subject/session). A specific increase in the gamma band can be observed. There were considerable differences in power induction between MEG, LFP and spikes.

\section{Spectral properties of gamma MEG data in human visual cortex}

In 8 of 9 participants, the peak of the MEG beamformer image was found in visual cortex contralateral to the stimulus presentation, consistent with many previous studies using similar stimulation [Duncan et al., 2010; Hall et al., 2005a; Muthukumaraswamy et al., 2010; Swettenham et al., 2009]. The mean pseudo-t value (over all contrasts) was 4.5 (SD = 2.8). We note that in participant 2 (Figure 2, middle 
panel top row) the gamma SNR was very low. The weights of the beamformer for the peak voxel were extracted and used for the reconstruction of the source time series (see methods). All analyses performed were based on this source reconstructed signal, which beyond a localization to V1 also offers a higher SNR compared to the sensor data [Brookes et al., 2009b; Hadjipapas et al., 2007].

Figure 2 shows the effect of contrast on the power spectra obtained in the MEG experiment. In Figure 2A, trial-averaged Time-Frequency Representations (TFRs) from an example participant (participant 8, middle panel bottom row Figure $2 \mathrm{~B}$ ) exhibiting a strong gamma response are shown for the full range of contrasts used. A gamma band was discernible at stimulus contrasts of $36 \%$ and higher (Figure $2 \mathrm{~A}$ ). For these contrasts, there was a transient power increase in the gamma range briefly after stimulus onset, followed by weaker power as long as the stimulus remained in view. This is consistent with previous observations of the MEG response in the human [Hadjipapas et al., 2007; Muthukumaraswamy et al., 2010; Swettenham et al., 2009] and the ECoG and LFP response in the macaque (Rols et al., 2001; Xing et al., 2012 respectively). Figure 2A also shows that both relative gamma power and gamma peak frequency increased with contrast. Figure $2 \mathrm{~B}$ shows the spectra for each participant. As illustrated in Figure $1 \mathrm{E}$, each power spectrum represents the relative power change ratio between a spectrum corresponding to the stimulus period and a spectrum corresponding to the baseline period; spectra were obtained using Fourier analysis and DPSS tapering (see Materials and Methods for details). Despite individual variation, these data overall are in line with the average power increase and the shift of the gamma band towards higher frequencies with increasing stimulus contrast, as shown in Figure 2A. However, some participants showed very weak gamma power across all contrasts. This is a common observation [Hadjipapas et al., 2007; Muthukumaraswamy et al., 2010; Swettenham et al., 2009] and may be due to a number of non-physiological factors (including distance between brain and sensors, source geometry and orientation with respect to sensors). Of particular interest in Figures $2 \mathrm{~A}$ and $\mathrm{B}$ was the peak-power frequency of the gamma spectrum, also referred to as 'peak frequency'. When averaging the spectral data over participants the effect of power dominates (Figure 2C, top), although the frequency shift of the spectra is still evident. To better visualize the peak frequency shift, we normalized all spectra to the range $[0,1]$ for each contrast (Figure 2C, bottom). From 36\% to $96 \%$ contrast, the peak frequency averaged across participants increased by $\sim 8 \mathrm{~Hz}$ (see quantifications below). 


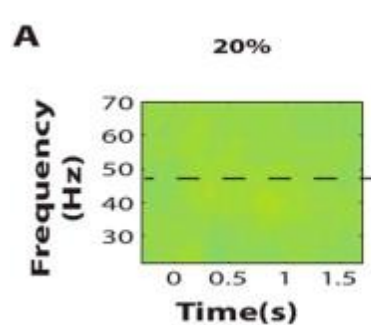

B


Michelson Contrast (\%)
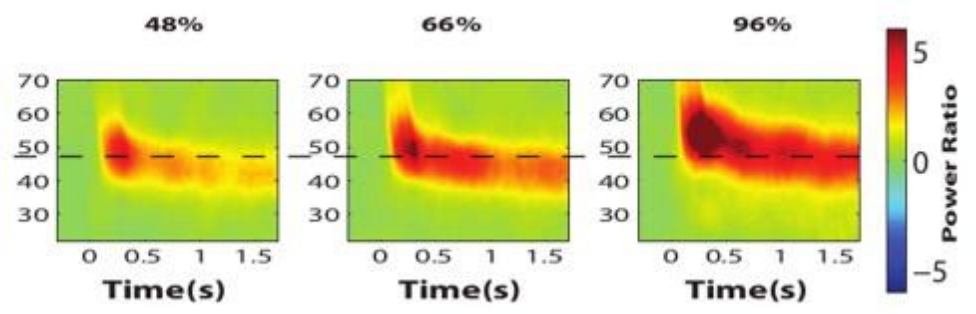

c
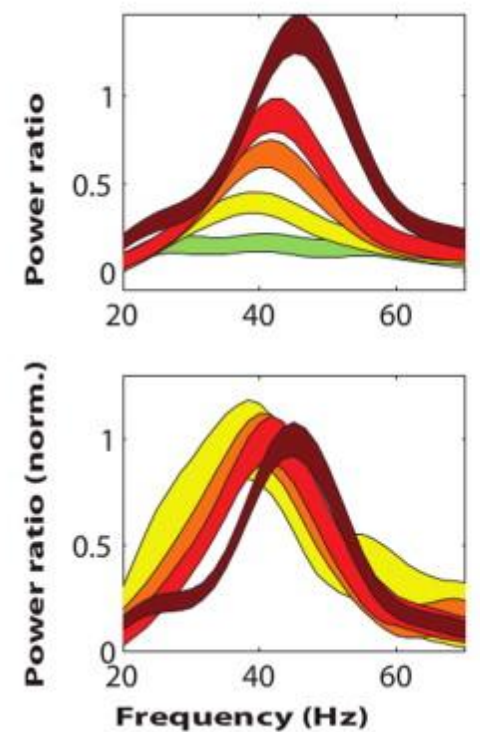

Michelson

Contrast

$20 \%$

$36 \%$

$48 \%$

$66 \%$

$96 \%$

Figure 2. Human MEG results A) Time frequency representation (TFR, time window for baseline correction -2 to 0 sec) for different stimulus contrast conditions of a participant 8. B) The power spectra (relative power) for the individual participants (1 to 9 ordered from left upper to right lower panel). Error bars represent \pm 1 standard error of the mean. Participants had different relative power levels ( $\mathrm{Y}$ axis values). C) Average of individual power spectra before (top) and after normalization per contrast of power spectra of the individual participants.

\section{Gamma spectral properties of neurophysiological data in macaque V1}

For comparison with the human MEG data, Figure 3 shows electrophysiological data from area V1 of two macaque monkeys. Figure 3A shows the TFR of a single example session from Monkey $\mathrm{S}$ (59 trials per contrast on average). There was observable gamma power at a contrast as low as $6 \%$, with a frequency of $\sim 28 \mathrm{~Hz}$. With increasing contrast, the peak frequency of sustained gamma increased to $45 \mathrm{~Hz}$ at $72 \%$ contrast. Figures $3 \mathrm{~B}$ and $3 \mathrm{E}$ show the LFP gamma spectra for monkey $\mathrm{S}$ ( 15 sessions with on average 59 trials per session and contrast) and monkey $\mathrm{K}$ (13 sessions with on average 43 trials per session and contrast). The spectral data were averaged over the whole laminar probe (see Materials and Methods). The LFP spectra showed a large shift in frequency as a function of contrast, as well as a large modulation in power. Figures $3 \mathrm{C}$ and $3 \mathrm{~F}$ show the contrast-response function for the two monkeys based on population spike rate for all sessions combined, normalized by baseline (120 and 104 multiunit spike channels in monkey $\mathrm{S}$ and $\mathrm{K}$ respectively). Spike rate increased as a function of contrast, reaching asymptotic levels at the highest contrasts, in agreement with previous observations [Hamilton, 1982; Sclar et al., 1990]. Spike train spectral analysis in Figures 3D and G was based on a Fourier 
transform applied directly to the spike trains of a total of 7 single well-isolated (spike-sorted) neurons ( 5 neurons in monkey S, 2 in monkey K). By working on the spike trains only rather than using Spike Field Coherence (SFC) we wanted to make sure that we have a measure of single unit rhythmicity , which was independently derived from the LFP. The low number of neurons used for spike spectral power analysis was due to the requirement of a strong spectral gamma component (see Materials and Methods). We should note that the majority of units exhibited only weakly oscillatory spike trains. Previous experimental studies [Buzsáki and Wang, 2012; Fries et al., 2007; Tiesinga and Sejnowski, 2009; Vinck et al., 2013] have shown that single units are typically only weakly locked to the population rhythm, that is the majority of units skip cycles. The neurons we focus on here however, fired spikes on every gamma cycle and therefore exhibited strong gamma periodicity. Here we tested the hypothesis of whether these single units exhibited the same spectral modulations as a function of contrast as those observed in population signals (LFP and MEG). Despite the fact that spike train spectral properties were derived independently from the LFP, the results from spike spectral power analysis resembled those from LFP analysis, especially in monkey S. In both the LFP and spiking spectral analysis, monkey S showed a lack of saturation of the frequency shift at high contrasts, and a decrease in gamma power for contrasts exceeding $35.9 \%$. Comparing LFP and spiking analysis in monkey $\mathrm{K}$ shows a similar pattern of gamma frequency increases and clear saturation of gamma power at higher contrasts (with a decrease in spikebased spectral power at the highest contrast). Hence, within monkeys there was close similarity in LFP and single unit spiking spectral analysis (in Figure 3 compare B, D and E, G), but both analyses showed consistent differences between monkeys (in Figure 3 compare $B, E$ and $D, G$ ). Despite these differences in spectral data, spike rate as a function of contrast was similar between the two monkeys (in Figure 3 compare C, F). This indicates that spectral power of LFP oscillations was not tightly related to spiking in the majority of recorded neurons (see[Logothetis et al., 2001]). The LFP predominantly reflects synaptic rather than spiking events, particularly when these result in synchronous fluctuations of the membrane potentials across many neurons (thus typically during oscillations)[Buzsáki et al., 2012]. Thus, changes in synchronization between neurons can change the power of the LFP in the absence of changes in firing rate. Direct experimental evidence for this has been obtained recently in an analogous situation although at a different spatial scale (spatial synchronization of LFPs affecting EEG power)[Musall et al., 2014]. Thus one possibility for the observed dissociation is that while firing rates continue to go up with increased contrast (increased afferent excitatory drive), neurons become less synchronous resulting in lower LFP power. 
A

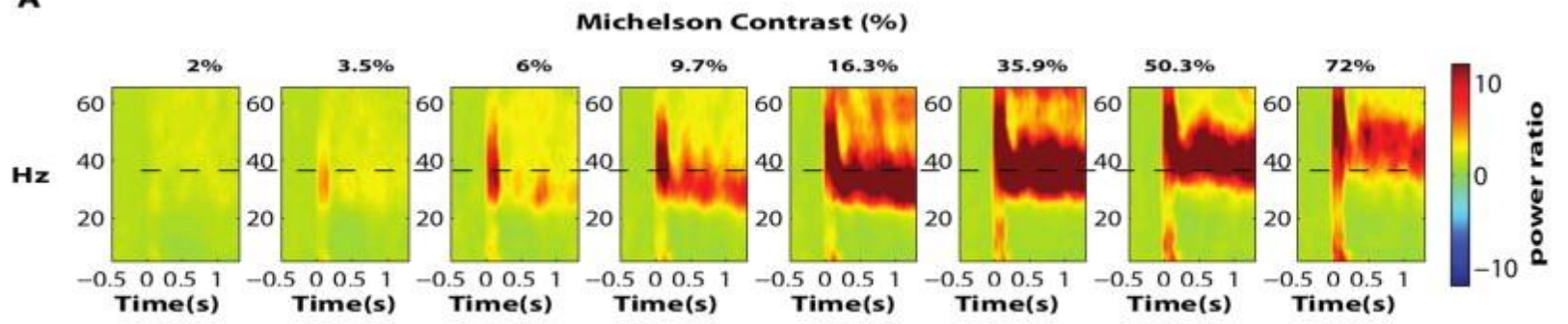

B

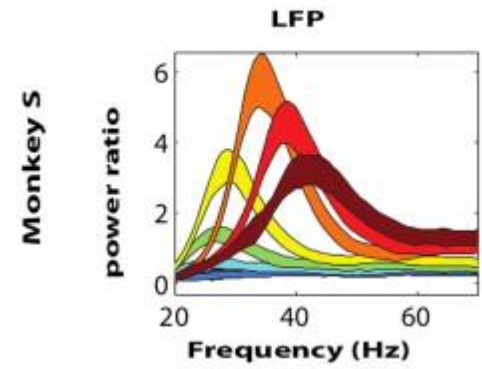

E

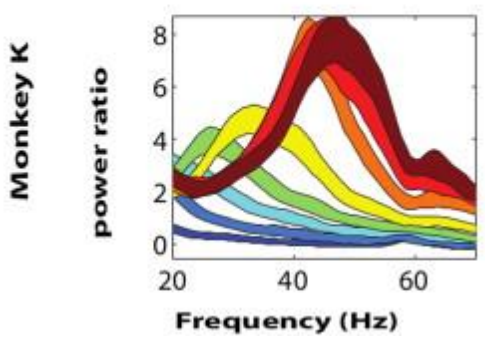

C

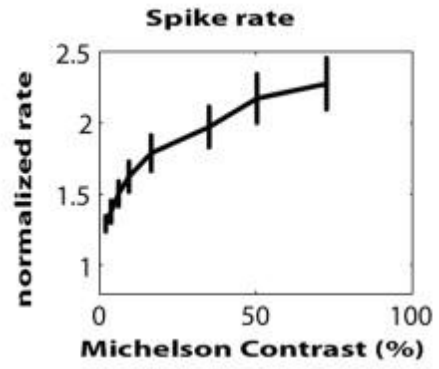

$\mathbf{F}$

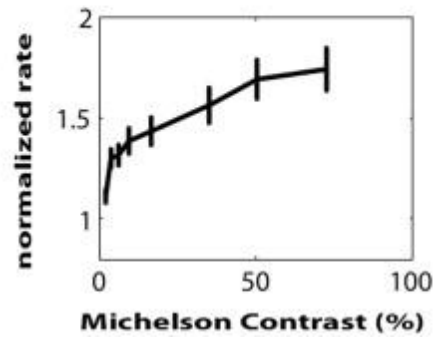

D
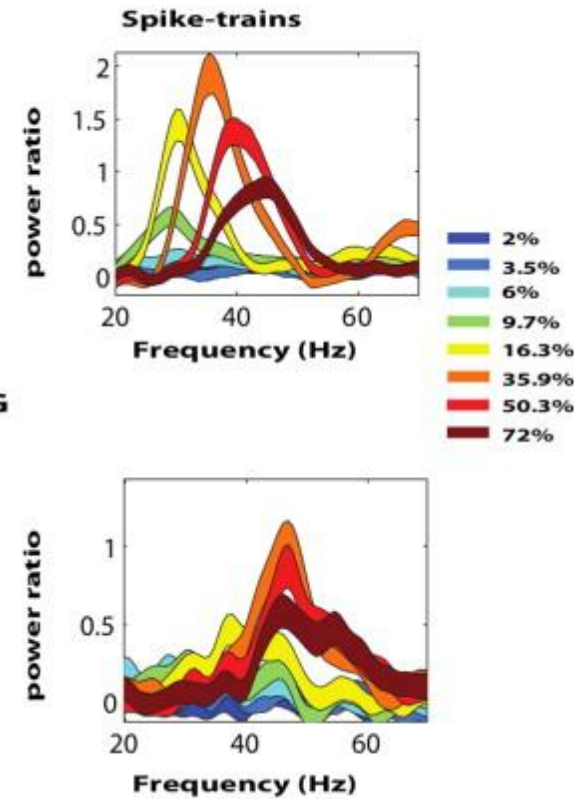

Figure 3. Results from monkey V1 recordings A) TFR from a session example from Monkey S for eight different contrasts (time window for baseline correction $-1-0 \mathrm{sec}$ ). B, E) LFP relative power spectra for monkeys $\mathrm{K}$ and $\mathrm{S}$. Error bars represent \pm 1 standard error of the mean. Contrast modulated the total power, peak frequency and asymmetry of the spectra. C, F) Mean spike rate is shown as a function of contrast for monkeys S (120 multi units) and K (104 multi-units), respectively. In both monkeys average spike rate increased with a stronger slope at lower contrast ranges. D, G) The power spectra of single neuron spike trains for the different contrast conditions in monkey $\mathrm{S}$ ( 5 neurons) and monkey $\mathrm{K}$ ( 2 neurons). The spectra for these spiking data were similar to the LFP power spectra.

\section{Comparison of contrast-induced changes in gamma spectra between spikes and LFPs in the macaque and human MEG}

In Figure 4, we summarize the effects of luminance contrast on the spectral parameters for the three signal domains (MEG, LFP and spikes). Figures 4A-C show the peak frequency in the MEG, LFP and the frequency of the periodic component of single unit spike trains respectively; Figures 4D-F show the power ratio relative to baseline. Figures $4 A$ and $D$ represent human MEG signal, Figures $4 B$ and $E$ show monkey LFP, and Figures $4 \mathrm{C}$ and $\mathrm{F}$ shows monkey single unit spike trains, as a function of contrast. Notice that the contrasts included differ in the different figure panels (for details, see Materials and Methods, Data analysis common for human MEG and monkey microelectrode data, and next two paragraphs). In monkey LFP data, the peak frequency shifted from $\sim 26 \mathrm{~Hz}$ at $9.7 \%$ contrast to $\sim 45 \mathrm{~Hz}$ at 
$72 \%$ contrast, hence a shift of $\sim 19 \mathrm{~Hz}$. In human MEG, the shift from $32 \%$ contrast to $96 \%$ contrast was $\sim 8 \mathrm{~Hz}$. This is in good agreements with the $7.6 \mathrm{~Hz}$ shift in peak frequency observed in monkeys for the most comparable contrast variation from $36 \%$ to $72 \%$ contrast. The frequency of the gamma periodic component of the spike trains estimated based on the 7 single units (pooled over monkeys, from figures 3D and G) increased with contrast over a similar frequency range as the LFP peak frequency. Note that, contrary to power, the relationship between contrast and frequency was monotonic across all recording modalities, species and spatial scales (spikes, LFP, MEG). Figures 4D-F show contrast-induced shifts in power ratios for the MEG, LFP and spiking signals. Interestingly, despite some differences between the monkeys (Figure 3), the average spectral power of monkey LFP and spiking signals saturated at high contrasts, while average MEG power showed a nearly linear relation over the whole contrast range. The latter observation is in line with the individual subject data in Figures 2B and with a previous MEG study [Hall et al., 2005a]. The main observations in each panel of Figure 4 were tested statistically by regression analyses, which are described below but not illustrated.
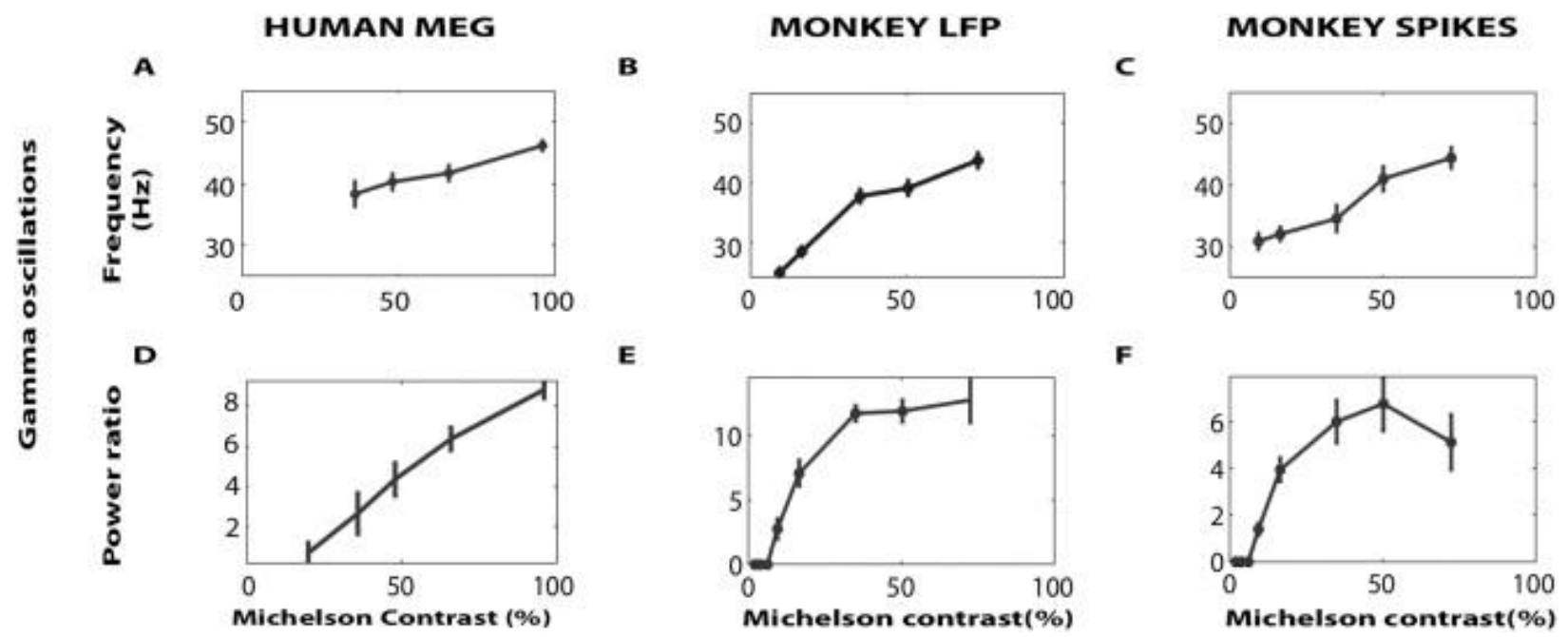

Figure 4. Quantification of gamma spectral effects in humans and monkeys. A-C) The gamma frequency plotted as a function of contrast for human MEG data (A) monkey LFP data (B) and monkey spiking data (C). D-F) Gamma power plotted as a function of contrast for human MEG data (D), Monkey LFP data (E) and monkey spiking data (F). Monkey neurophysiological data are analyzed for sessions that showed significant gamma signal and likewise human MEG data are analyzed in participants with significant changes of gamma power from baseline (7 of 9) (details in text). Means represent the mean of session averages in the monkey neurophysiology data (22 sessions total), and of participant averages $(N=7)$ in the human MEG data. Error bars represent \pm 1 standard error of the mean.

Across-species effects of contrast on gamma peak frequency: We first statistically tested the relationship between peak frequency and contrast in human participant data on the data shown in Figure 4A. However, note that peak frequency could not be estimated when the gamma spectrum did not differ significantly from baseline (if t-test $p$-value $(25-60 \mathrm{~Hz})>0.05)$. Participants in which this was the case for $>3$ contrast conditions ( 2 of 9 participants) were excluded from the individual analysis of spectral power and peak-power frequency (participants 2 and 3, see Figure 2). In the 7 remaining participants, we also excluded the lowest contrast from analysis as the stimulus-induced gamma spectrum did not differ significantly from the baseline, leaving 4 contrasts for analysis. In these 7 participants, we estimated 
peak frequency based on fitted asymmetric Gaussian in single trials. The relationship between singletrial estimates of peak frequency and contrast was well fitted by a linear regression in each participant (all $p<0.05$, average $R^{2}=0.072$, range $R^{2}: 0.09$ to 0.264 ). Using a single-trial approach, the explained variance was however only a fraction of the total trial-to-trial frequency variation. A part of the unexplained variance was likely accounted for by non-physiological noise inherent to the MEG measurements. In addition, gamma frequency is known to vary widely from moment to moment and from trial to trial even under constant conditions in the monkey [Brunet et al., 2013; Brunet et al., 2014; Burns et al., 2011a; Roberts et al., 2013]. Most of this unexplained variability in the regression analysis was removed when averaging the single trial estimates within contrast conditions per participant (SE bars in Figure 4A correspond to this analysis). Using the 4 (contrasts) $\times 7$ (participants) averages for the regression, the linear relationship of the peak frequency of the spectrum to luminance contrast was again highly significant in human MEG but this time with much higher explained variance ( $F=29.7$, $\mathrm{R}^{2}=0.534, \mathrm{df}=26, \mathrm{p}<10^{-5}$ ). There was no substantial increase in $\mathrm{R}^{2}$ when comparing a quadratic to a linear fit (the change of explained variance $R^{2}$ when going from linear to a quadratic model $\left(\Delta \mathrm{R}^{2}(\right.$ linear $\rightarrow$ quadratic $\left.)=0.0001\right)$. The regression slope corresponded to an increase of $0.16 \mathrm{~Hz}$ per $\%$ contrast.

We then statistically tested the relationship between peak frequency and contrast in the two monkeys on data shown in Figure 4B. Sessions that had no significant gamma power induction in at least three contrasts were excluded (if t-test $\mathrm{p}$-value $(25-60 \mathrm{~Hz})>0.05)$, leaving 11 sessions in Monkey $\mathrm{S}$ and 11 sessions in Monkey $\mathrm{K}$ (for details, see Materials and Methods). Because of low power in the lowest contrasts used, the contrast-frequency relationship was tested only over the 5 highest contrasts in the monkey data (Figure 4B). In a single trial linear regression analysis of LFP in the 22 available sessions, we found a robust relationship between peak frequency and contrast in each session estimate in both monkey S LFP $\left(R^{2}(\right.$ mean $)=0.452$, all $\left.p<10^{-3}\right)$ and monkey $K\left(R^{2}(\right.$ mean $)=0.448$, all $\left.p<10^{-5}\right)$. We then entered the averages per contrast and per session into the regression analysis (sample of 22 sessions $\times 5$ contrasts). We found a strong linear relationship for the influence of contrast on the LFP frequency ( $F=$ $159, R^{2}=0.63, d f=112, p<10^{-5}, \Delta R^{2}$ (linear $\rightarrow$ quadratic) $\left.=0.045\right)$. The regression slope over the whole contrast range corresponded to an increase of $0.254 \mathrm{~Hz}$ (over both monkeys) per \% contrast.

We continued by testing the relationship between gamma frequency derived from single neuron spike trains and contrast (Figure 4C) in 7 isolated single units collected in the same contrast conditions and sessions as used for LFP regression analysis. For the spiking data we tested the linear regression immediately on the averages per neuron and per contrast ( 7 neurons $x 5$ contrasts), yielding an effect size comparable to that found for the LFP data $\left(F=47.8, R^{2}=0.61, d f=32, p<10^{-5}, \Delta R^{2}\right.$ (linear $\rightarrow$ quadratic) $=$ 0.02 ). The regression slope over the whole contrast range corresponded to an increase of $0.192 \mathrm{~Hz}$ (over both monkeys) per \% contrast.

Across species effects of luminance contrast on gamma power: We found a strong modulation of gamma power (estimated by the area under the fitted Gaussian) as a function of contrast. In human MEG (Figure 4D), linear regression fits on single trial estimates were performed in each of the 7 subjects also included in the analysis of frequency, this time however including the lowest contrast. In all participants, linear regression yielded significant results $\left(p<0.05\right.$; mean $R^{2}=0.11$, range $R^{2}=0.003$ to 0.33 , 
$\Delta \mathrm{R}^{2}$ (linear $\rightarrow$ quadratic $\left.)=0.007\right)$. Analysis on data averaged per contrasts (5) per participant $(7)$ yielded a robust linear relationship $\left(F=32.9, R^{2}=0.56, d f=26, p<10^{-5}\right)$.

For monkey LFP data (Figure 4E), we first did a single trial based regression analysis of power on the 11 sessions from each monkey used in the previous section for analysis of frequency, but including all 8 contrasts in the analysis. We found significant linear relationships, but also found that adding a quadratic term improved the fit of the gamma power versus contrast relationship. This held in monkey $S$ (all $p<10^{-5}, R^{2}$ (mean) $=0.16, \Delta R^{2}$ (linear $\rightarrow$ quadratic) $=0.12$ ) and monkey $\mathrm{K}$ (all $p<10^{-5} R^{2}($ mean $)=0.155$, $\Delta R^{2}$ (linear $\rightarrow$ quadratic) $=0.027$ ). Quadratic fitting on the sample of data averaged at session level for 22 sessions $\times 8$ contrasts gave a good fit with a substantial improvement against a linear fit $\left(F=68, R^{2}=0.58\right.$, $\mathrm{df}=112, \mathrm{p}<10^{-5}, \Delta \mathrm{R}^{2}$ (linear $\rightarrow$ quadratic) $=0.2$ ). We also performed quadratic regression on the power of the periodic component of single unit spike trains (data averaged for 7 neurons and 8 contrasts, Figure $4 F)$ and found a good quadratic fit, which was superior to a linear fit $\left(F=8.67, R^{2}=0.35, d f=31, p=1.1^{*} 10^{-}\right.$ $3, \Delta R^{2}$ (linear $\rightarrow$ quadratic) $=0.344$ ). Therefore, the analyses of power in Figures $4 D-F$ confirmed the monotonic linear relationship between contrast and power in human MEG and the non-linear relationship between contrast and power in monkey LFP and single unit spike train data.

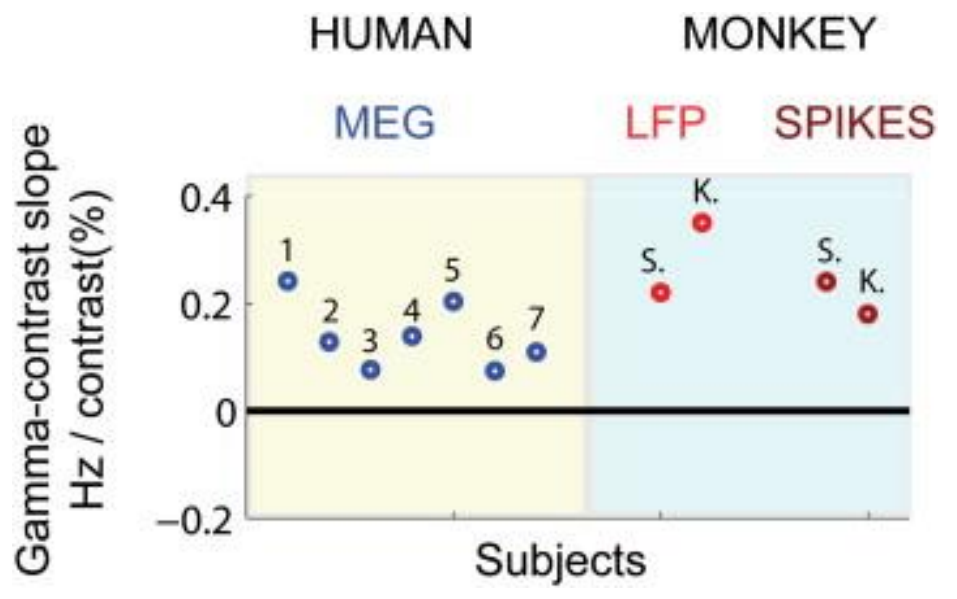

Figure 5. Contrast- Gamma Frequency regression slopes at different spatial scales. The regression slopes between the frequency of spectral gamma peak as a function of luminance contrast for the 7 human subjects with significant gamma power increase from baseline (details in text) and the two monkeys were computed. For robustness considerations, the slope fitting was based on trial-averaged estimates. For the human subjects the slopes were quantified based on the MEG power spectra, whereas for the monkeys quantifications were based on the LFP and spike power spectra.

Effects of luminance contrast on the asymmetry (skewness) of the spectral gamma distribution: Beyond the effect on the power and the peak frequency of the spectra, contrast also had an effect on the shape of the spectra which could be expressed in terms of spectral asymmetry. In Figure 5, the top row shows the spectral distributions for examples of lower and higher contrasts for MEG (Figure 5A), LFP (5B) and single unit spike trains $(5 C)$, using averages of all available trials from respectively all participants, monkeys, or neurons. Considering the monkey LFP and spiking data first (Figures 5B, C), the spectral distribution for a $16 \%$ contrast stimulus (red) was skewed to the right, while the spectral distribution for a $50.3 \%$ contrast stimulus (blue) appeared more symmetric. This change in skewness from lower to 
higher contrasts was weaker in the average MEG data (Figure 5A), but visible in single subjects (Figure 2B). The skewness of the spectral distribution was assessed by fitting an asymmetric Gaussian, and then comparing the fit's peak frequency (mode) with the centre of weight frequency (mean), using a skewness index (see Materials and Methods for details). When the mean is larger than the peak frequency, the distribution is skewed to the right (positive skewness index), and when the reverse is true it is skewed to the left (negative skewness index). Figures 5D-F show the changes in skewness as a function of contrast in human MEG data, as well as monkey LFP and single unit spiking data on the same data sets as used for the analysis of contrast-induced shifts in peak frequency. In MEG, LFP, and spiking data, skewness evolved from positive (skewed to the right) to zero (symmetrical) and even slightly negative at the highest contrasts (skewed to the left). The change in skewness with contrast was quantified by linear regression on MEG data averaged at the participant level $(N=7)\left(F=16.8, R^{2}=0.4\right.$, $\left.d f=26, p=0.4 \times 10^{-3}\right)$, LFP data averaged at the sessions level pooled over monkeys $(N=22)\left(F=21, R^{2}=0.17\right.$, $\left.d f=113, p=0.1 \times 10^{-3}\right)$ and single unit spiking data averaged at the neuron level $(N=7)\left(F=8.3, R^{2}=0.22\right.$, $\mathrm{df}=32, \mathrm{p}=0.72 \times 10^{-2}$ ). In summary, independent of data modality, an increase in contrast had three effects on gamma spectra, namely an increase in peak frequency, an increase in power (which however in monkeys showed saturation), and a decrease in right-sided skewness.

A

HUMAN MEG

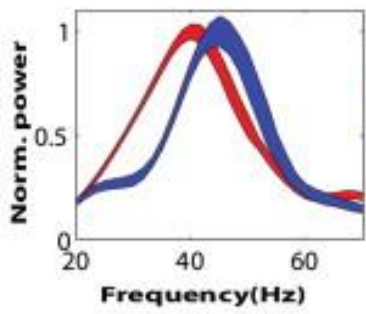

D

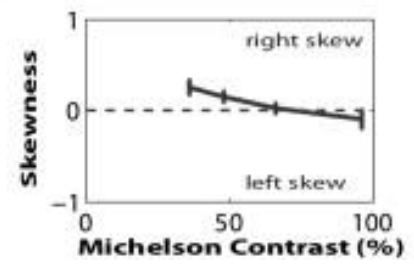

B

MONKEY LFP
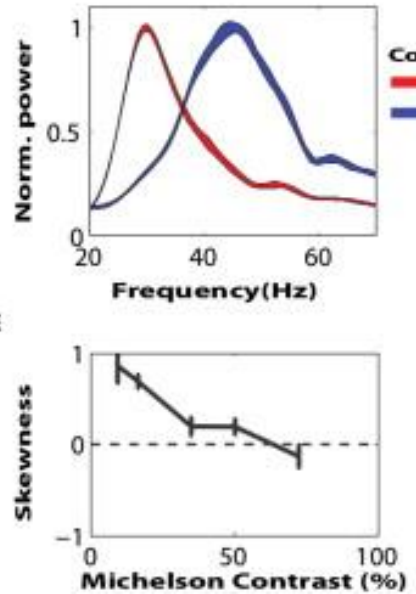

C

MONKEY SPIKES
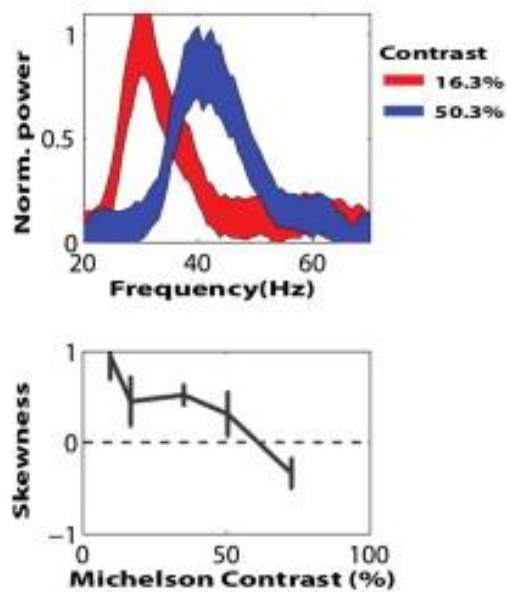

Figure 6. Spectral asymmetry modulated by contrast. The three columns represent MEG, LFP and Spikes respectively. The top row (A-C) depicts two relative power spectra of lower (red) and higher (blue) contrast in the different modalities. The spectra were normalized to [0 1] for each contrast to emphasize shape rather than power differences. Note the skewness to the right at lower contrasts and the relative symmetry at higher contrasts. The bottom row (D-F) shows the quantification of the spectral skewness. Error bars represent \pm 1 standard error of the mean. In all modalities the right-sided skewness decreased with contrast; in the case of spikes and MEG at high contrast there is even a reversal to left-sided skewness. The same data samples are used as in Figure 4. 


\section{Discussion}

In this paper, we present human MEG and monkey electrophysiological (LFP and spiking) recordings obtained in V1, where gamma oscillations were studied under similar stimulation parameters. MEG beamformer source reconstructions are well-suited to make this comparison due to their capacity to localize sources to visual cortex and to improve signal SNR (Brookes et al., 2009; Duncan et al., 2010; Hadjipapas et al., 2007). We used a paradigm where luminance contrast is varied parametrically. Because contrast is a proxy for afferent excitatory drive to visual cortex, this lead to a theoretical prediction about changes in gamma oscillation frequency. Gamma frequency is a fundamental network parameter that affects both internal structuring of units within the network and also longer-distance communication between populations (Bosman et al., 2012; Fries, 2009; Fries et al., 2007; Gregoriou et al., 2009; Roberts et al., 2013). The approach illustrated here permits testing the extent to which network mechanisms underlying gamma in non-human primates can be extended to humans. We report three main findings: First that in human MEG data gamma frequency increased over a substantial range as a function of luminance contrast, both on the population and single subject level. This replicates and extends recent findings (Perry et al., 2014), and fits well with our monkey LFP and single unit spike data. Second, the MEG data showed a monotonic contrast-dependent increase in gamma power consistent with previous MEG studies (Hall et al., 2005a; Perry et al., 2014), which contrasted with the power saturation and decay observed in our macaque V1 data at higher contrasts. Third, we present the first experimental evidence for contrast-induced modulations of spectral asymmetry, observed in human MEG as well as monkey LFP and single unit spikes. Below, we will discuss the implications of the three main observations, especially with respect to the extent to which these constitute support for common gamma mechanisms between the two species and for generalization of gamma spectral features across the different measurement scales (single neuron spikes, LFP and MEG signals).

\section{Frequency modulation of MEG and LFP gamma oscillations as well as single unit spikes by stimulus contrast}

In the MEG participants there was a systematic, approximately linear change in the dominant gamma oscillation frequency with stimulus contrast over the range of contrasts where estimations were possible. The frequency shift was significant in 7 of 9 participants, in which the 2 participants with no significant effect also had very low induced gamma power, limiting frequency estimations. All participants with significant gamma power induction had a frequency change in the same direction. The frequency modulations were very similar to the one observed in the LFP of our rhesus macaque experiments and in other studies in the macaque (Jia et al., 2013; Ray and Maunsell, 2010) and also to a recent study of human MEG (Perry et al., 2014).

We aimed to test whether results found in population signals could be extended to behaviour of single neurons. To this end we analyzed spike trains in isolation, as opposed to the more commonly used spike-field coherence (SFC) measures, in order to have a local measure of gamma periodicity in spikes independent of LFP properties. We quantified spectral parameters (peak frequency and skewness index) that required a sufficiently strong gamma power induction in the spectrum for reliable estimation. 
Previous studies (Buzsáki and Wang, 2012; Fries et al., 2007; Tiesinga and Sejnowski, 2009; Vinck et al., 2013) have shown that neurons, and in particular pyramidal cells, display only weak locking to network population oscillations and further, that spike rates are often lower than the population gamma frequency. That is, single units regularly skip cycles. Consistently with this, the majority of units we recorded exhibited only weakly oscillatory spike trains. The selected neurons ( $n=5$ in monkey $S$ and $n=2$ in monkey K) however, fired spikes on many gamma cycles and thus exhibited a strong periodic component in their spike trains. We must emphasize that these neurons are not representative for the whole population of cortical cells but rather of that subclass of cells that exhibits strong gamma periodicity. Here we show that these single units exhibited the same frequency modulations and shifts in spectral asymmetry as a function of contrast as those observed in population signals (LFP and MEG).

\section{Gamma peak frequency as a robust parameter of the visual cortical network}

The observation of contrast-dependent gamma frequency modulations fits with studies linking gamma frequency with other visual features such as stimulus size (Gieselmann and Thiele, 2008; Ray and Maunsell, 2011), eccentricity (van Pelt and Fries, 2013), motion speed (Friedman-Hill et al., 2000; Swettenham et al., 2009), and motion direction (Feng et al., 2010). Gamma peak frequency, but not power, shows correlations with perceptual performance, cortical structure, and age, and has been shown to be highly heritable (Edden et al., 2009; Gaetz et al., 2012; Schwarzkopf et al., 2012; van Pelt et al., 2012). This emphasizes the importance of frequency measures in understanding human network function. Our results of strikingly similar contrast-induced modulation in gamma frequency in macaque LFP, single unit spike trains and human MEG (see regression slopes in figure 5) suggest that gamma frequency may be a spectral parameter that is robust across vastly different spatial scales (from single units to MEG) and thus provide a valuable tool in future investigations of human network function.

\section{Power modulation of gamma oscillations by stimulus contrast}

We observed luminance contrast-dependent gamma power modulation in the human MEG as well as in monkey LFP and single unit spike trains. In both human MEG and monkey LFP and spike trains, gamma power increased strongly in the lower-to-middle contrast ranges. However, we observed a substantial difference in the saturation of power at high contrasts. Whereas gamma power in the LFP and singleunit spike saturated (Figures 3,4), despite increasing spike rates (Figure 3), MEG gamma power increased approximately linearly with contrast (Figures 2, 4). The monotonic increase in induced gamma power with contrast in human MEG is in line with previous human MEG (Hall et al., 2005b; Perry et al., 2014) and EEG (Koch et al., 2009) studies. In addition, the finding of non-monotonic increase in gamma power in V1 LFP is in line with two other studies in awake macaques (Bosman et al., 2012 (Suppl. Fig.S5); Ray \& Maunsell, 2010), although mixed results are reported by studies in anaesthetized macaques (Henrie and Shapley, 2005b; report lack of saturation, whereas saturation is reported by Jia et al., 2013). Computational modeling of gamma-generating networks indicates that contrast-dependent modulation of gamma power with a non-monotonic component might arise by changing afferent E-drive to the excitatory-inhibitory spiking network (see figure 7B in Roberts et al., 2013), but the exact mechanisms underlying the relationship between power and contrast (or even excitatory input strength) are still not well established (Jadi and Sejnowski, 2014; Jia et al., 2013; Roberts et al., 2013). A possibly critical 
component for explaining non-monotonic power modulations is neural resonance (Moca et al., 2014), where there is enhancement of power at particular frequencies and not others. Optogenetic studies have provided experimental evidence for resonance in the gamma range (Cardin et al., 2009), with particular involvement of fast-spiking inhibitory neurons.

It is not clear what underlies the difference of the contrast - gamma power response function between MEG and macaque electrophysiological recordings. The observed difference in contrast response functions of power may be rooted in cross-species differences, in the different signals/recording techniques used or in the differences in experimental setups despite our efforts to minimize such differences. It could be the case that the differences observed are due to small differences in size and eccentricity of the stimuli. The most likely hypothesis in this vein is that a larger contribution of the transient pathway in the monkey, due to slightly more peripheral stimulus presentation than in the human participants, has led to greater saturation of gamma power with increased contrast in the monkeys. However, the typical saturation effect in the spiking rate seen for eccentric stimuli (Sclar et al., 1990) is not present in our data, which suggests that a much greater contribution of the transient system in the monkeys than in the humans is not a plausible explanation for the differences in contrastinduced power change seen between monkeys and humans. Hence, cross-species differences cannot be ruled out, although such conclusion would require an exclusion of the possibility that differences in the contrast-dependency of gamma power between monkeys and humans is due to the different manners in which LFP and MEG signals are generated. The LFP is comparatively local while the MEG signal is based on aggregation over space, causing it to be dominated by a much larger relative contribution of synchronous compared to asynchronous meso-sources (Nunez and Srinivasan, 2006). For instance, it is possible that increasing contrast may result in increasing longer range spatial synchronization, which may outweigh local saturation effects as picked up with LFP. The relevance of aggregation effects is supported by a recent electrophysiological study in monkeys from Mussal et al. (2014) in which spatial LFP synchrony across electrodes was shown to be a major determinant of simultaneously recorded EEG power. If this can be confirmed, it would indicate that LFP power and MEG power are complimentary signals.

\section{A comparison of the effect of contrast modulations on spike rates, LFP frequency and LFP power}

Luminance contrast, a central visual parameter, is a proxy for excitatory drive (E-drive) to primary visual cortex, increasing the spike rates of the majority of neurons in retino-geniculate pathway as well as early visual cortex (Contreras and Palmer, 2003; Sclar et al., 1990). Plausible biophysical mechanisms for Edrive dependent oscillation frequency shift have been described in a number of computational modeling studies of gamma generating networks (Brunel and Wang, 2003; Roberts et al., 2013; Traub et al., 1996). The theoretical models predict a close link between the precise gamma frequency and the overall excitation state of the underlying network. This view is supported by our finding of similar monotonic increases in gamma frequency and multi-unit spike rates as a function of stimulus contrast. Similar observations have been reported by other macaque V1 studies (Jia et al., 2013; Ray and Maunsell, 2010). In contrast to LFP gamma frequency, LFP gamma power saturated or even decreased at high 
contrasts despite monotonically increasing spike rates. This indicates that LFP gamma power was not tightly related to spiking in the majority of recorded neurons (Logothetis et al., 2001). The LFP predominantly reflects synaptic, rather than spiking events, particularly when these result in synchronous fluctuations of the membrane potentials across many neurons (Buzsáki et al., 2012). Thus, changes in synchronization between neurons can change the power of the LFP in the absence of changes in firing rate. Thus, one possibility for the observed dissociation is that while firing rates continue to go up with increased contrast (increased afferent excitatory drive), locally neurons become less synchronous resulting in lower LFP power.

\section{Contrast-induced modulations of the asymmetry of gamma spectral distributions}

We have observed in both human and macaque visual cortex and at all measurement scales (MEG, LFP and single-unit spikes) systematic asymmetries in the gamma spectral distribution (right/left skewness of the gamma power spectra), which were modulated by stimulus contrast. To our knowledge, this has not been reported so far. The shape of the gamma power distribution may reveal further properties of the underlying oscillatory process. Asymmetry may be a meaningful characteristic of the dynamical process underlying gamma; an idea that is supported by its systematical changes with stimulus contrast.

The asymmetry measure we used here was derived from baseline-normalized spectra. One concern is that because the baseline spectra are characterized by an $1 / f$ power scaling (Miller et al., 2009; Pritchard, 1992), the baseline-normalization could lead to asymmetry of the gamma power distribution artificially. Several observations contradict this possibility. First, the asymmetries observed in spike train data are similar to the ones observed in LFP/MEG data, yet, spike train power spectra in the baseline were not characterized by $1 / f$ characteristics. Second, similar effect ranges between MEG and LFP were observed, although the power ratio of induced power to baseline power was considerably different (gamma peak power in human MEG 1.5-2 and monkey LFP 5-10). We would expect that a lower power ratio would yield larger asymmetries; however, this was not observed. Further, the $1 / f$ characteristic of the spectra in the baseline cannot explain the reversal of asymmetry at higher contrasts. As to the underlying causes of the empirically-observed spectral asymmetries, these are still unknown. The moment-to-moment variation in gamma oscillation power and frequency has been reported as being highly complex (Burns et al., 2011, 2010) and shown to be modulated by multiple other rhythmic neuronal processes (Bosman et al., 2009; Jensen and Colgin, 2007; Lakatos et al., 2005; Osipova et al., 2008; Schroeder and Lakatos, 2009). Slow microsaccadic rhythms that modulate the gamma oscillations may also contribute to such asymmetries. A further possible factor for explaining power asymmetries may be neural resonance (Moca et al., 2014), where particular frequencies lead to enhancement of power. The understanding of the dynamical properties of gamma oscillations in vivo, their appropriate quantification as well as their neurocomputational significance are still far from being fully elucidated (Burns et al., 2011, 2010; Cohen, 2014; Lakatos et al., 2005; Roberts et al., 2013; Schroeder and Lakatos, 2009). Future detailed modeling work will need to investigate possible mechanisms behind gamma power asymmetries. 


\section{Measurement sensitivity of gamma oscillations with non-invasive MEG in humans}

A prominent difference between human MEG measured gamma power and gamma power in macaque V1 measured microelectrodes is the large difference in overall induced power indicating low measurement sensitivity (Hadjipapas et al., 2007; Muthukumaraswamy et al., 2010; Swettenham et al., 2009). This was true despite the fact that stimulus parameters were optimized for the MEG experiment. The low gamma SNR becomes exacerbated for small cortical stimulus representations, i.e. when eccentricity is high (Swettenham et al., 2009; van Pelt and Fries, 2013) or stimulus size small (Gieselmann and Thiele, 2008; Perry et al., 2013). Hence, to increase SNR in the MEG experiment the stimuli were placed at a smaller eccentricity and presented in a square aperture to restrict their presentation to one hemifield. Additionally, the contrast manipulation was shifted to higher contrast values. Furthermore, we used a beamforming technique to improve the localization and SNR of gamma oscillations. Still, we did not observe significant MEG gamma power at the lowest contrast used (20\% contrast), yet we observed substantial induced power in the gamma range as low as $6 \%$ contrast in both monkeys. Reports in rhesus macaque studies have indicated that gamma oscillations can even be detected in conditions of no visual stimulation (Spaak et al., 2012; Vinck et al., 2013).

A critical point to consider is the different scale of measurement involved in the MEG compared to microelectrodes. Even when the MEG signal stems from a well-reconstructed source, it still reflects aggregated neural activity of orders of magnitudes larger than the local field potential and spiking activity measured by microelectrodes. The MEG signal is therefore very sensitive to the neural synchrony across larger cortical distances. However, the synchrony of gamma oscillation has been shown to be spatially limited (Eckhorn et al., 2004). Hence, the more local spatial scale of gamma-band synchronization makes MEG/EEG recordings less sensitive to gamma oscillations.

Another important point to consider is the cellular/cortical origin of the MEG signal. Due to their dominant somatodendritic axis, pyramidal neurons collectively give rise to current summation and resulting fields that can be measured at a distance from their source (so-called 'open-field' configurations (Buzsáki et al., 2012; Murakami and Okada, 2006)). The MEG signal is thought to predominantly reflect currents running along the somatodendritic axis that arise from opposing charges at the apical dendrites and the soma of pyramidal neurons (Hämäläinen et al., 1993). Quantitative studies taking into account neuronal and dendritic geometry indicate that layer $\mathrm{V}$ neurons make a larger contribution than layer II-III neurons (Murakami and Okada, 2006). This is likely because superficial layer pyramidal neurons are smaller and have much shorter dendrites, a smaller separation between soma and dendrites and therefore a less pronounced open field configuration, and a lesser dipole moment (Jones et al., 2007). Several macaque studies have indicated that the strongest source of gamma oscillations are observed in the superficial and middle cortical layers (Buffalo et al., 2011; Roberts et al., 2013; Xing et al., 2012). Hence, assuming a layer dependency in the human that is similar to that in the macaque, the contribution of superficial layer gamma to the MEG signal would be small compared to that from the layer $V$ neurons (Jones et al., 2007). 


\section{Conclusions}

Our results in primary visual cortex show that contrast-induced modulations in gamma frequency and gamma spectral asymmetry in human MEG matched the results from macaque microelectrode recordings, including results from well-isolated single unit recordings. However, there were discrepancies in gamma power. The latter is more complex to understand not in the least because gamma power in the MEG depends on longer-range spatial synchrony. Thus, while oscillation frequency was found to be more stable across spatial scales (figure 5) and to convey similar information about the stimulus, LFP and MEG power seemed to convey complementary information. Our results and theoretical considerations on spatial aggregation lead us to the conclusion that in spite of many similar findings between human MEG and monkey LFP/spike data, there are also differences and one needs to be prudent in translating macaque microelectrode findings to human MEG, in particular for oscillatory power. To fully understand the similarities and differences in findings from invasive recordings in monkeys and non-invasive measurements in humans, and in the underlying cortical mechanisms, future work will need to involve detailed modeling studies taking into account contributions of functional and structural properties of single neurons, of different layers, of within- and across-layer connectivity, as well as effects of overall network size and stimulus drive.

\section{References}

Adjamian P, Holliday IE, Barnes GR, Hillebrand A, Hadjipapas A, Singh KD (2004): Induced visual illusions and gamma oscillations in human primary visual cortex. Eur J Neurosci 20:587-92.

Barardi A, Sancristóbal B, Garcia-Ojalvo J (2014): Phase-Coherence Transitions and Communication in the Gamma Range between Delay-Coupled Neuronal Populations. Ed. Christopher J. Honey. PLoS Comput Biol 10:e1003723. http://dx.plos.org/10.1371/journal.pcbi.1003723.

Berens P, Keliris GA, Ecker AS, Debakey ME, Affairs V (2008): Feature selectivity of the gamma-band of the local fi eld potential in primate primary visual cortex. Neuroscience 2:199-207.

Börgers C, Kopell N (2005): Effects of noisy drive on rhythms in networks of excitatory and inhibitory neurons. Neural Comput 17:557-608.

Bosman CA, Schoffelen J-M, Brunet N, Oostenveld R, Bastos AM, Womelsdorf T, Rubehn B, Stieglitz T, De Weerd $P$, Fries $P$ (2012a): Attentional stimulus selection through selective synchronization between monkey visual areas. Neuron 75:875-888. http://dx.doi.org/10.1016/j.neuron.2012.06.037.

Bosman CA, Womelsdorf T, Desimone R, Fries P (2009): A microsaccadic rhythm modulates gamma-band synchronization and behavior. J Neurosci 29:9471-9480. http://dx.doi.org/10.1523/JNEUROSCl.1193-09.2009.

Bosman CA, Schoffelen J-M, Brunet N, Oostenveld R, Bastos AM, Womelsdorf T, Rubehn B, Stieglitz T, De Weerd P, Fries P (2012b): Attentional Stimulus Selection through Selective Synchronization between Monkey Visual Areas. Neuron.

Brainard DH (1997): The Psychophysics Toolbox. Spatial Vision. Brill.

Brookes MJ, Vrba J, Mullinger KJ, Geirsd\{'o\}ttir G (2009a): Source localisation in concurrent EEG/fMRI: applications at 7T. Neuroimage 45:440-452.

Brookes MJ, Vrba J, Mullinger KJ, Geirsdóttir GB, Yan WX, Stevenson CM, Bowtell R, Morris PG (2009b): Source localisation in concurrent EEG/fMRI: applications at 7T. Neuroimage 45:440-52. 
Brunel N, Wang X-J (2003): What determines the frequency of fast network oscillations with irregular neural discharges? I. Synaptic dynamics and excitation-inhibition balance. J Neurophysiol 90:41530. http://jn.physiology.org/content/90/1/415.

Brunet N, Bosman C a, Roberts M, Oostenveld R, Womelsdorf T, De Weerd P, Fries P (2013): Visual Cortical Gamma-Band Activity During Free Viewing of Natural Images. Cereb Cortex:1-9. http://www.ncbi.nlm.nih.gov/pubmed/24108806.

Brunet NM, Bosman CA, Vinck M, Roberts M, Oostenveld R, Desimone R, De Weerd P, Fries P (2014): Stimulus repetition modulates gamma-band synchronization in primate visual cortex. Proc Natl Acad Sci U S A 111:3626-31.

Buffalo EA, Fries P, Landman R, Buschman TJ, Desimone R (2011): Laminar differences in gamma and alpha coherence in the ventral stream. Proc Natl Acad Sci U S A 108:11262-7. http://www.pnas.org/content/108/27/11262.short.

Buia C, Tiesinga $\mathrm{P}$ (2006): Attentional modulation of firing rate and synchrony in a model cortical network. J Comput Neurosci 20:247-64. http://www.ncbi.nlm.nih.gov/pubmed/16683206.

Burns SP, Xing D, Shapley RM (2011a): Is gamma-band activity in the local field potential of V1 cortex a "clock" or filtered noise? J Neurosci 31:9658-64.

Burns SP, Xing D, Shapley RM (2011b): Is gamma-band activity in the local field potential of V1 cortex a "clock" or filtered noise? J Neurosci 31:9658-9664. http://dx.doi.org/10.1523/JNEUROSCI.066011.2011.

Burns SP, Xing D, Shelley MJ, Shapley RM (2010): Searching for autocoherence in the cortical network with a time-frequency analysis of the local field potential. J Neurosci 30:4033-4047. http://dx.doi.org/10.1523/JNEUROSCI.5319-09.2010.

Buzsáki G (2006): Rhythms of the Brain. Oxford University Press, USA.

Buzsáki G, Anastassiou CA, Koch C (2012): The origin of extracellular fields and currents--EEG, ECoG, LFP and spikes. Nat Rev Neurosci 13:407-20. http://dx.doi.org/10.1038/nrn3241.

Buzsáki G, Draguhn A (2004): Neuronal oscillations in cortical networks. Science 304:1926-9.

Buzsáki G, Wang X-J (2012): Mechanisms of gamma oscillations. Annu Rev Neurosci 35:203-25.

Cardin JA, Carlén M, Meletis K, Knoblich U, Zhang F, Deisseroth K, Tsai L-H, Moore Cl (2009): Driving fastspiking cells induces gamma rhythm and controls sensory responses. Nature 459:663-667.

Cohen MX (2014): Fluctuations in oscillation frequency control spike timing and coordinate neural networks. J Neurosci 34:8988-98.

Colgin LL, Denninger T, Fyhn M, Hafting T, Bonnevie T, Jensen O, Moser M-B, Moser El (2009): Frequency of gamma oscillations routes flow of information in the hippocampus. Nature 462:353-7. http://dx.doi.org/10.1038/nature08573.

Contreras D, Palmer L (2003): Response to Contrast of Electrophysiologically Defined Cell Classes in Primary Visual Cortex. J Neurosci 23:6936-6945. http://www.jneurosci.org/content/23/17/6936.short.

Csicsvari J, Hirase H, Czurkó a, Mamiya a, Buzsáki G (1999): Oscillatory coupling of hippocampal pyramidal cells and interneurons in the behaving Rat. J Neurosci 19:274-87.

Doane DP, Seward LE (2011): Measuring Skewness : A Forgotten Statistic? J Stat Educ 19:1-18.

Duncan KK, Hadjipapas A, Li S, Kourtzi Z, Bagshaw A, Barnes G (2010): Identifying spatially overlapping local cortical networks with MEG. Hum Brain Mapp 31:1003-16.

Edden RAE, Muthukumaraswamy SD, Freeman TCA, Singh KD (2009): Orientation discrimination performance is predicted by GABA concentration and gamma oscillation frequency in human primary visual cortex. J Neurosci 29:15721-6.

Engel AK, Fries P, König P, Brecht M, Singer W (1999): Temporal binding, binocular rivalry, and consciousness. Conscious Cogn 8:128-51.

http://www.sciencedirect.com/science/article/pii/S1053810099903896. 
Feng W, Havenith MN, Wang P, Singer W, Nikolić D (2010): Frequencies of gamma/beta oscillations are stably tuned to stimulus properties. Neuroreport 21:680-4.

Friedman-Hill S, Maldonado PE, Gray CM (2000): Dynamics of striate cortical activity in the alert macaque: I. Incidence and stimulus-dependence of gamma-band neuronal oscillations. Cereb Cortex 10:1105-16.

Frien a, Eckhorn R, Bauer R, Woelbern T, Gabriel a (2000): Fast oscillations display sharper orientation tuning than slower components of the same recordings in striate cortex of the awake monkey. Eur J Neurosci 12:1453-65.

Fries $\mathrm{P}$ (2005): A mechanism for cognitive dynamics: neuronal communication through neuronal coherence. Trends Cogn Sci 9:474-80. http://www.sciencedirect.com/science/article/pii/S1364661305002421.

Fries $P$ (2009): Neuronal gamma-band synchronization as a fundamental process in cortical computation. Annu Rev Neurosci 32:209-24.

Fries P, Nikoli? D, Singer W (2007): The gamma cycle. Trends Neurosci 30:309-316. http://dx.doi.org/10.1016/j.tins.2007.05.005.

Gaetz W, Roberts TPL, Singh KD, Muthukumaraswamy SD (2012): Functional and structural correlates of the aging brain: relating visual cortex (V1) gamma band responses to age-related structural change. Hum Brain Mapp 33:2035-46.

Gieselmann $\mathrm{M}$ a, Thiele a (2008): Comparison of spatial integration and surround suppression characteristics in spiking activity and the local field potential in macaque V1. Eur J Neurosci 28:447-59.

Gray CM, Singer W (1989): Stimulus-specific neuronal oscillations in orientation columns of cat visual cortex. Proc Natl Acad Sci U S A 86:1698-702.

Gregoriou GG, Gotts SJ, Zhou H, Desimone R (2009): High-frequency, long-range coupling between prefrontal and visual cortex during attention. Science 324:1207-1210.

Hadjipapas A, Adjamian P, Swettenham JB, Holliday IE, Barnes GR (2007): Stimuli of varying spatial scale induce gamma activity with distinct temporal characteristics in human visual cortex. Neuroimage 35:518-30.

Hall SD, Holliday IE, Hillebrand A, Singh KD, Furlong PL, Hadjipapas A, Barnes GR (2005a): The missing link: analogous human and primate cortical gamma oscillations. Neuroimage 26:13-7.

Hall SD, Holliday IE, Hillebrand A, Singh KD, Furlong PL, Hadjipapas A, Barnes GR (2005b): The missing link: analogous human and primate cortical gamma oscillations. Neuroimage 26:13-17. http://dx.doi.org/10.1016/j.neuroimage.2005.01.009.

Hämäläinen M, Hari R, Ilmoniemi RJ, Knuutila J, Lounasmaa O V. (1993): Magnetoencephalographytheory, instrumentation, and applications to noninvasive studies of the working human brain. Rev Mod Phys 65:413-497.

Hamilton DGA and DB (1982): Striate cortex of monkey and cat: contrast response function. J Neurophysiol 48.

Henrie JA, Shapley R (2005a): LFP power spectra in V1 cortex: the graded effect of stimulus contrast. J Neurophysiol 94:479-90.

Henrie JA, Shapley R (2005b): LFP power spectra in V1 cortex: the graded effect of stimulus contrast. J Neurophysiol 94:479-490. http://dx.doi.org/10.1152/jn.00919.2004.

Hoogenboom N, Schoffelen J-M, Oostenveld R, Parkes LM, Fries P (2006): Localizing human visual gamma-band activity in frequency, time and space. Neuroimage 29:764-73. http://www.sciencedirect.com/science/article/pii/S1053811905006361.

Jadi MP, Sejnowski TJ (2014): Cortical oscillations arise from contextual interactions that regulate sparse coding. Proc Natl Acad Sci U S A 111:6780-5. http://www.ncbi.nlm.nih.gov/pubmed/24742427. 
Jensen O, Colgin LL (2007): Cross-frequency coupling between neuronal oscillations. Trends Cogn Sci 11:267-9. http://www.sciencedirect.com/science/article/pii/S1364661307001271.

Jia X, Xing D, Kohn A (2013): No consistent relationship between gamma power and peak frequency in macaque primary visual cortex. J Neurosci 33:17-25. http://dx.doi.org/10.1523/JNEUROSCI.168712.2013.

Jones SR, Pritchett DL, Stufflebeam SM, Hämäläinen M, Moore Cl (2007): Neural correlates of tactile detection: a combined magnetoencephalography and biophysically based computational modeling study. J Neurosci 27:10751-64.

Kleiner M, Brainard D, Pelli D, Ingling A, Murray R, Broussard C (2007): What's new in Psychtoolbox-3. Perception 36:1.

Koch SP, Werner P, Steinbrink J, Fries P, Obrig H (2009): Stimulus-induced and state-dependent sustained gamma activity is tightly coupled to the hemodynamic response in humans. J Neurosci 29:13962-13970.

Koelewijn L, Dumont JR, Muthukumaraswamy SD, Rich AN, Singh KD (2010): Orientation selectivity in primary visual cortex using MEG: an inverse oblique effect? J Vis 10:928-928.

Lakatos P, Shah AS, Knuth KH, Ulbert I, Karmos G, Schroeder CE (2005): An oscillatory hierarchy controlling neuronal excitability and stimulus processing in the auditory cortex. J Neurophysiol 94:1904-1911. http://dx.doi.org/10.1152/jn.00263.2005.

Logothetis NK, Pauls J, Augath M, Trinath T, Oeltermann A (2001): Neurophysiological investigation of the basis of the fMRI signal. Nature 412:150-7.

Miller KJ, Sorensen LB, Ojemann JG, Den Nijs M (2009): Power-law scaling in the brain surface electric potential. PLoS Comput Biol 5.

Moca V V., Nikolić D, Singer W, Mureşan RC (2014): Membrane resonance enables stable and robust gamma oscillations. Cereb Cortex 24:119-142.

Murakami S, Okada Y (2006): Contributions of principal neocortical neurons to magnetoencephalography and electroencephalography signals. J Physiol 575:925-36.

Musall S, von Pföstl V, Rauch A, Logothetis NK, Whittingstall K (2014): Effects of neural synchrony on surface EEG. Cereb Cortex 24:1045-53.

Muthukumaraswamy SD, Edden R a E, Jones DK, Swettenham JB, Singh KD (2009): Resting GABA concentration predicts peak gamma frequency and $\mathrm{FMRI}$ amplitude in response to visual stimulation in humans. Proc Natl Acad Sci U S A 106:8356-61.

Muthukumaraswamy SD, Singh KD (2008): Spatiotemporal frequency tuning of BOLD and gamma band MEG responses compared in primary visual cortex. Neuroimage 40:1552-1560.

Muthukumaraswamy SD, Singh KD, Swettenham JB, Jones DK (2010): Visual gamma oscillations and evoked responses: variability, repeatability and structural MRI correlates. Neuroimage 49:3349-57.

Nunez PL, Srinivasan R (2006): Electric Fields of the Brain. Oxford University Press.

Oostenveld R, Fries P, Maris E, Schoffelen J-M (2011): FieldTrip: Open source software for advanced analysis of MEG, EEG, and invasive electrophysiological data. Comput Intell Neurosci 2011:156869.

Osipova D, Hermes D, Jensen O (2008): Gamma power is phase-locked to posterior alpha activity. PLoS One 3:e3990.

Pelli DG (1997): The VideoToolbox software for visual psychophysics: transforming numbers into movies. Spatial Vision. Brill.

Van Pelt S, Boomsma DI, Fries P (2012): Magnetoencephalography in twins reveals a strong genetic determination of the peak frequency of visually induced $y$-band synchronization. J Neurosci 32:3388-92.

Van Pelt S, Fries P (2013): Visual stimulus eccentricity affects human gamma peak frequency. Neuroimage 78C:439-447. 
Pikovsky A (2003): Synchronization: A Universal Concept in Nonlinear Sciences. Cambridge University Press. Vol. 45.

Pritchard WS (1992): The brain in fractal time: 1/f-like power spectrum scaling of the human electroencephalogram. Int J Neurosci 66:119-129.

Quiroga RQ, Nadasdy Z, Ben-Shaul Y (2004): Unsupervised spike detection and sorting with wavelets and superparamagnetic clustering. Neural Comput 16:1661-87.

Ray S, Maunsell JHR (2010a): Differences in gamma frequencies across visual cortex restrict their possible use in computation. Neuron 67:885-96.

Ray S, Maunsell JHR (2010b): Differences in gamma frequencies across visual cortex restrict their possible use in computation. Neuron 67:885-896. http://dx.doi.org/10.1016/j.neuron.2010.08.004.

Ray S, Maunsell JHR (2011): Different origins of gamma rhythm and high-gamma activity in macaque visual cortex. PLoS Biol 9:e1000610.

Roberts MJ, Lowet E, Brunet NM, Ter Wal M, Tiesinga P, Fries P, De Weerd P (2013): Robust gamma coherence between macaque V1 and V2 by dynamic frequency matching. Neuron 78:523-36. http://www.ncbi.nlm.nih.gov/pubmed/23664617.

Robinson S, Vrba J (1999): Functional neuroimaging by synthetic aperture magnetometry (SAM). Recent ...:2-5.

Rols G, Tallon-Baudry C, Girard P, Bertrand O, Bullier J (2001): Cortical mapping of gamma oscillations in areas V1 and V4 of the macaque monkey. Vis Neurosci 18:527-40.

Schroeder CE, Lakatos P (2009): The gamma oscillation: master or slave? Brain Topogr 22:24-26.

Schwarzkopf DS, Robertson DJ, Song C, Barnes GR, Rees G (2012): The frequency of visually induced $\gamma^{-}$ band oscillations depends on the size of early human visual cortex. J Neurosci 32:1507-12.

Sclar G, Maunsell JH, Lennie P (1990): Coding of image contrast in central visual pathways of the macaque monkey. Vision Res 30:1-10.

Spaak E, Bonnefond M, Maier A, Leopold D a, Jensen O (2012): Layer-specific entrainment of gammaband neural activity by the alpha rhythm in monkey visual cortex. Curr Biol 22:2313-8. http://www.ncbi.nlm.nih.gov/pubmed/23159599.

Swettenham JB, Muthukumaraswamy SD, Singh KD (2009): Spectral properties of induced and evoked gamma oscillations in human early visual cortex to moving and stationary stimuli. J Neurophysiol 102:1241-1253.

Tiesinga PH, Sejnowski TJ (2010): Mechanisms for Phase Shifting in Cortical Networks and their Role in Communication through Coherence. Front Hum Neurosci 4:196. http://dx.doi.org/10.3389/fnhum.2010.00196.

Tiesinga P, Sejnowski TJ (2009): Cortical enlightenment: are attentional gamma oscillations driven by ING or PING? Neuron 63:727-32.

Traub RD, Whittington M a, Colling SB, Buzsáki G, Jefferys JG (1996): Analysis of gamma rhythms in the rat hippocampus in vitro and in vivo. J Physiol 493 ( Pt 2:471-84.

Vinck M, Womelsdorf T, Buffalo EA, Desimone R, Fries P (2013): Attentional Modulation of Cell-ClassSpecific Gamma-Band Synchronization in Awake Monkey Area V4. Neuron 80:1077-1089.

Vrba J, Robinson SE (2001): Signal processing in magnetoencephalography. Methods 25:249-71.

Xing D, Yeh C-I, Burns S, Shapley RM (2012): Laminar analysis of visually evoked activity in the primary visual cortex. Proc Natl Acad Sci U S A 109:13871-6. http://www.pnas.org/content/109/34/13871.short. 


\section{Chapter 4}

\section{Areas V1 and V2 show microsaccade-related 3-4 Hz covariation in gamma power and frequency}




\begin{abstract}
Neuronal gamma-band synchronization $(25-80 \mathrm{~Hz})$ in visual cortex appears sustained and stable during prolonged visual stimulation when investigated with conventional averages across trials. Yet, recent studies in macaque visual cortex have used single-trial analyses to show that both power and frequency of gamma oscillations exhibit substantial moment-by-moment variation. This has raised the question whether these apparently random variations might limit the functional role of gamma-band synchronization for neural processing. Here, we studied the moment-by-moment variation of gamma oscillation power and frequency, as well as inter-areal gamma synchronization by simultaneously recording local field potentials in V1 and V2 of two macaque monkeys. Our analyses demonstrate that gamma-band synchronization is not stationary and sustained but undergoes moment-by-moment variations in power and frequency. Those variations are not random and thereby a possible obstacle to neural communication, but they are highly structured, shared between areas, and shaped by a microsaccade-related $3-4 \mathrm{~Hz}$ theta rhythm. Our findings provide experimental support for the suggestion that cross-frequency coupling might structure and facilitate the information flow between brain regions.
\end{abstract}




\section{Introduction}

Visually induced neuronal responses are commonly assessed by averaging trials aligned to the onset of the visual stimulus (stimulus-triggered averaging). Likewise, stimulus-triggered averaging is a standard approach to study gamma-band oscillations $(25-80 \mathrm{~Hz})$. Stimulus-triggered time-frequency representations (TFRs) typically show strong, 'transient' modulation of gamma, which is described as 'stimulus-evoked', followed by 'sustained' gamma (Hoogenboom et al., 2006; Swettenham et al., 2009), which is often the target of experimental manipulations and analysis. However, recent V1 recordings indicate that single-trial gamma oscillations in the so-called 'sustained' period preserve neither frequency nor amplitude over time, but seem to have random, 'burst'-like characteristics (Burns et al., 2010, 2011; Ray and Maunsell, 2010; Roberts et al., 2013). These findings refute the view of a stationary oscillation as could be suggested by trial-averaged data. Moreover, the apparent randomness of gamma may impede its contribution to neural computation (Burns et al., 2011). Gamma randomness may also impede neural communication (Ray and Maunsell, 2010), possibly in part by preventing the frequency matching among neural populations necessary for communication (Roberts et al., 2013). However, it is challenging to distinguish noise from complexity in experimental data. Hence, it is crucial to investigate whether fluctuations of gamma frequency and power are structured, regulated and exploited by other brain processes.

In a number of brain areas, it has been shown that gamma variation depends on slower rhythmic fluctuations that include delta, theta and alpha/beta frequencies (Lakatos et al., 2005; Jensen and Colgin, 2007; Osipova et al., 2008; Schroeder and Lakatos, 2009; Canolty and Knight, 2010; Jutras et al., 2013) and that these slower rhythms can affect gamma synchronization among brain areas (Colgin et al., 2009; Bosman et al., 2012; Schomburg et al., 2014). The dependence of a fast rhythm on a slower rhythm is often referred to as cross-frequency coupling (CFC). A particular type of CFC represents the linkage between gamma oscillations and (often rhythmic) movements of sensory organs. For example, in the visual system, gamma modulations have been linked to saccadic eye movements (Rajkai et al., 2008; Bosman et al., 2009; Ito et al., 2011; Brunet et al., 2013). CFC has been studied mainly in terms of phaseto-power interactions. However, phase-to-frequency interactions, where the precise frequency of gamma depends on a slower oscillation phase have been rarely discussed despite the fact that frequency is a critical factor for enabling synchronization (Pikovsky et al., 2002).

We therefore studied in more detail whether moment-by-moment variation in gamma frequency is temporally structured by slower rhythms in macaque V1 and V2, and whether it affects gamma synchronization between these cortical areas. To this aim, we analyzed simultaneous microelectrode recordings of local field potentials (LFPs) from V1 and V2 sites with (near-) overlapping receptive fields (RFs) from two macaque monkeys and from an additional monkey with an EcoG grid covering V1. 


\section{Materials and Methods}

Surgical procedure and electrophysiological methods for monkeys $\mathrm{S}$ and $\mathrm{K}$ have been described in detail in Roberts et al. (2013), and for monkey A in (Bosman et al., 2012; Brunet et al., 2013). All three monkeys were adult, male Maccaca Mulatta $(7-10 \mathrm{~kg})$. In monkeys $\mathrm{S}$ and $\mathrm{K}$, recordings were done with a depth probe in V1 and one in V2, separated by a distance of 4-6mm. Recording sites were assigned to V1 or V2 using conventional retinotopic mapping relative to the vertical meridian representation (Gattass et al., 1981); details in Suppl.Mat. (Roberts et al., 2013). Based on this mapping procedure, we also chose the probe positions such that RFs in V1 and V2 were overlapping or near-overlapping (Roberts et al., 2013), as retinotopic projections between V1 and V2 (Lund, 2003) predict that this enhances the possibility of finding V1-V2 coherence (Nowak et al., 1999; Bosman et al., 2012). Note that we did not greatly vary the distance between probes (RFs) and therefore in our own data did not confirm the decrease in coherence for increasing probe distances (Suppl.Mat. (Roberts et al., 2013)). Note furthermore that for the purposes of the present analysis, we averaged across all cortical layers. In monkey A, recordings were obtained by means of an EcoG grid (Rubehn et al., 2009) covering most of the right hemisphere (Bosman et al., 2012; Brunet et al., 2013). V1 recordings were analyzed from the bipolar electrode pair that yielded the strongest gamma oscillatory response compared to baseline to allow for robust instantaneous frequency and power estimations. In this monkey, we could not find electrodes with strong gamma signal that could be assigned to V2 with sufficient confidence, which may be due to the limited exposure of V2 at the surface just posterior from the lunate sulcus (Gattass et al., 1981 ) in this monkey.

The depth probe V1/V2 data for the theta-triggered gamma oscillation were acquired from recording chambers implanted above the left hemisphere in monkeys $S$ and $K$. Depth probe data for microsaccadetriggered analysis in monkey $\mathrm{S}$ were acquired from a V1/V2 chamber above the right hemisphere after

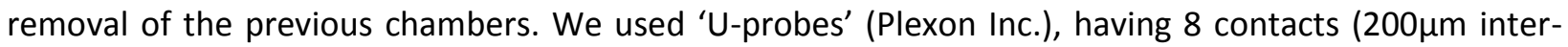
contact spacing) or 16 contacts $(150 \mu \mathrm{m}$ inter-contact spacing). LFPs were filtered $(0.7-300 \mathrm{~Hz})$ and recorded at $1 \mathrm{KHz}$ (Plexon MAP system). In monkey A, an 252-electrode EcoG grid (Rubehn et al., 2009) was used covering a large part of the right hemisphere including V1 (Bosman et al., 2012). Signals were amplified by a factor 20 using eight Plexon headstage amplifiers (Plexon, USA). The signals were then low-pass filtered at $8 \mathrm{kHz}$, followed by digitization at $32 \mathrm{kHz}$ by a Neuralynx Digital Lynx system (Neuralynx, USA). LFP signals were lowpass filtered (Bosman et al., 2012) at $200 \mathrm{~Hz}$ and downsampled to $1 \mathrm{kHz}$. In all three monkeys, recordings were done while gratings were presented and while the monkeys directed their gaze to a fixation point. Trials were aborted after fixation errors. The data for monkeys $S$ and $\mathrm{K}$ used for theta-triggered analysis of gamma were obtained with stationary square wave gratings (2cpd, $3-5^{\circ}$ diameter), shown on an isoluminant gray background. Stimuli were presented at 8 different luminance contrasts $(2 \%, 3.5 \%, 6 \%, 9.7 \%, 16.3 \%, 35.9 \%, 50.3 \%, 72 \%)$. We present the analysis for a middle contrast (35.9\%) which gave strong gamma responses in both monkeys. We obtained comparable results at other contrasts. The behavioral task was to hold fixation on a fixation spot in the middle of the computer screen during stimulus presentation $(1.500-4.500 \mathrm{~ms}$ only trials with $>1800 \mathrm{~ms}$ were included). The data represent baseline data for an ongoing perceptual learning project. 
The data for microsaccade-triggered analysis from Monkey $\mathrm{S}$ was also acquired during a different phase of the perceptual learning experiment. Here, the monkey had to report a color change at fixation with an upward eye movement to a target. Stimulus gratings of different contrasts $(73.6 \%, 54.5 \%, 37.4 \%$, $26.7 \%, 18.7 \%, 12.7 \%, 8.5 \%, 7.3 \%, 6.1 \%, 4.9 \%$ ) were shown (with $>1.5$ s duration). For the present study, we chose the data from the $37.4 \%$ contrast grating because it induced the strongest gamma response, thus facilitating an investigation of its relation with slower rhythms and microsaccades. For monkey $A$, LFP data were acquired in which the monkey had to fixate while a whole-field square-wave grating (63\% contrast) was shown (1sec fixation and $2 \mathrm{sec}$ fixation with stimulus). For clarity, the present study in monkey A was conducted after the study reported in (Brunet et al., 2013), and hence stimuli and task used in the present study differ from those in (Brunet et al., 2013).

For analysis, we used the FieldTrip MATLAB toolbox (Oostenveld et al., 2011) for spectral and coherence analysis. For theta-triggered (Fig. 2) and microsaccade-triggered data (Fig.3-4), we applied wavelet transforms (complex Morlet) to compute time-frequency representations (TFR). LFPs are characterized by $1 / f$ power scaling (Miller et al., 2009). We derived the scaling factor in our data from the slope of the line fitted onto the log-log power spectra. We then used the factor to correct the TFRs for the $1 / f$ scaling. To calculate the moment-to-moment (instantaneous) variation in gamma frequency, we estimated the frequency, for a given time point, with the highest power within the gamma frequency range $[25-60 \mathrm{~Hz}]$ of the wavelet TFRs (corrected for $1 / \mathrm{f}$ ). We obtained similar results, yet not as robust, by using filtering and applying the Hilbert transform (Le Van Quyen et al., 2001).

The locking between V1 and V2 gamma oscillations was estimated by spectral coherence applied to the wavelet transform (Fig.1E, Fig.2E). Microsaccade-field coherence (Fig.2C and Fig3C) is a measure of locking between microsaccades and the local field potential in the frequency domain. It was calculated by averaging the Fourier-transformed LFP segments aligned to microsaccades and normalizing by the average power in those LFP segments.

For the theta-triggered analysis, we filtered the LFP signal (two-pass Butterworth filter) around the maximum theta peak $( \pm 0.5 \mathrm{~Hz})$ observed in the power spectra. For each probe, we used the contact with the strongest theta power peak as the theta reference for all other contacts. The filtered signal was Hilbert transformed and the moment-by-moment phase derived. Around zero-phase, we detected the maximum amplitude peak of the theta wave around which we triggered time-windows $[ \pm 0.25 \mathrm{~s}]$. Only data above the lower $25^{\text {th }}$ percentile of the amplitude distribution were included in further analysis as phase estimates for data with low amplitude were unreliable.

We used two eye tracking systems in our experiments. In all three monkeys and in all recording sessions, fixation behavior was monitored using a low-resolution eye tracker directed at one eye (Arrington, $60 \mathrm{~Hz}$ ). In addition, in a subset of sessions, we additionally used a higher resolution eye tracking system (Thomas Recording, $240 \mathrm{~Hz}$ ) to measure microsaccades in one eye. This equipment was acquired after recording in monkey $\mathrm{K}$ had stopped, therefore Thomas eye tracking was done only in monkeys $\mathrm{S}$ and $\mathrm{A}$. For the microsaccade-triggered analysis in monkeys $S$ and $A$, we smoothed horizontal and vertical eye signals (rectangular window of $\pm 5 \mathrm{~ms}$ ) and differentiated the signals over time points separated by $10 \mathrm{~ms}$ to obtain robust eye speed signals. Horizontal and vertical eye speeds were summed. As a threshold for 
microsaccade detection, we used $4 * 20^{\text {th }}$ percentile of the eye speed distribution. Time windows were trigged to the microsaccade onset [0-0.3s]. We assessed the statistical significance of theta- or microsaccade-triggered modulations by fitting a cosine function to each triggered window, where the phase (positive) amplitude and offset were free parameters (EzyFit Toolbox, F.Moisy, http://www.fast.upsud.fr/ezyfit/). To assess whether the fitted parameter distribution of interest, here the phase parameter, was different from noise, we estimated a distribution from randomly-triggered data (with the same probability of windows per trial). The difference between these two distributions was tested with a Wilcoxon signed rank test on population data. In addition, we also applied a linear-circular correlation approach for frequency and power variation (Berens, 2009). The amount of data included in the analyses is documented in Table 1.

\begin{tabular}{|c|c|c|}
\hline & $\begin{array}{l}\text { Theta-triggered analysis } \\
\text { Figure } 1\end{array}$ & $\begin{array}{l}\text { Microsaccade-triggered analysis } \\
\text { Figure } 2,3 \text { and } 4\end{array}$ \\
\hline $\begin{array}{l}\text { Monkey S } \\
\text { (V1/V2) }\end{array}$ & $\begin{array}{l}10 \text { sessions } X 2 \text { areas } X 8 \text { contacts } X \\
53 \text { trials (mean) } X 1.8 \text { seconds } \\
\text { Left hemisphere }\end{array}$ & $\begin{array}{l}12 \text { sessions } X 2 \text { areas } X 16 \text { contacts } \times 42 \text { trials } \\
\text { (mean) } \times 1.5 \text { seconds } \\
\text { Right hemisphere }\end{array}$ \\
\hline $\begin{array}{l}\text { Monkey K } \\
\text { (V1/V2) }\end{array}$ & $\begin{array}{l}11 \text { session } X 2 \text { area } X 8 \text { contacts } X \\
43 \text { trials (mean) } \times 1.8 \text { seconds } \\
\text { Left hemisphere }\end{array}$ & $\begin{array}{l}\text { Not recorded because monkey no longer } \\
\text { available }\end{array}$ \\
\hline $\begin{array}{l}\text { Monkey A } \\
\text { (V1) }\end{array}$ & & $\begin{array}{l}10 \text { sessions } \times 1 \text { bipolar contact (highest induced } \\
\text { gamma power) } \times(34.5 \text { trials (mean) } \times 1.8 \mathrm{sec} \\
\text { Left hemisphere }\end{array}$ \\
\hline
\end{tabular}

Table.1 Observations comprised in population data per monkey/area (rows) and per type of analysis (columns).

\section{Results}

First, we computed the standard trial-averaged stimulus-onset triggered TFR $(20-60 \mathrm{~Hz})$. Fig.1A shows a single session example from monkey S in V1. Shortly after stimulus onset, the gamma band 'settled' around a dominant frequency for the remaining part of the trial. However, in the LFP of single-trial TFRs (Fig.1B), we observed 'bursts' of gamma oscillations (Burns et al., 2011) that occurred roughly at a low theta frequency $(3-4 \mathrm{~Hz})$ and in parallel in both V1 and V2. The relationship between V1/V2 gamma bursts and the $3-4 \mathrm{~Hz}$ rhythm was evident from the alignment of gamma bursts visible in the raw LFP to theta peaks that emerged after filtering the LFP in a $3-4 \mathrm{~Hz}$ band (Fig.1B). To gain further insight into theta-related gamma modulations, we computed theta-triggered TFRs. To this end, we selected the peak of each theta cycle as a trigger around which to center time-windows $( \pm 0.25 \mathrm{sec})$ for averaging gamma (Fig.1C). Fig.1D shows population-averaged theta-triggered TFRs from V1 and V2 of monkeys $\mathrm{S}$ and K. Below each TFR in Fig.1D, the corresponding time courses are shown of raw LFP amplitude, of gamma frequency, and of gamma-band power. The theta phase modulated gamma response properties: gamma frequency reached its peak shortly after the trough of the theta cycle, and then decayed slowly. Median gamma frequency modulations in V1 were $6.2 \mathrm{~Hz}$ in Monkey S and $5.1 \mathrm{~Hz}$ in Monkey $\mathrm{K}$. In V2, the modulation was $4.7 \mathrm{~Hz}$ in monkey $\mathrm{S}$ and $4.9 \mathrm{~Hz}$ in monkey $\mathrm{K}$. These frequency modulations were all 
significant ( $p<0.005$ in each session) and showed considerable effect size $\left(r^{2} \sim 0.16\right.$ in monkey $S$ over all contacts and $r^{2} \sim 0.07$ in monkey $\mathrm{K}$ ). Notice the strong asymmetry in frequency modulation with sharp frequency increases followed by slower decreases, observable in both monkeys and areas. The theta rhythm also modulated gamma band power (Lakatos et al., 2005) in both areas of both monkeys in the same order of magnitude (all $r^{2} \sim 0.1, p<0.005$ ). To assess the theta-triggered gamma synchronization between areas V1 and V2, we computed spectral coherence using small time windows for both monkeys, taking the peak of the $\mathrm{V} 1$ theta as an alignment trigger. Fig. $1 \mathrm{E}$ shows population average TFRs of coherence and below them the coherence peak frequency and the gamma-band coherence strength. Theta phase significantly modulated the peak frequency of gamma coherence in monkeys $\mathrm{S}(4.9 \mathrm{~Hz})$ and monkey $\mathrm{K}(4.8 \mathrm{~Hz})$ (median, $\mathrm{p}<0.01$, both monkeys, Wilcoxon signed rank test here and in the following tests) and weakly the median gamma coherence strength ( $p<0.05$ both monkeys). The results so far indicate that gamma-band activity during the so-called sustained period occurred in theta-rhythmic periods within which gamma frequency and power were systematically modulated.

A

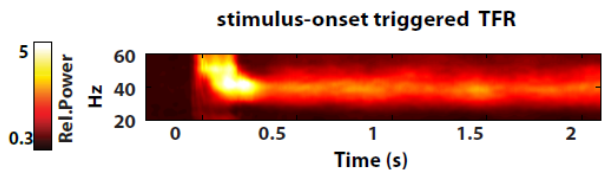

B

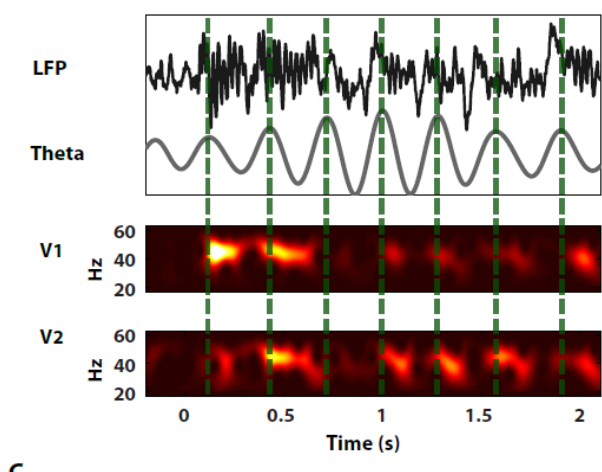

C

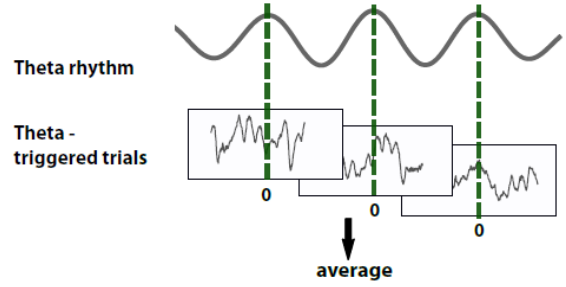

D

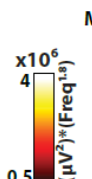

Monkey S

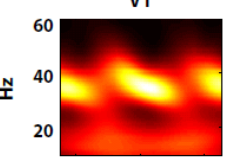

论可 0.5

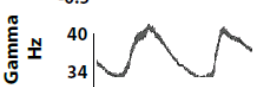

๕ั
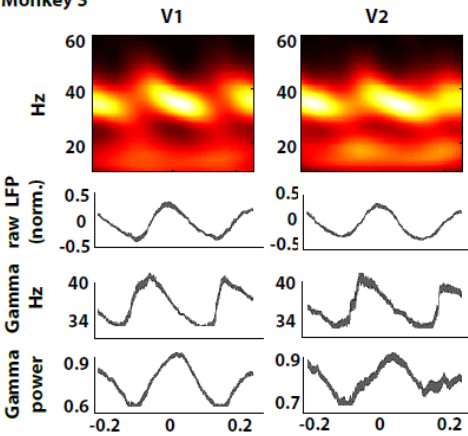

0.


Monkey K
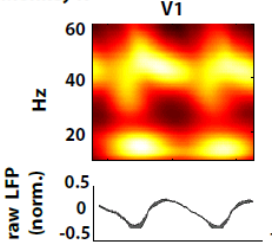

约 N
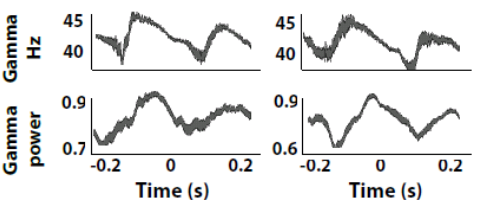

E
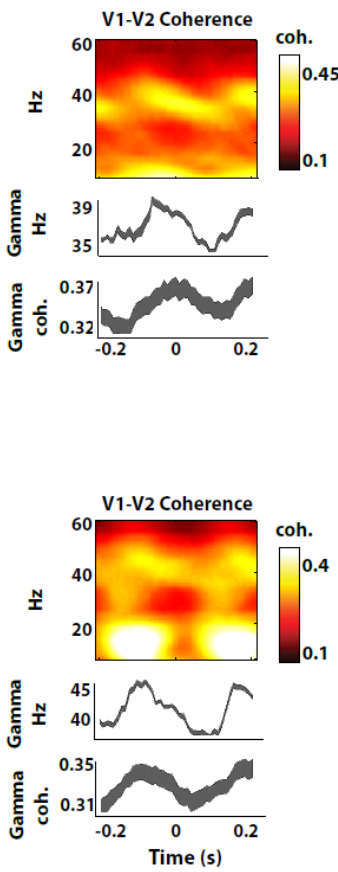

Figure 1. Stimulus and theta triggered analysis of visual gamma oscillatory responses (A) standard stimulus-onset triggered averaged time-frequency-representation (TFR) (single session, one contact). (B) Single-trial example. Raw LFP with filtered LFP in the theta range $(3-4 \mathrm{~Hz})$ at the top. Below, TFR of a V1 and V2 contact. The green dashed lines represent the peak of the theta signal. Notice that the gamma bursts in V1 and V2 show locking with respect to the theta peaks. (C) Peaks of LFP theta rhythm were used as triggers to align the center of $500 \mathrm{~ms}$ data snippets (theta-triggered trials). (D) Theta-triggered TFRs are shown for V1 and V2 of both monkeys. Below each TFR, the averaged LFP response (normalized) and modulations in frequency as well as power (normalized) of gamma oscillations are shown. (E) Theta-triggered TFR of coherence between V1 and V2 sites. Below, quantifications of the modulation in frequency and the strength of gamma coherence are shown. (D-E) Plot thickness represents standard error (population data). 
Although not the focus of the present report, Figures 1D and $E$ show that theta triggering also reveals structure in the oscillatory power and coherence in the alpha-beta range, in addition to that described in the gamma range. Qualitative observations suggest that relative to the theta trigger, moments of highest power (coherence) in gamma and in alpha-beta occur at different phases, perhaps in line with the idea of a role of alpha in gating neural processing (Jensen and Mazaheri, 2010). Note the larger coherence in the alpha-beta range in monkey $\mathrm{K}$ (Fig. 1E, lower panel). Although we cannot be sure what the difference in the magnitude of coherence in alpha-beta range between monkeys is due to, we can assert it is unlikely to be due to task differences as the monkeys were only required to fixate.

A

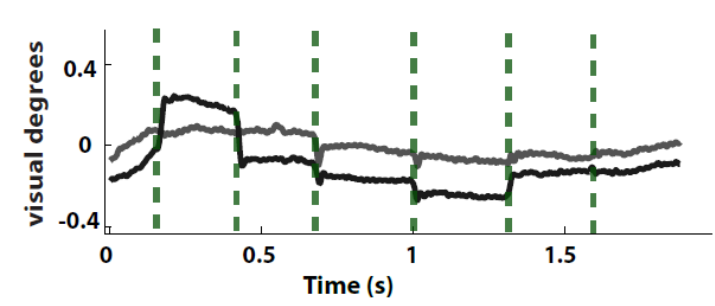

B

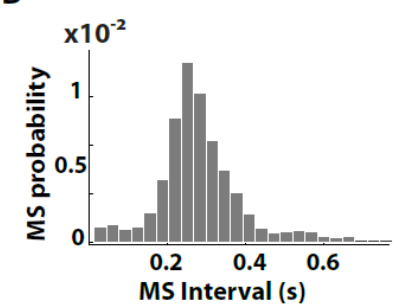

C

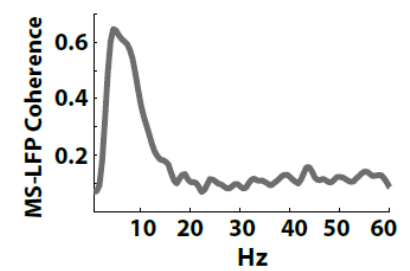

D

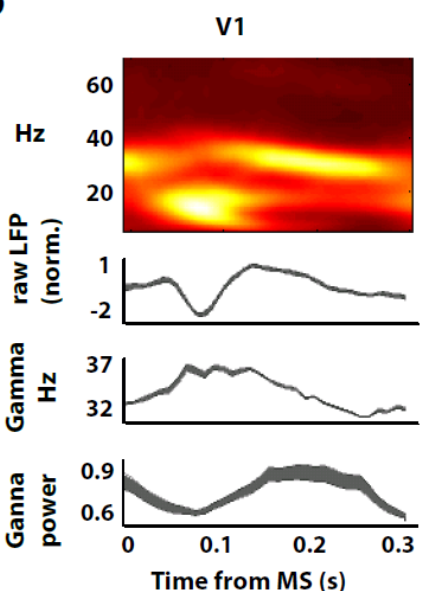

V2

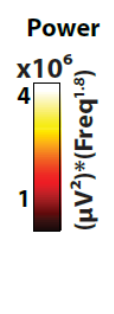

E
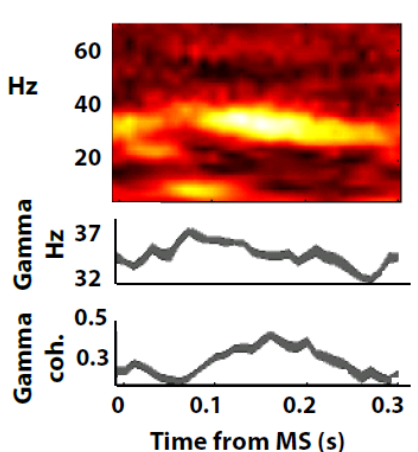

$\mathrm{V} 1 \longrightarrow \mathrm{V} 2$

Coherence
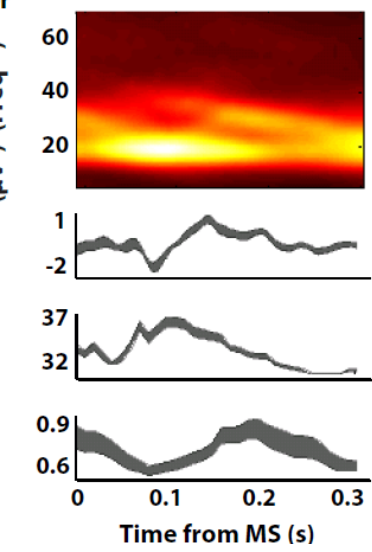
0.35

Figure 2. Microsaccade-triggered analysis in Monkey $S$ (population data). (A) Example trial showing the $X$ and $Y$ eye position clearly showing small saccadic deflections. (B) Histogram of microsaccade intervals (C) The MS-LFP-coherence. Similar to spikeLFP coherence but with MSs instead of spikes. A peak in the lower frequency range can be observed with a maximum in the 3$5 \mathrm{~Hz}$ range. (D) Microsaccade-triggered time-frequency power analysis of LFP for V1 and V2 contacts. Top to bottom: TFRs, averaged raw LFP, estimated frequency of gamma and the power. (E) Microsaccade-triggered time-resolved V1/V2 coherence spectrum, with below gamma frequency showing peak coherence, and total gamma coherence.

Previous studies have indicated that the theta rhythm is linked to (micro)saccades (Bosman et al., 2009; Ito et al., 2011; Brunet et al., 2013). Microsaccades are small, involuntary eye movements that occur during fixation. Although they are transient and typically smaller than 1 degree of visual angle (Fig.2A), they induce strong modulations of neural activity (Leopold and Logothetis, 1998; Martinez-Conde et al., 2000, 2009; Bosman et al., 2009; Rolfs, 2009). We tested the relationship between the theta phase modulation of gamma, demonstrated above, and microsaccades in monkey $\mathrm{S}$, and in an additional monkey A with an EcoG covering V1, where we had high-resolution eye data available. We found that 
the inter-microsaccade interval histogram peaked at $270 \mathrm{~ms}$ (Fig.2B, Fig.3B), corresponding to the 3-4 $\mathrm{Hz}$ theta frequency (see Fig.1B). In addition, microsaccade probability was linked to the LFP theta phase. This was revealed by an analysis of microsaccade-field coherence in monkey S (Fig.2C) and A. (Fig.3C). The microsaccade-field coherence peaked at $3-4 \mathrm{~Hz}$, indicating a rhythmic modulation of microsaccade probability in the theta band. We then tested the link between microsaccades and gamma by computing microsaccade-triggered TFRs. Fig.2D shows microsaccade-triggered TFRs for V1 and V2 in monkey S, with bottom panels showing the corresponding visually evoked response (VEP), frequency modulation, and power modulation. We found a systematic relationship between gamma periods and microsaccades and a strong resemblance between microsaccade-triggered TFRs (Fig.2D, 3D) and theta-triggered TFRs (Fig.1D). Within the period of high gamma power, the peak frequency of gamma decayed by (median) $5.2 \mathrm{~Hz}$ in $\mathrm{V} 1$ and $4.6 \mathrm{~Hz}$ in $\mathrm{V} 2$ (both $\mathrm{p}<0.0001$ ). In monkey A. in which we used EcoG recording (Fig.3A), microsaccade-triggered TFR of bipolar LFP revealed similar modulations in the frequency (median=3.2 Hz) and power of the V1 gamma-band (both $\mathrm{p}<0.01$ ). Further, in monkey $\mathrm{S}$ microsaccadetriggered coherence analysis (Fig.2E) confirmed a microsaccade-dependent modulation of V1-V2 gamma coherence in frequency and strength (both $p<0.01$ ) similar to theta-triggered coherence analysis in monkeys $\mathrm{S}$ and $\mathrm{K}$. These results support the view that microsaccades play a critical role in theta-rhythmic gamma dynamics (Bosman et al., 2009; Ito et al., 2011).

A Data from EcoG Monkey A

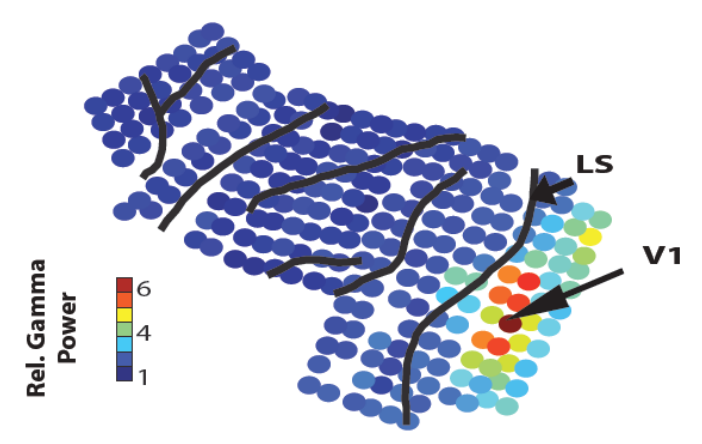

D

B

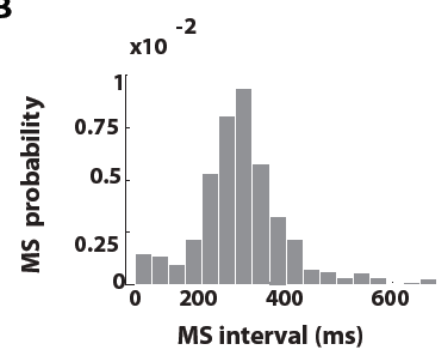

C

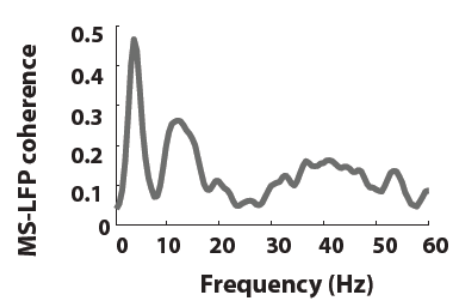



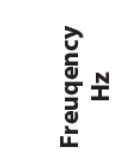

E
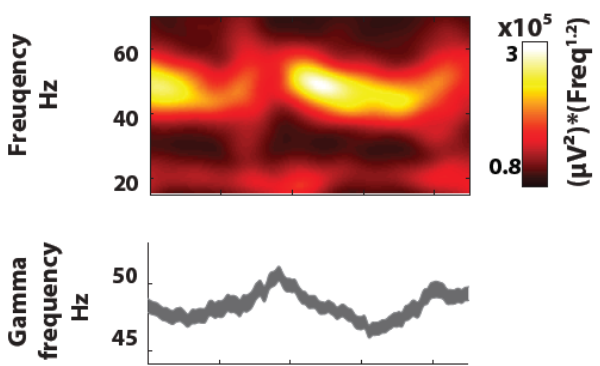

$\mathbf{F}$

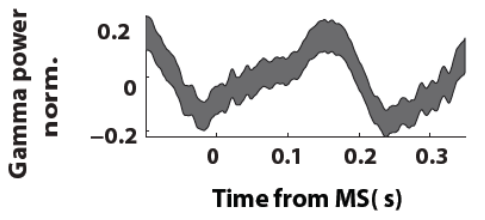

Figure 3. Microsaccade-triggered analysis in Monkey A (population data). (A) The 256-channel EcoG grid is depicted where each dot represents the position of a bipolar differentiation between two sensors. The color of the dot represent the relative induced gamma power by the stimulus compared to baseline. The stimulus induced strong gamma power in sensors covering V1 (posterior to the lunate sulcus, LS). For the microsaccade-triggered TFR we used the bipolar LFP from the sensors with the higher relative gamma power. (B) Histogram of microsaccade intervals (C) The MS-LFP-coherence. (D) Microsaccade-triggered time-frequency power analysis of the bipolar LFP from V1 .Top to bottom: TFRs, estimated frequency of gamma and the power. 
The microsaccade-triggered analysis revealed intricate structure in oscillatory activity that was hidden in commonly-used stimulus-onset triggered analysis (Fig.1A). We highlight this by directly comparing stimulus-onset triggered trial averaging with microsaccade-triggered trial averaging (Fig.4), using a single session from Monkey S with 667 trials (at a single contrast). From those trials, we selected trials that contained their second microsaccade within the period of 400-500ms after stimulus-onset (leaving 105 trials) and then realigned those trials to the second MS of the trial. This procedure permitted the observation of gamma modulations over a 1s time period (Fig.4, right), which were absent after

A

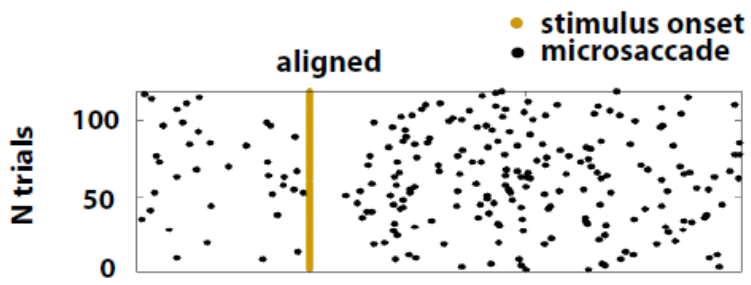

B

C
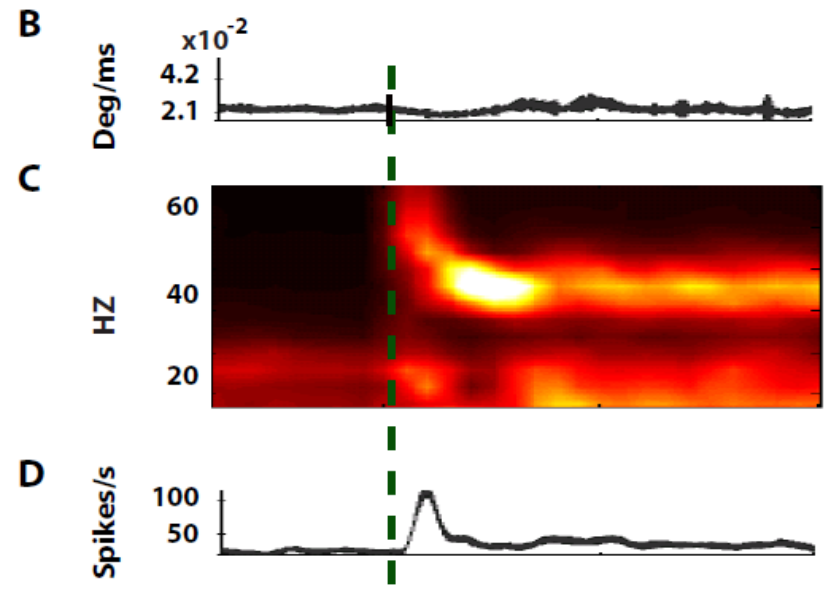

E



$\mathbf{F}$

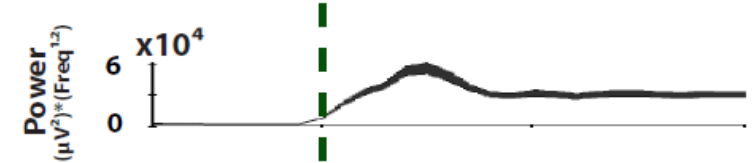

G

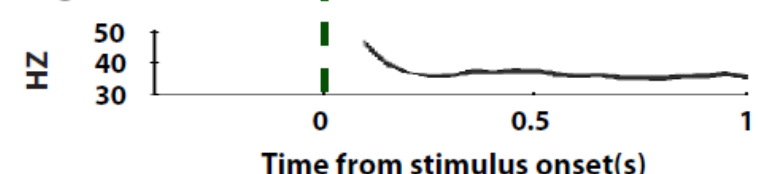

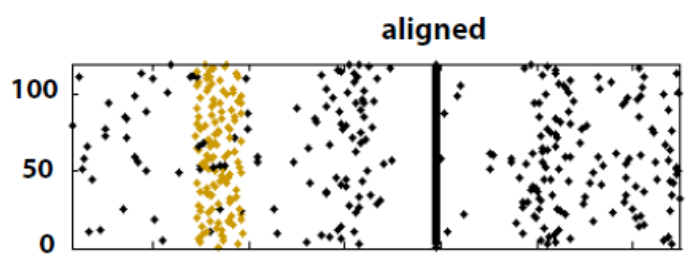
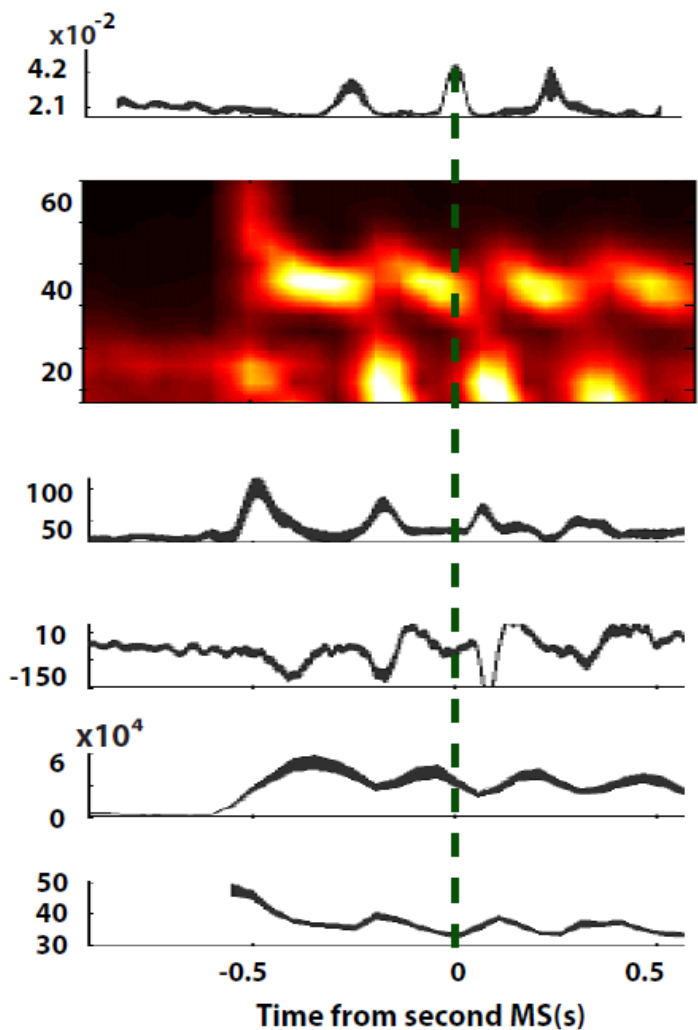

Time from second MS(s)

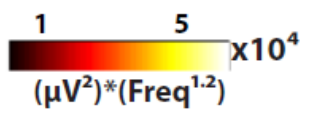

Fig.4 Comparison of stimulus-onset triggered and microsaccade-triggered trial averaging (monkey $\mathrm{S}$, single session, $\mathrm{N}$ trials= 105). (A) Raster plots of microsaccades (black dots) and stimulus onsets (yellow dots). The left column represents trials defined in relation to stimulus onset, whereas the right column represents trials defined in relation to the occurrence of the second microsaccade after stimulus onset. In each panel, the stimulus-onset triggered average (left) and microsaccade triggered average (right) are shown as a function of time for (B) monocular eye speed (C) time-frequency representation of LFP power, (C) spike density, (E) averaged local field potential (LFP), (F) gamma power, and (G) gamma frequency. In B, D-G line thickness represents standard error. 
conventional triggering by stimulus onset (Fig.4, left). Figure 4 illustrates the profound differences in the effects of stimulus-onset versus microsaccade-triggered averaging (see Fig.4A/B) on TFR (Fig.4C), LFP (Fig.4D), spike probability (Fig.4E), gamma power (Fig.4F), and gamma frequency (Fig.4G).

\section{Discussion}

Recent studies (Burns et al., 2010, 2011) have shown that gamma oscillations are not stationary ('clocklike'). They found that V1 gamma dynamics were fitted better by a (stochastic) model of 'filtered noise' and clearly deviated from a fixed-frequency model. However, as indicated by these authors, their analysis could not distinguish a stochastic process from a deterministic, yet complex, process. Hence, while potentially problematic for neural communication, Burns' (2010, p9663) findings do not per definition exclude a role of gamma in communication, and they even suggested that brief gamma power enhancements could act as a sync pulse to briefly achieve neural communication/synchronization in the gamma range. Our awake macaque V1 and V2 data confirm that gamma oscillations occur in bursts with rapidly changing frequencies. However, in line with the possibility left open by previous authors (Burns et al., 2010, 2011), we found that a substantial part of the gamma fluctuations reflected complexity, rather than randomness, as those fluctuations were systematically related to a microsaccade-linked theta rhythm. Thus, part of the gamma power and frequency variation arose due to cross-frequency interaction. This is in line with gamma oscillation dynamics described in other brain structures like the hippocampus (Belluscio et al., 2012; Lisman and Jensen, 2013; Schomburg et al., 2014), where crossfrequency coupling between theta phase and gamma power/frequency has been intensively studied. The modulation strength by the $3-4 \mathrm{~Hz}$ theta phase in V1 and V2 was of similar magnitudes $\left(r^{2}\right.$ values of 0.1, see Result section) for CFC phase-frequency interaction compared to the more commonly estimated phase-power interaction. We therefore argue that from an experimental as well as from a theoretical viewpoint (Cohen, 2014), there is no reason to favor reporting one type of interaction over the other. In fact, oscillation frequency, defining the window length of spiking probability (Fries, 2009), is a critical factor for enabling synchronization between interacting oscillations (Pikovsky et al., 2002; Roberts et al., 2013). Importantly, we show that not only gamma power variations (Bosman et al., 2009, 2012) but also frequency variations are linked to a $3-4 \mathrm{~Hz}$ microsaccade-related rhythm, thus supporting what was reported as a tentative observation in (Bosman et al., 2009).

Furthermore, we found that V1-V2 gamma coherence was modulated by a $3-4 \mathrm{~Hz}$ theta rhythm, suggesting the relevance of CFC in framing communication between cortical areas. Our findings are in line with other studies (Colgin et al., 2009; Bosman et al., 2012), which indicate that gamma-mediated inter-areal communication (Fries, 2009) is neither continuous nor sustained during stimulus processing, but instead occurs in 'bursts' that are structured by slower rhythms. As these slower rhythms are shared among V1 and V2, they may help in establishing co-occurrence and alignment of phase and frequency of gamma in the two areas. Confirming this idea, the simultaneous recordings in V1 and V2 revealed that inter-areal gamma coherence was modulated in its preferred frequency and strength at the rhythm of microsaccades. These findings support the view that microsaccades play a critical role in visual cortical 
temporal dynamics and fit with observations of the strong impact of microsaccades on spiking activity in visual cortical as well as subcortical areas (Martinez-Conde, 2013). Notably, Xing et al.'s (2012) finding of low frequency gamma band modulations in V1 of anesthetized monkeys does not invalidate this idea, as the driving force of the low frequency gamma modulations is likely at least in part generated internally (Melloni et al., 2009), and does not just reflect a feedforward effect due to displacement of the retinal image.

Our findings underline the view that cortical gamma oscillations are highly non-stationary, and exhibit marked transient modulations that should be given more attention in both theoretical as well as experimental analysis. In conclusion, our findings indicate that gamma variation, rather than being random and thereby a potential obstacle to neural communication, are highly structured, shared between areas, and regulated by a microsaccade-related theta rhythm. Moreover, our findings fit with the important contribution of cross-frequency coupling to structuring and facilitating information flow as shown in other domains (Jensen and Colgin, 2007; Canolty and Knight, 2010).

\section{References}

Belluscio MA, Mizuseki K, Schmidt R, Kempter R, Buzsaki G (2012) Cross-Frequency Phase-Phase Coupling between Theta and Gamma Oscillations in the Hippocampus. J Neurosci 32:423-435.

Berens P (2009) CircStat: A MATLAB toolbox for circular statistics. J Stat Softw 31:1-21 Available at: http://kyb.mpg.de/publications/attachments/J-Stat-Softw-2009-Berens_6037[0].pdf.

Bosman CA, Schoffelen J-M, Brunet N, Oostenveld R, Bastos AM, Womelsdorf T, Rubehn B, Stieglitz T, De Weerd $P$, Fries $P$ (2012) Attentional stimulus selection through selective synchronization between monkey visual areas. Neuron 75:875-888 Available at: http://dx.doi.org/10.1016/j.neuron.2012.06.037.

Bosman CA, Womelsdorf T, Desimone R, Fries P (2009) A microsaccadic rhythm modulates gamma-band synchronization and behavior. J Neurosci 29:9471-9480 Available at: http://dx.doi.org/10.1523/JNEUROSCI.1193-09.2009.

Brunet N, Bosman C a, Roberts M, Oostenveld R, Womelsdorf T, De Weerd P, Fries P (2013) Visual Cortical Gamma-Band Activity During Free Viewing of Natural Images. Cereb Cortex:1-9 Available at: http://www.ncbi.nlm.nih.gov/pubmed/24108806.

Burns SP, Xing D, Shapley RM (2011) Is gamma-band activity in the local field potential of V1 cortex a "clock" or filtered noise? J Neurosci 31:9658-9664 Available at: http://dx.doi.org/10.1523/JNEUROSCI.0660-11.2011.

Burns SP, Xing D, Shelley MJ, Shapley RM (2010) Searching for autocoherence in the cortical network with a time-frequency analysis of the local field potential. J Neurosci 30:4033-4047 Available at: http://dx.doi.org/10.1523/JNEUROSCI.5319-09.2010.

Canolty RT, Knight RT (2010) The functional role of cross-frequency coupling. Trends Cogn Sci 14:506515 Available at: http://www.sciencedirect.com/science/article/pii/S1364661310002068 [Accessed January 21, 2014].

Cohen MX (2014) Fluctuations in oscillation frequency control spike timing and coordinate neural networks. J Neurosci 34:8988-8998.

Colgin LL, Denninger T, Fyhn M, Hafting T, Bonnevie T, Jensen O, Moser M-B, Moser El (2009) Frequency of gamma oscillations routes flow of information in the hippocampus. Nature 462:353-357 Available at: http://dx.doi.org/10.1038/nature08573 [Accessed July 11, 2014]. 
Fries P (2009) Neuronal gamma-band synchronization as a fundamental process in cortical computation. Annu Rev Neurosci 32:209-224.

Gattass R, Gross CG, Sandell JH (1981) Visual topography of V2 in the macaque. J Comp Neurol 201:519539 Available at: http://www.ncbi.nlm.nih.gov/pubmed/7287933 [Accessed February 25, 2015].

Hoogenboom N, Schoffelen J-M, Oostenveld R, Parkes LM, Fries P (2006) Localizing human visual gamma-band activity in frequency, time and space. Neuroimage 29:764-773 Available at: http://www.sciencedirect.com/science/article/pii/S1053811905006361 [Accessed January 29, 2014].

Ito J, Maldonado P, Gruen S (2011) Cross-frequency coupling of eye-movement related LFP activities of freely viewing monkeys. BMC Neurosci 12:P132.

Jensen O, Colgin LL (2007) Cross-frequency coupling between neuronal oscillations. Trends Cogn Sci 11:267-269 Available at: http://www.sciencedirect.com/science/article/pii/\$1364661307001271 [Accessed January 23, 2014].

Jensen O, Mazaheri A (2010) Shaping functional architecture by oscillatory alpha activity: gating by inhibition. Front Hum Neurosci 4:186.

Jutras MJ, Fries P, Buffalo EA (2013) Oscillatory activity in the monkey hippocampus during visual exploration and memory formation. Proc Natl Acad Sci U S A 110:13144-13149.

Lakatos P, Shah AS, Knuth KH, Ulbert I, Karmos G, Schroeder CE (2005) An oscillatory hierarchy controlling neuronal excitability and stimulus processing in the auditory cortex. J Neurophysiol 94:1904-1911 Available at: http://dx.doi.org/10.1152/jn.00263.2005.

Le Van Quyen M, Foucher J, Lachaux J, Rodriguez E, Lutz A, Martinerie J, Varela FJ (2001) Comparison of Hilbert transform and wavelet methods for the analysis of neuronal synchrony. J Neurosci Methods 111:83-98.

Leopold DA, Logothetis NK (1998) Microsaccades differentially modulate neural activity in the striate and extrastriate visual cortex. Exp Brain Res 123:341-345 Available at: http://link.springer.com/10.1007/s002210050577 [Accessed February 14, 2014].

Lisman JE, Jensen O (2013) The theta-gamma neural code. Neuron 77:1002-1016 Available at: http://dx.doi.org/10.1016/j.neuron.2013.03.007.

Lund JS (2003) Anatomical Substrates for Functional Columns in Macaque Monkey Primary Visual Cortex. Cereb Cortex 13:15-24 Available at: http://cercor.oxfordjournals.org/content/13/1/15.short [Accessed February 25, 2015].

Martinez-Conde $S$ (2013) The impact of microsaccades on vision: towards a unified theory of saccadic function. Nat Rev ... Available at: http://www.nature.com/nrn/journal/v14/n2/abs/nrn3405.html [Accessed February 13, 2014].

Martinez-Conde S, Macknik SL, Hubel DH (2000) Microsaccadic eye movements and firing of single cells in the striate cortex of macaque monkeys. Nat Neurosci 3:251-258 Available at: http://dx.doi.org/10.1038/72961 [Accessed February 14, 2015].

Martinez-Conde S, Macknik SL, Troncoso XG, Hubel DH (2009) Microsaccades: a neurophysiological analysis. Trends Neurosci 32:463-475.

Melloni L, Schwiedrzik CM, Rodriguez E, Singer W (2009) (Micro)Saccades, corollary activity and cortical oscillations. Trends Cogn Sci 13:239-245.

Miller KJ, Sorensen LB, Ojemann JG, Den Nijs M (2009) Power-law scaling in the brain surface electric potential. PLoS Comput Biol 5.

Nowak LG, Munk MHJ, James AC, Girard P, Bullier J (1999) Cross-Correlation Study of the Temporal Interactions Between Areas V1 and V2 of the Macaque Monkey. J Neurophysiol 81:1057-1074 Available at: http://jn.physiology.org/content/81/3/1057.short [Accessed February 25, 2015].

Oostenveld R, Fries P, Maris E, Schoffelen J-M (2011) FieldTrip: Open source software for advanced analysis of MEG, EEG, and invasive electrophysiological data. Comput Intell Neurosci 2011:156869. 
Osipova D, Hermes D, Jensen O (2008) Gamma power is phase-locked to posterior alpha activity. PLoS One 3:e3990.

Pikovsky A, Rosenblum M, Kurths J, Hilborn RC (2002) Synchronization: A Universal Concept in Nonlinear Science. Am J Phys 70:655 Available at: http://link.aip.org/link/AJPIAS/v70/i6/p655/s1\&Agg=doi [Accessed August 28, 2014].

Rajkai C, Lakatos P, Chen C-M, Pincze Z, Karmos G, Schroeder CE (2008) Transient cortical excitation at the onset of visual fixation. Cereb Cortex 18:200-209.

Ray S, Maunsell JHR (2010) Differences in gamma frequencies across visual cortex restrict their possible use in computation. Neuron 67:885-896 Available at: http://dx.doi.org/10.1016/j.neuron.2010.08.004.

Roberts MJ, Lowet E, Brunet NM, Ter Wal M, Tiesinga P, Fries P, De Weerd P (2013) Robust gamma coherence between macaque V1 and V2 by dynamic frequency matching. Neuron 78:523-536 Available at: http://www.ncbi.nlm.nih.gov/pubmed/23664617.

Rolfs M (2009) Microsaccades: small steps on a long way. Vision Res 49:2415-2441.

Rubehn B, Bosman C, Oostenveld R, Fries P, Stieglitz T (2009) A MEMS-based flexible multichannel ECoGelectrode array. J Neural Eng 6:036003.

Schomburg EW, Fernández-Ruiz A, Mizuseki K, Berényi A, Anastassiou CA, Koch C, Buzsáki G (2014) Theta Phase Segregation of Input-Specific Gamma Patterns in Entorhinal-Hippocampal Networks. Neuron 84:470-485 Available at: http://www.sciencedirect.com/science/article/pii/S0896627314007818 [Accessed September 25, 2014].

Schroeder CE, Lakatos P (2009) The gamma oscillation: master or slave? Brain Topogr 22:24-26.

Swettenham JB, Muthukumaraswamy SD, Singh KD (2009) Spectral properties of induced and evoked gamma oscillations in human early visual cortex to moving and stationary stimuli. J Neurophysiol 102:1241-1253 Available at: http://dx.doi.org/10.1152/jn.91044.2008.

Xing D, Shen Y, Burns S, Yeh C-I, Shapley R, Li W (2012) Stochastic generation of gamma-band activity in primary visual cortex of awake and anesthetized monkeys. J Neurosci 32:13873-13880a Available at: http://www.jneurosci.org/content/32/40/13873.short [Accessed February 14, 2015]. 


\section{Chapter 5}

\section{Input-dependent frequency modulation of cortical gamma oscillations shapes spatial synchronization and enables phase coding}

Based on:

E. Lowet, M. Roberts, A. Hadjipapas, A. Peter, J. van der Eerden, and P. De Weerd, "Input-Dependent Frequency Modulation of Cortical Gamma Oscillations Shapes Spatial Synchronization and Enables Phase Coding.," PLoS Comput. Biol., vol. 11, no. 2, p. e1004072, Feb. 2015. 


\section{ABSTRACT}

Fine-scale temporal organization of cortical activity in the gamma range $(\sim 25-80 \mathrm{~Hz})$ may play a significant role in information processing, for example by neural grouping ('binding') and phase coding. Gamma synchronization has been shown to be local, matching the spatial connectivity extent of cortical neurons. Moreover, the precise frequency of gamma oscillations varies with input drive (e.g. visual contrast) and can differ among nearby cortical locations. This has challenged theories assuming widespread cortical gamma synchronization at a common frequency. In the light of those findings, we constructed a biophysically realistic excitatory-inhibitory network model able to express different gamma oscillation frequencies at nearby spatial locations. Similarly to cortical networks, the model was topographically organized with spatially local connectivity and spatially-varying input drive. We investigated the underlying principles of gamma-mediated temporal organization with respect to phaselocking, phase-relations and frequency differences, and quantified the stimulus-related information represented by gamma phase and frequency. By stepwise simplification of our models, we found that the gamma-mediated temporal organization could be reduced to basic synchronization principles of weakly coupled oscillators, where input drive determines the intrinsic (natural) frequency of oscillators. The gamma phase-locking, the precise phase relation and the emergent (measurable) frequencies were determined by two principal factors: the detuning (intrinsic frequency difference, i.e. local input difference) and the coupling strength. Further, in addition to frequency coding, gamma phase contained complementary stimulus information. Crucially, the phase code reflected input differences, but not the absolute input level. This property of relative input-to-phase conversion, contrasting with latency codes or slower oscillation phase codes, and may resolve conflicting experimental observations on gamma phase coding. Our modeling results offer clear testable experimental predictions. We conclude that input-dependency of gamma frequencies could be essential rather than detrimental for meaningful gamma-mediated temporal organization of cortical activity. 


\section{AUTHOR SUMMARY}

Almost 350 years ago the physicist and polymath Christiaan Huygens first observed the synchronization between two pendulum clocks attached to a common support. Since then synchronization has been recognized as a universal phenomenon from astronomy to biology. The phase-locking (synchrony) and the phase-relation between the two pendulums are determined by two principal forces: the synchronization force exerted over the connection and the tendency to desynchronize due to frequency (speed) differences. We propose that gamma synchronization $(25-80 \mathrm{~Hz})$ among oscillating cortical neurons in the brain can be understood according to the same principles - like a field of many connected pendula - with the critical addition that input changes the frequency of gamma oscillations, as shown by recent experimental studies. It has been assumed that input-dependent changes in oscillation frequency are detrimental for a meaningful role of gamma synchronization in neural processing. To the contrary, our theoretical analysis demonstrates that because input can change the frequency of the oscillation, phase-locking and phase-relations among neurons relate systematically to input. By analogy, it is because a local push to a pendulum will change its frequency, that resulting changes in phase-locking and phase-relation among the pendula can be used to derive the external force applied. 


\section{Introduction}

How the millions of neurons in the brain are coordinated to permit meaningful computations is one of the fundamental questions of neuroscience. Spike synchrony and relative spike timing play important roles in dynamically coordinating neural activity (Bressler and Kelso, 2001; Ritz and Sejnowski, 1997; Singer, 1999a, 1999b; Stanley, 2013; Tiesinga et al., 2008; Tsukada et al., 1996) with substantial impact on neuronal function (Branco and Häusser, 2011; Branco et al., 2010; Caporale and Dan, 2008; London and Häusser, 2005; Markram et al., 2012). Synchronization often goes hand in hand with neural oscillations, of which gamma-band oscillations $(\sim 25-80 \mathrm{~Hz})$ have received broad attention (Buzsáki and Wang, 2012; Fries, 2009; Fries et al., 2007). Gamma oscillations occur in various brain regions and species (Buzsáki and Wang, 2012; Fries, 2009; Fries et al., 2007; Stopfer et al., 1997). Gamma oscillations arise locally from mainly direct interactions between inhibitory and excitatory neurons (Buzsáki and Wang, 2012; Fries et al., 2007; Tiesinga and Sejnowski, 2009, 2010). Modulations of gamma oscillation properties (power, frequency) have been found for various cognitive functions including perception (Bertrand and Tallon-Baudry, 2000; Brunet et al., 2013; Engel et al., 1999), attention (Bosman et al., 2012; Jensen et al., 2007; Womelsdorf and Fries, 2007), working memory (Jensen et al., 2007) as well as in psychiatric disorders like psychosis (Herrmann and Demiralp, 2005; Lee et al., 2003) and ADHD (Lenz et al., 2008; Yordanova et al., 2001). At the neuronal level, different roles (that are not mutually exclusive) have been attributed: They include neural grouping by phase-locking within (Eckhorn, 2000, 1999a; Engel et al., 1999; Gail et al., 2000) and between cortical areas (Fries, 2009, 2005; Roberts et al., 2013), phase coding (Fries et al., 2007; Havenith et al., 2011; Jensen et al., 2014; Timothee Masquelier et al., 2009; Tiesinga and Sejnowski, 2010; Vinck et al., 2010), neuronal plasticity (Lee et al., 2009; Traub et al., 1998), gain control (Tiesinga and Sejnowski, 2010), and normalization (Ray et al., 2013).

However, the role of gamma oscillations in neural computation is controversial, with judgments ranging from fundamental (Buzsáki and Wang, 2012; Engel et al., 1999; Fries, 2009) to epiphenomenal (Jia et al., 2013b; Merker, 2013; Ray and Maunsell, 2010). Experimental studies have given conflicting evidence on the role of gamma phase coding of input drive. For example, Vinck et al. (Vinck et al., 2010) have shown that visual cortical neurons receiving different input drive (through varying stimulus orientation) can exhibit reliable spike timing differences in the gamma oscillation range. However, Montemurro et al. (Montemurro et al., 2008) using natural stimuli could not find any contribution of visual cortical gamma phase to the encoding of the input. Similarly, McLelland and Paulsen (McLelland and Paulsen, 2009) did not find a rate-to-phase transform for gamma oscillations, which would assign a specific level of input to a specific phase of gamma. Moreover, although various experimental studies (Eckhorn, 1999a; Gail et al., 2000; Ray and Maunsell, 2010) have shown input(stimulus)-dependent changes in gamma synchronization, theoretical models (Eckhorn et al., 1990; Engel et al., 1999; König and Schillen, 1991a) have fallen short in convincingly including the local and variable nature of gamma oscillations. For example, the dependence of gamma oscillation frequency on stimulus attributes (e.g. visual contrast (Jia et al., 2013b; Ray and Maunsell, 2010; Roberts et al., 2013)) as well as the limited spread of gamma phase-locking over cortical distance (Eckhorn, 1999b; Palanca and DeAngelis, 2005) are seen as 
conflicting with a functional role of gamma oscillations in neural processing (Hermes et al., 2014; Jia et al., 2013b; Merker, 2013; Ray et al., 2013).

Here, we used computational modeling techniques to develop a deeper understanding of inputdependent cortical gamma synchronization. We focused on the underlying organization principles of phase and frequency coding of input drive and its relation to spatial synchronization and network connectivity. Mathematically, the synchronization of interacting limit-cycle oscillators (and other types, (Rosenblum et al., 1996)) is well understood (Ermentrout and Kleinfeld, 2001; Hoppensteadt and Izhikevich, 1998, 1996). In particular, the theory of weakly coupled oscillators (TWCO) (see (Pikovsky et al., 2002) for review) has proven to be useful and has been applied in many scientific domains, including neuroscience (Bendels and Leibold, 2007; Breakspear et al., 2010; Ermentrout and Kleinfeld, 2001; Galán et al., 2005; Hoppensteadt and Izhikevich, 1998, 1996). In TWCO the phase of an oscillator (neuron, group of neurons) is defined by an intrinsic (natural) frequency. The interaction with other oscillators is characterized by the phase response curve (PRC, (Schwemmer and Lewis, 2012)) which defines how the phase is modified by this interaction. Crucially, the phase-locking between oscillators depends on the intrinsic frequency difference (described as the detuning level) as well as interaction strength (or coupling strength), defining the so called Arnold tongues (region of synchronization defined by the interplay of detuning and coupling) (Coombes and Bressloff, 1999; Pikovsky et al., 2002; Tiesinga and Sejnowski, 2010). Note that in TWCO, the coupling strength is considered to be 'weak', meaning that the interactions among oscillators mainly change the phases but not the oscillation amplitudes.

A few prior studies have concretely considered TWCO for explaining input-dependent cortical gamma synchronization (Barardi et al., 2014; Ermentrout and Kleinfeld, 2001; Hopfield and Brody, 2001; Hoppensteadt and Izhikevich, 1996; Markowitz et al., 2008; Tiesinga and Sejnowski, 2010). Of most relevance here, Tiesinga and Sejnowski (Tiesinga and Sejnowski, 2010) first applied TWCO in a biophysically realistic gamma network for explaining gamma phase coding in visual cortex (Vinck et al., 2010). Several interconnected pyramidal-interneuron-gamma networks (PING) synchronized on a common frequency, despite receiving different levels of input currents, and converted input differences into phase-differences. Here, we extended Tiesinga and Sejnowski's (Tiesinga and Sejnowski, 2010) important study in several ways. We studied primarily spatially-continuous PING-type gamma networks (no assumption of columns) with local connectivity decaying as a function of spatial distance and different network locations receiving different input drives. Gamma synchronization was mainly local within the network. We used input drive ranges that induced different gamma oscillation frequencies and phase-relations allowing us to investigate the relationship between frequency and phase coding as a function of input. The gamma frequency ranges were set to match our own observations in awake monkey V1. We quantified the encoding performance of input by phase and frequency systematically with both simple stimuli and complex natural stimuli.

In short, we observed that phase-locking, phase-relation and frequency among neurons resulted from an interplay between detuning ( $\Delta$ intrinsic frequency) and coupling strength, in accord with TWCO and the Arnold tongue. Critical for the behavior was the property of gamma oscillations to shift their preferred frequency with input drive. Phase and frequency coding of input was largely complementary in accordance to the Arnold tongue concept, whereby conditions inside the Arnold tongue lead to phase 
coding, and conditions outside lead to frequency coding. A combined frequency and phase coding could best reconstruct the stimulus input. Importantly, the Arnold-tongue based phase coding implied a relative $\Delta$ rate-to-phase transform and therefore gamma phase told little about absolute input levels. Our work has clear theoretical implications leading to experimentally testable predictions that are elaborated on in the Discussion.

\section{Methods}

Experimental procedures. Experimental observations in Fig. 1 and associated methods of data collection shown have been described in a previous publication (Roberts et al., 2013). We show here only V1 data from monkey $S$ for illustration purposes only. We re-analyzed the LFP spectra obtained during stimulation (Stim.) with static square-wave grating ( 2 cycles per degree), using a multi-taper method with discrete prolate spheroid sequences for frequencies 20 to $60 \mathrm{~Hz}$ (smoothing $\pm 3 \mathrm{~Hz}$ ) in nonoverlapping $500 \mathrm{~ms}$ windows starting $350 \mathrm{~ms}$ after stimulus onset. LFP power in the pre-stimulus baseline (Base) was calculated from the $500 \mathrm{~ms}$ period before stimulus onset. Relative power was calculated as (Stim.-Base)/Base, where Stim and Base were calculated separately after averaging over trials. In Fig.1B the quantifications of maximum of peak gamma power as well as frequency of peak power is shown for the Michelson contrast conditions $6.1 \%, 9.7 \%, 16.3 \%, 35.9 \%, 50.3 \%$ and $72 \%$. The stimulus contrast conditions $2.5 \%$ and $3.7 \%$ had very low induced gamma power and no clear peak in the power spectrum.

Computational resource. Intel(R) Xeon(R) CPU E5-1620 0 @3.6GHz with 16GB RAM.

Hodgkin-Huxley network. Minimal single-compartment Hodgkin-Huxley models (Pospischil et al., 2008) were used to construct E-cells (regular-spiking excitatory neurons, RS) and I-cells (fast-spiking inhibitory interneurons, FS). For the network simulations shown in Fig. 1 and 2 the networks consisted of $80 \mathrm{E}-$ cells and $20 \mathrm{I}$-cells. For Fig. 3 the network consisted of $160 \mathrm{E}$-cells and $40 \mathrm{I}$-cells neurons. The E-cells in Fig.3 had particularly high firing rate matching the network gamma frequencies. This was done to increase oscillatory stability of the small network which was limited in size due to computational constraints. We use Izhikevich-type neurons [] for replicating our findings in larger E-and I-cells networks (see Suppl. Method). In Fig. 3 neurons were ordered along a ring to avoid network border effects (continuous connectivity). Numerical simulations were computed using a variable step size Runge-Kutta method of order 8 according to the Dormand and Prince algorithm (Hairer and Wanner, 1991). The simulation code was written in FORTRAN95. Analysis of the simulation output was performed with Matlab (MathWorks, R2012b). 


\section{Model summary:}

Regular-spiking (RS) E-cell:

$$
C_{m} \frac{d V}{d t}=-g_{\text {Leak }}\left(V-E_{\text {Leak }}\right)-I_{N a}-I_{K}-I_{M}
$$

Fast-spiking (FS) I-cell:

$$
C_{m} \frac{d V}{d t}=-g_{\text {Leak }}\left(V-E_{\text {Leak }}\right)-I_{N a}-I_{K}
$$

The leakage reversal potential and conductance were $E_{\text {Leak }}=-70 \mathrm{mV}$ and $g_{\text {Leak }}=0.0205 \mathrm{mS} / \mathrm{cm}^{2}$ for E-cells and $g_{\text {Leak }}=0.015 \mathrm{mS} / \mathrm{cm}^{2}$ for l-cells. The membrane capacitance was $C_{m}=1 \mathrm{mF} / \mathrm{cm}^{2}$. All kinetic parameters were according to a temperature of $36^{\circ} \mathrm{C}$ using standard conductance equations.

\section{Conductances}

Sodium current $I_{\mathrm{Na}}$ :

RS: $E_{N a}=50 \mathrm{mV}, \bar{g}_{N a}=50 \mathrm{mS} / \mathrm{cm}^{2} \quad V_{T}=-61.5 \mathrm{mV}$

FS: $E_{N a}=50 \mathrm{mV}, \bar{g}_{N a}=46 \mathrm{mS} / \mathrm{cm}^{2} V_{T}=-61.84 \mathrm{mV}$

$$
\begin{gathered}
I_{N a}=\bar{g}_{N a} m^{3} h\left(V-E_{N a}\right) \\
\frac{d m}{d t}=\alpha_{m}(V)(1-m)-\beta_{m}(V) m \\
\frac{d h}{d t}=\alpha_{h}(V)(1-h)-\beta_{h}(V) h \\
\alpha_{m}=\frac{-0.32\left(V-V_{T}-13\right)}{\exp \left[-\frac{V-V_{T}-13}{4}\right]-1} \\
\beta_{m}=\frac{0.28\left(V-V_{T}-40\right)}{\exp \left[-\frac{V-V_{T}-40}{5}\right]-1} \\
\alpha_{h}=0.128 \exp \left[-\frac{V-V_{T}-17}{18}\right]
\end{gathered}
$$


Chapter5

$$
\beta_{h}=\frac{4}{1+\exp \left[-\frac{V-V_{T}-40}{5}\right]}
$$

Delayed-rectifier potassium current $I_{K d}$ :

RS: $E_{K d}=-90 \mathrm{mV}, \bar{g}_{K d}=4.8 \mathrm{mS} / \mathrm{cm}^{2} \quad V_{T}=-61.5 \mathrm{mV}$

FS: $E_{K d}=-90 \mathrm{mV}, \bar{g}_{K d}=5.1 \mathrm{mS} / \mathrm{cm}^{2} V_{T}=-61.84 \mathrm{mV}$

$$
\begin{gathered}
I_{K d}=\bar{g}_{K d} n^{4}\left(V-E_{k}\right) \\
\frac{d n}{d t}=\alpha_{n}(V)(1-n)-\beta_{n}(V) n \\
\alpha_{n}=\frac{-0.032\left(V-V_{T}-15\right)}{\exp \left[-\frac{V-V_{T}-15}{5}\right]-1} \\
\beta_{n}=0.5 \exp \left[-\frac{V-V_{T}-10}{40}\right]
\end{gathered}
$$

Slow non-inactivating potassium current $I_{M}$ :

RS: $E_{K m}=-90 m V, \bar{g}_{K m}=0.15 m S / \mathrm{cm}^{2} \quad V_{T}=-61.5 m V, \tau_{\max }=1123.5 \mathrm{~ms}$

$$
\begin{gathered}
I_{M}=\bar{g}_{M} p\left(V-E_{k}\right) \\
\frac{d p}{d t}=\left(p_{\infty}(V)-p\right) / \tau_{p(V)} \\
p_{\infty}(V)=\frac{1}{1+\exp \left[-\frac{V+35}{10}\right]} \\
\tau_{p}(V)=\frac{\tau_{\max }}{3.3 \exp \left[\frac{V+35}{20}\right]+\exp \left[-\frac{V+35}{20}\right]}
\end{gathered}
$$


Synapses. Synaptic excitatory AMPA and inhibitory GABA-A potentials were modeled based on (Jensen et al., 2005). The synaptic current into neuron $\alpha$ was:

$$
I_{s y n, \alpha}=G_{i n, \alpha}\left(V_{\alpha}-V_{i n}\right)+G_{e x, \alpha}\left(V_{\alpha}-V_{e x}\right)
$$

Here, the total synaptic conductance from inhibitory presynaptic neurons was:

$$
G_{i n, \alpha}=\sum_{\beta i n} g_{\beta \rightarrow \alpha} s_{\beta}
$$

The expression for the excitatory synaptic conductance was of the same form. It was assumed that the dynamics of all synapses of a given (presynaptic) neuron were perfectly synchronized. Hence a synaptic gate, though physically located on the post-synaptic neuron $\alpha$, followed the potential $V_{\beta}$ of the presynaptic neuron $\beta$ with parameters shown in Table 3. For maximum conductance values $g_{\beta \rightarrow \alpha}$ see above.

$$
\frac{\mathrm{ds}_{\beta}}{\mathrm{dt}}=\mathrm{a}_{\beta}\left(1+\tanh \left(\frac{\mathrm{V}_{\beta}}{4}\right)\right)\left(1-\mathrm{s}_{\beta}\right)-\frac{\mathrm{s}_{\beta}}{\tau_{\beta}}
$$

\begin{tabular}{llll}
\hline & Reversal potentials $(\mathrm{mV})$ & $\boldsymbol{\alpha}_{\boldsymbol{\beta}}\left(\mathbf{m s}^{-\mathbf{1}}\right)$ & $\boldsymbol{T}_{\boldsymbol{\beta}}(\mathbf{m s})$ \\
\hline AMPA & $\mathrm{V}_{\mathrm{in}}=0$ & 1 & 2.4 \\
GABA-A & $\mathrm{V}_{\mathrm{ex}}=-80$ & 2 & 20 \\
\hline
\end{tabular}

From left to right: The reversal potential (in $\mathrm{mV}$ ), the rising time constant $\alpha_{\beta}$ and decay time constant $\mathrm{T}_{\beta}$ are shown for AMPA (middle row) and GABA-A (bottom row).

doi:10.1371/journal.pcbi.1004072.t001

Connectivity. For Fig. 1 - 3 the network connectivity parameters (in $\mathrm{mS} / \mathrm{cm}^{2}$ ) are listed in Table 1.. The connectivity matrix network was based on the number of neighbor connections $\mathrm{m}$. For example, $\mathrm{m}=8$ meant that a neuron connected to the closest 8 other neurons with unit connection strength. For E-I connections, $m$ meant that I-cell received input from $m$ E-cells (afferent). For I-E, $m$ meant that I-cell sent input to $\mathrm{m}$ E-cells (efferent). The connectivity parameters were normalized (divided) by the number of connections $\mathrm{m}$. The chosen parameters are listed in Table 2. We describe effects of changing coupling parameters in Suppl.Fig.1. 


\begin{tabular}{lll}
\hline Within network & Number of connections $\mathbf{~ m}$ & Connection strength $\mathbf{~}_{\boldsymbol{\beta} \rightarrow \boldsymbol{\alpha}}\left(\mathbf{m S} / \mathbf{c m}^{2}\right)$ \\
\hline$E \rightarrow I$ (afferent) & 20 & 0.6 \\
$E \rightarrow E$ (afferent) & 35 & 0.05 \\
$\mathrm{I} \rightarrow \mathrm{E}$ (efferent) & 20 & 0.8 \\
$\mathrm{I} \rightarrow \mathrm{I}$ (efferent) & 15 & 0.5 \\
Between network & & \\
$E \rightarrow E$ (afferent) & 10 & $0-0.07$ \\
$E \rightarrow I$ (afferent) & 10 & $0-0.07$ \\
\hline
\end{tabular}

Left column specifies which cell-types are connected. 'Afferent' means that the connection number m defines how many connections from the sending neuron population a neuron receives. 'Efferent' means how many connections a sending neuron has with the receiving neuron population. Middle column shows the number of connection $m$ per neuron. The right column shows the synaptic connection strength $(E \rightarrow I$ or $E \rightarrow E:$ AMPA, $I \rightarrow E$ or $I \rightarrow I:$ GABA-A).

doi:10.1371/journal.pcbi.1004072.t002

\begin{tabular}{lll}
\hline & Number of connections $\mathbf{~}$ & Connection strength $\mathbf{g}_{\boldsymbol{\beta} \rightarrow \boldsymbol{\alpha}}\left(\mathbf{m S} / \mathbf{c m}^{2}\right)$ \\
\hline$E \rightarrow I$ (afferent) & 10 & 0.23 \\
$E \rightarrow E$ (afferent) & 25 & 0.03 \\
$\mathrm{I} \rightarrow \mathrm{E}$ (efferent) & 10 & 0.13 \\
$\mathrm{I} \rightarrow \mathrm{I}$ (efferent) & 4 & 0.1 \\
\hline
\end{tabular}

Left column specifies which cell-types are connected. 'Afferent' means that the connection number m defines how many connections from the sending neuron population a neuron receives. 'Efferent' means how many connections a sending neuron has with the receiving neuron population. Middle column shows the number of connection $m$ per neuron. The right column shows the synaptic connection strength ( $\mathrm{E} \rightarrow \mathrm{I}$ or $\mathrm{E} \rightarrow \mathrm{E}:$ AMPA, $\mathrm{I} \rightarrow \mathrm{E}$ or $\mathrm{I} \rightarrow \mathrm{I}:$ GABA-A).

doi:10.1371/journal.pcbi.1004072.t003

Network input. The input to each neuron consisted of external excitatory input plus internal excitatory and inhibitory input via network connections. The external input consisted of a train of AMPA synaptic conductance spikes (double exponentials: rising constant $=1 \mathrm{~ms}$, decaying constant $=5.2 \mathrm{~ms}$ ) with Poisson statistics at a rate of $800 \mathrm{~Hz}( \pm S D=100)$ and spike amplitudes of default $0.02 \mathrm{mS} / \mathrm{cm}^{2}( \pm S D=0.002)$. The default mean AMPA input level to each neuron was $0.01 \mathrm{mS} / \mathrm{cm}^{2}$ for FS neurons. For Fig. 1 the mean amplitude of the AMPA synaptic potentials were modulated from 0.02 to $0.08 \mathrm{mS} / \mathrm{cm}^{2}$ for RS by modulating the spike amplitude. In Fig. 2 the mean AMPA conductance input level was $0.06 \mathrm{mS} / \mathrm{cm}^{2}$. In Fig.3 each neuron received a spatially specific input level depending on its position in the ring architecture. The amplitude of the sinusoidally modulated AMPA conductance was of $0.006 \mathrm{mS} / \mathrm{cm}^{2}$ and the mean conductance AMPA input $0.06 \mathrm{mS} / \mathrm{cm}^{2}$ and $0.055 \mathrm{mS} / \mathrm{cm}^{2}$ for Suppl. Fig 1. 
Spike detection and network signal. The voltage used as the spike detection level was $-17.5 \mathrm{mV}$ for both E- and I-cells. The local field potential ( LFP) was estimated in units of microVolt ( $\mu \mathrm{V}$ ) for Fig.1 \& 2 as an overall network signal. The LFP was the extracellular electrical field potential $\operatorname{LFP}=\operatorname{LFP}\left(\mathbf{r}_{0}, t\right)$ at an electrode position $\mathbf{r}_{0}$. We treated neuron $i$ at position $\mathbf{r}_{\mathbf{i}}$ as a point-current source $\mathbf{I}=\mathrm{I}\left(\mathbf{r}_{\mathbf{i}}, t\right)$ (total transmembrane current into the neuron) in a homogeneous extracellular medium with conductivity $\sigma$ $(1 / \sigma=0.3 \mathrm{k} \Omega m$, taken from (Logothetis, 2003): $0.2-0.4 \mathrm{k} \Omega \mathrm{cm})$. We summed the individual neuron contributions according to the quasistatic Maxwell equations:

$$
L F P=\frac{1}{4 \pi \sigma} \sum_{i}^{N} \frac{I\left(\boldsymbol{r}_{i}, t\right)}{R}
$$

with $R=\left|\boldsymbol{r}_{\boldsymbol{i}}-\boldsymbol{r}_{0}\right|$ the distance of the point source to the electrode $(\mathrm{R}=\sim 1 \mathrm{~mm})$. The extracellular voltage signal was smoothed with a pseudo-Gaussian function (width $=4 \mathrm{~ms}$ ).

For computing local synchronous rhythmic activity of local population of neurons in Suppl. Fig.1 to show effects on noise on rhythmic population activity and rhythmic single neuron activity, we derived a local population average signal (LPA) based on the spike trains of the neurons. For each position in the network, we aggregated the spike activity of the whole network weighted by a spatially exponentially decaying function.

$$
L P A_{i}=\frac{1}{N} \sum_{j=1}^{N} r_{i} \exp ^{-D_{i, j} / \mathrm{s}}
$$

With $r_{i}$ being the binary spiking variable, $D_{i, j}$ being the spatial distance between neurons (defined circularly on the ring in radians). S corresponds to the spatial decay constant which was chosen to be 0.4. The rationale of the value (similar results were observed for a large range of values) was that it was large enough to allow for sufficient aggregation to quantify oscillatory activity and spatially specific enough to reveal the spatial change of phase-locking and phase-relation. The LPA was further smoothed with a pseudo-Gaussian function (width=4ms).

Natural contrast images and intrinsic frequency map. We obtained natural images from the Berkeley segmentation dataset (BSDS500, (Martin et al., 2001)). We took the first 100 gray-scale natural images (comprising the Berkeley training dataset). The natural images were first resampled and squared to fit it to the $100 \times 100$ lattice. Then the local root-mean squared contrast $C=C\left(x_{c}, y_{c}\right)$ at image position $\left(x_{c}, y_{c}\right)$ (RMS, (Frazor and Geisler, 2006)) was computed:

$$
\begin{gathered}
C=\sqrt{\sum_{i=1}^{N} w_{i} \frac{\left(L_{i}-L\right)^{2}}{L^{2}} / \sum_{i=1}^{N} w_{i}} \\
w_{i}=0.5\left(\cos \left(\frac{\pi}{p} \sqrt{\left(x_{i}-x_{c}\right)^{2}+\left(y_{i}-y_{c}\right)^{2}}\right)+1\right)
\end{gathered}
$$


$L$ is luminance and $\mathbf{i}$ is the pixel index. The summation was over pixels within a patch radius $p$ of 3 pixels. The local contrast values $C$ were then transformed into intrinsic frequencies $v$ by approximating the experimentally observed relationship $v=25+0.25 C$ between gamma frequency and contrast. We defined a minimum $(25 \mathrm{~Hz})$ and slope of $0.25 \mathrm{~Hz}$ per contrast value (estimated over both monkeys,(Roberts et al., 2013)). Gamma power was not taken into account in the phase-oscillator model. 20 of 100 images were excluded because not enough segmentation borders (see criteria below) suitable for phase-locking analysis (minimum of $>10$ per image) could be obtained.

Phase-Oscillator model. We used a modified version of the Kuramoto model (Breakspear et al., 2010) as a basic model of the dynamics of a limit-cycle oscillators that has been used to investigated synchronization between coupled oscillators. The network input was set by natural images transformed into local contrast. The intrinsic frequency of each oscillator was set by the local contrast at the oscillator's corresponding pixel. For each image, the simulation run was $10 \mathrm{~s}$ with a time step of $2 \mathrm{~ms}$. Each oscillator started with a random phase. During the simulation run the phase of each oscillator was determined by an intrinsic (natural) frequency $(\omega)$, a noise term $(\zeta)$ and an interaction term describing the impact (phase response curve, PRC) by other coupled oscillators depending on the coupling constant (K).

$$
\frac{\mathrm{d} \theta_{\mathrm{i}}}{\mathrm{dt}}=\omega_{\mathrm{i}}+\zeta_{\mathrm{i}}+\sum_{\mathrm{j}=1}^{\mathrm{N}} \mathrm{K}_{\mathrm{i}, \mathrm{j}}\left(-\sin \left(\theta_{\mathrm{j}}-\theta_{\mathrm{i}}\right)\right), \quad \mathrm{i}=1 \ldots \mathrm{N}
$$

The interaction term (infinitesimal PRC, (Breakspear et al., 2010)) was a sinusoidal function such that the coupled oscillators tended to engage in zero-phase synchrony. The coupling constant was an exponential function of distance (D) (in contrast to the all-to-all connectivity in the Kuramoto model), with a scaling constant ( $s=0.4$ for ring-network (radians) and $s=0.5$ for $2 \mathrm{D}$ lattice network (pixel)) and strength $(C=1.65)$.

$$
\mathrm{K}_{\mathrm{i}, \mathrm{j}}=\mathrm{C} \exp ^{-\mathrm{D}_{\mathrm{i}, \mathrm{j}} / \mathrm{s}}
$$

The noise term was pink noise with a power scaling exponent of 1 . The strength $C$ was scaled at a sufficient level for the model to reach near-zero coherence when oscillators were uncoupled. The noise was spatially correlated (smoothed with spatial kernel of 3 pixels), to reduce spurious phase locking over the lattice. This step eased the computation of 'true' phase-locking between distant clusters having very close frequencies (without the use of phase-perturbation techniques), because synchronous clusters cannot easily average out noise (correlated between members of a cluster). We also included a timedelay term as function of distance as conduction delay of cortical horizontal connections can be significant for longer cortical distances (Angelucci and Bullier, 2003; Boucsein et al., 2011).

$$
\mathrm{T}_{\mathrm{i}, \mathrm{j}}=\mathrm{vD}_{\mathrm{i}, \mathrm{j}}+\mathrm{v}_{\mathrm{o}}
$$

Where the time-delay $T_{i, j}$ was a linear function of distance (pixel units). The slope $v$ was 0.4 and $v_{0}$ was $2 m s . T_{i, j}$ was then made then discrete to change in steps of $2 \mathrm{~ms}$ (simulation time step). The inclusion of the time-delay factor was not critical for the results of the paper. Natural images and segmentations by human observers were taken from the Berkeley segmentation dataset (Martin et al., 2001). Images were 
downsampled from $350 \times 450$ pixels to $100 \times 100$ pixels using the Matlab in-built 'imresize' function, to fit the size of the lattice model.

Spectral power estimations. In Fig.1 as well as Suppl. Fig.2 we used the Matlab in-built power spectral density function ( $p s d)$ with multitaper estimation for estimating the power spectrum. For the timefrequency representation (TFR) in Fig.2c we used the Matlab in-built spectrogram function (Short-time Fourier transform).

Instantaneous phase and frequency estimations. The instantaneous phase (IP) was derived for the LFP (Fig.1-2) or LPA (Suppl.Fig.2) signals by taking the Hilbert-transform (HT, (Picinbono, 1997)) of the signal. The HT gives the analytical signal (complex numbers) from which the IP can be obtained by taking the argument of the complex number. The HT is well defined for signals characterized by a single oscillation (mono-component) which was the case in our simulations. The IP was the output variable of the phaseoscillator model. The instantaneous frequency (IF) was obtained by taking the derivative of the IP. IF was estimated by unwrapping the IP, then first applying smoothing (half-cycle rectangular points smoothing) followed by computing the first derivative. For the phase-oscillator model (Fig.5-8), we could directly use the output phase-traces to compute IF. We averaged the IF estimation of each time point over the whole simulation (excluding the first $200 \mathrm{~ms}$ ) period to obtain a mean frequency. For single neurons spike trains we used the spike rate, computed as $\mathrm{n}$ spikes per second, as our frequency estimation.

Phase-locking and phase relation estimations.

\section{A. Based on Instantaneous phase (LFP,LPA and phase-oscillators)}

The phase relation was defined as the mean circular phase difference between two signals (averaged in the complex domain).

$$
\theta_{i j}=\arg \left(\frac{1}{N} \sum_{i=1}^{N} \exp \left(i\left(\varphi_{i}-\varphi_{j)}\right)\right)\right.
$$

with a range of $[-\pi, \pi]$. Arg is the argument function and $\varphi$ is the IP. For estimating phase-locking we computed the phase-locking value (PLV, (Lachaux et al., 1999)). The PLV was computed by averaging the complex values with unit amplitude

$$
\psi_{i j}=\mid \frac{1}{N} \sum_{i=1}^{N} \exp \left(i\left(\varphi_{i}-\varphi_{j}\right) \mid\right.
$$

The PLV ranges from 1, corresponding to full phase consistency, to 0 , corresponding to fully random.

\section{B. Based on spike trains}

For computing the phase-relation and locking between two neurons we applied cross-correlation.

$$
C C_{i j}(l)=\frac{1}{N}\left(\sum_{n=0}^{N-1} r_{i}^{*}[n] r_{j}[n+l]\right)
$$

with $r_{i}^{*}$ being the complex conjugate $\left({ }^{*}\right)$ of spike train $(r)$ of neuron $\mathrm{i}$. The cross-correlation $C C_{i j}$ between neuron I and $\mathrm{j}$ was computed with lags not exceeding +/- half mean rate (time window is assumed to be the period of the oscillation the neurons are locked to). The spike timing difference (in ms) was defined as 


$$
s t_{i j}=\arg \left(\max \left(C C_{i j}\right)\right)
$$

and the locking as

$$
\psi_{i j}=\max \left(C C_{i j}\right)
$$

We converted the spike timing differences into phase-values by dividing twice timing difference by the mean spike rate of the respective neurons and then multiplied by $\pi$.

$$
\theta_{i j}=\pi\left(\frac{2 \mathrm{st}_{\mathrm{ij}}}{\left(\mathrm{r}_{\mathrm{i}}+\mathrm{r}_{\mathrm{j}}\right) / 2}\right)
$$

Phase-locking and phase-relation matrix. The matrices represent the phase-locking or the phaserelation between all possible pairs of neurons or oscillators. The diagonal is always 1 (phase-locking with itself) in the phase-locking matrix and 0 in the phase-relation matrix. For the phase-locking matrix the color were from 0 (black) to 1 (yellow-white), if not otherwise stated. For the phase-relation matrix the color were - pi/2 (blue) to pi/2 (red). A negative phase relation (blue) means that the neuron/oscillator $X$ from the $x$-axis has an earlier/leading phase compared to the neuron/oscillator $Y$ from the $y$-axis. The phase-relation matrix was threshold for illustration purposes, because phase-relations from nonsynchronized neurons/oscillators are randomly distributed over -pi to pi making the plot difficult to interpret visually. The threshold was defined as being equal to 3 times the mean phase-locking value between uncoupled neurons/oscillators.

Segmentation border analysis. We used the image segmentations performed by several human observers ( $n=30)$ from the Berkeley segmentation dataset (BSDS500,(Martin et al., 2001)). All subjects did not segment all the images, instead, segmentations from a subset of the observers was available $(n \sim 5)$ for each image. For each image the segmentation-border analysis based on different observers was averaged. We selected 1-dimensional spatial windows of \pm 15 pixels centered on segmentation lines that fulfilled the following criteria: (1). A vertical or horizontal segmentation line should consist of three consecutive pixels. (2) Within the spatial window no other line should be present. For the analysis, the horizontal and vertical line segments were concatenated. We then computed the averaged phaselocking matrix (Suppl.Fig.4) between all oscillators as well as the averaged absolute spatial derivative of contrast values. For computing significance thresholds (permutation testing, (Maris and Oostenveld, 2007)) we constructed a null distribution by choosing random positions for the same number of spatial windows.

Stimulus reconstruction. The stimulus to be reconstructed $S_{\text {orig }}(i)$ for each network position $i$ was the excitatory (AMPA) input drive to E-cells for the PING networks and the intrinsic frequency for the phaseoscillator model. For ring-networks the spatial variation of $\mathrm{S}_{\text {orig }}$ was defined by a sinusoidal function and for the 2D phase-oscillator lattice network by natural image local contrast, where each network position corresponded to one pixel. A seemingly easy way of estimating the stimulus $\mathrm{S}_{\text {orig }}$ is by using frequency coding $S_{e s t}(\omega)$. If it is defined at single neuron level, it is often termed spike rate. At neuronal population level, the code might be based on the oscillation frequency. In the Discussion section we discuss these different type of frequency coding and their relation (see also Suppl. Fig. 1). The spike rate 
is defined as the number of spikes per second for a given time window (spike count code,[]). This was used for Fig. 3. The oscillation frequency was determined as the mean instantaneous frequency [] over the simulation period. This was used for Fig.2 (LFP), Fig. 6 and 7 (phase-oscillator) and Suppl. Fig. 1 (LPA). The $S_{e s t}(\omega)$ was simply defined as the frequency of the neuron i

$$
S_{\text {est }}(\omega)=\omega_{i}
$$

The stimulus $\mathrm{S}_{\text {orig, }}$ estimated by phase differences between neurons, was defined as follows:

$$
S_{\text {est }}(\theta)=\left(\sum_{j=1}^{j=n} \theta_{i j} \psi_{i j} K_{i j}\right) / n
$$

$\theta_{i j}$ is the phase-relation, $\psi_{i j}$ the phase-locking and $K_{i j}$ the connectivity strength between the reference neuron $\mathrm{i}$ and the neighbor neuron j. $\theta_{i j}$ was determined using the whole simulation period. For analysis based on spike trains, the phase-relation and strength was determined by cross-correlation analysis (see above), whereas for LPA or phase-oscillator analysis it was based on the instantaneous phase variable (for LPA determined by Hilbert transform). The connectivity strength was defined prior to simulation (see above) and was an exponential function decaying over distance (phase-oscillator model) or was of nearest neighbor type with unit strengths (PING network). For all network types, the interaction strength (determined by direct and indirect connections) decayed over distance approximated as an exponential decay function over space with the same parameter used for all network types. Including the connectivity term improved the $S_{\text {est }}$ in particular for the 2D phase-oscillator lattice model $(\mathrm{MI}=0.60$ to 0.67$)$. This is because the detuning-to-phase conversion is coupling dependent. The phase value of each neuron $\mathrm{i}$, was computed by averaging phase-relations to all other neurons in the network weighted by phase-locking strength and coupling strength. $S_{e s t}(\theta)$ is an assembly code using the spike relation between neurons to obtain more information about the stimulus.

For the combined frequency and phase code $S_{e s t}(\omega, \theta)$ the stimulus level $S_{\text {est }}$ for a given neuron was then given as

$$
S_{e s t}(\omega, \theta)=S_{e s t}(\omega)+S_{e s t}(\theta) * F
$$

where $F$ is a scaling factor determining the contribution of the phase code. The scaling factor $F$ which maximized the stimulus reconstruction performance was chosen. Intuitively, the optimal scaling factor is the slope of the function between intrinsic frequency and the phase variable (see red line in Fig. 2J \& $6 \mathrm{IV})$ for a given coupling value. One common scaling factor was chosen for all 80 natural image simulations. 
Measure of stimulus reconstruction performance. We estimated the reconstruction performance as the Shannon mutual information I(X; Y) (Duncan, 1970) between the intrinsic frequency image $X$ and the reconstructed intrinsic frequency image $\mathrm{Y}$. The direct method approach gives:

$$
\mathrm{I}(\mathrm{X} ; \mathrm{Y})=\sum_{\mathrm{y} \in \mathrm{Y} \in \mathrm{X} \in \mathrm{X}} \mathrm{p}(\mathrm{x}, \mathrm{y}) \log \left(\frac{\mathrm{p}(\mathrm{x}, \mathrm{y})}{\mathrm{p}(\mathrm{x}) \mathrm{p}(\mathrm{y})}\right)
$$

where $p(x, y)$ is the joint probability, $p(x)$ and $p(y)$ are the marginal distributions. We normalized $I(X ; Y)$ by dividing by $I(X ; X)$. We compare the phase-code, frequency-code and the combined frequency/phase code. For the natural images, we computed a baseline reconstruction performance, for each image we computed the normalized MI between the simulation output from that image with the intrinsic frequency maps from all other images. We averaged the $79 \mathrm{MI}$ values $(79 \mathrm{MI}$ values per 80 images, each image compared with all other images) to get an estimate of baseline reconstruction for each image. We used a repeated measures ANOVA to test for significant effect of coding types. For posthoc pairwise comparisons (Jaccard et al., n.d.) between different coding types we used the Tukey's HSD (honest significant difference) test (which corrects for multiple comparisons). The Tukey's HSD was computed as follows:

$$
H S D=\frac{\left(\mu_{1}-\mu_{2}\right)}{\sqrt{M S E / N}}
$$

With $\mu$ the mean value of a condition, MSE is the mean sum squared error and $\mathrm{N}$ is the number of values within a condition.

\section{Results}

\section{The frequency of gamma oscillations depends on excitatory input drive.}

During active information processing, a cortical network will receive variable afferent input drive reflecting sensory variables. By input drive we mean the net excitatory drive to a population of neurons resulting from the sum of afferent excitatory and inhibitory connections. The dependence of gamma oscillations on the input drive is central for understanding its role in neural processing. Theoretical (Jia et al., 2013b; Roberts et al., 2013; Tiesinga and Sejnowski, 2009) and experimental observations (Jia et al., 2013b; Ray and Maunsell, 2010; Roberts et al., 2013; Steriade et al., 1996; Traub et al., 1996) have shown that excitatory drive increases the frequency of gamma oscillations. For example, recent experimental studies on gamma oscillations in primate visual cortex have shown a striking relationship between visual contrast, which is considered a proxy for excitatory drive (Jia et al., 2013b; Ray and Maunsell, 2010; Roberts et al., 2013), and the frequency and power of gamma oscillations. Results from our own experimental work (Roberts et al., 2013) demonstrated the effect of contrast on gamma oscillations in primary visual cortex V1 and in V2 of rhesus monkeys (Fig.1A). We found a monotonic increase in the frequency at which the gamma frequency spectrum peaks (Fig. 1B, top) with increased contrast, and a non-monotonic modulation of gamma power (Fig.1B, bottom). 

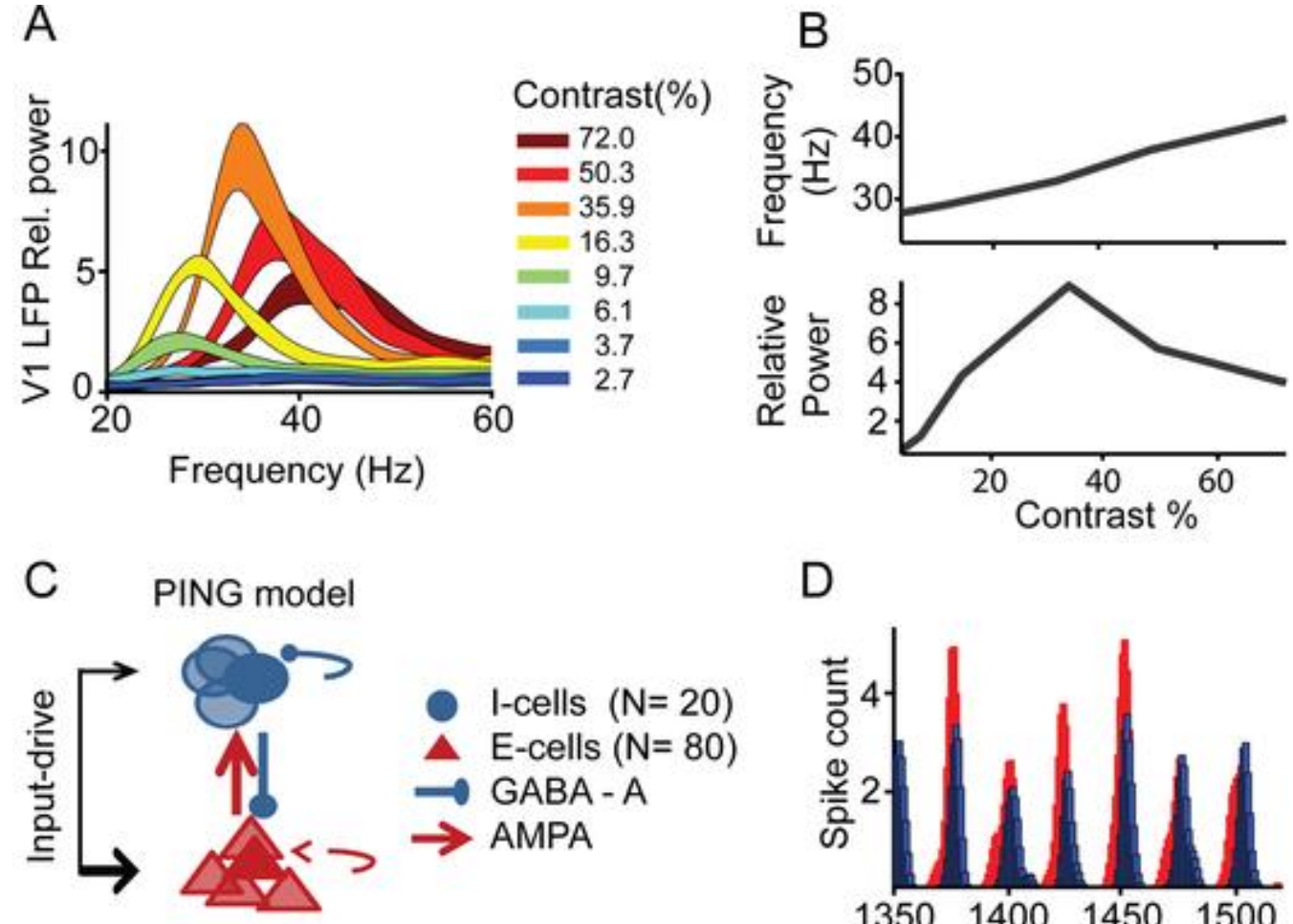

$\mathrm{D}$
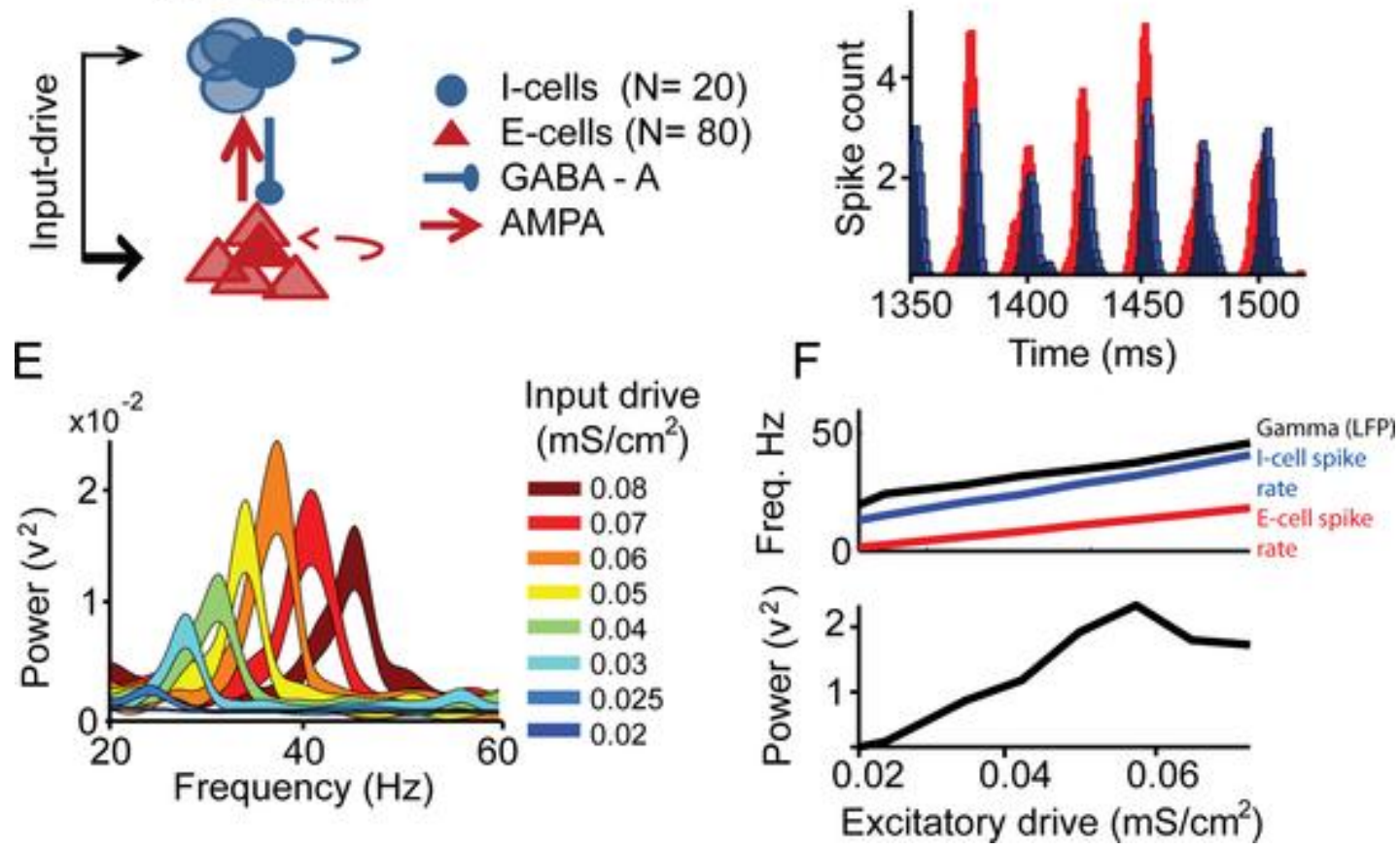

Figure 1: Luminance contrast and input-drive dependent gamma oscillation frequency (A) V1 LFP mean relative power spectra $(20-60 \mathrm{~Hz}$, line thickness represents \pm 1 SEM) during presentation of square wave gratings of 8 different luminance contrasts (line color), mean data from Monkey S [8]. (B) Gamma band peak frequency (top) and power (bottom) as a function of contrast (only the 6 highest contrast conditions). (C) Schematic architecture of the pyramidal (red) - interneuron (blue) gamma network (PING). (D) Example time period of population spike histogram ( $2 \mathrm{~ms}$ bins) during steady excitatory drive input (0.06 $\mathrm{mS} / \mathrm{cm}^{2}$ ). Spikes of the excitatory (red) neurons occurred earlier than from inhibitory (blue) neurons within a gamma cycle. (E) The absolute power spectra for different input excitation levels (mimicking contrast). (F) Quantification of (upper panel) gamma frequency (black), I-cell spike rate (blue), E-cell spike rate (red) and (lower panel) gamma power as a function of excitatory input

\section{The frequency of gamma oscillations depends on excitatory input drive}

During active information processing, a cortical network will receive variable afferent input drive reflecting sensory variables. By input drive we mean the net excitatory drive to a population of neurons resulting from the sum of afferent excitatory and inhibitory connections. The dependence of gamma 
oscillations on the input drive is central for understanding its role in neural processing. Theoretical (Jia et al., 2013b; Roberts et al., 2013; Tiesinga and Sejnowski, 2009) and experimental observations (Jia et al., 2013b; Ray and Maunsell, 2010; Roberts et al., 2013; Steriade et al., 1996; Traub et al., 1996) have shown that excitatory drive increases the frequency of gamma oscillations. For example, recent experimental studies on gamma oscillations in primate visual cortex have shown a striking relationship between visual contrast, which is considered a proxy for excitatory drive (Jia et al., 2013b; Ray and Maunsell, 2010; Roberts et al., 2013), and the frequency and power of gamma oscillations. Results from our own experimental work (Roberts et al., 2013) demonstrated the effect of contrast on gamma oscillations in primary visual cortex V1 and in V2 of rhesus monkeys (Fig.1A). We found a monotonic increase in the frequency at which the gamma frequency spectrum peaks (Fig. 1B, top) with increased contrast, and a non-monotonic modulation of gamma power (Fig.1B, bottom).

These findings fit with theoretical studies of the two most common gamma oscillation generating mechanisms (Tiesinga and Sejnowski, 2009), the interneuron-gamma network (ING, e.g. (Brunel and Wang, 2003a; Wang and Buzsaki, 1996)) and the pyramidal-interneuron gamma network (PING, e.g. (Buia and Tiesinga, 2006; Roberts et al., 2013)), which are characterized by increasing oscillation frequency with increasing excitatory drive. We replicated this relationship in a model network consisting of $20 \mathrm{l}$-cells (fast-spiking type) and 80 E-cells (regular spiking type) using model neurons based on the Hodgkin-Huxley formalism (Fig.1C, see Methods,(Pospischil et al., 2008)). Model neurons interacted through model synapses (Jensen et al., 2007) that included AMPA and GABA-A connections. Each neuronal class received independent external excitatory input, yet the main excitatory input for I-cells was internally generated by E-cells (Fig.1C). The network exhibited pyramidal-interneuron gamma oscillations (PING) characterized by I-cell spikes lagging E-cell spikes (Fig.1D). We then (Fig.1E) systematically modulated external excitatory input to the network (modeled as a train of AMPA-spikes), with the mean level of input ranging from 0.02 to 0.08 milliSiemens per area $\left(\mathrm{ms} / \mathrm{cm}^{2}\right)$. GABA-A decay time constant $(20 \mathrm{~ms})$ was defined such that frequencies were in the range as observed in our own experimental V1 LFP recordings. However, the exact frequency range is not critical for the conclusions of the paper. We observed input-dependent effects on the model power spectra based on the estimated LFP (from transmembrane currents, see Methods). Gamma oscillation frequency increased monotonically with input-drive over a range of $\sim 25 \mathrm{~Hz}$ (Roberts et al., 2013), as did the spike rates (Fig.1F, top). Oscillation power (Fig.1F bottom) showed a nonlinear relationship with oscillation frequency, with peak power at intermediate levels of input (Roberts et al., 2013). In line with previous work (for review (Tiesinga and Sejnowski, 2009)), the main time constant of the PING network oscillation was set by the inhibitory GABA-A decay time constant and by the time needed for the E-cells to escape the inhibition, the latter being reduced by higher excitatory drive. We assume here that synaptic time constants and connectivity strengths did not change within the time-scale considered here for the stimulation (several $100 \mathrm{~ms}$ to a few seconds). 


\section{Role of detuning and coupling in regulating synchronization and phase relations between two interacting gamma PING networks}

As described above, there is substantial evidence that gamma oscillations adapt their frequency as a function of input-drive. But what happens if input-drive varies over cortical space? An experimental study in macaque V1 (Ray and Maunsell, 2010) with contrast-varying stimuli has shown that the frequency of gamma oscillation can vary over a short cortical distance, with higher contrast producing higher frequencies. Hence, nearby cortical location can show different oscillation frequencies. This supports older studies in V1 (Eckhorn et al., 2001; Gail et al., 2000) that showed that gamma phaselocking decayed rapidly over cortical distance at the spatial scale of horizontal connectivity. In the light of those findings, a theoretical model of cortical gamma oscillation should be able to express different oscillation frequencies at nearby spatial locations. Such results cannot be simulated in gamma network models that are characterized by global synchronization and express one dominant frequency at a time (Wang and Terman, 1995). Gamma oscillation networks with predominantly local spatial connectivity with locally varying input drive could thus be a promising framework for cortical gamma oscillations. We therefore aimed to gain an understanding of the underlying principles that cause these networks to organize themselves depending on spatially varying input drive.

A

B

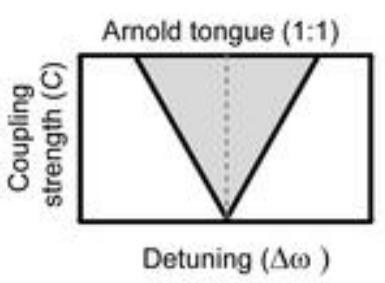

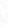

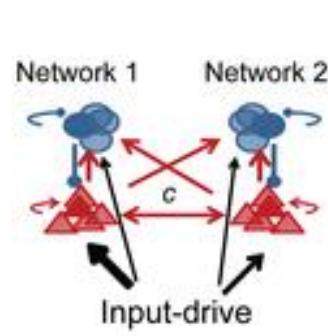

C - Estimated LFP



E

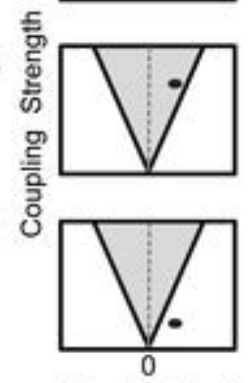

Detuning $(\Delta \omega)$
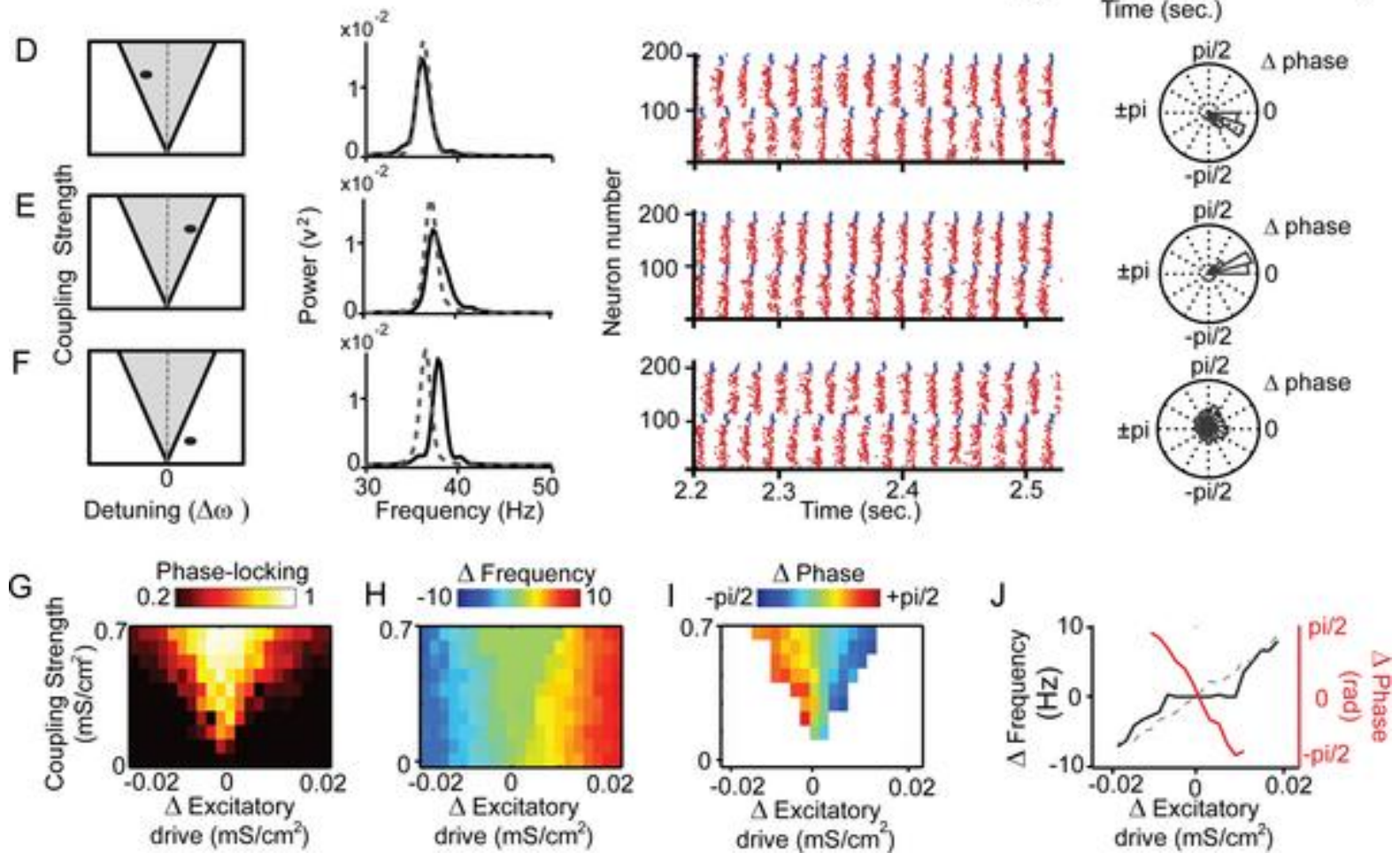
Figure 2: Impact of oscillation frequency on the interaction between two PING gamma networks. (A) Illustration of the Arnold tongue. The potential for two oscillators to synchronize (grey area) is positively correlated with coupling strength and negatively correlated with the difference in intrinsic frequency. (B) The main structure of the two coupled PING gamma networks. Excitatory drive difference (detuning, $\Delta \omega$ ) and the coupling strength $C$ between the two networks were modulated. (C) Example simulation output from the networks, upper) smoothed LFP signal, arbitrary scaling, lower) time-frequency representation. (D-F) Example simulations with different network parameters: Left to Right: Arnold tongue: Parameters $\Delta \omega$ and Coupling Strength are indicated as black dot in Arnold tongue diagram. Power spectra, shown in black line for simulated LFP for network 1 and in dashed grey line for network 2. Population raster plots shown for simulation window [2.2sec to 2.55sec]: network 1= neuron 1-100, network 2 = neurons 101-200. E-cells spikes are indicated by red dots, I-cells spikes by blue dots. Polar plot of phase difference are shown to the right. Bar length indicates percentage of time (from $5 \mathrm{~s}$ trial) within each phase bin. G-I) Reconstruction of Arnold Tongue when manipulating coupling strength and detuning (detuning $=$ input-drive $_{\text {network1 }}-$ input-drive $_{\text {network2 }}$ ). Arnold Tongue corresponds to a region with strong phase locking (G, bright colors), common emergent frequency $(H$, green color), and systematic phase difference ( $\mathrm{l}$, color-coded) between coupled networks, Network 1 lagged in phase compared to network 2 (red in I) when network 1 received a lower drive. Conversely, network 1 had a leading phase relationship with network 2 (blue in I) when network 1 received the higher excitatory drive $\Delta$ phase is only shown for conditions of substantial phase locking ( $>0.3)(\mathrm{J})$ Overlaid representation of emergent frequency difference (black line), intrinsic frequency difference (dashed line) and phase difference (red line) for simulations with inter-network excitatory connections of $0.6 \mathrm{mS} / \mathrm{cm}^{2}$.

A theoretical framework for understanding the self-organization principles of such a network with spatially local emerging oscillations is offered by the theory of weakly coupled oscillators (TWCO). The TWCO describes under which conditions interacting (coupled) oscillators synchronize. The ability of a population of coupled oscillators (Breakspear et al., 2010) to synchronize is controlled by two opponent forces (Pikovsky et al., 2002): their detuning ( $\Delta$ intrinsic frequency) and their interaction strength (here through synaptic interactions), to which we refer as coupling strength. The region in the twodimensional parameter space of coupling strength and detuning within which synchronization occurs is called the 'Arnold tongue' (Pikovsky et al., 2002). For conditions within the Arnold tongue (Fig.2A), the oscillators converge on a common emergent frequency. Within the Arnold tongue, the initial (intrinsic) frequency difference between the pair is replaced by a consistent phase difference, where the oscillator with the higher intrinsic frequency leads in phase. Outside the Arnold tongue, intrinsic frequency differences are maintained, precluding a consistent phase relationship (i.e., phase precession instead of synchronization).

We first illustrate these ideas in simulations from a gamma model consisting of two interconnected PING networks (Fig.2B, see also (Tiesinga and Sejnowski, 2010)). The two PING networks were both identical to the network introduced in Fig.1C-F, with inhibitory neurons only projecting locally within their own network to excitatory $(I \rightarrow E)$ and inhibitory cells $(I \rightarrow I)$. The inter-network connectivity was comprised of excitatory-to-inhibitory connections $(E \rightarrow I, I$ receiving input from $8 \mathrm{E}$ ) and excitatory-toexcitatory connections ( $\mathrm{E} \rightarrow \mathrm{E}, 8$ per neuron). In different model simulations, the two inter-network connection types (coupling) were modulated jointly from 0 to $0.07 \mathrm{~ms} / \mathrm{cm}^{2}$ (note that these values are an order of magnitude lower than in intra-network coupling, see Methods). Fig. $2 \mathrm{C}$ shows an example of simulation output with estimated LFP traces (top) and corresponding time-frequency representations (TFRs, bottom). In this example, the drive to the two coupled networks (here coupled with $0.004 \mathrm{mS} / \mathrm{cm}^{2}$ ) was very similar (network $1 / 2=0.069 / 0.0635 \mathrm{~ms} / \mathrm{cm}^{2}$ ). This resulted in closely matching 
oscillation behavior. We then used this model to study the effects of varying input drive differences and of varying coupling strength.

Fig.2D-F shows the detailed effects of three combinations of coupling strength and detuning (intrinsic frequency difference) on the ability of coupled oscillating networks to synchronize. Phase locking was estimated here based on the population response of each PING network (here LFP, see Methods). In Fig.2D, network 2 (neurons 101-200 in simulated spike histograms) received (network $1 / 2=$ $0.0598 / 0.0635 \mathrm{mS} / \mathrm{cm}^{2}$ ) more excitatory input than network 1 (neurons 1-100). Because of a sufficiently small difference in input and intrinsic frequency at the chosen coupling strength (a parameter constellation falling within the Arnold tongue, $0.02 \mathrm{mS} / \mathrm{cm}^{2}$ ), the networks synchronized at a common emergent frequency $(\sim 35.5 \mathrm{~Hz})$. This is visible (from left to right) in the overlapping power spectra, in the consistent time difference between spikes of network 1 and 2 in the population spike raster, and in the narrow phase difference distribution (see Methods). The spike raster and the phase difference distribution also show that spikes in network 2 were leading spikes in network 1. In Fig.2E, the excitation level difference in the networks were approximately reversed (network $1 / 2=0.069 / 0.0635 \mathrm{mS} / \mathrm{cm}^{2}$ ) while keeping coupling strength constant. Again, the networks synchronized at a common frequency $(\sim 37 \mathrm{~Hz})$, but spikes of network 2 now lagged network 1. In Fig.2F the coupling (cross E-E, E-I) between networks 1 and 2 was decreased from $0.02 \mathrm{mS} / \mathrm{cm}^{2}$ to $0.004 \mathrm{mS} / \mathrm{cm}^{2}$, while keeping the detuning constant (network $1 / 2=0.069 / 0.0635 \mathrm{~ms} / \mathrm{cm}^{2}$ ), creating a condition falling outside the Arnold tongue region. The two networks therefore did not synchronize but oscillated at different frequencies (network $1 / 2=\sim 36 \mathrm{~Hz} / \sim 38 \mathrm{~Hz}$ ). By systematically modulating the coupling strength and the detuning between the two networks, the Arnold tongue could be fully reconstructed: it emerged as a region of high phaselocking (Fig.2G) characterized by a common emergent frequency (Fig.2H) and systematic phase differences (Fig.2l). Fig.2J shows a comparison of the intrinsic frequency of one network observed in the absence of coupling (dashed line), and its emergent frequency when coupling was set to $0.04 \mathrm{mS} / \mathrm{cm}^{2}$ (black solid line). As in Fig.1F, the intrinsic frequency depended linearly on the input level. However, the emergent frequency during coupling displayed a non-linear function, whereby frequency was constant within the range of the Arnold tongue. Within that range synchronization was observed; meaning that a consistent phase relationship emerged ( $\Delta$ phase, red line). The phase relationship was linearly related to the input level difference.

\section{Input-dependent self-organization of a spatially extended gamma network}

We described above how the behavior of two interacting PING-networks can be understood in the framework of TWCO. However, to understand the self-organization principles of gamma oscillation activity in a cortical area, one needs to take into account interactions among large numbers of interconnected neurons that constitute multiple potential local PING networks. The local networks may be more easily comparable to anatomically distinct 'columns' (which may or may not underlie

functionally defined columns) in some sensory systems (e.g., barrel cortex) than in others (e.g., visual cortex), but this correspondence is not critical to our argument. In this study, we used continuous local connectivity and spatially specific input drive as the more general case. We chose a model architecture in which neurons were organized along a ring (Fig $3 \mathrm{~A}$, see Methods), to avoid border effects and thus 
facilitate analysis. For the generation of the PING mechanism, E-cells and I-cells were designed to have relatively strong local interactions $(E-I, I-E)$ between neighbors $\left(E-I=0.23 \mathrm{mS} / \mathrm{cm}^{2}, 10\right.$ per neuron, I-E $=0.13 \mathrm{mS} / \mathrm{cm}^{2}, 10$ per neuron ). Inhibitory-to inhibitory connections (I-I) further supported the PING
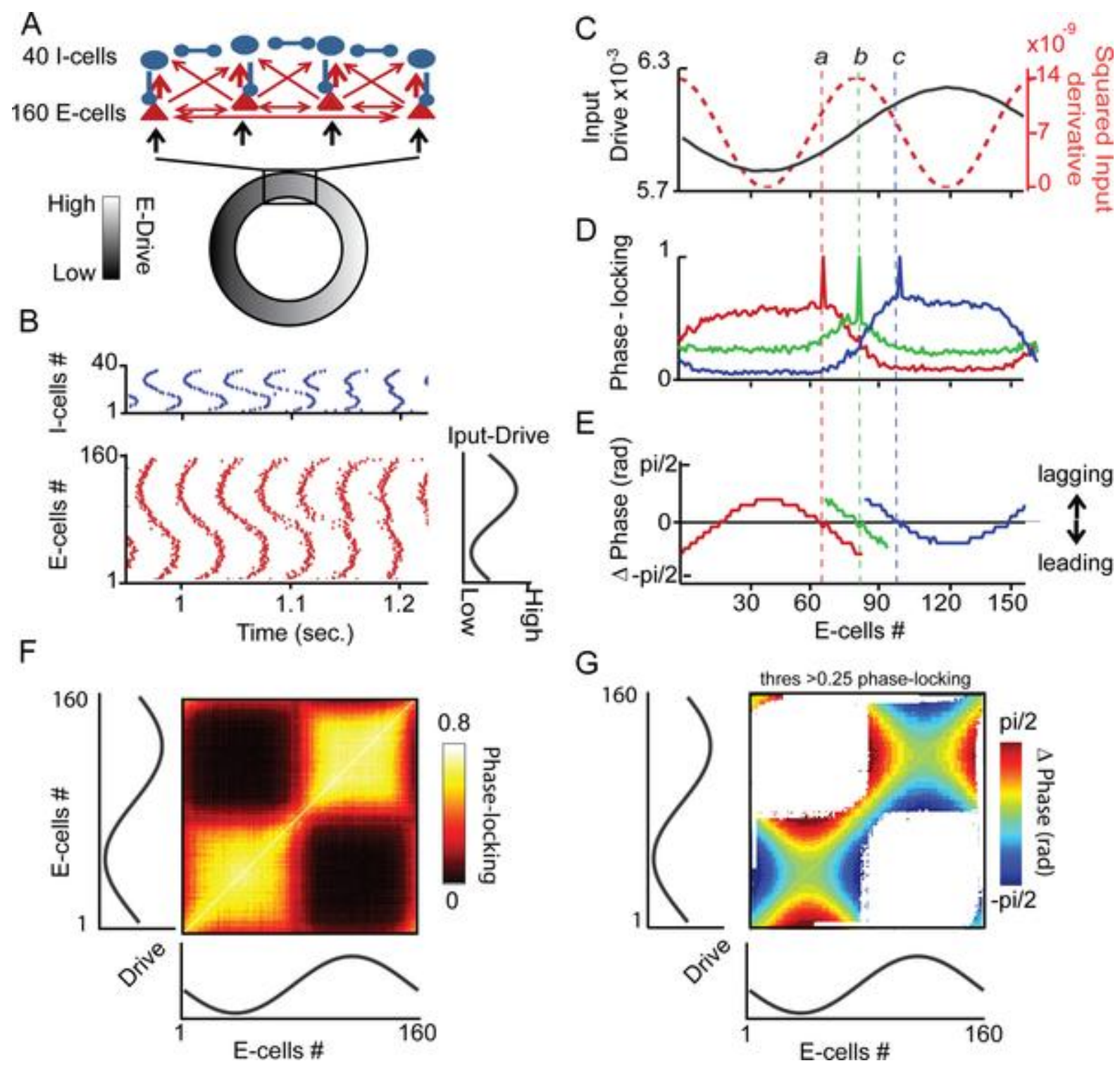

Figure 3: Assembly formation and complementary rate/phase code $(A)$ The overall network ring structure of the PING model with nearest-neighbor connections (B) Example of a simulation population spike raster output (top I-cells, bottom E-cells), variation in E-cell excitatory drive is indicated to the right. ( $C$ to $E$ ) Detailed results for three example E-cells $\boldsymbol{a}, \boldsymbol{b}$ and $\boldsymbol{c}$. (C) Location of each example E-cell along the ring structure is indicated by the level of input-drive (black) as well as the squared derivative of input (red). (D) Phase-locking values between each example E-cell and all other E-cells (estimated by crosscorrelation peak). (E) Phase difference between example E-cell and all other E-cells with phase-locking threshold $>0.25$ (for illustration, see Methods). (F) Matrix showing phase-locking between all possible pairs of E-cell pairs, location in the ring is indicated by the level of input-drive, as in C. (G) Phase difference between all possible E-cell pairs with same phase-locking threshold as above. Blue indicates that the $\mathrm{X}$-axis neuron leads the $\mathrm{Y}$-axis neuron, red indicates the reverse. 
mechanism (I-I =0.1 mS/cm², 4 per neuron), yet they were not critical (Tiesinga and Sejnowski, 2009, 2010). In addition, weak but numerous RS to RS excitatory connections ( $E-E)$ were added ( $E-E=0.03$ $\mathrm{mS} / \mathrm{cm}^{2}, 25$ per neuron) [16]. The topographic input to RS neurons was modulated sinusoidally around the ring (shading in Fig. 3A). I-cells received most of their input drive from nearby located E-cells (for details, see Methods). In the ring-network simulations E-cells had similar spike rates to I-cells and both had spike rates close to the gamma oscillation frequency. This allowed us to use smaller but stably synchronized networks to increase computational efficiency. However, we will describe below that our results can be extended to large sparse-firing gamma oscillation networks in which E-cells (RS) fire much less than the I-cells (FS) and below the gamma frequency

In Fig.3B, an example simulation output is shown, displaying the spike raster for the entire network (red, E-cells; blue, I-cells). Neuronal spiking was synchronized in the gamma range ( $25-35 \mathrm{~Hz}$ ), but individual neurons displayed spike timing differences relative to each other that were related to the input-drive differences. Fig. 3C-F describes the detailed relationship between synchronization and input. This relationship will be described both in terms of the strength of phase locking among neurons and of the phase differences among synchronized neurons. Phase locking was estimated by the peak of the crosscorrelation histogram computed over the respective simulated spike trains, and the phase difference by the lag of the cross-correlation peak (divided by cycle length). We focus here on E-cells but all observations for E-cells have been replicated for I-cells.

We found that the spatial extent of phase-locking in the network differed along the sinusoidal input function (Fig 3C) in close relation to the level of detuning approximated here by the squared input derivative (red line). Neurons receiving their input around the trough and the peak of the spatial sinusoidal input engaged in spatially larger ensembles of synchronized rhythmic activity compared to neurons along the slope of the sinusoidal input (Fig 3D). To illustrate this, phase locking strength distributions are shown for three reference neurons. Each distribution refers to locking between a reference neuron and all other neurons in the network. The three reference neurons were labeled as neuron $\boldsymbol{a}$ (red), neuron $\boldsymbol{b}$ (green), and $\boldsymbol{c}$ (blue), located respectively near the trough, slope, and near the peak of the sinusoidal input function. The spatial extent of phase-locking (Fig.3D) decreased with increases in the slope of the sinusoidal input, corresponding to increases in detuning, yielding much larger distributions for neurons $\boldsymbol{a}$ and $\boldsymbol{c}$ than for $\boldsymbol{b}$. Specific features of the input also influenced the distribution of phase locking strength. The spatial distribution of phase-locking for neuron $\boldsymbol{b}$, situated where the slope of the sinusoid was steepest, was not only small but also symmetric. By contrast, the larger distributions for neurons $\boldsymbol{a}$ and $\boldsymbol{c}$, were asymmetric, with a skew towards neurons receiving more similar input drive. Hence, despite the symmetric synaptic spatial coupling for each reference neuron with its neighbors, their spatial phase-locking distributions with neighboring neurons differed. This reflected the spatial variation of input drive to neurons in the vicinity of reference neurons $\boldsymbol{a}, \boldsymbol{b}$ and $\boldsymbol{c}$. Moreover, the relation between input drive and synchronization also led to characteristic phase differences among synchronized neurons (Fig 3E). This is illustrated for each of the same three reference neurons (only phase-relations shown if $>.25$ phase-locking, see Methods). The reference neurons had 
systematically leading phase relationships with neurons receiving a lower input drive, and a lagging phase relationship with respect to neurons receiving a higher input drive.

We now consider the combined results of all E-cells in the network. Fig.3F shows a phase-locking matrix in which the phase-locking values between all possible pairs of E-cells in the network $(160 \times 160 \mathrm{E}$-cell pairs) are shown. Neurons around the peak or trough of the sinusoidal input function formed large assemblies of synchronized units. Neurons along the steepest slope of the input function only synchronized with their immediate neighbors (narrow regions of bright color at the centre and extreme ends of the diagonal). Note that neurons close to, but not exactly on, the peak/trough had asymmetric distributions of phase-locking values, in spite of their symmetric connectivity.

In Fig.3G, phase differences are also shown for all E-cell pair combinations. Within regions of high synchronization, neurons with higher input drive (negative lag, blue) led neurons with lower input drive (positive lag shown in red). Both the behavior of phase-locking and phase-relation as a function of detuning are in agreement with TWCO. The detuning magnitude (large at the sinusoidal slope and small around the peak/trough) strongly determined whether neurons could synchronize. If synchronized (within the Arnold tongue), the sign and magnitude of detuning defined the phase-relation.

The synchronization properties of the ring-PING network in Fig. 3 depended on the connectivity patterns. First, sufficiently strong $\mathrm{E} \rightarrow \mathrm{I}$ as well as $\mathrm{I} \rightarrow \mathrm{E}$ connections were required to allow for PING type synchronization (Tiesinga and Sejnowski, 2009). Further, we observed that the exact synchronization properties depended on the number of excitatory connections in relation to the number of inhibitory connections (Suppl. Fig.1). In the case of more numerous (or stronger) E-E connectivity, the spatial extent of synchronization was larger around the sinusoidal peak compared to the trough. In contrast, in case of strong I-I or I-E connectivity, the spatial extent of synchrony was larger around the trough compared to the peak. This seemingly odd result can be understood if one considers the influence of excitatory vs. inhibitory input in terms of the phase response curve (PRC). Whereas excitatory connectivity will tend to advance the phase of a next spike, inhibitory connectivity will delay the occurrence of a next spike. Neurons synchronizing to other neurons with excitatory connections alone will most optimally entrain neurons with intrinsically lower frequencies (as excitatory connections speed them up) and hence synchronization extends further around the peak. In comparison, inhibitory connections entrain best neurons with intrinsically higher frequencies and will therefore lead to stronger synchronization around the trough of the sinusoidal input. Therefore the balance of inhibitory and excitatory interaction is critical for understanding how PING networks will self-organize depending on input-drive.

In the simulation analysis presented in Fig 3, network performance was analyzed in terms of simulated spike output, where phase-locking strength and phase differences were derived from spike cross correlations. In experimental studies, gamma oscillation properties are often investigated in terms of the Local Field Potentials (LFP), which is a population aggregate signal (mainly reflecting synaptic potentials, (Buzsáki et al., 2012)). We therefore conducted a similar analysis on E-cell population activity (representing a LFP-like signal) to test whether the same phase-locking and phase-relation behavior could be observed. Further, we were interested whether input noise affected single neuron spike rates 
differently than the local population oscillation frequency. To estimate the local LFP-like measure, we aggregated the network spiking activity at each E-cell reference position with an exponentially decaying spatial function (see Methods). We termed this the local population average (LPA), to make clear that it is not the LFP, yet sharing the property of being a population signal. Results are shown in Suppl.Fig.2. We observed the same behavior of phase-locking and phase-relation patterns for the LPA estimates. The properties of the network were relatively robust against input noise (Suppl.Fig.2). Generally, the higher the input noise, the smaller the extent of synchrony (Pikovsky et al., 2002; Tiesinga and Sejnowski, 2010). Further, at higher input noise levels, we observed that the spike rates were no longer highly locked to the local gamma frequency (estimated based on LPA) and, if rates were estimated over a long time window (here $5 \mathrm{sec}$ ), the single spike rates could reflect input differences between neurons, despite being locked to the same gamma oscillation frequency. The relationship between population gamma frequency and single neuron spike rates as well as important issues related to noise and the encoding time window will be elaborated further in the Discussion section.

One limitation of the above presented ring-PING network was that the E-cells and I-cells had similar spike rates, both in the range of the local population gamma frequency. However, experimental studies suggest that neurons, in particular pyramidal neurons (RS-type, (Pospischil et al., 2008)), have spikes rates lower (sparser) than the gamma oscillation frequency (they do not spike each gamma cycle). It has been shown in theoretical studies that sparsely firing PING network regimes exist as long as the number of neurons is sufficiently large (Brunel and Wang, 2003b). We therefore replicated the findings shown in Suppl.Fig.3 in a larger network with Izhikevich neuron models (Izhikevich, 2003), which have higher computational efficiency than the Hodgkin-Huxley neuronal model, but still generate realistic RS and FS spiking patterns. The ring-PING network consisted of $4000 \mathrm{E}$-cells and 1000 I-cells. Whereas the I-cells still spiked close to the gamma range being around $\sim 40 \mathrm{~Hz}$, the E-cells had spike rates around $\sim 12 \mathrm{~Hz}$. Although the E-cells showed spike rates much lower than the gamma oscillation frequency, we still observed the phase-locking and phase-relation among E-cells as described in Fig. 3.

\section{Detuning is transformed into a complementary gamma phase and frequency code}

The results from the two interacting PING-networks (Fig.2) suggested that reliable phase differences corresponded to small local differences in input (small detuning), whereas frequency differences reflected larger input differences. The same could be observed for the ring-PING network driven by spatially varying input (Fig. 3). Neurons interacting with small detuning (at the peak or trough of the sinusoidal input) exhibited reliable phase differences, whereas neurons interacting at larger detuning values (at the steepest slope of the input function) showed reduced synchrony and large (emergent) frequency differences. This indicates that information about input drive differences might be present both in frequency and phase in a complementary manner. We therefore extended our analysis of the ring-PING network to investigate neural coding by quantifying explicitly the relationship between the input patterns and the neuronal responses in terms of their frequency and phase-relation. We will first describe the coding types and their derivations. The stimulus $\left(\mathrm{S}_{\text {orig }}\right)$ was the spatially-defined sinusoidal excitatory drive to the E-cells. The first coding type was the (emergent) 'frequency code' $\left(S_{\text {est }}(\omega)\right.$ ). We explicitly mean the frequency that would be (experimentally) measurable in a network. In our ring-PING network described above, single neuron spike frequencies (rates) were close to the (LPA) gamma 
frequency and neurons were strongly locked to the rhythm. Therefore LPA gamma frequencies or single spike rates gave here similar estimates (see Discussion below). The second coding type is the 'phase code' $\left(S_{\text {est }}(\theta)\right)$. We described above the phase-relations between neurons in the ring-PING network as function of the spatial sinusoidal input. When neurons were synchronized, hence sharing a common frequency, the neuron with higher drive occupied a leading (earlier) phase. In our network, multiple oscillatory frequencies were present and we therefore had to define the common oscillation frequency by the group of neurons to which it had substantial phase-locking. This was implemented by weighting each phase-relation between neurons by their phase-locking strength. The Arnold tongue relationship states also that a phase-difference between two oscillators depends on their coupling strength. Hence, to achieve more exact estimates of the input differences from the phase differences, we needed to make them independent of coupling. This was implemented by multiplying a given phase-difference by the coupling strength between neurons (see Methods and Discussion). This operation was necessary as a phase-difference between strongly coupled neurons corresponds to a higher input difference than the same phase-difference between more weakly coupled neurons. To summarize, the phase-code was calculated for each neuron as the average phase-relation to all other neurons weighted by their phaselocking strength and coupling strength. In the combined 'frequency and phase code' $\left(S_{\text {est }}(\theta, \omega)\right)$ both the phase code and the frequency code were summated.

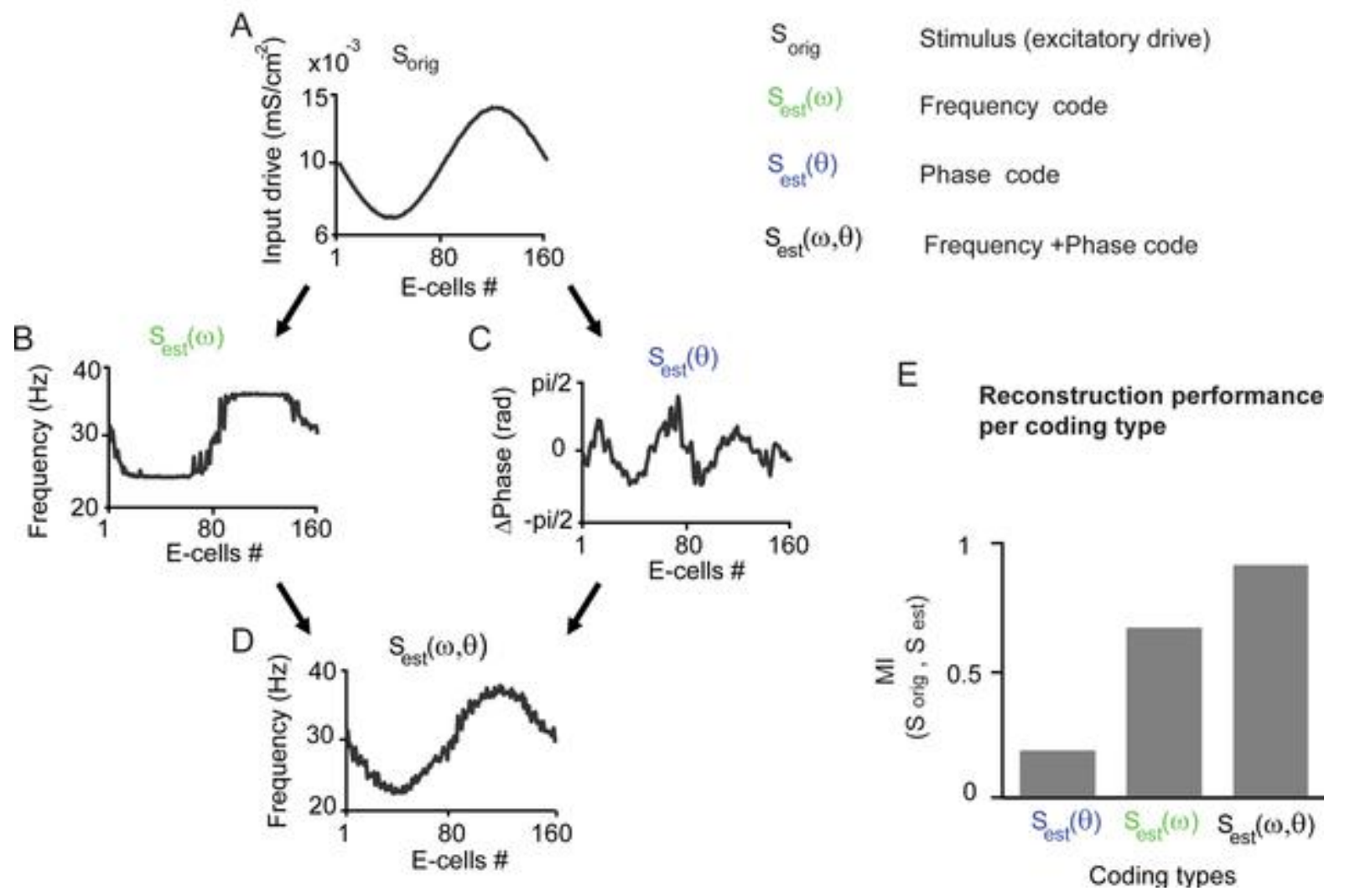

Figure 4: Reconstruction of stimulus input based on phase and frequency coding. See Methods for derivations of the coding schemes. A) The stimulus input $S_{\text {orig }}$ to be reconstructed B) Reconstruction based on frequency $S_{\text {est }}(\omega)$ (here E-ell rate) alone C) based phase-differences among E-cells $S_{\text {est }}(\theta)$ D) based on a combined frequency and phase code $S_{\text {est }}(\omega, \theta)$. E) The reconstruction performance, measured by mutual information (MI), was from lowest to highest $\mathrm{MI}=0.18$ for $\mathrm{S}_{\text {est }}(\theta)$, $\mathrm{MI}=0.65$ for frequency code $S_{\text {est }}(\omega)$ and $\mathrm{Ml}=0.92$ for combined code $S_{\text {est }}(\omega, \theta)$. 
In Fig.4B, we reconstructed the spatial sinusoidal input $\left(\mathrm{S}_{\text {ori }}\right)$ of the ring-PING network based on the E-cell spike count (frequency code $S_{\text {est }}(\omega)$ ), the phase-relation between E-cell spike trains (phase code $S_{\text {est }}(\theta)$ ) or by combining both sources of information (combined code $S_{\text {est }}(\theta, \omega)$ ). The frequency code exhibited plateaus around the peak and trough of the sinusoid, where synchronization was strongest. The phase code followed the variation around the peak and trough of the sinusoid, but could not follow the larger input differences (e.g. overall difference between peak and trough). By combing both coding types the variation of the original sinusoidal input was well reconstructed. This was quantified by computing the mutual information (MI, (Duncan, 1970)) between $\mathrm{S}_{\text {orig }}$ and $\mathrm{S}_{\text {est }}$. The lowest reconstruction performance was achieved by the phase-code $(\mathrm{Ml}=0.18)$, followed by the frequency code $(\mathrm{Ml}=0.65)$, with the highest $\mathrm{MI}$ for the combined code ( $\mathrm{Ml}=0.92)$. The contribution of each coding type depended on the exact input characteristics. For example, the higher the synchrony within a network (e.g. by lower amplitude of the sinusoidal input function) the more information the phase code added to the frequency code (data not shown; see discussion for further considerations). These results indicate 1) that phase coding is most suited to resolve fine (small input differences) and local (high coupling) input variation; 2) that phase coding represents a relative $\Delta$ rate-phase transform (McLelland and Paulsen, 2009)); 3) that phase coding depends on both input difference and coupling strength (Arnold tongue); and 4) that phase coding can add complementary information to frequency coding.

\section{Extension to a neural phase-oscillator ring-network model}

The Hodgkin-Huxley PING network simulations in Fig. 1-4 have shown that the input-dependent gamma synchronization can be well understood within the TWCO and the Arnold tongue (Pikovsky et al., 2002). In the following result section, we show that the PING spiking neural network can be successfully reduced to a basic model (Hoppensteadt and Izhikevich, 1998, 1996) of weakly-coupled oscillator networks, the phase-oscillator model (Kuramoto model, (Breakspear et al., 2010)).The reduction to the phase-oscillator model allowed us to investigate the oscillatory properties of much larger topographic networks exposed to natural complex input patterns due to the computational efficiency. However, first we will describe the rationale of reducing local PING networks to abstract phase-oscillators. We will then show that the exact same behavior of the ring-PING network can be reproduced by a ring-phaseoscillator network.

A single phase-oscillator is characterized by an intrinsic (natural) frequency that determines how fast the phase-variable (the central variable of the model) evolves over time. The intrinsic frequency is the frequency that characterizes the oscillator in the absence of interactions with nearby phase-oscillators. A local PING network consisting of a few E- and I- cells is considered here as equivalent to one phaseoscillator (Fig.5A). The population frequency of the local PING network (LPA, Fig.5B) would be similar to the frequency of the phase-oscillator. The instantaneous phase (Picinbono, 1997) of the local population rhythm (Fig.5C) would be equal to the output-variable of the phase-oscillator model (Fig.5D). In a network of connected (coupled) oscillators, there is not only a reduction in terms of units (from a number of E- and I- cells to a single phase-oscillator), but also a reduction in the complexity of connectivity. Connectivity (Coupling) is defined here in terms of one oscillator advancing or delaying the phase of the other in a manner that is defined by the phase relation between them. Coupling strength refers to the magnitude of the modulation of the phase-variable in two oscillators which is a function of 
the ongoing phase-relation between the phase-oscillators. This function is referred to as the phaseresponse curve (PRC). It is a sinusoidal function as defined in the Kuramoto model of weakly coupled oscillators, with a clear attractor at phase 0 . For example, if oscillator 1 at a given moment is trailing oscillator 2, oscillator 2 will push the phase of oscillator 1 forward while oscillator 1 will delay the phase of oscillator 2 (assuming sufficiently similar frequencies). This means that given equal intrinsic frequencies, a network initialized with random phases in each oscillator will tend to converge towards

\section{A}

Hodgkin-Huxley simulations
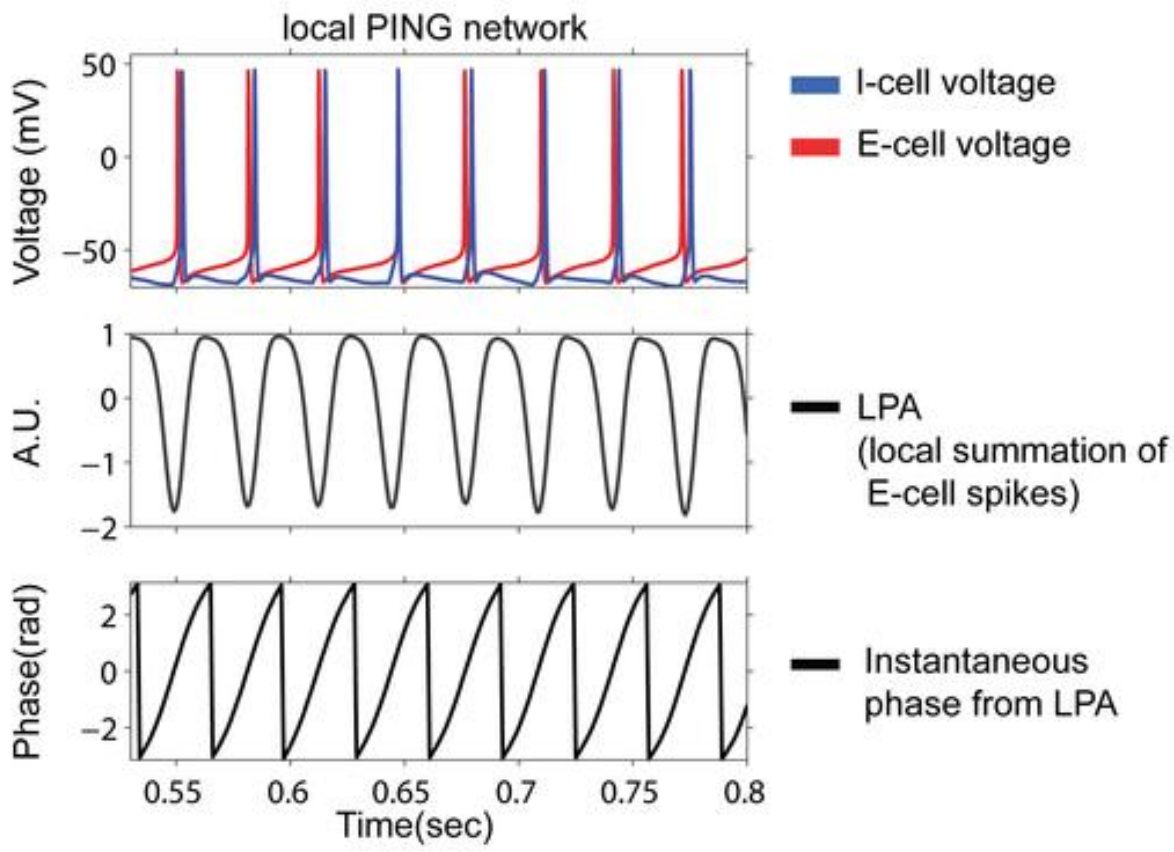

B

Phase-oscillator simulations

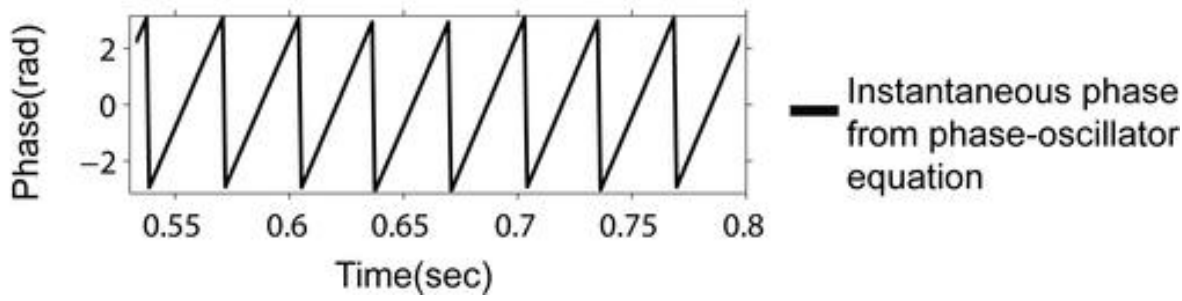

Figure 5: Comparison of Hodgkin-Huxley networks $(\mathrm{HH})$ and phase-oscillator model. A) The voltage membrane of an E-cell and I-cell is shown as modeled by the HH-dynamical equations. The generative mechanism of PING gamma oscillation is the rhythmic interaction of E- and I-cells. B) The LPA (local population average of E-cell spikes, see Methods) is shown. The fluctuations in the LPA represents the synchronous rhythmic interactions among the local population of E- and I-cells. C) Using the Hilbert transform, one can easily derive the instantaneous phase of the LPA. D) The instantaneous phase of a phaseoscillator is shown. In the phase-oscillator model the phase-variable is modeled directly by one simple dynamical equation (see Methods) mainly governed by the intrinsic frequency and interactions by other oscillators. Our assumption in this study is that the instantaneous phase derived from local LPA in the HH-PING network can be approximated by phase-oscillators. 
the attractor phase. In the case of a local-PING network, the interactions with nearby neurons are exerted through excitatory connections (E-E, E-I) and inhibitory connections (I-E, I-I) which together determine the effective coupling strength and the phase response curve (PRC) of our network. A further difference between oscillator networks and PING networks is the manner in which input modulates ongoing interactions in the network. In PING networks, the oscillation frequency emerged from the interaction between network properties and excitatory input drive. In phase oscillator networks, the intrinsic frequency of a phase-oscillator was set based on a function (as established experimentally) linking input strength to oscillation frequency. Further, whereas in the PING network the gamma rhythm might be not sustainable in some conditions, e.g. due to low input drive, a phase-oscillator will always oscillate at any arbitrary frequency. Overall, it must be emphasized that even though not all complexities of the PING network can be captured by a phase-oscillator model; we argue that it captures the most characteristic properties of PING network behavior.

To illustrate that phase-oscillator networks capture the behavior of the PING model, Fig. 6 describes a ring-phase oscillator neural network similar to the ring PING network (Fig.6A), in which 160 phaseoscillators were locally coupled along a ring (see Methods). The intrinsic frequency of each phaseoscillator was set by a sinusoidal input function (defined over the ring). The simulation output can be seen in Fig.6B, which shows the output phase-traces of the 160 phase-oscillators. In Fig.6 C-D, we computed the phase-locking and the phase-relation matrix between all phase-oscillators. The results shown resembled those obtained for the ring-PING network (Fig.3 F-G). The spatial extent of phaselocking was larger around the peak and trough of sinusoidal function. The phase-relation patterns within the region of phase locking were the same as in the ring-PING-network, so that phase-oscillators with higher intrinsic frequency had a leading phase compared to other phase-oscillators. In Fig. 6 E-F, we tested the contribution of the different coding types to input reconstruction (see Fig.4 B-C for comparison). Notice that $\mathrm{S}_{\text {orig }}$ (stimulus input) corresponds here to the intrinsic frequency set by an input function and not to excitatory drive. The same results (here with sinusoidal intrinsic frequency fluctuation of $+/-3 \mathrm{~Hz}$ ) were obtained as in the ring-PING network. The best input reconstruction was given by the combined frequency and phase code $(\mathrm{Ml}=0.95)$, followed by the frequency code $(\mathrm{MI}=0.73)$, and lastly the phase-code ( $\mathrm{Ml}=0.17)$. We also reproduced the asymmetries in spatial synchronization (around peak and trough of sinusoid)m which in the ring-PING network were induced by changing the amount of excitatory connections in relation to the amount of inhibitory connections (see Suppl.Fig.1). The asymmetries in spatial extent of synchronization were obtained in the phase oscillator network by modifying the phase-response curve (PRC). To model a dominance of excitatory connections, we set all values below zero (phase delay) to zero, and to model a dominance of inhibitory connections we did the opposite. This resulted in the same asymmetries in synchronization around the peak or trough of the sinusoidal function (Suppl. Fig.2D). 
A Ring-phaso-oscillator network model



B

Simulation output examplo
C

Phase-locking matrix

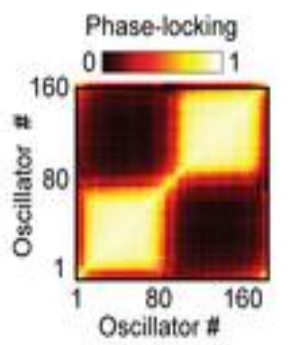

D

Phase-relation matrix

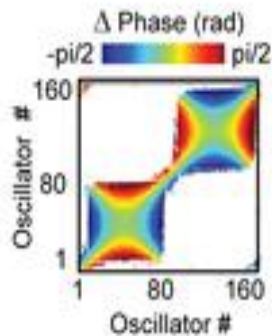

E

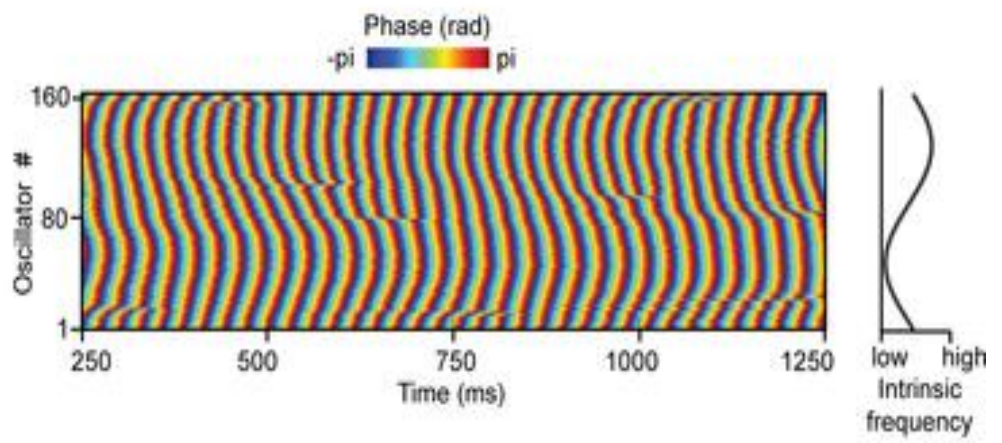

Stimulus reconstruction from Ring-phase-oscillator network

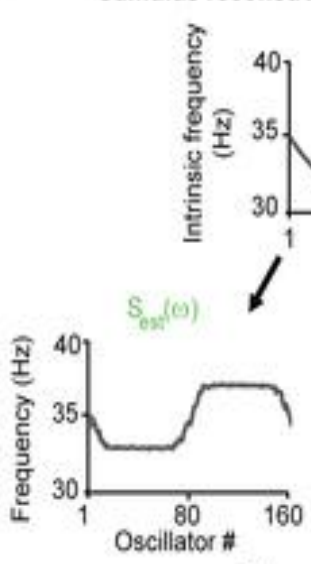

$S_{\text {orig }}$

$S_{\text {esi }}(6) \quad$ Frequency code

$S_{\text {est }}(\theta) \quad$ Phase code

$\mathrm{S}_{\text {eyi }}(0, \theta) \quad$ Frequency + Phase code

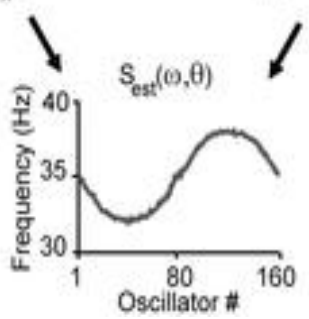

F

Reconstruction performance per coding type

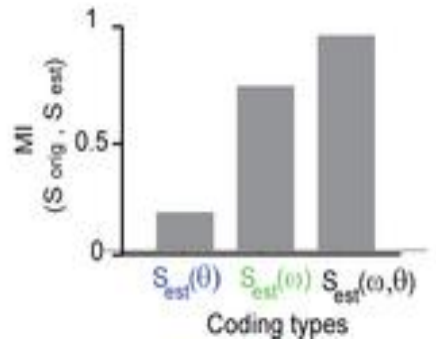

Figure 6: Reproduction of Hodgkin-Huxley results of Figure 3 and 4 by a phase oscillator model (A) Replication of Figure 3 Hodgkin-Huxley network architecture, here with 160 phase-oscillators with the same connectivity structure. B) Example extract of simulation output. Color represents the current phase of individual oscillators. The location of each oscillator in the ring architecture is indicated by the relative input level (intrinsic frequency) C) Matrix of phase-locking values between all possible oscillator pairs, equivalent to Figure 3F. D) Phase relation between all possible oscillator pairs. Pairs with phase-locking $<\sim 0.3$ (see Method) are masked for illustrative reasons. Blue indicates that the $\mathrm{X}$-axis oscillator leads the $\mathrm{Y}$-axis oscillator, red indicates the reverse. E) Stimulus (I) reconstruction $S_{\text {orig }}$ (intrinsic frequency) based on (II) the frequency code $S_{\text {est }}(\omega)$, (III) phase code $S_{\text {est }}(\theta)$ and (IV) the combined frequency and phase code $\left.S_{\text {est }}(\omega, \theta) F\right)$ The reconstruction performance, measured by mutual information ( $\mathrm{Ml}$ ), from lowest to highest $\mathrm{Ml}=0.17$ for phase code $S_{\text {est }}(\theta), \mathrm{Ml}=0.75$ for frequency code $\mathrm{S}_{\text {est }}(\omega)$ and $\mathrm{Ml}=0.95$ for combined code $S_{\text {est }}(\omega, \theta)$. 


\section{Large 2D topographic phase-oscillator networks driven by natural spatial input-patterns self-}

organizes along the same Arnold tongue principles with implications for coding and spatial synchronization

We took advantage of the computational efficiency of the phase-oscillator model and extended our analysis to a 2D 100X100 lattice networks. The network consisted of 10.000 phase-oscillators with a total of $10^{8}$ possible connections. Connections among phase-oscillators decreased exponentially in strength as a function of distance on the lattice as an approximation for cortical horizontal connectivity (e.g. V1, (Boucsein et al., 2011)). Note that the lattice network was an abstract model of a cortical area aimed to reflect only the essential characteristics of a sensory cortical area (topographical map, spatially local connectivity, feature map). The model was aimed to capture the essential input-dependent selforganization principles of cortical oscillations, in particular gamma oscillations. We tested the network behavior using visual images representing natural and complex intrinsic frequency variation better (Fig.7A). Inspired by experimental observations of a close link between visual contrast and gamma oscillation frequency in macaque visual cortex V1 and V2 (Roberts et al., 2013), we used the local contrast of natural visual stimuli to define the intrinsic frequencies of the phase-oscillators. To that aim, we used 80 (grayscale) natural images from an online database (Berkeley segmentation dataset, see Methods). The images were down-sampled such that each pixel of the image corresponded to one phase-oscillator. Local contrast was estimated using a root-mean square measure [25] with a spatial kernel of 3 pixels. From the online database, information on the location of boarders between objects, or segments in the image (segmentation borders), defined by 30 human observers (Martin et al., 2001), was also available.
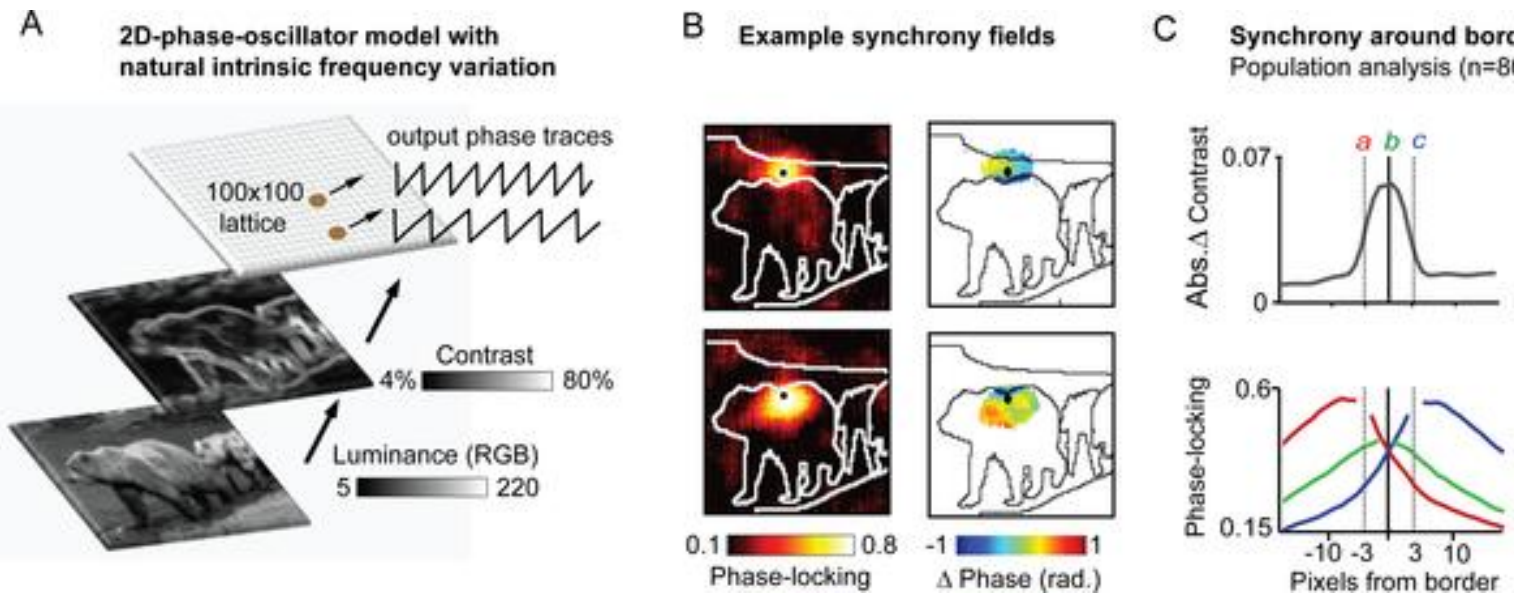

Figure 7: Phase-oscillator model with natural image input. A) The general approach: The natural image was compressed to $100 \times 100$ pixels and transformed from a luminance image into a contrast image. Contrast values were used to define the intrinsic gamma-frequencies of the $100 \times 100$ phase-oscillator lattice on a one-to-one pixel to oscillator basis. B) (I) Example synchrony fields (color) of two reference oscillators to all other oscillators (color) overlaid onto border segmentation of the corresponding image. One example (black dot) was located outside of the main object (top row) and the other within the object (bottom row). (II) Phase relation maps of example oscillators representing the phase relation to all oscillators with phaselocking $>0.3$ (see Methods). Blue (red) indicates that the oscillator leads (lags) compared to the reference oscillator. C) (I-II) Segmentation-border triggered analysis. From the online image database (Martin et al., 2001) segmentation borders as 
indicated by 30 human subjects are available. We used these segmentation borders to analyze spatial synchronization around them (see Methods). Borders are thought to be associated with high contrast variation (and hence detuning) (Marr, 1982). (I) shows the mean absolute contrast spatial derivative (averaged over the 80 images) confirming that segmentation borders are indeed associated with higher contrast/detuning. (II) Mean synchronization profile for reference oscillators a and c located 3 pixels on each side of the window's center (i.e., the boundary location) and reference oscillator b located at the boundary location. Spatial synchronization is reduced over the segmentation border in line with the higher detuning at the segmentation borders (see Suppl. Fig.4 for more details).

In Fig.7B, we show in the left column the synchronization (black= no phase-locking, yellow/white= $\max$ phase-locking of 1 ) and in the right column the phase-relations (blue= earlier phase, red=later phase, white= below threshold, see Methods) of two reference oscillators (black dots) as compared to all other oscillators in response to an example image. We will use these examples to show how the synchronization fields and phase-relations adapt to local changes of contrast/intrinsic frequency. The 'synchronization field' refers to the spatial extent over which a reference oscillator synchronizes with neighboring oscillators (Eckhorn et al., 1990). The synchronization fields were asymmetric, despite symmetric coupling in the network. Within the synchronization fields, off-zero phase-relations could be observed. This was due to the positions of the two example reference phase-oscillators, which were chosen to be close to an object border (here a bear) with one reference oscillator located just outside the main object (top row Fig.7B) and the other just within (bottom row Fig.7B). The object border was associated with high local contrast variation (hence large detuning). The asymmetry of the synchronization fields followed from the fact that synchronization drops with the rapid increase of local contrast when moving from the reference oscillator towards the border (increasing detuning) while the converse was true when moving from the reference oscillator away from the border (decreasing detuning). Here, synchronization extended far from the reference oscillator towards the interior or ulterior surfaces (because the small detuning within surfaces permits synchronization over larger spatial extents). Furthermore, within the synchronization fields, the oscillators closer to the border led in phase compared to the reference oscillator, because the border had higher contrast/intrinsic frequency, while the converse was true when considering oscillators away from the border. These example synchrony fields indicate that phase-oscillators in the 2D lattice model behaved similarly to those in the ring-phase oscillator and ring-PING network models. Moreover, the data suggest that the synchronization fields might capture specific aspects of the statistics of contrast distributions in natural images. To further explore this point, we tested systematically in Fig.7C how synchronization was affected around segmentation borders in 80 natural images (available from the Berkeley segmentation dataset (Martin et al., 2001), see Methods for more details). We first tested whether contrast variation was significantly modulated around segmentation borders (see Methods). To quantify these effects, we defined 1dimensional spatial windows of \pm 15 pixels centered on segmentation borders. We then aligned the different windows and averaged them for each image separately (see Methods for more details). Population statistics are based on these average windows per image. We then calculated the averaged absolute spatial derivative of contrast values (equivalent to detuning) along each window. Fig. 7C (top) shows a steep change in contrast as a function of distance to the border. Mean contrast variability at the center of the window, at the border, was significantly different from the extremities of the window (paired t-test: $t=7.35, d f=79, p<0.001$ ). We then quantified the change in synchronization as a function 
of distance to a border. For initial population analysis $(\mathrm{N}=80)$, we selected three reference oscillators. Reference oscillators $\boldsymbol{a}$ and $\boldsymbol{c}$ (Fig. 7C bottom) were located 3 pixels away from each side of the border, and reference oscillator $\boldsymbol{b}$ was on the border. Relative to reference oscillators $\boldsymbol{a}$ and $\boldsymbol{c}$, synchronization fields showed a much more rapid decline of phase-locking strength towards the border than away from the border (see Suppl. Fig.4 for more details). Thus overall, the asymmetry of synchronization fields around reference oscillators $\boldsymbol{a}$ and $\boldsymbol{c}$ in Fig. 7E matched with the asymmetry of synchronization fields around neurons $\boldsymbol{a}$ and $\boldsymbol{c}$ in Fig.3C-E of the ring-PING network model. This input-dependency of spatial synchronization suggests, in line with previous studies (Eckhorn, 1999a; Eckhorn et al., 1990; Engel et al., 1999; König and Schillen, 1991a; Ranganath and Kuntimad, 1994; Wang and Terman, 1997), that oscillatory synchronization might be a useful tool for clustering operations, for example for visual segmentation.

A Arnold tongue reconstruction from 2D-phase-oscillator network Population analysis $(n=80)$
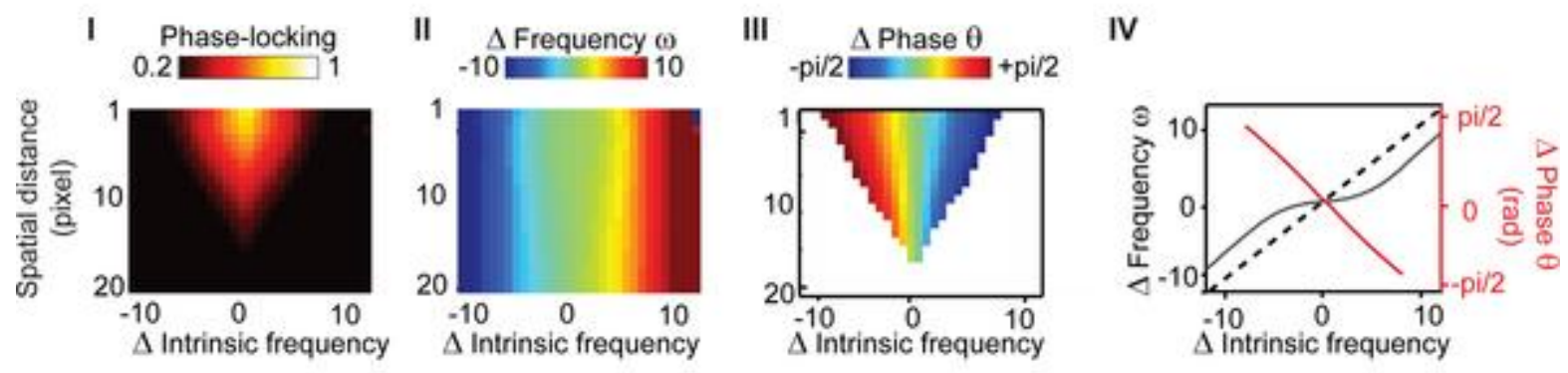

B

Stimulus reconstruction from 2D-phase-oscillator network Population analysis $(n=80)$
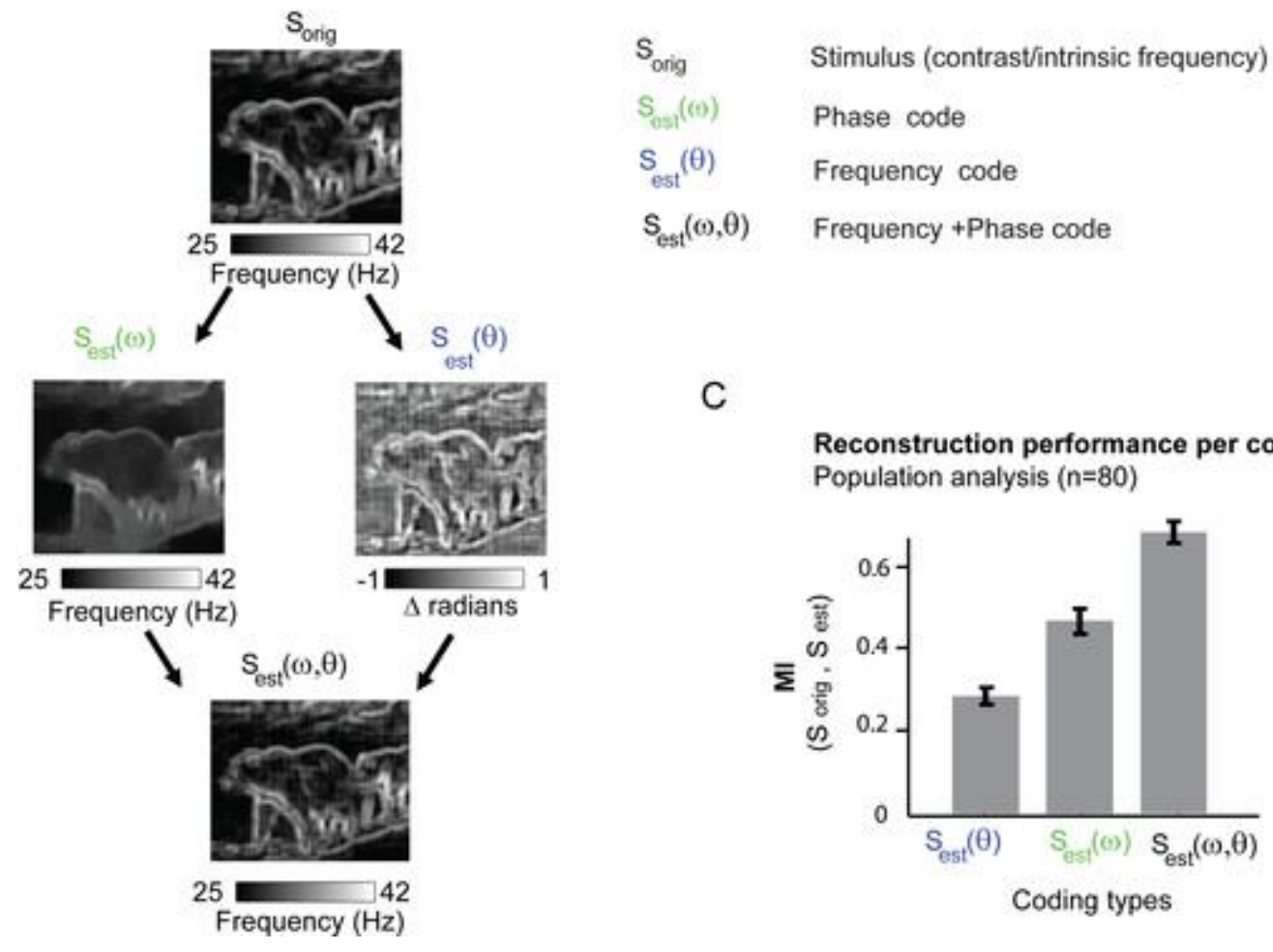

Reconstruction performance per coding type Population analysis $(n=80)$

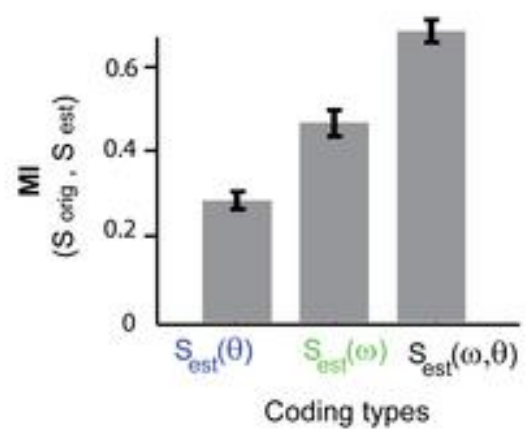


Figure 8: Arnold reconstruction and information content of the frequency, phase and combined codes for natural image input. A) Arnold tongue reconstruction from the natural images $(n=80)$ by using detuning and coupling between all possible pairs of oscillators in the lattice network. We used lattice distance here as approximation for coupling (see Methods). The resulting Arnold tongue in terms of (I) phase-locking (II) phase-relation and (III) (emergent) frequency difference is shown. In (IV) a cross-section of the Arnold-tongue with overlaid representation of phase-locking (black), phase-relation (red) and intrinsic frequency (dashed) for an oscillator-pair of a lattice distance of 3 pixels (direct coupling strength=0.62). B) Stimulus reconstruction of the natural image contrast. Intrinsic frequency of all phase oscillators determined by (I) local contrasts of example image $S_{\text {orig }}$ based on (II) the frequency code $S_{\text {est }}(\omega)$, (III) phase code $S_{\text {est }}(\theta)$ and (IV) the combined frequency and phase code $S_{\text {est }}(\omega, \theta)$ C) The reconstruction performance, measured by mutual information $(\mathrm{MI})$, was from lowest to highest $\mathrm{MI}=0.28$ for $S_{\text {est }}(\theta), M I=0.46$ for frequency code $S_{\text {est }}(\omega)$ and $M I=0.67$ for combined code $S_{\text {est }}(\omega, \theta)$. Error bars give \pm 3 SEM $(n=80)$.

So far, we have described the behavior of the phase-oscillator model using particular image examples. We now analyze the network behavior in terms of the principles that underlie its self-organization behavior independent of the specific image providing the input. If the input-dependent self-organization of the phase-oscillators is mainly governed by principles of the TWCO, then one should be able to reconstruct the Arnold tongue from the simulation output. To test this, we determined for each phaseoscillator pair its coupling strength (direct connections) and detuning (as derived from the 80 input images). Fig.8A shows that in the two-dimensional parameter space of detuning and coupling strength an Arnold tongue could be observed in terms of phase-locking (Fig 8A I), frequency difference (Fig 8A II) and phase-difference (Fig 8A III). Fig. 8A IV represents a horizontal cross-section of the Arnold tongue (coupling strength $=0.62$ ) where frequency differences (black) and reliable phase differences (red) are plotted. The dashed line denotes the intrinsic frequencies. As described in Fig.2 for PING networks, phase differences better resolved smaller intrinsic frequency variation, whereas frequency differences reflected best the larger differences. The Arnold tongue reconstruction was reliable and reconstructions from individual images looked very similar to the averaged one shown in Fig. 8A. The Arnold tongue properties were similar to the one described from the two interacting PING networks (Fig.2 G-J). This analysis confirmed that the phase-oscillator lattice model with complex natural detuning (intrinsic frequency variation) behaved very similar to the ring-PING network model driven by simple sinusoidal excitatory drive. Hence, information about the natural image stimulus should be available in a complementary manner at the level of frequency variation as well as phase variation. We therefore quantified the amount of information present in the above defined coding types, frequency and phase coding, as well as a combined coding type (Fig.8). We used the same approach as used for the ring-PING network and ring- phase oscillator network. The stimulus $S_{\text {orig }}$ to be reconstructed was the intrinsic frequency image defined by the local natural image contrast. The frequency code $S_{\text {est }}(\omega)$ was the mean (emergent) frequency of a phase-oscillator. The phase code $S_{\text {est }}(\theta)$ for a given phase-oscillator was the phase differences with all other phase-oscillators weighted by phase-locking and coupling strength (see Methods). The combined code $S_{\text {est }}(\omega, \theta)$ was the summation of both former coding types. In Fig.8B, the stimulus and the reconstruction estimates are shown for an example image. The stimulus reconstruction based on frequencies $S_{\text {est }}(\omega)$ appeared smoothed compared to the original stimulus $S_{\text {orig }}$ reflecting the loss of fine spatial details. The reconstruction based on phase-relations $S_{\text {est }}(\theta)$ resembled a second derivative of the original stimulus $\mathrm{S}_{\text {orig. }}$. The phase code reflected well local and fine details but it did not reflect the absolute contrast/intrinsic frequency level. A fair reconstruction of the original stimulus was achieved by the combined frequency and phase code $S_{\text {est }}(\omega, \theta)$, which indicates that information from 
the frequency code and phase code were complementary. In Fig 8C, we quantified information content, by estimating the (normalized) mutual information (MI) between the intrinsic frequency image $\mathrm{S}_{\text {orig }}$ and the reconstructed image estimates $S_{\text {est }}$. For the example image in Fig. $8 \mathrm{~B}$, the MI was 0.44 for $S_{\text {est }}(\omega)$, the $\mathrm{MI}$ for $\mathrm{S}_{\text {est }}(\theta)$ was 0.31 , and the $\mathrm{MI}$ for $S_{\text {est }}(\omega, \theta)$ was 0.69 . Over the population of 80 natural images (Fig.8c), the MI was $0.46\left(\mathrm{SEM}= \pm 9 * 10^{-3}\right)$ for the frequency code $\mathrm{S}_{\text {est }}(\omega), 0.28\left(\mathrm{SEM}= \pm 5 * 10^{-3}\right)$ for the phase code $S_{\text {est }}(\theta)$ and $0.67\left(S E M= \pm 7^{*} 10^{-3}\right)$ for the combined frequency and phase code $S_{\text {est }}(\omega, \theta)$. A repeated measures ANOVA showed that all three codes were significantly different from one another $(F(2,158)=881, p<0.001)$, and all pair-wise comparisons were highly significant according to the Tukey's HSD tests).

\section{Discussion}

In the following, we will first discuss the underlying assumptions and limitations of our cortical gamma network model and relate them to previous modeling approaches. Then, we will turn to the implications of our findings for gamma phase coding and its relation to frequency/rate coding, stressing the distinction between the coding of larger versus smaller input variations, the effect of noise, encoding time window and connectivity. We will further discuss the experimental and theoretical implications of the relative rate-to-phase transform, as proposed here for gamma synchronization, compared to an absolute rate-to-phase transform. This is followed by considerations on input-dependent spatial synchronization, in particular in the context of the phase-oscillator network to natural images, and comparisons to related modeling approaches more specifically designed for image segmentation. We end with testable experimental predictions that follow directly from the present study.

\section{Underlying model of cortical gamma oscillations}

\section{'Single oscillator model' vs. 'multiple oscillator model'}

The exact underlying mechanism of gamma oscillations is still under debate, even though significant advances have been achieved over the last decades. A primary distinction (Tiesinga and Sejnowski, 2009) has been made between interneuron-network gamma (ING) versus pyramidal-interneuron network gamma (PING). However, we will first focus on another key model distinction that has received much less attention so far. In one class of models, inhibition acts as reference clock to which excitatory neurons at different cortical locations are entrained at different phases (Buzsáki and Wang, 2012; Fries et al., 2007; Lisman and Jensen, 2013), whereas in another class of models I- and E-cells at different cortical locations represent different 'clocks' (oscillators) that synchronize at different phases (Ermentrout and Kleinfeld, 2001; Hoppensteadt and Izhikevich, 1998; Tiesinga and Sejnowski, 2010). We call the former the 'single-oscillator' model and the latter the 'multiple-oscillator model'. The critical distinction is whether I-cells receive spatially local excitatory drive or whether the l-cell network acts as a (indiscriminative) single unit. In the latter case, the network can express only one dominant gamma frequency at a time. In principle, both types of models can be implemented in either PING or ING mode. Reliable phase-coding in a single oscillator model regime is not easy to achieve (Tiesinga and Sejnowski, 2010) and requires strong inhibition and relatively high spike timingprecision. Phase-coding is 
determined mainly by how fast E-cells recover from inhibition (similar to latency coding, (Timothée Masquelier et al., 2009)). In the 'multiple-oscillator model' a precise phase-locking between E-cells to their nearby I-cells is not essential as long as they produce a gamma rhythm. Phase-relations are established between nearby gamma rhythmic E- and I-cells through synchronization (Pikovsky et al., 2002), during which phase-relations are determined by detuning and coupling strength. In our network models, we observed that the behavior of the PING network was largely consistent with the multipleoscillator model. However, the two models do not necessarily exclude each other. Predictions can be formulated that can be experimentally tested to distinguish these two underling models of gamma phase coding (see below).

\section{ING versus PING}

It has been shown that an ING (Traub et al., 1996; Wang and Buzsaki, 1996) network of mutually interacting inhibitory neurons can produce robust gamma oscillations, if the network is driven with sufficient excitatory input. In this model, local pyramidal neurons receive rhythmic inhibition from the Icells. An extension of the model is the PING model, where the excitatory drive to the I-cells originates from the local pyramidal E-cells themselves (Buia and Tiesinga, 2006; Fries et al., 2007). In the PING model, the excitatory state of the pyramidal neurons influences the network rhythm, in particular the frequency. In comparison, in the ING model the frequency is determined solely by the excitability of the inhibitory neurons.

Both ING and PING mechanism likely coexist in cortical networks (Brunel and Wang, 2003b) and the dominance of one to the other may switch depending on network state (Vinck et al., 2013). Experimental studies suggest that cortical gamma oscillation in a stimulus-driven state show properties consistent with the PING model (Fries et al., 2007; Vinck et al., 2013). However, the essential TWCO properties we described in our networks are not restricted to PING networks. An ING network with locally defined connectivity and excitatory drive will exhibit similar behavior. Hence, our modeling results are expected to be independent of specific PING and ING network configurations. However, in the case of E-cells receiving rhythmic inhibition from an ING network that either does not receive a spatially-defined drive or is coupled in a manner such that the ING network acts as a single unit, then the regime is not expected to be in agreement with TWCO ('single-oscillator model', see above). The same is expected for a PING network, where the $\mathrm{E} \rightarrow \mathrm{I}$ connections are all-to-all, (such that all I-cells receive the same mean input from E-cells).

\section{Assumptions of the theory of weakly coupled oscillators}

Hoppenstaedt and Izhikevich (Hoppensteadt and Izhikevich, 1998) formulated two basic assumptions in which oscillatory interactions in the cortex would be expected to occur in the regime of weakly coupled oscillators. First, they assumed that oscillations are internally (autonomously) generated and second, they assumed that the coupling is weak between oscillating neural populations.

The first assumption was fulfilled by the PING mechanism. The mean network input had no rhythmic components. Oscillations were generated by the interaction between E-and I-cells. The second 
assumption of weak coupling can be understood as oscillatory interactions between units leading to phase shifts (as defined by the PRC), but not to substantial amplitude changes or perturbation of the rhythm-generating mechanism (or quenching, (Pikovsky et al., 2002)). In the case of the two PING networks (Fig.2), the cross-network connections were an order of magnitude smaller than the withinnetwork connections. We observed small phase-dependent amplitude fluctuations (partial synchronized state), but, they did not substantially affect the phase-trajectory of the network oscillations. In the ringPING network (Fig.3) connectivity was spatially continuous and no columnar structures were assumed, thus there was no distinction between within and cross network connectivity. The synaptic connectivity strengths were in the range as normally used for PING networks (Tiesinga and Sejnowski, 2010) and the behavior was stable for a large range of different connections strengths. As described in Fig.5, for the reduction of the ring-PING network to the ring-phase-oscillator model we assumed that single phaseoscillators could be equated to pairs of E-cells and I-cells of the PING network. Further, we assumed that the complex interactions (E-E, I-E, E-I, I-I) could be approximated by a single PRC-defined connection type. Weak coupling in this context means that the synaptic coupling between neurons did shift the spike timing and did neither increase the firing rate susbtantially nor interfered with the spike generation mechanism. The comparison of the ring-PING network with the ring-phase oscillator network indeed revealed striking similarities (compare Fig 3 with 6). In conclusion, we showed that our weakly coupled oscillator network conformed to the assumptions of the TWCO. In addition, our modeling data indicates that discrete columnar network structure (coupling of many individual PING networks) is not a necessary condition to investigate PING-type oscillations in the weakly coupled oscillator regime.

\section{Networks with sparsely firing neurons}

In Fig.3, the E-cells had firing rates close to the gamma oscillation frequency $(\sim 30-40 \mathrm{~Hz})$, which might be considered as unusual for regular spiking pyramidal neurons (RS, (Pospischil et al., 2008)). E-cells often spike at much lower rates than the gamma frequency, and in the hippocampus spike rates can be as low as a few $\mathrm{Hz}$ despite gamma oscillations in the $20 \mathrm{to} 80 \mathrm{~Hz}$ range (Sirota et al., 2008). It has also been described in the neocortex that Layer $2 / 3$ networks display more sparse-firing properties compared to Layer 4 (Ainsworth et al., 2011). In contrast, I-cells of the fast-spiking type (FS) have firing rates that can be close to the network gamma rhythm (Fries et al., 2007).To demonstrate that the network behavior described above was not restricted to networks with fast firing E-cells, we constructed a network in which the E-cells had much lower spike rates. It has been described that such 'sparse' networks need a sufficient number of neurons to reach stability (Brunel and Wang, 2003b), as each cell contributes a spike only every few cycles. For computational efficiency we used Izhikevich-type neurons (Izhikevich, 2003) instead of Hodgkin-Huxley $(\mathrm{HH})$ type to increase the network size by an order of magnitude. We were able to replicate the findings from the smaller HH-network in a large Izhikevich-type network where E-cells had 3-4x lower firing rates than the gamma oscillation frequency. Hence, the essential behavior described generalizes to sparser-firing networks. 


\section{Topographic phase-oscillator lattice model}

The topographic phase-oscillator neural network was aimed to represent a simplified sensory cortical area (inspired by V1) (Boucsein et al., 2011) with predominantly local connectivity where input features are smoothly represented over cortical space (feature map). We emphasize however that our topographic network model is not a strict model of a particular sensory area (such as V1 with columns and hyper-columns, layers). Moreover, we used natural visual contrast stimuli as an example for natural input pattern to a visual topographic network, but the described principles of self organization may well apply across different sensory systems. However, we suggest that the main principles underlying the network self-organization are perhaps most easily experimentally testable in a cortical area like V1.

\section{Conduction delay and asymmetric connectivity}

In the PING networks the conduction delay in the interaction was mainly determined by the synaptic postsynaptic potentials [1-2ms]. The conduction delay did not change with distance between neurons. However, conductional delays of horizontal interactions over cortical space can be significant ( $\sim 0.3$ $\mathrm{mm} / \mathrm{ms}$, (Angelucci and Bullier, 2003; Boucsein et al., 2011)). Conduction delays may affect the phaserelation as well as phase-locking. For the phase-oscillator lattice model we included a time-delay term as a linear function of spatial distance (slope of $0.4 \mathrm{~ms} /$ pixel, offset $1 \mathrm{~ms}$ ). By including this term we observed that the spread of spatial synchronization became more limited helping to restrict the 'synchronization fields'. We did not include the time-delay term explicitly in the reconstruction coding formula, but because it affected the phase-locking strength, it was implicitly included in the weighting of phase-relations by phase-locking. An additional factor that is of importance in our models was the assumption of symmetric coupling, and how it interacts with time-delays. Whereas under conditions of symmetric coupling we expect time-delays to affect phase-locking but not phase-relations, under asymmetric coupling we expect pronounced effects on phase-relation. This reflects the fact that symmetric coupling tends to annihilate phase-shifts due to time-delays. More research will be needed to investigate the effect of time delays and asymmetries in connectivity on input-dependent gamma synchronization.

\section{Implications for neural coding}

\section{Background}

The problem of neural coding of sensory signals is a central topic in neuroscience with a long history (for review [1,88-90]). Despite substantial advances over the last decades, many fundamental issues remain unresolved. We will give a short review of different perspectives on this issue. A first important conceptual opposition in the literature is that between rate and time coding. The 'rate-coding hypothesis' has a long tradition founded on early discoveries of a close relationship between spike rate (frequency) and sensory variables. Rate-codes however are constrained by the length of the encoding time window (integration time constant), which defines its resolution. Another limitation is represented by the saturation properties of many spiking neurons in the low and high input range, that is, the limited dynamic range of neuronal spike rate. In this framework, variability in spike times over time is 
considered as noise to be averaged out either over time, or over many neurons. As an alternative to rate coding, the 'time-coding hypothesis' was introduced later, stating that precise spike timing contains a significant amount of information about the stimulus (Timothe Masquelier et al., 2009; VanRullen et al., 2005). Time-codes require short integration time windows (coincidence detectors, (König et al., 1996)). It is well established that single-neuron spike timing contains additional information about time-varying stimuli (Rieke et al., 1997). However, the idea that spike-timing relation between different neurons is meaningful has received less acceptance in the neuroscience community (Singer, 1999a). A further distinction has been made between 'independent single-neuron coding' and 'assembly/ensemble coding'. In an 'independent' scenario, each neuron codes its input by its rate and position within a feature map (position coding) independent of other neurons. All the information is represented in the rate of the single neuron (input-rate transform) and the position it has within the network. However, experimental observations have shown in many studies (Salinas and Sejnowski, 2001) that correlations exists between spike trains at various spatial-and temporal-scales. We consider here precise correlations of spiking timing between neurons (synchrony) in contrast to slow time varying (trial-bytrial) fluctuations in spike rate (known as noise correlation, (Pouget et al., 2000)). There have been various experimental studies showing precise spike synchrony between neurons on a fast-time scale (Diesmann et al., 1999; Engel et al., 1999; Jia et al., 2013a). The observations of spike synchrony indicates that additional information about the stimulus might be present in the relative spike-timing between neurons, which has led to the formulation of the 'assembly/ensemble coding' hypothesis (König and Schillen, 1991a; Sakurai, 1996; Singer, 1999b; Tsukada et al., 1996). Information is represented here in the exact spiking pattern of several neurons. The relationship between oscillation phase coding (assembly code) and single spike rates or population frequency has not been well studied. In the following sections, we will discuss in more depth the implications of our theoretical results for the understanding of gamma phase coding and its relationship to rate/frequency coding.

\section{Complementary coding between frequency and phase}

Our theoretical analysis has shown that oscillating neural networks with local connectivity represent input patterns in frequency and phase according to the TWCO. Of particular importance is the Arnold tongue that describes, as a function of coupling strength and input difference, the transition of frequency coding to phase coding. Reliable phase coding can only exist if neurons are synchronized (phase-locked, (Tiesinga and Sejnowski, 2010)), or in other words converge on a common frequency. That is the reason why frequency and phase coding are in principle complementary, because the process of synchronization 'transfers' the information (detuning magnitude) represented at the level of frequency differences into phase differences (outside vs. inside the Arnold tongue).

We have shown in our models that phase coding can add significant information about the stimulus. The level of contribution of phase coding will depend both on the input as well as on the coupling characteristics. A network will rely more on frequency coding if coupling (interaction) between neuron is weak/sparse and input variability is high, whereas it will rely more on phase coding if coupling is strong and input variability is low. We argue that in many cases the network will be situated between these two extremes and would profit from combining both coding types. 
Previous theoretical studies have already suggested the TWCO as the underlying model of phase-coding. Hoppenstaedt and Izhikevich (Hoppensteadt and Izhikevich, 1998) discussed the theory of weakly coupled oscillators in the context sensory cortical columnar processing. Moreover, Tiesinga and Sejnowski (Tiesinga and Sejnowski, 2010) discussed the behavior of multiple interconnected PING networks using TWCO. They were able to reproduce the experimentally observed gamma phase coding of stimulus orientation in primary visual cortex (Vinck et al., 2010). In their modeling study, each PING network had a different orientation tuning. During 'presentation' of a particular stimulus orientation, the PING networks synchronized and the PING network with strongest input (optimal orientation) led in phase. In other words, the PNG networks operated within the Arnold tongue regime (in which detuning was translated into phase differences). They also described that PING networks with weak coupling showed stronger phase shifts than PING networks with stronger coupling, in line with experimental data (Vinck et al., 2010). We replicated the main observations of Tiesinga and Sejnowski (Tiesinga and Sejnowski, 2010), but also extended their results. First, we confirmed their observations also in networks with continuous spatial connectivity (no assumption of columns). Second, we described in a systematic way networks which 'translated' input/intrinsic frequencies into phase-relations as well as (emergent) frequencies. Moreover, we quantified explicitly the contribution of frequency and phase information to the encoding of simple as well as complex natural stimuli.

We described above the fundamental complimentary nature of frequency and phase coding, as predicted by the Arnold tongue. In assessing the complementary aspects of frequency and phase coding, however several factors need to be taken into account. First, the transition between frequency (asynchrony) and phase coding (synchrony) is not sharp, but characterized by a state of partial synchrony in which both frequency and phase coding can be expressed. Second, noise has an important effect on the level of synchrony / partial synchrony. Third, the encoding time window is critical for the assessment of the contribution of phase and frequency coding. These points are also critical to evaluate the relation of oscillation frequency to single spike rates as described below.

\section{Noise and partial synchrony}

Our study indicates that oscillators with different input strengths/intrinsic frequencies, are not necessarily precluded from synchronization, however in order to synchronize they must first arrive at an emergent 'compromise' frequency. This is apparently at odds with experimental studies showing that different neurons can be phase-locked to a rhythm, yet still express different spike rates (Vinck et al., 2010). Further, this seems at odd with a study that reported significant gamma synchronization between separate neuronal populations despite different (mean) gamma frequencies (Ray and Maunsell, 2010). Importantly, their findings can be accounted for within the TWCO by 'partial synchrony' (Pikovsky et al., 2002), corresponding to an attraction towards a synchronous state, including the adoption of a common frequency, during brief time periods interspersed with periods of non-synchrony and separate frequencies.

Noise plays an important role. As reported also by Tiesinga\&Sejnowski(Tiesinga and Sejnowski, 2010), noise shrinks the border of the Arnold tongue, and increases the amount of partial synchrony. Arnold tongue reconstruction from self-organization in both the PING-networks (Fig.2) and in the phase- 
oscillator lattice model (Fig.8) exhibits large regions of partial synchrony with only a very small region where phase-locking really reaches to locking of 1 . Therefore, although information in noisy networks will be present (on average) in both frequency as well as in phase differences, a strict complementarity will not be present within most of the Arnold tongue. .

\section{Phase coding, single spike rates and encoding time window}

Synchronization of spike rates between single neurons to a common rate has so far not been reported in experimental work, with the exception of an in-vitro study (Markowitz et al., 2008). Theoretically, a single neuron spike rate will be close or equal the population frequency if the neuron is highly synchronized to the population rhythm. In noisy networks however, the synchronization can be low and a single neuron spike rate can be independent of the population frequency (yet still exhibit phase coding, (Tiesinga and Sejnowski, 2010)). This is particularly true for neurons with spike rates much lower than the gamma oscillation frequency, because higher-order 1: $\mathrm{N}$ Arnold tongues are narrow and neurons adapt their spiking rates also by skipping gamma cycles. This fits with experimental data where a rather low locking of single neurons to an oscillation rhythm is observed, especially for pyramidal neurons (Vinck et al., 2013). In this regime, single neuron spike rates contain additional information compared to the local population rhythm (see Suppl.Fig.2) and both phase-relation and single neuron spike rate might contain overlapping ('redundant') information.

Another important aspect however, is how much time is needed to reliably retrieve the relevant information (Gautrais and Thorpe, 1998; Timothe Masquelier et al., 2009; VanRullen et al., 2005). Compared to a rate code, the phase-code can retrieve the information in much shorter time windows (in one or a few oscillation cycles). For example, considering a rate code, a $1 \mathrm{~Hz}$ spike rate difference can be retrieved with a minimal $1000 \mathrm{~ms}$ encoding (integration) time window, whereas with the phase-code a time window of $25-100 \mathrm{~ms}$ might be sufficient for a $40 \mathrm{~Hz}$ oscillation (1-4cycles) to get reliable estimates. Hence, although information about small input differences might be present in both phase-relation and spike rates over longer time windows, a phase-code could be particularly beneficial for stimulus reconstruction on a fast-time scale (e.g. within typical saccade intervals of $\sim 300 \mathrm{~ms}$ ).

\section{Drate-phase transform}

Analogous to the common input-rate transform of single neurons, it was assumed that oscillations implement a rate-phase transform (Fries et al., 2007; McLelland and Paulsen, 2009; Montemurro et al., 2008). That means that the higher the input strength to a neuron, the earlier in phase the neuron will spike within the oscillation cycle. Therefore the phase gives a direct estimate of the absolute input levels. There is experimental evidence for this type of transform for slower frequency oscillations, in particular the delta/theta rhythm (McLelland and Paulsen, 2009; Montemurro et al., 2008). Strong experimental evidence has been obtained from hippocampal theta oscillations, where neurons spike at different phases depending on their input (Buzsáki, 2002). An explicit experimental test for a rate-phase transform was carried out by McLelland\&Paulsen (McLelland and Paulsen, 2009) for the hippocampal theta oscillations. They found that whereas theta oscillations implemented a rate-phase transform, they failed to find it for gamma oscillations. This seemed to be in line with other observations that failed to 
find a systematic relation between the input level and the gamma phase under natural stimulation conditions (Montemurro et al., 2008). In addition, stimulus contrast modulations did not yield phase shifts in macaque V1 (Ray and Maunsell, 2010). However, other experimental studies in macaque V1 have shown that gamma phase can code for orientation tuning (Vinck et al., 2010), suggesting a role of gamma phase in stimulus encoding Hence there are conflicting results whether gamma phase coding can represent input in the same way as has been reported for slower oscillations. Our computational analysis can resolve these seemingly contradictory results. We have shown, in line with previous studies (Ermentrout and Kleinfeld, 2001; Hoppensteadt and Izhikevich, 1998; Tiesinga and Sejnowski, 2010), that the gamma phase coding can be understood in terms of the Arnold tongue, where phase-relations depends on the input difference/detuning (for a given coupling value). Hence, the essential parameter is not the absolute input level, but the input difference between interacting neurons. We term this coding the ' $\Delta$ rate-phase transform'. This transform represents a relative encoding of relative input differences (detuning) between nearby neurons, irrespective of mean input levels. Changes in absolute input levels are in turn represented in the frequency of gamma oscillations. This is in line with the lack of rate-phase transform findings in the gamma range (McLelland and Paulsen, 2009), with the finding of no phaseshifting with contrast (Ray and Maunsell, 2010) and the finding of phase-coding with orientation (Vinck et al., 2010). Orientation tuning is locally defined in visual cortex where nearby neurons are slightly differently driven by a given stimulus orientation. In this case, as shown in Tiesinga and Sejnowski (Tiesinga and Sejnowski, 2010), phase-coding should reflect the input differences between synchronized columns, independently however of overall input drive. We predict therefore that the gamma phasecoding of orientation should be insensitive to overall input strength, e.g. stimulus contrast.

\section{Linkage between coupling and phase coding}

According to the Arnold tongue, phase-relations between oscillating neurons are determined by input (intrinsic frequency) differences as well as coupling strength. This implies that for an exact interpretation of input differences, knowledge about the coupling values is required. In our reconstruction formula we multiplied the phase-differences with coupling values, such that phase-differences of more strongly coupled oscillators were weighted more strongly than from more weakly coupled oscillators. We conceptualize the included coupling term as a 'prior' (representing the general connectivity structure of a network) that might be used by the brain to optimize the input reconstruction performance based on phase. In general, we argue that the dependence of gamma phase coding on connectivity is of high interest and should be investigated in future studies, because it makes the code also sensitive to information (e.g. memory) imprinted in the network connectivity structure.

\section{Input-dependent spatial synchronization}

We observed synchronization between nearby locations and the formation of gamma synchronization fields which were shaped by anatomical connectivity constraints and by the spatial pattern of input. In our simulations, synchronization fields emerged in regions of high local input similarity (low detuning) where nearby neurons shared similar input properties. The shapes of those fields were input-specific; being small in regions of high local input variance and large in regions of low local variance. Their shapes 
were asymmetric around reference oscillators close to large discontinuities in input such as borders in a visual image. An analysis of the topographic network's response to natural images showed that synchronization fields extended away from segmentation borders (as indicated by human observers), and did not cross them, in agreement with the network behavior described above. This network response matches with the statistics of natural images, in which segmentation borders are often associated with large local contrast changes (Marr, 1982), whereas the interior of surfaces often shows more modest variations in local contrast. This indicates that input-dependent spatial synchronization may be meaningful way to cluster/integrate nearby neurons based on input similarity.

The potential of oscillating neural networks for meaningful segmentation of input patterns has been well established in computational neuroscience studies (Chen and Wang, 2002; Kuntimad and Ranganath, 1999; Kuzmina et al., 2004) (in particular visual segmentation), which were inspired by experimental studies on stimulus-specific gamma synchronization over the last decades (Eckhorn, 2000; Engel et al., 1999; Singer, 1999a). However, the proposed segmentation mechanisms differ between studies. In some studies, the clustering is based on a phase-code only (Eckhorn, 1999b; Kuntimad and Ranganath, 1999; Wang and Terman, 1997, 1995), whereas in others it is mainly based on de/synchronization (König and Schillen, 1991a). Clustering capacity of a neuronal network model similar to the one proposed in our study has been demonstrated (König and Schillen, 1991b). Our model architecture differs strongly from model networks characterized by global synchrony, like the LEGION model (local excitatory global inhibitory oscillator network, (Wang and Terman, 1995)) or the PCNN (pulse-coupled neural network, (Kuntimad and Ranganath, 1999)), where clustering is based on phase alone and the network has a single main frequency at any given moment. LEGION and PCNN are powerful for image segmentation tasks, yet, they are not accurate models of cortical gamma oscillations, which are characterized by local synchrony and variable oscillation frequencies. However, they might be more appropriate model for slower oscillation phase (Lisman and Jensen, 2013) or latency coding (Guyonneau et al., 2004). In our simulations we did not explicitly investigate the clustering/segmentation performance of the neural network per se. However, our results give a new perspective into that matter. In particular, the TWCO offers a more precise understanding on how frequency/synchrony and phase in a self-organizing network relate to stimulus input characteristics and network connectivity. According to the Arnold tongue, input variations are transformed into both frequency (synchrony) and phase variations, and therefore both might be useful for clustering. We

further suggest that clustering based on phase and synchrony/frequency would be complementary and represent fine and coarse spatial scales respectively. Finally, the Arnold tongue is an appropriate framework in linking clustering based on connectivity with clustering based on synchronization.

\section{Experimental predictions}

From our theoretical analysis clear predictions can be derived that can be tested experimentally. We offer three key predictions:

1. For measuring the information content of gamma phase coding, e.g. during natural image stimulation in visual cortex, we predict that it is critical to measure the excitation differences 
between nearby locations, because we predict that gamma phase coding does capture absolute input. We also expect that the frequency of gamma oscillations will contain a significant amount of information about the stimulus.

2. By manipulating both excitatory drive independently in two nearby cortical locations as well as the distance between the locations, one should be able to reconstruct an Arnold tongue, assuming that cortical distance is correlated with a declining probability of connectivity. We expect that large excitation differences are expressed in oscillations frequency differences, whereas lower excitation differences will permit synchronization and a translation of the excitation differences into phase-differences. This idea could be tested with optogenetic technology (Cardin et al., 2009) by driving pyramidal cells with different intensities at different locations or by manipulating sensory stimulus features known to manipulate excitatory drive (e.g. contrast in V1).

3. Our model predicts that I-cells (e.g. FS) will exhibit phase-differences, not just E-cells.

Acknowledgments: We thank Bart Gips, Maarten Leenders, Iris Grothe, Pascal Fries, Margarita Zachariou, Joscha Schmiedt and Cristiano Micheli for commenting on the manuscript. This research was supported by NWO VICl grant 453-04-002 (to P.D.W.), NWO VENI grant 451-09-025 (to M.R.), and Maastricht University graduate funding in cooperation with the Donders Institute for Brain Behavior and Cognition in Nijmegen (to P.D.W.).

\section{References}

Ainsworth, M., Lee, S., Cunningham, M.O., Roopun, A.K., Traub, R.D., Kopell, N.J., Whittington, M.A., 2011. Dual Gamma Rhythm Generators Control Interlaminar Synchrony in Auditory Cortex. J. Neurosci. doi:10.1523/JNEUROSCI.2209-11.2011

Ainsworth, M., Lee, S., Cunningham, M.O., Traub, R.D., Kopell, N.J., Whittington, M.A., 2012. Rates and rhythms: a synergistic view of frequency and temporal coding in neuronal networks. Neuron 75 , 572-583. doi:10.1016/j.neuron.2012.08.004

Angelucci, A., Bullier, J., 2003. Reaching beyond the classical receptive field of V1 neurons: Horizontal or feedback axons? J. Physiol. Paris. doi:10.1016/j.jphysparis.2003.09.001

Barardi, A., Sancristóbal, B., Garcia-Ojalvo, J., 2014. Phase-Coherence Transitions and Communication in the Gamma Range between Delay-Coupled Neuronal Populations. PLoS Comput. Biol. 10, e1003723. doi:10.1371/journal.pcbi.1003723

Bendels, M.H.K., Leibold, C., 2007. Generation of theta oscillations by weakly coupled neural oscillators in the presence of noise. J. Comput. Neurosci. 22, 173-89. doi:10.1007/s10827-006-0006-6

Bertrand, O., Tallon-Baudry, C., 2000. Oscillatory gamma activity in humans: a possible role for object representation. Int. J. Psychophysiol. 38, 211-223. doi:10.1016/S0167-8760(00)00166-5

Borst, A., Theunissen, F.E., 1999. Information theory and neural coding. Nat. Neurosci. 2, 947-57. doi:10.1038/14731

Bosman, C.A., Schoffelen, J.-M., Brunet, N., Oostenveld, R., Bastos, A.M., Womelsdorf, T., Rubehn, B., Stieglitz, T., De Weerd, P., Fries, P., 2012. Attentional stimulus selection through selective 
synchronization between monkey visual areas. Neuron $75,875-888$.

doi:10.1016/j.neuron.2012.06.037

Boucsein, C., Nawrot, M.P., Schnepel, P., Aertsen, A., 2011. Beyond the cortical column: abundance and physiology of horizontal connections imply a strong role for inputs from the surround. Front Neurosci 5, 32. doi:10.3389/fnins.2011.00032

Branco, T., Clark, B.A., Häusser, M., 2010. Dendritic discrimination of temporal input sequences in cortical neurons. Science 329, 1671-5. doi:10.1126/science.1189664

Branco, T., Häusser, M., 2011. Synaptic integration gradients in single cortical pyramidal cell dendrites. Neuron 69, 885-92. doi:10.1016/j.neuron.2011.02.006

Breakspear, M., Heitmann, S., Daffertshofer, A., 2010. Generative models of cortical oscillations: neurobiological implications of the kuramoto model. Front Hum Neurosci 4, 190. doi:10.3389/fnhum.2010.00190

Bressler, S.L., Kelso, J.A.S., 2001. Cortical coordination dynamics and cognition. Trends Cogn Sci 5, 26-36.

Brunel, N., Wang, X.-J., 2003a. What determines the frequency of fast network oscillations with irregular neural discharges? I. Synaptic dynamics and excitation-inhibition balance. J. Neurophysiol. 90, 41530. doi:10.1152/jn.01095.2002

Brunel, N., Wang, X.-J., 2003b. What determines the frequency of fast network oscillations with irregular neural discharges? I. Synaptic dynamics and excitation-inhibition balance. J Neurophysiol 90, 415430. doi:10.1152/jn.01095.2002

Brunet, N., Bosman, C. a, Roberts, M., Oostenveld, R., Womelsdorf, T., De Weerd, P., Fries, P., 2013. Visual Cortical Gamma-Band Activity During Free Viewing of Natural Images. Cereb. Cortex 1-9. doi:10.1093/cercor/bht280

Buia, C., Tiesinga, P., 2006. Attentional modulation of firing rate and synchrony in a model cortical network. J. Comput. Neurosci. 20, 247-64. doi:10.1007/s10827-006-6358-0

Buzsáki, G., 2002. Theta oscillations in the hippocampus. Neuron. doi:10.1016/S0896-6273(02)00586-X

Buzsáki, G., Anastassiou, C.A., Koch, C., 2012. The origin of extracellular fields and currents--EEG, ECoG, LFP and spikes. Nat. Rev. Neurosci. 13, 407-20. doi:10.1038/nrn3241

Buzsáki, G., Wang, X.-J., 2012. Mechanisms of gamma oscillations. Annu Rev Neurosci 35, 203-225. doi:10.1146/annurev-neuro-062111-150444

Caporale, N., Dan, Y., 2008. Spike timing-dependent plasticity: a Hebbian learning rule. Annu. Rev. Neurosci. 31, 25-46. doi:10.1146/annurev.neuro.31.060407.125639

Cardin, J.A., Carlén, M., Meletis, K., Knoblich, U., Zhang, F., Deisseroth, K., Tsai, L.-H., Moore, C.I., 2009. Driving fast-spiking cells induces gamma rhythm and controls sensory responses. Nature 459, $663-$ 667. doi:10.1038/nature08002

Chen, K., Wang, D., 2002. A dynamically coupled neural oscillator network for image segmentation. Neural Networks 15, 423-439. doi:10.1016/S0893-6080(02)00028-X

Coombes, S., Bressloff, P.C., 1999. Mode locking and Arnold tongues in integrate-and-fire neural oscillators. Phys. Rev. E. Stat. Phys. Plasmas. Fluids. Relat. Interdiscip. Topics 60, 2086-2096. doi:10.1103/PhysRevE.60.2086

Diesmann, M., Gewaltig, M.O., Aertsen, A., 1999. Stable propagation of synchronous spiking in cortical neural networks. Nature 402, 529-33. doi:10.1038/990101

Duncan, T.E., 1970. On the Calculation of Mutual Information. SIAM J. Appl. Math. 19, 215-220. doi:10.1137/0119020

Eckhorn, R., 1999a. Neural mechanisms of scene segmentation: recordings from the visual cortex suggest basic circuits for linking field models. IEEE Trans Neural Netw 10, 464-479. doi:10.1109/72.761705 
Eckhorn, R., 1999b. Neural mechanisms of scene segmentation: recordings from the visual cortex suggest basic circuits for linking field models. IEEE Trans. Neural Netw. 10, 464-79. doi:10.1109/72.761705

Eckhorn, R., 2000. Cortical synchronization suggests neural principles of visual feature grouping. Acta Neurobiol Exp 60, 261-269.

Eckhorn, R., Bruns, A., Saam, M., Gail, A., Gabriel, A., Brinksmeyer, H.J., 2001. Flexible cortical gammaband correlations suggest neural principles of visual processing. Vis. cogn. 8, 519-530. doi:10.1080/13506280143000098

Eckhorn, R., Reitboeck, H.J., Arndt, M., Dicke, P., 1990. Feature Linking via Synchronization among Distributed Assemblies: Simulations of Results from Cat Visual Cortex. Neural Comput. 2, 293-307. doi:10.1162/neco.1990.2.3.293

Engel, A.K., Fries, P., König, P., Brecht, M., Singer, W., 1999. Temporal binding, binocular rivalry, and consciousness. Conscious. Cogn. 8, 128-51. doi:10.1006/ccog.1999.0389

Ermentrout, G.B., Kleinfeld, D., 2001. Traveling electrical waves in cortex: insights from phase dynamics and speculation on a computational role. Neuron 29, 33-44.

Frazor, R.A., Geisler, W.S., 2006. Local luminance and contrast in natural images. Vis. Res 46, 1585-1598. doi:10.1016/j.visres.2005.06.038

Fries, P., 2005. A mechanism for cognitive dynamics: neuronal communication through neuronal coherence. Trends Cogn. Sci. 9, 474-80. doi:10.1016/j.tics.2005.08.011

Fries, P., 2009. Neuronal gamma-band synchronization as a fundamental process in cortical computation. Annu Rev Neurosci 32, 209-224. doi:10.1146/annurev.neuro.051508.135603

Fries, P., Nikolić, D., Singer, W., 2007. The gamma cycle. Trends Neurosci. 30, 309-16. doi:10.1016/j.tins.2007.05.005

Gail, A., Brinksmeyer, H.J., Eckhorn, R., 2000. Contour decouples gamma activity across texture representation in monkey striate cortex. Cereb Cortex 10, 840-850.

Galán, R., Ermentrout, G., Urban, N., 2005. Efficient Estimation of Phase-Resetting Curves in Real Neurons and its Significance for Neural-Network Modeling. Phys. Rev. Lett. 94, 158101. doi:10.1103/PhysRevLett.94.158101

Gautrais, J., Thorpe, S., 1998. Rate coding versus temporal order coding: a theoretical approach. Biosystems 48, 57-65.

Guyonneau, R., Vanrullen, R., Thorpe, S.J., 2004. Temporal codes and sparse representations: a key to understanding rapid processing in the visual system. J Physiol Paris 98, 487-497. doi:10.1016/j.jphysparis.2005.09.004

Hairer, E., Wanner, G., 1991. Solving ordinary differential equations II: Stiff and differential-algebraic problems, SpringerVerlag. doi:10.1007/978-3-642-05221-7

Havenith, M.N., Yu, S., Biederlack, J., Chen, N.-H., Singer, W., Nikolić, D., 2011. Synchrony makes neurons fire in sequence, and stimulus properties determine who is ahead. J. Neurosci. 31, 8570-84. doi:10.1523/JNEUROSCI.2817-10.2011

Hermes, D., Miller, K.J., Wandell, B. a, Winawer, J., 2014. Stimulus Dependence of Gamma Oscillations in Human Visual Cortex. Cereb. Cortex 1-9. doi:10.1093/cercor/bhu091

Herrmann, C.S., Demiralp, T., 2005. Human EEG gamma oscillations in neuropsychiatric disorders. Clin. Neurophysiol. 116, 2719-33. doi:10.1016/j.clinph.2005.07.007

Hopfield, J.J., Brody, C.D., 2001. What is a moment? Transient synchrony as a collective mechanism for spatiotemporal integration. Proc Natl Acad Sci U S A 98, 1282-1287. doi:10.1073/pnas.031567098

Hoppensteadt, F.C., Izhikevich, E.M., 1996. Synaptic organizations and dynamical properties of weakly connected neural oscillators. I. Analysis of a canonical model. Biol Cybern 75, 117-127.

Hoppensteadt, F.C., Izhikevich, E.M., 1998. Thalamo-cortical interactions modeled by weakly connected oscillators: could the brain use FM radio principles? Biosystems 48, 85-94. 
Izhikevich, E.M., 2003. Simple model of spiking neurons. IEEE Trans Neural Netw 14, 1569-1572. doi:10.1109/TNN.2003.820440

Jaccard, J., Becker, M.A., Wood, G., n.d. Pairwise multiple comparison procedures: A review.

Jensen, O., Gips, B., Bergmann, T.O., Bonnefond, M., 2014. Temporal coding organized by coupled alpha and gamma oscillations prioritize visual processing. Trends Neurosci. 37, 357-369. doi:10.1016/j.tins.2014.04.001

Jensen, O., Goel, P., Kopell, N., Pohja, M., Hari, R., Ermentrout, B., 2005. On the human sensorimotorcortex beta rhythm: sources and modeling. Neuroimage 26, 347-355. doi:10.1016/j.neuroimage.2005.02.008

Jensen, O., Kaiser, J., Lachaux, J.-P., 2007. Human gamma-frequency oscillations associated with attention and memory. Trends Neurosci. 30, 317-24. doi:10.1016/j.tins.2007.05.001

Jia, X., Tanabe, S., Kohn, A., 2013a. $\gamma$ and the coordination of spiking activity in early visual cortex. Neuron 77, 762-74. doi:10.1016/j.neuron.2012.12.036

Jia, X., Xing, D., Kohn, A., 2013b. No consistent relationship between gamma power and peak frequency in macaque primary visual cortex. J Neurosci 33, 17-25. doi:10.1523/JNEUROSCI.1687-12.2013

König, P., Engel, A.K., Singer, W., 1996. Integrator or coincidence detector? The role of the cortical neuron revisited. Trends Neurosci. doi:10.1016/S0166-2236(96)80019-1

König, P., Schillen, T.B., 1991a. Stimulus-Dependent Assembly Formation of Oscillatory Responses: I. Synchronization. Neural Comput. 3, 155-166. doi:10.1162/neco.1991.3.2.155

König, P., Schillen, T.B., 1991b. Stimulus-Dependent Assembly Formation of Oscillatory Responses: I. Synchronization. Neural Comput. 3, 155-166. doi:10.1162/neco.1991.3.2.155

Kuntimad, G., Ranganath, H.S., 1999. Perfect image segmentation using pulse coupled neural networks. IEEE Trans. Neural Netw. 10, 591-8. doi:10.1109/72.761716

Kuzmina, M., Manykin, E., Surina, I., 2004. Oscillatory network with self-organized dynamical connections for synchronization-based image segmentation. Biosystems. 76, 43-53. doi:10.1016/j.biosystems.2004.05.005

Lachaux, J.P., Rodriguez, E., Martinerie, J., Varela, F.J., 1999. Measuring phase synchrony in brain signals. Hum Brain Mapp 8, 194-208.

Lee, K.-H., Williams, L.M., Breakspear, M., Gordon, E., 2003. Synchronous Gamma activity: a review and contribution to an integrative neuroscience model of schizophrenia. Brain Res. Rev. 41, 57-78. doi:10.1016/S0165-0173(02)00220-5

Lee, S., Sen, K., Kopell, N., 2009. Cortical gamma rhythms modulate NMDAR-mediated spike timing dependent plasticity in a biophysical model. PLoS Comput Biol 5, e1000602. doi:10.1371/journal.pcbi.1000602

Lenz, D., Krauel, K., Schadow, J., Baving, L., Duzel, E., Herrmann, C.S., 2008. Enhanced gamma-band activity in ADHD patients lacks correlation with memory performance found in healthy children. Brain Res. 1235, 117-32. doi:10.1016/j.brainres.2008.06.023

Lisman, J.E., Jensen, O., 2013. The ?-? neural code. Neuron 77, 1002-1016. doi:10.1016/j.neuron.2013.03.007

Logothetis, N.K., 2003. The Underpinnings of the BOLD Functional Magnetic Resonance Imaging Signal. J. Neurosci. 23, 3963-3971.

London, M., Häusser, M., 2005. Dendritic computation. Annu. Rev. Neurosci. 28, 503-32. doi:10.1146/annurev.neuro.28.061604.135703

Maris, E., Oostenveld, R., 2007. Nonparametric statistical testing of EEG- and MEG-data. J. Neurosci. Methods 164, 177-190. doi:10.1016/j.jneumeth.2007.03.024

Markowitz, D.A., Collman, F., Brody, C.D., Hopfield, J.J., Tank, D.W., 2008. Rate-specific synchrony: using noisy oscillations to detect equally active neurons. Proc Natl Acad Sci U S A 105, 8422-8427. doi:10.1073/pnas.0803183105 
Markram, H., Gerstner, W., Sjöström, P.J., 2012. Spike-timing-dependent plasticity: a comprehensive overview. Front. Synaptic Neurosci. 4, 2. doi:10.3389/fnsyn.2012.00002

Marr, D., 1982. Vision. book.

Martin, D., Fowlkes, C., Tal, D., Malik, J., 2001. A database of human segmented natural images and its application to evaluating segmentation algorithms and measuring ecological statistics. Proc. Eighth IEEE Int. Conf. Comput. Vision. ICCV 2001 2. doi:10.1109/ICCV.2001.937655

Masquelier, T., Hugues, E., Deco, G., Thorpe, S.J., 2009. Oscillations, phase-of-firing coding, and spike timing-dependent plasticity: an efficient learning scheme. J Neurosci 29, 13484-13493. doi:10.1523/JNEUROSCI.2207-09.2009

Masquelier, T., Hugues, E., Deco, G., Thorpe, S.J., 2009. Oscillations, phase-of-firing coding, and spike timing-dependent plasticity: an efficient learning scheme. J. Neurosci. 29, 13484-93. doi:10.1523/JNEUROSCI.2207-09.2009

McLelland, D., Paulsen, O., 2009. Neuronal oscillations and the rate-to-phase transform: mechanism, model and mutual information. J. Physiol. 587, 769-785. doi:10.1113/jphysiol.2008.164111

Merker, B., 2013. Cortical gamma oscillations: the functional key is activation, not cognition. Neurosci. Biobehav. Rev. 37, 401-17. doi:10.1016/j.neubiorev.2013.01.013

Montemurro, M.A., Rasch, M.J., Murayama, Y., Logothetis, N.K., Panzeri, S., 2008. Phase-of-firing coding of natural visual stimuli in primary visual cortex. Curr. Biol. 18, 375-80. doi:10.1016/j.cub.2008.02.023

Palanca, B.J.A., DeAngelis, G.C., 2005. Does neuronal synchrony underlie visual feature grouping? Neuron 46, 333-46. doi:10.1016/j.neuron.2005.03.002

Picinbono, B., 1997. On instantaneous amplitude and phase of signals. IEEE Trans. Signal Process. 45, 552-560. doi:10.1109/78.558469

Pikovsky, A., Rosenblum, M., Kurths, J., Hilborn, R.C., 2002. Synchronization: A Universal Concept in Nonlinear Science. Am. J. Phys. 70, 655. doi:10.1119/1.1475332

Pospischil, M., Toledo-Rodriguez, M., Monier, C., Piwkowska, Z., Bal, T., Fregnac, Y., Markram, H., Destexhe, A., 2008. Minimal Hodgkin-Huxley type models for different classes of cortical and thalamic neurons. Biol Cybern 99, 427-441. doi:10.1007/s00422-008-0263-8

Pouget, a, Dayan, P., Zemel, R., 2000. Information processing with population codes. Nat. Rev. Neurosci. 1, 125-32. doi:10.1038/35039062

Ranganath, H.S., Kuntimad, G., 1994. Image segmentation using pulse coupled neural networks, in: Proceedings of 1994 IEEE International Conference on Neural Networks (ICNN'94). IEEE, pp. 12851290. doi:10.1109/ICNN.1994.374369

Ray, S., Maunsell, J.H.R., 2010. Differences in gamma frequencies across visual cortex restrict their possible use in computation. Neuron 67, 885-896. doi:10.1016/j.neuron.2010.08.004

Ray, S., Ni, A.M., Maunsell, J.H.R., 2013. Strength of gamma rhythm depends on normalization. PLoS Biol. 11, e1001477. doi:10.1371/journal.pbio.1001477

Rieke, F., Warland, D., De Ruyter Van Steveninck, R., Bialek, W., 1997. Spikes: Exploring the Neural Code, Computational Neuroscience. doi:10.1016/S0065-230X(09)04001-9

Ritz, R., Sejnowski, T.J., 1997. Synchronous oscillatory activity in sensory systems: new vistas on mechanisms. Curr Opin Neurobiol 7, 536-546.

Roberts, M.J., Lowet, E., Brunet, N.M., Ter Wal, M., Tiesinga, P., Fries, P., De Weerd, P., 2013. Robust gamma coherence between macaque $\mathrm{V} 1$ and $\mathrm{V} 2$ by dynamic frequency matching. Neuron $78,523-$ 36. doi:10.1016/j.neuron.2013.03.003

Rosenblum, Pikovsky, Kurths, 1996. Phase synchronization of chaotic oscillators. Phys Rev Lett 76, 18041807.

Sakurai, Y., 1996. Population coding by cell assemblies--what it really is in the brain. Neurosci Res 26, 116. 
Salinas, E., Sejnowski, T.J., 2001. Correlated neuronal activity and the flow of neural information. Nat Rev Neurosci 2, 539-550. doi:10.1038/35086012

Schwemmer, M.A., Lewis, T.J., 2012. Phase Response Curves in Neuroscience, in: Phase Response Curves in Neuroscience. pp. 3-31. doi:10.1007/978-1-4614-0739-3

Singer, W., 1999a. Neuronal Synchrony: A Versatile Code for the Definition of Relations? Neuron 24, 4965. doi:10.1016/S0896-6273(00)80821-1

Singer, W., 1999b. Time as coding space? Curr. Opin. Neurobiol. 9, 189-194. doi:10.1016/S09594388(99)80026-9

Sirota, A., Montgomery, S., Fujisawa, S., Isomura, Y., Zugaro, M., Buzsáki, G., 2008. Entrainment of neocortical neurons and gamma oscillations by the hippocampal theta rhythm. Neuron 60, 683697. doi:10.1016/j.neuron.2008.09.014

Stanley, G.B., 2013. Reading and writing the neural code. Nat. Neurosci. 16, 259-63. doi:10.1038/nn.3330

Steriade, M., Amzica, F., Contreras, D., 1996. Synchronization of fast $(30-40 \mathrm{~Hz})$ spontaneous cortical rhythms during brain activation. J. Neurosci. 16, 392-417.

Stopfer, M., Bhagavan, S., Smith, B.H., Laurent, G., 1997. Impaired odour discrimination on desynchronization of odour-encoding neural assemblies. Nature 390, 70-74. doi:10.1038/36335

Tiesinga, P., Fellous, J.-M., Sejnowski, T.J., 2008. Regulation of spike timing in visual cortical circuits. Nat. Rev. Neurosci. 9, 97-107. doi:10.1038/nrn2315

Tiesinga, P., Sejnowski, T.J., 2009. Cortical enlightenment: are attentional gamma oscillations driven by ING or PING? Neuron 63, 727-732. doi:10.1016/j.neuron.2009.09.009

Tiesinga, P.H., Sejnowski, T.J., 2010. Mechanisms for Phase Shifting in Cortical Networks and their Role in Communication through Coherence. Front Hum Neurosci 4, 196. doi:10.3389/fnhum.2010.00196

Traub, R.D., Spruston, N., Soltesz, I., Konnerth, A., Whittington, M.A., Jefferys, J.G.., 1998. Gammafrequency oscillations: a neuronal population phenomenon, regulated by synaptic and intrinsic cellular processes, and inducing synaptic plasticity. Prog. Neurobiol. 55, 563-575. doi:10.1016/S0301-0082(98)00020-3

Traub, R.D., Whittington, M.A., Colling, S.B., Buzsaki, G., Jefferys, J.G., 1996. Analysis of gamma rhythms in the rat hippocampus in vitro and in vivo. J. Physiol. 493, 471-484.

Tsukada, M., Ichinose, N., Aihara, K., Ito, H., Fujii, H., 1996. Dynamical Cell Assembly Hypothesis Theoretical Possibility of Spatio-temporal Coding in the Cortex. Neural Netw 9, 1303-1350.

VanRullen, R., Guyonneau, R., Thorpe, S.J., 2005. Spike times make sense. Trends Neurosci 28, 1-4. doi:10.1016/j.tins.2004.10.010

Vinck, M., Lima, B., Womelsdorf, T., Oostenveld, R., Singer, W., Neuenschwander, S., Fries, P., 2010. Gamma-phase shifting in awake monkey visual cortex. J Neurosci 30, 1250-1257. doi:10.1523/JNEUROSCI.1623-09.2010

Vinck, M., Womelsdorf, T., Buffalo, E.A., Desimone, R., Fries, P., 2013. Attentional Modulation of CellClass-Specific Gamma-Band Synchronization in Awake Monkey Area V4. Neuron 80, 1077-1089. doi:10.1016/j.neuron.2013.08.019

Wang, D., Terman, D., 1995. Locally excitatory globally inhibitory oscillator networks. IEEE Trans. Neural Netw. 6, 283-6. doi:10.1109/72.363423

Wang, D., Terman, D., 1997. Image Segmentation Based on Oscillatory Correlation. Neural Comput. 9 , 805-836. doi:10.1162/neco.1997.9.4.805

Wang, X.-J., Buzsaki, G., 1996. Gamma Oscillation by Synaptic Inhibition in a Hippocampal Interneuronal Network Model. J. Neurosci. 16, 6402-6413.

Womelsdorf, T., Fries, P., 2007. The role of neuronal synchronization in selective attention. Curr. Opin. Neurobiol. 17, 154-60. doi:10.1016/j.conb.2007.02.002 
Yordanova, J., Banaschewski, T., Kolev, V., Woerner, W., Rothenberger, A., 2001. Abnormal early stages of task stimulus processing in children with attention-deficit hyperactivity disorder - evidence from event-related gamma oscillations. Clin. Neurophysiol. 112, 1096-1108. doi:10.1016/S1388-

2457(01)00524-7

\section{Supporting Text S1}

\section{Method text S1}

\section{Izhikevich neural network.}

In Fig.3 the E-cells spiked regularly close to the network population rhythm. However, the E-cells' spike rate is often reported to be lower than the gamma rhythm (Fries et al., 2007; Vinck et al., 2013). The minimum number of neurons needed to sustain stable gamma oscillations is higher for a sparsely firing network where E-cells fire only every second or third gamma cycle. To demonstrate that the same principles shown in Fig. 3 also apply to more sparsely firing networks, we simulated $(15 \mathrm{sec})$ a ring gamma network with 4000 E-cells (regular-spiking type) and 1000 I-cells (fast-spiking type) based on an Izhikevich-type neuron model (IZ, (Izhikevich, 2003)). Parameters are shown in Table S1.

$$
\begin{gathered}
\frac{d v}{d t}=0.04 v^{2}+5 v+140-u+I \\
\frac{d u}{d t}=a(b v-u) \\
\text { if } v \geq 30 m V \text {, then }\left\{\begin{array}{c}
v \leftarrow c \\
u \leftarrow u+d
\end{array}\right.
\end{gathered}
$$

Network input was similarly constructed as with the Hodgkin-Huxley type network with FS receiving external input current $\mathrm{I}=3.5 \mathrm{mV}( \pm S D=1.5)$ and $\mathrm{RS}$ a sinusoidal modulation of the mean input current $7 \mathrm{mV}$ $( \pm S D=1.5)$ with a spatial sinusoidal amplitude of $1 \mathrm{mV}$. The network connectivity was defined similarly to the phase-oscillator network model

$$
\mathrm{K}_{\mathrm{i}, \mathrm{j}}=\mathrm{C} \exp ^{-\mathrm{D}_{\theta} / \mathrm{s}}
$$

where $C$ is maximal connection strength, $D$ is the distance matrix based on circular distance and $\mathrm{s}$ is the spatial scaling constant. Simulations based on connectivity structure as implemented in the HodgkinHuxley network revealed similar findings in terms of the phase-locking and phase-relation matrix (Fig.S3). The simulation MALTAB code for the IZ-type network as well as the ring phase-oscillator network) are provided in the addendum. 
Table S1. Parameter values of the Izhikevich-type network.

\begin{tabular}{|l|l|l|}
\hline parameter & Regular spiking neurons (RS) & Fast spiking interneuron (FS) \\
\hline a & 0.02 & 0.1 \\
\hline b & 0.2 & 0.2 \\
\hline c (mV) & -65 & -65 \\
\hline$d$ & 8 & 2 \\
\hline Connectivity & Spatial constant s & Max connection strength (C) \\
\hline$E \rightarrow E$ & 0.4 & 0.004 \\
\hline$E \rightarrow I$ & 0.3 & 0.07 \\
\hline$I \rightarrow E$ & 0.3 & -0.04 \\
\hline$I \rightarrow I$ & 0.3 & -0.015 \\
\hline
\end{tabular}

Upper half: Neuron types are defined by the four parameter a, b, c and $d$ in the Izhikevich neuron model (Izhikevich, 2003). These values are defined for regular spiking neurons (RS) in the middle column and for fast-spiking neurons (FS) in the right column. Lower Half: The connectivity matrix of the network. The spatial constant defines the rate of the exponential decay function of connectivity over space (middle column). The maximum connectivity strength is defined in the rightmost column.

\section{Code S1}

\section{A. The ring-Izhikevich-type PING network}






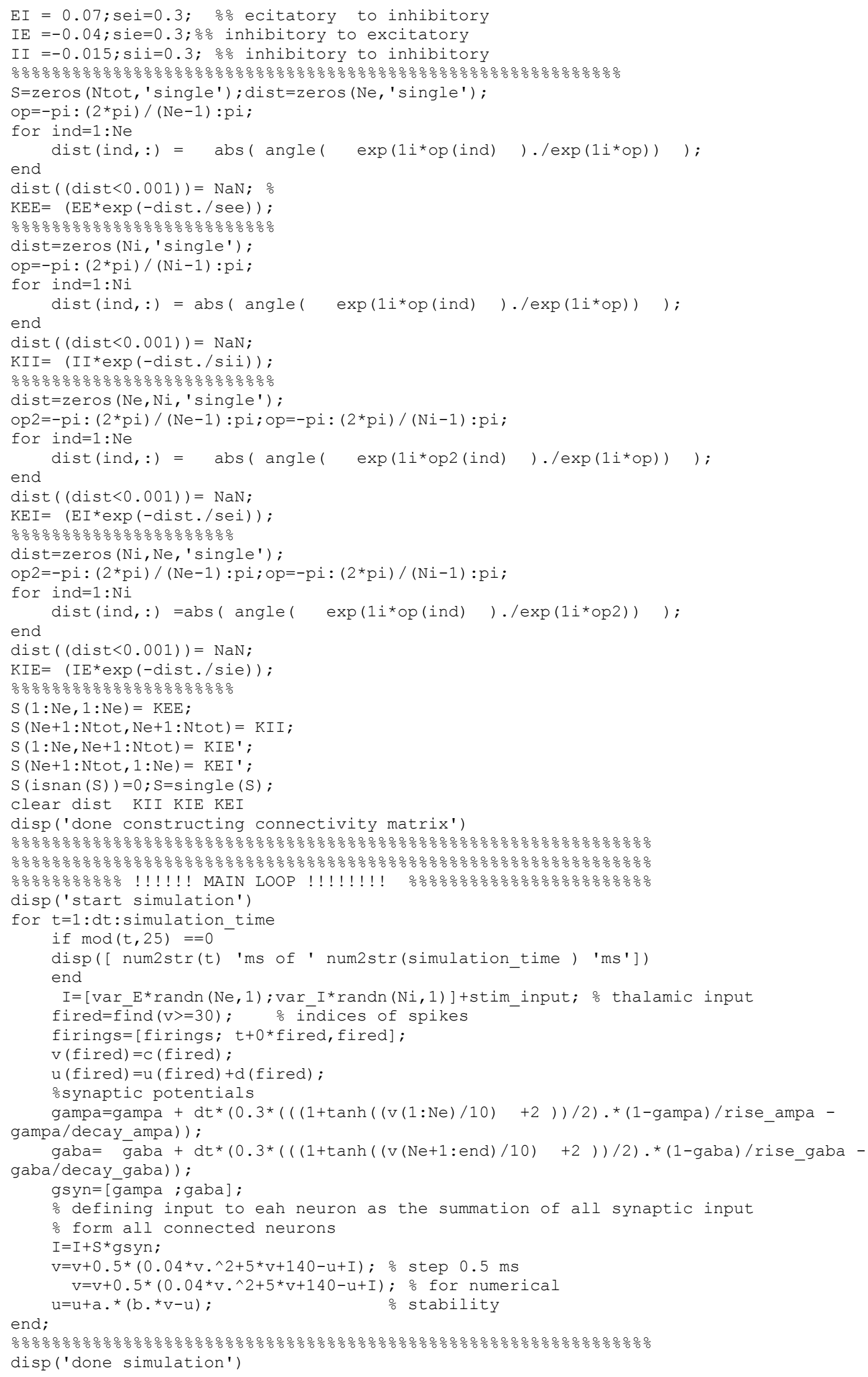




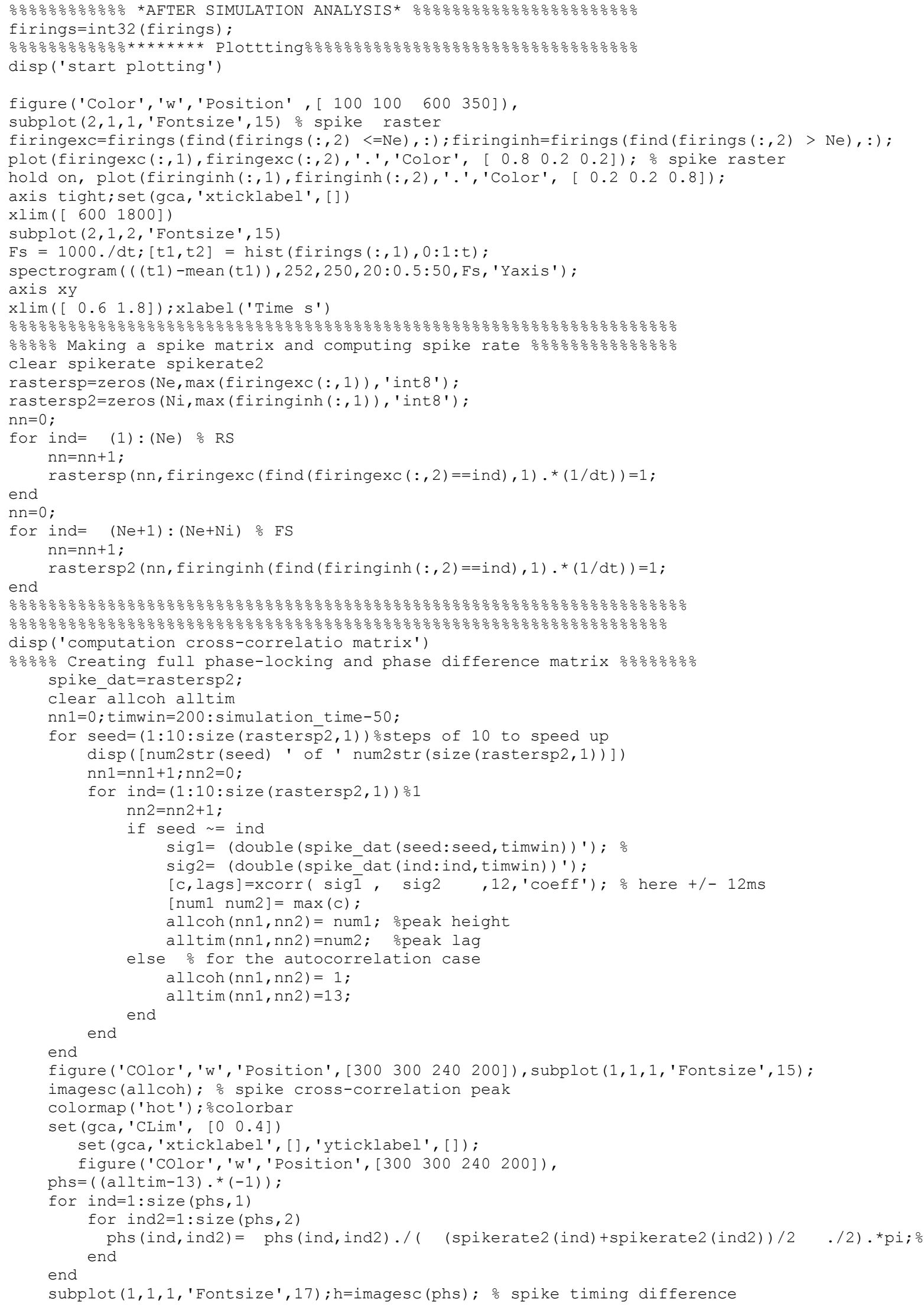




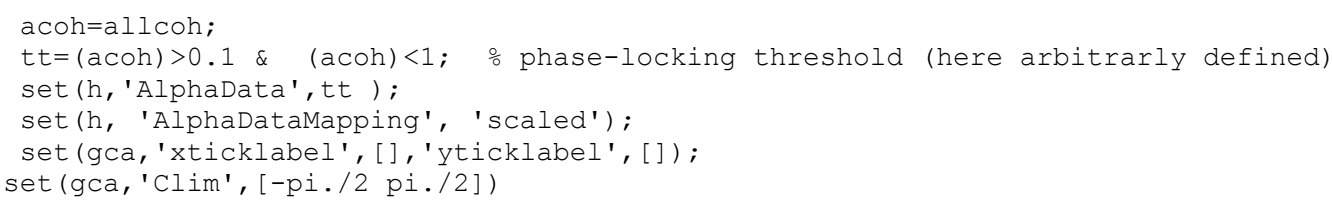

\section{B. The ring-phase-oscillator network}

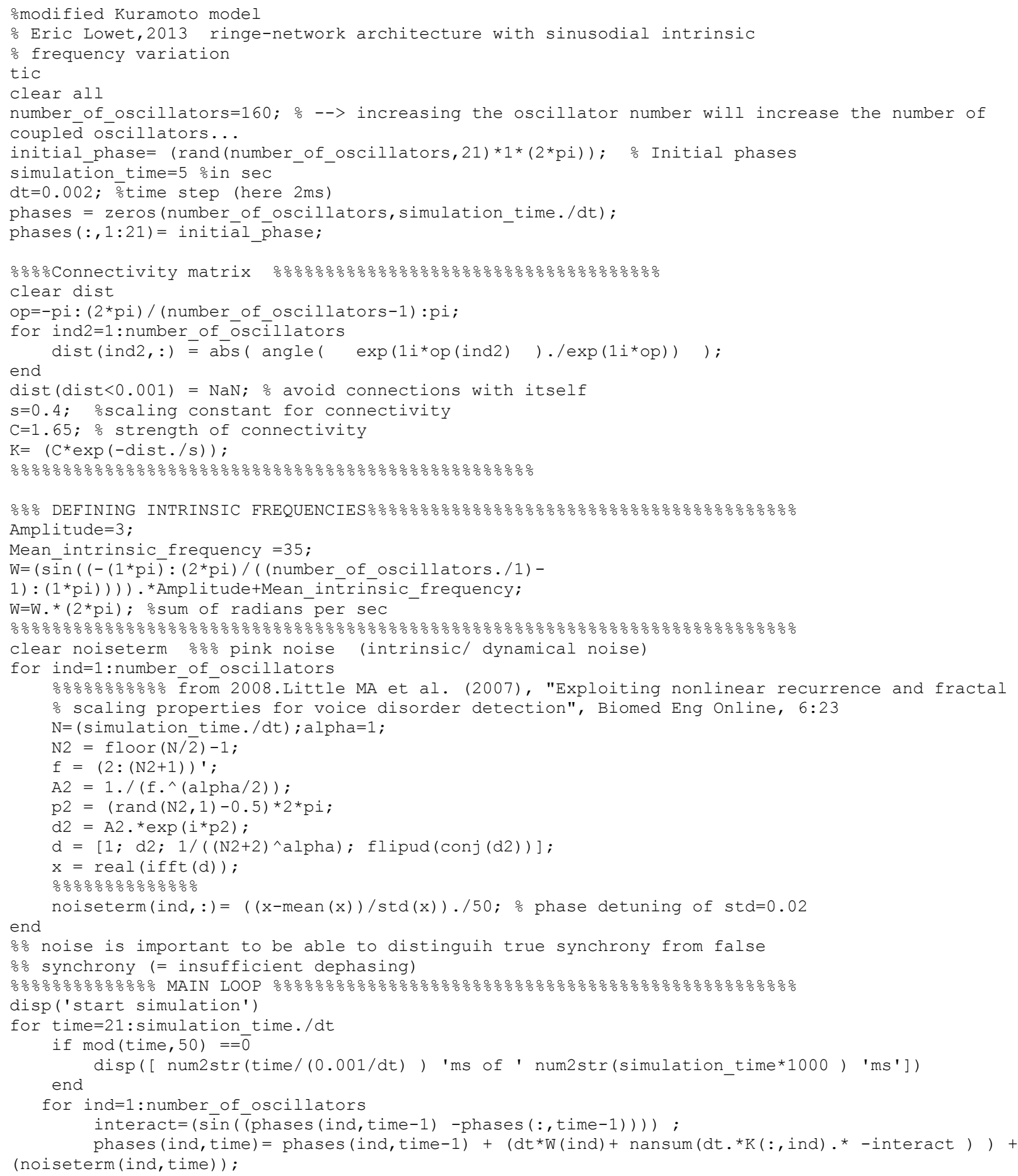


end

disp ('done simulation')



$\mathrm{ph}=\left(\bmod \left(\right.\right.$ phases' $\left.\left.^{\prime}, 2 * \mathrm{pi}\right)\right)$ '; \%phases

$\mathrm{xx}=\left(\exp (1 i * \operatorname{ph}(:, 100: 1: \text { end }))^{\prime}\right)$;

$\mathrm{ab}=\mathrm{xx}^{\prime} * \mathrm{xx}$;

allcoh=abs (ab); allcoh=allcoh./max(allcoh(:)); \%phase locking matrix

allph=angle (ab); $\quad$ ophase relation matrix

응 Plotting the phase locking matrix

figure ('Color', 'w', 'Position', [300 300300 200]), subplot(1,1,1, 'Fontsize', 17); imagesc(allcoh)

set (gca, 'clim', [ 01 )

colormap ('hot')

set (gca, 'xticklabel', [], 'yticklabel', [ ] ) ; colorbar

axis $x y$

응 Plotting the phase relation matrix

coh thres $=0.25$; $\frac{\circ}{0}$ theshold for illustration

figure('Color', 'w', 'Position', [640 300300 200]),

subplot $(1,1,1$, 'Fontsize', 17); h=imagesc (allph)

acoh=allcoh;

$t t=($ acoh $)>$ coh thres; o phase-lcoking threshold

set (h, 'AlphaDāta', tt );

set (h, 'AlphaDataMapping', 'scaled') ;

set (gca, 'xticklabel', [], 'yticklabel', [ ] )

set(gca,' 'clim', [ -pi/2 pi/2])

colorbar

axis $x y$

toc

\section{A Connectivty types}

I

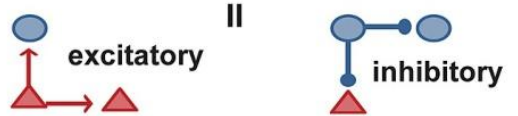

B Phase response curve (PRC)

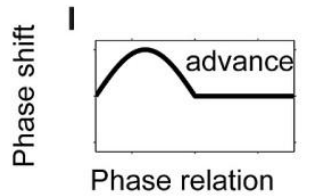

C Arnold tongue

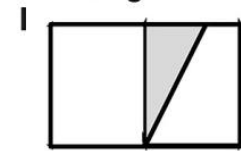

$\Delta \omega$
II

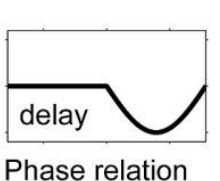

Phase relation

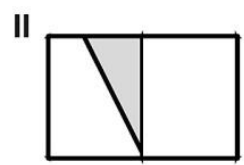

$\Delta \omega$

D Phase-locking matrix (phase-oscillators)

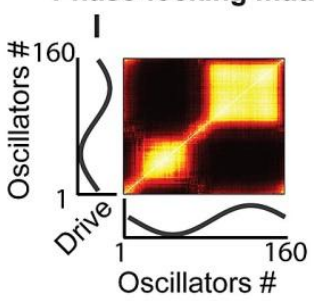

II

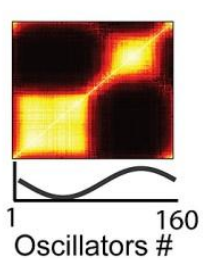

E Higher E / / connection ratio
High $\mathrm{E}<->\mathrm{E}$
$\left(0.075 \mathrm{mS} / \mathrm{cm}^{2}, \mathrm{n}=25\right)$
No $I<->\mid$
$\left(0 \mathrm{mS} / \mathrm{cm}^{2}, \mathrm{n}=0\right)$
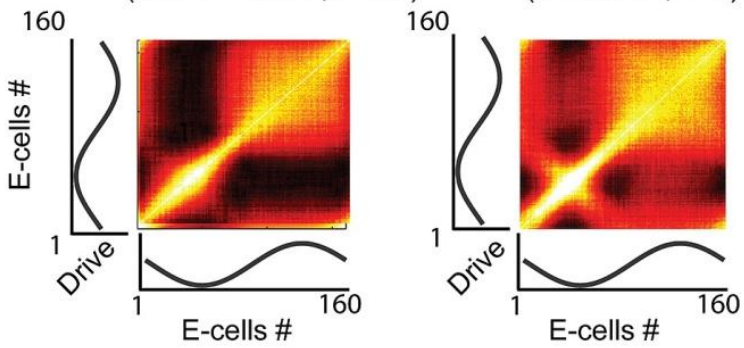

F

Lower E / I connection ratio

No $E<->E$

$\left(0 \mathrm{mS} / \mathrm{cm}^{2}, \mathrm{n}=0\right.$ )

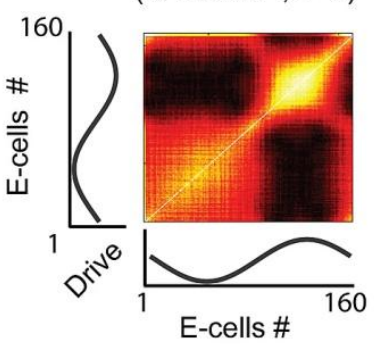

0.7



High I <-> I $\left(0.3 \mathrm{mS} / \mathrm{cm}^{2}, \mathrm{n}=6\right.$ )



Figure S1. The effect of changing connection parameters in the ring-PING network. The main results are that we observed changes in the spatial synchronization properties as a function of different relative strengths of E-E, E-I, I-E and I-I synaptic connections. We present in this figure a heuristic for understanding these changes. In A) we show the two main types of connection in the PING networks, (I) the excitatory type (AMPA) and (II) the inhibitory (GABA-A) type. In B) we associate these two connection types with respective advancing (I) and delaying (II) part of the phase-response curve (PRC) assuming type 1 
PRC (Schwemmer and Lewis, 2012). Type 1 PRC means that excitatory synaptic input will advance the next spike (occurs earlier) and inhibitory synaptic input delays the next spike (occurs later). In C) the respective resultant Arnold tongues are depicted if only either excitatory (I) or inhibitory (II) would be present. In D) we show the resultant phase-locking matrix of the ring-phaseoscillator network simulation. In (I) we kept only an advancing component of the (infinitesimal) PRC, whereas in (II) only the delaying part. It can be observed that synchrony is enhanced around the peak of the sinusoidal input and reduced at the trough (I) and vice versa for (II). Advancing PRC (I) connections are good for entraining a lower frequency oscillation by a higher frequency oscillation which therefore leads to stronger synchronization around the higher frequencies of the sinusoidal input (peak). In E) we show the results of the ring PING network where we strengthened the dominance of excitatory connections (I) by either strengthening E-E connections (left) or reducing I-I connections (right). The phase-locking matrix resembled the matrix resulting from phase-oscillator simulations. In F) we strengthened the dominance of inhibitory connections by either decreasing E-E connections (left) or strengthening I-I connections. Again, the phase-locking matrix looks similar to the one with phaseoscillator networks with delaying PRC only.
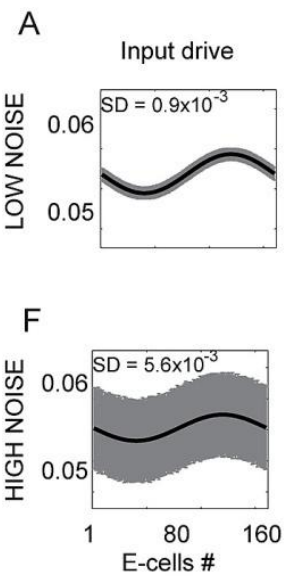

B


C
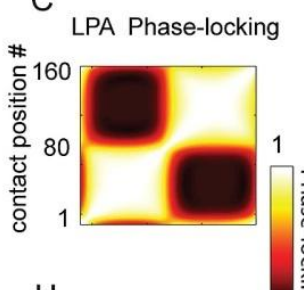

$0^{-10}$

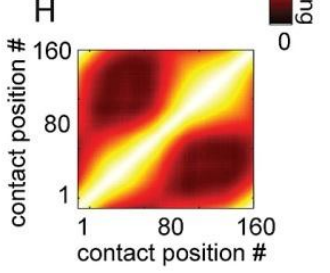

D

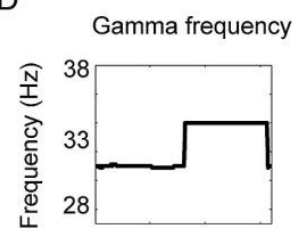

E
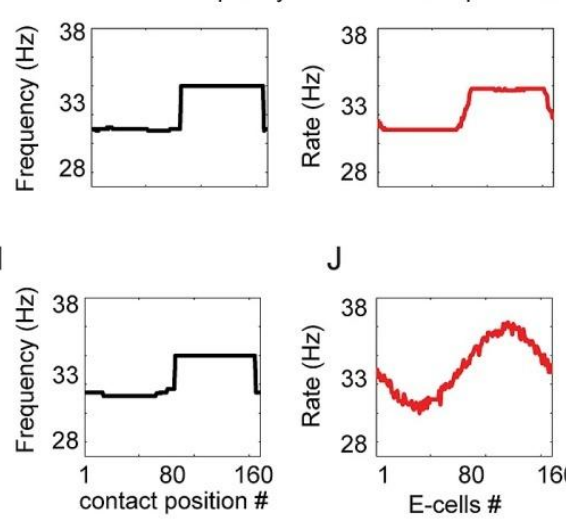

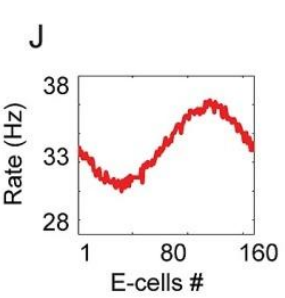

Figure S2. The effect of noise on the ring-PING network. Main results are that noise reduced synchronization (shrinking Arnold tongue, see (Tiesinga and Sejnowski, 2010)) and dissociated single E-cell spike rate from the population gamma oscillation (as measured with LPA). Noise here was a Gaussian fluctuation of AMPA input rate over time added to the spatially defined

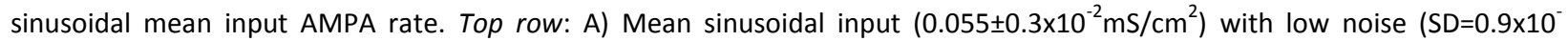
${ }^{3} \mathrm{mS} / \mathrm{cm}^{2}$ ). B) The power spectra of the LPA contact points (see Methods) clearly showed gamma power with mainly two synchronous 'components' around the trough and peak of the sinusoidal input. In C) the phase-locking among all LPAs are shown in phase-locking matrix. In D) we quantified the gamma frequency (frequency of the maximal power peak). In E) the single E-cell spike rates are shown. Notice that D) and E) are highly similar. Bottom row: F) High noise input ( $\mathrm{SD}=5.6 \times 10^{-3}$ $\mathrm{mS} / \mathrm{cm}^{2}$ ). G) The power spectra of the LPAs still show gamma power with two main 'components', however reduced in size. In $\mathrm{H})$ the LPA phase-locking matrix is shown. The synchronization between oscillators over larger distances is strongly reduced. In I) the gamma frequency is depicted still exhibiting frequency plateaus. The E-cell spikes rates in J) however lost these plateaus completely and have spike rates similar to the rates they would have if unconnected (intrinsic frequency). 
A

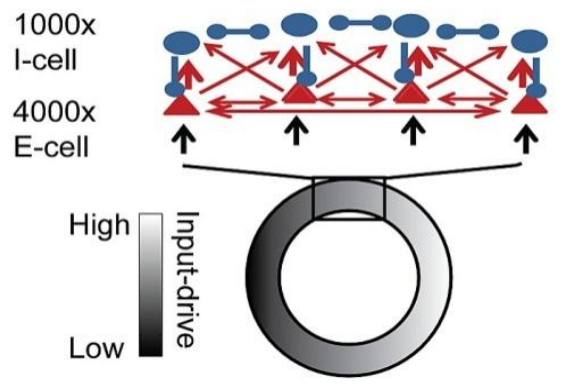

C



B



D

Phase- locking

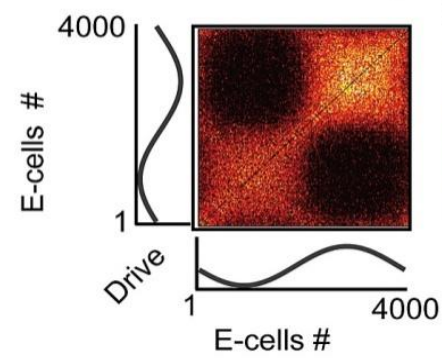

Phase-relation



Phase-relation

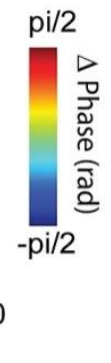

Figure S3. Replication of Hodgkin-Huxley $(\mathrm{HH})$ results in Figure 3 using Izhikevich (IZ) neuron types (E-cell = regular-spiking type, I-cell = fast-spiking type). In the $\mathrm{HH}$-network the E-cells had firings rates close to the gamma rhythm. However, pyramidal cells (E-cell) often have spikes rates lower than the gamma rhythm (Brunel and Wang, 2003b; Buzsáki and Wang, 2012; Fries et al., 2007). We therefore used IZ-type neurons with E-cells having lower rates than the gamma frequency. We used IZ-types because of their computational efficiency as it is known that low E-cell rates needs larger networks (Brunel and Wang, 2003a).(A) The general network architecture of the Izhi-network, replicating the architecture of main text Figure 3, however with $80 x$ more cells. (B) Extract of a simulation, where the black line (top) is a LPA signal, blue line is the voltage membrane of a I-cell (middle) and the red line is voltage membrane of an E-cell (bottom). Notice that the E-cell is skipping many gamma cycles. (C) Here the mean gamma frequency (black) estimated from the LPA, the mean E-cell spike rate (red) and the mean I-cell spike rate (blue) is shown. Whereas the I-cell had spike rates close to the gamma frequency $(\sim 35-40 \mathrm{~Hz})$, the E-cell had spike rates much lower $(\sim 10-15 \mathrm{~Hz})$. In D) the phase-locking (left) as well as the phase-relation (right) matrix is shown between all E-cell pairs in the matrix. The phase-relation values were threshold $(0.3>)$ for illustrative reasons. The network behaved in similar manner as previously described even though individual E-cells had lower firing rates than the gamma rhythm.

A



B



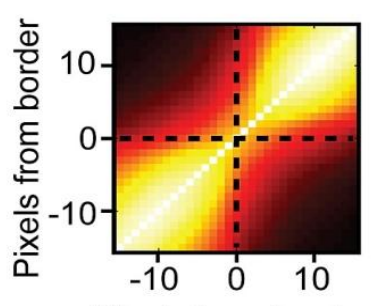

Pixels from border
C

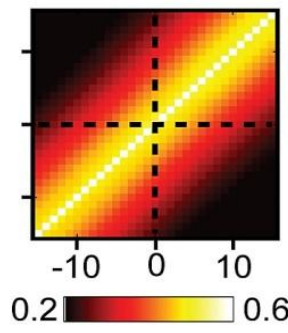

Phase-locking
D

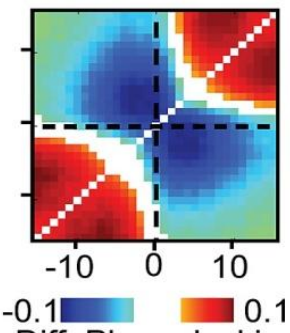

Diff. Phase-locking $p<0.01$

Figure S4. Segmentation-border analysis. A) Capturing spatial windows (sized 31X1 pixels) for quantitative analysis illustrated in an example image. Windows were centered on a border and could be horizontal or vertical. All windows were then aligned to each other and concatenated. B) Mean phase-locking between all possible pairs of oscillators along border-centered spatial windows. Dashed line represents the 0 -axis (position of the border). C) As for B, but phase-locking matrix was computed from spatial windows located randomly on the image to construct a null distribution. D) Differences in phase-locking between $B$ and $C$, red colors indicate higher than expected phase-locking, blue lower than expected. White indicates that differences were not significant (permutation test, (Maris and Oostenveld, 2007)). 


\section{Chapter 6}

\section{Problems of spectral coherence for quantifying oscillatory synchronization}

Based on:

E.Lowet, M. J. Roberts, P.Bonizzi, J.Karel and P. De Weerd, "Problems of spectral coherence for quantifying oscillatory synchronization" (in review) 


\begin{abstract}
Phase synchronization (phase-locking) between oscillating neuronal groups is considered to be important for understanding how information is coordinated and shared among cortical networks. Spectral phase-locking measures like coherence are still largely used to quantify phase locking. They are based on the assumption of the stationarity of signals. We systematically explored the validity of coherence measures applied for quantifying synchronization among neural oscillators. To that aim, we simulated coupled oscillatory signals that exhibited realistic synchronization dynamics. We found that within a large parameter range the spectral phase-locking measure deviated substantially from the expected phase-locking and did not converge to the true value with increasing signal-to-noise. We found that it particularly failed with oscillators being in the partially synchronized state due to the fast changing frequency and amplitude changes induced by synchronization forces. The partially synchronized state, in which oscillators are neither completely phase coupled nor asynchronous, is a likely scenario for neural synchronization. As an alternative we discuss phase-locking estimation approaches that are based on the reconstruction of the instantaneous phase. Here we computed the singular spectrum decomposition (SSD) of the signal for noise-reduction and to assure that the signal is mono-componental, followed by the extraction of instantaneous phases of the signal using the HilbertTransform. Phase-locking estimates based on the instantaneous phase have broad applicability as they do not rely on stationarity, and, contrary to coherence, have the desirable property of more accurate estimations of oscillatory synchronization across a wide range of different synchronization regimes.
\end{abstract}




\section{Introduction}

Neuronal oscillatory synchronization is widespread in the nervous system (Buzsáki and Draguhn, 2004; Buzsáki, 2006; Steriade et al., 1993) and it has been suggested to play a critical role in the coordination of spiking activity across neuronal populations (Fries, 2009; Salinas and Sejnowski, 2001; Tiesinga et al., 2008). In particular, the phase synchronization (Pikovsky et al., 2002) of local or distant oscillatory neuronal populations has received substantial scientific interest (Bosman et al., 2012; Buschman and Miller, 2007; Colgin et al., 2009; Gail et al., 2000; Gregoriou et al., 2009; Ray and Maunsell, 2010; Roberts et al., 2013; Saalmann et al., 2012; Sirota et al., 2008; Womelsdorf et al., 2007) and is central to influential theories like 'Communication through coherence' (CTC, (Bosman et al., 2012; Fries, 2005)) and the 'Binding by synchrony theory' (Eckhorn et al., 1990; Engel et al., 1999). It is therefore of critical importance to have a valid approach for measuring the phase consistency (phase-locking) between neural oscillations to be able to accurately test the theoretical models with experimental observations.

A popular approach to measure phase consistency is to apply spectral coherence analysis (Bosman et al., 2012; Gregoriou et al., 2009; Ray and Maunsell, 2010; Roberts et al., 2013; Saalmann et al., 2012). This analysis estimates the linear phase-consistency between neuronal oscillations (Carter et al., 1973). Because coherence is also sensitive to amplitude correlations (Lachaux et al., 1999; Srinath and Ray, 2014), a modified index was devised yielding a spectral phase-locking value (termed here as PLV $\mathrm{FFT}_{\text {, }}$ (Bastos et al., 2014; Vinck et al., 2010) ), which is only sensitive to the phase information of the spectrum. A critical property of spectral phase-locking measures is that they assume (wide-sense) stationarity which means that the autocorrelation structure of a signal is not dependent on the reference time point. It has been shown not to hold for single trial data, which is characterized by moment-by-moment fluctuations in oscillation amplitude and frequency (Atallah and Scanziani, 2009; Burns et al., 2011, 2010; Lakatos et al., 2005; Roberts et al., 2013). These non-stationarities may partly reflect the dynamic nature of synchronization, during which oscillations in interacting neural populations mutually adapt their rhythms (Pikovsky et al., 2002). Since in an extended network, there are likely to be complex and changing interactions between synchronizing and desynchronizing forces, neural networks are typically characterized by continuously ongoing non-stationarities (Bressler and Kelso, 2001), as revealed in single trial analysis (Atallah and Scanziani, 2009; Burns et al., 2011, 2010; Lakatos et al., 2005; Roberts et al., 2013).

The aim of the present study was to study the problems that arise when applying spectral phase locking estimation to oscillatory synchronization data that most likely exhibit non-stationary properties. To that goal, we simulated oscillatory signals with plausible synchronization dynamics (Pikovsky et al., 2002). We manipulated the phase-locking strength between the oscillations predictably by changing their detuning parameter (initial frequency difference). We found that spectral-based PLV deviated from the expected PLV, and it did not converge to the true value with increasing signal-to-noise ratio (SNR). The deviation was particularly strong in case when oscillators were not completely synchronized to 1 , either because of detuning (intrinsic frequency difference) or intrinsic noise fluctuations. This is because synchronization in this regime leads to systematic fast-changing frequency fluctuations. Moreover, spectral PLV was sensitive to phase-relation dependent amplitude fluctuations showing that it is not a 
pure phase-locking measure. As alternative, we aimed to test another approach, by estimating the phase-locking strength on the instantaneous phases of the oscillatory signals, as had been suggested originally for PLV (Lachaux et al., 1999; LeVanQuyen et al., 2001). Neuronal field signals like the local field potential (LFP) are complex multi-component signals and hence need to be decomposed to extract meaningful phase estimates. We here used the singular spectrum decomposition (SSD, (Bonizzi et al., 2014)) to extract the oscillation signal, which is as a completely data-driven decomposition technique able to track linear as well non-linear components. The results showed that phase-locking methods based on the instantaneous phase gave more accurate estimations of phase synchronization robustly across a wide range of synchronization regimes.

\section{Methods and Results}

\section{NOTATIONS}

\begin{tabular}{|c|c|}
\hline$X, Y$ & oscillator $\mathrm{X}$ or $\mathrm{Y}$ \\
\hline $\mathrm{t}$ & time variable \\
\hline $\mathrm{T}$ & Time length \\
\hline$\omega$ & frequency (intrinsic) \\
\hline A & amplitude \\
\hline$\phi$ & phase \\
\hline$\theta$ & Phase difference \\
\hline$\Delta \omega$ & frequency difference (detuning) \\
\hline K & interaction strength (coupling) \\
\hline$\varepsilon$ & phase offset between oscillators \\
\hline PrFM & phase-relation dependent frequency modulation \\
\hline PrAM & phase-relation dependent amplitude modulation \\
\hline$\alpha$ & modulation strength of PrAM \\
\hline PRC & phase response curve \\
\hline PLV & phase locking value \\
\hline $\mathrm{PLV}_{\mathrm{T}}$ & true phase locking value (analytically derived) \\
\hline Coh & spectral coherence \\
\hline$S_{x}, S_{y}$ & Fourier spectra of oscillator $\mathrm{X}$ or $\mathrm{Y}$ \\
\hline $\mathrm{S}_{\mathrm{xx}}, \mathrm{S}_{\mathrm{yy}}$ & power spectra of oscillator $\mathrm{X}$ or $\mathrm{Y}$ \\
\hline$S_{x y}$ & Fourier cross-spectrum of $X$ and $Y$ \\
\hline SM $M_{\text {PrFM,PrAM }}$ & modulation sideband induced by PrFM or/and PrAM \\
\hline SNR & signal-to-noise ratio \\
\hline$\chi$ & chi distribution \\
\hline Nw & white noise process \\
\hline$\theta_{w}$ & uniform phase distribution (white noise) \\
\hline $\mathrm{PLV}_{\mathrm{FFT}}$ & spectral-based PLV estimate \\
\hline $\mathrm{PLV}_{\text {INST }}$ & PLV estimate based on instantaneous phases \\
\hline $\mathrm{PLV}_{\mathrm{u}}^{2}$ & unbiased population estimate of $\mathrm{PLV}^{2}$ \\
\hline
\end{tabular}




$\begin{array}{ll}\text { Arg } & \text { principal value of argument function (=atan2) } \\ \text { Np } & \text { pink noise process } \\ \text { Rel.Pow } & \text { relative power ratio } \\ \text { HT } & \text { Hilbert-Transform } \\ \text { SSA } & \text { singular spectrum analysis } \\ \text { SSD } & \text { singular spectrum decomposition } \\ \text { IMF } & \text { intrinsic mode function } \\ \text { EMD } & \text { empirical mode decomposition } \\ \text { HHT } & \text { Hilbert-Huang Transform } \\ \text { TWCO } & \text { Theory of weakly coupled oscillators }\end{array}$

\section{Model of phase synchronization}

Phase synchronization is the process in which oscillators adjust their rhythms (Pikovsky et al., 2002), a phenomenon that has been first described by Huygens in $17^{\text {th }}$ century between pendulum clocks. Phase synchronization means that the oscillators have a preferred phase-relation to each other and that the oscillators adjust their phases as a function of phase difference. The adjustment of phase is defined by the 'phase response curve' (PRC), which has been investigated in various neuroscience domains (Schwemmer and Lewis, 2012). The PRC defines how much the phase of an oscillation is delayed or advanced by (e.g., from another oscillator) as a function of the phase the oscillator had when a force was exerted. Further, it defines which phase-relations among oscillators occur preferentially, thus representing fixed attractor points in the phase-relations among oscillators. The theory of weakly coupled oscillators (TWCO) (Breakspear et al., 2010; Hoppensteadt and Izhikevich, 1996) describes mathematically the phase dynamics among weakly interacting oscillators. 'Weak' means that interactions lead to phase adjustments without strong perturbations of the oscillatory generative mechanism. 'Strong' coupling can lead to chaotic regimes (Battaglia and Hansel, 2011; Rosenblum et al., 1996) or to 'oscillation death/quenching' (Pikovsky et al., 2002; Zou et al., 2009). TWCO has been applied in many neuroscience fields, including in gamma-generating neural network models (Tiesinga and Sejnowski, 2010). To simulate oscillatory synchronization data, we used a basic model of phaseoscillators (Breakspear et al., 2010) that is simple, yet exhibits plausible phase synchronization dynamics.

\section{Phase-oscillator model}

An oscillator is defined here as a periodic process that is defined by its phase $\theta$, frequency $\omega$ and amplitude $A$. If amplitude is unity and the oscillator is unperturbed the evolution of an oscillator can be defined as

$$
\text { (1) } \frac{d \varphi_{X}(t)}{d t}=\omega_{X}(t)
$$

where the phase of a oscillator $X$ is defined by its (intrinsic) frequency. In case of two interacting oscillators $X$ and $Y$, the exact phase trajectory of oscillator $X$ and $Y$ depends on the interaction term. 


$$
\text { (2) } \frac{d \varphi_{X}(t)}{d t}=\omega_{X}(t)+\kappa_{X Y}\left(\sin \left(\varphi_{Y}(t)-\varphi_{X}(t)\right)\right)
$$

The phase of oscillator $X$ (or $Y$ ) is determined by an intrinsic (natural) frequency $(\omega)$ and an interaction term that describes the phase adjustments (phase response curve, PRC) induced by the other oscillator $Y$ (or $\mathrm{X}$ ) depending on the coupling constant $\mathrm{k}\left(\mathrm{\kappa}_{\mathrm{X}, \mathrm{Y}}\right.$ or $\left.\mathrm{\kappa}_{\mathrm{Y}, \mathrm{X}}\right)$. As the interaction term we used a sinusoidal function with an attractor fixed point (in-phase) and repeller fixed point (anti-phase) (Kuramoto model, (Breakspear et al., 2010)). It has been shown that the evolution of the phase relation between the two oscillators can be described with a single equation, referred to as the Adler equation (Pikovsky et al., 2002):

$$
\begin{aligned}
& \text { (3) } \frac{d \theta}{d t}=\Delta \omega+K * \sin (\theta) \\
& \text { (4) } \theta=\varphi_{Y}-\varphi_{X}
\end{aligned}
$$

The equation shows that the time evolution of the phase relation $\theta(t)$ is a function of the frequency difference $\Delta \omega(t)$ at that time, and the coupling strength $\kappa$ of the sinusoidal interaction function. We show below how the phase-locking strength between two coupled phase-oscillators can be derived.

\section{Synchronization properties}

The synchronization properties of the coupled phase-oscillators are governed by two factors: the level of detuning $\Delta \omega(t)$ (intrinsic frequency difference) and the coupling strength $\kappa$. The detuning $\Delta \omega(t)$ determines the phase precession frequency (de-synchronization force) and the coupling $\kappa$ determines the strength of phase adjustments (synchronization force). Both parameters define a two-dimensional space ( $x$-axis: detuning, $y$-axis: coupling strength) in which the phase-locking between oscillators can be determined. In this space one can observe inverted triangles that define the phase-locking region in the detuning versus coupling space. The triangular phase-locking regions look like tongues, and are referred to as 'Arnold' tongues(Pikovsky et al., 2002)). The triangular shape derives from the fact that oscillators with stronger coupling strength $\kappa$ can phase-lock with larger detuning $\Delta \omega(t)$.

The classical definition of phase-locking is that the phase-differences is constant over time, hence no phase precession and full synchrony (Pikovsky et al., 2002)

$$
\text { (5) }\left\|n \varphi_{Y}(t)-m \varphi_{X}(t)\right\|=c \forall t \quad c \in \mathbb{R}, \quad m, n \in \mathbb{Z} \text {. }
$$

The $n$ and $m$ are integers and represent the frequency ratios $(1: 2,2: 3, \ldots$, (Pikovsky et al., 2002; Tiesinga and Sejnowski, 2010)) in which the oscillators can satisfy the condition of phase-locking despite different frequencies. Therefore one can observe several (higher-order) Arnold tongues. Here, we focused on phase-locking with $n=m=1$ (Arnold tongue 1:1). This is because we were interested in the quantification 
of phase synchronization among oscillators with nearby frequencies (oscillators within a 'frequency band', e.g. the gamma band).

Phase-locking is often not complete, and can be considered present if the phase-relation distribution is non-uniform. This means that specific phase-relations are more likely than others, despite some phase precession being present. This implies that phase-locking can be of different magnitudes. This is in line with the idea that biological systems are inherently noisy and complex (i.e. variable frequencies over time) and thus unlikely to engage in complete synchrony.

In agreement with the continuous variation of phase locking, one can observe that outside of the Arnold tongues the phase-locking does not vanish abruptly to 0 (uniform distribution). Instead there is a decay of phase-locking with distance from the Arnold tongue. The incomplete phase-locking is called the partially synchronized state (Pikovsky et al., 2002) in which oscillators still exhibit a preference for particular phase-relations intermixed with periods of phase precession. The only case where the phaserelation distribution is strictly uniform (full phase precession) is when the coupling strength $k$ equals 0 .

A

$$
\text { oscillator } X \text { oscillator } Y
$$

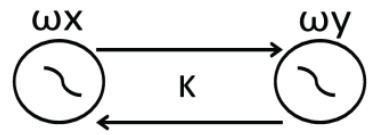

$\Delta \omega(\omega y-\omega x)$
B

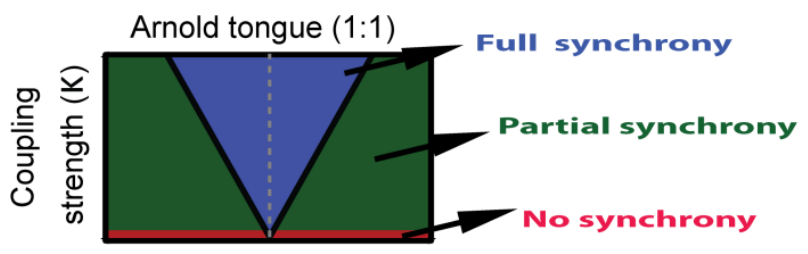

Detuning $\Delta \omega$

C
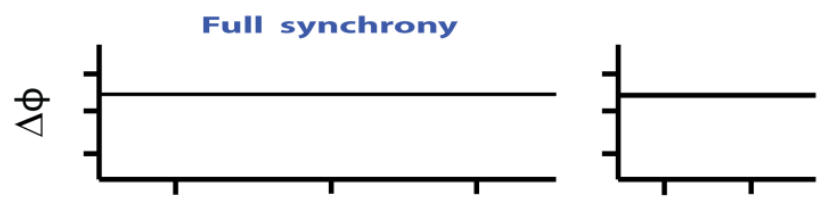

Properties:

- same frequency

- no phase precession

- perfect locking

D
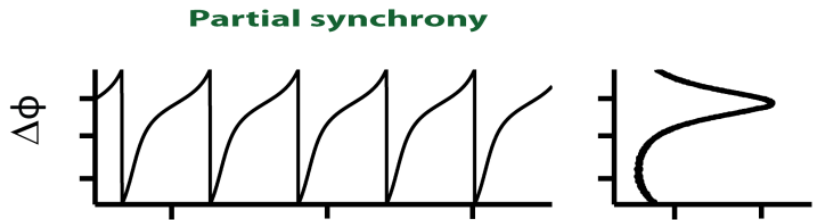

\section{Properties:}

- different frequency

- phase precession

- locking between 0 and 1

- phase-relation dependent fluctuations

E No synchrony

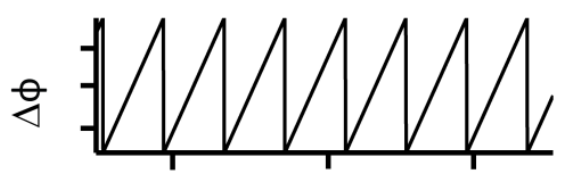

Time

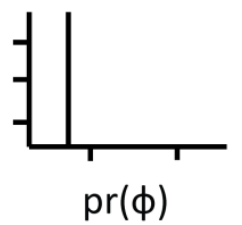

\section{Properties:}

- different frequency

- phase precession

- no locking

Figure 1: Basic properties of oscillatory phase synchronization. A) The underlying model of this study was a phase-oscillator model governed by two principal factors: The intrinsic (natural) frequency $\omega$ and the coupling strength $\mathrm{k}$. The intrinsic frequency difference (detuning $\Delta \omega$ ) between oscillators determines the phase precession. The coupling strength $\mathrm{K}$ determines the interaction strength, which is a function of the phase-relation (defined by the phase response curve, PRC). B-E). The 
detuning $\Delta \omega$ and the coupling strength $\mathrm{k}$ definesd a 2-dimensional space, in which phase-locking occurs within certain ranges. In a noiseless system, full synchrony (phase locking of 1) occurs in a limited area of detuning and coupling strength that appears as inverted triangle (Arnold tongue). The stronger the coupling strength, the more detuning is possible while still reaching full synchrony. Full synchrony (C) occurs if the oscillators converge on a common frequency (no phase precession). The phase difference distribution exhibits a strong peak at the attractor phase relation. Partially synchrony takes place outside of the Arnold tongue. (D) The state of partial synchrony is characterized by phase-locking between 0 and 1 . In most regions in the $\Delta \omega$ by $\mathrm{k}$ space, phase-locking might be close to 0 . Yet, close to the Arnold tongue the phase-locking might still be substantial. The oscillators do not converge to a common frequency, but exhibit phase precession. The phase precession does not have a smooth trajectory, but is modulated depending on the phase-relation. This leads to non-uniform phase-difference distribution with a peak at the phase-relation in which the oscillators have the closest frequency difference. E) Complete asynchrony is only possible when the oscillators are uncoupled. The phase-precession is smooth and the phase-difference distribution uniform.

\section{Deriving phase-locking value from two coupled phase-oscillators}

In this study we used a commonly applied measure to test for a non-uniform phase-relation distribution which is defined as the mean vector length, the phase locking value (PLV, (Lachaux et al., 1999)).

$$
\text { (6) } P L V=\left\|\frac{1}{N} \sum_{t=1}^{N} e^{i \theta(t)}\right\|
$$

where $N$ is number of sample points and $t$ is the time variable. If the phase-relation distribution is uniform, the PLV will give a value of 0 (in practice, due to finite data, PLV will be always slightly larger than zero), while if there is a particular peak in the distribution, the PLV will give a value $>0$. There are also different measures that might be more favorable (Tort et al., 2010), yet we used PLV here because it is currently a widely used measure (in addition to coherence) in neuroscience. The PLV characteristics described in the present study are also valid for coherence.

The true phase locking value $\mathrm{PLV}_{\mathrm{T}}$ between two coupled oscillators is related in a simple manner to the sinusoidal coupling function defining the PRC (Hoppensteadt and Izhikevich, 1998). If the detuning $|\Delta \omega|$ is smaller or equal than the amplitude of the coupling function $K$ then the phase locking value is 1 . This is the case when full synchrony among oscillators is achieved. In this case, the instantaneous phase relation is constant over time and no phase precession is present. This is because the detuning $\Delta \omega$ and the associated tendency to phase precess, cannot overcome the 'attractor' defined by the PRC with the strength $\kappa$. The true phase locking value $\mathrm{PLV}_{\mathrm{T}}$ then equals to:

\section{(7) $\mathrm{PLV}_{\mathrm{T}}=1$ if $|\Delta \omega| \leq x \forall t>t_{0}$}

However, in the case in which frequency detuning $\Delta \omega(\mathrm{t})$ is stronger than the coupling strength $(\kappa: \Delta \omega(\mathrm{t})$ $>K$ ), the attractor is not strong enough to prevent phase precession. Hence, full synchrony is no longer possible. Yet, the phase relation distribution is not uniform and oscillators are still more likely to be in their preferred relationship. This is because the rate of phase precession (or instantaneous frequency difference) changes as a function of phase-relation. When the oscillators are at their preferred relationship, the phase precession speed is minimized (the point of the PRC with the strongest phase 
adjustment opposite to the phase precession), whereas at the non-preferred phase-relation, the phase precession speed is maximal. The modulation of the instantaneous phase relation leads to non-uniform phase-relations and thus to phase-locking of $>0$.

For deriving the $\mathrm{PLV}_{\mathrm{T}}$ for $|\Delta \omega|>\mathrm{k}$, we computed the resultant vector length of the phase-relation probability density distribution (pdf), which can be derived from the Adler equation (see equation 3 ) that describes the time derivative of the phase-relation as a function of the phase-relation and a frequency difference constant. Notice that the time derivative of the phase-relation is the instantaneous frequency difference. In the case of $|\Delta \omega|>k$ the time derivative is always non-zero (no fixed point). To derive the pdf, we first rearranged the terms of equation 3 to

$$
\text { (8) } d t=\frac{1}{\Delta \omega+\kappa \sin (\theta)} d \theta
$$

, where the time derivative is a function of phase derivative and the inverse of the Adler function. Integrating this function over the phase space gives the characteristic time period $\mathrm{T}$ for which the oscillators need to make a full phase precession (Pikovsky et al., 2002). Because the (noiseless) oscillators repeat exactly the same patterns at intervals $T$, considering only period $T$ is sufficient for the full determination of the pdf.

$$
\text { (9) } T=\int_{0}^{2 \pi} \frac{1}{\Delta \omega+\pi \sin (\theta)} d \theta
$$

It can be easily seen that integrating around a small phase interval, divided by $\mathrm{T}$, gives the probability value (the amount of time the phase-relation $\theta$ spent in the phase interval $[a, b]$ within the time scale T).

$$
\text { (10) } \operatorname{Pr}[a \leq \theta \leq b]=\frac{1}{T} \int_{a}^{b} \frac{1}{\Delta \omega+\kappa \sin (\theta)} d \theta
$$

The inverse of the Adler function therefore describes the probability density function.

$$
\text { (11) } p d f(\theta)=\frac{1}{\Delta \omega+\kappa \sin (\theta)}
$$

To compute the true phase-locking value $\mathrm{PLV}_{\mathrm{T}}$, one needs to integrate over all the phases with their associated probability densities.

$$
\text { (12) } \mathrm{PLV}_{T}=\| \frac{1}{T} \int_{0}^{2 \pi} p d f(\theta) e^{i \theta} d \theta \mid \quad \text { if } 0<\kappa<|\Delta \omega|
$$

Complete asynchrony is only possible when the oscillators are not coupled and hence there are no phase-relation dependent modulations of phase precession speed (pdf is described by a constant).

$$
\text { (13) } \mathrm{PLV}_{T}=0 \text { if } \mathrm{K}=0,|\Delta \omega|>0
$$




\section{Partially synchronized state: PrFM and PrAM}

Here, we describe in more detail the partially synchronized state and the implications for the oscillatory dynamics. The partial synchronized state is characterized by incomplete phase-locking and therefore the presence of phase precession. This means that oscillators will traverse all possible phase-relations over time. As we have described above, the PRC defines the phase adjustments in terms of positive or negative delays as a function of phase-relation and time (Fig.2A). The phase change over time, the time derivative of phase, is the definition of the instantaneous frequency (IF) of an oscillator (Pikovsky et al., 2002). Close to the preferred phase-relation, the IF difference between phase-oscillators is minimized (phase precession slows down), whereas in non-preferred phase-relations the IF difference is maximal (phase precession is faster and IF approaches the intrinsic frequency). This will lead to systematic phaserelation-dependent IF fluctuations (PrFM) (Fig.2B-C). The main frequency of PrFM equals the phase precession frequency (Pikovsky et al., 2002).

A

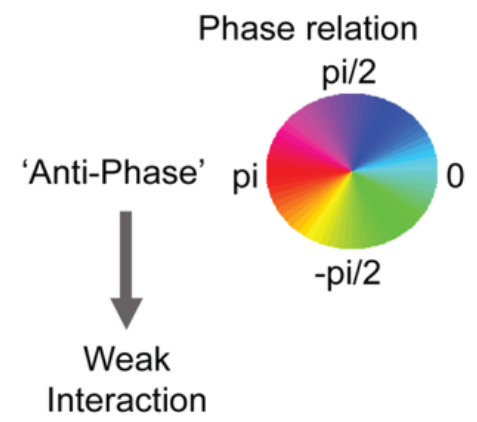

B

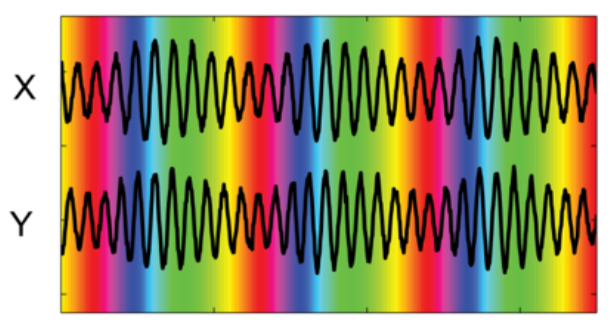

'In -Phase' $\quad$ ஸ્

C


phase relation (PrFM)
Type I : Frequency modulation by

\section{Strong} Interaction
D

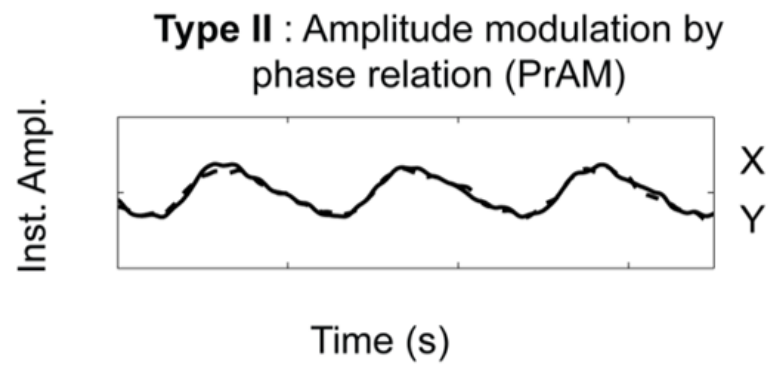

Figure 2: Effect of partially synchronized state. A) Polar representation of possible phase-relations [-pi to pi] between two oscillators. Phase synchronization occurs if two oscillators have a particular phase-relation that 'attracts' them (here In-phase) and in which they start to interact. Other phase-relations do not 'attract' and the oscillators interact weakly. B) Phase-relation dependent interactions lead to phase-relation dependent fluctuation in frequency and/or power $\mathrm{C}$ ) Phase-relation dependent (instantaneous) Frequency modulation (PrFM) are a defining property of partially synchronized states. When the phase-relation is close to the preferred phase-relation ('attractor'), the frequency of oscillators tends to converge (slower phase precession). Far from the preferred phase-relation, the frequency diverges (faster phase precession). Because the frequency is modulated, the phase-locking will vary between 0 and 1. D) Phase-relation dependent amplitude modulation (PrAM) does not necessarily arise with partially synchronized states, yet we expect that it will likely occur in neural networks. This is because phase-relation modulates the gain of transmitted spikes between networks, which in turn influences the excitability/synchrony of the networks. Fluctuations in excitability/synchrony will likely change the power (see Discussion). In the phase-oscillator simulations we introduced PrAM post-hoc. 
In the model, the phase-oscillators had unit amplitude, which did not change during the synchronization dynamics (following the theory of weakly coupled theory). However, we included a form of phaserelation dependent amplitude modulation (PrAM) post-hoc to the simulated oscillatory signals (Fig.2bD). We included PrAM based on our experience with Hodgkin-Huxley based simulations of gammaoscillation generating excitatory-inhibitory neural networks, where we observed PrAM (beside PrFM) between two interacting networks in partially synchronized states, yet still being well describable by TWCO. The Phase-relation dependent frequency modulations (PrFM) in our simulation of coupled phase-oscillators could be described with the following equation:

$$
\text { (14) } \operatorname{PrFM}(\theta)=\kappa \sin (\theta)
$$

where $\theta$ is the phase-relation between the two oscillators, sin the sinusoidal modulation function (PRC) and $K$ the coupling strength. Hence, the modulation function of PrFM related directly to the PRC, whereas the strength of the fluctuation depends on the coupling strength $\kappa$.

The Phase-relation dependent amplitude modulations (PrAM) were defined as:

$$
\text { (15) } \operatorname{PrAM}(\theta)=\alpha \cos (\theta)
$$

where $\theta$ is the phase-relation between two oscillators, $\cos$ is the cosine modulation function (maximal amplitude at phase 0 ) and $\alpha$ is the amplitude modulation strength. Note that $\alpha$ was defined as percentage modulation of oscillation amplitude. For example, a $20 \%$ modulation means that the amplitude of the sinusoidal modulation function represent $20 \%$ of the oscillation amplitude.

\section{Spectral phase-locking measures}

We assumed here that the measure of interest is phase-synchronization among neural oscillations. Moreover, we assumed that the underlying process is oscillatory and that phase-locking is achieved through the process of synchronization.

\section{Spectral phase-locking measures}

Spectral phase-locking measures are used in many experimental studies and also offered by widely-used analysis toolbox in neuroscience as the principal method to quantify phase-locking (Fieldtrip (Oostenveld et al., 2011), Chronux (Bokil et al., 2010)). Commonly used spectral-based measures are the coherence index (Carter et al., 1973) or its modification to spectral phase-locking value (Vinck et al.,

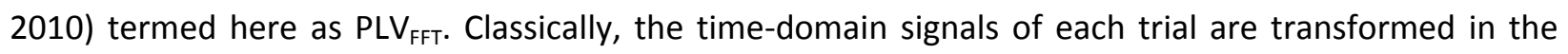
frequency-domain (e.g. Fourier transform) and phase-coupling is assessed frequency-by-frequency. The advantage is that one can observe, in a computationally efficient manner, frequency-resolved peaks in phase-locking, which yield a quick overview of phase locking over relevant frequencies. However, the coherence index and spectrally based phase locking values assume (wide-sense) stationary processes. We describe below spectral coherence, and the spectral-based PLV, which is similar to spectral coherence, but is not sensitive to amplitude correlations. 


\section{Fourier-based Phase-locking value ( $\left.P L V_{F F T}\right)$}

First, to estimate the spectral phase, we computed the (discrete) Fourier transform of the time series of the oscillatory signal $X(t)(t=0, \ldots T-1)$ of a given trial with length $T$.

$$
\text { (16) } S_{x}(\omega)=\sum_{t=0}^{T-1} X(t) e^{-\mathrm{i} 2 \pi \omega t / P}
$$

where $S_{x}(f)$ is the complex-valued Fourier coefficient at frequency $\omega$. The spectral power is defined as:

$$
\text { (17) } S_{x x}(\omega)=S_{x}(\omega) S_{x}(\omega)^{*}
$$

The spectral coherence and PLV between two signals $x$ and $y$ are based on the Fourier cross-spectral density estimate in the form of the cross-spectral periodogram of $S_{x}(\omega)$ and $S_{y}(\omega)$, defined as follows:

$$
\text { (18) } S_{x y}(\omega)=S_{x}(\omega) S_{y}(\omega)^{*}
$$

where $S_{x y}(\omega)$ is the complex-valued Fourier cross-spectral periodogram at frequency $\omega$, and where $S_{y}(\omega)^{*}$ is the complex conjugate of $S_{y}(\omega)$. The cross-spectral density reflects both the mean phasedifference as well as the power correlation between $S_{x}(\omega)$ and $S_{y}(\omega)$. The spectral coherence Coh $(\omega)$ (Vinck et al., 2010)) is therefore normalized by the respective spectral power and is defined as follows:

$$
\text { (19) } \operatorname{Coh}(\omega)=\frac{\left|\sum_{n=1}^{N} S x y(\omega)\right|}{\sqrt{\left(\sum_{n=1}^{N} S x x(\omega)\right)\left(\sum_{n=1}^{N} S y y(\omega)\right)}}
$$

where $n$ is the trial number $(n=1, . . N)$. A critical point of spectral coherence is that the $S x y(\omega)$ value depends on the phase as well as the amplitude correlation. Each trial therefore contributes as a function of amplitude correlation making spectral coherence sensitive to amplitude correlation values (Lachaux et al., 1999; Srinath and Ray, 2014). The phase-locking value (PLV) was introduced as being insensitive to amplitude correlation and hence to provide a better phase-locking measure (Lachaux et al., 1999). Note that the original definition was based on the instantaneous phase (Lachaux et al., 1999). Spectral PLV is computed as follows:

$$
\text { (20) } \operatorname{PLV}_{\mathrm{FFT}}(\omega)=\left|\frac{1}{N} \sum_{n=1}^{N} \frac{S x y(\omega)}{\sqrt{S x x(\omega) S_{y y}(\omega)}}\right|
$$

Each absolute cross-spectral product for a given trial $\mathrm{n}$ and frequency $\omega$ is normalized with square-root product of the autospectra $\operatorname{Sxx}(\omega)$ and $\operatorname{Syy}(\omega)$. Therefore different levels of amplitude correlations do not affect the phase-locking measure. It has been therefore been assumed that spectral PLV (PLV $\mathrm{FFT}_{\text {) }}$ is a pure phase-locking measure (Oostenveld et al., 2011; Vinck et al., 2010). 
The results shown in this study are very similar for spectral coherence (equation 19) or spectral phaselocking value (equation 20), because the amplitude correlation are, if PrAM is included, small. The effect of amplitude correlation has been systematically studied recently (Srinath and Ray, 2014). We chose the spectral phase-locking value $\left(\mathrm{PLV}_{\mathrm{FFT}}\right)$ for the following results as it promises to better estimate the phase-locking than spectral coherence, because coherence is not a pure phase-locking measure. However it needs to be noted that spectral coherence is still a very popular oscillatory phase coupling measure (Baldauf and Desimone, 2014; Bosman et al., 2012; Gregoriou et al., 2009; Ray and Maunsell, 2010; Saalmann et al., 2012)). It can be expected that coherence will be worse or equal than the estimation accuracy of $\mathrm{PLV}_{\mathrm{FFT}}$. Our results can be generalized to any measure that bases the estimation on cross-spectral periode-gram (where an estimate is done for each frequency bin separately). We define this class of phase-locking measures with the term 'spectral phase-locking measures'. As we describe below the underlying problems reside in the spectral estimation itself and not at the level of the quantification of the phase-relation distribution. Therefore applying other measures like information theoretic approaches (Tort et al., 2010) will not resolve the problems as described below.

\section{Effects of applying spectral phase-locking measures on data reflecting partially synchronized states}

Oscillatory processes that exhibit phase synchronization without perfect phase locking, are in a partially synchronized state, and will show characteristic PrFM and likely also PrAM. These systematic modulations of the oscillation frequency and power occur at the frequency of phase precession (equal to the frequency difference between oscillators). These types of modulations can be seen as a form of cross-frequency coupling (CFC, (Jensen and Colgin, 2007)), here between oscillators of nearby frequencies and with the phase-relation as the modulation variable. More common is the notion of CFC between the phase of a slower frequency oscillation (e.g. delta $3 \mathrm{~Hz}$ ) and the power of a higher frequency oscillation (e.g. (low) gamma-band, $45 \mathrm{~Hz}$ ). In that case, the modulation variable is the phase of the slower oscillation and not the phase-relationship (compare Fig.3A with Fig3E).

The Fourier transform of the slow and high frequency oscillations gave expected peaks in the power spectrum at $3 \mathrm{~Hz}$ and $45 \mathrm{~Hz}$ respectively (Fig.3B-C). Yet, the power spectrum $S_{y y}$ of the high frequency oscillation showed two additional peaks, here at $42 \mathrm{~Hz}$ and $48 \mathrm{~Hz}$ (Fig3C). These 'modulation sidebands' have been previously described (Aru et al., 2014) which occur through systematic power modulation by a slow oscillation phase. A systematic amplitude modulation (AM) of $3 \mathrm{~Hz}$ in the high frequency oscillation locked to the phase of the lower frequency signal induces modulation sidebands at $\pm 3 \mathrm{~Hz}$ from

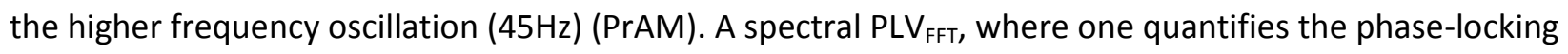
frequency-by frequency (Fig.3D) is not meaningful as $X$ and $Y$ did not share power at common frequencies. The PLV $\mathrm{FFT}_{\text {Was }}$ trivially 0.

The CFC between two oscillations of nearby frequencies (i.e. 42 and $45 \mathrm{~Hz}$ ) in which frequency is modulated as a function of their phase-relation at phase precession frequency $(3 \mathrm{~Hz})$ also lead to modulation sidebands (Fig3G) that are located $\pm 3 \mathrm{~Hz}$ of the highest gamma frequency (PrFM). Here we are interested in computing the CFC phase-phase locking between the two oscillatory signals (Belluscio 
et al., 2012). To that aim, one would need to compute the relationship of the phases at the higher frequency $(45 \mathrm{~Hz})$ to the phases at the lower frequency $(42 \mathrm{~Hz})$, because here the PLV $\mathrm{F}_{\mathrm{FT}}$ is not meaningful because the oscillators $X$ and $Y$ did not share power at common frequencies. Yet, when computing spectral PLV $\mathrm{FFT}_{\text {it }}$ gave values of 1 . This is because one of the spectral sidebands next to the power peak at the higher frequency oscillation overlapped with the power peak in the spectrum of the lower frequency oscillation. Note that this phase locking estimate is bound to be incorrect, because it computes PLV frequency per frequency, yet here, one would need to compute PLV across frequencies. Below we explain in more detail why the modulation sidebands are induced by PrFM and PrAM and how phaselocking computed with PLV FFT leads to erroneous estimates of phase locking under these conditions.

\section{Modulation sideband induced by PrFM}

For demonstration purposes, we assume as first step that the oscillatory processes have no intrinsic (dynamical) and extrinsic (measurement) noise. Further, we assume that oscillator $X$ is unperturbed and unidirectionally coupled to oscillator $Y$ with a coupling strength of $\mathrm{k}=1$. Oscillator $Y$ exhibited $\operatorname{PrFM}$. The oscillators have a frequency difference such that $\left|\omega_{X}-\omega_{Y}\right|>\mathrm{k}$. The parameter $\varepsilon$ is the initial phaseoffset between the oscillators. Oscillator $Y$ is defined as in equation (1). Oscillator $X$ is defined as follows:

$$
\text { (21) } \frac{d \varphi_{X}(t)}{d t}=\omega_{X}(t)+\sin \left(\varphi_{Y}(t)-\varphi_{X}(t)\right)+\varepsilon
$$

We now demonstrate that the (true) PLV between the oscillators is equal to the absolute Fourier transform (amplitude) of the modulated oscillator $Y$ evaluated at frequency $\omega_{Y}$.

The PLV equation (6) can be changed to the equivalent form of:

$$
\text { (22) } \mathrm{PLV}=\left|\frac{1}{N} \sum_{\mathrm{t}=1}^{N} e^{\mathrm{i}\left(\varphi_{X}(t)+\varepsilon\right)} e^{-\mathrm{i}\left(\varphi_{Y}(t)\right)}\right|
$$

The absolute (discrete) Fourier coefficient (amplitude), evaluated at frequency $\omega_{Y}$, can be formulated as follows:

$$
\text { (23) } \begin{aligned}
\left|S_{X}\left(\omega_{Y}\right)\right| & =\left|\frac{1}{N} \sum_{t=1}^{N} X(t) e^{\left.-\mathrm{i} i \varphi_{Y}(t)\right)}\right| \\
& \text { with } \varphi_{Y}(t)=2 \pi \omega_{Y} \mathrm{t}
\end{aligned}
$$

We transformed the phase evolution $\varphi_{X}(t)$ of oscillator $X$ into an equivalent complex notation (complex sinusoid with unit amplitude).

$$
\text { (24) } X(t)=\mathrm{e}^{\mathrm{i}\left(\varphi_{x}(\mathrm{t})+\varepsilon\right)}
$$


A

\section{CFC phase-to-power}

Modulation of $3 \mathrm{~Hz}$ by phase $\mathrm{X}$

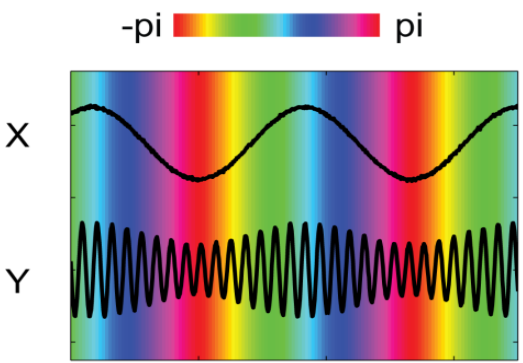

B

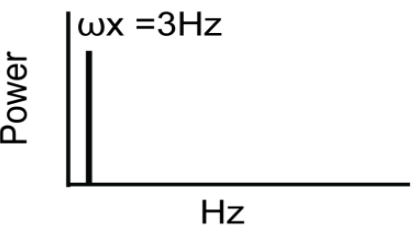

E

CFC
phase-relation -to-power

Modulation of $3 \mathrm{~Hz}$ by phase precession between $X$ and $Y$ -pi

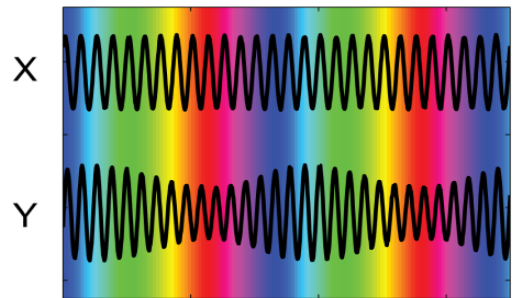

F

G
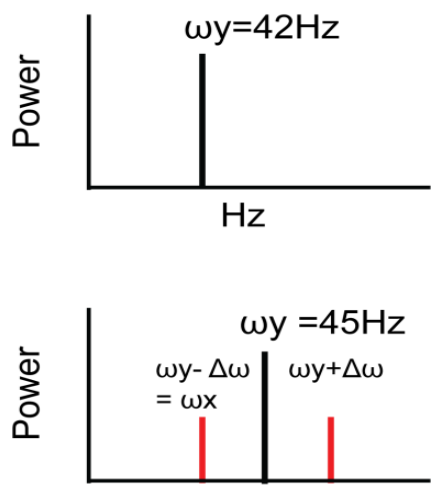

$\mathrm{H}$
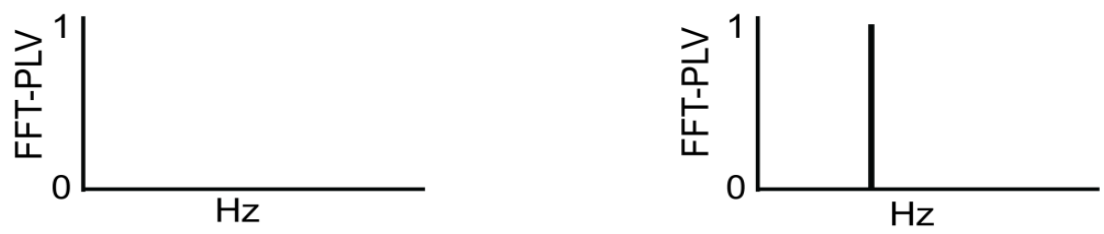

Figure 3: Comparison between a standard case of cross-frequency coupling (CFC, left column), and phase-relation dependent amplitude modulation (PrAM, right column). The spectra illustrated for PrAM show the basis for erroneous phase locking estimates when PLV $V_{\text {FFT }}$ computation is applied. Note that PrFM has similar spectral effects and similar consequences for the outcome of PLV $V_{F F T}$ estimations. The present figure is limited to PrAM as it is easier to illustrate. A) Typical phase-to-power CFC between the phase (polar color) of a slower oscillator $\mathrm{X}$ and the power of a higher frequency oscillation $\mathrm{Y}$. B) The schematic power spectrum of $X$ with a power peak at $3 \mathrm{~Hz}$. C) The power spectrum of $Y$ with a power peak at $45 \mathrm{~Hz}$ and additional modulation sidebands at $45 \pm 3 \mathrm{~Hz}$. The modulation sidebands occur due to the systematic power modulation at $3 \mathrm{~Hz}$ in the faster oscillating signal by the phase of the slower oscillation. D) Applying phase-locking value (PLV) based on the Fourier crossspectrum (PLV $\mathrm{FFT}_{\text {) }}$ ) would result in a value of 0 , because no frequency has common power between $\mathrm{X}$ and $\mathrm{Y}$. To quantify the interaction, cross-frequency coupling measures need to be applied. E) CFC between oscillators of nearby frequencies where the modulation is dependent on the phase-relation (polar colors). Here $\mathrm{X}$ modulates the power of $\mathrm{Y}$ as a function of their phaserelation. The modulation frequency in the power of Yis equal to the phase-precession frequency. F) The power spectrum of $X$ with a power peak at $42 \mathrm{~Hz}$. G) The power spectrum of $Y$ with a power peak at $45 \mathrm{~Hz}$ and modulation sidebands at $45 \pm 3 \mathrm{~Hz}$. The $3 \mathrm{~Hz}$ is the phase precession frequency, which equals the frequency difference between $\mathrm{X}$ and $\mathrm{Y}$. This implies that one modulation sideband of $Y$ will be at the same frequency as $X$. H) When applying PLV $V_{F F T}$, a phase-locking of 1 will emerge at the frequency which $X$ shares with the modulation sideband of $Y$. At that frequency, phase-locking is 1 , because the modulation sideband of $Y$ has a fixed phase-relationship to $X$ (determined by the modulation function) that is independent of phase-shifts over trials. 
This gives the following Fourier coefficient magnitude:

$$
\text { (25) }\left|S_{X}\left(\omega_{Y}\right)\right|=\left|\frac{1}{N} \sum_{t=1}^{N} e^{i\left(\varphi_{X}(t)+\varepsilon\right)} e^{-i t\left(\varphi_{Y}(t)\right)}\right|
$$

which is equivalent to the PLV equation (22).

$$
(26) \rightarrow\left|S_{x}\left(\omega_{y}\right)\right|=\text { PLV }
$$

The $\operatorname{PLV} V_{F F T}$ between $S_{X}\left(\omega_{Y}\right)$ and $S_{Y}\left(\omega_{X}\right)$ will be 1 assuming the modulation function does not vary across trials (consistent preferred relationship across trials/time), because the relationship between $S_{X}\left(\omega_{Y}\right)$ and $S_{X}\left(\omega_{Y}\right)$ is then consistent independent of the actual true PLV. This shows that the phase-locking of oscillator $X$ and the modulated oscillator $Y$ is equal to the amplitude induced in the Fourier-spectrum of oscillator $Y$ at the frequency of $\omega_{\gamma}$, hence the amplitude of the modulation sideband. Generally, we derived the modulation sideband amplitude (in the case PrAM=0) as follows:

$$
\text { (27) } \mathrm{SM}_{\mathrm{PrFM}}=A \mathrm{PLV}_{T}
$$

where $A$ is the amplitude of the oscillator that is modulated.

\section{Modulation sideband induced by PrAM}

The relation between PrAM and modulation sideband can be shown using trigonometry, assuming no phase-locking, as follows (Aru et al., 2014):

(28) $Y(t)=\sin \left(\omega_{Y} t+\varepsilon\right)$

$$
\begin{aligned}
& \text { (29) } X(t)=(1+\cos (\Delta \omega t+\varepsilon)) \sin \left(\omega_{X} t\right) \\
& \text { with } \Delta \omega t=\omega_{Y} t-\omega_{X} t
\end{aligned}
$$

The cosines function represents the phase-relation dependent amplitude modulation function (PrAM). The variable $\varepsilon$ is the phase-relation offset between the two oscillators. The equation $X(t)$ can be rewritten in the following form:

$$
\begin{gathered}
\text { (30) } \left.X(t)=\sin \left(\omega_{X} t\right)+\frac{1}{2} \sin \left(\omega_{Y} t+\varepsilon\right)+\frac{1}{2} \sin \left(\omega_{X} t+\Delta \omega t+\varepsilon\right)\right) \\
\text { with } \omega_{Y} t=\omega_{X} t-\Delta \omega t
\end{gathered}
$$

The second and third term represent the two symmetric $( \pm \Delta \omega)$ modulation sidebands. Because the second term, the modulation sideband at frequency $\omega_{Y}$ of oscillator $X$, representing the amplitude 
modulation function, the $\mathrm{PLV}_{\mathrm{FFT}}$ at frequency $\omega_{Y}$ between oscillator $\mathrm{X}$ and $\mathrm{Y}$ will be 1 , despite a true PLV of 0 , because the phase-relation offset $\varepsilon$ does not change the phase relation between the modulation sideband of oscillator $X$ and oscillator $Y$. The phase-relation offset $\varepsilon$ affects them both in the same way. If no PLV is present (no PrFM), the amplitude of the modulation sideband can be computed as:

$$
\text { (31) } \mathrm{SM}_{\mathrm{PrAM}}=A \frac{a}{2} \text {, if } \mathrm{PLV}=0
$$

\section{Modulation sideband induced by PrAM and PrFM combined}

If both PrAM and PrFM are present, we derive the amplitude of the modulation sideband as follows:

$$
\text { (32) } \mathrm{SM}_{\mathrm{PrFM}, \mathrm{PrAM}}=A\left|\int_{\theta=-\pi}^{\theta=\pi} M \sin (\theta) \operatorname{pr}(\theta) e^{i \theta} d \theta\right|
$$

where $\operatorname{pdf}(\theta)$ is defined as in equation (11) and $A$ represents the amplitude of the modulated oscillator.

\section{Testing of PLV $V_{F F}$ on phase-oscillators with different levels of extrinsic noise}

So far we have shown schematically the problem that arises when PLV $\mathrm{FFT}_{\text {is }}$ applied to non-stationary data showing PrAM or PrFM. Moreover, we demonstrated mathematically in the case of noiseless

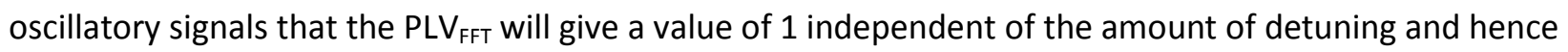
the true phase-locking. However, measured oscillatory brain signals are not noiseless. We therefore extended our analysis to oscillatory signals with different levels of uncorrelated white noise (which did not affect dynamical properties of the signals). This manipulation of noise was done to mimic the different Signal-to-Noise Ratio (SNR) that occurs under experimental conditions. The SNR is defined as follows:

$$
\text { (33) } \mathrm{SNR}=\frac{\text { Power }(\text { Signal })}{\text { Power }(\text { Noise })}
$$

Hence, we investigated systematically the behavior of $\mathrm{PLV}_{\mathrm{FFT}}$, for different SNRs, as a function of detuning frequency $\Delta \omega$ (PrFM) and different level of PrAM. For this we compared in our simulations the analytical derived true PLV of two interacting phase-oscillators with the analytical derived spectral PLV $V_{\mathrm{FFT}}$ as well as the numerical estimate $\mathrm{PLV}_{\mathrm{FFT}}$ using computer simulations.

\section{Expected PLV $V_{\text {FFT }}$}

Adding uncorrelated white noise ('measurement error') decreased the PLV values in general. The amount of reduction was a function of the ratio between the signal power and the noise power (the SNR).The essential ratio determining the Fourier-based PLV is the ratio between the amplitude of the modulation sideband and the white noise amplitude. There are four variables affecting the true phase 
difference between the two oscillators: the amplitudes of two independent white noise processes, in relation to the signal amplitudes, and their phase values. The power from a white noise process is known to have a chi-square distribution of order 2 (Gilgen, 2006) with mean power being equal to its variance. For amplitudes it corresponds to a chi-distribution of order 2 , for which the probability density function is given by:

$$
\begin{aligned}
& \text { (34) } \chi(A, k)=\frac{2^{1-k / 2} A^{k-1} e\left(\frac{A}{2}\right)^{2}}{\Gamma_{\frac{k}{2}}} \\
& \text { (35) } \rightarrow \chi(A, 2)=A e^{-\frac{A^{2}}{2}}
\end{aligned}
$$

The phase distribution of a white noise process is the uniform distribution:

$$
\text { (36) } \theta_{W}=\frac{1}{2 \pi}
$$

The actual phase of each oscillator is the complex vector addition of the signal and noise. The product of these complex values between the two oscillators gives the actual phase difference. The PLV $\mathrm{FFT}_{\text {is }}$ evaluated at the frequency where the oscillation $X$ shares power with the modulation sideband of oscillation $Y$.

(37) $\mathrm{PLV}_{\mathrm{FFT}}=$



where $A_{X}$ and $A_{Y}$ represent the amplitudes of the white noise. $\theta_{X W}$ and $\theta_{Y W}$ represent the phases of the white noise of the oscillator $X$ and $Y$ respectively. $S_{X}$ is the amplitude of oscillator $X$ and $S_{Y}$ is the amplitude of the modulation sideband of $Y$ (see equation 32). We assumed for simplicity that the oscillator $X$ and the sideband modulation of oscillator $Y$ have a constant phase relationship $\left(\theta_{x}\right.$ and $\theta_{y}$ are constants), therefore assuming that the modulation by PrAM and PrFM has constant characteristics.

\section{Numerical derived PLV}

We also computed the PLV $\mathrm{FFT}_{\text {f }}$ on simulation data of two coupled phase-oscillators. We simulated two phase-oscillators with unidirectional coupling $\left(\kappa_{\mathrm{X}, \mathrm{Y}}=1, \kappa_{\mathrm{Y}, \mathrm{X}}=0\right)$ for simplicity. Yet, the same results were observed with mutual interactions. One phase-oscillator had an intrinsic frequency of $40 \mathrm{~Hz}$, whereas the frequency of other oscillator was systematically modulated in a range of $40 \mathrm{~Hz} \pm 8 \mathrm{~Hz}(\operatorname{PrFM})$. In another set of simulations the phase-oscillators were not phase-coupled, yet we included different level of PrAM. Each particular condition consisted of 500 trials. Each trial was 6 sec long with a time-step size of $1 \mathrm{~ms}$. For the computation of phase-locking we only considered the last $4 \mathrm{sec}$ of the simulation giving a resolution of $0.25 \mathrm{~Hz}$. The first $2 \mathrm{sec}$ were used to let the phase-oscillators reach a steady state. The initial (phase) conditions were taken from a uniform distribution. To the simulated signal we added white noise which was uncorrelated between the oscillators. We then converted the simulated phaseoscillators into real-valued oscillatory signals by taking the cosine. 
After we simulated the trials, we computed the (Fast) Fourier transform (FFT). We did not use tapering or padding. Then we computed the $\mathrm{PLV}_{\mathrm{FFT}}$ as described above (equation 20). To compare the estimates we relied on adapted squared PLV, which is more robust for the inflation due to finite numbers of trials (Vinck et al., 2010). In the analysis below we used lower trial numbers. The unbiased estimator of square PLV is the following (Aydore et al., 2013):

$$
\text { (38) } \mathrm{PLV}_{u}^{2}=\frac{1}{N-1}\left(\left(\mathrm{PLV}^{2} * N\right)-1\right)
$$

where $N$ is the number of sample points.

\section{Systematic modulation in the detuning frequency (PrFM)}

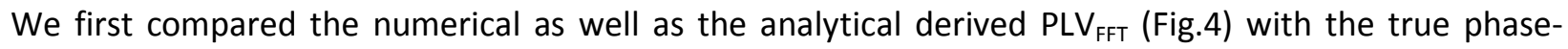
locking between the two interacting phase-oscillators, for different SNR, as function of detuning frequency $\Delta \omega$. The oscillator $X$ was coupled to oscillator $Y$ by $k=0.75$. Oscillator $X$ had a frequency of $40 \mathrm{~Hz}$. The frequency of oscillator $Y$ shifted from 40 to $48 \mathrm{~Hz}$ with step size of $0.25 \mathrm{~Hz}$ (detuning $\Delta \omega 0$ to $8 \mathrm{~Hz}$ ). The values chosen corresponded to a half cross-section of the (1:1) Arnold tongue. We evaluated SNR of 500, 50, 10, 5 and 2 . The numerically and analytically derived PLV conditions, and we therefore do not distinguish them further.

For low detuning the oscillators were in full synchrony and had a phase-locking of 1 . The PLV the phase-locking correctly for higher SNR. For lower SNR the noise started to affect the PLV $\mathrm{FFT}_{\text {T }}$ estimate more substantially. As white noise was uncorrelated, the white noise tended to decrease the PLV towards 0. At SNR of 2 the $\mathrm{PLV}_{\mathrm{uFFT}}^{2}$ gave an estimate reduced by $50 \%$. At a particular detuning frequency $(\Delta \omega=1.5 \mathrm{~Hz})$ the phase-locking between the oscillators started to drop (partially synchronized state). The oscillators were therefore not frequency-frequency locked and had disparate frequencies.

The behavior of $\mathrm{PLV}_{\mathrm{uFFT}}{ }^{2}$ deviated strongly from the true $\mathrm{PLV}_{\mathrm{u}}{ }^{2}$ (Fig.4A). The estimates were strongly dependent on the SNR. As we have shown above, without any additive white noise the $\operatorname{PLV}_{\mathrm{uFFT}}{ }^{2}$ estimates were 1 . The lower the SNR, the more noise affected the estimate and the more it evolved towards 0 . Importantly, with higher SNR, the estimates converge not on the true phase-locking, but to a phase-locking of 1 . Nevertheless, the $\mathrm{PLV}_{\mathrm{uFFT}}{ }^{2}$ estimates reflect to a certain degree the underlying changes in the true PLV ${ }^{2}$ as a function of detuning. This is because, as we have shown above, the amplitude of the modulation sideband (induced by PrFM) is a function of the true PLV. The noise therefore unmasks this dependence.

\section{Systematic modulation in PrAM}

We then investigated the dependence of $\mathrm{PLV}_{\mathrm{uFFT}}{ }^{2}$ estimates on the presence of PrAM. For simplicity, we used conditions where the phase-oscillators were uncoupled and hence the true phase-locking was 0 for all conditions. The oscillators had a detuning of $3 \mathrm{~Hz}$. We evaluated different level of PrAM ranging from 0 to $100 \%$. We used the same SNR conditions as before. Again, the numerical and analytical derived $\mathrm{PLV}_{\mathrm{uFFT}}^{2}$ estimates matched well. 
We observed that $\mathrm{PLV}_{\mathrm{uFFT}}{ }^{2}$ estimates deviated from the true phase-locking as a function of both the level of PrAM and the level of SNR (Fig.4B). The higher the PrAM was set, the higher the estimate (deviation from true locking) resulted. This is because higher PrAM leads to higher amplitude of the modulation sideband making it more dominant regarding the noise. Further, the higher SNR, the higher the estimate and hence the more deviation there was from the true phase-locking. Similar to the case with PrFM, the $\mathrm{PLV}_{\mathrm{uFFT}}{ }^{2}$ estimates did not converge towards the true phase-locking, but to a phase-locking of 1 . These results show that $\mathrm{PLVu}_{\mathrm{FFT}}{ }^{2}$ is not a pure phase-locking measure, but also reflects phase-relation dependent amplitude fluctuations.

\section{Testing of PLV $V_{F F}$ on phase-oscillators with intrinsic noise and with different levels of extrinsic noise}

So far we have shown for oscillations with a fixed intrinsic frequency (with synchronization-induced FM and $A M)$ that PLV $_{F F T}$ can deviate wildly from the underlying true phase-locking. The oscillations were very narrow-banded and the frequency distributions of the two coupled oscillations in most cases nonoverlapping. Measured neural oscillations however (e.g. gamma-band) have broader spectral power peaks (Brunet et al., 2014; Burns et al., 2011, 2010; Roberts et al., 2013). This indicates that neural oscillations exhibit rapid frequency dynamics, which can be expected from noisy and complex networks (Bressler and Kelso, 2001) of which brain networks are prime examples. Sources of the variable oscillation frequencies might be e.g. intrinsic noise/instability within a network (Burns et al., 2011), perturbations from other networks (Womelsdorf et al., 2007) and cross-frequency interactions (Lakatos et al., 2005). 
A

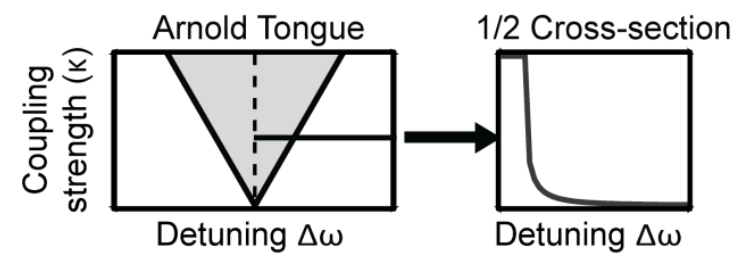

Type I : Fluctuation in Frequency

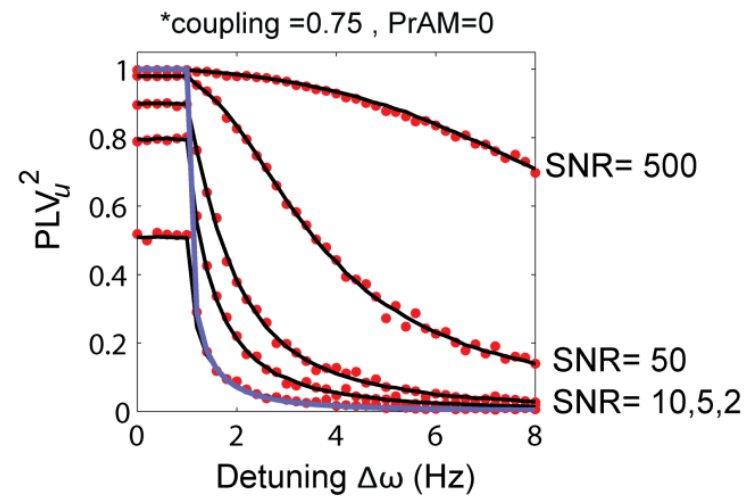

B

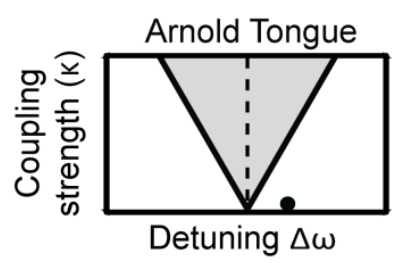

Type II : Fluctuation in Power

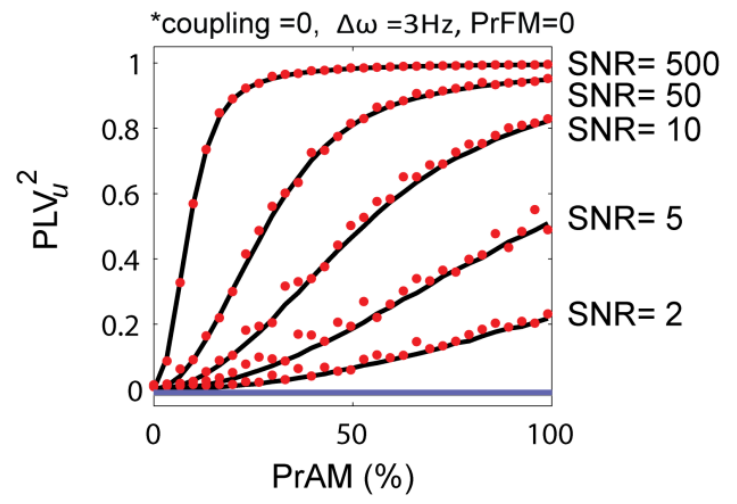

\footnotetext{
- numerical FFT-PLV

- analytical FFT- PLV

— true phase locking (analytical)
}

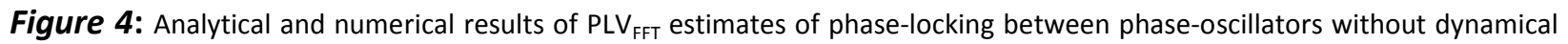
noise, but with different levels of extrinsic (measurement) noise (uncorrelated between oscillators). Noise manipulations were carried out without (A) and with (B) phase-relation dependent amplitude modulation (PrAM) A) We compared the numerically

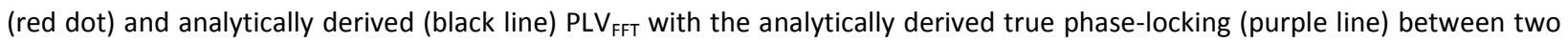
oscillators as a function of frequency detuning $(\Delta \omega)$ and different levels of SNR (Power (Oscil. Signal) / Power(External Noise)). We compared the square PLV values to minimize inflation due to a finite number of trials. A) rendering of the Arnold tongue, shown with a $1 / 2$ cross-section at the level of a 0.75 coupling strength (dashed line), for which phase locking values are plotted as a function of positive, increasing intrinsic frequency differences between oscillators $X$ and $Y(\Delta \omega)$. Here, we did not add PrAM to the oscillatory signal. In the partial synchronized states associated with different $\Delta \omega$ values in the selected coupling condition, we observed strong deviations of PLV FFT $_{\text {from the true locking. The PLV }}$ FFT sbecame more inflated with higher SNR and detuning frequency $\Delta \omega$. The numerically computed PLV FFT matched with the analytically derived PLV FFT. B) The impact of different levels of PrAM is shown with different level of SNR. The oscillators were uncoupled and hence asynchronous (in the condition indicated by the fat dot at the bottom of the Arnold tongue) and the true locking was therefore 0 . The oscillators had a phase precession of $3 \mathrm{~Hz}$ (chosen condition is located off the midline of the Arnold tongue). We observed strong deviations from the true locking with increasing PrAM and SNR. The numerically and analytically derived values matched.

As a next step, we therefore extended our analysis to coupled phase-oscillators that exhibited intrinsic (dynamic) noisy fluctuations. In comparison to the previous simulations, now the phase variable of oscillator $X$ and $Y$ was also influenced by a pink noise process $N p$ (scaling factor of $1, S D=\sim 1.5 \mathrm{~Hz}$ ). The equation of $X$ (and $Y$ ) was:

$$
\text { (39) } \frac{d \varphi_{X}}{d t}=\omega_{X}+\kappa_{X, Y}\left(\sin \left(\varphi_{Y}-\varphi_{X}\right)\right)+\mathrm{Np}_{X}
$$


We show data where $X$ was unidirectionally coupled with oscillator $Y\left(\kappa_{X, Y}=1, \kappa_{Y, X}=0\right)$, yet the same conclusions could be drawn with other connectivity configurations. The pink noise (applied to $X$ and $Y$ ) was uncorrelated between the oscillators. Including the pink noise term had two effects: It broadened the range of frequencies an oscillator occupied. Second, because the intrinsic frequency varies due to the noise term, the oscillators did not have a precise position on the detuning dimension, but varied over time. That makes full synchrony very difficult to achieve, because strong noise fluctuation kick the oscillators out of the 'attractor' phase-relation (Pikovsky et al., 2002). The oscillators will reach full synchrony only if their coupling strength is strong enough to suppress any effects of the strongest possible fluctuations in the noise process. This means that although they have a common mean oscillation frequency, the phase-locking will likely not be 1 and phase precession will occur.

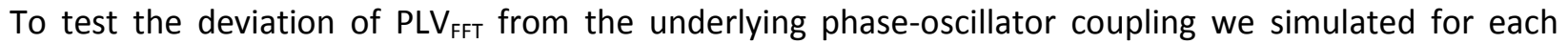
condition 100 trials of $4 \mathrm{~s}$ length. The first $2 \mathrm{~s}$ were discarded of each trial. We analyzed 5 different SNR conditions (5,18,45,100 and 200). Further, we evaluated conditions without any PrAM and conditions with $20 \%$ PrAM. For these simulations we did not derive the analytical phase-locking between the two oscillators, but relied on a numerical estimation. To that goal, we computed the PLV of the noise-free instantaneous phases resulting from the phase-oscillator equations. We concatenated the trials prior to the estimation yielding a large number of time points. From prior simulations we observed that the analytical derived true PLV and the estimated PLV matched closely (mean square error (MSE) $=1.4 \mathrm{e}^{-5}$ ).

In Fig.5A we show the relative power spectra of one oscillator for different SNR. The relative power (Rel.Pow) was defined as:

$$
\text { (40) Rel.Pow }(\omega)=\frac{S_{\mathrm{NS}}(\omega)^{2}}{S_{\mathrm{N}}(\omega)^{2}}
$$

where $S_{\mathrm{NS}}(\omega)^{2}$ stands for spectral power of the oscillatory signal plus white noise term at frequency $\omega$ and $S_{\mathrm{N}}(\omega)^{2}$ stands for spectral power of just the white noise term as applied at frequency $\omega$. Notice that the spectral power distribution was broader due to the intrinsic frequency variability of the oscillators. Experimentally reported relative power values in the gamma band range from $\geq 1$ to $\geq 20$, depending on the method used and neuronal structure investigated. It can be expected that with the advance of new recording techniques and shielding, the SNR might increase in future studies.

The expected $\mathrm{PLV}_{\mathrm{u}}{ }^{2}$ was at $\sim 0.3$ at the mean detuning of $\mathrm{OHz}$ (Fig.5B). The mean frequency of the oscillators matched. Yet, because of dynamic noise the oscillators could not reach full synchrony, but exhibited phase precession. As in previous simulations, the $\mathrm{PLV}_{\mathrm{uFFT}}{ }^{2}$ estimates were dependent on the SNR. With higher SNR it did not converge to the expected $\mathrm{PLV}_{\mathrm{u}}{ }^{2}$, but exceeded it. Including a $20 \% \operatorname{PrAM}$ led to a further inflation of the $\mathrm{PLV}_{\mathrm{uFFT}}{ }^{2}$ estimates. Hence, despite the oscillators having a matching mean frequency, cross-frequency interactions PrFM and PrAM still had a substantial effect on PLV $_{\text {uFFT }}{ }^{2}$ estimates. Increasing the detuning frequency $\Delta \omega$ led to a smooth decrease of the expected phaselocking. The PLV $\mathrm{UFFT}^{2}$ estimate of the highest SNR did not decrease at all. Middle SNR levels (100, 45 and 
18) showed a very slow decrease of $\mathrm{PLV}_{\mathrm{uFFT}}{ }^{2}$ estimates leading to strong deviation from the expected $\mathrm{PLV}^{2}$. At low SNRs the effect of the white noise became dominant and $\mathrm{PLV}_{\text {uFFT }}{ }^{2}$ estimates were always lower than the expected $\mathrm{PLV}_{\mathrm{u}}{ }^{2}$. Applying the 20\% PrAM led to an overall inflation of the $\mathrm{PLV}_{\mathrm{uFT}}{ }^{2}$ estimates. Note that the inflation by the PrAM increased with increasing SNR (as expected from Fig.4B).

These results show that also under more realistic oscillatory dynamics the spectral based PLV ${ }_{\mathrm{uFFT}}^{2}$ exhibit $^{2}$ strong deviation from the expected PLV. With increasing the SNR the deviation becomes stronger and might become dissociated from the underlying expected PLV.
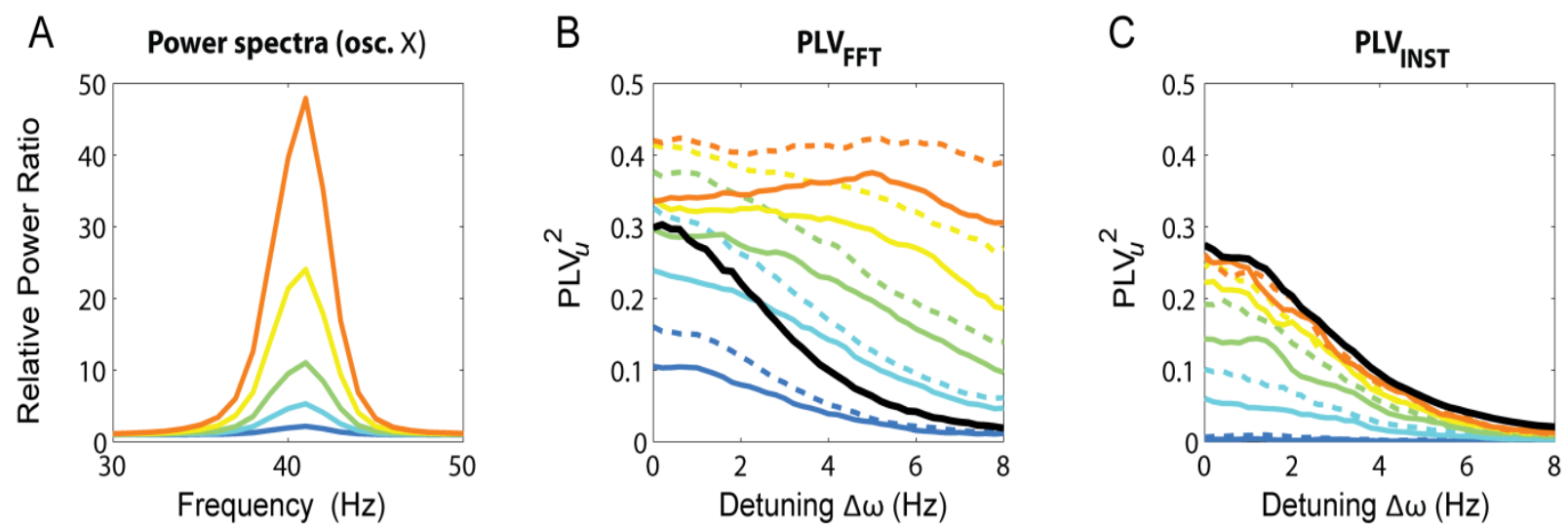

Phase-relation dependent amplitude modulation (PrAM)

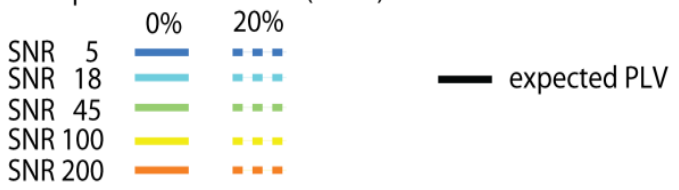

Figure 5: Comparison of spectrally and non-spectrally based approaches for the estimation of phase locking. The figure shows numerical results of PLV $\mathrm{FFT}_{\text {T }}$ estimates of phase-locking between phase-oscillators with both dynamical noise (more broadband) and different level of extrinsic (measurement) noise (uncorrelated between oscillators). Under these conditions, we compared the PLV $_{\text {FFT }}$ with an alternative method based on the estimated instantaneous phase $\left(P_{\text {L }} V_{\text {INST }}\right)$. We used singular spectrum decomposition (SSD) to decompose the signal in a mono-component signal and to reduce the effect of noise. Based on the SSDsignal we applied Hilbert-Transform (HT) to derive the instantaneous phase. From the instantaneous phase difference between the two oscillators we computed the $\mathrm{PLV}_{\text {NST }}$. A) The relative power spectra [Power(Noise+Signal) /Power(Noise)] of $\omega 1$ are shown for different signal-to-noise (SNR) levels. B) A $1 / 2$ cross-section of the Arnold tongue, similar to Fig.4a, is shown. The continuous lines represent simulations without PrAM and the dashed lines represent simulation with a PrAM of $20 \%$. We compared the PLV FFT to the expected phase-locking. We used the noise-free instantaneous phases of the phase-oscillators to compute the expected PLV, which was a good estimator of the analytically derived true phase-locking. We observed that the $\mathrm{PLV}_{\mathrm{FFT}}$ values deviated strongly from the expected phase-locking. The exact deviation depended on the detuning frequency and SNR. Including a PrAM of $20 \%$ led to a further inflation of the PLV FFT $_{\text {values. Note also the deviations of PLV }}$ fFT from the expected PLV at a zero detuning frequency. C) The same analysis as in B) but with PLV values estimated by the SSA-HT method. We observed that for higher SNR the estimate behaved better and remained close to the expected phase locking. At lower SNR the PLV INST showed lower than expected values due to the effect of (uncorrelated) noise. Including a PrAM of $20 \%$ led to an inflation of PLV INST values in the lower SNR only. 


\section{An alternative for spectral coherence methods}

Given the fact that in the brain signals are likely not stationary, and given demonstrations here that nonstationarity leads to problems in the interpretation of spectral-based PLV, we can assume that in a majority of conditions encountered in neuroscience, spectral-based PLV will not be a preferable method to measure phase relationships. The central problem is rooted in the stationarity assumption of the spectral-based synchronization measures applied on experimental trials with lengths varying from a few $100 \mathrm{~ms}$ to several seconds. Phase-synchronization is a non-stationary process which requires methods that do not rely on stationarity and that allow for cross-frequency phase-locking estimates.

Various methods have been proposed that quantify phase-locking based on the instantaneous phase (Picinbono, 1997). The main challenge is to decompose the often complex multi-component measured brain signal in well-defined oscillatory components (e.g. through filtering or wavelet decomposition techniques) from which the instantaneous phase is extracted (i.e., after a Hilbert-Transform or directly from a time-frequency spectrum TFR, (Le Van Quyen et al., 2001)). Below we propose an alternative method that is based on the singular spectrum decomposition (Bonizzi et al., 2014) and HilbertTransform. We then applied this method to the simulated two coupled phase-oscillator signals having intrinsic and extrinsic noise. Our main aim in this study is not to compare different methods of instantaneous phase extraction for computing phase-locking estimates, yet to show that the phaselocking methods based on instantaneous phase estimates do not exhibit the striking problems that arise by applying coherence measures. The findings below (Fig.5c) will be generalizable to any method that can robustly approximate the instantaneous phases of the underlying neural oscillators.

\section{Singular Spectrum Decomposition and Hilbert-Transform}

Singular spectrum decomposition (SSD) is a recently proposed method for decomposition of nonlinear and non-stationary time series (Bonizzi et al., 2014, 2012). Here, the method is applied to reduce the influence of noise and to provide a PLV estimate that unlike the FFT-based one is able to handle nonstationary signals. Additionally the method is also able to deal with nonlinear signals unlike waveletbased approaches. The key ideas underlying the method are introduced in the following. The interested reader is referred to (Bonizzi et al., 2014) for additional details. The method originates from singular spectrum analysis (SSA), a nonparametric spectral estimation method used for analysis and prediction of time series. The advantage of SSA-derived components over Fourier-derived sines and cosines is that SSA-components are model free (empirical/data driven) and therefore are not necessarily harmonic functions. Being data adaptive, SSA components can capture highly non-harmonic oscillatory shapes, making them suitable for the analysis of nonlinear and non-stationary time series. In the SSD method the choice of the main SSA parameters, the embedding dimension and the selection of the principal components for the representation of a specific component series have been made fully data-driven and automated. This makes SSD an adaptive decomposition method. Similar to empirical mode decomposition (EMD) (Huang, 2005), the decomposition is based on the extraction of the energy associated with various intrinsic time scales. One advantage of SSD over EMD is that it tends to avoid mixing components with different frequency bands and provides accurate separation between intermittent components at the transition points (Bonizzi et al., 2014). Given a zero-mean time series 
$x(n), n=1, \ldots, N$, the SSD algorithm iteratively extracts a set of component series from $x(n)$ until the total variance of the extracted components reaches a user-defined threshold. Each iteration consists of the following steps:

Embedding. The time series $x(n)$ is embedded in a vector space of dimension $M$. Given an embedding dimension $M$, with $1<M<N$, the embedding procedure forms $M$ lagged vectors $x_{i}=(x(i), \ldots, x(N)$; $x(1), \ldots, x(i-1))$, with $i=1, \ldots, N-M+1$. For instance, given the time series $x(n)=\{1,2,3,4,5\}$, and an embedding dimension $M=3$, the corresponding trajectory matrix will be:

$$
\text { (41) } X=\left[\begin{array}{lll|ll}
1 & 2 & 3 & 4 & 5 \\
2 & 3 & 4 & 5 & 1 \\
3 & 4 & 5 & 1 & 2
\end{array}\right]
$$

such that this is a Hankel matrix (constant cross-diagonals) of size $(M \times N)$. This embedding procedure is different from the standard one used by SSA, and it has the advantage of enhancing its oscillatory content. Possible discontinuities generated by the periodic extension in matrix $X$ are negated by projection on the subspace of the principal components. The embedding dimension $M$ is automatically estimated as $F_{s} / f_{\max }$, with $f_{\max }$ being the dominant frequency in the power spectral density (PSD) of $x(n)$, and $F_{s}$ the sampling frequency.

Decomposition. The singular value decomposition of the trajectory matrix $X$ is then computed, providing $X=U D V^{T}$, with $U=(M \times M)$ and $V=(N \times N)$ being orthogonal matrices containing the left and right singular vectors, respectively, and $D=(M \times N)$ being a matrix containing the singular values on the main diagonal and zeros elsewhere.

Grouping. Out of the $M$ principal components of $X$, a subset $(P<M)$ is selected that corresponds to a dominant frequency in the range [fmax $-\delta f ; f \max +\delta f]$. Practically this is determined by selecting the components whose left eigenvectors show a dominant frequency in that range. This allows the component series, which will be reconstructed from this subset, to describe a well-defined time scale. The width of the dominant peak $\delta f$ is estimated by means of a Gaussian interpolation of the PSD of the time series $x(n)$.

Reconstruction. Only the selected $P$ principal components are used to generate a rank- $P$ approximation of $X$. The corresponding time series is then reconstructed by suitable diagonal averaging of this matrix along the cross-diagonals. In order to carry out the average along the $i$-th cross-diagonal of $X$, the wrapped part of the right hand block must be correctly appended to the top right of the left hand block. For the example above:

$$
\text { (42) } X=\left[\begin{array}{rrr|rrr} 
& & 1 & & \\
& 1 & 2 & & \\
1 & 2 & 3 & 4 & 5 \\
2 & 3 & 4 & 5 & * \\
3 & 4 & 5 & * & *
\end{array}\right]
$$


where the asterisks mark the former locations of the elements composing the wrapped part moved to the right top of the left hand block. With this approach, each cross-diagonal contains the same number of elements $M$ (with $M=3$ in this example; the empty locations should be ignored).

The estimated component series is then subtracted from $x(n)$, and the procedure is iterated for the residual until a stopping criterion is met, when the energy of the residual falls below a pre-defined threshold.

The Hilbert-Huang transform (HHT, (Huang et al., 1998)) is a method to obtain instantaneous frequency data, suitable for the analysis of nonlinear and non-stationary time series. EMD is applied to a time series to decompose it in intrinsic mode functions (IMFs), simple amplitude and frequency modulated components with only one instantaneous frequency per instant. Hilbert spectral analysis is then applied to each IMF to obtain its instantaneous frequency. To achieve that, the analytic signal of the IMF is obtained $\left(\mathrm{IMF}_{\mathrm{a}}\right)$, given by:

$$
\text { (43) } \mathrm{IMF}_{a}=\mathrm{IMF}+i \mathrm{HT}(\mathrm{IMF})
$$

where HT(IMF) is the Hilbert-Transform of the IMF component, and the instantaneous frequency $\omega$ is computed as the derivative of the phase of the analytical signal:

$$
\text { (44) } \theta=\arg \left(\mathrm{IMF}_{a}\right) \text { and } \frac{d \theta}{d t}=\omega
$$

The Hilbert spectrum can be used on SSD components when interpreting its outcome with caution. Indeed, SSD-components contain several frequencies, with no clear indication about how many instantaneous frequencies per time instant may be present. Hence, they cannot be supposed to be IMFs. However, the narrow-banded frequency content of each SSD-component allows considering the results of the Hilbert spectrum as sufficiently reliable under most conditions (Bonizzi et al., 2014).

In Fig.5C we show the SSD-HT-PLV ${ }_{\mathrm{u}}^{2}$ estimates compared with the $\mathrm{PLV}_{\mathrm{uFFT}}{ }^{2}$ estimates in Fig 5B, both figures being based on the same dataset. We observed that for higher SNR (also for the noiseless case) the SSD-HT-PLV ${ }_{u}^{2}$ estimates converged to the expected $\mathrm{PLV}_{\mathrm{u}}{ }^{2}$ over the whole detuning frequency range $\left(0\right.$ to $8 \mathrm{~Hz}$ ). For lower SNRs the $\mathrm{PLV}_{\mathrm{u}}{ }^{2}$ estimates were lower than the expected $\mathrm{PLV}^{2}$, because the extrinsic noise started to affect the estimates more substantially. At the lowest SNR, the SSD-HT-PLV ${ }_{u}{ }^{2}$ estimates were very close to 0 .. The addition of a $20 \%$ PrAM affected the lower SNR condition, but not the higher SNR conditions. Overall, the SSD-HT-PLV² estimates behaved much more appropriately in the conditions tested. 


\section{Discussion and Conclusions}

The first aim of this paper was to investigate whether spectral-based phase-locking estimations were appropriate for quantifying phase-synchronization among oscillatory processes. Spectral phase-locking estimations, including spectral coherence (Carter et al., 1973) or the spectral phase-locking value (PLV or PPC, (Vinck et al., 2010)), are commonly used in electrophysiological data analysis (Bokil et al., 2010; Oostenveld et al., 2011) for measuring the phase-locking between neural oscillatory signals (e.g., (Bosman et al., 2012; Gregoriou et al., 2009; Ray and Maunsell, 2010; Roberts et al., 2013; Saalmann et al., 2012)). The appropriate quantification of the oscillatory phase-locking and phase-relation is central to theories like the 'Communication through Coherence' (CTC) hypothesis (Fries, 2005; Fries et al., 2007; Womelsdorf et al., 2007) or the 'Binding by synchrony' hypothesis (Eckhorn, 2000; Engel et al., 1999; Singer, 1999). Central to these theoretical frameworks is that (1) neural signals are oscillatory and (2) that the oscillatory signals coordinate through neural interaction, in particular by synchronization. For the testing of these theoretical models, it is necessary to use methods that can extract the essential parameters in a valid manner. Yet, we found striking misestimations of the actual phase-locking by spectral phase-locking methods in a large parameter range. We suggest that in many studies investigating phase synchronization of neuronal oscillatory activity, the oscillatory data likely do not satisfy the stationarity assumption, in which cases spectral coherence or phase-locking value estimations should not be used. The second aim of the paper was to provide an alternative approach for datasets that violate the stationarity assumption. We found that using singular spectrum decomposition (SSD) of the oscillatory signals followed by the Hilbert-Transform provided a more robust means of estimating phase locking, with the desirable property that increased signal-to-noise reduces estimation errors. Since this alternative approach yields good estimates irrespective of whether the stationarity assumption is met or not, it could be argued that it is in general preferable over Fourier-based methods. To test the behavior of the spectral-based and SSD/Hilbert-based phase-locking estimates, we used a basic model of oscillatory phase synchronization, the phase-oscillator model, to simulate oscillatory signals with plausible phase synchronization properties (Breakspear et al., 2010; Ermentrout and Kleinfeld, 2001; Hoppensteadt and Izhikevich, 1996; Pikovsky et al., 2002). The simplicity of the model allowed us to mathematically derive the true phase-locking. We observed that spectral-based PLVs gave strikingly disparate values in comparison to the expected phase-locking between the oscillations. The divergence depended on the signal-to-noise (SNR) level, the frequency difference between oscillators (detuning) and the level of intrinsic frequency variability. In the extreme (theoretical) case of no measurement noise, the spectral-based PLV value became totally unrelated to the actual phase-locking of the oscillators. Furthermore, we observed that phase-relation dependent amplitude fluctuations (PrAM), a phenomenon that can be expected from interacting neuronal networks, lead to strong deviations from the expected PLV values. This shows that spectral-based PLV is not a pure phase-locking measure when the stationarity assumption is not met.

To show the relevance of this finding, we will discuss the phenomenon of synchronization, and in particular the partially synchronized state, in more detail. This will permit a better understanding, on the one hand, of the fact that partial synchronization (and non-stationarity) can be expected to be a common network state, and, on the other hand, of the reasons underlying the divergent estimations of 
phase-locking by spectral-based PLV. The synchronization principles of interacting oscillatory processes (limit-cycle as well as other types, (Rosenblum et al., 1996)) have been described mathematically in detail (Ermentrout and Kleinfeld, 2001; Hoppensteadt and Izhikevich, 1998, 1996). In particular, the theory of weakly coupled oscillators (TWCO) (see (Pikovsky et al., 2002) for review) has proven to be useful and has been applied in many scientific domains, including neuroscience (Bendels and Leibold, 2007; Breakspear et al., 2010; Burwick, 2008, 2007; Ermentrout and Kleinfeld, 2001; Galán et al., 2005; Hoppensteadt and Izhikevich, 1998, 1996). In TWCO the phase of an oscillator is determined by an intrinsic (natural) frequency. The interaction with other oscillators is characterized by the phase response curve (PRC, (Schwemmer and Lewis, 2012)), which defines how the phase is modified by the interaction. Crucially, the phase-locking between oscillators depends on the intrinsic frequency difference (described as the detuning level) as well as interaction strength (or coupling strength), defining the so called Arnold tongues (regions of synchronization defined by the interplay of detuning and coupling) (Coombes and Bressloff, 1999; Pikovsky et al., 2002; Tiesinga and Sejnowski, 2010). According to this scheme, oscillation can be fully phase synchronized (Pikovsky et al., 2002) with a phase-locking of 1 or fully asynchronous with a phase-locking of 0 . However, more likely, the oscillators will be partially synchronized (phase-locking between 0 and 1), in which the oscillators phase precess and have a frequency difference (detuning). That interacting neural oscillations can have close but different frequencies (within the same frequency-band) has been observed experimentally for gamma oscillations (Bosman et al., 2012; Gregoriou et al., 2009; Ray and Maunsell, 2010). Due to phase precession, the phase-locking is lower than 1 , but higher than 0 because the oscillators spend more time in some phase-relations than in others. Hence, under these conditions, the phase-precession is not smooth (Pikovsky et al., 2002), which results in (instantaneous) frequency fluctuations that are phaserelation dependent (PrFM). We also included phase-relation dependent amplitude fluctuations (PrAM) in our study. We expect that small but systematic amplitude fluctuations will occur because network oscillation amplitude (e.g. gamma oscillations) is sensitive to the amount and effectiveness of input spikes (Jia et al., 2013; Ray and Maunsell, 2010; Roberts et al., 2013; Tiesinga and Sejnowski, 2010) and phase-relations are thought to modulate the effectiveness/gain of incoming spikes (Fries, 2005; Tiesinga and Sejnowski, 2010; Womelsdorf et al., 2007). Hence, it can be expected that the partially synchronized state, characterized by PrAM and PrFM is the rule, rather than the exception, in the brain. This is because neural network oscillations are inherently noisy (variable) and complex, making perfect phaselocking unlikely.

In the case of partial synchronization, oscillations have divergent moment-by-moment frequencies and therefore their phase-relations need to be evaluated across frequencies. A phase-locking estimation across frequencies is in essence a cross-frequency coupling (CFC) measure. CFC measures are usually applied to quantify interactions between oscillations of different frequencies (Jensen and Colgin, 2007). CFC interactions between a lower frequency band (e.g. theta or alpha) and a higher frequency band (e.g. gamma) have been extensively studied, especially for phase-to-amplitude interactions, but also, although to a lesser extent, for phase-to-phase or phase-to-frequency interactions. The current study shows that the CFC approach also applies for oscillations that have nearby frequencies, yet are labeled to be within the same 'frequency band' (i.e. gamma band). In addition, the CFC idea also applies for oscillations with matching average frequencies but that have moment-by-moment frequency 
differences (see Figure 5B). CFC interactions are by definition non-stationary processes, because the oscillatory dynamics (power, frequency) are time-dependent.

The focus of the present study has been on the CFC interactions arising from synchronizing oscillations at nearby frequencies. Hence, instead of studying how a lower frequency-band oscillation phase modulates the amplitude or frequency of a higher frequency-band oscillation, here, our point of departure is that the phase-relation between the oscillatory signals modulates their own amplitude or frequency. Hence, here the process of synchronization itself produces non-stationarities (PrFM, PrAM). We investigated the effects of these non-stationarities on spectrally based phase-locking estimates. As shown in the result section, a consequence of systematic cross-frequency interactions ( $\operatorname{PrFM}, \operatorname{PrAM}$ ) is the induction of modulation sideband peaks in the power spectra at a given +/- offset from the modulation frequency (Aru et al., 2014; Berman et al., 2012). For example, if a slower oscillation of $3 \mathrm{~Hz}$ modulates the amplitude of a higher frequency oscillation at $40 \mathrm{~Hz}$, the power spectra will show peaks at $37 \mathrm{~Hz}$ and $43 \mathrm{~Hz}$ that are induced by the CFC interactions (Aru et al., 2014; Berman et al., 2012). As we have shown, these modulation sidebands also arise when two oscillators with nearby frequencies (e.g. $37 \mathrm{~Hz}$ and $41 \mathrm{~Hz}$ ) synchronize, as the synchronization process induces PrFM and possibly PrAM. Here, the modulation frequency is the frequency difference between oscillators (phase precession speed, e.g. $41 \mathrm{~Hz}-37 \mathrm{~Hz}=4 \mathrm{~Hz}$ ). This leads to spectra wherein the modulation sideband of one oscillation will match the peak frequency of the other oscillators. As we discuss below, this is bound to affect spectral PLV (or coherence) measures.

Spectral coherence or PLV estimation is a common approach to assess phase-locking between neural oscillatory signals of the same frequency-band, including for trial-based estimations (Bokil et al., 2010; Oostenveld et al., 2011). The coherence or the PLV is then computed based on the Fourier crossspectral densities (representing trials) for each frequency bin separately. This has the advantage that one obtains a frequency-resolved coherence/PLV plot which can be easily represented and compared to the power spectrum in a straightforward way. It therefore gives the phase-locking only for the same frequency bin (i.e. $42 \mathrm{~Hz}$ ), but not across frequency bins (e.g. $35 \mathrm{~Hz}$ and $42 \mathrm{~Hz}$ ). The Fourier based PLV as applied to experimental data can approach correctness as long as the data sufficiently obey the stationarity assumption. However, the assumption of stationarity is rarely tested in studies applying Fourier-based PLV to electrophysiological data. In regard to gamma oscillations, the studies that have investigated time-dependent variation in in-vivo cortical gamma oscillations have found consistently strong evidence for fast time-scale and systematic variation in frequency and power (Bosman et al., 2012, 2009; Burns et al., 2011, 2010; Roberts et al., 2013)), indicating that at the level of single trials there is no stationarity. This could in part be related to the possibility that many of the recordings in the mentioned studies may have probed ensembles characterized by partial synchronization, which by definition entails timedependent fluctuations in frequency and power.

If two interacting oscillations exhibit synchronization, while having a frequency difference, one could naively assume that the PLV $\mathrm{FFT}_{\text {s }}$ should give an estimate close to 0 , because it cannot give meaningful estimates of non-stationary oscillatory signals for cross-frequency bins. Yet, we observed that the spectral PLV gave inflated PLV estimates. In the worst case, it gave values of 1 independent of the detuning (and of the expected PLV). The inflation was substantial even when the mean power spectra of 
oscillator $X$ and $Y$, having intrinsic frequency variability, would be exactly overlapping. This means that the absence of a frequency difference at the level of (commonly reported) averaged power spectra is not sufficient to rule out inflated spectral-based PLV values.

In experimental settings, a substantial amount of measurement noise is present and therefore it can be expected that the PLV $V_{\mathrm{FFT}}$ estimation will be related to some extent to the underlying true phase-locking. Nevertheless, we suggest that the trial-averaged spectral PLV (or coherence) applied to oscillatory brain signals has significant downsides, because in a number of conditions a 'blind' application of spectral PLV (or coherence) (i.e., without knowing the extent of non-stationarities in the data) will lead to unreasonable estimates of phase locking. In addition, amplitude fluctuations that are phase-relation locked will affect the estimation. Further, we anticipate that with the improvement of data acquisition techniques and thus enhanced signal to noise in neurophysiological measurements, the probability that PLV $_{\mathrm{FFT}}$ leads to inflated estimates will increase (see Fig.4 and 5). The limitations of applying spectral PLV to oscillatory data that show non-stationarity, and that show realistic synchronization properties (Pikovsky et al., 2002) are equally likely to be encountered for other questions of interest in neuroscience and other fields, such as, for example, directionality measures (e.g. spectral granger methods (Ding et al., 2006)).

If spectral coherence measures in many conditions may lead to estimations that are difficult to interpret in terms of pure phase locking, then what could be the alternative? In line with the original definition of PLV (Lachaux et al., 1999), we propose to quantify the phase-locking value based on the instantaneous phases of the oscillations (Picinbono, 1997). So, instead of representing the phase trajectory of an oscillatory signal by a sinusoid with a fixed frequency and a phase offset, the moment-by-moment phase is estimated with a flexible (instantaneous) frequency. Estimating the moment-by-moment phase trajectory of an oscillation allows one to characterize properly the cross-frequency phase-locking and also to disentangle the phase-locking from PrFM and PrAM. There are two main approaches to extract the moment-by-moment phase trajectory of experimental oscillatory data (Le Van Quyen et al., 2001). The first is based on the time-frequency representation (TFR) of the signal, e.g., by using wavelet decomposition. The second is based on the Hilbert-Transform, which due to the multi-component nature of measured brain signals, is often preceded by a decomposition step, such as spectral filtering, empirical mode decomposition (EMD, (Huang et al., 1998)), or singular spectrum decomposition (SSD, (Bonizzi et al., 2014, 2012)).

We have shown that extracting the instantaneous phases using the Hilbert-Transform preceded by SSD (that also reduces the impact of noise) gave more accurate estimations of phase locking (Fig.5C) than estimations based on spectral coherence measures (Fig.5B) for a large parameter range. In contrast to spectral coherence, the estimations converged to the true value with increasing SNR. Nevertheless, at very high measurement noise levels (uncorrelated between oscillations), the SSD-HT-PLV method underestimated the PLV and was also sensitive to PrAM. A strong advantage of the method however is that PrFM and PrAM can be estimated from the data and considered for the interpretation of the phaselocking estimations. Overall, we suggest that the non-spectrally-based PLV computation proposed here is safer to use than spectral coherence. 


\section{Acknowledgements}

We thank Avgis Hadjipapas, Martin Vinck and Pascal Fries for comments.

\section{References}

Aru, J., Priesemann, V., Wibral, M., Lana, L., Pipa, G., Singer, W., Vicente, R., 2014. Untangling crossfrequency coupling in neuroscience, bioRxiv. doi:10.1101/005926

Atallah, B. V, Scanziani, M., 2009. Instantaneous modulation of gamma oscillation frequency by balancing excitation with inhibition. Neuron 62, 566-577. doi:10.1016/j.neuron.2009.04.027

Aydore, S., Pantazis, D., Leahy, R.M., 2013. A note on the phase locking value and its properties. Neuroimage 74, 231-244. doi:10.1016/j.neuroimage.2013.02.008

Baldauf, D., Desimone, R., 2014. Neural mechanisms of object-based attention. Science 344, 424-7. doi:10.1126/science.1247003

Bastos, A.M., Briggs, F., Alitto, H.J., Mangun, G.R., Usrey, W.M., 2014. Simultaneous recordings from the primary visual cortex and lateral geniculate nucleus reveal rhythmic interactions and a cortical source for gamma-band oscillations. J. Neurosci. 34, 7639-44. doi:10.1523/JNEUROSCI.421613.2014

Battaglia, D., Hansel, D., 2011. Synchronous chaos and broad band gamma rhythm in a minimal multilayer model of primary visual cortex. PLoS Comput. Biol. 7. doi:10.1371/journal.pcbi.1002176

Belluscio, M.A., Mizuseki, K., Schmidt, R., Kempter, R., Buzsaki, G., 2012. Cross-Frequency Phase-Phase Coupling between Theta and Gamma Oscillations in the Hippocampus. J. Neurosci. doi:10.1523/JNEUROSCI.4122-11.2012

Bendels, M.H.K., Leibold, C., 2007. Generation of theta oscillations by weakly coupled neural oscillators in the presence of noise. J. Comput. Neurosci. 22, 173-89. doi:10.1007/s10827-006-0006-6

Berman, J.I., McDaniel, J., Liu, S., Cornew, L., Gaetz, W., Roberts, T.P.L., Edgar, J.C., 2012. Variable Bandwidth Filtering for Improved Sensitivity of Cross-Frequency Coupling Metrics. Brain Connect. doi:10.1089/brain.2012.0085

Bokil, H., Andrews, P., Kulkarni, J.E., Mehta, S., Mitra, P.P., 2010. Chronux: A platform for analyzing neural signals. J. Neurosci. Methods 192, 146-151. doi:10.1016/j.jneumeth.2010.06.020

Bonizzi, P., Karel, J., De Weerd, P., Lowet, E., Roberts, M., Westra, R., Meste, O., Peeters, R., 2012. Singular spectrum analysis improves analysis of local field potentials from macaque $V 1$ in active fixation task. Conf. Proc. IEEE Eng. Med. Biol. Soc. 2012, 2945-8. doi:10.1109/EMBC.2012.6346581

Bonizzi, P., Karel, J.M.H., Meste, O., Peeters, R.L.M., 2014. Singular spectrum decomposition: A new method for time series decomposition. Adv. Adapt. Data Anal. 1450011. doi:10.1142/S1793536914500113

Bosman, C.A., Schoffelen, J.-M., Brunet, N., Oostenveld, R., Bastos, A.M., Womelsdorf, T., Rubehn, B., Stieglitz, T., De Weerd, P., Fries, P., 2012. Attentional stimulus selection through selective synchronization between monkey visual areas. Neuron 75, 875-888.

doi:10.1016/j.neuron.2012.06.037

Bosman, C.A., Womelsdorf, T., Desimone, R., Fries, P., 2009. A microsaccadic rhythm modulates gammaband synchronization and behavior. J Neurosci 29, 9471-9480. doi:10.1523/JNEUROSCI.119309.2009 
Breakspear, M., Heitmann, S., Daffertshofer, A., 2010. Generative models of cortical oscillations: neurobiological implications of the kuramoto model. Front Hum Neurosci 4, 190. doi:10.3389/fnhum.2010.00190

Bressler, S.L., Kelso, J.A.S., 2001. Cortical coordination dynamics and cognition. Trends Cogn Sci 5, 26-36. Brunet, N.M., Bosman, C.A., Vinck, M., Roberts, M., Oostenveld, R., Desimone, R., De Weerd, P., Fries, P., 2014. Stimulus repetition modulates gamma-band synchronization in primate visual cortex. Proc. Natl. Acad. Sci. U. S. A. 111, 3626-31. doi:10.1073/pnas.1309714111

Burns, S.P., Xing, D., Shapley, R.M., 2011. Is gamma-band activity in the local field potential of V1 cortex a "clock" or filtered noise? J Neurosci 31, 9658-9664. doi:10.1523/JNEUROSCI.0660-11.2011

Burns, S.P., Xing, D., Shelley, M.J., Shapley, R.M., 2010. Searching for autocoherence in the cortical network with a time-frequency analysis of the local field potential. J Neurosci 30, 4033-4047. doi:10.1523/JNEUROSCI.5319-09.2010

Burwick, T., 2007. Oscillatory neural networks with self-organized segmentation of overlapping patterns. Neural Comput. 19, 2093-2123. doi:10.1162/neco.2007.19.8.2093

Burwick, T., 2008. Temporal coding: assembly formation through constructive interference. Neural Comput. doi:10.1162/neco.2008.09-06-342

Buschman, T.J., Miller, E.K., 2007. Top-down versus bottom-up control of attention in the prefrontal and posterior parietal cortices. Science 315, 1860-2. doi:10.1126/science.1138071

Buzsáki, G., 2006. Rhythms of the Brain. Oxford University Press, USA.

Buzsáki, G., Draguhn, A., 2004. Neuronal oscillations in cortical networks. Science 304, 1926-9. doi:10.1126/science.1099745

Carter, G., Knapp, C., Nuttall, A., 1973. Estimation of the magnitude-squared coherence function via overlapped fast Fourier transform processing. IEEE Trans. Audio Electroacoust. 21. doi:10.1109/TAU.1973.1162496

Colgin, L.L., Denninger, T., Fyhn, M., Hafting, T., Bonnevie, T., Jensen, O., Moser, M.-B., Moser, E.I., 2009. Frequency of gamma oscillations routes flow of information in the hippocampus. Nature 462, $353-$ 7. doi:10.1038/nature08573

Coombes, S., Bressloff, P.C., 1999. Mode locking and Arnold tongues in integrate-and-fire neural oscillators. Phys. Rev. E. Stat. Phys. Plasmas. Fluids. Relat. Interdiscip. Topics 60, 2086-2096. doi:10.1103/PhysRevE.60.2086

Ding, M., Chen, Y., Bressler, S.L., 2006. Granger Causality: Basic Theory and Application to Neuroscience, in: Handbook of Time Series Analysis. pp. 451-474. doi:10.1002/9783527609970.ch17

Eckhorn, R., 2000. Cortical synchronization suggests neural principles of visual feature grouping. Acta Neurobiol Exp 60, 261-269.

Eckhorn, R., Reitboeck, H.J., Arndt, M., Dicke, P., 1990. Feature Linking via Synchronization among Distributed Assemblies: Simulations of Results from Cat Visual Cortex. Neural Comput. 2, 293-307. doi:10.1162/neco.1990.2.3.293

Engel, A.K., Fries, P., König, P., Brecht, M., Singer, W., 1999. Temporal binding, binocular rivalry, and consciousness. Conscious. Cogn. 8, 128-51. doi:10.1006/ccog.1999.0389

Ermentrout, G.B., Kleinfeld, D., 2001. Traveling electrical waves in cortex: insights from phase dynamics and speculation on a computational role. Neuron 29, 33-44.

Fries, P., 2005. A mechanism for cognitive dynamics: neuronal communication through neuronal coherence. Trends Cogn. Sci. 9, 474-80. doi:10.1016/j.tics.2005.08.011

Fries, P., 2009. Neuronal gamma-band synchronization as a fundamental process in cortical computation. Annu Rev Neurosci 32, 209-224. doi:10.1146/annurev.neuro.051508.135603

Fries, P., Nikolić, D., Singer, W., 2007. The gamma cycle. Trends Neurosci. 30, 309-16. doi:10.1016/j.tins.2007.05.005 
Gail, A., Brinksmeyer, H.J., Eckhorn, R., 2000. Contour decouples gamma activity across texture representation in monkey striate cortex. Cereb Cortex 10, 840-850.

Galán, R., Ermentrout, G., Urban, N., 2005. Efficient Estimation of Phase-Resetting Curves in Real Neurons and its Significance for Neural-Network Modeling. Phys. Rev. Lett. 94, 158101. doi:10.1103/PhysRevLett.94.158101

Gilgen, H., 2006. Univariate time series in geosciences: Theory and examples, Univariate Time Series in Geosciences: Theory and Examples. doi:10.1007/3-540-30968-3

Gregoriou, G.G., Gotts, S.J., Zhou, H., Desimone, R., 2009. High-frequency, long-range coupling between prefrontal and visual cortex during attention. Science 324, 1207-1210. doi:10.1126/science.1171402

Hoppensteadt, F.C., Izhikevich, E.M., 1996. Synaptic organizations and dynamical properties of weakly connected neural oscillators. I. Analysis of a canonical model. Biol Cybern 75, 117-127.

Hoppensteadt, F.C., Izhikevich, E.M., 1998. Thalamo-cortical interactions modeled by weakly connected oscillators: could the brain use FM radio principles? Biosystems 48, 85-94.

Huang, N.E., 2005. Introduction to the Hilbert-Huang Transform and its related mathematical problems. Hilbert-Huang Transform Its Appl. 1-26. doi:doi:10.1142/9789812703347_0001

Huang, N.E., Shen, Z., Long, S.R., Wu, M.C., Shih, H.H., Zheng, Q., Yen, N.-C., Tung, C.C., Liu, H.H., 1998. The empirical mode decomposition and the Hilbert spectrum for nonlinear and non-stationary time series analysis. Proc. R. Soc. A Math. Phys. Eng. Sci. doi:10.1098/rspa.1998.0193

Jensen, O., Colgin, L.L., 2007. Cross-frequency coupling between neuronal oscillations. Trends Cogn. Sci. 11, 267-9. doi:10.1016/j.tics.2007.05.003

Jia, X., Xing, D., Kohn, A., 2013. No consistent relationship between gamma power and peak frequency in macaque primary visual cortex. J Neurosci 33, 17-25. doi:10.1523/JNEUROSCI.1687-12.2013

Lachaux, J.P., Rodriguez, E., Martinerie, J., Varela, F.J., 1999. Measuring phase synchrony in brain signals. Hum Brain Mapp 8, 194-208.

Lakatos, P., Shah, A.S., Knuth, K.H., Ulbert, I., Karmos, G., Schroeder, C.E., 2005. An oscillatory hierarchy controlling neuronal excitability and stimulus processing in the auditory cortex. J Neurophysiol 94, 1904-1911. doi:10.1152/jn.00263.2005

Le Van Quyen, M., Foucher, J., Lachaux, J., Rodriguez, E., Lutz, A., Martinerie, J., Varela, F.J., 2001. Comparison of Hilbert transform and wavelet methods for the analysis of neuronal synchrony. J. Neurosci. Methods 111, 83-98. doi:http://dx.doi.org/10.1016/S0165-0270(01)00372-7

LeVanQuyen, M., Foucher, J., Lachaux, J.-P., Rodriguez, E., Lutz, A., Martinerie, J., Varela, F., 2001. Comparison of Hilbert transform and wavelet methods for the analysis of neuronal synchrony. J. Neurosci. Methods 111, 83-98.

Oostenveld, R., Fries, P., Maris, E., Schoffelen, J.-M., 2011. FieldTrip: Open source software for advanced analysis of MEG, EEG, and invasive electrophysiological data. Comput. Intell. Neurosci. 2011, 156869. doi:10.1155/2011/156869

Picinbono, B., 1997. On instantaneous amplitude and phase of signals. IEEE Trans. Signal Process. 45, 552-560. doi:10.1109/78.558469

Pikovsky, A., Rosenblum, M., Kurths, J., Hilborn, R.C., 2002. Synchronization: A Universal Concept in Nonlinear Science. Am. J. Phys. 70, 655. doi:10.1119/1.1475332

Ray, S., Maunsell, J.H.R., 2010. Differences in gamma frequencies across visual cortex restrict their possible use in computation. Neuron 67, 885-896. doi:10.1016/j.neuron.2010.08.004

Roberts, M.J., Lowet, E., Brunet, N.M., Ter Wal, M., Tiesinga, P., Fries, P., De Weerd, P., 2013. Robust gamma coherence between macaque V1 and V2 by dynamic frequency matching. Neuron 78, 52336. doi:10.1016/j.neuron.2013.03.003

Rosenblum, Pikovsky, Kurths, 1996. Phase synchronization of chaotic oscillators. Phys Rev Lett 76, 18041807. 
Saalmann, Y.B., Pinsk, M.A., Wang, L., Li, X., Kastner, S., 2012. The Pulvinar Regulates Information Transmission Between Cortical Areas Based on Attention Demands. Science (80-. ). doi:10.1126/science.1223082

Salinas, E., Sejnowski, T.J., 2001. Correlated neuronal activity and the flow of neural information. Nat Rev Neurosci 2, 539-550. doi:10.1038/35086012

Schwemmer, M.A., Lewis, T.J., 2012. Phase Response Curves in Neuroscience, in: Phase Response Curves in Neuroscience. pp. 3-31. doi:10.1007/978-1-4614-0739-3

Singer, W., 1999. Neuronal Synchrony: A Versatile Code for the Definition of Relations? Neuron 24, 4965. doi:10.1016/S0896-6273(00)80821-1

Sirota, A., Montgomery, S., Fujisawa, S., Isomura, Y., Zugaro, M., Buzsáki, G., 2008. Entrainment of neocortical neurons and gamma oscillations by the hippocampal theta rhythm. Neuron 60, 683697. doi:10.1016/j.neuron.2008.09.014

Srinath, R., Ray, S., 2014. Effect of amplitude correlations on coherence in the local field potential. J. Neurophysiol. 112, 741-51. doi:10.1152/jn.00851.2013

Steriade, M., McCormick, D.A., Sejnowski, T.J., 1993. Thalamocortical oscillations in the sleeping and aroused brain. Science 262, 679-685. doi:10.1126/science.8235588

Tiesinga, P., Fellous, J.-M., Sejnowski, T.J., 2008. Regulation of spike timing in visual cortical circuits. Nat. Rev. Neurosci. 9, 97-107. doi:10.1038/nrn2315

Tiesinga, P.H., Sejnowski, T.J., 2010. Mechanisms for Phase Shifting in Cortical Networks and their Role in Communication through Coherence. Front Hum Neurosci 4, 196. doi:10.3389/fnhum.2010.00196

Tort, A.B.L., Komorowski, R., Eichenbaum, H., Kopell, N., 2010. Measuring phase-amplitude coupling between neuronal oscillations of different frequencies. J. Neurophysiol. 104, 1195-1210. doi:10.1152/jn.00106.2010

Vinck, M., van Wingerden, M., Womelsdorf, T., Fries, P., Pennartz, C.M.A., 2010. The pairwise phase consistency: A bias-free measure of rhythmic neuronal synchronization. Neuroimage 51, 112-122. doi:10.1016/j.neuroimage.2010.01.073

Womelsdorf, T., Schoffelen, J.-M., Oostenveld, R., Singer, W., Desimone, R., Engel, A.K., Fries, P., 2007. Modulation of neuronal interactions through neuronal synchronization. Science 316, 1609-1612. doi:10.1126/science.1139597

Zou, W., Wang, X.G., Zhao, Q., Zhan, M., 2009. Oscillation death in coupled oscillators. Front. Phys. China 4, 97-110. doi:10.1007/s11467-009-0009-7

\section{Supporting Text S1}

\section{Code S1}

function [parameter,data,true_inst_ph] $=$ phase_oscil_plv_plos(cfg)

$\% \% \% \% \% \% \% \% \% \% \% \% \% \% \% \% \% \% \% \% \% \% \% \% \% \% \% \% \% \% \% \% \%$

$\%$ Generates the data (two coupled phase osicllators)

$\%$ and estimates the FFT-PLV

$\%$ Output is generated to be easy to use for fieldtrip functions

$\%$ cfg.SNR white noise level added to the signal (measurment noise).

$\% \quad$ If intrinisc noise is added, then this SNR definition will differ from the

$\% \quad$ relative power ratio, because power is spreaded over frequencies.

$\%$ cfg.trial number number of trials

$\%$ cfg.coupling symmetric coupling values between phase oscillators (coupling function is sinusoidal (defining phase response curve) 


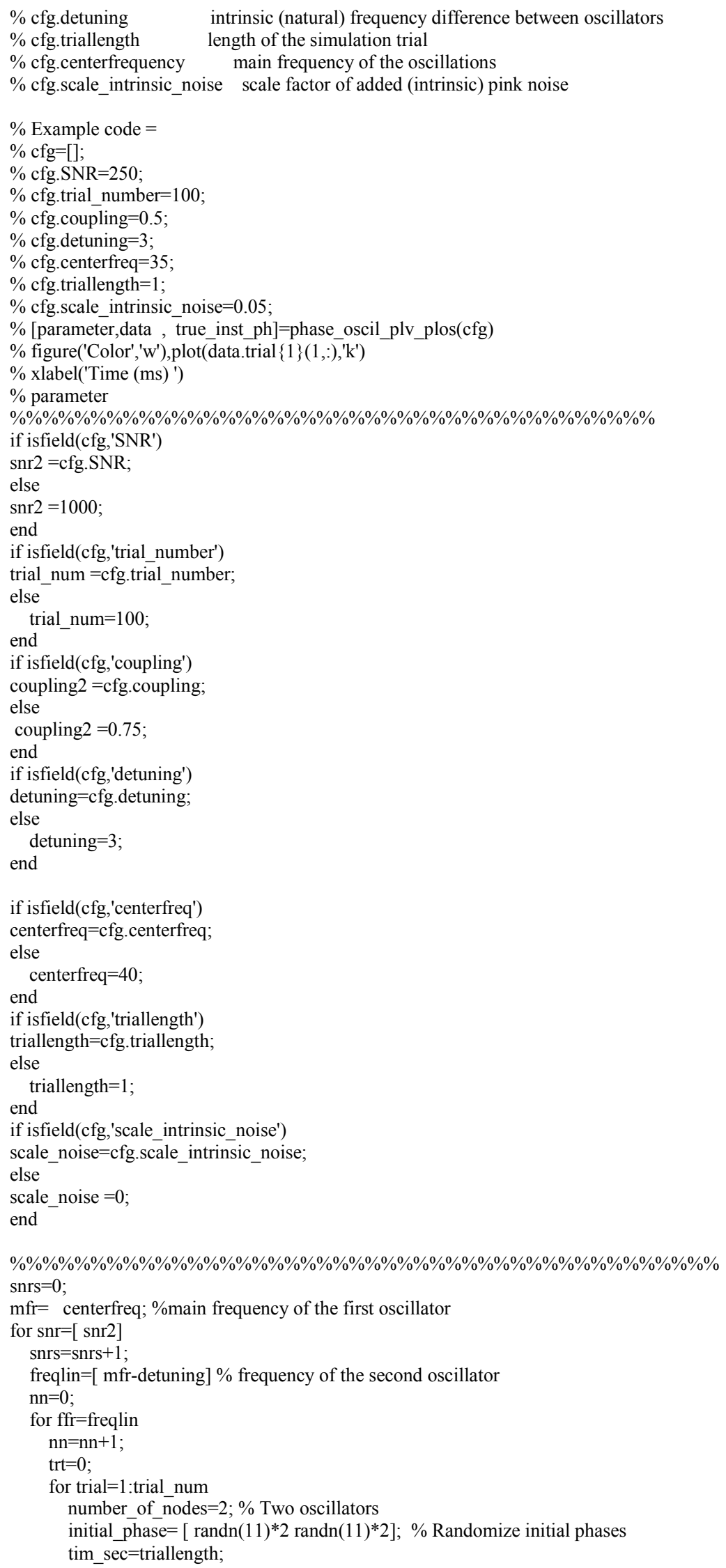


time_steps $=$ tim_sec $+2 ; \%$ The two extra seconds is due to transient dynamics at the beginning

$\mathrm{dt}=0.001 ; \%$ step size (here $1 \mathrm{~ms}$ )

phases $=$ zeros(number of nodes,time steps./dt);

phases $(1: 2,1)=$ initial_phase $(1:$ number_of_nodes, 1$) . * 1$;

clear noiseterm

for ind $=1: 2$

noiseterm(ind,:)=powernoise(1,(time_steps./dt)+1,'normalize').*scale_noise;

end

$\mathrm{f} 2=0$;

for freqs $2=\mathrm{ffr}$

$\mathrm{f} 2=\mathrm{f} 2+1$

cops $=0$;

for coupling = coupling2

cops $=$ cops +1 ;

$\mathrm{K}=$ [ coupling coupling $] ; \%$ coupling matrix (here symmetric)

$\mathrm{W}=[\mathrm{mfr}$ freqs 2$]$;

$\mathrm{W}=\mathrm{W} . *(2 * \mathrm{pi}) ; \%$ radians per sec

$\mathrm{K}=\mathrm{K} . *(2 * \mathrm{pi}) ; \%$ scaling of coupling

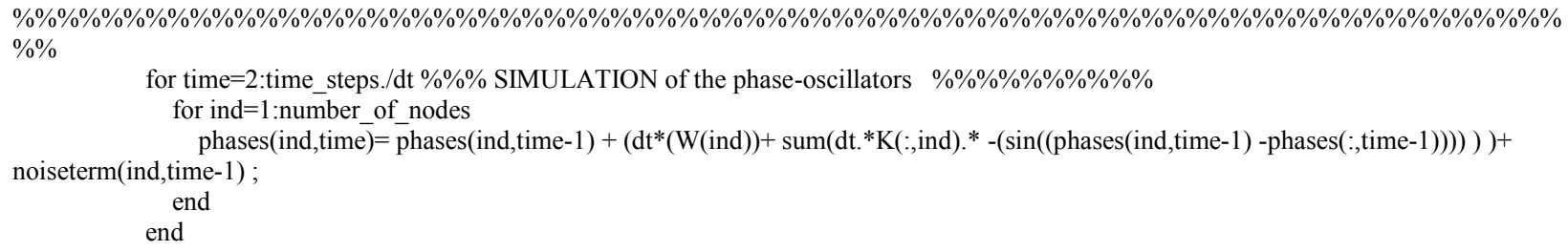






function $\mathrm{x}=$ powernoise(alpha, $\mathrm{N}$, varargin)

$\%$ Generate samples of power law noise. The power spectrum

$\%$ of the signal scales as $\mathrm{f}^{\wedge}$ (-alpha).

$\%$ Useage:

$\% \mathrm{x}=$ powernoise(alpha, $\mathrm{N}$ )

$\% \mathrm{x}=$ powernoise(alpha, $\mathrm{N}$, 'option 1', 'option2', ...)

$\%$ Inputs:

$\%$ alpha - power law scaling exponent

$\% \mathrm{~N}$ - number of samples to generate 


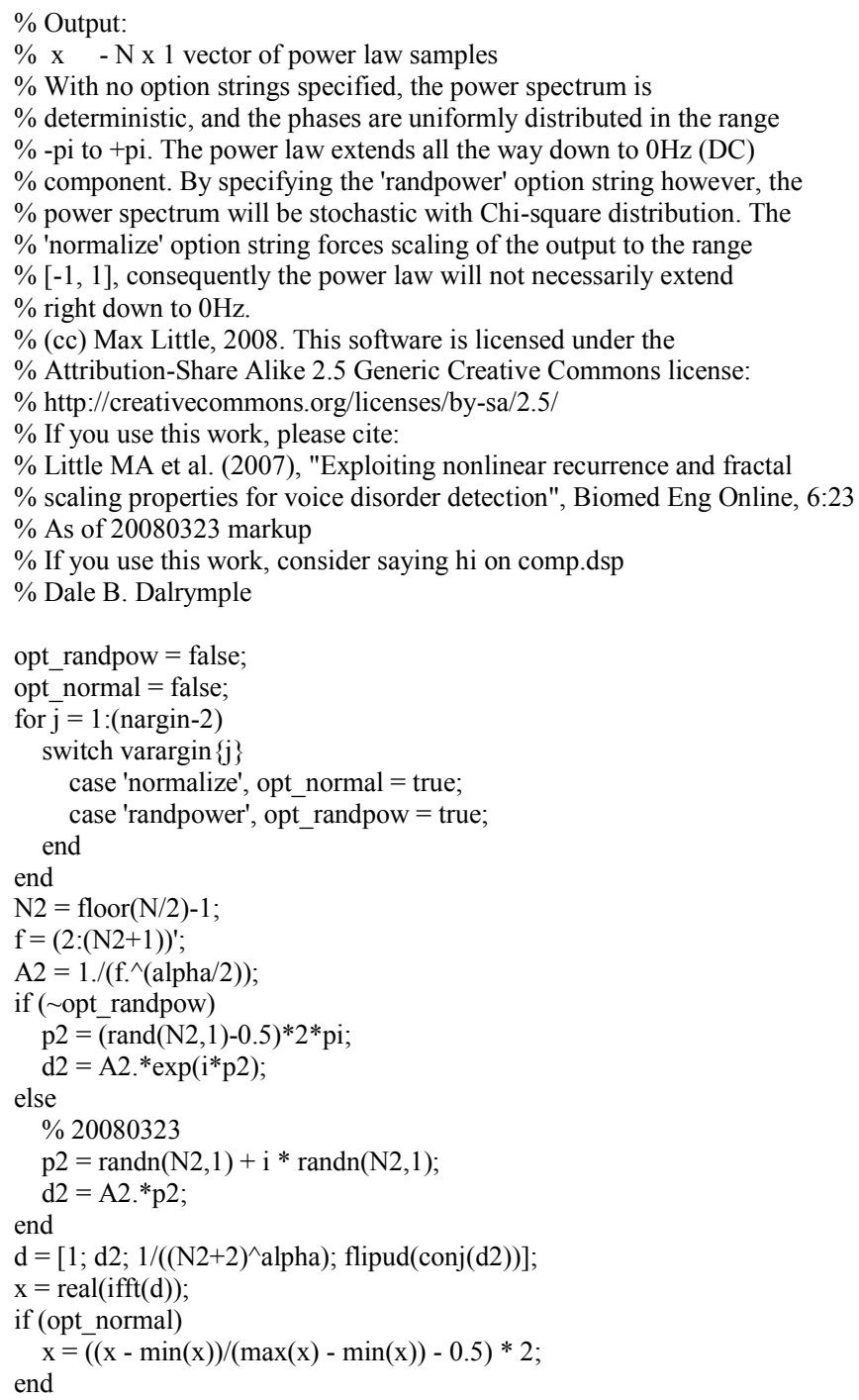




\section{Chapter 7}

\section{Gamma-band coordination of synchrony and spike timing in visual cortex predicted by the theory of weakly coupled oscillators}

Based on:

E.Lowet, M. J. Roberts, P. De Weerd, "Gamma-band coordination of synchrony and spike timing in visual cortex predicted by the theory of weakly coupled oscillators" (in preparation) 


\section{ABSTRACT}

In the visual system, stimulus-specific oscillations in the gamma range $(25-80 \mathrm{~Hz})$ have been described (Gray and Singer, 1989; Hermes et al., 2014; Jia et al., 2013b) and suggested to play a role in visual processes (Brunet et al., 2013; Eckhorn, 1999; Singer and Gray, 1995; Womelsdorf and Fries, 2007). Yet, it is has so far been unclear what principles underlie gamma synchronization in visual cortex. Here we present theoretical analysis and experimental recordings in macaque visual cortex V1, and show that the phase-locking and the phase-relation among local gamma oscillations was well predicted by the theory of weakly coupled oscillators(Breakspear et al., 2010; Ermentrout and Kleinfeld, 2001; Hoppensteadt and Izhikevich, 1998, 1996; Pikovsky et al., 2002) when input drive is mapped onto preferred (intrinsic) frequency. In empirical recordings, we presented the monkey with full-screen gratings in which the local contrast varied over space such that the receptive fields of the three laminar probes were exposed to variable contrast differences. Thus we manipulated the local gamma frequency at each probe location in order to parametrically vary the frequency difference between the recorded neural populations. We found that the regulation of gamma synchrony and phase-relation was dependent both on the gamma frequency difference (detuning) and the interaction strength, assessed as distance between receptive fields. After combining all recorded conditions we were able to reconstruct the Arnold tongue of locally interacting V1 gamma oscillations. Further, we here demonstrate for the first time the presence of the partially (intermittent) synchronized state in macaque visual cortex (Gong et al., 2007; Kozma and Freeman, 2008) characterized by phase-relation-dependent instantaneous frequency modulations (Pikovsky et al., 2002). The partially synchronized state allows intriguing view onto the underlying synchronization dynamics and experimental access to the phase-response curve (PRC) critical for

understanding synchronization properties. Our results indicate that gamma synchronization among visual cortical neurons, operating in partially synchrony regime, can support meaningful coordination, integrating information from input drive (intrinsic frequency) and interaction strength (connectivity) and hence, in principle, well situated to support information processing and plasticity. 


\section{Introduction}

Synchronization, the ability of oscillators to mutually adapt their rhythm, is a ubiquitous phenomena observed in many field of sciences founded in the first observation of Huygens of mutual entrainment of pendula (Pikovsky et al., 2002). Synchronization has been suggested to play a role in the coordination of neural activity (Eckhorn, 2000; Singer and Gray, 1995; Singer, 1999).

In the visual system, stimulus-specific oscillations in the gamma range $(25-80 \mathrm{~Hz})$ have been described (Gray and Singer, 1989; Hermes et al., 2014; Jia et al., 2013b) and suggested to play a role in visual processes (Brunet et al., 2013; Eckhorn, 1999; Singer and Gray, 1995; Womelsdorf and Fries, 2007). Stimulus-induced gamma oscillations in primate early visual areas have been found to emerge locally in cortex and to be highly sensitive to visual input parameter (Gail et al., 2000; Gray and Singer, 1989). Recent studies have shown that the precise frequency of gamma oscillations can shift systematically with stimulus input parameter e.g. contrast, (Jia et al., 2013a; Ray and Maunsell, 2010; Roberts et al., 2013), such that nearby V1 neural populations can engage slightly in different gamma frequencies (Ray and Maunsell, 2010) supporting the view that locally generated gamma oscillations adapt their frequency to the level of excitatory drive. It has been suggested that the sensitivity of the precise gamma frequency on sensory input drive might be detrimental for a functional role of gamma synchronization in visual cortical processing (Ray and Maunsell, 2010). In this study, we investigated using theoretical as well experimental techniques the significance of frequency variation for the synchronization properties of gamma oscillations and the implication for the spatio-temporal coordination of neural activity.

A theoretical framework for understanding the self-organization principles of a network with spatially local emerging oscillations of different frequencies is offered by the theory of weakly coupled oscillators (TWCO). The TWCO describes, for conditions of relatively weak interaction strength (Pikovsky et al., 2002), under which conditions interacting (coupled) oscillators synchronize. The ability of coupled oscillators to synchronize is controlled by two opponent forces (Pikovsky et al., 2002): their detuning, or intrinsic frequency difference, and interaction strength. The exact underlying interaction function is described by the phase response curve (PRC, Schwemmer and Lewis, 2012). The PRC defines the phase adjustments in terms of positive or negative delays as a function of phase-relation (Schwemmer and Lewis, 2012). We derived several predictions from the TWCO framework that we tested experimentally for gamma synchronization in macaque visual cortex V1 using locally contrast-varying grating stimulus and three nearby inserted multi-contact depth probes: 1 ) The phase-relation shifts systematically with detuning, 2) the slope of the phase shifting depends on the interaction strength (i.e. by synaptic connectivity) 3) phase-locking decreases with detuning 4) phase-locking increases with interaction strength and 5) interactions will be according to partial synchrony, characterized by PrFM.

The local nature of intrinsically generated V1 gamma oscillations combined with the established relationship between gamma frequency and contrast formed the basis of empirical recording experiments in V1 of two macaque monkeys. In these experiments, we used three depth probes 
separated by several $\mathrm{mm}$, while full-screen gratings were presented in which the local contrast varied over space, such that the receptive fields of neurons recorded by the three laminar probes were exposed to different contrasts. This induced a different local gamma frequency at each probe location. By manipulating the contrast difference, we could hence control the gamma frequency difference between the recorded neural populations. We also varied the separation (over sessions) between neighboring probes to manipulate interaction using the established relation of decreasing synaptic connection probability with cortical distance (Stettler, Das, Bennett, \& Gilbert, 2002). We found that the regulation of gamma synchrony and phase-relation, was dependent both on the gamma frequency difference (detuning) and the interaction strength, assessed as the distance between receptive fields.

After combining all recorded conditions, we were able to reconstruct the Arnold tongue of locally interacting V1 gamma oscillations. Further, we demonstrated here for the first time the presence of a partially (intermittent) synchronized state in macaque visual cortex (Gong et al., 2007; Kozma and Freeman, 2008) characterized by phase-relation-dependent instantaneous frequency modulations (Pikovsky et al., 2002) allowing an intriguing view onto the underlying synchronization dynamics and the underlying phase-response curve (PRC).

These results suggest that spatio-temporal gamma-band coordination of V1 cortical activity can be described within the framework of weakly coupled oscillators.

\section{Results}

\section{Experimental frequency manipulation of local V1 gamma oscillations}

For experimental testing of the theoretical predictions, we recorded gamma rhythmic activity using acute laminar probe recordings in the visual cortical area V1 in two awake macaque monkeys (Figure 1A). Three 16-contact laminar depth probes were inserted in parafoveal V1. From the three probes we analyzed gamma rhythmic activity in local field potentials (LFP), current-source density (CSD, Pettersen et al., 2011) and spiking activity. The distances between the probes could varied sessions and was on the order of 2 to $4 \mathrm{~mm}$. The three probes had distinct but nearby visual receptive fields (RF, see Figure 1B). The task of the monkey was to fixate a fixation dot situated at the center of the screen. At the same time, a whole-field square-wave grating was presented (1sec baseline, 2 sec stimulus), which had spatial modulations of luminance contrast (Figure 1A, see Methods for details). The modulations were such that each RF received a different level of contrast. The grating stimulus induced substantial gamma power. We observed a systematic cortical depth profile (Figure $2 \mathrm{C}$ ). In middle-superficial layers, we found the strongest increase in gamma power, whereas around Layer 4C-5 the gamma power was low (dashed line corresponds to top of layer 4C). We also found gamma power peak in Layer 6 . In the following analysis we report results from the middle-superficial layers of V1 (see further analysis in Suppl. Materials).

To test the TWCO-derived predictions, we needed a measure of detuning (intrinsic frequency difference) and of interaction strength. In both cases, the critical variables cannot be measured directly, however they can be well approximated. For interaction strength, the receptive field (RF) distance between probes is closely related to the cortical distance for the probe positions we have used (Stettler, Das, Bennett, \& Gilbert, 2002). We used this measure instead of the physical distance between the probes 
because it was difficult to control whether due to insertion angle or slight bending the probes might approach or deviate from each other in the cortex. It has been established by previous studies (Angelucci \& Bullier, 2003; Boucsein, Nawrot, Schnepel, \& Aertsen, 2011; Stettler et al., 2002) that the connectivity among neurons decreases on average with cortical distance. Within and between recording session we had different RF distances covering from $\sim 0.8 \mathrm{deg}$ to $3.5 \mathrm{deg}$ (Fig.2B). We hence had pairs with a substantial range of different connectivity values.

A

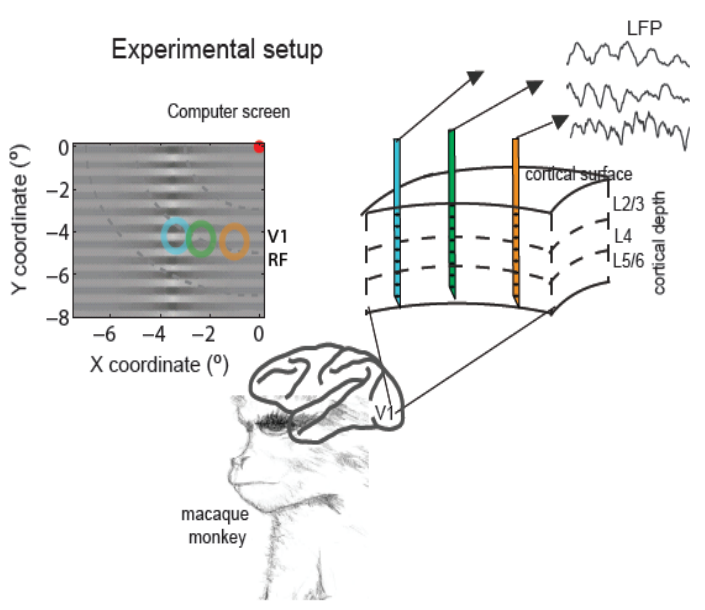

D
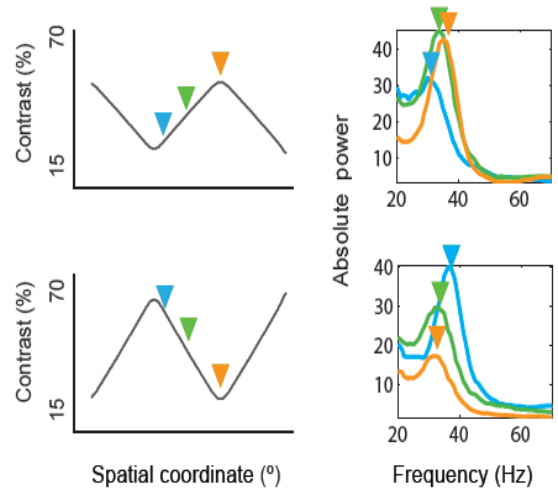

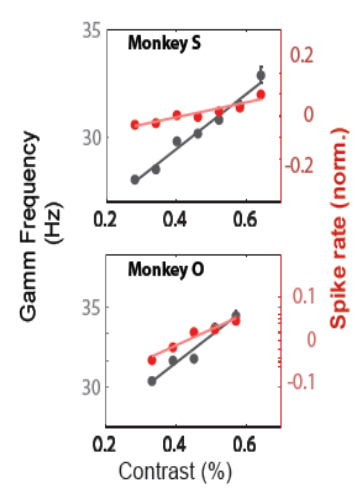

B
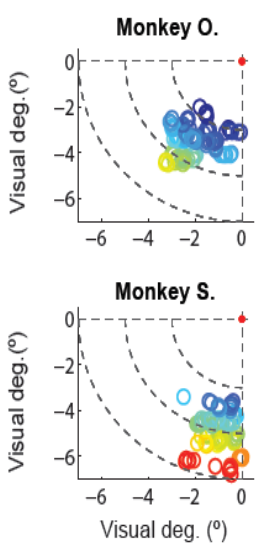

$\mathbf{F}$

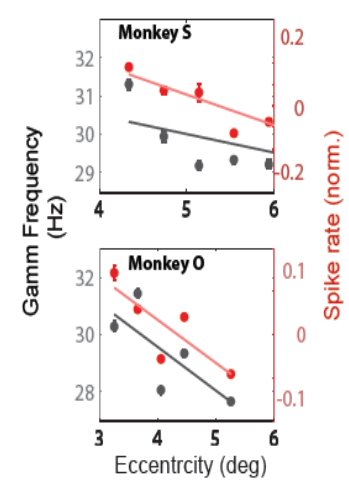

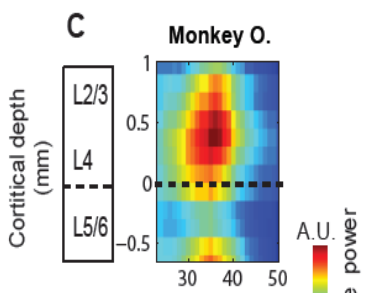

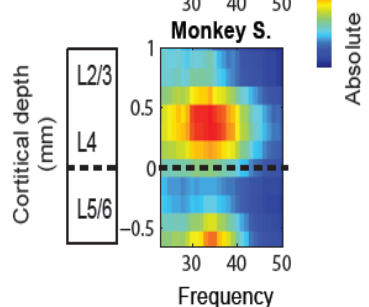

$(\mathrm{Hz})$

G



Figure 1: The experimental set-up and manipulations to test the predictions from the theoretical model. A) The experimental set-up used using awake macaque monkeys and acute laminar probe recordings. Three equi-distant 16-contact laminar depth probes were inserted in parafoveal V1. The three probes had distinct but nearby visual receptive fields (RF, colored circles). The task of the monkey was to fixate on a fixation dot situated at the center of the screen (red dot).. B) The distribution of eccentricities over the populations of probes used in the experiment where each ring represents average RF position of a probe. Color represent eccentricity value (blue -low, red-high) dashed curves show 2, 4 and 6 degrees of eccentricity. Top plot is Monkey O. and bottom plot is Monkey S. C) The spectral gamma properties of LFP as a function of cortical depth (average over all probes and sessions (62\% contrast condition). (E-G) Quantifications of experimental manipulations of contrast and eccentricity on gamma oscillation frequency and spike rate. E) The effect of luminance grating contrast (local modulation) on gamma frequency and spike rate. F) Correlation between eccentricity and frequency as well spike rate. Both $E$ and $F$ are in line with previous published experiments. G) Relationship between the gamma frequency difference of the gamma rhythms and the spike rate difference between $\mathrm{V} 1$ locations in both monkeys. 
For manipulating detuning, we used our previously established observations that the frequency of V1 gamma can be shifted with visual contrast. Here, we manipulated the contrast spatially while keeping the overall mean contrast of the stimulus the same (Figure 1D, left). As indicated in previous studies (Jia, Xing, \& Kohn, 2013; Ray \& Maunsell, 2010; Roberts et al., 2013), and shown in Figure 1D (right), we found that experimental manipulation of visual grating contrast modulated systematically the precise frequency of gamma $\left(R^{2}=0.31(0.25), p<10^{-10}, n=558(468)\right.$. In this notation, statistical values of Monkey $S$ are followed by statistical values from monkey $\mathrm{O}$. in brackets, and this convention is followed in the remainder of the text. Similarly to gamma frequency, also the spike rate was modulated in a monotonic manner by contrast $\left(R^{2}=0.12(0.14), p<10^{-10}, n=558(468)\right.$, Figure $\left.1 E\right)$. In addition, we found that the visual eccentricity of the inserted probes (Figure $1 F)$ had systematic effect on the frequency $\left(R^{2}=0.05(0.09)\right.$, $\left.p<10^{-10}, n=558(468)\right)$ as well as on spike rate $\left(R^{2}=0.04(0.08), p<10^{-10}, n=558(468)\right.$, Fig.2E). This had also been established previously (van Pelt \& Fries, 2013). The eccentricity effect may be due to the constant spatial frequency of the stimulus and the known shifts of spatial frequency tuning with eccentricity. We found that within recording sites there was a clear relationship between spike rate difference between conditions and the gamma frequency difference $\left(R^{2}=0.36(0.1), p<10^{-10}, n=558(468)\right)$, which supports the view that the preferred gamma frequency of a local V1 neural population shifts with the local excitatory drive, here as a function of visual contrast and eccentricity. The frequency manipulations occurred separately in the three probes such that gamma oscillations recorded from the three probes had different frequencies for a given experimental condition. We found a strong relationship between detuning parameter (see Methods) and the spike rate differences among V1 locations $\left(R^{2}=0.53(0.36)\right.$, $p<10^{-10}, n=1116(734)$, Figure $\left.1 G\right)$, as well with contrast difference $\left(R^{2}=0.24(0.37), p<10^{-10}, n=1116(734)\right.$, Figure $1 \mathrm{E})$. These findings confirmed that our experimental manipulation was successful in parametrically varying gamma frequency locally in V1.

\section{The theory of weakly coupled oscillators: The phase response curve and the partial synchrony state}

The TWCO offers a framework for a precise description of the synchronization of weakly coupled oscillators (Ermentrout and Kleinfeld, 2001; Hoppensteadt and Izhikevich, 1996; Schwemmer and Lewis, 2012). 'Weak' coupling means that the interaction leads to phase adjustments (changes in instantaneous frequency), yet not to strong power fluctuations and destabilization of the oscillationgenerating mechanism. The assumption of 'weak' coupling is plausible for interacting local V1 gamma rhythms as horizontal synaptic connections have been described as modulatory rather than strong or dominant (Hoppensteadt and Izhikevich, 1998; Stettler et al., 2002). TWCO has been applied to explain the synchronization behavior of gamma-generating excitatory-inhibitory conductance-based modeling networks (Lowet et al., 2015; Tiesinga and Sejnowski, 2010).

A central concept in the theory is the phase response curve (PRC). The PRC (Pikovsky et al., 2002) describes how interacting oscillators with slightly different intrinsic frequencies (Figure $2 \mathrm{~A}$ left) adjust their phases as a function of the phase-relation between them (Figure 2A right). For phase-locking to occur between two oscillators, the phase-relation needs to be kept around a preferred phase-relation. When there is no frequency difference, interacting oscillators will settle for a constant phase difference. 
When there is an intrinsic frequency difference (detuning), there will be variation on their phase relation between the oscillators, referred to as phase precession, which will vary between a minimum and a maximum at a frequency matching the frequency difference between the two oscillators. Note that when two oscillators with different detuning would not be connected and would not interact, free phase precession would occur in which the two oscillators would show repetitive linear traversing of all possible phase relations from 0 to $2 \pi$. When the oscillators are connected and do interact, for a limited range of intrinsic frequency differences there will be a force that will lead to a preferential phase relation and a limitation of the variations around that that phase relation. In these cases, the frequency difference will therefore be minimized around the phase-relation of preference.

The shape of the PRC is crucial for understanding under which conditions oscillators will synchronize, at what strength and with what phase-relation. The PRC might occur in different forms that may include accelerating (phase advance) and decelerating (phase delay) parts (Schwemmer and Lewis, 2012). Phase-relations around which the phase-advance component flips to a phase-delay component are fixed attractor points (the derivative of the PRC is negative). A fixed attractor point is the phase-relation to which the oscillators converge, and can be seen as the deepest point in the energy landscape of the oscillator system. A simple form of a PRC is a sinusoidal shape, which has one stable fixed point and symmetric advance and delay components (Figure 2A, right). Sinusoidal PRCs have been used widely in the phase-oscillator literature (Breakspear et al., 2010), due to their simple yet plausible synchronization characteristics. They appear to be a good candidate to understand synchronized gamma rhythms interacting via inhibitory and excitatory connections (Lowet et al., 2015). The amplitude of the PRC defines the interaction strength and how strong it can counteract detuning.

We now illustrate the synchronization regimes that can occur in a TWCO model (Fig. 2B). We represent the PRC and synchronization dynamics, referring to Pikovsky et al., (2002) as a 'particle' sliding along an inclined potential. The particle represents the phase-relation state. The attractor represents here a minimum in potential. Without an additional force, the particle will remain trapped in the potential minimum (Fig. 2, $\Delta \mathrm{w} \leq \mathrm{K}$ ). In this case, the phase relation remains constant and complete synchrony is achieved. Detuning (intrinsic frequency difference) induces a potential gradient along which the particle slides down. If the interaction strength is larger than the gradient induced by detuning $(K>\Delta \mathrm{w})$, then complete synchrony is preserved. This would be the case if the potential landscape is slightly titled, but not enough to have the particle roll down. Without an attractor (Fig. 2, K=0), the particle would slide down at the same speed (smooth phase precession) and each phase would have the same probability (complete asynchrony), which is the case when there is no coupling between oscillators. In the case the detuning gradient is larger than the interaction strength $(\Delta \mathrm{w}>\mathrm{K})$, the particle cannot be kept in place and it will slide down the gradient. However, the speed will vary, being slowed down when passing the attractor and sped up around the opposite phase. The phase-relation distribution in this case will not be uniform, showing a clear peak around the preferred phase-relation. This regime is called the partially synchronized state. In noiseless oscillator systems the complete synchrony regime can be large and the partially synchronized regime limited. In noisy oscillatory systems, however, the partially synchronized regime can be large or even be the most dominant one. This is because (phase) noise can kick the particle out of the attractor minimum potential (Fig $2 B, \Delta w+\xi>K$ ) with a likelihood determined by the 
noise probability distribution. The dynamics of biological oscillators are often noisy and complex and it is therefore plausible to assume that the partially synchronized regime is the most likely scenario between interacting neural gamma oscillations. In Fig $2 \mathrm{C}$ we show the cumulative phase relation trajectories of two phase-oscillators with different coupling values $(k)$ for a detuning frequency of $-2.2 \mathrm{~Hz}$. If $k$ is 0 , the phase-relation trajectory is smooth. If $\mathrm{k}<2.2$, partial synchrony can be observed with varying phaserelation trajectories (resulting in corresponding variations in frequency difference). For $k>2.2$ the phase-relation is constant and synchrony is complete.

We will now describe how the fluctuations in phase-relation precession speed (instantaneous frequency difference) associated with the partial synchrony regime can be experimentally assessed with the goal of determining the PRC including detuning and interaction strength. In the partially synchronized state, the instantaneous frequency (IF) difference between two oscillators fluctuates as a function of their phaserelation, with a main frequency of IF fluctuations related to the detuning, and the amplitude related to the interaction strength of the PRC (Figure 2D left \& middle). In Figure 2D (right) the instantaneous frequency difference is plotted as a function of phase-relation. We term this fluctuation the phaserelation dependent frequency modulation (PrFM). It has the shape of the PRC, the averaged IF over all phase-relations (-pi to pi) being the detuning (see Methods) and the amplitude being twice the interaction strength $\mathrm{k}$. There is a strict relationship between the PrFM and the PRC. Increasing the interaction strength $\mathrm{k}$ leads to a proportional increase in the PrFM amplitude (Figure 2E). Changing the detuning shifts the mean of the PrFM accordingly (Figure 2F), as long the oscillators do not reach complete synchrony. The phase-relation of minimal frequency difference (preferred phase-relation) shifts with detuning frequency (Figure 2F).

The underlying PRC, and its main parameters, namely the interaction strength and the detuning frequency, are not directly accessible. However, the instantaneous phase (and its derivative, the instantaneous frequency) can be experimentally estimated using appropriate techniques. Moreover, interaction strength can be estimated from the PrFM amplitude. Hence, we aimed to estimate the parameters detuning and interaction strength to investigate gamma synchronization in macaque visual cortex. The detuning parameter was estimated from the mean of the PrFM. The detuning frequency achieved during synchronization is strongly related, but not identical, to the mean frequency difference between two oscillations. However, the stronger the interaction strength and phase-locking, the stronger they will diverge. This is because synchronization tries to minimize the frequency difference between oscillators. Hence, with strong interaction and phase locking, a large intrinsic frequency difference between two cortical sites during synchronization will be reduced, and the emergent or observable frequency difference will be smaller than the intrinsic one. Hence, the stronger the synchronization, the stronger the difference between the detuning and the mean (observable) frequency difference will become. The interaction strength was estimated as half of the amplitude of the PrFM. This estimate is strictly related to the amplitude of the PRC. In simulations of gammagenerating excitatory-inhibitory modeling networks, the PrFM amplitude is related to synaptic connection strength (Suppl.Fig.1). However, the PrFM estimate decreases if the signal-to-noise (SNR) gets low, hence the empirically estimated values likely underestimate the true interaction strength. 
(i) TWCO model : PRC \& partial synchronization

A

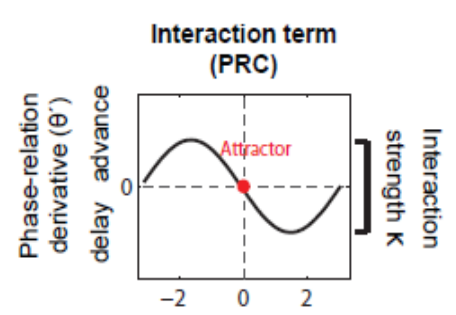

Phase-relation $\theta$
B

Complete synchrony $\Delta \omega<=K \quad$ Partial synchrony(i) $\Delta \omega>K$

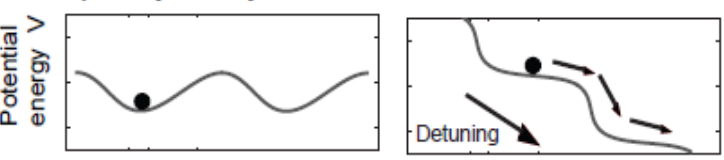

Complete asynchrony $K=0 \quad$ Partial synchrony(ii) $\Delta \omega+\xi>K$



$\theta / 2 \pi$

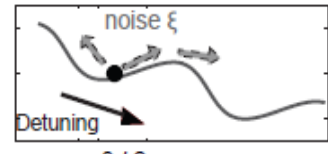

$\theta / 2 \pi$
C

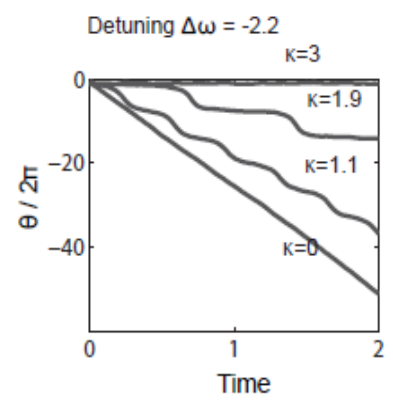

F

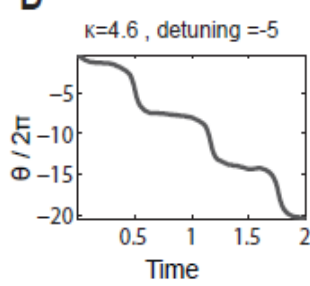

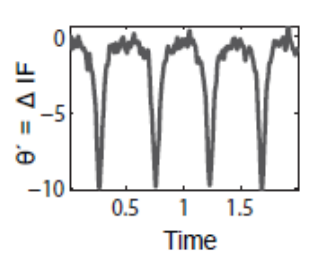

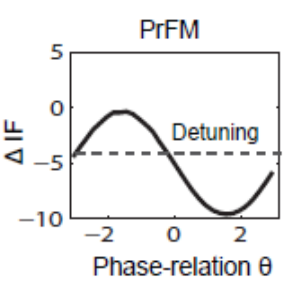

E

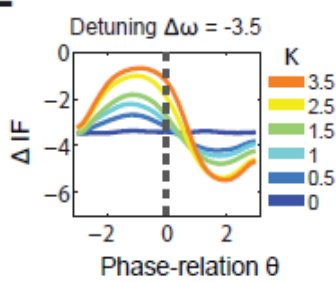

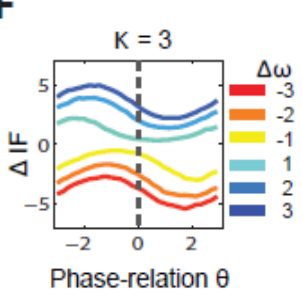

(ii) V1 gamma data

G


H
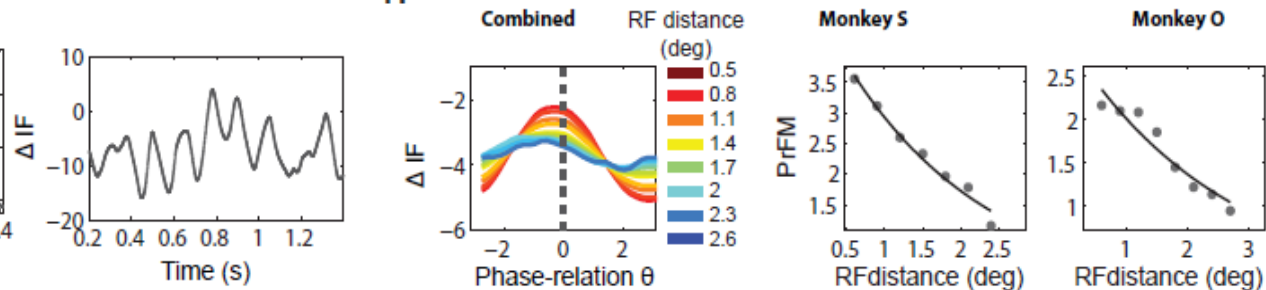

I

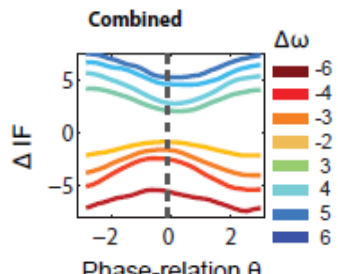



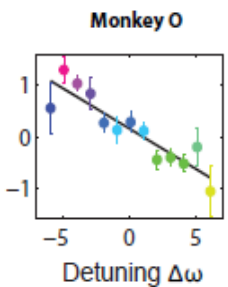

Figure 2: A-F) Predictions from the theory of weakly coupled oscillators. A) The phase-dependent interaction terms, the phase-response curve (PRC). B) The schematic explanation of the synchronization regimes (Pikovsky et al., 2002). The black dot is a 'particle' that follows a 'potential gradient'. C) The (cumulative) phase precession over for different interaction values $\mathrm{k}$ inducing different synchronization regimes $D$ ) The derivation of the phase-relation dependent frequency modulation (PrFM) function. The derivative of the cumulative phase precession is the instantaneous frequency difference ( $\triangle \mathrm{IF}$ ). For a giving contact pair, plotting the $\triangle \mathrm{IF}$ as a function of phase-relation gives PrFM which is closely linked to the underlying PRC. E) The PrFM plotted for different interaction strengths $\mathrm{k}$. The PrFM amplitude scales proportionally to $\mathrm{k}$. F) The PrFM plotted for different detuning $\Delta \mathrm{w}$. The mean of the PrFM shifts in accordance to the detuning. The phase of minimal frequency difference (i.e., closest to zero) shifts with detuning. G-I) Partial synchrony and the derivation of the PrFM (PRC) in V1 gamma synchronization data. G) Example reconstruction of a PrFM from a contact pair similar to D). H) The PrFM plotted as a function of receptive field 
distance (RF) distance. Here combined over the monkeys (PrFM of both monkeys were very similar).Quantifications of the interaction strength (amplitude of the PrFM) plotted as a function of RF distance is shown to the right. I) PrFM plotted as a function of detuning. To the right the quantifications of the phase of minimal frequency difference as a function of detuning are plotted.

For estimating PRC and its parameters in our empirical data recorded in V1, we needed to reconstruct the instantaneous phases of $\mathrm{V} 1$ gamma oscillations. To isolate the gamma oscillation component from the multi-component CSD signal, we applied singular-spectrum decomposition SSD ( Bonizzi, Karel, Meste, \& Peeters, 2014; Pietro Bonizzi et al., 2012), which decomposes a non-linear and non-stationary signal into different spectral components. From the different spectral components, we selected the component containing the gamma-band power. We then applied Hilbert-Transform to compute the analytical signal from which the instantaneous phase could be retrieved. Other methods (band-pass filtering or wavelet-based methods) did not change the results reported in this paper. The derivative of the instantaneous phase gave the instantaneous frequency.

Using this approach, we were able for any given contact pair between two laminar probes to estimate for each time point the instantaneous phase difference as well as the instantaneous frequency difference. In both monkeys, we found a substantial amount of phase-relation dependent (instantaneous) frequency modulation (PrFM), a signature of the partially synchronized state (Pikovsky et al., 2002). In Fig.2G-H we show empirical examples of systematic fluctuations in the instantaneous frequency difference. These fluctuations were locked to the phase-relation. Plotting the instantaneous frequency as a function of phase-relation yielded clear PrFMs. The PrFM shape had a clear peak and through and approximated a sinusoidal shape.

From the PrFM, the critical parameter detuning and interaction strength were estimated for further analysis. According to the definition of the PRC in the context of TWCO, the mean of the PrFM was defined as the experimentally estimated detuning frequency. The half amplitude of the PrFM shape was defined as the experimentally estimated interaction strength.

In Fig. $2 \mathrm{H}$, the population estimate of PrFM over two monkeys is shown (the PrFM between the two monkeys was very similar). It confirmed that the PrFM was approximately sinusoidal and hence we concluded that the PRC of V1 gamma synchronization approximated a unimodal sinusoidal-shaped function. Further, in accordance to theory, we observed that the PrFM amplitude decreased systematically with RF distance $\left(R^{2}=0.41(0.29), p<10^{-10}, n=1116(734)\right)$. This means the larger the distance between V1 gamma oscillations was the lower the amplitude of PrFM. This was expected, because it is known that the synaptic probability among V1 cortical neurons decreases as a function of spatial distance (Stettler et al., 2002). This measure validates that PrFM is indeed reflecting interaction strength among gamma-synchronized neurons. We found further that the phase of minimal frequency difference shifted systematically with detuning $\left(R^{2}=0.21(0.21), p<10^{-10}, n=1116(734)\right.$, Fig.2I) in concordance with the preferred phase-relation. This is because the preferred phase-relation, a compromise of the PRC and the detuning level, is counteracting phase-precession (frequency difference) most strongly. 


\section{Gamma-band phase coordination: Coding of detuning and interaction strength}

Gamma-band phase coding in visual cortex has been reported before (Maris et al., 2013; Vinck et al., 2010). It has been shown that neurons with higher spike rates (manipulated by stimulus orientation) occur at an earlier phase respectively to the gamma field potential with reported shifts of 0.6-0.7rad (Vinck et al., 2010). A central concept in these studies is the 'rate-to-phase transform' (McLelland and Paulsen, 2009) in which neurons with higher input drive spikes earlier, because these neurons overcome earlier the gamma rhythmic inhibition (Fries et al., 2007). To test that hypothesis, it is common practice to relate the spikes to the field potential of the same cortical location. This is an interesting concept, but studies from the hippocampus (McLelland and Paulsen, 2009), auditory (Panzeri et al., 2010) and visual cortex (Montemurro et al., 2008) suggest that the link between higher spike rate and earlier phase does not always apply. The TWCO provides a very different framework to understand gamma phase coding mediated by synchronization. Here, the phase-relations during synchronization among nearby oscillators are regulated by detuning and interaction strength. As we have shown (e.g., see Fig 1G), the detuning is related to the spike rate difference (or the difference in input drive). Hence, the gamma phase-relation between neurons is a dependent on 'rate difference', and not strictly dependent on the absolute rate level. Furthermore, the gamma phase code is sensitive to interaction strength, meaning that for a given detuning or rate difference, the established phase-relation will change with interaction strength. To test the phase-relation predictions form TWCO model, the gamma phase relation among spatially nearby located neurons has to be investigated taking into consideration their detuning as well as their interaction strength. We will first describe the theory in more detail to allow for a better appreciation of the experimental results presented.

In the TWCO model, the phase-relation is a function of the PRC shape at a given level of detuning (intrinsic frequency difference) and interaction strength. Without detuning, for a given interaction strength, the preferred phase relation will be 0 (Fig. 3A). Irrespective of the initial phase-relation, the oscillators will converge to the preferred phase-relation (attractor fixed point). With a specific level of detuning, there is an extra force towards phase precession (gray arrow in Figure 3B), which needs to be counter-balanced. The resulting preferred phase-relation will be a function of the detuning strength and the counter-balancing synchronization force defined by the PRC (Figure. 3B). The stronger the detuning, the larger the shift in the preferred phase relation will be. For a given detuning, it will be the case that lowering interaction strengths, will lead to increasing phase shifts (Fig.3C). The maximum phase shifts are expected to be at $\pm \mathrm{pi} / 2$ at the peak or trough of the PRC sinusoidal function. If the detuning is larger than the interaction strength $(\Delta \mathrm{w}>\mathrm{k})$, than the preferred phase-relation stays at $\pm \mathrm{pi} / 2$. If oscillators experience noise, than the transition between synchrony $(\Delta \mathrm{w}<\mathrm{k})$ and partial synchrony $(\Delta \mathrm{w}>\mathrm{k})$ is smoothed (intermixed). From the model, we derived two critical predictions: 1$)$ The phase-relation between two contact points depends on the detuning (Fig.3D); 2) The slope of the relation between phase relation and detuning systematically decreases (exponentially) with interaction strength (inverse RF distance, Fig.3E). 
(i) TWCO model : Phase relation prediction

A

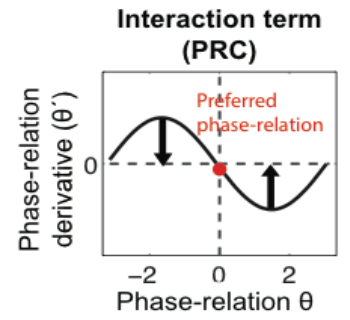

C

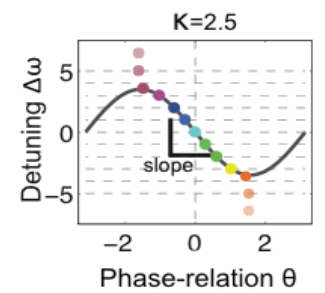

B
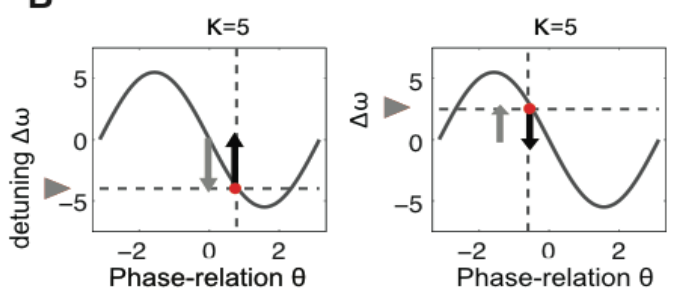

Phase-relation $\theta$

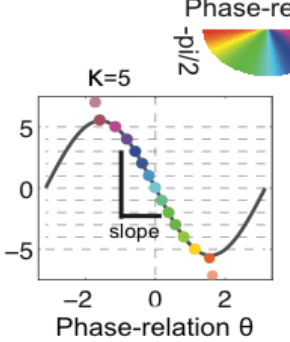

D

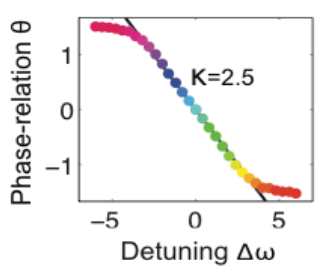

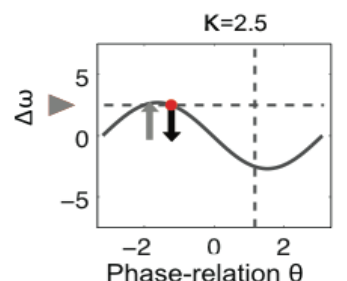

E

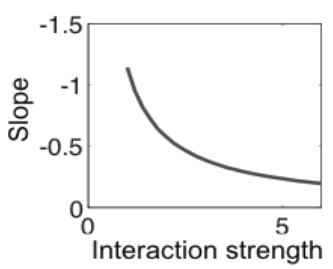

(ii) V1 gamma data

F

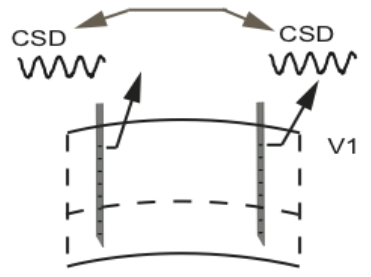

G

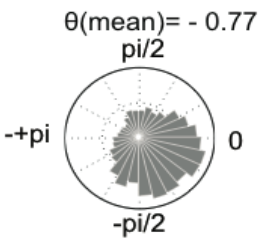

J

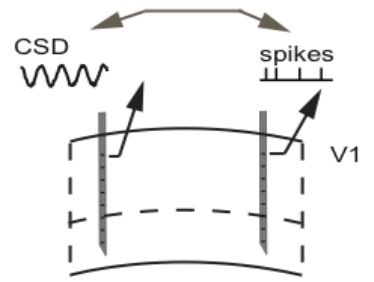

H Phase-relation $\theta$ (CSD-CSD) |
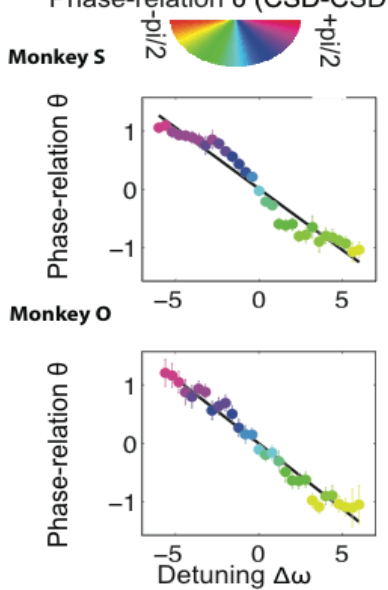

Phase-relation $\theta$ (Spike-CSD)
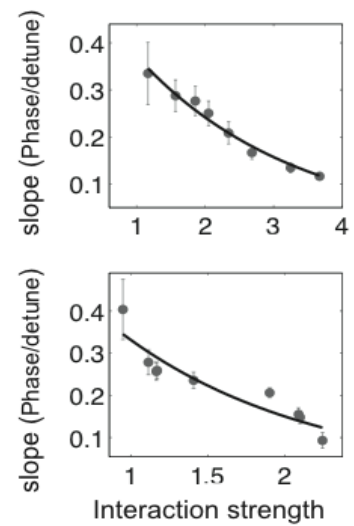

(PrFM)

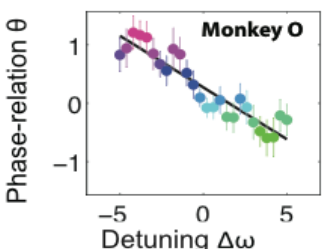

Figure 3: A-E) Predictions from the theory of weakly coupled oscillators (TWCO).A) the phase response curve (PRC) with black arrows show the phase-precession counter-balancing force. B) Detuning $\Delta \mathrm{w}$ leads to an additional force pushing the oscillator for phase precession. This force needs to be counter-balanced. The preferred phase-relation results from the neutralization of the detuning-induced phase-precession force and the PRC synchronization force. C) The preferred phaserelation for different detuning (dashed lines) are plotted along the PRC. Different phase vales are color coded. We show two PRC with different interaction strengths $k$ to stress that the slope of the function phase-relation and detuning scales with $\mathrm{k}$. D) Similar than C) just with inverted axis. E) the quantification of the regression slope as a function of interaction strength $\mathrm{K}$. F-J) V1 gamma synchronization data. F) We computed the mean (instantaneous) phase-relation of the CSD field potential for a given contact pair between two laminar probes. G) Examples of the instantaneous phase-relation distributions. H) The phase-relation 
as a function of detuning $\Delta \mathrm{w}$ plotted for monkey $\mathrm{S}$ and $\mathrm{O}$. I) The regression slope of phase-relation and detuning is plotted as a function of interaction strength (estimated as the amplitude of the PrFM). Notice that the interaction strength scales inversely with receptive field (RF) distance. J) We also computed the phase of the spike-CSD Field locking for a given contact pair from two probes. The preferred phase of spiking is plotted as a function of detuning $\Delta \mathrm{W}$ for monkey $\mathrm{S}$ and $\mathrm{O}$.

To test these model predictions, we computed the phase of the mean resultant vector length of the instantaneous phase differences for a given contact pair (Fig.3F). The results are shown for CSD signals (CSD was used to eliminate volume conduction, see Methods). In both monkeys, we found a strong overall relationship between detuning and phase-relation, where phase-relation shifted from near $-1 / 2 \mathrm{pi}$ to near $1 / 2$ pi $\left(R^{2}=0.4(0.4), p<10^{-10}, n=1116(734)\right.$, Fig.3G). This means that higher detuning levels (intrinsic frequency differences) led to increased offsets of the phase-relation from zero. Notice further that the relation between detuning and phase-relation was not exactly linear, but showed systematic deviations from it similar to those predicted by the TWCO model. Specifically, the non-linearity is in line with the PRC between $-\mathrm{pi} / 2$ and $+\mathrm{pi} / 2$ being a half sinusoid. The slope of the relationship between detuning and phase relationship is predicted to be dependent on interaction strength, here approximated with $\mathrm{RF}$ distance. We found a significant relationship (Fig. $3 \mathrm{H}$ ) in the predicted direction in both monkeys $\left(R^{2}=0.46(0.09), p<10^{-10}(0.03), n=68(41)\right)$. We could replicate the findings from CSD-CSD phase-relations also in spike-CSD phase-relations (Fig.3J)

These results show that 1) gamma phase-relation mapped clearly on the detuning parameter 2) gamma phase-relation was sensitive to interaction strength (spatial distance). The total phase-relation shifts reported here are of $\sim 2.3$ rad in both monkeys.

\section{Gamma-band synchrony is sensitive to detuning and interaction strength}

Stimulus-input dependent phase-locking (or coherence) of gamma oscillation in V1 has attracted large attention across neuroscience community (Eckhorn et al., 2001; Gray and Singer, 1989), because it was proposed to play a role in perceptual grouping or segmentation, and to be a solution to the 'binding problem' (Malsburg, 1995). Several studies have challenged this conception by showing 1) that gammaband phase-locking is local in V1 (corresponding to the extent of horizontal connectivity) and cannot encompass the whole visual object representation (Palanca and DeAngelis, 2005); and 2) that local frequency variation of gamma oscillation might limit functional phase-locking in general (Ray and Maunsell, 2010). The TWCO framework predicts that 1) phase-locking is indeed critically dependent on interaction strength (in V1 corresponding to spatial specificity), but 2) it also predicts that detuning (frequency difference), which is tightly linked to spike rate and sensory drive, is an important regulatory factor for establishing input-dependent gamma phase-locking (Lowet et al., 2015).

The phase-locking strength is a function of detuning and interaction strength. The larger the interaction strength, the larger the detuning value can be under which the oscillator still synchronize (reach a common frequency). If one plots the phase-locking strength in a two-dimensional plot as a function of detuning value and interaction (Fig.4A) one can observe an inverse triangle, the so-called Arnold-tongue (Pikovsky et al., 2002). Within the Arnold tongue $(\Delta \mathrm{w}<=\mathrm{k})$, oscillators are called phase-locked $\left(\varphi^{\prime}=0\right)$ and outside of the Arnold tongue they are not (perfectly) phase-locked $\left(\varphi^{\prime} \neq 0\right)$. 
Here, we will define phase-locking strength in the context of the (instantaneous) phase-relation distribution. We will consider oscillators as phase-locked as long as the phase-relation distribution differs from a uniform distribution (such that a peak in the phase-relation distribution can be determined). We measured the strength of the phase locking using the phase-locking value (PLV) which represents the mean resultant vector length (Lachaux et al., 1999) and quantifies how pronounced the peak is in the phase-relation distribution. This is critical, because in the brain states of intermediate phase-locking strength may also play an important role in synchronization and neural communication. Noise (Fig.4B) narrows the (complete) phase-locking $\left(\varphi^{\prime}=0\right)$ or eliminates it completely. However, the PLV might still reach high values (up to close 1 ) and if one plots the PLV as a function of detuning and interaction, an Arnold tongue can still be observed. We define the Arnold tongue therefore in terms of detuning and interaction strength $(\Delta \mathrm{w}<=\mathrm{k})$ and not in terms of complete phase-locking.

In Fig4C we plot the TWCO prediction of PLV as a function of (absolute) detuning without noise (left) and with noise (right). The PLV decreases as a function of detuning. In the noiseless case, the PLV is $1\left(\varphi^{\prime}=0\right)$ within the Arnold tongue $(\Delta \mathrm{w}<\mathrm{k})$ and rapidly decreases towards to 0 with further detuning. With noise, the maximal PLV reached depended on the precise interaction strength $\mathrm{k}$. The transition from $(\Delta \mathrm{w}<=\mathrm{k})$ to $(\Delta \mathrm{w}>\mathrm{k})$ was more smooth in terms of PLV change. A similar pattern was observed with PLV as a function of interaction strength shown in Fig.4D. The PLV is predicted to increase with interaction strength.

For the experimental V1 gamma data, we computed the PLV on the instantaneous phase-relation distribution for a given contact pair (see examples in Fig.4E). We found that the PLV decreased significantly with absolute detuning value $\left(R^{2}=0.23(0.17), p<10^{-10}, n=1116(734)\right)$ in both monkeys in accordance with theory. Notice that the PLV decreases non-linearly (and more strongly so for higher interaction strengths) indicating that despite the intrinsic (dynamic) noisy nature of V1 gamma oscillations (Xing et al., 2012) and measurement noise, the border of the Arnold tongue could still be observed. We also observed systematic PLV decrease with $R F$ distance $\left(R^{2}=0.28(0.27), p<10^{-10}\right.$, $\mathrm{n}=1116(734)$ ), in agreement with previously published study (Gail, Brinksmeyer, \& Eckhorn, 2000; Ray \& Maunsell, 2010). 
(i) TWCO model : Phase locking prediction

A

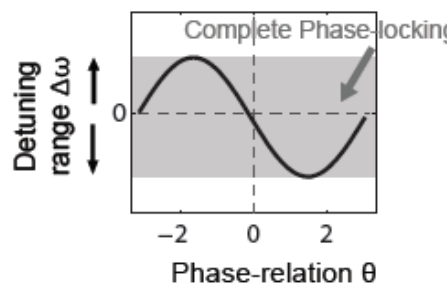

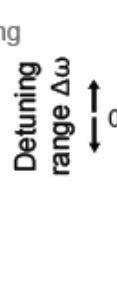

B

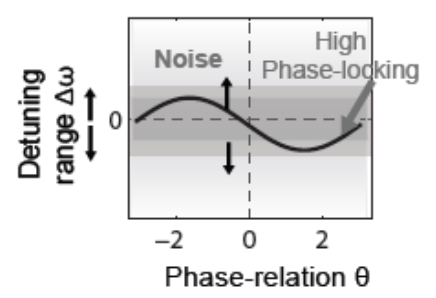

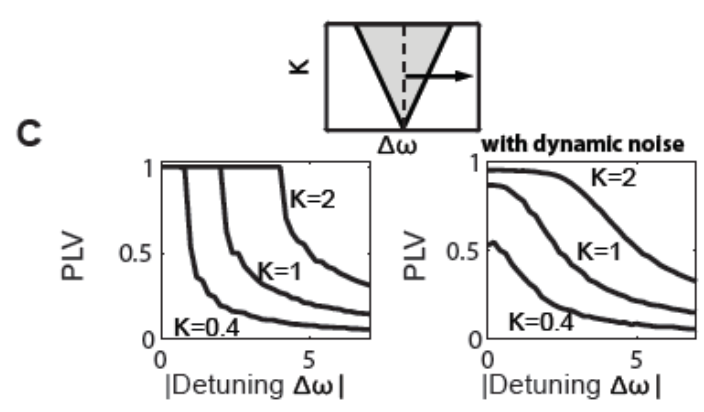

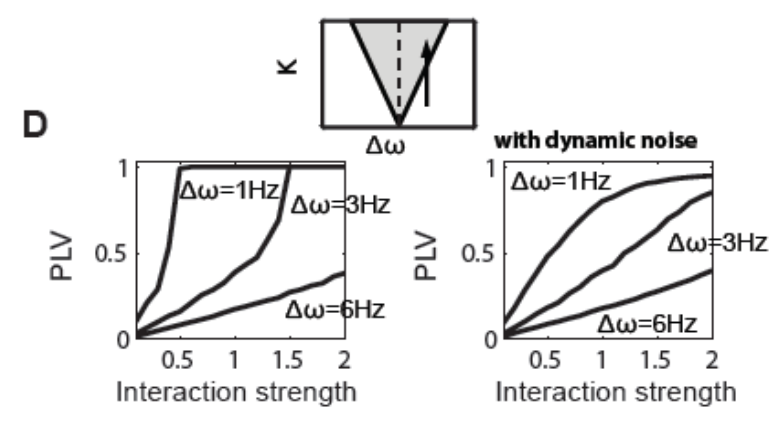

(ii) V1 gamma data (CSD)

E
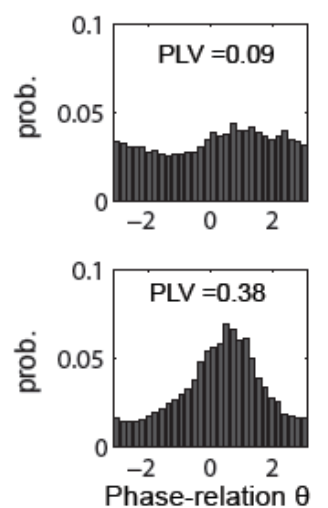

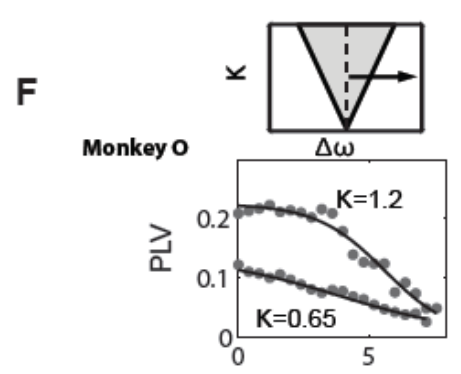

Monkey S

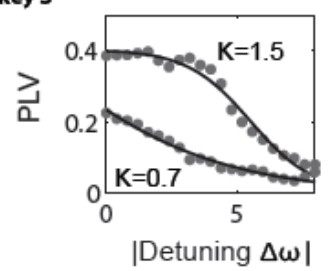

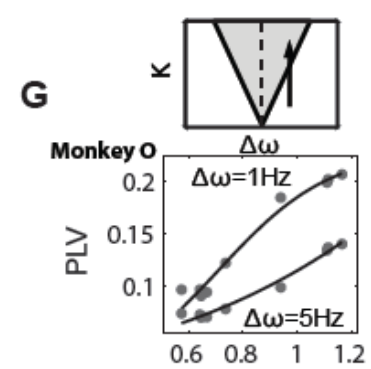

Monkey S

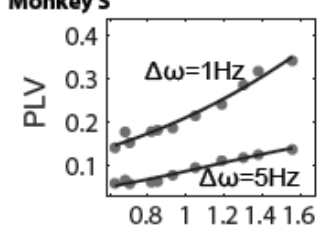

Interaction strength

(PrFM)

Figure 4: A-D) Predictions from the theory of weakly coupled oscillators (TWCO). A) The phase response curve (PRC) with gray area showing level of detuning range where the (noiseless) oscillator still synchronize completely $\left(\theta^{\prime}=0\right)$. The larger the interaction strength (amplitude of the PRC), the larger the detuning range. If the synchronization range is plotted as a function of detuning $\Delta \mathrm{w}$ and interaction strength $\mathrm{k}$, then one observes the Arnold tongue. B) Similar to A), but with noisy oscillators. Here the border between high-locking and low locking is smoothed. Further, complete phase-locking $\left(\theta^{\prime}=0\right)$ is not possible, yet high phase-locking (phase-locking value PLV approximating 1 ) is still possible. C) The PLV (mean resultant vector length) as function of absolute detuning $|\Delta w|$ is shown for noiseless case (left) noisy case (right). Different lines represent different interaction strengths. D) Same as in C), but here as a function of interaction strength $\mathrm{K}$. Different lines represent different detuning values. E-G) V1 gamma synchronization data. E) Examples of instantaneous phase-relation distribution are shown with the resultant PLV values. F) The PLV as a function of absolute detuning $|\Delta w|$ is shown for two different interaction strength values (estimated by half PrFM amplitude). G) Similar than $\mathrm{H}$ ), but as a function of interaction strength. 
These results demonstrate that gamma phase-locking strength between nearby V1 locations, as measured by PLV, depends critically on both interaction strength (RF distance) and detuning (spike rate/contrast difference). Although our results confirm the finding that gamma phase-locking is local in V1 and dependent on connectivity (Gail et al., 2000; Palanca and DeAngelis, 2005; Ray and Maunsell, 2010), they on the other hand also support the view that gamma-band phase-locking allows for input(detuning)-dependent phase-locking (Eckhorn et al., 2001; Gray and Singer, 1989) in which frequency variation is critical. The TWCO therefore provides an exact mathematical framework to improve our understanding of neural synchronization phenomena in neural networks, and permits making precise predictions to investigate functional phase-locking among nearby visual cortical neurons in the gamma range.

\section{Gamma-band synchronization: The reconstruction of the Arnold tongue}

Using both parameter dimensions 'detuning' (manipulated by local stimulus contrast and eccentricity of the recording probe) and 'interaction strength' (manipulated by RF distance), we reconstructed the Arnold tongue (combined over both monkeys) in terms of phase-locking PLV and phase-relation. This is, to our knowledge, the first time that the Arnold tongue has been reconstructed from in-vivo cortical data, delineating the conditions limiting gamma synchronization, as predicted by the TWCO model.

\section{DISCUSSION}

The gamma synchronization data from macaque visual area V1 are in agreement with the predictions derived from the theory of weakly coupled oscillators (TWCO). The TWCO is exemplified in the so-called Arnold tongue, which represents the synchronization region within a two-dimensional space of detuning and interaction strength. As a demonstration of the agreement between theory and data, we could reconstruct the Arnold tongue from our V1 neural recordings. This suggests that gamma-range synchronization integrates information from (sensory) input drive as well as integration strength (presumably mediated by synaptic connectivity). 
(i) TWCO model : Arnold tongue

A

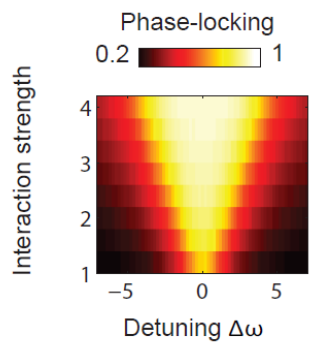

B

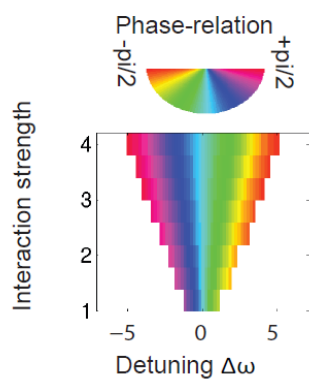

(ii) V1 gamma-band data: Arnold tongue

C

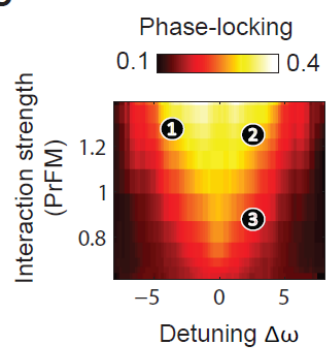

D

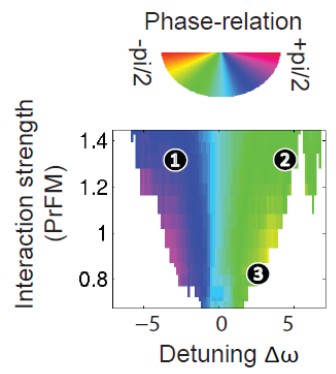

E
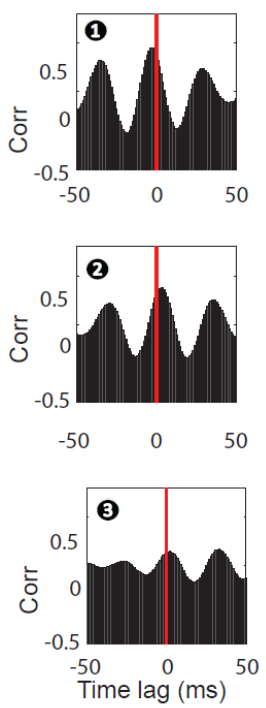

Figure 5: A-B) Predictions from the theory of weakly coupled oscillators (TWCO). A) The Arnold tongue as predicted of the TWCO (noisy oscillators) where hot color represents high PLV between the oscillators. The Y-axis is interaction strength (with top high and bottom low). The X-axis is detuning parameter between the two oscillators. B) The mean phase-relation (in HSV color) is plotted onto the Arnold tongue (PLV $>0.3$, arbitrary threshold and for illustration purposes only). C-E) V1 gamma synchronization data. C) The Arnold tongue reconstruction where hot color represent high PLV between V1 cortical locations. The $\mathrm{Y}$-axis is interaction strength (as estimated by half PrFM amplitude). The interaction is inversely related to RF distance. The $\mathrm{X}$-axis is detuning parameter (estimated as the mean of PrFM). D) The Arnold tongue reconstruction with HSV-color and same axis definition as in C. E) Three examples (1-3) where the cross-correlation is shown between two V1 location CSD SSD gamma signals (See Methods for Details). The positions of the examples are depicted on the Arnold tongues of C and D as small numbers.

The Arnold tongue captures the fundamental mathematical rules that determine how nearby cortical neurons will synchronize. This has important implications for the structure of spatio-temporal spiking patterns, which in turn are critical for understanding neural computation and plasticity (Brette, 2012; Dan and Poo, 2004; Ermentrout and Kleinfeld, 2001; Heitmann et al., 2013; Hinton, 2000; Hopfield and Brody, 2001; London and Häusser, 2005; Masquelier et al., 2009; Ritz and Sejnowski, 1997; Salinas and Sejnowski, 2001; Singer, 1999; Tiesinga et al., 2008). Moreover, we report for the first time in visual cortex that gamma synchronization operates mainly in the partially (intermittent) synchronized regime (Ahn et al., 2011; Gong et al., 2007; Kozma and Freeman, 2008). By using the synchronization-induced instantaneous frequency fluctuations associated with the partial synchronization regime, we could retrieve and quantify the underlying phase-response curve (PRC). The PRC is the critical interaction function underlying the synchronization characteristics (Schwemmer and Lewis, 2012).

In line with a previous study in macaque visual cortex ( Vinck et al., 2010), input variation was translated into a gamma phase code. The phase shifts reported in this study are larger ( $\sim 140$ degrees) compared to the previous study ( 40-50dergree, (Vinck et al., 2010)). In agreement with TWCO, we showed that phase-relations among nearby neurons are critically linked to their detuning (intrinsic frequency 
difference) in the gamma range. In addition, we confirmed reports of phase shifts depending on cortical distance (Gail et al., 2000; Vinck et al., 2010), which were accounted for by the vertical (interaction strength) dimension of the Arnold tongue (Tiesinga and Sejnowski, 2010). Specifically, we showed that the translation (slope) of input variation (detuning) to phase decreased with interaction strength (inversely related to receptive field distance). Overall, the results suggests that the gamma phaserelation code among nearby neurons is not a rate-to-phase transform (McLelland and Paulsen, 2009), but a 'rate-difference to phase-difference transform' and hence might represent a fundamentally different form of phase coding than reported for slower oscillations (Buzsáki, 2002; McLelland and Paulsen, 2009). In accordance, shifts in the overall drive to a gamma network only weakly changed the preferred phase of neurons (Ray and Maunsell, 2010), yet changed the preferred oscillation frequency instead. Hence, gamma phase mainly represents a 'relational code' (Singer, 1999).

We also showed that the phase-locking strength (measured by PLV) was sensitive to detuning (and visual contrast differences). This indicates that gamma synchronization is sensitive to input/feature similarity (Eckhorn et al., 2001; Singer and Gray, 1995), and this supports the notion that gamma synchronization might be implicated in functional grouping/clustering of local visual cortical activity (Burwick, 2007; Eckhorn, 1999; Kuntimad and Ranganath, 1999; Wang and Terman, 1997) at the level of horizontal connectivity spread/surround receptive fields (Boucsein et al., 2011; Palanca and DeAngelis, 2005; Stettler et al., 2002). Note that this potential clustering function of gamma synchronization is a fundamental consequence of the fact that gamma frequency is input-dependent (Lowet et al., 2015), in contrast to a previous suggestion that the stimulus dependency of gamma would be detrimental to its assumed role in neural communication (Ray and Maunsell, 2010).

Our empirical results suggest that efficient gamma synchronization and phase coding in V1 will occur mainly locally and will be limited to input spatial patterns of low heterogeneity (low detuning) and hence not all input patterns occurring during visual natural processing might induce the same amount of gamma synchronization (Brunet et al., 2013; Hermes et al., 2014; Kayser, 2003). The exact spatial extent

and shape of spatial gamma synchronization and phase-relation distributions (also described as traveling waves in the spatial dimension) can be better understood within the TWCO framework (Ermentrout and Kleinfeld, 2001).

In conclusion, our results indicate that gamma synchronization among visual cortical neurons, operating in a partial synchrony regime, can support meaningful coordination of neural activity. During gamma synchronization, information from input drive (intrinsic frequency) is integrated with effects of connection strength and hence, in principle, gamma is well positioned to contribute to neural information processing and plasticity.

\section{Methods}

\section{Surgical procedures}

Two adult male rhesus monkeys (Macaca mulatta) took part in this experiment. Two chambers were implanted above early visual cortex, one positioned over V1/ V2 and the second over V4. For the 
experiment reported here we used data from V1/V2 chamber only. A head post was implanted to headfix the monkey during the experiment. All the procedures were in accordance with the European council directive 2010/63/EU, the Dutch 'experiments on animal acts' (1997) and approved by the Radboud University ethical committee on experiments with animals (Dier-Experimenten-Commissie, DEC).

\section{Recording techniques}

V1 recordings were made with Plexon U-probes (Plexon Inc.) consisting of 16 contacts $(10 \mu \mathrm{m}$ diameter, $0.5-1 \mathrm{~m} \Omega$ impedance, and $150 \mu \mathrm{m}$ inter-contact spacing). Three probes were inserted through a sharp guide tube, which was lowered through granulation tissue to just above the level of the dura surface. The probes were arranged in a linear manner separated from each other by $1-3 \mathrm{~mm}$. The probes were then advanced by a separate microdrive (Nan Instruments LTD.). The probes were connected to headstages of high input impedance, and data were acquired via the Plexon 'Multichannel Acquisition system' (MAP, Plexon Inc.). The measured extracellular signal was filtered online between $150 \mathrm{~Hz}$ and $8 \mathrm{kHz}$ to extract spiking activity and filtered between $0.7 \mathrm{~Hz}$ and $300 \mathrm{~Hz}$ to obtain the 'local field potential' (LFP). The signal was amplified and digitized with $1 \mathrm{kHz}$ for the LFP and $40 \mathrm{kHz}$ for the spike signal.

\section{Receptive field mapping}

Receptive fields (RFs) were mapped by presenting high contrast black and white squares at pseudorandom locations on a $10 \times 10$ grid while monkeys fixated centrally on the screen. Square size was adjusted between $1^{\circ}$ and $0.1^{\circ}$ to optimally map concurrently recorded RFs (150ms presentation time, $150 \mathrm{~ms}$ inter-stimulus interval). We used two approached to define RFs. The first was based on the multiunit spike rate response. We computed a relative spike rate response, where the max rate response $+40-70 \mathrm{~ms}$ after stimulus onset divided by the mean response during the time window $-100-0 \mathrm{~ms}$ before stimulus onset. In a second approach we used CSD signals filtered in the broad gamma range $(30 \mathrm{~Hz}-$ $200 \mathrm{~Hz}$ ). A relative response was computed with the same time-windows as defined above. Both approaches results in very similar RFs. However, the CSD-approach gave robust RFs in sessions with noisy spiking channels. Therefore, if spiking responses were not reliable enough to determine clear RFs, we chose the second approach for compensation

\section{Visual stimulation and experimental conditions}

The monkeys were head-fixed and placed in a Faraday-isolated darkened box at a distance of $57 \mathrm{~cm}$ from a computer screen. Stimuli were presented on a Samsung TFT screen (SyncMaster $940 \mathrm{bf}, 38^{\circ} \times 30060 \mathrm{~Hz}$ ). The screen was calibrated to linearize luminance as function of RGB values. During stimulation and prestimulus time the monkey maintained eye position (measured by infra-red camera, Arrington $60 \mathrm{~Hz}$ sampling rate) within a square window of $2 \times 2^{\circ}$. This window was relatively large to allow for noise associated with the camera. The monkey was rewarded if keeping gaze within the eye window during the whole trial. Stimuli were whole-screen square-wave gratings which entailed spatial modulation of contrast. The spatial modulation of contrast $\mathrm{C}$ was defined in Monkey $\mathrm{S}$ and $\mathrm{O}$ as:

$$
\complement(x)=C_{m}+S\left(2 A F\left(\left|\bmod \left(x-x_{c}, S c\right)-\frac{S c}{2}\right|\right)+\frac{A}{2}\right)
$$


with $\mathrm{x}$ being the spatial variable in visual degrees and $\mathrm{x}_{\mathrm{c}}$ being the center point. The spatial modulation is defined by the 'modulation sign' $\mathrm{S}$ and the 'contrast modulation amplitude' $\mathrm{A}$. We had five different contrast modulation values $( \pm 23 \%, 17 \%, 12.2 \%, 6.8 \%, 0 \%$ Michelson luminance contrast). The modulation amplitude was defined how strong the modulation of contrast is over spatial distance and the direction indicates whether the modulation is positive or negative to symmetrically modulate contrast around the middle contrast value $\mathrm{C}_{\mathrm{M}}(39.2 \%$ Michelson luminance contrast). $\mathrm{F}$ is a particular modulation function which we tested. It was either linear ( $F=1$, Monkey 0.$)$, logarithmic ( $F=\log$, Monkey S) or exponential ( $F=e x p$, Monkey S). We explored different modulation functions in order to find the best method to achieve the desired modulations of local gamma frequency, however the particular modulation function used in each session was not relevant for the theoretical and experimental results described here and all three types gave same conclusions. Sc defines the spatial scale of the modulation function (the spatial frequency). The spatial scale Sc differed between sessions to fit the modulation to the receptive distances of the three probes. For example, if the outer probes had a RF distance of 3deg, then the contrast modulation was set such that the trough and peak of the modulation function was separated by $3 \mathrm{deg}$. If the RF distance was $2 \mathrm{deg}$, then the spatial contrast modulation was changed in accordance. We also included 4 gratings with four different spatially constant contrast $(21 \%, 35 \%, 43 \%$, $62 \%$ Michelson luminance contrast). The stimuli were phase-jittered trial-by-trial.

\section{Determination of the contrast value observed by a receptive field}

To estimate the contrast value for a given laminar probe we first estimated the receptive field (RF) of the probe. We then estimated the center and width of the RF (see above). Given the center and width, we computed the mean of contrast values, defined by the contrast modulation function, within the RF.

\section{Current source density (CSD)}

The laminar current source density (CSD)(Pettersen et al., 2011) was computed as a second spatial derivative

$$
\frac{\vartheta^{2} \varphi}{\vartheta z^{2}}=\frac{\phi_{(\mathrm{z}+\mathrm{n} \Delta \mathrm{z})}-2 \phi_{(\mathrm{z})}+\phi_{(\mathrm{z}+\mathrm{n} \Delta \mathrm{z})}}{(\mathrm{n} \Delta \mathrm{z})^{2}}
$$

The LFP signals were spatially (zero-phase) filtered with a Gaussian kernel of $s d=1$. We used the 'Vakninapproach' to approximate the CSD for the outer channels(Vaknin et al., 1988).

\section{Laminar alignment and assignment}

Each recording day we inserted the three laminar probes. The exact laminar positions of the three probes differed within and between sessions and hence we depth-aligned the probes based on their stimulus-evoked response and phase-coupling characteristics.

For depth-alignment (to assign each contact a particular cortical depth value) we chose the following procedure: 
1. We computed the CSD-evoked response profile to checkerboard flash stimuli (Roberts et al., 2013).

2. The different sink-source profile (time-window) were aligned using a parallel-tempering technique(Frenkel and Smit, 2001). Parallel-tempering minimized the error measure defined as follows(Godlove et al., 2014) :

$$
\varepsilon(\Delta)=\sum_{i=1}^{N-1} \sum_{j=j+1}^{N} \frac{1}{\alpha_{\Delta(i, j)}} \sum_{d}^{n d} \sum_{t}^{n t}\left(C S D_{i, d+\Delta(i), t}-C S D_{j, d+\Delta(j), t}\right)^{2}
$$

3. We the computed the within laminar probe coherence(Carter et al., 1973) matrix. It has been shown that there is sharp decrease in coherence around the L4/L5 border(Maier et al., 2010). We chose this to refine the depth alignments of step 2 using the coherence matrix and again parallel tempering with the initial values defined by the output of step 2 . An advantage of the coherence matrix is that it is a robust feature and insensitive to possible gain differences among contacts.

4. After step we had a manual checking for outliers. If outliers were found, the matching was done by eye (using CSD response profile, coherence matrix, dura-cortex border, V1-V2 border).

\section{Definition of the V1-White Matter - V2 border}

The depth probes often collected signals beyond the lower V1 layer 6 border and often reached the deep V2 infragranular layers. When the probes reached deep V2 the RFs shifted abruptly several degrees as expected form V1-V2 retinotopy (Gattass et al., 1981). The white matter situated between the two areas were relatively small, often comprising 1-2 contacts (150-300microns).

To estimate the lower V1 Layer 6 boundary, we first used spiking RFs to determine the transition. We computed an Rf center distance, referenced to L4-15 border, measure to determine at which contact the transition to deep V2 occured. Before the transition, often 1 or 2 contacts did not show spike RFs at all (White matter). V1 Layer 6 border was then defined as the contact with the last low RF center distance (threshold $<0.5 \mathrm{deg}$ ).In probes with low spiking quality, we used CSD signals (filtered in the gamma range $(30-150 \mathrm{~Hz})$ for determining the V1 L6 border.

\section{Singular spectrum decomposition (SSD)}

Singular spectrum decomposition (SSD) is a recently proposed method for decomposition of nonlinear and non-stationary time series (Bonizzi et al., 2014; Bonizzi et al., 2012). Here, the method is applied to reduce the influence of noise and to extract the gamma oscillation component form the LFP or CSD signals in a data-driven manner. SSD provides an estimate that unlike an FFT-based one is able to handle non-stationary signals. Similar to empirical mode decomposition (EMD) (Huang, 2005), the decomposition is based on the extraction of the energy associated with various intrinsic time scales. One advantage of SSD over EMD is that it tends to avoid mixing components with different frequency bands and provides accurate separation between intermittent components at the transition points (Bonizzi et al., 2014). The interested reader is referred to (Bonizzi et al., 2014) for additional details. We selected the SSD-component with the spectral power in the gamma frequency range between $25 \mathrm{~Hz}$ and $60 \mathrm{~Hz}$.

\section{Hilbert-Huang transform}

The Hilbert-Huang transform (HHT, (Huang et al., 1998)) is a method to obtain instantaneous frequency data, suitable for the analysis of nonlinear and non-stationary time series. EMD is applied to a time 
series to decompose it in intrinsic mode functions (IMFs), simple amplitude and frequency modulated components with only one instantaneous frequency per instant. Hilbert spectral analysis is then applied to each IMF to obtain its instantaneous frequency. To achieve that, the analytic signal of the IMF is obtained $\left(\mathrm{IMF}_{\mathrm{a}}\right)$, given by:

$$
\mathrm{IMF}_{a}=\mathrm{IMF}+i \mathrm{HT}(\mathrm{IMF})
$$

where HT(IMF) is the Hilbert-Transform of the IMF component, and the instantaneous frequency $\omega$ is computed as the derivative of the phase of the analytical signal:

$$
\theta=\arg \left(\mathrm{IMF}_{a}\right) \text { and } \frac{d \theta}{d t}=\omega
$$

The Hilbert spectrum can be used on SSD components when interpreting its outcome with caution. Indeed, SSD-components contain several frequencies, with no clear indication about how many instantaneous frequencies per time instant may be present. Hence, they cannot be supposed to be IMFs. However, the narrow-banded frequency content of each SSD-component allows considering the results of the Hilbert spectrum as sufficiently reliable under most conditions (Bonizzi et al., 2014).

\section{Phase-locking and phase relation estimations.}

The phase relation was defined as the mean circular phase difference between two signals (averaged in the complex domain).

$$
\theta_{i j}=\arg \left(\frac{1}{N} \sum_{t=1}^{N} \exp \left(i\left(\varphi_{i}-\varphi_{j}\right)\right)\right.
$$

with a range of $[-\pi, \pi]$. Arg is the argument function and $\varphi$ is the IP. For estimating phase-locking we computed the phase-locking value (PLV, (Lachaux et al., 1999)). The PLV was computed by averaging the complex values with unit amplitude

$$
\psi_{i j}=\mid \frac{1}{N} \sum_{t=1}^{N} \exp \left(i\left(\varphi_{i}-\varphi_{j}\right) \mid\right.
$$

The PLV ranges from 1, corresponding to full phase consistency, to 0 , corresponding to fully random.

\section{Estimation of instantaneous frequency}

The instantaneous frequency (IF) can be easily determined as the derivative of the instantaneous phase. The phases need to be unwrapped before applying the derivative. However, the IF might exhibit strong outliers if the signal is noisy or having multi-components. We used therefore the Savitzky-golay filter (Schafer, 2011) to smooth the phase trajectory (and hence the IF) using a polynomial fitting approach. 


\section{Partial synchrony and phase-relation dependent frequency modulation (PrFM)}

If oscillators are not completely synchronized (phase locking of 1), yet not asynchronous, oscillators are in the partially synchronized state, where they exhibit phase precession (non-zero frequency difference). Synchronization will try to counter-act the phase precession by either accelerating or decelerating the precession depending on the form of the phase-response curve. Hence, phase-relation depended instantaneous frequency modulations are expected from synchronization theory. For quantify strength of phase-relation dependent frequency modulation (PrFM) we first computed for each phase-relation bin (bin size $=0.1 \mathrm{rad}$ ) the mean frequency difference for a contact pair. The (instantaneous) frequency differences were rectified to avoid cancellation of fluctuations around zero (which is a problem for small detuning). This resulted in a phase-relation dependent mean frequency difference trajectory. For each contact pair, the (min-max) $/ 2$ gave the amplitude of the modulation.

\section{Estimation of detuning frequency (intrinsic frequency)}

The intrinsic frequency, the frequency an oscillator would have without interactions with other oscillators, could here not be directly experimentally measured. However, the intrinsic frequency can be approximated from the phase-relation dependent instantaneous frequency (PrFM) fluctuations. If there are no interactions among oscillators, the measured frequency is equal to the intrinsic frequency. However, if the oscillators interact, the instantaneous frequency (IF) will fluctuate as a function of the phase-relation. At the preferred phase-relation the IF difference between oscillators is minimal, whereas at the anti-preferred phase it is maximal. If the oscillators interact, the preferred phase-relation will be more likely to occur and the mean measured frequency will be mostly affected by the frequencies occurring during the preferred phase. To derive the intrinsic frequency, we first assumed that the phaseresponse curve (describing the modulation as a function of phase-relation) was symmetric and sinusoidal, which appeared to be a good approximation for the observed modulations. For each phaserelation bin we estimated the mean frequency. The intrinsic frequency was then obtained by computing the mean over the phase-relation depended mean frequencies. In the Hodgkin-Huxley network this method could correctly estimate the intrinsic frequency. The detuning parameter was taken as the difference between estimated intrinsic frequencies.

\section{Phase-oscillator model (noiseless case)}

An oscillator is defined here as a periodic process that is defined by its phase $\theta$, frequency $\omega$ and amplitude $A$. If amplitude is unity and the oscillator is unperturbed the evolution of an oscillator can be defined as

$$
\frac{d \varphi_{X}(t)}{d t}=\omega_{X}(t)
$$

where the phase of a oscillator $X$ is defined by its (intrinsic) frequency. In case of two interacting oscillators $X$ and $Y$, the exact phase trajectory of oscillator $X$ and $Y$ depends on the interaction term.

$$
\frac{d \varphi_{X}(t)}{d t}=\omega_{X}(t)+\kappa_{\mathrm{X}, \mathrm{Y}}\left(\sin \left(\varphi_{Y}(t)-\varphi_{X}(t)\right)\right)
$$


The phase of oscillator $\mathrm{X}$ (or $\mathrm{Y}$ ) is determined by an intrinsic (natural) frequency $(\omega)$ and an interaction term that describes the phase adjustments (phase response curve, PRC) induced by the other oscillator $Y$ (or $\mathrm{X}$ ) depending on the coupling constant $\mathrm{k}\left(\kappa_{\mathrm{X}, \mathrm{Y}}\right.$ or $\left.\kappa_{\mathrm{Y}, \mathrm{X}}\right)$. As the interaction term we used a sinusoidal function with an attractor fixed point (in-phase) and repeller fixed point (anti-phase) (Kuramoto model, (Breakspear et al., 2010)). It has been shown that the evolution of the phase relation between the two oscillators can be described with a single equation, referred to as the Adler equation (Pikovsky et al., 2002):

$$
\begin{gathered}
\frac{d \theta}{d t}=\Delta \omega+\kappa^{*} \sin (\theta) \\
\theta=\varphi_{Y}-\varphi_{X}
\end{gathered}
$$

The equation shows that the time evolution of the phase relation $\theta(t)$ is a function of the frequency difference $\Delta \omega(t)$ at that time, and the coupling strength $\kappa$ of the sinusoidal interaction function. We show below how the phase-locking strength between two coupled phase-oscillators can be derived.

\section{Deriving phase-locking value from two coupled phase-oscillators}

In this study we used a commonly applied measure to test for a non-uniform phase-relation distribution which is defined as the mean vector length, the phase locking value (PLV, (Lachaux et al., 1999)).

$$
\operatorname{PLV}=\left|\frac{1}{N} \sum_{t=1}^{N} \mathrm{e}^{\mathrm{i} \theta(\mathrm{t})}\right|
$$

where $N$ is number of sample points and $t$ is the time variable. If the phase-relation distribution is uniform, the PLV will give a value of 0 (in practice, due to finite data, PLV will be always slightly larger than zero), while if there is a particular peak in the distribution, the PLV will give a value $>0$. There are also different measures that might be more favorable (Tort et al., 2010), yet we used PLV here because it is currently a widely used measure (in addition to coherence) in neuroscience. The PLV characteristics described in the present study are also valid for coherence.

The true phase locking value $\mathrm{PLV}_{\mathrm{T}}$ between two coupled oscillators is related in a simple manner to the sinusoidal coupling function defining the PRC (Hoppensteadt and Izhikevich, 1998). If the detuning $|\Delta \omega|$ is smaller or equal than the amplitude of the coupling function $\kappa$ then the phase locking value is 1 . This is the case when full synchrony among oscillators is achieved. In this case, the instantaneous phase relation is constant over time and no phase precession is present. This is because the detuning $\Delta \omega$ and the associated tendency to phase precess, cannot overcome the 'attractor' defined by the PRC with the strength $\kappa$. The true phase locking value $\mathrm{PLV}_{\mathrm{T}}$ then equals to:

$$
\mathrm{PLV}_{\mathrm{T}}=1 \quad \text { if }|\Delta \omega| \leq \kappa \forall t>t_{0}
$$


However, in the case in which frequency detuning $\Delta \omega(t)$ is stronger than the coupling strength ( $\kappa: \Delta \omega(t)$ $>\kappa)$, the attractor is not strong enough to prevent phase precession. Hence, full synchrony is no longer possible. Yet, the phase relation distribution is not uniform and oscillators are still more likely to be in their preferred relationship. This is because the rate of phase precession (or instantaneous frequency difference) changes as a function of phase-relation. When the oscillators are at their preferred relationship, the phase precession speed is minimized (the point of the PRC with the strongest phase adjustment opposite to the phase precession), whereas at the non-preferred phase-relation, the phase precession speed is maximal. The modulation of the instantaneous phase relation leads to non-uniform phase-relations and thus to phase-locking of $>0$.

For deriving the $\mathrm{PLV}_{\mathrm{T}}$ for $|\Delta \omega|>k$, we computed the resultant vector length of the phase-relation probability density distribution (pdf), which can be derived from the Adler equation (see equation 3 ) that describes the time derivative of the phase-relation as a function of the phase-relation and a frequency difference constant. Notice that the time derivative of the phase-relation is the instantaneous frequency difference. In the case of $|\Delta \omega|>k$ the time derivative is always non-zero (no fixed point). To derive the pdf, we first rearranged the terms of equation 3 to

$$
d t=\frac{1}{\Delta \omega+\kappa \sin (\theta)} d \theta
$$

where the time derivative is a function of phase derivative and the inverse of the Adler function. Integrating this function over the phase space gives the characteristic time period $\mathrm{T}$ for which the oscillators need to make a full phase precession (Pikovsky et al., 2002). Because the (noiseless) oscillators repeat exactly the same patterns at intervals $\mathrm{T}$, considering only period $\mathrm{T}$ is sufficient for the full determination of the pdf.

$$
T=\int_{0}^{2 \pi} \frac{1}{\Delta \omega+\kappa \sin (\theta)} d \theta
$$

It can be easily seen that integrating around a small phase interval, divided by $\mathrm{T}$, gives the probability value (the amount of time the phase-relation $\theta$ spent in the phase interval $[a, b]$ within the time scale T).

$$
\operatorname{Pr}[a \leq \theta \leq b]=\frac{1}{T} \int_{a}^{b} \frac{1}{\Delta \omega+\kappa \sin (\theta)} d \theta
$$

The inverse of the Adler function therefore describes the probability density function.

$$
p d f(\theta)=\frac{1}{\Delta \omega+\kappa \sin (\theta)}
$$


To compute the true phase-locking value $\mathrm{PLV}_{\mathrm{T}}$, one needs to integrate over all the phases with their associated probability densities.

$$
\operatorname{PLV}_{T}=\left|\frac{1}{T} \int_{0}^{2 \pi} p d f(\theta) e^{i \theta} d \theta\right| \quad \text { if } 0\langle\kappa\langle|\Delta \omega|
$$

Complete asynchrony is only possible when the oscillators are not coupled and hence there are no phase-relation dependent modulations of phase precession speed ( $p d f$ is described by a constant).

$$
\mathrm{PLV}_{T}=0 \quad \text { if } \kappa=0,|\Delta \omega|>0
$$

\section{REFERENCES}

Ahn, S., Park, C., Rubchinsky, L.L., 2011. Detecting the temporal structure of intermittent phase locking. Phys. Rev. E 84, 016201. doi:10.1103/PhysRevE.84.016201

Angelucci, A., Bullier, J., 2003. Reaching beyond the classical receptive field of V1 neurons: Horizontal or feedback axons? J. Physiol. Paris. doi:10.1016/j.jphysparis.2003.09.001

Bonizzi, P., Karel, J., De Weerd, P., Lowet, E., Roberts, M., Westra, R., Meste, O., Peeters, R., 2012. Singular spectrum analysis improves analysis of local field potentials from macaque $\mathrm{V} 1$ in active fixation task. Conf. Proc. ... Annu. Int. Conf. IEEE Eng. Med. Biol. Soc. IEEE Eng. Med. Biol. Soc. Annu. Conf. 2012, 2945-8. doi:10.1109/EMBC.2012.6346581

Bonizzi, P., Karel, J.M.H., Meste, O., Peeters, R.L.M., 2014. Singular spectrum decomposition: A new method for time series decomposition. Adv. Adapt. Data Anal. 1450011. doi:10.1142/S1793536914500113

Boucsein, C., Nawrot, M.P., Schnepel, P., Aertsen, A., 2011. Beyond the cortical column: abundance and physiology of horizontal connections imply a strong role for inputs from the surround. Front Neurosci 5, 32. doi:10.3389/fnins.2011.00032

Breakspear, M., Heitmann, S., Daffertshofer, A., 2010. Generative models of cortical oscillations: neurobiological implications of the kuramoto model. Front Hum Neurosci 4, 190. doi:10.3389/fnhum.2010.00190

Brette, R., 2012. Computing with neural synchrony. PLoS Comput. Biol. 8, e1002561. doi:10.1371/journal.pcbi.1002561

Brunet, N., Bosman, C. a, Roberts, M., Oostenveld, R., Womelsdorf, T., De Weerd, P., Fries, P., 2013. Visual Cortical Gamma-Band Activity During Free Viewing of Natural Images. Cereb. Cortex 1-9. doi:10.1093/cercor/bht280

Burwick, T., 2007. Oscillatory neural networks with self-organized segmentation of overlapping patterns. Neural Comput. 19, 2093-2123. doi:10.1162/neco.2007.19.8.2093

Buzsáki, G., 2002. Theta oscillations in the hippocampus. Neuron. doi:10.1016/S0896-6273(02)00586-X 
Carter, G., Knapp, C., Nuttall, A., 1973. Estimation of the magnitude-squared coherence function via overlapped fast Fourier transform processing. IEEE Trans. Audio Electroacoust. 21. doi:10.1109/TAU.1973.1162496

Dan, Y., Poo, M.M., 2004. Spike timing-dependent plasticity of neural circuits. Neuron. doi:10.1016/j.neuron.2004.09.007

Eckhorn, R., 1999. Neural mechanisms of scene segmentation: recordings from the visual cortex suggest basic circuits for linking field models. IEEE Trans Neural Netw 10, 464-479. doi:10.1109/72.761705

Eckhorn, R., 2000. Cortical synchronization suggests neural principles of visual feature grouping. Acta Neurobiol Exp 60, 261-269.

Eckhorn, R., Bruns, A., Saam, M., Gail, A., Gabriel, A., Brinksmeyer, H.J., 2001. Flexible cortical gammaband correlations suggest neural principles of visual processing. Vis. cogn. 8, 519-530. doi:10.1080/13506280143000098

Ermentrout, G.B., Kleinfeld, D., 2001. Traveling electrical waves in cortex: insights from phase dynamics and speculation on a computational role. Neuron 29, 33-44.

Frenkel, D., Smit, B., 2001. Understanding molecular simulation: from algorithms to applications. Academic Press.

Fries, P., Nikolic, D., Singer, W., 2007. The gamma cycle. Trends Neurosci 30, 309-316. doi:10.1016/j.tins.2007.05.005

Gail, A., Brinksmeyer, H.J., Eckhorn, R., 2000. Contour decouples gamma activity across texture representation in monkey striate cortex. Cereb Cortex 10, 840-850.

Gattass, R., Gross, C.G., Sandell, J.H., 1981. Visual topography of V2 in the macaque. J. Comp. Neurol. 201, 519-39. doi:10.1002/cne.902010405

Godlove, D.C., Maier, A., Woodman, G.F., Schall, J.D., 2014. Microcircuitry of agranular frontal cortex: testing the generality of the canonical cortical microcircuit. J. Neurosci. 34, 5355-69. doi:10.1523/JNEUROSCI.5127-13.2014

Gong, P., Nikolaev, A.R., van Leeuwen, C., 2007. Intermittent dynamics underlying the intrinsic fluctuations of the collective synchronization patterns in electrocortical activity. Phys. Rev. E 76, 011904. doi:10.1103/PhysRevE.76.011904

Gray, C.M., Singer, W., 1989. Stimulus-specific neuronal oscillations in orientation columns of cat visual cortex. Proc. Natl. Acad. Sci. U. S. A. 86, 1698-702.

Havenith, M.N., Yu, S., Biederlack, J., Chen, N.-H., Singer, W., Nikolić, D., 2011. Synchrony makes neurons fire in sequence, and stimulus properties determine who is ahead. J. Neurosci. 31, 8570-84. doi:10.1523/JNEUROSCI.2817-10.2011

Heitmann, S., Boonstra, T., Breakspear, M., 2013. A dendritic mechanism for decoding traveling waves: principles and applications to motor cortex. PLoS Comput. Biol. 9, e1003260. doi:10.1371/journal.pcbi.1003260

Hermes, D., Miller, K.J., Wandell, B. a, Winawer, J., 2014. Stimulus Dependence of Gamma Oscillations in Human Visual Cortex. Cereb. Cortex 1-9. doi:10.1093/cercor/bhu091

Hinton, G.E., 2000. Computation by neural networks. Nat. Neurosci. 3 Suppl, 1170. doi:10.1038/81442 Hopfield, J.J., Brody, C.D., 2001. What is a moment? Transient synchrony as a collective mechanism for spatiotemporal integration. Proc Natl Acad Sci U S A 98, 1282-1287. doi:10.1073/pnas.031567098 
Hoppensteadt, F.C., Izhikevich, E.M., 1996. Synaptic organizations and dynamical properties of weakly connected neural oscillators. I. Analysis of a canonical model. Biol Cybern 75, 117-127.

Hoppensteadt, F.C., Izhikevich, E.M., 1998. Thalamo-cortical interactions modeled by weakly connected oscillators: could the brain use FM radio principles? Biosystems 48, 85-94.

Huang, N.E., 2005. Introduction to the Hilbert-Huang Transform and its related mathematical problems. Hilbert-Huang Transform Its Appl. 1-26. doi:doi:10.1142/9789812703347_0001

Huang, N.E., Shen, Z., Long, S.R., Wu, M.C., Shih, H.H., Zheng, Q., Yen, N.-C., Tung, C.C., Liu, H.H., 1998. The empirical mode decomposition and the Hilbert spectrum for nonlinear and non-stationary time series analysis. Proc. R. Soc. A Math. Phys. Eng. Sci. doi:10.1098/rspa.1998.0193

Jia, X., Tanabe, S., Kohn, A., 2013a. $y$ and the coordination of spiking activity in early visual cortex. Neuron 77, 762-74. doi:10.1016/j.neuron.2012.12.036

Jia, X., Xing, D., Kohn, A., 2013b. No consistent relationship between gamma power and peak frequency in macaque primary visual cortex. J Neurosci 33, 17-25. doi:10.1523/JNEUROSCI.1687-12.2013

Kayser, C., 2003. Responses to Natural Scenes in Cat V1. J. Neurophysiol. 90, 1910-1920. doi:10.1152/jn.00195.2003

Kozma, R., Freeman, W.J., 2008. Intermittent spatio-temporal desynchronization and sequenced synchrony in ECoG signals. Chaos An Interdiscip. J. Nonlinear Sci. 18, 037131. doi:10.1063/1.2979694

Kuntimad, G., Ranganath, H.S., 1999. Perfect image segmentation using pulse coupled neural networks. IEEE Trans. Neural Netw. 10, 591-8. doi:10.1109/72.761716

Lachaux, J.P., Rodriguez, E., Martinerie, J., Varela, F.J., 1999. Measuring phase synchrony in brain signals. Hum Brain Mapp 8, 194-208.

London, M., Häusser, M., 2005. Dendritic computation. Annu. Rev. Neurosci. 28, 503-32. doi:10.1146/annurev.neuro.28.061604.135703

Lowet, E., Roberts, M., Hadjipapas, A., Peter, A., van der Eerden, J., De Weerd, P., 2015. InputDependent Frequency Modulation of Cortical Gamma Oscillations Shapes Spatial Synchronization and Enables Phase Coding. PLoS Comput. Biol. 11, e1004072. doi:10.1371/journal.pcbi.1004072

Maier, A., Adams, G.K., Aura, C., Leopold, D.A., 2010. Distinct superficial and deep laminar domains of activity in the visual cortex during rest and stimulation. Front. Syst. Neurosci. 4. doi:10.3389/fnsys.2010.00031

Malsburg, C. von der, 1995. Binding in models of perception and brain function. Curr. Opin. Neurobiol. 5, 520-526. doi:10.1016/0959-4388(95)80014-X

Maris, E., Womelsdorf, T., Desimone, R., Fries, P., 2013. Rhythmic neuronal synchronization in visual cortex entails spatial phase relation diversity that is modulated by stimulation and attention. Neuroimage 74, 99-116. doi:10.1016/j.neuroimage.2013.02.007

Masquelier, T., Hugues, E., Deco, G., Thorpe, S.J., 2009. Oscillations, phase-of-firing coding, and spike timing-dependent plasticity: an efficient learning scheme. J Neurosci 29, 13484-13493. doi:10.1523/JNEUROSCI.2207-09.2009

McLelland, D., Paulsen, O., 2009. Neuronal oscillations and the rate-to-phase transform: mechanism, model and mutual information. J. Physiol. 587, 769-785. doi:10.1113/jphysiol.2008.164111 
Montemurro, M.A., Rasch, M.J., Murayama, Y., Logothetis, N.K., Panzeri, S., 2008. Phase-of-firing coding of natural visual stimuli in primary visual cortex. Curr. Biol. 18, 375-80. doi:10.1016/j.cub.2008.02.023

Palanca, B.J.A., DeAngelis, G.C., 2005. Does neuronal synchrony underlie visual feature grouping? Neuron 46, 333-46. doi:10.1016/j.neuron.2005.03.002

Panzeri, S., Brunel, N., Logothetis, N.K., Kayser, C., 2010. Sensory neural codes using multiplexed temporal scales. Trends Neurosci. 33, 111-20. doi:10.1016/j.tins.2009.12.001

Pettersen, K.H., Dale, A.M., Einevoll, G.T., Lindén, H., 2011. Extracellular spikes and CSD. Handb. Neural Act. Meas. 92 - 135. doi:10.1017/СB09780511979958

Pikovsky, A., Rosenblum, M., Kurths, J., Hilborn, R.C., 2002. Synchronization: A Universal Concept in Nonlinear Science. Am. J. Phys. 70, 655. doi:10.1119/1.1475332

Ray, S., Maunsell, J.H.R., 2010. Differences in gamma frequencies across visual cortex restrict their possible use in computation. Neuron 67, 885-896. doi:10.1016/j.neuron.2010.08.004

Ritz, R., Sejnowski, T.J., 1997. Synchronous oscillatory activity in sensory systems: new vistas on mechanisms. Curr Opin Neurobiol 7, 536-546.

Roberts, M.J., Lowet, E., Brunet, N.M., Ter Wal, M., Tiesinga, P., Fries, P., De Weerd, P., 2013. Robust gamma coherence between macaque V1 and V2 by dynamic frequency matching. Neuron 78, $523-$ 36. doi:10.1016/j.neuron.2013.03.003

Salinas, E., Sejnowski, T.J., 2001. Correlated neuronal activity and the flow of neural information. Nat Rev Neurosci 2, 539-550. doi:10.1038/35086012

Schafer, R.W., 2011. What is a savitzky-golay filter? IEEE Signal Process. Mag. 28, 111-117. doi:10.1109/MSP.2011.941097

Schwemmer, M.A., Lewis, T.J., 2012. Phase Response Curves in Neuroscience, in: Phase Response Curves in Neuroscience. pp. 3-31. doi:10.1007/978-1-4614-0739-3

Singer, W., 1999. Neuronal Synchrony: A Versatile Code for the Definition of Relations? Neuron 24, 4965. doi:10.1016/S0896-6273(00)80821-1

Singer, W., Gray, C.M., 1995. Visual Feature Integration and the Temporal Correlation Hypothesis. Annu Rev Neurosci. doi:10.1146/annurev.ne.18.030195.003011

Stettler, D.D., Das, A., Bennett, J., Gilbert, C.D., 2002. Lateral Connectivity and Contextual Interactions in Macaque Primary Visual Cortex. Neuron 36, 739-750. doi:10.1016/S0896-6273(02)01029-2

Tiesinga, P., Fellous, J.-M., Sejnowski, T.J., 2008. Regulation of spike timing in visual cortical circuits. Nat. Rev. Neurosci. 9, 97-107. doi:10.1038/nrn2315

Tiesinga, P.H., Sejnowski, T.J., 2010. Mechanisms for Phase Shifting in Cortical Networks and their Role in Communication through Coherence. Front Hum Neurosci 4, 196. doi:10.3389/fnhum.2010.00196

Tort, A.B.L., Komorowski, R., Eichenbaum, H., Kopell, N., 2010. Measuring phase-amplitude coupling between neuronal oscillations of different frequencies. J. Neurophysiol. 104, 1195-1210. doi:10.1152/jn.00106.2010

Vaknin, G., DiScenna, P.G., Teyler, T.J., 1988. A method for calculating current source density (CSD) analysis without resorting to recording sites outside the sampling volume. J. Neurosci. Methods 24, 131-135. doi:10.1016/0165-0270(88)90056-8 
Van Pelt, S., Fries, P., 2013. Visual stimulus eccentricity affects human gamma peak frequency. Neuroimage 78, 439-447. doi:10.1016/j.neuroimage.2013.04.040

Vinck, M., Lima, B., Womelsdorf, T., Oostenveld, R., Singer, W., Neuenschwander, S., Fries, P., 2010. Gamma-phase shifting in awake monkey visual cortex. J Neurosci 30, 1250-1257. doi:10.1523/JNEUROSCI.1623-09.2010

Wang, D., Terman, D., 1997. Image Segmentation Based on Oscillatory Correlation. Neural Comput. 9 , 805-836. doi:10.1162/neco.1997.9.4.805

Womelsdorf, T., Fries, P., 2007. The role of neuronal synchronization in selective attention. Curr. Opin. Neurobiol. 17, 154-60. doi:10.1016/j.conb.2007.02.002

Xing, D., Shen, Y., Burns, S., Yeh, C.-I., Shapley, R., Li, W., 2012. Stochastic generation of gamma-band activity in primary visual cortex of awake and anesthetized monkeys. J. Neurosci. 32, 13873-80a. doi:10.1523/JNEUROSCI.5644-11.2012 


\section{Supporting Materials}

A

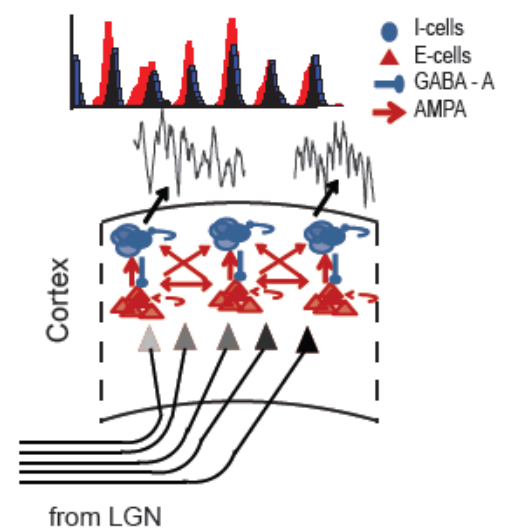

B

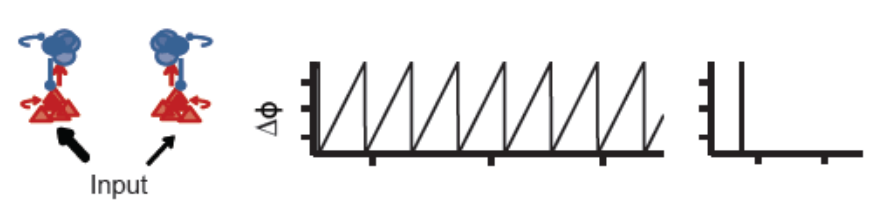

C

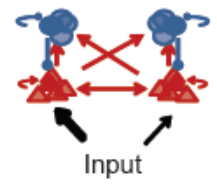

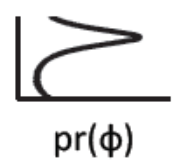

D



E



F


Time lag (ms)
G
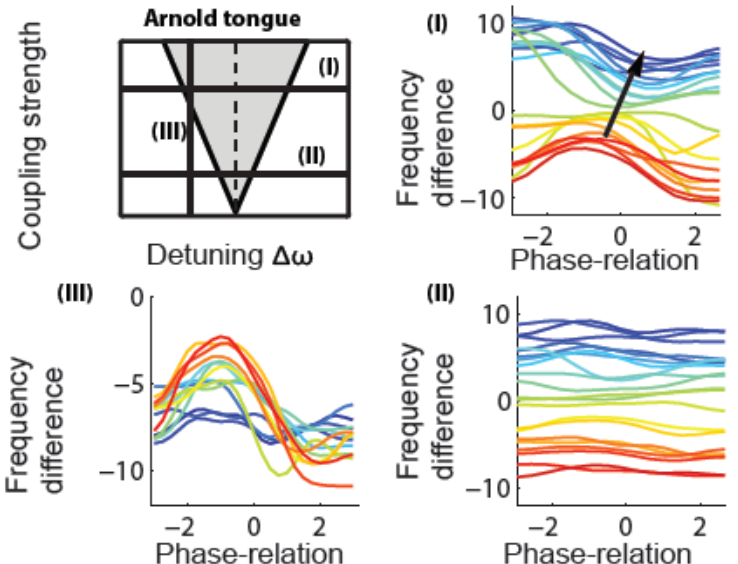

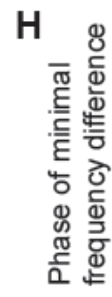

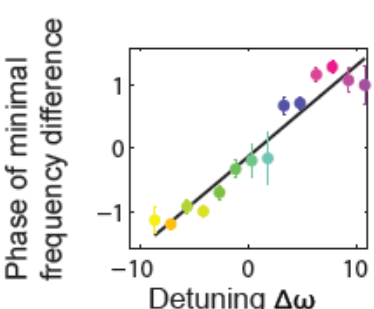

I

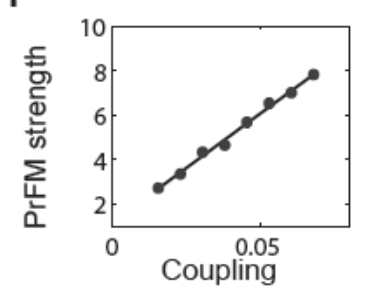

Suppl. Figure 1: Simulation of two interacting Hodgkin-Huxley excitatory-inhibitory networks. A) General model conception of visual cortical gamma synchronization like V1. Visual input-dependent from the LGN drives neural activity in V1. The strength of the drive (here colored form light to dark) depend on visual input parameter (e.g. visual contrast). The LGN drive affects the dynamics excitatory-inhibitory networks as presented in the superficial layers. The oscillation frequency increases with increasing drive. Nearby cortical locations produces different gamma rhythms, however the different locations interact through horizontal interactions. In B) und C) the effect of horizontal interactions on the gamma rhythms is shown. B) With no horizontal interactions between two networks, the phase-relation distribution is uniform and hence completely asynchronous. C) With horizontal interactions the phase-relation distribution is non-uniform and hence there is synchronization. Due to intrinsic noise and frequency differences (= Detuning) the phase-locking is not complete, but the networks exhibit phase precession yet also a preferred phase-relation at the same time. Further, the phase precession speed (freq2uency difference) is not 
constant anymore, but systematically changes with phase-relation. This regime is called the partially synchronized state. (D-F) Plotting PLV and phase-relation as a function of coupling strength and detuning. The area within the 2D parameter space of higher phase-locking is called the Arnold Tongue. D) The Arnold tongue reconstruction where hot color represent the PLV between the two networks. The Y-axis is synaptic coupling strength (with top high and bottom low). The X-axis is excitatory input drive difference between the two networks (detuning). E) The Arnold tongue reconstruction with hsv-color and same axis definition representing phase-relation. F) Three examples (1-3) where the cross-correlation is shown of the networks signals. The positions of the examples are depicted on the Arnold tongues of $\mathrm{H}$ and $\mathrm{J}$ as small numbers. (G-I) The quantification of the partially synchronized state. G) A characteristic property of partially synchronized state is phase-relation dependent frequency modulations (PrFM). Here we plotted the modulation of the instantaneous frequency difference between the two networks as a function of their phase-relation. We plotted in (I-III) three cross-sections of the Arnold tongue, where each point along the cross-sections represents a line color. H) Quantification of the phase shifting of the minimal absolute frequency difference as a function of detuning. I) The quantification of the PrFM value as a function of synaptic coupling strength. 


\section{Chapter 8: Summary and Conclusions}

The primary aim of this thesis is to understand the mechanisms and the significance of the frequency variations of gamma oscillation for its synchronization behavior and for information processing, using theoretical as well as experimental techniques. We had to integrate our theoretical approaches with empirical targeted neurophysiological experiments in monkeys, to test model predictions in empirical data. Contrary to currently emerging views that seemingly random variations in frequency render gamma useless for encoding and neuronal communication (Burns et al., 2011; Ray and Maunsell, 2010), our work indicates that frequency variation in the visual cortical gamma rhythm is essential for understanding how synchronization may contribute to the encoding of input (as well as to neural communication within and between cortical areas).

In the experimental work using LFP/CSD simultaneous recording in macaque visual areas V1 and V2 (Chapter 2), we show that the frequency of gamma oscillations in V1 and V2 shifts in a similar manner with stimulus grating contrast. Moreover, the frequency of V1 and V2 gamma oscillations always matched over the different conditions and on a moment-by-moment basis (single trial dynamics). Importantly, despite stimulus-driven changes in gamma frequency and despite momentary frequency variations at a fixed contrast, V1 and V2 gamma showed substantial coherence. The manner in which coherence emerged was in line with anatomical expectations. In particular, V1 gamma oscillations influenced V2 gamma in a feed-forward manner and exhibited a laminar-specific coherence pattern across the cortical areas in line with anatomically described feedforward connectivity. This work showed that despite of the important variation of gamma frequency, spatially separated cortical network can maintain coherence. This contradicts previous suggestions and views that this variation would prevent meaningful inter-areal communication (Burns et al., 2011; Ray and Maunsell, 2010). Instead, our data suggested an active mechanism by which gamma frequency was matched in order to permit synchronization and neural communication.

The gamma frequency dependency on visual contrast, demonstrated in monkey microelectrode recording, could be confirmed in the human primary visual cortex using magneto-encephalography (MEG) as well (Chapter 3). Similar to the monkey early visual cortex, the frequency increased with visual contrast in human visual cortex. In addition, we showed that gamma power increased with contrast, yet

in contrast to macaque cortex, without a saturation component. Furthermore, we described for the first time systematic asymmetries in the power spectral profile of gamma oscillations. This work extends the work done on monkey visual cortex to human visual cortex, and is important as theoretical models on gamma synchronization described in monkeys (see chapters 5 and 7) are likely to also apply to humans.

One of the features of gamma brought to the foreground in the empirical work in the first two chapters, pointed towards the presence of strong V1/V2 gamma frequency variation over time within a particular condition. To improve understanding of these fast time-scale variation, we analyzed the V1-V2 neural data from Chapter2 with the aim of understanding whether the seemingly random frequency fluctuations (as described by (Burns et al., 2011) is instead structured by other brain processes (Chapter 
4). We found that gamma frequency fluctuations are temporally structured with a rhythmic component showing peak power in the $3-4 \mathrm{~Hz}$ range (low theta band). By theta-windowing analysis, we showed that the frequency, the power as well as the coherence between V1 and V2 gamma is modulated by a low theta rhythm. In addition we showed that this low theta rhythm is closely related to fixational eye movements (micro-saccades) that occur 3-4 times per second. This work showed that rapid fluctuations of gamma frequency are not random, but instead are complex and systematically depending on slower time-scale rhythmic fluctuations.

In Chapter 5 numerical simulation techniques were used to study the role and implication of systematically changing oscillation frequency. We used conductance-based neuronal models (HodgkinHuxley equations) to simulate excitatory-inhibitory networks. In addition, we used an abstract phaseoscillator model (that can be used for analytical analysis) to represent the essential dynamics occurring in the complex excitatory-inhibitory networks. We found that oscillation frequency, modulated by input variations among neighboring neuronal pools, is a critical variable to determine phase-locking as well as phase-relation among those locally connected neurons. We systematically described the relation of phase-locking, frequency and phase-relation and found that the weakly coupled oscillatory theory (TWCO) could well explain the synchronization patterns emerging in response to distributed patterns of input to the network. Our simulations showed that the specific synchronization response to distributed input to a neural network, which consisted of predictable frequency and phase relations among local units in the network, effectively captured the properties of the input. Hence, the performed simulations show that frequency variations, rather than being detrimental, are useful for information processing. Moreover, a subset of the simulations also directly demonstrated how the TWCO based networks showed self-organization into synchronization fields that could be useful feature integrations in natural visual scenes. This simulation hence supports the idea of a 'local' version of binding theory (see Introduction).

The TWCO based simulations, the assumption is made that weak coupling can be compared to horizontal connectivity among columns, and that input sets intrinsic frequency. While the performance I these networks in encoding input seemed promising in simulations, we aimed to test the clear predictions on the properties of synchronization in experimental data. As our recordings and simulations revealed the non-stationary nature of gamma, we anticipated that we would need a new method for analyzing phase relations in empirical recording data. To that aim, we first tested whether standard oscillatory synchronization detection methods are trustable for fast-varying oscillations. Hence, in Chapter 6 we studied the implication of fast-varying frequency and power fluctuation of gamma oscillations for commonly used phase-locking measures (coherence) that are based on the Fourier Transform. Commonly used Fourier-based phase-locking methods assume stationarity which is violated by these rapid fluctuations. We show how badly Fourier-based phase-locking methods fail when applied to non-stationary data, and document the underlying reasons why under these circumstances these commonly used methods fail. In this work, the concept of non-stationarity is developed, and demonstrated in the form of the partially synchronized state, which is characterized by the occurrence of phase-relation dependent frequency fluctuations (PrFM). Although in general it may be considered a 
given that Fourier based phase locking methods should not be applied to non-stationary data, these methods are commonly used without verification of non-stationarity, and in the face of the large probability that in fact the data will be non-stationary. As an alternative approach, we suggest to use non-stationary methods based on the Hilbert Transform or Wavelet Transform. We show as a concrete alternative the results from a combined application of singular-spectrum decomposition (SSD) and Hilbert Transform. This approach clearly yielded superior results compared to common stationary methods in determining the correct phase-locking value.

In Chapter 7, we used experimentally controlled spatial contrast variations to manipulate intrinsic frequency variations across the retinotopic map of macaque V1, and measured the effects of these frequency variations on synchronization behavior for varying distances among local pools of neurons exposed to these different input levels. This was achieved by simultaneously recording with three laminar probes in macaque visual cortex V1 for various variations in local stimulus contrast. In this experimental setup, we aimed to test the precise predictions from TWCO regarding the emergence of specific frequencies as well as modulations of phase-locking and phase-relations of gamma oscillations as a function of intrinsic frequency differences (i.e. set by stimulus input). The results show for the first time that cortical gamma oscillations behave in accordance to the predictions of the weakly coupled oscillator theory (by reconstructing the Arnold tongue). In addition, we show for the first time the existence of partially synchronized state in gamma oscillations and the presence of phase-relation dependent frequency fluctuations (PrFM), necessitating the use of non-linear approaches for the estimation of phase locking.

The most important contributions of the thesis to the current literature are the following. First, the work presented in the thesis demonstrates, in accordance with recent reports from other labs (Burns et al., 2011; Jia et al., 2013; Ray and Maunsell, 2010), that oscillation frequency rapidly fluctuates over time and changes systematically with stimulus input and other experimental manipulations (and induced cognitive states). However, we found that the frequency fluctuations are structured, shaped by the oculomotor system, and shared among interacting visual cortical areas. This underlines the importance of distinguishing truly (non-deterministic) random processes from processes which are highly complex, yet deterministic. Second, our work underlines the necessity to use spectral data analysis methods that can capture the fast dynamics and complexity that we have shown to be present in what can be considered typical LFP data. These methods, such as the SSD/Hilbert-based methods we contributed, should not assume stationarity and preferably should capture linear as well as non-linear components. Third, starting from our own observations and that of other labs showing that neural gamma oscillations adapt their frequency in an input-specific manner, we investigated according to which theoretical models this input dependency of gamma could be exploited by the brain for computation. We showed theoretically as well as experimentally that frequency variations are critical for determining the phaselocking and phase-relation among synchronizing neurons. The combined theoretical and experimental analysis performed, identified the mathematical principles of TWCO as determining synchronization behavior. This represents an important step forward in moving from a descriptive analysis of synchronization towards an analysis of mathematical principles with predictive power. Fourth, as a 
direct result of identifying a mathematical model that provides principles of synchronization, the presented work also puts a more realistic scope on the contribution of gamma in encoding, limiting it to (local) interactions among neurons that are closely connected to each other. Fifth, with respect to the discussion in the literature whether synchronization of spikes or spike time differences are important for neural coding, the present work shows that both parameters, namely phase-locking (spike synchrony) and phase-relation (spike timing), can be seen as important for understanding the mechanism of neural coding (Buehlmann and Deco, 2010; Caporale and Dan, 2008; Hopfield and Brody, 2001; Ritz and Sejnowski, 1997; Stanley, 2013; Tiesinga et al., 2008). Sixth, we directly demonstrated in our empirical data the existence of the partially synchronized state, which is associated with small frequency differences. This has not only implications for data analysis (see point two), but also invites a rethinking of the concept of synchronization and how it contributes to neural processing and coding. In the partially synchronized synchronization regime, which is predicted by TWCO, oscillators are neither completely synchronized nor asynchronous, but exhibit periods of 'phase-locking' intermixed with periods of fast phase precession. This type of synchronization induces rapid phase-relation dependent frequency and amplitude fluctuations. This synchronization regime, in line with the concept of criticality (Beggs, 2008; Daido, 1990; Yang et al., 2012), is not an undesirable or imperfect state of synchronization, but rather a regime (between complete order and disorder) that permits fast and flexible changes in synchronization patterns when input of brain state is changing. The system is neither in complete synchrony (as occurring during epilepsy) where differences are minimized and the information capacity is small, nor in complete asynchrony (random phase-relations) where information cannot be encoded. This in-between regime, which on the surface appears complex and random-like, is instead inherently systematic and structured, and might be considered 'meaningful', as suggested in various other systems (Bak et al., 1988). The flexibility of the partially synchronized state can also be understood in the context of attractor states in neural network functioning. In a partially synchronized state, the activity pattern in the system is influenced by an 'attractor state' but not completely captured by it, so that it can more easily switch from one attractor state to another (Eliasmith, 2005).

Taking all of these points together, our work demonstrates, seemingly counter-intuitively, that small frequency differences among interacting oscillations, is not detrimental but instead critical for establishing meaningful synchronization patterns that are flexible enough to represent fluctuating inputs and brain states, and hence that are useful for neural computation. We suggest that the conceptual advances made by the work included in this thesis will provide a basis to better understand the role of oscillatory synchronization for information processing, transmission and learning in visual and other systems. 


\section{References}

Bak, P., Tang, C., Wiesenfeld, K., 1988. Self-organized criticality. Phys. Rev. A 38, 364-374. doi:10.1103/PhysRevA.38.364

Beggs, J.M., 2008. The criticality hypothesis: how local cortical networks might optimize information processing. Philos. Trans. A. Math. Phys. Eng. Sci. 366, 329-43. doi:10.1098/rsta.2007.2092

Buehlmann, A., Deco, G., 2010. Optimal information transfer in the cortex through synchronization. PLoS Comput. Biol. 6. doi:10.1371/journal.pcbi.1000934

Burns, S.P., Xing, D., Shapley, R.M., 2011. Is gamma-band activity in the local field potential of V1 cortex a "clock" or filtered noise? J Neurosci 31, 9658-9664. doi:10.1523/JNEUROSCI.0660-11.2011

Caporale, N., Dan, Y., 2008. Spike timing-dependent plasticity: a Hebbian learning rule. Annu. Rev. Neurosci. 31, 25-46. doi:10.1146/annurev.neuro.31.060407.125639

Daido, H., 1990. Intrinsic fluctuations and a phase transition in a class of large populations of interacting oscillators. J. Stat. Phys. 60, 753-800. doi:10.1007/BF01025993

Eliasmith, C., 2005. A unified approach to building and controlling spiking attractor networks. Neural Comput. 17, 1276-1314. doi:10.1162/0899766053630332

Hopfield, J.J., Brody, C.D., 2001. What is a moment? Transient synchrony as a collective mechanism for spatiotemporal integration. Proc Natl Acad Sci U S A 98, 1282-1287. doi:10.1073/pnas.031567098

Jia, X., Xing, D., Kohn, A., 2013. No consistent relationship between gamma power and peak frequency in macaque primary visual cortex. J Neurosci 33, 17-25. doi:10.1523/JNEUROSCI.1687-12.2013

Ray, S., Maunsell, J.H.R., 2010. Differences in gamma frequencies across visual cortex restrict their possible use in computation. Neuron 67, 885-896. doi:10.1016/j.neuron.2010.08.004

Ritz, R., Sejnowski, T.J., 1997. Synchronous oscillatory activity in sensory systems: new vistas on mechanisms. Curr Opin Neurobiol 7, 536-546.

Stanley, G.B., 2013. Reading and writing the neural code. Nat. Neurosci. 16, 259-63. doi:10.1038/nn.3330

Tiesinga, P., Fellous, J.-M., Sejnowski, T.J., 2008. Regulation of spike timing in visual cortical circuits. Nat. Rev. Neurosci. 9, 97-107. doi:10.1038/nrn2315

Yang, H., Shew, W.L., Roy, R., Plenz, D., 2012. Maximal variability of phase synchrony in cortical networks with neuronal avalanches. J. Neurosci. 32, 1061-72. doi:10.1523/JNEUROSCI.277111.2012 


\section{Appendix}

\section{Knowledge Valorization}

The work presented in this thesis was aimed to deepen our understanding of the underlying principles of cortical gamma synchronization which is a basic element of neural dynamics and computation. The work should be categorized as being 'fundamental research' that is directed to generate new knowledge underlying a natural phenomena. Fundamental research establishes the knowledge on which 'applied research' can solve concrete problems arising from society. Both, fundamental and applied research, necessitate each other and need both to be pursued equally (Poyago-Theotoky, 2002).

Because it was fundamental research, no direct valorization for society (beside increase in knowledge) can be scientifically demonstrated here. Future application-directed research will need to evaluate how this knowledge can be used for concrete problems. However, we will shortly discuss which applications fields could potentially use the insights from the thesis in a fruitful manner.

In clinical neuroscience, gamma synchronization has been discussed in the context of various psychiatric illnesses (Herrmann and Demiralp, 2005), for example in the context of psychosis/schizophrenia (Lee et al., 2003; Symond et al., 2005). It was found that gamma oscillations are profoundly affected in patients with psychosis(Haig et al., 2000). The causes of gamma oscillation deterioration is not well established nor the role of these deterioration for explaining the cognitive disabilities (Lee et al., 2003). Previous work has concentrated on gamma power and gamma coherence for investigating the role of gamma oscillation in psychotic patients. Our work suggests that frequency variations and synchronization principles (sensitivity to coupling and detuning) are critical variables for understanding how gamma oscillations operate. Future research might gain further insights by taking these variables into account.

Another example is the understanding of epilepsy which arise when brain regions get over-synchronized (da Silva et al., 2003; Parra et al., 2003; van Drongelen et al., 2005). Our work suggests that inputdependent frequency variation is critical for controlling synchronization among locally coupled neurons. Understanding the (intrinsic) frequency variations in an epileptic brain region might reveal further insights. In addition, medication or devices that increase the intrinsic frequency variation might be developed to counteract acute epileptic attacks.

The gamma synchronization principles and its linkage to the theory of weakly coupled oscillators have interesting computational properties, as described in chapter 5. For example, oscillatory synchronization has been studied as image segmentation technique and shown to be very powerful (Kuntimad and Ranganath, 1999; Kuzmina et al., 2004; Wang and Terman, 1997). It is therefore likely that the thesis work might inspire development of new or more efficient algorithms for artificial intelligence problems. For example, image segmentation (or generally as clustering algorithm) is useful for example for recognition tasks (e.g. tumor image recognition software). Further, it might help to improve significantly devices developed for blind or half-blind people (Dowling, 2005). 
In summary, despite this work represents fundamental research and the benefits for society will be concretized only in the next years, it is reasonable to expect the improved knowledge will have positive effects in various applications fields including psychiatry and artificial intelligence.

\section{REFERENCES}

Da Silva, F.L., Blanes, W., Kalitzin, S.N., Parra, J., Suffczynski, P., Velis, D.N., 2003. Epilepsies as Dynamical Diseases of Brain Systems: Basic Models of the Transition Between Normal and Epileptic Activity. Epilepsia 44, 72-83. doi:10.1111/j.0013-9580.2003.12005.x

Dowling, J., 2005. Artificial human vision. Expert Rev. Med. Devices 2, 73-85. doi:10.1586/17434440.2.1.73

Haig, A.R., Gordon, E., De Pascalis, V., Meares, R.A., Bahramali, H., Harris, A., 2000. Gamma activity in schizophrenia: evidence of impaired network binding? Clin. Neurophysiol. 111, 1461-1468. doi:10.1016/S1388-2457(00)00347-3

Herrmann, C.S., Demiralp, T., 2005. Human EEG gamma oscillations in neuropsychiatric disorders. Clin. Neurophysiol. 116, 2719-33. doi:10.1016/j.clinph.2005.07.007

Kuntimad, G., Ranganath, H.S., 1999. Perfect image segmentation using pulse coupled neural networks. IEEE Trans. Neural Netw. 10, 591-8. doi:10.1109/72.761716

Kuzmina, M., Manykin, E., Surina, I., 2004. Oscillatory network with self-organized dynamical connections for synchronization-based image segmentation. Biosystems. 76, 43-53. doi:10.1016/j.biosystems.2004.05.005

Lee, K.-H., Williams, L.M., Breakspear, M., Gordon, E., 2003. Synchronous Gamma activity: a review and contribution to an integrative neuroscience model of schizophrenia. Brain Res. Rev. 41, 57-78. doi:10.1016/S0165-0173(02)00220-5

Parra, J., Kalitzin, S.N., Iriarte, J., Blanes, W., Velis, D.N., Lopes da Silva, F.H., 2003. Gamma-band phase clustering and photosensitivity: is there an underlying mechanism common to photosensitive epilepsy and visual perception? Brain 126, 1164-1172. doi:10.1093/brain/awg109

Poyago-Theotoky, J., 2002. Universities and Fundamental Research: Reflections on the Growth of University-Industry Partnerships. Oxford Rev. Econ. Policy 18, 10-21. doi:10.1093/oxrep/18.1.10

Symond, M.P., Symond, M.B., Harris, A.W.F., Gordon, E., Williams, L.M., 2005. "Gamma synchrony" in first-episode schizophrenia: a disorder of temporal connectivity? Am. J. Psychiatry 162, 459-65. doi:10.1176/appi.ajp.162.3.459

Van Drongelen, W., Lee, H.C., Hereld, M., Chen, Z., Elsen, F.P., Stevens, R.L., 2005. Emergent epileptiform activity in neural networks with weak excitatory synapses. IEEE Trans. Neural Syst. Rehabil. Eng. 13, 236-41. doi:10.1109/TNSRE.2005.847387

Wang, D., Terman, D., 1997. Image Segmentation Based on Oscillatory Correlation. Neural Comput. 9, 805-836. doi:10.1162/neco.1997.9.4.805 


\section{Acknowledgments}

I would like acknowledge first my love Aafke van den Berg for her support in all difficult moments to finish my thesis.

I would like to thank deeply my family. My parents Nicole and Mark Lowet to have supported me so much during school and university, also during difficult times. Without their belief in me, I would not be here. Also my sisters Florence Hein and Cecile Lowet for their endless support and encouragements. I would like to generally thank my whole family.

Special thank I would like to give to my supervisors Prof.Dr.Peter de Weerd and Dr.Mark Roberts who made my first steps in science possible. I always enjoyed working in the group and appreciated much the scientific discussions as well as the creativity and open-mindedness. I am immensely grateful for their support during my Master and PhD.

I would like to give a particular thank to colleagues who were important for my development as a scientist that includes Alina Peter, Dr.Avgis Hadjipapas, Dr.Jan van der Eerden, Bart Gips, Prof.Dr. Pascal Fries and Dr.Cristiano Michaelis, Maarten Leenders and many more. I would also like to thank Dr.Christine Schiltz, my former bachelor supervisor.

Much thanks also to my school friends Oliver Pelzer, David Schuster, Moritz Michels, Lukas Bauer and David Haas.

Last but not least: a big thank to all my colleagues in Maastricht and Nijmegen for the nice moments, particularly in 'cultuur café '. 


\section{Curriculum Vitae}

Eric Lowet was born the $6^{\text {th }}$ November 1984 in Valenciennes in France at the same time with his twin sister Florence and three years after his sister Cecile. The proud parents are Mark and Nicole Lowet. After a few years in France his family moved to Germany. There he got his elementary and high-school education. He received his Abitur in 2005. From 2005-2008 he followed a Bachelor program in Psychology, with a focus on Bio-psychology, in Luxembourg. He moved then to Maastricht in the Netherlands to join the research master program in cognitive neuroscience (2008-2010). In 2010 he moved to Nijmegen for a master internship to work on monkey neurophysiology and computational neuroscience under the supervision of Peter de Weerd and Mark Roberts. He continued to work on in the research group, by starting a PhD which should last till 2015. 


\section{Publications}

Bonizzi, P., Karel, J., De Weerd, P., Lowet, E., Roberts, M., Westra, R., .. Peeters, R. (2012). Singular spectrum analysis improves analysis of local field potentials from macaque V1 in active fixation task. Conference Proceedings : ... Annual International Conference of the IEEE Engineering in Medicine and Biology Society. IEEE Engineering in Medicine and Biology Society. Annual Conference, 2012, 2945-8. doi:10.1109/EMBC.2012.6346581

E.Lowet, M. J. Roberts, P. De Weerd. (n.d.). Gamma-band coordination of synchrony and spike timing in visual cortex predicted by the theory of weakly coupled oscillators. In Preparation.

E.Lowet, M. J. Roberts, C.A.Bosman, P. Fries and P. De Weerd. (n.d.). Areas V1 and V2 show microsaccade-related 3-4 Hz covariation in gamma power and frequency. In Review.

E.Lowet, M. J. Roberts, P.Bonizzi, J. Karel and P. De Weerd. (n.d.). Problems of spectral coherence for quantifying oscillatory synchronization. In Review.

Hadjipapas, A., Lowet, E., Roberts, M. J., Peter, A., \& De Weerd, P. (2015). Parametric variation of gamma frequency and power with luminance contrast: A comparative study of human MEG and monkey LFP and spike responses. NeuroImage. doi:10.1016/j.neuroimage.2015.02.062

Lowet, E., Roberts, M., Hadjipapas, A., Peter, A., van der Eerden, J., \& De Weerd, P. (2015). InputDependent Frequency Modulation of Cortical Gamma Oscillations Shapes Spatial Synchronization and Enables Phase Coding. PLoS Computational Biology, 11(2), e1004072. doi:10.1371/journal.pcbi.1004072

Roberts, M. J., Lowet, E., Brunet, N. M., Ter Wal, M., Tiesinga, P., Fries, P., \& De Weerd, P. (2013). Robust gamma coherence between macaque V1 and V2 by dynamic frequency matching. Neuron, 78(3), 523-36. doi:10.1016/j.neuron.2013.03.003 


\section{Conference presentations and invited talks}

E.Lowet, M.Roberts, A.Hadjipapas, J.v.d.Eerden, P.Fries and P. De Weerd. Robust and narrow-band inter-areal coupling in the gamma to beta range is mediated by a theta rhythm in macaque visual cortex. Washington, DC., US, (SFN) Society for Neuroscience, 2011

E.Lowet, M.Roberts, A.Hadjipapas, J.v.d.Eerden, P.Fries and P. De Weerd. The role of oscillation frequency in network interaction and network state. PIRE PICCS Summer school, 2011

E.Lowet, M.Roberts, P. De Weerd. Gamma frequency dependent horizontal interaction in macaque V1. New Orleans, LA, US, (SFN) Society for Neuroscience, 2012

E.Lowet, A.Hadjipapas, M.Roberts P.Alina, and P. De Weerd. Marked Spectral Shifts Of Gamma Spectra With Stimulus Contrasts: A Comparison Of Human Meg And Macaque Lfp/Spike Data. Barcelona, Spain FENS (Federation of all European neuroscience societies), 2012

E.Lowet. Input-dependent frequency modulation of cortical gamma oscillations shapes spatial synchronization and phase coding. ERNST STRÜNGMANN LECTURES. Frankfurt, Germany ,2014 